\title{
Das Diskriminierungsverbot im Zivilrecht
}

und seine Auswirkungen auf das rechtsgeschäftliche und quasi-

rechtsgeschäftliche Handeln

\section{Dissertation}

\author{
zur Erlangung des Doktorgrades \\ der Juristischen Fakultät \\ der Georg-August-Universität zu Göttingen
}

vorgelegt

von

Matthias Zeckei

aus Wernigerode

Göttingen 2008 
Berichterstatterin: Professor Dr. Christiane Wendehorst Mitberichterstatter: Professor Dr. Rüdiger Krause

Tag der mündlichen Prüfung: 19.05.2008

Gleichzeitig erschienen in elektronischer Form an der Niedersächsischen Staats- und Universitätsbibliothek Göttingen (SUB) - D 7 


\section{Inhaltsverzeichnis:}

Seite

Das Diskriminierungsverbot im Zivilrecht und seine Auswirkungen auf das rechtsgeschäftliche und quasi-rechtsgeschäftliche Handeln

Einleitung, Fragestellung und Gang der Arbeit

Teil 1: Antidiskriminierungsmaßnahmen auf internationaler und nationaler Ebene

$\begin{array}{ll}\text { A. Allgemeiner Gleichheitssatz } & 6\end{array}$

$\begin{array}{ll}\text { B. Geschlechtergleichbehandlung } & 8\end{array}$

C. Besonderer Schutz der (werdenden) Mutter 15

D. Gleichbehandlung der „Rassen“ und nationalen Minderheiten 16

E. Gleichbehandlung von Menschen unterschiedlicher Staatsangehörigkeit 19

F. Gleichbehandlung Behinderter 22

G. Gleichbehandlung in Beschäftigung und Beruf 25

Teil 2: Diskriminierung - Begriffsbestimmung $\quad 31$

A. Diskriminierungsrelevantes Verhalten 31

I. Benachteiligende Ungleichbehandlung - Unmittelbare bzw. direkte $\begin{array}{ll}\text { Diskriminierung } & 31\end{array}$

1. Offene Diskriminierung 32

2. Verdeckte Diskriminierung 33

3. Diskriminierungsdrittwirkung 33

II. Einseitig belastende Behandlung 34

1. Individuelle Vertragsgestaltung 34

a) Hypothetische Ungleichbehandlung 35

b) Resümee - Diskriminierung durch einseitig belastende $\begin{array}{ll}\text { Behandlung } & 37\end{array}$

2. Exkurs: (Sexuelle) Belästigung 38

a) Belästigungsschutz = Diskriminierungsschutz? 39

b) Tatbestandsmerkmal „bezwecken oder bewirken“ 42 
c) Feindliche Umfeldprägung

d) Zurechnung fremden Verhaltens

III. Benachteiligende Gleichbehandlung - Mittelbare bzw. indirekte Diskriminierung

1. (Potenzielle) Benachteiligung

2. Überwiegende Betroffenheit

IV. Anweisung zur Diskriminierung

V. Zusammenfassung

B. Diskriminierungsmerkmale

I. Unbeeinflußbares Merkmal

II. Status als Merkmal

1. Status und statusbedingtes Verhalten

2. Staatsangehörigkeit

III. Verhalten als Merkmal

IV. Unbegrenzte Anzahl potenzieller Diskriminierungsmerkmale

1. „Entstehung“ neuer Diskriminierungsmerkmale

2. „Merkmalsbezogenes Merkmal“

3. Geschützte Merkmale im Rahmen des Allgemeinen Gleichheitssatzes

4. Geschützte Merkmale im Rahmen des $§ 611$ a BGB-A

5. Geschützte Merkmale im Rahmen der mittelbaren Diskriminierung

6. Offener Merkmalskatalog

V. Tatsächlicher und vermeintlicher Merkmalsträger

VI. Zusammenfassung

C. Rechtswidrigkeit des diskriminierungsrelevanten Verhaltens

I. Privatautonomie contra Diskriminierungsschutz

II. Rechtswidrigkeitsprüfung als Ausdruck praktischer Konkordanz 68

III. „Wesentlich und entscheidend“ - Wortbedeutung 70

IV. Diskriminierungsmerkmal als objektive Anforderung 72

$\begin{array}{ll}\text { V. Diskriminierungsmerkmal als rechtliche Anforderung } & 73\end{array}$

$\begin{array}{ll}\text { 1. Beschäftigungsverbote } & 74\end{array}$

2. Fördermaßnahmen $\quad 74$

a) Arten von Fördermaßnahmen $\quad 74$

aa) Relative Quoten $\quad 74$

bb) Absolute Quoten $\quad 75$ 
cc) Nichtdiskriminierende Fördermaßnahmen

b) Exkurs: Zulässigkeit ,positiver Diskriminierung“

c) Resümee

3. Diskriminierungsverbot und Sozialstaatsprinzip

4. Verstoß der nationalen Vorschrift gegen europäisches Recht

VI. Diskriminierungsmerkmal als subjektive Anforderung

1. „Wesentlich- und Entscheidendmachung“

2. Objektive Grenze

3. Resümee - Unsachliche Behandlung rechtswidrig

VII. Zusammenfassung

D. Sachlicher Geltungsbereich des Diskriminierungsverbotes

I. Rechtsgeschäftliches und quasi-rechtsgeschäftliches Handeln

II. Vorfeld des Vertragsschlusses

1. Merkmalsneutrale Ausschreibung

2. Merkmalsneutrale Bewerbungsunterlagen

3. Fragerecht

III. Öffentlich angebotene Verträge

IV. Entgeltlichkeit - Betrachtungen am Beispiel letztwilliger Verfügungen

1. Unbegrenzte Anzahl potenzieller Diskriminierungsmerkmale

2. Diskriminierungsverbot im Rahmen letztwilliger Verfügungen?

a) Sozialpflicht des Erblassers

b) Rechtmäßiges Alternativverhalten

c) Nachweis des Erblassermotivs

d) Pflichtteilsrecht contra Diskriminierungsschutz

e) Recht auf willkürliche Entscheidung oder Diskriminierungsschutz

3. Resümee

V. Gewerbsmäßiges und nichtgewerbsmäßiges Handeln

VI. Verträge ohne engen persönlichen Kontakt zum Vertragspartner

1. Intimsphäre

2. Privatsphäre

3. Sozialsphäre - Betrachtungen am Beispiel arbeitsrechtlicher Kündigungen

a) Unbegrenzte Anzahl potenzieller Diskriminierungsmerkmale

b) Rechtswidrigkeit - Bereichs-abhängige Interessenabwägung 
c) Resümee 117

$\begin{array}{ll}\text { VII. Zusammenfassung } & 118\end{array}$

Teil 3: Auswirkungen des Diskriminierungsverbotes auf das (quasi-)rechtsgeschäftliche

\section{Handeln}

A. Vorbemerkungen 121

I. Sachlicher Grund - Wertausfüllungsfähiger und -bedürftiger Rechtsbegriff 121

II. Rechtswidrigkeitsprüfung einzelfallabhängig 122

III. Kein abschließender Katalog sachlicher Gründe - Rechtsunsicherheit 123

IV. Kriterien für Abwägungsentscheidung bestimmbar 124

B. Unterscheidung zwischen unbeeinflußbarem Merkmal, Status und Verhalten 125

C. „Diskriminierung“ als bloßer Selbstzweck 125

D. Zeitlich (un-)begrenzter Ausschluß 127

E. (Nicht) vertragsbezogene Gründe 128

F. Vorurteile und Generalisierungen $\quad 129$

G. (Vorwerfbare) Störung des Vertragsverhältnisses 130

H. Zusammentreffen der Träger von Diskriminierungsmerkmalen 132

I. Grundsatz des freien Auswahlermessens 133

1. Einschränkung durch das Diskriminierungsverbot? 133

2. „Wie“ der Einschränkung 134

II. § 15 II 2 AGG 136

III. Mittelbare Diskriminierung 137

IV. Relativität des Begriffs der Bestqualifizierung 138

$\begin{array}{ll}\text { V. Zusammenfassung } & 139\end{array}$

J. Authentizitätswahrung 140

K. Interessen Dritter 141

I. Sachlich gerechtfertigte Kundenwünsche 141

II. Diskriminierende Kundenwünsche 143

1. Mindereinnahmen - Zumutbarer Grad der wirtschaftlichen Einbuße 144

2. Nichtbeachtung des diskriminierenden Kundenwunsches nicht sanktionierbar 145

3. Mehreinnahmen 146

III. Öffentliche Interessen $\quad 147$

$\begin{array}{ll}\text { IV. Zusammenfassung } & 147\end{array}$ 
L. Zusammentreffen von Diskriminierungsmerkmalen in einer Person 148

$\begin{array}{ll}\text { I. Unbewußtes Zusammentreffen } & 148\end{array}$

1. Absolute Differenzierungsverbote 149

a) „Wegen eines in $\S 1$ genannten Grundes“ - § 7 I AGG 149

b) Kein Recht auf gleichen Lohn für gleiche/gleichwertige Arbeit $\quad 150$

c) „Wegen eines [anderen als] in 1 AGG genannten Grundes“ 151

2. „Diskriminierung“ gestützt auf den (un-)sachlichen Grund? 152

II. Bewußtes Zusammentreffen - Motivbündel 153

1. Kausalität des (un-)sachlichen Grundes für die Entscheidung 155

2. Keine Sanktionierung des diskriminierenden Motivs 155

III. Zeitlich versetztes Zusammentreffen - § 15 II AGG 157

IV. Nachschieben von sachlichen Gründen $\quad 159$

1. Beweisrechtliches Problem 159

2. Voraussetzungen zulässigen Nachschiebens sachlicher Gründe $\quad 160$

3. Keine Offenlegungspflicht der Entscheidungsgründe 161

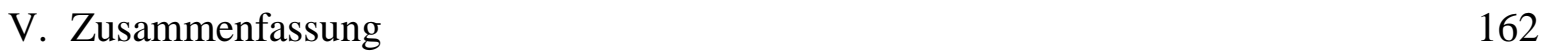

$\begin{array}{ll}\text { M. Tendenzschutz } & 163\end{array}$

I. Geltung des Diskriminierungsverbotes in Tendenzbetrieben 164

1. Art. 137 III, VII WRV i. V. m. Art. 140 GG 164

2. Art. 4 II Richtlinie 2000/78/EG 165

II. Tendenz als Anknüpfungspunkt 166

1. Kernbereich der Tätigkeit des Tendenzbetriebes 167

2. Randbereich der Tätigkeit des Tendenzbetriebes 167

a) Keine Privilegierung gegenüber dem „Nicht-Tendenzbetrieb“ 168

b) Tendenzträgerschaft 168

III. Sonstiges Merkmal als Anknüpfungspunkt 169

1. Kernbereich der Tätigkeit des Tendenzbetriebes 169

2. Randbereich der Tätigkeit des Tendenzbetriebes 170

IV. Pflicht zu tendenzdefensivem Verhalten 172

V. Tendenzschutz außerhalb des Arbeitsrechts 173

1. Tendenz als Anknüpfungspunkt 173

2. Sonstiges Merkmal als Anknüpfungspunkt 174

VI. Tendenzschutz im „Nicht-Tendenzbetrieb“ 174

$\begin{array}{ll}\text { VII. Zusammenfassung } & 176\end{array}$ 
N. Unmöglichkeit der Vertragsdurchführung

I. Ermöglichungspflicht

1. Art. 5 Richtlinie 2000/78/EG

2. $\S 81$ IV SGB IX

3. $§ 4$ I Nr. 2 a Gaststättengesetz

II. Grenzen der Ermöglichungspflicht

III. Zusammenfassung

O. Vertragsanpassungspflicht

I. Diskriminierungsschutz nicht auf formale Gleichbehandlung beschränkt

II. Vertragsanpassungspflichten de lege lata

1. $\S \S 2,16 \mathrm{MuSchG}$

2. $\S 554$ a BGB

3. $\S 242 \mathrm{BGB}, 106 \mathrm{GewO}$

III. Zusammenfassung

Teil 4: Gesetzestechnische Umsetzung des Diskriminierungsverbotes

A. Notwendigkeit legislativer Maßnahmen

I. Auswirkungen der Antidiskriminierungsmaßnahmen auf das Privatrechtsverhältnis

1. Innerstaatliche Geltung der internationalen Antidiskriminierungsmaßnahmen

a) Völkerrechtliche Verträge

aa) Transformationstheorie

bb) Inkorporations-, Adoptions-, Vollzugstheorie

cc) Stellungnahme

b) Völkergewohnheitsrecht

c) EU-Verordnungen

d) EU-Richtlinien

e) ,Soft-Law“

2. Unmittelbare Anwendbarkeit der Antidiskriminierungsmaßnahmen

a) Völkerrechtliche Verträge

aa) Unmittelbare Verpflichtung der Vertragsstaaten

bb) Unmittelbare Berechtigung Privater

cc) Unmittelbare Verpflichtung Privater 
dd) Zusammenfassung

b) Völkergewohnheitsrecht

c) EU-Verordnungen

d) EU-Richtlinien

e) „Soft-Law“

f) Grundrechte des Grundgesetzes

aa) Unmittelbare Drittwirkung

bb)Zurechnung privat bewirkter Beeinträchtigung zum Staat

cc) Mittelbare Drittwirkung

dd) Stellungnahme

3. Mittelbare Drittwirkung der Antidiskriminierungsmaßnahmen

a) Völkerrechtliche Verträge

b) EU-Richtlinien

c) ,Soft-Law“

d) Grundrechte des Grundgesetzes

II. Resümee - Legislativer Handlungsbedarf

B. Das Allgemeine Gleichbehandlungsgesetz und alternative Gesetzesentwürfe

I. Allgemeines Gleichbehandlungsgesetz (AGG) von 2006

1. Diskriminierungsrelevantes Verhalten - §3 AGG

2. Diskriminierungsmerkmale $-\S 1 \mathrm{AGG}$

3. Sachlicher Geltungsbereich - $§ 2$ i.V.m. $§ \S 7,19$ AGG

a) Benachteiligungsverbot des $\S 19$ AGG

b) Benachteiligungsverbot des $\S 7$ AGG

4. Rechtfertigung des diskriminierungsrelevanten Verhaltens

a) Zulässige unterschiedliche Behandlung gem. § 20 AGG

b) Zulässige unterschiedliche Behandlung gem. $\S \S 8-10$ AGG

c) Positive Maßnahmen - $§ 5$ AGG

5. Resümee

II. Gesetzesentwürfe von 1998

1. Diskriminierungsrelevantes Verhalten

2. Diskriminierungsmerkmale

3. Sachlicher Geltungsbereich

4. Rechtfertigung des diskriminierungsrelevanten Verhaltens

5. Resümee 
C. Vorschlag für die gesetzestechnische Umsetzung des Diskriminierungsverbotes

I. Tatbestand $-\S 115 \mathrm{I}$, II BGB-E

II. Rechtsfolgen

1. Schadensersatzanspruch

a) $\S 115$ BGB-E als Schutzgesetz im Sinne des $\S 823$ II BGB

b) Materielle Belastung als Folge des Gesetzesverstoßes

c) Vermögens- und Nichtvermögensschaden

d) Verschulden

e) $\S 253$ II BGB-E

f) $\S \S 280,826 \mathrm{BGB}$

2. Beseitigungs- und Unterlassungsanspruch $-\S 1004$ BGB analog

3. Anspruch auf Vertragsschluß

a) §611 a II 2. Halbsatz BGB-A

b) $\S 15$ VI AGG und $\S 15$ VI, 21 II ADG-E-2005

c) $\S 1004$ BGB analog

4. Nichtigkeit der diskriminierenden Willenserklärung - § 115 III BGB-E

III. Beweislast

a) „Vermuten lassen“ im Sinne des $§ 22$ AGG

b) Überwiegende Wahrscheinlichkeit

c) Hilfstatsachen im Sinne des $§ 22$ AGG

d) Vollbeweis der Hilfstatsachen

e) Entkräftung der glaubhaften Diskriminierung Beweis des Gegenteils

f) Nicht bzw. schwer beweisbare Tatsachen Beweislastentscheidung

a) Diskriminierung - $\$ 115$ IV 1 BGB-E

b) Verschulden - $§ 115$ IV 2, 3 BGB-E 561

c) Praktische Bedeutung des $\S 115$ IV BGB-E 
$-\mathrm{XI}-$

Literaturverzeichnis

XIII

Lebenslauf des Verfassers

XXV 



\section{Das Diskriminierungsverbot im Zivilrecht und seine Auswirkungen auf das rechtsgeschäftliche und quasi-rechtsgeschäftliche Handeln}

„Denn man soll um des Bartes willen den Kopf nicht verachten, an dem er wächst.“1

\section{Einleitung, Fragestellung und Gang der Arbeit}

Am 11.12.2003 berichtete die Presse von einem Personalmanager, dem gekündigt worden war, weil er sich geweigert hatte, Bewerbungen von Türkinnen bei künftigen Einstellungen nicht mehr zu berücksichtigen. $^{2}$ Der Personalmanager argumentierte, es sei seine Bürgerpflicht gewesen, Menschenrechte zu wahren und die diskriminierende Anweisung abzulehnen. Außerdem, so der Manager, könne man dann genausogut jemandem wegen dessen Haarfarbe oder Schuhgröße den Arbeitsplatz verweigern. Der Anwalt des Managers führte Art. 3 GG sowie Antidiskriminierungsrichtlinien der EU ins Feld. Der Arbeitgeber verteidigte demgegenüber sein Verhalten mit dem Argument, daß er als Unternehmer selbst entscheiden dürfe, wer bei ihm arbeite. Der Richter folgte dieser Argumentation. Außerdem wies er darauf hin, daß es - außer bezogen auf Diskriminierungen wegen des Geschlechts - noch kein Antidiskriminierungsgesetz gebe.

Die Europäische Union hat im Rahmen ihrer gesetzgeberischen Befugnisse Maßnahmen getroffen, die den Schutz von Personen bezwecken, die aufgrund der Tatsache, daß sie Träger bestimmter Merkmale, sogenannter Diskriminierungsmerkmale sind, im gesellschaftlichen Leben benachteiligt werden. Vor allem durch den Erlaß von Richtlinien sollten die Mitgliedstaaten angehalten werden, diesbezügliche Vorgaben in nationales Recht umzusetzen. Besondere Erwähnung verdienen in diesem Zusammenhang Richtlinie 2000/43/EG zur Anwendung des Gleichbehandlungsgrundsatzes ohne Unterschied der Rasse oder der ethnischen Herkunft, Richtlinie 2000/78/EG zur Festlegung eines allgemeinen Rahmens für die Verwirklichung der Gleichbehandlung in Beschäftigung und Beruf sowie Richtlinie 2004/113/EG zur Verwirklichung des Grundsatzes der Gleichbehandlung von Männern und Frauen beim Zugang $\mathrm{zu}$ und bei der Versorgung mit Gütern und Dienstleistungen.

Nachdem in Deutschland mehrere Entwürfe für ein allgemeines Antidiskriminierungsgesetz zur Diskussion gestellt und verworfen worden sind - der erste Entwurf stammt aus dem Jahr 1995 -, trat am 18.08.2006 das Gesetz zur Umsetzung europäischer Richtlinien zur Verwirklichung des

Aus der Novelle „Moses Mendelsohn“ von Johann Peter Hebel.

Müller-Münch in Frankfurter Rundschau vom 11.12.2003, S. 1, unter dem Titel: „Türkinnen unerwünscht - und das Gericht gab dem Chef Recht". 
Grundsatzes der Gleichbehandlung in Kraft, welches in seinem Art. 1 ein Allgemeines Gleichbehandlungsgesetz (AGG) enthält. ${ }^{3}$

Um das Thema „Antidiskriminierungsgesetz“ ist eine kontroverse Diskussion auf politischer wie rechtswissenschaftlicher Ebene entbrannt. Für Zündstoff hat dabei vor allem die Tatsache gesorgt, daß die EU-Richtlinien Diskriminierungsverbote auch in Privatrechtsbeziehungen statuieren. In diesem Sinne verpflichtet das Allgemeine Gleichbehandlungsgesetz beispielsweise Arbeitgeber dazu, Auswahlentscheidungen nicht von bestimmten Merkmalen, wie der Rasse oder ethnischen Herkunft des Bewerbers, abhängig zu machen. Das Allgemeine Gleichbehandlungsgesetz könnte demnach bewirken, daß - um auf den Ausgangsfall zurïckzukommen - dem Arbeitgeber in Zukunft verwehrt wäre, Türkinnen per se vom Auswahl- und Einstellungsverfahren auszuschließen.

Die vorliegende Arbeit dient dem Ziel zu ergründen, welche Bedeutung dem Begriff „Diskriminierung“ im Zivilrecht zukommt bzw. zukommen soll. Hat der Arbeitgeber in dem Ausgangsfall türkische Bewerberinnen dadurch diskriminiert, daß er seinen Personalmanager anwies, selbige nicht einzustellen?

Im Zusammenhang mit der Bestimmung des Diskriminierungsbegriffs stellen sich folgende Fragen: Welche Verhaltensweisen sind geeignet, den Tatbestand der Diskriminierung zu erfüllen? Welches sind die Merkmale, aufgrund derer diskriminiert werden kann (Diskriminierungsmerkmale)? Ist das Verhalten, das als diskriminierungsrelevant eingestuft wurde, rechtswidrig? Gibt es einen sachlichen Bereich, innerhalb dessen diskriminiert werden kann? Besteht auf der anderen Seite ein solcher, in dem ein etwaiges Diskriminierungsverbot nicht gelten darf?

Mit dem Ziel, Antworten auf die gestellten Fragen zu finden, untersucht die Arbeit in ihrem ersten Teil Antidiskriminierungsmaßnahmen auf internationaler wie nationaler Ebene. Konkret herausgearbeitet wird dabei, in welchen Bereichen und für welche Merkmale besagte Maßnahmen Schutz vor Diskriminierung bieten.

Die (inter-)nationalen Antidiskriminierungsmaßnahmen - insbesondere die eingangs erwähnten Antidiskriminierungsrichtlinien - bilden den Ausgangspunkt für die im zweiten Teil der Arbeit vorzunehmende konkrete Bestimmung des Diskriminierungsbegriffs. Den Ausgangspunkt bilden sie deshalb, weil besagte Maßnahmen indizieren, in welchen Bereichen und für welche Merkmale Diskriminierungsschutz notwendig ist. Insofern erfüllen sie die Funktion einer wichtigen Orientierungshilfe für die Beantwortung der aufgeworfenen Fragen. Darüber hinaus sind sie insofern zwingend zur Grundlage aller weiteren Überlegungen zu machen, als sie - wie im Fall der Antidiskriminierungsrichtlinien - verbindlichen Charakter für Deutschland haben. 
Welche Verhaltensweisen sind geeignet, den Tatbestand der Diskriminierung zu erfüllen? Im Rahmen der Beantwortung dieser Frage werden die in den Antidiskriminierungsrichtlinien enthaltenen Begriffe „unmittelbare Diskriminierung“, „mittelbare Diskriminierung“ sowie „(sexuelle) Belästigung“ zu klären sein. Der Verfasser wird der Frage nachgehen, ob die Richtlinien damit alle diskriminierungsrelevanten Verhaltensweisen abdecken, oder ob der Diskriminierungsbegriff insofern erweiterungsbedürftig ist.

Welches sind die Merkmale, aufgrund derer diskriminiert werden kann (Diskriminierungsmerkmale)? Die untersuchten Antidiskriminierungsmaßnahmen sind teilweise beschränkt auf den Schutz einzelner Diskriminierungsmerkmale. Teilweise enthalten sie aber auch offene Merkmalskataloge. Vor dem Hintergrund dieser Polarität stellt sich die Frage, ob eine Unterteilung in schutzwürdige und nicht schutzwürdige Merkmale möglich ist, oder ob nicht vielmehr alle Eigenschaften oder Verhaltensweisen eines Menschen grundsätzlich Anknüpfungspunkt für eine Diskriminierung sein können. Ist es - um wiederum auf den Ausgangsfall zurückzukommen - möglich, daß jemand aufgrund seiner Haarfarbe oder Schuhgröße diskriminiert wird?

Ist das Verhalten, das als diskriminierungsrelevant eingestuft wurde, rechtswidrig? Qualifizierte man jedes diskriminierungsrelevante Verhalten per se als Diskriminierung, ließe man unberücksichtigt, daß sich in den hier interessierenden Fallkonstellationen Private gegenüberstehen. Das führt dazu, daß in jedem Fall geprüft werden muß, ob den Rechten des „Diskriminierten“4 Rechte des „Diskriminierenden“ gegenüberstehen, die dessen diskriminierungsrelevantes Verhalten rechtfertigen. Zu diesem Zweck werden im dritten Teil der Arbeit anhand zahlreicher Fallbeispiele Kriterien für die erforderliche Interessenabwägung entwickelt.

Die Ausführungen zur Bestimmung des Diskriminierungsbegriffs schließen mit der Definition des sachlichen Geltungsbereichs des entsprechenden Diskriminierungsverbotes.

Der vierte und letzte Teil der Arbeit beschäftigt sich schließlich mit der gesetzestechnischen Umsetzung des Diskriminierungsverbotes im Zivilrecht. Dabei wird zunächst der Frage nachgegangen, ob legislativer Handlungsbedarf vor Inkrafttreten des AGG für den deutschen Gesetzgeber überhaupt bestand. Um diese Frage beantworten zu können, ist zunächst zu klären, wie sich die im ersten Teil der Arbeit untersuchten Antidiskriminierungsmaßnahmen auf das Privatrechtsverhältnis auswirken. Im Anschluß daran werden das Allgemeine Gleichbehandlungsgesetz und alternative Gesetzesentwürfe untersucht und gewürdigt. Daran knüpft ein eigener Vorschlag für die Umsetzung des Diskriminierungsverbotes an, der neben dem

4 „Diskriminierung“, „Diskriminierter“, „Diskriminierender“ $=$ potenzielle Diskriminierung bzw. potenziell Diskriminierter/Diskriminierender; Die Anführungszeichen sollen verdeutlichen, daß das Vorliegen einer Diskriminierung in dem Stadium, in dem die Wörter in dieser Form gebraucht werden, noch nicht bejaht worden ist. 
Diskriminierungstatbestand auch auf die Sanktionen, die ein Verstoß gegen das Diskriminierungsverbot zu Folge hat, sowie auf Fragen der Beweislast eingeht. 
Teil 1: Antidiskriminierungsmaßnahmen auf internationaler und nationaler Ebene

Eine Vielzahl von Maßnahmen auf völkerrechtlicher Ebene propagiert ein Recht auf Gleichbehandlung ohne Unterscheidung aufgrund bestimmter Merkmale. So setzen sich die Vereinten Nationen in Art. 1 III der Charta der Vereinten Nationen ${ }^{1}$ vom 26.06.1945 das Ziel

„eine internationale Zusammenarbeit herbeizuführen, um [...] die Achtung vor den Menschenrechten und Grundfreiheiten für alle ohne Unterschied der Rasse, des Geschlechts, der Sprache oder der Religion zu fördern und zu festigen“.

Im Gegensatz zu dieser Bestimmung, die lediglich ein in Zukunft zu erreichendes Ideal, die Achtung der Menschenrechte und Grundfreiheiten ohne Unterscheidung aufgrund bestimmter Merkmale, beschreibt, enthält die Konvention zum Schutze der Menschenrechte und Grundfreiheiten $^{2}$ vom 04.11.1950 in ihrem Art. 14 ein Diskriminierungsverbot. Die Bestimmung lautet:

„Der Genuß der in dieser Konvention anerkannten Rechte und Freiheiten ist ohne Diskriminierung insbesondere wegen des Geschlechts, der Rasse, der Hautfarbe, der Sprache, der Religion, der politischen oder sonstigen Anschauung, der nationalen oder sozialen Herkunft, der Zugehörigkeit zu einer nationalen Minderheit, des Vermögens, der Geburt oder eines sonstigen Status zu gewährleisten.“

Ähnlich formulieren es auch die Allgemeine Erklärung der Menschenrechte ${ }^{3}$ vom 10.12 .1948 in Art. 2, der Internationale Pakt über bürgerliche und politische Rechte ${ }^{4}$ vom 19.12.1966 in Art. 2 I sowie der Internationale Pakt über wirtschaftliche, soziale und kulturelle Rechte ${ }^{5}$ vom 19.12.1966 in Art. 2 II.

Der Wortlaut des Art. 14 der Konvention zum Schutze der Menschenrechte und Grundfreiheiten macht deutlich, daß es sich bei dieser Norm lediglich um ein akzessorisches Diskriminierungsverbot handelt. ${ }^{6}$ Allein aus Art. 14 kann der einzelne somit keine Ansprüche herleiten. Aber auch in Verbindung mit anderen Bestimmungen der Konvention ergeben sich keine für die vorliegende Arbeit relevanten Rechte einzelner. Etwas anderes gilt insofern für das ebenfalls akzessorische Diskriminierungsverbot des Art. 2 I des Internationalen Paktes über bürgerliche und

BGB1. 1973 II 1, S. $431 \mathrm{ff}$.

BGB1. 1952 II, S. 685 ff., Berichtigung S. 953; Neubek. der Konvention in BGB1. 2002 II, S. 1054.

Am 10. 12. 1948 von der Generalversammlung der Vereinten Nationen beschlossen (Sartorius II, Internationale Verträge, Europarecht, Nr. 19, S. 1).

BGB1. 1973 II, S. 1534 ff.

BGBl. 1973 II, S. $1570 \mathrm{ff}$.

6 So auch Nowak, CCPR-Kommentar, Art. 2, Rn. 31; Gleiches gilt für die genannten Bestimmungen der Allgemeinen Erklärung der Menschenrechte, des Internationalen Paktes über bürgerliche und politische Rechte sowie des Internationalen Paktes über wirtschaftliche, soziale und kulturelle Rechte. 
politische Rechte i. V. m. den in den Artt. 6 - 27 besagten Paktes niedergelegten subjektiven Rechten.

\section{A. Allgemeiner Gleichheitssatz}

Besonders hervorzuheben ist in diesem Zusammenhang Art. 26, der wie folgt lautet:

„Alle Menschen sind vor dem Gesetz gleich und haben ohne Diskriminierung Anspruch auf gleichen Schutz durch das Gesetz. In dieser Hinsicht hat das Gesetz jede Diskriminierung zu verbieten und allen Menschen gegen jede Diskriminierung, wie insbesondere der Rasse, der Hautfarbe, des Geschlechts, der Sprache, der Religion, der politischen oder sonstigen Anschauung, der nationalen oder sozialen Herkunft, des Vermögens, der Geburt oder des sonstigen Status, gleichen und wirksamen Schutz zu gewährleisten. “7

Art. 26 S. 1 statuiert zunächst ein Recht auf Gleichheit vor dem Gesetz, also den formellen Anspruch, daß bestehende Gesetze auf alle Rechtsunterworfenen in gleicher Weise angewendet werden müssen. ${ }^{8}$ Dieser sogenannte allgemeine Gleichheitssatz ist auch Gemeinschaftsgrundrecht ${ }^{9}$ im Sinne des Art. 6 II des EU-Vertrages (EUV) ${ }^{10}$ vom 07.02.1992 sowie des Art. 20 der am 07.12.2000 proklamierten Charta der Grundrechte der $E U^{11}$ und zudem gem. Art. 3 I Grundgesetz. (GG) Bestandteil der deutschen Verfassung. ${ }^{12}$

Aus Art. 26 S. 1 ergibt sich ferner der Anspruch auf gleichen Schutz durch das Gesetz, der den Gesetzgeber in zweifacher Hinsicht verpflichtet. Er hat einerseits beim Erlaß von Gesetzen jede Diskriminierung zu unterlassen. ${ }^{13}$ Auf der anderen Seite trifft den Gesetzgeber aus Art. 26 S. 1 in Verbindung mit S. 2 die Verpflichtung, durch Erlaß besonderer Gesetze Diskriminierungen zu verbieten und gegen Diskriminierungen wirksamen Schutz zu gewährleisten. ${ }^{14}$ Die primäre

Beachte, daß Art. 2 I von „Unterschied“ spricht, während Art. 26 das Wort „Diskriminierung“ gebraucht. Ein sachlicher Unterschied soll sich aus den unterschiedlichen Formulierungen jedoch nicht ergeben. (Nowak, CCPRKommentar, Art. 2, Rn. 32)

Eine dem Art. 26 vergleichbare Regelung enthält Art. 7 der Allgemeinen Erklärung der Menschenrechte.

8 Nowak, CCPR-Kommentar, Art. 26, Rn. 14; Das Recht auf Gleichheit vor dem Gesetz soll verletzt sein, wenn ein Gericht oder eine Verwaltungsbehörde ein für alle gleichermaßen geltendes Gesetz offensichtlich willkürlich anwendet, also bei gleichlautendem Sachverhalt aus Gründen persönlicher Sympathie oder Antipathie oder sonstiger offensichtlich unsachlicher Motive unterschiedliche Rechtsfolgen bestimmt. (Nowak, CCPR-Kommentar, Art. 26, Rn. 12) Willkür liege vor allem dann vor, wenn Personen lediglich aufgrund eines oder mehrerer der in Art. 26 S. 2 genannten Unterscheidungsmerkmale diskriminiert würden. (Nowak, CCPR-Kommentar, Art. 26, Rn. 15).

9 Kingreen in Calliess/Ruffert, Art. 6 EUV, Rn. 170; Hirsch, RdA 1998, S. 194 (195).

10 BGBl. 1992 II, S. 1253 ff.; in seiner durch den Vertrag von Nizza (26.02.2001) geänderten Fassung BGB1. 2001 II S. $1667 \mathrm{ff}$.

11 Abl. EG 2000, C 364, S. 1 ff.; in ihrer am 12.12.2007 angepaßten Fassung Abl. EG 2007, C 303, S. 1 ff.

12 Gemeinschaftsgrundrecht und nationales Grundrecht stimmen inhaltlich praktisch überein (Heun in Dreier, Band I, Art. 3, Rn. 10) und beeinflussen sich gegenseitig. (Huster in Friauf/Höfling, Band 1, C Art. 3, Rn. 5).

13 Nowak, CCPR-Kommentar, Art. 26, Rn. 17.

14 Nowak, CCPR-Kommentar, Art. 26, Rn. 17. 
Bedeutung des Diskriminierungsschutzes bestehe dabei in der Verpflichtung der Vertragsstaaten, ihre Rechtsunterworfenen gegen Diskriminierungen durch Private wirksam zu schützen. ${ }^{15}$

Einen Anspruch auf gleichen Schutz durch das Gesetz statuiert Art. 3 I GG zwar nicht explizit, jedoch ist in Deutschland auch der (Zivil-)Gesetzgeber wie alle öffentliche Gewalt aufgrund des Art. 1 III GG an den Gleichheitssatz aus Art. 3 I GG gebunden. ${ }^{16}$

Es wird diskutiert, ob auch der allgemeine Gleichheitssatz des Art. 3 I GG dem Gesetzgeber Schutzpflichten für Fälle von Diskriminierung im Privatrechtsverkehr auferlegt, was jedoch letztlich verneint wird. ${ }^{17}$ Gefragt wird in diesem Zusammenhang zudem, ob der Staat aus Art. 3 I GG verpflichtet sei, faktische Gleichheit, also die faktische Durchsetzung rechtlicher Gleichheit und sogar soziale Gleichstellung zu schaffen, ${ }^{18}$ was aber mit dem Argument abgelehnt wird, es sei wenig überzeugend, ein und derselben Norm - gemeint ist der Gleichheitssatz - ein Prinzip und sein Gegenteil zu entnehmen, nämlich ein Recht auf Gleichbehandlung einerseits und ein Recht auf Herstellung faktischer, sozialer Gleichheit auf der anderen Seite. ${ }^{19}$ Rechtliche Gleichbehandlung ermögliche und bewahre gerade Freiheit und damit faktische Ungleichheit. ${ }^{20}$

Dieselbe Debatte wird mit dem gleichen Ergebnis auch im Rahmen des Art. 3 III 1 GG geführt, ${ }^{21}$ der den allgemeinen Gleichheitssatz konkretisiert und spezialisiert ${ }^{22}$ und bestimmt, daß

„,[n]iemand [...] wegen seines Geschlechts, seiner Abstammung, seiner Rasse, seiner Sprache, seiner Heimat und Herkunft, seines Glaubens, seiner religiösen oder politischen Anschauungen benachteiligt oder bevorzugt werden [darf]. “23

Nowak, CCPR-Kommentar, Art. 26, Rn. 30; a.A. aber Tomuschat in Festschrift für Schlochauer, S. 691 (710 ff. u. 716).

16 Starck in von Mangoldt/Klein/Starck, Band 1, Art. 3 Abs. 1, Rn. 2 und 224; Heun in Dreier, Band I, Art. 3, Rn. 6.

17 Heun schließt das Bestehen von Schutzpflichten aus Art. 3 I gerade im Hinblick auf Diskriminierungen im Privatrechtsverkehr nicht grundsätzlich aus (Heun in Dreier, Band I, Art. 3, Rn. 66), hält jedoch eine Konstruktion, die Art. 3 I GG ausschließlich als Anspruch auf Gleichbehandlung interpretiert, für vorzugswürdig und verfassungsdogmatisch klarer. (Heun in Dreier, Band I, Art. 3, Rn. 68); Starck erkennt eine Schutzpflicht nur für bestimmte strenge Differenzierungsgebote an. So müsse der Staat durch Gesetzgebung und Rechtsprechung bspw. gleiche Rechtsfähigkeit, die formale Gleichheit der Teilnehmer am Privatrechtsverkehr, Gleichheit im Deliktsrecht, im Prozessrecht usw. garantieren. (Starck in von Mangoldt/Klein/Starck, Band 1, Art. 3 Abs. 1, Rn. 265); Für Osterloh kommt dem Art. 3 I GG allenfalls eine flankierende Schutzfunktion zu: „Wo Freiheit geschützt werden muß oder geschützt wird, muß sie gleichheitsgerecht geschützt werden.“(Osterloh in Sachs, Art. 3, Rn. 67).

Heun in Dreier, Band I, Art. 3, Rn. 67.

Heun in Dreier, Band I, Art. 3, Rn. 116 und 138; Starck in von Mangoldt/Klein/Starck, Band 1, Art. 3 Abs. 1, Rn. 343; Dieses Ergebnis ergebe sich nicht zuletzt durch einen Umkehrschluß zu Art. 3 II, dessen über das Diskriminierungsverbot des Art. 3 III GG hinausgehender Regelungsgehalt darin bestehe, daß er ein Gleichberechtigungsgebot aufstelle, welches er auch auf die gesellschaftliche Wirklichkeit erstrecke. (BVerfGE 85, S. 191 (206 f.)); Nickel gibt zu bedenken, aus dem Vergleich zu Art. 3 II GG könne zumindest der Schluß gezogen werden, ,daß im Lichte des Gleichbehandlungsanspruchs aus Art. 3 III GG die Betroffenen jedenfalls vor Benachteiligungen im Privatrechtsverkehr geschützt werden können.“ (Nickel, NJW 2001, S. 2668 (2669))

Eingedenk der gegenüber Art. 3 II GG beschränkten Reichweite des Art. 3 III GG wird darauf hingewiesen, daß Diskriminierungsverbote in internationalen Menschenrechtskonventionen eine über Art. 3 III GG hinausgehende Bedeutung erlangen können, soweit sie die Vertragsstaaten ausdrücklich verpflichten, gegen Diskriminierungen im gesellschaftlichen Bereich vorzugehen. (Huster in Friauf/Höfling, Band 1, C Art. 3, Rn. 12).

22 
Die rechtliche Gleichstellung soll „nur“ zu einer Verbesserung der Chancen führen, auf deren Grundlage sich im Laufe der Zeit auch eine annähernde tatsächliche Gleichheit einstellen könne. ${ }^{24}$ Festgehalten werden kann an dieser Stelle, daß aus Art. 3 I u. III GG keine Verpflichtung des Gesetzgebers zur Schaffung eines Diskriminierungsschutzgesetzes folgt.

Die bislang erwähnten Maßnahmen zeichnen sich zum einen dadurch aus, daß sie in bezug auf die verpönten Unterscheidungsmerkmale einen großzügigen und nicht immer abschließenden Maßstab anlegen. $^{25}$ Zum anderen sind sie auch in bezug auf die Frage, welcher sachliche Bereich von dem Diskriminierungsverbot umfaßt sein soll, nicht eingeschränkt bzw. sehr allgemein formuliert. Die im folgenden vorgenommene Einteilung soll verdeutlichen, daß es auf internationaler wie nationaler Ebene auch und sogar größtenteils Maßnahmen gibt, die Diskriminierungsschutz für ganz bestimmte Unterscheidungsmerkmale und/oder in einem speziellen sachlichen Bereich anstreben.

\section{B. Geschlechtergleichbehandlung}

Das Ziel, die Gleichbehandlung der Geschlechter, mithin die Überwindung der Schlechterstellung der Frau in einer Vielzahl von gesellschaftlichen Bereichen zu erreichen, hat zu einer fast unüberschaubaren Fülle von Antidiskriminierungsmaßnahmen auf völkerrechtlicher Ebene geführt. In Art. 2 sowie Art. 3 II des EG-Vertrages $(E G V)^{26}$ vom 25.03.1957 setzt sich die Gemeinschaft das Ziel, die Gleichstellung von Männern und Frauen zu fördern und im Rahmen ihres Tätigkeitsbereiches ${ }^{27}$ daraufhin $\mathrm{zu}$ wirken, Ungleichbehandlungen $\mathrm{zu}$ beseitigen. Auch die Gemeinschaftscharta der Sozialen Grundrechte der Arbeitnehmer ${ }^{28}$ vom 09.12.1989 verfolgt gem. Titel I Nr. 16 das Ziel der Gleichbehandlung von Männern und Frauen. Gleiches gilt gem. ihres Art. 23 für die Charta der Grundrechte der Europäischen Union. Der EuGH hat schließlich dem Grundsatz der Gleichbehandlung der Geschlechter über seine inhaltliche Ausprägung in Art. 141 EGV hinaus Grundrechtsqualität im Sinne des Art. 6 II EUV zuerkannt. ${ }^{29}$

23 Die Charta der Grundrechte der EU statuiert in Art. 21 I ein dem Art. 3 III GG vergleichbares Diskriminierungsverbot auf europäischer Ebene, dessen Katalog verpönter Unterscheidungsmerkmale allerdings umfassender ist als der jener Norm. Erwähnenswert ist zudem, daß einige Bundesländer in ihren Verfassungen auch ein Verbot der Diskriminierung aufgrund der sexuellen Identität enthalten. (Huster in Friauf/Höfling, Band 1, C Art. 3, Rn. 14).

24 Starck in von Mangoldt/Klein/Starck, Band 1, Art. 3 Abs. 1, Rn. 342.

25 Vergleiche dazu Art. 26 des Internationalen Paktes über bürgerliche und politische Rechte: ,[...] oder des sonstigen Status $[\ldots]^{“}$.

26 BGB1. 1957 II 1, S. 766 ff.; in seiner durch den Vertrag von Nizza (26.02.2001) geänderten Fassung, BGB1. 2001 II, S. 1667.

27 Vergleiche dazu Art. 3 I EGV.

28 KOM (89) 248 endg.

29 EuGH, verb. Rs. C-75 und 117/82, Slg. 1984 1, S. 1509 (1530, Rn. 16). 
Besondere Erwähnung verdient das Übereinkommen zur Beseitigung jeder Form von Diskriminierung der Frau ${ }^{30}$ vom 18.12.1979. Die Vertragsstaaten des Übereinkommens verpflichten sich in Art. 2 lit. b)

„durch geeignete gesetzgeberische und sonstige Maßnahmen, gegebenenfalls auch Sanktionen, jede Diskriminierung der Frau zu verbieten“.

Art. 2 lit. e) macht dabei deutlich, daß sich die Antidiskriminierungsmaßnahmen auch auf den Privatrechtsverkehr erstrecken sollen, indem er die Vertragsstaaten verpflichtet,

,alle geeigneten Maßnahmen zur Beseitigung der Diskriminierung der Frau durch Personen, Organisationen oder Unternehmen zu ergreifen“.

In Deutschland ist der Gesetzgeber bereits aus Art. 3 II GG verpflichtet, seine Rechtsunterworfenen vor Diskriminierung aufgrund des Geschlechts durch Private zu schützen. ${ }^{31}$ Gemäß dieser Norm sind

„Männer und Frauen [...] gleichberechtigt. Der Staat fördert die tatsächliche Durchsetzung der Gleichberechtigung von Frauen und Männern und wirkt auf die Beseitigung bestehender Nachteile hin.“

Die Verpflichtung des Gesetzgebers aus Art. 3 II GG geht damit weiter als die aus Art. 3 III GG. Art. 3 II GG statuiert nicht nur wie jene Norm ein Diskriminierungsverbot, sondern stellt darüber hinaus ein Gleichberechtigungsgebot auf und erstreckt dieses auch auf die gesellschaftliche Wirklichkeit. ${ }^{32}$ Dieses Gleichberechtigungsgebot, daß mittlerweile ausdrücklich in Art. 3 II 2 GG verankert ist, ${ }^{33}$ zielt auf die tatsächliche Durchsetzung der Gleichberechtigung der Geschlechter und auf eine diesbezügliche Angleichung der Lebensverhältnisse. ${ }^{34}$

Die Tatsache, daß Frauen gerade im Arbeitsleben, also in einem für die Entwicklung eines jeden Menschen besonders relevanten Bereich, Ungleichbehandlung erfahren, hat dazu geführt, daß internationale wie nationale Antidiskriminierungsmaßnahmen überwiegend die Gleichbehandlung der Frau in Beschäftigung und Beruf zum Gegenstand haben.

Gemäß Art. 137 I lit. i EGV

BGB1. 1985 II 1, S. 648 ff.

BVerfGE 88, S. 203 (260); Heun in Dreier, Band I, Art. 3, Rn. 114; Osterloh in Sachs, Art. 3, Rn. 261.

$B V e r f G E$ 85, S. 191 (206 f.).

$B$ VerfGE 92, S. 91 (109).

BVerfGE 85, S. 191 (207). 
"unterstützt und ergänzt die Gemeinschaft die Tätigkeit der Mitgliedstaaten [auf dem Gebiet der] Chancengleichheit von Männern und Frauen auf dem Arbeitsmarkt und [der] Gleichbehandlung am Arbeitsplatz“ ${ }^{35}$

Gemäß Art. 11 I lit. b und d des Übereinkommens zur Beseitigung jeder Form von Diskriminierung der Frau haben Frauen ein

„Recht auf dieselben Arbeitsmöglichkeiten einschließlich der Anwendung derselben Auswahlkriterien bei der Einstellung“ [sowie] ,auf gleiches Entgelt, einschließlich sonstiger Leistungen, und auf Gleichbehandlung bei gleichwertiger Arbeit sowie bei der Arbeitsqualität“.

Vor allem das Gebot der Entgeltgleichheit der Geschlechter hat im völkerrechtlichen Antidiskriminierungsrecht eine herausgehobene Bedeutung erlangt, was dadurch zum Ausdruck kommt, daß sich eine Reihe von Maßnahmen ausschließlich oder zumindest auch diesem Problem widmen.

So werden die Mitglieder der „International Labour Organisation“ (ILO) ${ }^{36}$ in Art. 2 I des Übereinkommens der ILO Nr. 100 über die Gleichheit des Entgelts männlicher und weiblicher Arbeitskräfte für gleichwertige Arbeit ${ }^{37}$ vom 29.06.1951 verpflichtet,

„[...] die Anwendung des Grundsatzes der Gleichheit des Entgelts männlicher und weiblicher Arbeitskräfte für gleichwertige Arbeit auf alle Arbeitnehmer zu fördern und [...] sicherzustellen.“

Gleichartige Vorschriften enthalten Art. 4 I Nr. 3 der Europäische Sozialcharta ${ }^{38}$ vom 18.10.1961 sowie Art. 11 I lit. d des Übereinkommens zur Beseitigung jeder Form von Diskriminierung der Frau.

Von besonderer Bedeutung sind in diesem Zusammenhang Art. 141 I EGV sowie die durch Art. 141 III EGV legitimierte Richtlinie 75/117/EWG zur Angleichung der Rechtsvorschriften der Mitgliedstaaten über die Anwendung des Grundsatzes des gleichen Entgelts für Männer und Frauen $^{39}$ vom 10.02.1975, da sie zu gesetzgeberischem Handeln in Deutschland geführt haben. In

35 Zu diesem Zweck sieht Art. 137 II lit. b die Möglichkeit für den Rat der Europäischen Gemeinschaft vor, Richtlinien in den Bereichen zu erlassen, in denen die Gemeinschaft gem. Art. 137 I die Tätigkeit der Mitgliedstaaten unterstützt und ergänzt.

36 International Labour Organisation = Internationale Arbeitsorganisation (IAO); Die ILO ist eine Sonderorganisation der Vereinten Nationen. (Ipsen, Völkerrecht, § 33, Rn. 69).

37 BGB1. 1956 II, S. 23 ff.

38 BGBl. 1964 II, S. $1262 \mathrm{ff}$.

39 AB1. EG 1975 Nr. L 45/19; Richtlinie 75/117/EWG ist zwar nicht auf Grundlage des Art. 141 III EGV erlassen worden, da jene Bestimmung erst durch den Vertrag von Amsterdam vom 02.10.1997 in den EG-Vertrag aufgenommen wurde. (BGB1. 1998 II, S. 387 ff., ber. BGB1. 1999 II, S. 416 ff.) Die Richtlinie soll dennoch wie auch einige andere, die auf zweifelhafter Grundlage zustande gekommen sind, durch Art. 141 III EGV legitimiert sein. (Eichenhofer in Streinz, Art. 141 EGV, Rn. 22); so auch Rebhahn, der gewisse, bereits bestehende Richtlinien dem Art. 141 III zuordnet. (Rebhahn in Schwarze, § 141 EGV, Rn. 39). 
Umsetzung des Art. 141 EGV sowie der Richtlinie 75/117/EWG bestimmte $\S 612$ III 1 BGB bevor er am 18.08.2006 mit Inkrafttreten des Gesetzes zur Umsetzung europäischer Richtlinien zur Verwirklichung des Grundsatzes der Gleichbehandlung ${ }^{40}$ gem. dessen Art. 3 XIV aufgehoben wurde -, daß

,[bei] einem Arbeitsverhältnis [...] für gleiche oder gleichwertige Arbeit nicht wegen des Geschlechts des Arbeitnehmers eine geringere Vergütung vereinbart werden [darf] als bei einem Arbeitnehmer des anderen Geschlechts.“" ${ }^{41}$

Entgegen seines Wortlauts, der auf ein reines Benachteiligungsverbot schließen läßt, ${ }^{42}$ enthielt $\S$ 612 III BGB-A ${ }^{43}$ eine eigenständige Rechtsgrundlage für einen Anspruch auf Entgeltgleichheit. ${ }^{44}$ Der Arbeitnehmer hatte mithin einen unmittelbaren Erfüllungsanspruch darauf, daß ihm gleicher (d.h. mehr) Lohn gezahlt wird. ${ }^{45}$

Eine dem $§ 612$ III 1 BGB-A inhaltlich entsprechende Regelung enthält nunmehr $§ 7$ AGG i.V.m. § 2 I Nr. 2 AGG, wobei der Grundsatz des gleichen Entgelts für gleiche oder gleichwertige Arbeit hier nicht auf das Merkmal „Geschlecht“ beschränkt ist. ${ }^{46}$ Gemäß $§ 7$ I AGG dürfen Beschäftigte

„,...] nicht wegen eines in $\S 1$ genannten Grundes [ - d. h. nicht aus Gründen der Rasse oder wegen der ethnischen Herkunft, des Geschlechts, der Religion oder Weltanschauung, einer Behinderung, des Alters oder der sexuellen Identität - ] benachteiligt werden; dies gilt auch, wenn die Person, die die Benachteiligung begeht, das Vorliegen eines in $\S 1$ genannten Grundes bei der Benachteiligung nur annimmt. “47

$\S 2$ I Nr. 1 bis 3 AGG bestimmt den sachlichen Geltungsbereich, in dem Benachteiligungen im Sinne des § 7 I AGG unzulässig sind. Gemäß $§ 2$ I Nr. 2 AGG gilt das Benachteiligungsverbot dabei unter anderem in bezug auf das Arbeitsentgelt.

Erwähnung finden muß im Zusammenhang mit dem Verbot der Entgeltdiskriminierung schließlich noch Richtlinie 86/378/EWG zur Verwirklichung des Grundsatzes der Gleichbehandlung von Männern und Frauen bei den betrieblichen Systemen der sozialen Sicherheit ${ }^{48}$ vom 24.07.1986.

$\mathrm{Zu}$ beachten ist, daß die Richtlinie 75/117/EWG gem. Art. 34 I der Richtlinie 2006/54/EG mit Wirkung vom 15.08.2009 aufgehoben und durch jene Richtlinie ersetzt wird.

40 BGB1. 2006 I, S. 1897 ff.; Beachte in diesem Zusammenhang die Übergangsvorschrift des § 33 I AGG.

$41 \quad B A G$, NJW 1993, S. 3091 (3093); Preis in Erfurter Kommentar zum Arbeitsrecht, $\S 612$ BGB, Rn. 46; Raab in Soergel, Band 4/1, § 612, Rn. 43.

$42 \quad$ Putzo in Palandt, $\S 612, \mathrm{Rn} .11$.

43 Aufgehobene Bestimmungen werden im folgenden durch den Zusatz ,-A“ gekennzeichnet.

44 Preis in Erfurter Kommentar zum Arbeitsrecht, § 612 BGB, Rn. 46.

45 Putzo in Palandt, $\S 612$, Rn. 14.

46 Entwurfsbegründung, BT-Druck. 16/1780, S. 35 u. 57 f.

47 Vergleiche eingehend zu dem Benachteiligungsverbot des $§ 7$ AGG Teil 4, Prüfungspunkt B. I. 3. b).

48 ABl. EG 1986 Nr. L 225/40; in geänderter und jetzt gültiger Fassung Abl. EG 1999 Nr. L 151/39 
Aufgrund der Tatsache jedoch, daß der EuGH Ungleichbehandlungen in diesem Bereich unter das Verbot der Entgeltdiskriminierung i.S.d. Art. 119 a.F. (141 I n.F.) EGV subsumiert hat, ${ }^{49}$ erübrigt sich eine nähere Darstellung dieser Richtlinie. ${ }^{50}$

Richtlinie 76/207/EWG zur Verwirklichung des Grundsatzes der Gleichbehandlung von Männern und Frauen hinsichtlich des Zugangs zur Beschäftigung, zur Berufsausbildung und zum beruflichem Aufstieg sowie in Bezug auf die Arbeitsbedingungen ${ }^{51}$ vom 09.02 .1976 ergänzt den auf Entgeltgleichheit beschränkten Art. 141 EGV, indem sie gem. Art. 3 I - auch im privaten Bereich jede unmittelbare wie mittelbare Diskriminierung in folgenden Bereichen untersagt:

„a) die Bedingungen - einschließlich Auswahlkriterien und Einstellungsbedingungen - für den Zugang zu unselbständiger oder selbständiger Erwerbstätigkeit, unabhängig von Tätigkeitsfeld und beruflicher Position einschließlich des beruflichen Aufstiegs;

b) den Zugang zu allen Formen und allen Ebenen der Berufsberatung, der Berufsausbildung, der beruflichen Weiterbildung und der Umschulung einschließlich der praktischen Berufserfahrung;

c) die Beschäftigungs- und Arbeitsbedingungen einschließlich der Entlassungsbedingungen sowie das Arbeitsentgelt nach Maßgabe der Richtlinie 75/117/EWG;

d) die Mitgliedschaft und Mitwirkung in einer Arbeitnehmer- oder Arbeitgeberorganisation oder einer Organisation, deren Mitglieder einer bestimmten Berufsgruppe angehören, einschließlich der Inanspruchnahme der Leistungen solcher Organisationen.“

Die weite Fassung der Richtlinie ist der Tatsache geschuldet, daß Chancengleichheit der Geschlechter beim Zugang zur Betätigung, zur Berufsausbildung und zum beruflichen Aufstieg notwendige Voraussetzung für die Entgeltgleichheit für Männer und Frauen ist. ${ }^{52}$

Der deutsche Gesetzgeber ist seiner Umsetzungspflicht, die sich aus der Richtlinie 76/207/EWG ergeben hatte, durch Einführung des $\S 611$ a BGB-A $^{53}$ im Jahre 1980 nachgekommen. $^{54}$

Betriebliche Systeme der sozialen Sicherheit sind u.a. solche, die Schutz vor Krankheit, Invalidität, Alter (einschließlich vorzeitige Versetzung in den Ruhestand), Arbeitsunfall und Berufskrankheit, sowie Arbeitslosigkeit bieten (vgl. Art. 4 a).

49 Vergleiche dazu EuGH, Rs. C-262/88, Slg. 1990 I 2, S. 1889 (1950, Rn. 20; S. 1952, Rn. 30); Rs. C-110/91, Slg. 1993 I 6, S. 6591 (6616, Rn. 20; 6617, Rn. 24 u. 26).

50 Birk ist der Ansicht, die Rechtsprechung des EuGH habe die Richtlinie 86/378/EWG weitgehend ausgehebelt und überflüssig gemacht. (Birk in Münchener Handbuch zum Arbeitsrecht, Band 1, § 19, Rn. 362).

51 AB1. EG 1976 Nr. L 39/40; in der durch Richtlinie 2002/73/EG vom 23.09.2002 - Abl. EG Nr. L 269/15 geänderten und jetzt gültigen Fassung Abl. EG 2002 Nr. L 269/15.

$\mathrm{Zu}$ beachten ist, daß die Richtlinie 76/207/EWG gem. Art. 34 I der Richtlinie 2006/54/EG mit Wirkung vom 15.08.2009 aufgehoben und durch jene Richtlinie ersetzt wird.

52 So Eichenhofer in Streinz, Art. 141 EGV, Rn. 5; Rebhahn in Schwarze, § 141 EGV, Rn. 3.

53 Aufgehoben am 18.08.2006 mit Inkrafttreten des Gesetzes zur Umsetzung europäischer Richtlinien zur Verwirklichung des Grundsatzes der Gleichbehandlung - vgl. dessen Art. 3 XIV (BGB1. 2006 I, S. 1897 ff.); Beachte in diesem Zusammenhang die Übergangsvorschrift des § 33 I $A G G$.

54 Putzo in Palandt, $\S 611$ a, Rn. 1; Schlachter in Erfurter Kommentar zum Arbeitsrecht, § 611 a, Rn. 1; Junker, Grundkurs Arbeitsrecht, Rn. 162 
Gleichzeitig konkretisierte er durch die Normierung Art. 3 II GG für das Arbeitsrecht. ${ }^{55} \S 611$ a I 1 BGB-A lautete:

"Der Arbeitgeber darf einen Arbeitnehmer bei einer Vereinbarung oder einer Maßnahme, insbesondere bei der Begründung des Arbeitsverhältnisses, beim beruflichen Aufstieg, bei einer Weisung oder einer Kündigung, nicht wegen seines Geschlechts benachteiligen. “56

Eine dem $§ 611$ a I 1 BGB-A entsprechende Regelung enthält nunmehr $§ 7$ I AGG. ${ }^{57}$

Auch der ebenfalls 1980 ins BGB eingefügte $§ 611$ b BGB-A ${ }^{58}$ war Ergebnis der Verpflichtung Deutschlands aus der Richtlinie 76/207/EWG. ${ }^{59}$ Gemäß dieser Norm durfte

„,[d]er Arbeitgeber [...] einen Arbeitsplatz weder öffentlich noch innerhalb des Betriebs nur für Männer oder nur für Frauen ausschreiben, es sei den, daß ein Fall des § 611 a Abs. 1 Satz 2 [vorlag]. “60

$\S 611$ b BGB-A ist durch $\S 11$ AGG ersetzt worden. ${ }^{61}$ Gemäß jener Norm darf ein Arbeitsplatz

„[...] nicht unter Verstoß gegen $§ 7$ Abs. 1 ausgeschrieben werden.“

Richtlinie 86/613/EWG zur Verwirklichung des Grundsatzes der Gleichbehandlung von Männern und Frauen, die eine selbständige Erwerbstätigkeit - auch in der Landwirtschaft - ausüben, sowie über den Mutterschutz ${ }^{62}$ vom 11.12.1986 betrifft selbständig Erwerbstätige und deren mitarbeitende

$\S 611$ a BGB wurde wie $\S 612$ III BGB durch das Gesetz über die Gleichstellung von Männern und Frauen am Arbeitsplatz und über die Erhaltung von Ansprüchen bei Betriebsübergang (Arbeitsrechtliches EGAnpassungsgesetz) vom 13.08.1980 in das BGB eingefügt. (BGB1. 1980, I 2, S. 1308 f.)

In seiner ursprünglichen Fassung ist $\S 611$ a BGB nicht mehr erhalten, da die Norm - zuletzt 1998 - geändert werden mußte, um den Anforderungen des EuGH zu genügen. (Junker, Grundkurs Arbeitsrecht, Rn. 162).

55 Schlachter in Erfurter Kommentar zum Arbeitsrecht, § 611 a, Rn. 1; Junker, Grundkurs Arbeitsrecht, Rn. 162.

56 Die Formulierung der Norm schließt auch das Verbot der Entgeltdiskriminierung aufgrund des Geschlechts ein Putzo in Palandt, § 612, Rn. 11; Preis in Erfurter Kommentar zum Arbeitsrecht, § 612 BGB, Rn. 45 -, so daß sich die Frage nach der eigenständigen Bedeutung des § 612 III BGB-A stellte. Sie sollte in der besonderen Rechtsfolge bestehen, die (allein) § 612 III BGB-A statuiert habe. Gemeint ist der unmittelbare Erfüllungsanspruch des diskriminierten Arbeitnehmers auf gleichen Lohn. (Preis in Erfurter Kommentar zum Arbeitsrecht, § 612 BGB, Rn. 68; Putzo in Palandt, § 612, Rn. 11 und 14).

57 Entwurfsbegründung, BT-Druck. 16/1780, S. 57.

58 Aufgehoben am 18.08.2006 mit Inkrafttreten des Gesetzes zur Umsetzung europäischer Richtlinien zur Verwirklichung des Grundsatzes der Gleichbehandlung - vgl. dessen Art. 3 XIV (BGB1. 2006 I, S. 1897 ff.); Beachte in diesem Zusammenhang die Übergangsvorschrift des $§ 33$ I $A G G$.

59 Putzo in Palandt, $\S 611$ b, Rn. 1.

60 Zunächst als Sollvorschrift formuliert, erlangte die Norm ihren zwingenden Charakter durch Art. 7 Nr. 2 des 2. Gleichberechtigungsgesetzes (GleiBG) vom 24.06.1994 (BGBl. 1994 I 2, S. 1406 ff.)

Eine dem $\S 611$ b BGB-A entsprechende Bestimmung enthält $\S 6$ des Gesetzes zur Gleichstellung von Frauen und Männern in der Bundesverwaltung und in den Gerichten des Bundes (Bundesgleichstellungsgesetz - BGleiG) vom 30.11.2001. (BGB1. 2001, I 3, S. 3234 ff.) Das BGleiG hat indes für die vorliegende Arbeit keine unmittelbare Bedeutung, da es Private nicht verpflichtet.

61 Entwurfsbegründung, BT-Druck. 16/1780, S. 57.

62 Abl. EG 1986 Nr. L 359/56. 
Ehegatten. ${ }^{63}$ Sie statuiert ein Verbot der unmittelbaren oder mittelbaren Diskriminierung aufgrund des Geschlechts ${ }^{64}$ im Rahmen der

„Gründung, Ausrüstung und Erweiterung eines Unternehmens bzw. [der] Aufnahme oder Ausweitung jeder sonstigen Tätigkeitsform der selbständigen Erwerbstätigen und auch [der] finanziellen Fazilitäten“. ${ }^{65}$

Desweiteren thematisiert die Richtlinie die soziale Sicherung der Ehegatten selbständig Erwerbstätiger. ${ }^{66}$ Es wird die Ansicht vertreten, die praktische Bedeutung dieser Richtlinie sei gering, da einerseits die Vertragspartner von Selbständigen kaum nach dem Geschlecht differenzierten, mithin selten diskriminierten, und andererseits gesetzliche Differenzierungen bei der sozialen Sicherheit von der Richtlinie 79/7/EWG zur schrittweisen Verwirklichung des Grundsatzes der Gleichbehandlung von Männern und Frauen im Bereich der sozialen Sicherheit ${ }^{67}$ vom 19.10.1978 erfaßt seien. ${ }^{68}$

Die bisherigen Ausführungen haben gezeigt, daß internationale wie nationale Antidiskriminierungsmaßnahmen überwiegend die Gleichbehandlung der Frau in Beschäftigung und Beruf zum Gegenstand haben. Im Gegensatz dazu bezweckt die Richtlinie 2004/113/EG zur Verwirklichung des Grundsatzes der Gleichbehandlung von Männern und Frauen beim Zugang zu und bei der Versorgung mit Gütern und Dienstleistungen ${ }^{69}$ vom 13.12.2004 gem. ihres Art. 1

„[...] die Schaffung eines Rahmens für die Bekämpfung geschlechtsspezifischer Diskriminierungen beim Zugang zu und der Versorgung mit Gütern und Dienstleistungen zur Umsetzung des Grundsatzes der Gleichbehandlung von Männern und Frauen in den Mitgliedstaaten.“

Die Richtlinie gilt dabei gem. Art. 3 I

„[...] für alle Personen, die Güter und Dienstleistungen bereitstellen, die der Öffentlichkeit ohne Ansehen der Person zur Verfügung stehen, und zwar in öffentlichen und privaten Bereichen, einschließlich öffentlicher Stellen, und die

63 Vergleiche zum persönlichen Anwendungsbereich Art. 2 der Richtlinie.

64 Vergleiche Art. 3 der Richtlinie.

65 Vergleiche Art. 4 der Richtlinie.

66 Vergleiche Art. 6 der Richtlinie.

67 Abl. EG 1979, Nr. L 6, S. 24 f.

68 Rebhahn in Schwarze, $\S 141$ EGV, Rn. 50

Richtlinie 79/7/EWG setzt sich die schrittweise Verwirklichung des Grundsatzes der Gleichbehandlung von Männern und Frauen im Bereich der sozialen Sicherheit zum Ziel. (vgl. Art. 1) Systeme der sozialen Sicherheit sind u.a. solche, die Schutz vor Krankheit, Invalidität, Alter, Arbeitsunfall und Berufskrankheit, sowie Arbeitslosigkeit bieten. (vgl. Art. 3 a) Aufgrund der Beschränkung des sachlichen Anwendungsbereichs auf staatliche Sozialleistungssysteme (Rebhahn in Schwarze, § 141 EGV, Rn. 41; Rust in von der Groeben/Schwarze, Band 3, Art. 141 EGV, Rn. 236) - vgl. dazu Art. 3 - erlangt die Richtlinie 79/7/EWG für die vorliegende Arbeit keine Bedeutung und wird dementsprechend nicht vertieft behandelt. 
außerhalb des Bereichs des Privat- und Familienlebens und der in diesem Kontext stattfindenden Transaktionen angeboten werden.“

Auch in Umsetzung des Art. 3 I Richtlinie 2004/113/EG statuiert § 19 AGG ein zivilrechtliches Benachteiligungsverbot. ${ }^{70}$ Hervorzuheben ist, daß auch hier der gewährte Schutz nicht auf das Diskriminierungsmerkmal „Geschlecht“ beschränkt ist. Gemäß § 19 I AGG ist

„[e]ine Benachteiligung aus Gründen der Rasse oder wegen der ethnischen Herkunft, wegen des Geschlechts, der Religion, einer Behinderung, des Alters oder der sexuellen Identität bei der Begründung, Durchführung und Beendigung zivilrechtlicher Schuldverhältnisse, die

1. typischerweise ohne Ansehen der Person zu vergleichbaren Bedingungen in einer Vielzahl von Fällen zustande kommen (Massengeschäfte) oder bei denen das Ansehen der Person nach der Art des Schuldverhältnisses eine nachrangige Bedeutung hat und die zu vergleichbaren Bedingungen in einer Vielzahl von Fällen zustande kommen oder

2. eine privatrechtliche Versicherung zum Gegenstand haben,

[...] unzulässig.“ ${ }^{71}$

\section{Besonderer Schutz der (werdenden) Mutter}

Das Verbot der Ungleichbehandlung der Frau im Berufsleben steht in Zusammenhang mit besonderen Maßnahmen, die dem Schutz der (werdenden) Mutter dienen.

Gemäß Art. 11 lit. a des Übereinkommens zur Beseitigung jeder Form von Diskriminierung der Frau müssen die Vertragsstaaten u.a. Maßnahmen ergreifen, die das „Verbot der Entlassung wegen Schwangerschaft oder Mutterschaftsurlaubs“ durchsetzen. In diesem Sinne verbietet auch die Richtlinie 92/85/EG über die Durchführung von Maßnahmen zur Verbesserung der Sicherheit und des Gesundheitsschutzes von schwangeren Arbeitnehmerinnen, Wöchnerinnen und stillenden Arbeitnehmerinnen am Arbeitsplatz (zehnte Einzelrichtlinie im Sinne des Artikels 16 Absatz 1 der Richtlinie 89/391/EWG $)^{72}$ vom 19.10.1992 in Art. 10 Nr. 1

„die Kündigung der Arbeitnehmerinnen im Sinne des Artikels 2 [ - d.h. der schwangeren Arbeitnehmerinnen, Wöchnerinnen und stillenden Arbeitnehmerinnen - ] während der Zeit vom Beginn der Schwangerschaft bis zum Ende des Mutterschaftsurlaubs [...]; davon ausgenommen sind die nicht mit ihrem Zustand in Zusammenhang stehenden Ausnahmefälle, die entsprechend den einzelstaatlichen Rechtsvorschriften und / oder Gepflogenheiten zulässig sind, wobei gegebenenfalls die zuständige Behörde ihre Zustimmung erteilen muß.“

In Art. 8 Nr. 2 verpflichten sich die Vertragspartner der Europäischen Sozialcharta

71 Vergleiche eingehend zu dem Benachteiligungsverbot des § 19 AGG Teil 4, Prüfungspunkt B. I. 3. a).

Abl. EG 1992, Nr. L 348, S. 1 ff. 
„es als ungesetzlich zu betrachten, daß ein Arbeitgeber einer Frau während ihrer Abwesenheit infolge Mutterschaftsurlaubs oder so kündigt, daß die Kündigungsfrist während einer solchen Abwesenheit abläuft“”.

Richtlinie 86/613/EWG thematisiert den Schwangerschafts- und Mutterschutz für Frauen, die selbständig erwerbstätig oder Ehegatten von selbständig Erwerbstätigen sind. ${ }^{73}$ Auch die Richtlinie 76/207/EWG statuiert nunmehr ${ }^{74}$ besondere Rechte im Zusammenhang mit einer Schwangerschaft. So haben Frauen im Mutterschaftsurlaub gem. Art. 2 VII 2

„,nach Ablauf des Mutterschaftsurlaubs Anspruch darauf, an ihren früheren Arbeitsplatz oder einen gleichwertigen Arbeitsplatz unter Bedingungen, die für sie nicht weniger günstig sind, zurückzukehren, und darauf, daß ihnen auch alle Verbesserungen der Arbeitsbedingungen, auf die sie während ihrer Abwesenheit Anspruch gehabt hätten, zugute kommen.“75

Besonderer Kündigungsschutz der (werdenden) Mutter wurde in Deutschland erstmals 1927 durch Erlaß des Mutterschutzgesetzes (MuSchG) gesetzlich fixiert. ${ }^{76}$ Gemäß $§ 9$ I 1 MuSchG ist

„,[d]ie Kündigung gegenüber einer Frau während der Schwangerschaft und bis zum Ablauf von vier Monaten nach der Entbindung [...] unzulässig, wenn dem Arbeitgeber zur Zeit der Kündigung die Schwangerschaft oder Entbindung bekannt war oder innerhalb zweier Wochen nach Zugang der Kündigung mitgeteilt wird [...].“

Eine dem Art. 2 VII 2 der Richtlinie 76/207/EWG entsprechende Formulierung enthält $§ 9$ MuSchG nicht. Gleichwohl soll die Verpflichtung, die sich insoweit aus der Richtlinie ergibt, in Deutschland bereits geltendes Recht durch das MuSchG sein. ${ }^{77}$ Außerdem soll $\S 9$ MuSchG den Anforderungen des Art. 6 IV GG entsprechen, wonach jede Mutter „Anspruch auf den Schutz und die Fürsorge der Gemeinschaft“ hat. $^{78}$ Anzumerken ist, daß $\S 99$ MuSchG nicht nur als Antidiskriminierungsmaßnahme verstanden werden darf. Die Bestimmung wurde vor allem eingeführt, um psychologische wie physische Belastungen, die sich für Mutter und Kind abträglich auswirken könnten, zu vermeiden. ${ }^{79}$

\section{Gleichbehandlung der „Rassen“ und nationalen Minderheiten}

\footnotetext{
Vergleiche Art. 8 der Richtlinie.

Das heißt seit deren Änderung durch die Richtlinie 2002/73/EG (Abl. EG 2002, Nr. L 269, S. 15 ff.).

$\mathrm{Zu}$ beachten ist in diesem Zusammenhang auch Art. 2 VII 5, der eine ähnliche Regelung bei Vaterschafts- und Adoptionsurlaub vorsieht, soweit die Mitgliedstaaten solche Rechte anerkennen.

76 Buchner/Becker, Mutterschutzgesetz und Bundeserziehungsgeldgesetz, Vor $\S \S 9$ und 10 MuSchG, Rn. 3.

77 Hadeler, NZA 2003, S. 77 (79); Bauer, NJW 2001, S. 2672 (2676).

78 Buchner/Becker, Mutterschutzgesetz und Bundeserziehungsgeldgesetz, Vor $\S \S 9$ und 10 MuSchG, Rn. 3.

79 Buchner/Becker, Mutterschutzgesetz und Bundeserziehungsgeldgesetz, Vor $\S \S 9$ und 10 MuSchG, Rn. 2 und 9.
} 
Neben dem Merkmal Geschlecht hat das der „Rasse“ bzw. ethnischen Herkunft besondere Bedeutung im Rahmen des völkerrechtlichen Antidiskriminierungsschutzes erlangt.

In Art. 2 I lit. d des Internationalen Übereinkommens zur Beseitigung jeder Form von Rassendiskriminierung ${ }^{80}$ vom 07.03.1966 verpflichten sich die Vertragsstaaten,

„mit allen geeigneten Mitteln unverzüglich eine Politik der Beseitigung der Rassendiskriminierung in jeder Form [...] zu verfolgen [und] zu diesem Zweck [...] jede durch Personen, Gruppen oder Organisationen ausgeübte Rassendiskriminierung mit allen geeigneten Mitteln einschließlich der durch die Umstände erforderlichen Rechtsvorschriften, [zu verbieten und zu beenden .]“

Das Übereinkommen erfaßt, indem es jede Form der Rassendiskriminierung verbieten und beenden will, ausdrücklich auch die im Privatrechtsverkehr. ${ }^{81}$ In Konkretisierung des Art. 2 verpflichten sich die Vertragsstaaten in Art. 5 lit. f,

„das Recht [jedes einzelnen] auf Zugang zu jedem Ort oder Dienst, der für die Benutzung durch die Öffentlichkeit vorgesehen ist, wie Verkehrsmittel, Hotels, Gaststätten, Cafés, Theater und Parks [, zu gewährleisten.]“

Auch die Europäische Union hat sich zum Ziel gesetzt, Rassismus und Fremdenfeindlichkeit zu verhüten und zu bekämpfen. ${ }^{82}$ Gemäß Art. 13 des EG-Vertrages

„[...] kann der Rat [...] geeignete Vorkehrungen treffen, um Diskriminierungen aus Gründen des Geschlechts, der Rasse, der ethnischen Herkunft, der Religion oder der Weltanschauung, einer Behinderung, des Alters oder der sexuellen Ausrichtung zu bekämpfen.“

Durch Erlaß der Richtlinie 2000/43/EG zur Anwendung des Gleichbehandlungsgrundsatzes ohne Unterschied der Rasse oder der ethnischen Herkunft ${ }^{83}$ vom 29.06.2000 hat der Rat von seiner ihm durch Art. 13 EGV verliehenen Kompetenz (in eingeschränkter Weise) Gebrauch gemacht. ${ }^{84}$ Die Richtlinie erfaßt gem. Art. 2 I Diskriminierungen ,[...] aus Gründen der Rasse oder der ethnischen Herkunft $[\ldots . .]^{*}{ }^{85}$ Der sachliche Anwendungsbereich des Art. 3 I lit. a - d deckt sich mit dem des

80 BGB1. 1969 II, S. 962 ff.; BT-Druck. V/3960, S. 3 ff.

81 Stock, ZAR 99, S. 118 (118); Das Übereinkommen orientiert sich an Regelungen des innerstaatlichen Rechts Großbritanniens (,Race Relations Act“ von 1965) und der Vereinigten Staaten von Amerika („Civil Rigths Act“ von 1964), ,die den Ausschluß von der Benutzung bestimmter, der Allgemeinheit dienender, aber in privater Hand befindlicher Einrichtungen aus rassischen Gründen verhindern sollen.“ (Kühner, NJW 86, S. 1397 (1398)).

82 Vergleiche Art. 29 I EUV.

83 Abl. EG 2000 Nr. L 180, S. 22 ff.

84 Diskutiert wird, ob Art. 13 EGV neben seiner Funktion als Ermächtigungsgrundlage auch Gemeinschaftsgrundrecht ist. (dies verneinend: Nickel, Gleichheit und Differenz in der vielfältigen Republik, S. 113; Jochum, ZRP 99, S. 279 (280)) Eine unmittelbare Anwendbarkeit des Art. 13 EGV ziehen dagegen Szczekalla und Cirkel in Betracht. (Szczekalla, EuZW 1998, S. 215 (216); Cirkel, NJW 1998, S. 3332 (3333)).

85 Die Verwendung des Begriffs „Rasse“ ist nicht unproblematisch. So heißt es in Erwägungsgrund 6 der Richtlinie 2000/43/EG, ,[d]ie Europäische Union weist Theorien, mit denen versucht wird, die Existenz verschiedener 
Art. 3 I lit. a - d der Richtlinie 76/207/EWG ${ }^{86}$ mit dem Unterschied, daß die eine Richtlinie an das Diskriminierungsmerkmal Geschlecht, die andere an die Rasse bzw. ethnische Herkunft anknüpft. Der sachliche Anwendungsbereich der Richtlinie 2000/43/EG erstreckt sich darüber hinaus gem. Art. 3 I auf

„e) den Sozialschutz, einschließlich der sozialen Sicherheit und der Gesundheitsdienste;

f) die sozialen Vergünstigungen;

g) die Bildung;

h) den Zugang zu und die Versorgung mit Gütern und Dienstleistungen, die der Öffentlichkeit zur Verfügung stehen, einschließlich von Wohnraum.“

Wie bereits erwähnt, statuieren die $\S \S 7$ und 19 AGG Benachteiligungsverbote innerhalb und außerhalb von Beschäftigung und Beruf. In Umsetzung der Richtlinie 2000/43/EG sind dabei unter anderem Benachteiligungen ,aus Gründen der Rasse oder wegen der ethnischen Herkunft“ unzulässig.

Im Zusammenhang mit $\S 19$ I Nr. 2 AGG, der Benachteiligungen bei der Begründung, Durchführung und Beendigung zivilrechtlicher Schuldverhältnisse, die eine privatrechtliche Versicherung zum Gegenstand haben, für unzulässig erklärt, ${ }^{87}$ sei auch auf $\S 81$ e Versicherungsaufsichtsgesetz (VAG) hingewiesen:

„Als Mißstand im Sinne des $\S 81$ Abs. 2 sind auch Tarifbestimmungen und Prämienkalkulationen anzusehen, die auf die Staatsangehörigkeit des Versicherungsnehmers oder Versicherten oder auf deren Zugehörigkeit zu einer ethnischen Gruppe abstellen.“

Die Regelung wurde als Reaktion auf Abwehrpraktiken von Kfz-Versicherern gegenüber Ausländern eingeführt ${ }^{88}$ und hat in diesem Bereich ihre vorrangige Bedeutung. ${ }^{89}$

$\mathrm{Zu}$ erwähnen bleibt, daß zum Völkergewohnheitsrecht allgemeine Menschenrechte zählen, ${ }^{90}$ worunter auch das Recht auf Schutz vor rassistischer Diskriminierung fällt. ${ }^{91}$

menschlicher Rassen zu belegen, zurück. Die Verwendung des Begriffs „Rasse“ impliziert nicht die Akzeptanz solcher Theorien.“ (Abl. EG 2000 L 180, S. 22 (22)); Nickel ermahnt den deutschen Gesetzgeber, für den Fall gesetzgeberischen Tätigwerdens auf den Begriff „Rasse“ zu verzichten und stattdessen - wie in § 81 e VAG - von der „Zugehörigkeit zu einer ethnischen Gruppe“ zu sprechen. (Nickel, NJW 2001, S. 2668 (2669)).

86 Insoweit sei auf die Ausführungen zu dieser Richtlinie verwiesen.

87 Bereits Art. 3 I lit. h Richtlinie 2000/43/EG verpflichte u. a. auch die Versicherer. (Thüsing, NZA 2001, S. 1061 (1061)).

88 Kaulbach in Fahr/Kaulbach, $\S 81 \mathrm{e}, \mathrm{Rn} .1$; Sie ist nicht Konsequenz europarechtlicher Vorgaben. (Thüsing, NZA 2001, S. 1061 (1061, Fn. 7)).

89 Thüsing, NZA 2001, S. 1061 (1061, Fn. 7).

90 Rojahn in v. Münch/Kunig, Art. 25, Rn. 29; Streinz in Sachs, Art. 25, Rn. 68.

91 Herdegen in Maunz/Dürig, Art. 25, Rn. 48. 
Im Rahmenübereinkommen zum Schutz nationaler Minderheiten ${ }^{92}$ vom 01.02.1995 verpflichten sich die Vertragsstaaten in Art. 4 I,

„[...] jeder Person, die einer nationalen Minderheit angehört, das Recht auf Gleichheit vor dem Gesetz und auf gleichen Schutz durch das Gesetz zu gewährleisten. In dieser Hinsicht ist jede Diskriminierung aus Gründen der Zugehörigkeit zu einer nationalen Minderheit verboten." ${ }^{، 93}$

Die Verfassungen einiger deutscher Bundesländer enthalten Bestimmungen zur Anerkennung nationaler, ethnischer und kultureller Minderheiten. ${ }^{94} \mathrm{Zu}$ den Minderheiten zählen in Deutschland die Sorben, Dänen, Sinti und Roma ${ }^{95}$ sowie Friesen. Nicht unter diesen Begriff fallen hingegen Personen türkischer und ex-jugoslawischer Herkunft, ${ }^{96}$ mithin Personen, die in Deutschland in besonderem Maße von alltäglicher Diskriminierung betroffen sind. ${ }^{97}$

\section{E. Gleichbehandlung von Menschen unterschiedlicher Staatsangehörigkeit}

Diskriminierungsschutz aufgrund der Staatsangehörigkeit ist ein spezifisches Phänomen des EGVertrages, dessen Grund darin zu sehen ist, daß die Europäische Union in einer Vielzahl von Bereichen eine Einheit der Nationalstaaten anstrebt. Der Ausländer, der Bürger eines EUMitgliedstaates ist, soll in diesen Bereichen nicht anders als der Inländer behandelt werden.

In diesem Sinne bestimmt Art. 12 EGV, daß

92 BGB1. 1997 II, S. $1408 \mathrm{ff}$

93 Vergleiche in diesem Zusammenhang auch Art. 27 des Internationalen Paktes über bürgerliche und politische Rechte: „In Staaten mit ethnischen, religiösen oder sprachlichen Minderheiten darf Angehörigen solcher Minderheiten nicht das Recht vorenthalten werden, gemeinsam mit anderen Angehörigen ihrer Gruppe ihr eigenes kulturelles Leben zu pflegen, ihre eigene Religion zu bekennen und auszuüben oder sich ihrer eigenen Sprache zu bedienen.".

94 Huster in Friauf/Höfling, Band 1, C Art. 3, Rn. 14; vgl. Art. 25 BrandenbVerf; Art. 5, 6 SachsVerf; Art. 37 SachsAnhVerf; Art. 5 SchlHolstVerf; Art. 18 MecklVPVerf.

95 Auch der Begriff „Zigeuner“ wird in diesem Zusammenhang benutzt, der indes nicht unumstritten ist, wie die Auseinandersetzung um die Inschrift des Denkmals der während des Nationalsozialismus verfolgten und getöteten Angehörigen dieser Minderheit zeigt.

96 Hailbronner in Graf Vitzthum, 3. Abschnitt, Rn. 338, Fn. 493.

97 Befragungen des Zentrums für Türkeistudien (ZfT) aus den Jahren 1999 und 2000 haben ergeben, daß die Problemwahrnehmung türkischer Migranten in bezug auf erlebte Diskriminierung wächst. Diskriminierung sei dabei besonders in den von sozio-ökonomischer Konkurrenz geprägten Bereichen virulent und würde überhaupt viel weniger beim Kontakt mit öffentlichen Institutionen empfunden, als vielmehr in der zivilgesellschaftlichen Sphäre, namentlich auf dem freien Arbeits- und Wohnungsmarkt. (Sen/Sauer/Halm, ZAR 2001, S. 214 (216)) Auf dem Wohnungsmarkt sei die Ablehnung insbesondere gegenüber Türken und Jugoslawen gewachsen. (Hähnel, ZAR 2002, S. 356 (359) In diesem Zusammenhang wird darauf hingewiesen, daß die hohe Konzentration von Ausländern in bestimmten Stadtteilen nicht mit deren genereller Neigung begründet werden könne, sie lebten lieber unter sich. (Hoffmann, ZAR 2002, S. 63 (65, Fn. 9)). 
„[u]nbeschadet besonderer Bestimmungen dieses Vertrages [...] in seinem Anwendungsbereich jede Diskriminierung aus Gründen der Staatsangehörigkeit [verboten ist].“98

Konkretisiert wird Art. 12 durch die sog. „Grundfreiheiten“ des EGV. ${ }^{99} \mathrm{Zu}$ den Grundfreiheiten zählt die in Art. 39 EGV festgeschriebene Arbeitnehmerfreizügigkeit. Sie umfaßt gem. Art. 39 II EGV

„,die Abschaffung jeder auf der Staatsangehörigkeit beruhenden unterschiedlichen Behandlung der Arbeitnehmer der Mitgliedstaaten in bezug auf Beschäftigung, Entlohnung und sonstige Arbeitsbedingungen.“

\section{Gemäß Art. 40 EGV trifft der Rat}

„[...] durch Richtlinien und Verordnungen alle erforderlichen Maßnahmen, um die Freizügigkeit der Arbeitnehmer im Sinne des Artikels 39 herzustellen $[\ldots]^{\star}$. ${ }^{100}$

Die auf Grundlage dieser Ermächtigungsnorm erlassene Verordnung 1612/68 über die Freizügigkeit der Arbeitnehmer innerhalb der Gemeinschaft ${ }^{101}$ vom 15.10 .1968 sieht in Art. 7 I - der auch im Privatrechtsverhältnis gilt ${ }^{102}-$ vor, daß

„[e]in Arbeitnehmer, der Staatsangehöriger eines Mitgliedstaats ist, [...] auf Grund seiner Staatsangehörigkeit im Hoheitsgebiet der anderen Mitgliedstaaten hinsichtlich der Beschäftigungs- und Arbeitsbedingungen, insbesondere der im Hinblick auf Entlohnung, Kündigung und, falls er arbeitslos geworden ist, im Hinblick auf berufliche Wiedereingliederung oder Wiedereinstellung, nicht anders behandelt werden [darf] als die inländischen Arbeitnehmer.“

Darüber hinaus bestimmt Art. 7 IV, daß

„[a]lle Bestimmungen in Tarif- und Einzelarbeitsverträgen oder sonstigen Kollektivvereinbarungen betreffend Zugang zur Beschäftigung, Beschäftigung, Entlohnung und alle übrigen Arbeits- und Kündigungsbedingungen [...] von Rechts

98 Ähnlich formuliert es die Charta der Grundrechte der Europäischen Union in Art. 21 II.; Bereits jetzt soll dem Art. 12 EGV die Funktion eines Gemeinschaftsgrundrechts im Sinne des Art. 6 II EUV zukommen. (Szczekalla, Die sogenannten grundrechtlichen Schutzpflichten im deutschen und europäischen Recht, S. 477).

99 Der gegenüber besonderen Diskriminierungsverboten subsidiäre Art. 12 stellt gegenüber den Grundfreiheiten das allgemeine Diskriminierungsverbot dar. (Holoubek in Schwarze, Art. 12 EGV, Rn. 8 f.; Streinz, Europarecht, Rn. 668)

Die Grundfreiheiten werden teilweise auch als Gemeinschaftsgrundrechte bezeichnet. (siehe dazu die umfangreichen Nachweise bei Szczekalla, Die sogenannten grundrechtlichen Schutzpflichten im deutschen und europäischen Recht, S. 462 - 464).

100 Art. 12 enthält in seinem S. 2 ebenfalls eine Ermächtigungsgrundlage für den Rat, ,,[...] Regelungen für das Verbot solcher Diskriminierungen [zu] treffen." Auf der Grundlage des Art. 12 S. 2 sind jedoch bislang keine für die vorliegende Arbeit relevanten Maßnahmen erlassen worden.

101 Abl. EG 1968 Nr. L 257, S. 2; in der jetzt gültigen Fassung Abl. EG 1992 Nr. L 245, S. 1; Art. 49 EGV a.F., der in der Präambel der Verordnung vor allem als Ermächtigungsgrundlage genannt wird, entspricht Art. 40 EGV n.F..

102 Schneider/Wunderlich in Schwarze, § $39 \mathrm{EGV,} \mathrm{Rn.} \mathrm{71;} \mathrm{Birk} \mathrm{in} \mathrm{Münchener} \mathrm{Handbuch} \mathrm{zum} \mathrm{Arbeitsrecht,} \mathrm{Band} \mathrm{1,} \mathrm{§}$ 19, Rn. 36. 
wegen nichtig [sind], soweit sie für Arbeitnehmer, die Staatsangehörige anderer Mitgliedstaaten sind, diskriminierende Bedingungen vorsehen oder zulassen.“

Art. 8 statuiert einen

„[...] Anspruch auf gleiche Behandlung hinsichtlich der Zugehörigkeit zu Gewerkschaften und der Ausübung gewerkschaftlicher Rechte, einschließlich des Wahlrechts sowie des Zugangs zur Verwaltung oder Leitung von Gewerkschaften $[\ldots]^{\star .}{ }^{103}$

Da es sich bei Gewerkschaften um private Verbände handelt, ${ }^{104}$ potenzielle Diskriminierungen somit im Privatrechtsverhältnis stattfinden, erlangt Art. 8 im Rahmen dieser Arbeit Bedeutung. Schließlich bestimmt Art. 9 I, daß

„Arbeitnehmer, die die Staatsangehörigkeit eines Mitgliedstaats besitzen und im Hoheitsgebiet eines anderen Mitgliedstaats beschäftigt sind, hinsichtlich einer Wohnung, einschließlich der Erlangung des Eigentums an der von ihnen benötigten Wohnung, alle Rechte und Vergünstigungen [genießen] wie inländische Arbeitnehmer.“105

Anzumerken ist, daß die in Deutschland lebenden Türken, die besonders von Diskriminierung betroffen sind, nicht den Schutz der Grundfreiheiten genießen, da die Türkei bislang kein Unionsmitglied ist. Selbst wenn sie in Zukunft Mitglied sein sollte, steht nicht automatisch fest, daß ihre Bürger die Rechte aus Art. 39 EGV genießen werden. So hat die EU-Kommission im Oktober 2004 zwar die Aufnahme von Beitrittsverhandlungen mit der Türkei empfohlen, jedoch enthält die Empfehlung den Vorschlag, für türkische Staatsbürger längerfristig oder sogar auf Dauer die Arbeitnehmerfreizügigkeit einzuschränken. ${ }^{106}$

Potenziell auch bei Türken einschlägig ist hingegen die Richtlinie 2003/109/EG betreffend die Rechtsstellung der langfristig aufenthaltsberechtigten Drittstaatsangehörigen ${ }^{107}$ vom 25.11.2003. ${ }^{108}$ Gemäß Art. 11 I der Richtlinie werden langfristig Aufenthaltsberechtigte unter anderem auf folgenden Gebieten grundsätzlich wie eigene Staatsangehörige behandelt:

103 Der Begriff der „Gewerkschaft“ umfaßt alle Einrichtungen, die der Verteidigung und Vertretung von Arbeitnehmerinteressen dienen, also z.B. auch Berufskammern. (Brechmann in Calliess/Ruffert, Art. 39 EGV, Rn. 67).

104 Franzen in Streinz, Art. 39 EGV, Rn. 95, Fn. 357.

105 Art. 9 hat jedoch als Unterfall des Art. 7 II (Birk in Münchener Handbuch zum Arbeitsrecht, Band 1, § 19, Rn. 40), der seinerseits staatlich gewährte soziale Vergünstigungen betrifft (Brechmann in Calliess/Ruffert, Art. 39 EGV, Rn. 62), für die vorliegende Arbeit keine Bedeutung.

106 FAZ vom 07.10.2004, S. 1 unter dem Titel: „Die EU-Kommission empfiehlt Beitrittsverhandlungen mit der Türkei“.

107 Abl. EG 2004, Nr. L 16, S. 44 ff.

108 „Drittstaatsangehöriger“ ist gem. Art. 2 lit. a) der Richtlinie jede Person, die nicht Unionsbürger i.S.d. Art. 17 I EGV ist. Gemäß Art. 17 I 2 EGV ist Unionsbürger, wer die Staatsangehörigkeit eines Mitgliedstaates besitzt. 
„a) Zugang zu einer unselbständigen oder selbständigen Erwerbstätigkeit, wenn diese nicht, auch nicht zeitweise, mit der Ausübung öffentlicher Gewalt verbunden ist, sowie Beschäftigungs- und Arbeitsbedingungen, einschließlich Entlassungsbedingungen und Arbeitsentgelt;

f) Zugang zu Waren und Dienstleistungen sowie zur Lieferung von Waren und Erbringung von Dienstleistungen für die Öffentlichkeit und zu Verfahren für den Erhalt von Wohnraum“.

\section{F. Gleichbehandlung Behinderter}

Maßnahmen, die allein dem Schutz vor Diskriminierung aufgrund einer Behinderung dienen, existieren im Gegensatz zu den bereits erwähnten Merkmalen Geschlecht und Rasse bzw. ethnische Herkunft auf völkerrechtlicher Ebene nicht. Gleichwohl verdeutlichen einzelne völkerrechtliche Maßnahmen, daß auch das Unterscheidungsmerkmal Behinderung im Kampf gegen Diskriminierung eine besondere Stellung einnimmt.

So verpflichtet die Europäische Sozialcharta die Vertragsparteien in Art. 15 Nr. 2

„geeignete Maßnahmen zu treffen für die Vermittlung Behinderter auf Arbeitsplätze, namentlich [...] durch Maßnahmen, die den Arbeitgebern einen Anreiz zur Einstellung von Behinderten bieten.“

Die Gemeinschaftscharta der Sozialen Grundrechte der Arbeitnehmer verfolgt gem. ihres Titels I Nr. 26 neben der beruflichen auch die soziale Eingliederung behinderter Menschen. Gemäß Art. 25 der Charta der Grundrechte der Europäischen Union

„[...] anerkennt und achtet [die Union] den Anspruch von Menschen mit Behinderung auf Maßnahmen zur Gewährleistung ihrer Eigenständigkeit, ihrer sozialen und beruflichen Eingliederung und ihrer Teilnahme am Leben der Gemeinschaft.“

$\mathrm{Zu}$ beachten ist, daß Art. 25 keinen besonderen Gleichheitssatz enthält, sondern lediglich eine Aufgaben- und Zielbestimmung darstellt. ${ }^{109}$

Besondere Erwähnung verdient an dieser Stelle die Richtlinie 2000/78/EG zur Festlegung eines allgemeinen Rahmens für die Verwirklichung der Gleichbehandlung in Beschäftigung und Beruf $f^{10}$ vom 27.11.2000, die wie die bereits erwähnte Richtlinie 2000/43/EG auf Grundlage des Art. 13 EGV erlassen worden ist. ${ }^{111}$ Sie bezweckt gem. ihres Art. 1 unter anderem die Bekämpfung der Diskriminierung aufgrund einer Behinderung.

Ein Verbot der Diskriminierung behinderter Menschen enthält auch die deutsche Verfassung. Gemäß Art. 3 III 2 GG ,[darf n]iemand [...] wegen seiner Behinderung benachteiligt werden.“ 
Fraglich ist auch hier, ob die Norm den Staat verpflichtet, vor Diskriminierung durch Private zu schützen. Art. 3 III 2 GG statuiert im Gegensatz zu Art. 3 III 1 GG kein Bevorzugungsverbot. Dadurch soll Art. 3 III 2 GG ein Stück in Richtung des Art. 3 II GG rücken. ${ }^{112}$ Ganz überwiegend wird dennoch vertreten, daß Art. 3 III 2 GG den Staat zumindest nicht zu gesetzgeberischen Maßnahmen verpflichtet. ${ }^{113}$

Mit der Einfügung des am 01.07.2001 in Kraft getreten § 81 II des neunten Sozialgesetzbuchs (SGB IX) beabsichtigte der Gesetzgeber die Umsetzung der Richtlinie 2000/78/EG in bezug auf das Unterscheidungsmerkmal Behinderung. ${ }^{114}$ Gemäß § 81 II 1 SGB IX

„[dürfen] Arbeitgeber [...] schwerbehinderte Beschäftigte nicht wegen ihrer Behinderung benachteiligen.“115

In den folgenden Sätzen, die das Benachteiligungsverbot des Satzes 1 konkretisieren, enthielt $\S 81$ II SGB IX in seiner ursprünglichen Fassung Bestimmungen, die dem $\S 611$ a BGB-A nachgebildet waren. Die Kommentierung und Rechtsprechung zum Verbot der Benachteiligung aufgrund des Geschlechts sei aus diesem Grund nahezu vollständig auf das einer Behinderung übertragbar gewesen. ${ }^{116}$

Durch Art. $3 \mathrm{X}$ des Gesetzes zur Umsetzung europäischer Richtlinien zur Verwirklichung des Grundsatzes der Gleichbehandlung ${ }^{117}$ wurde $§ 81$ II 2 SGB IX wie folgt neu gefaßt:

„Im Einzelnen gelten hierzu die Regelungen des Allgemeinen Gleichbehandlungsgesetzes.“118

112 Heun in Dreier, Band I, Art. 3, Rn. 134.

113 Osterloh argumentiert, es fehle an einer dem Art. 3 II 2 GG entsprechenden Formulierung (Osterloh in Sachs, Art. 3, Rn. 307); Auch Starck verneint eine Verpflichtung des Privatgesetzgebers, wenn er formuliert, dieser könne Normen schaffen, die eine Gleichbehandlung Behinderter im Privatrechtsverkehr verlangen. (Starck in von Mangoldt/Klein/Starck, Band 1, Art. 3 Abs. 3, Rn. 388) Eine differnzierte Ansicht vertritt Casper, der dem Art. 3 III 2 GG i.V.m. dem Sozialstaatsprinzip ein objektiv-rechtliches Optimierungsgebot entnimmt, durch das insbesondere dem Gesetzgeber aufgegeben werde, den Unterschied zwischen behinderten und nichtbehinderten Personen möglichst zu beseitigen und auf eine Integration der Behinderten in die Gesellschaft hinzuwirken ohne jedoch ein unmittelbares subjektives Teilhaberecht des Behinderten auf konkrete staatliche Leistungen zu statuieren. (Casper, EuGRZ 2000, S. 135 (139 f.) Ähnlich formuliert es auch Frowein: „Eine Ausschaltung der Benachteiligung wegen Behinderung ist ganz offenbar nur dann ein sinnvolles und erreichbares Ziel, wenn damit nicht nur das Verhältnis zu dem Staat und seinen Organen gemeint ist, sondern ein allgemeines Prinzip ausgedrückt wird, dessen Förderung Sache des Staates ist. (Frowein in Festschrift für Hans F. Zacher, S. 157 (159)) Auch Jarass betont, Art. 3 III 2 GG verlange die Förderung Behinderter bzw. den Abbau von Benachteiligungen in der Gesellschaft, ohne jedoch eine Verpflichtung des Staates, Behinderte im Privatrechtsverkehr vor Diskriminierung zu schützen, zu konstatieren. (Jarass in Jarass/Pieroth, Art. 3, Rn. 131) Ebenso sieht Scholz in Art. 3 III 2 GG einen Auftrag an den Gesetzgeber zur Abwehr von Diskriminierungen Behinderter, der jedoch nicht so weit gehe, daß der Gesetzgeber zum Erlaß eines Gleichstellungsgesetzes für Behinderte sowie anderer Leistungen und Begünstigungen verpflichtet ist, da es in dessen pflichtgemäßem Ermessen stehe, auf welche Weise er das nunmehr ausdrücklich verankerte Benachteiligungsverbot für Behinderte konkret umsetzt. (Scholz in Maunz/Dürig, Band I, Art. 3 Abs. 3, Rn. 174 f.).

114 Junker, Grundkurs Arbeitsrecht, Rn. 165 a.

115 Wann ein Mensch schwerbehindert im Sinne des § 81 II SGB IX ist, ergibt sich aus § 2 II, III SGB IX.

116 Neumann in Neumann/Pahlen/Majerski-Pahlen, § 81, Rn. 11.

117 BGBl. 2006 I, S. 1897 ff.

118 Beachte in diesem Zusammenhang die Übergangsvorschrift des § 33 I AGG, die laut Entwurfsbegründung auch für Benachteiligungen nach $\S 81$ II SGB IX gelten soll. (Entwurfsbegründung, BT-Druck. 16/1780, S. 53). 
Grund für die Verweisung ins AGG bildet die Tatsache, daß nunmehr dieses Gesetz, wie bereits erwähnt, in den $\S \S 7$ und 19 Benachteiligungsverbote innerhalb und außerhalb von Beschäftigung und Beruf statuiert. Unzulässig sind dabei - in Umsetzung der Richtlinie 2000/78/EG - unter anderem Benachteiligungen ,wegen [...] einer Behinderung““

Besondere Erwähnung verdient $\S 81$ IV SGB IX, der den Arbeitgeber zu bestimmten Fördermaßnahmen zugunsten Behinderter, wie z.B. der behindertengerechten Einrichtung und Unterhaltung der Arbeitstätten (Nr. 4), verpflichtet. Eine ähnliche Norm enthält auch die Richtlinie 2000/78/EG. Gemäß ihres Art. 5 muß

„[...] der Arbeitgeber die geeigneten und im konkreten Fall erforderlichen Maßnahmen [ergreifen], um den Menschen mit Behinderung den Zugang zur Beschäftigung, die Ausübung eines Berufes, den beruflichen Aufstieg und die Teilnahme an Aus- und Weiterbildungsmaßnahmen zu ermöglichen, es sei denn, diese Maßnahmen würden den Arbeitgeber unverhältnismäßig belasten. Diese Belastung ist nicht unverhältnismäßig, wenn sie durch geltende Maßnahmen im Rahmen der Behindertenpolitik des Mitgliedstaates ausreichend kompensiert wird.“

Art. 5 der Richtlinie und $\S 81$ IV SGB IX deuten an, daß es im Falle des Unterscheidungsmerkmals Behinderung nicht ausreicht, ein bestimmtes (diskriminierendes) Verhalten zu verbieten. Erforderlich ist vielmehr, daß zunächst die Möglichkeit der Teilnahme Behinderter am gesellschaftlichen Leben gesichert wird.

Diesem Umstand versucht das Gesetz zur Gleichstellung behinderter Menschen (Behindertengleichstellungsgesetz $-B G G)^{119}$ vom 27.04.2002 durch Regelungen über die sog. Barrierefreiheit Rechnung $\mathrm{zu}$ tragen. ${ }^{120}$ Unmittelbare Bedeutung erlangt das BGG für die vorliegende Arbeit jedoch nicht, da es sich nicht an Privatpersonen richtet, selbige mithin nicht verpflichtet.

Der Diskriminierungsschutz reicht nicht soweit, daß der Behinderte einen Einstellungsanspruch hätte. § 15 VI AGG schließt einen derartigen Anspruch explizit aus. Zu beachten ist jedoch, daß Unternehmen einer bestimmten Größe gem. § 71 SGB IX einen gewissen Prozentsatz an Arbeitsplätzen schwerbehinderten Menschen zur Verfügung stellen müssen. Aus dieser Verpflichtung folgt aber kein Recht des einzelnen auf Einstellung, da die Beschäftigungspflicht des Arbeitgebers lediglich im Verhältnis zum Staat besteht. ${ }^{121}$

119 BGB1. 2002, I 1, S. 1468 ff.

120 Gemäß § 4 BGG sind barrierefrei ,[...] bauliche und sonstige Anlagen, Verkehrsmittel, technische Gebrauchsgegenstände, Systeme der Informationsverarbeitung, akustische und visuelle Informationsquellen und Kommunikationseinrichtungen sowie andere gestaltete Lebensbereiche, wenn sie für behinderte Menschen in der allgemein üblichen Weise, ohne besondere Erschwernis und grundsätzlich ohne fremde Hilfe zugänglich und nutzbar sind.“.

121 Kossens in Kossens/von der Heide/Maaß, § 71, Rn. 3; Neumann in Neumann/Pahlen/Majerski-Pahlen, § 71, Rn. 3; Gewarnt wird deshalb vor einer Fehlinterpretation des $\S 81$ III sowie des $\S 81$ IV 1 Nr. 1. Die Bestimmungen 
Gemäß $§ 81$ II 1 Nr. 1 SGB IX darf ein Schwerbehinderter unter anderem bei einer Kündigung nicht wegen seiner Behinderung benachteiligt werden. Zudem gewährt $\S 85$ SGB IX besagtem Personenkreis besonderen Kündigungsschutz. Gemäß dieser Norm bedarf

„[d]ie [ordentliche] Kündigung des Arbeitsverhältnisses eines schwerbehinderten Menschen durch den Arbeitgeber [...] der vorherigen Zustimmung des Integrationsamtes.“

Gleiches gilt gem. § 91 I i.V.m. § 85 SGB IX auch für die außerordentliche Kündigung. Wie im Falle des $\S 9 \mathrm{MuSchG}$ dürfte die Vorschrift nicht allein den Schutz vor Diskriminierung, sondern vor allem bezwecken, vor Ausspruch der Kündigung die besonderen Schutzinteressen Schwerbehinderter zu berücksichtigen und Kündigungen, die mit dem Schutzzweck des Gesetzes unvereinbar sind, zu vermeiden. ${ }^{122}$

\section{G. Gleichbehandlung in Beschäftigung und Beruf}

Wie bereits gezeigt, haben eine Vielzahl völkerrechtlicher Antidiskriminierungsmaßnahmen die Gleichbehandlung in Beschäftigung und Beruf zum Gegenstand. Der Grund dafür dürfte zum einen darin liegen, daß die Arbeitsstelle finanzielle wie soziale Existenzgrundlage des Menschen, mithin von herausragender Bedeutung für ihn ist, ${ }^{123}$ zum anderen darin, daß es sich bei Arbeitsverhältnissen um Dauerschuldverhältnisse handelt, also einen Bereich betrifft, der - wie sich im Laufe der Arbeit zeigen wird - besonders anfällig bzw. prädestiniert für Diskriminierung ist. In Art. 2 des Übereinkommens der International Labour Organisation (ILO) Nr. 111 über die Diskriminierung in Beschäftigung und Beruf ${ }^{124}$ vom 25.06 .1958 verpflichten sich die Mitgliedstaaten der Organisation

„[...] eine innerstaatliche Politik festzulegen und zu verfolgen, die darauf abzielt, [...] die Gleichheit der Gelegenheiten und der Behandlung in bezug auf Beschäftigung und Beruf zu fördern, um jegliche Diskriminierung auszuschalten.“

Unter Diskriminierung versteht das Übereinkommen gem. Art. 1 lit. a

,jede Unterscheidung, Ausschließung oder Bevorzugung, die auf Grund der Rasse, der Hautfarbe, des Geschlechts, des Glaubensbekenntnisses, der politischen Meinung, der nationalen Abstammung oder der sozialen Herkunft

könnten zu der Annahme verleiten, der Behinderte habe einen Anspruch auf Einstellung gegenüber dem Arbeitgeber.

122 Kossens in Kossens/von der Heide/Maaß, § 85, Rn. 1.

123 Auf den hohen Stellenwert des Arbeitsverhältnisses im Leben eines Menschen hat u.a. das BAG hingewiesen. (BAGE 48, S. $122(138))$.

124 BGBl. 1961 II 1, S. $97 \mathrm{ff}$. 
vorgenommen wird und die dazu führt, die Gleichheit der Gelegenheiten oder der Behandlung in Beschäftigung oder Beruf aufzuheben oder zu beeinträchtigen“.

Wie bereits erwähnt, bezwecken auch die Richtlinien 76/207/EWG, 2000/43/EG sowie 2000/78/EG Diskriminierungsschutz im Bereich des Arbeitslebens, wobei die zuletzt genannte in bezug auf die geschützten Diskriminierungsmerkmale die weitreichendsten Regelungen enthält. ${ }^{125}$

Neben dem speziellen Diskriminierungsschutz der $\S \S 7$ AGG und 81 II SGB IX gewährt das deutsche Arbeitsrecht auch allgemeinen, nicht auf bestimmte Unterscheidungsmerkmale beschränkten Diskriminierungsschutz in Form des sogenannten arbeitsrechtlichen Gleichbehandlungsgrundsatzes. ${ }^{126}$ Der allgemeine bzw. arbeitsrechtliche Gleichbehandlungsgrundsatz, der überwiegend als gewohnheitsrechtliche Umsetzung des Art. 3 I GG betrachtet wird, verbietet dem Arbeitgeber, Arbeitnehmer ohne sachlichen Grund von Begünstigungen auszunehmen oder ihnen Belastungen aufzuerlegen. ${ }^{127}$ Eine Verletzung des allgemeinen Gleichbehandlungsgrundsatzes soll jedoch immer nur dann in Betracht kommen, wenn der Arbeitgeber eine Regel aufstellt oder befolgt, die abstrakt an allgemeine Merkmale anknüpft und generell für eine Mehrzahl von Fällen gelten soll. ${ }^{128}$ Diese Voraussetzung sei beispielsweise dann erfüllt, wenn der Arbeitgeber, ohne tarif- oder arbeitsvertraglich dazu verpflichtet zu sein, eine Weihnachtsgratifikation zahlt. ${ }^{129}$ Das Gleichheitsprinzip soll demgegenüber dann nicht betroffen sein, wenn der Arbeitgeber mit den einzelnen Arbeitnehmern echte Individualabreden vereinbart. ${ }^{130}$ Grundsätzlich könne im Einzelarbeitsvertrag sogar wirksam festgelegt werden, daß ein Arbeitnehmer bestimmte Leistungen, die der Arbeitgeber bisher nach einer bestimmten Regel gewährt hat, nicht erhalten soll. ${ }^{131}$ Eine Ausnahme gelte insofern jedoch für zwingende Differenzierungsverbote wie sie unter anderem $\S 611$ a BGB-A aufgestellt habe. ${ }^{132}$ Dabei darf nicht übersehen werden, daß ein zwingendes Differenzierungsverbot die Gleichbehandlung nur insofern verlangt, als das verpönte Differenzierungsmerkmal nicht Anknüpfungspunkt für die unterschiedliche Behandlung sein darf. Der Arbeitgeber dürfe demgegenüber beispielsweise seinen Neffen aus familiären Gründen besser bezahlen als seine mitarbeitende Ehefrau. ${ }^{133}$ In diesem oder der Weltanschauung, einer Behinderung, des Alters oder der sexuellen Ausrichtung [...]“.

126 Es werden Überlegungen angestellt, den arbeitsrechtlichen Gleichbehandlungsgrundsatz ins Mietrecht zu übertragen. Der Grundsatz solle zumindest für Vermieter mit zahlreichen Mietparteien gelten. (Teichmann in Soergel, § 242, Rn. 48).

127 Junker, Grundkurs Arbeitsrecht, Rn. 58.

128 Junker, Grundkurs Arbeitsrecht, Rn. 58.

129 Junker, Grundkurs Arbeitsrecht, Rn. 58.

130 Otto, Einführung in das Arbeitsrecht, Rn. 167.

131 Richardi in Münchener Handbuch zum Arbeitsrecht, Band 1, § 14, Rn. 34.

132 Richardi in Münchener Handbuch zum Arbeitsrecht, Band 1, § 14, Rn. 35.

133 Otto, Einführung in das Arbeitsrecht, Rn. 167. 
Beispielsfall bevorzugt der Arbeitgeber seinen Neffen nicht aus dem Grund, weil dieser im Gegensatz zu seiner Frau ein Mann ist.

Anzumerken ist, daß das Institut des arbeitsrechtlichen Gleichbehandlungsgrundsatzes im Hinblick auf $\S 75$ I BetrVG teilweise als überflüssig angesehen wird. ${ }^{134}$ Gemäß jener Norm haben Arbeitgeber und Betriebsrat darüber zu wachen,

,[...] daß alle im Betrieb tätigen Personen nach den Grundsätzen von Recht und Billigkeit behandelt werden, insbesondere, daß jede Benachteiligung von Personen aus Gründen ihrer Rasse oder wegen ihrer ethnischen Herkunft, ihrer Abstammung oder sonstigen Herkunft, ihrer Nationalität, ihrer Religion oder Weltanschauung, ihrer Behinderung, ihres Alters, ihrer politischen oder gewerkschaftlichen Betätigung oder Einstellung oder wegen ihres Geschlechts oder ihrer sexuellen Identität unterbleibt. Sie haben darauf zu achten, daß Arbeitnehmer nicht wegen Überschreitung bestimmter Altersstufen benachteiligt werden.“135

Der Arbeitgeber ist unter anderem bei allen Maßnahmen im Rahmen des Einzelarbeitsvertrages, mithin bei dessen Abschluß, inhaltlichen Gestaltung und Beendigung sowie der Ausübung des Direktionsrechts, an die Verpflichtung aus $§ 75$ I BetrVG gebunden. ${ }^{136}$ Die Verpflichtung aus $§ 75$ BetrVG führe indes nicht zu korrespondierenden Ansprüchen des Arbeitnehmers gegenüber dem Arbeitgeber auf eine bestimmte Behandlung. ${ }^{137}$ Allenfalls beeinflusse die Norm mittelbar das Einzelarbeitsverhältnis dadurch, daß ihre Grundsätze arbeitsvertragliche Schutz- und Fürsorgepflichten des Arbeitgebers sowie den arbeitsrechtlichen Gleichbehandlungsgrundsatz mitprägten. ${ }^{138}$

Die Richtlinie 96/34/EG zu der von UNICE, CEEP und EGB geschlossenen Rahmenvereinbarung über Elternurlaub ${ }^{139}$ vom 03.06.1996 verbietet in Art. $1^{140}$

„[...] Entlassungen, die auf einem Antrag auf Elternurlaub oder auf der Inanspruchnahme des Elternurlaubs beruhen.“

134 Otto, Einführung in das Arbeitsrecht, Rn. 165.

135 § 75 I BetrVG ist zuletzt durch Art. 3 III des Gesetzes zur Umsetzung europäischer Richtlinien zur Verwirklichung des Grundsatzes der Gleichbehandlung - BGB1. 2006 I, S. 1897 ff. - modifiziert worden.

136 Kreutz in GK-BetrVG, Band II, §75, Rn. 22; Nicht unumstritten ist jedoch, ob § 75 BetrVG schon vor Vertragsschluß Wirkungen entfaltet, da man sich nicht einig darüber ist, ob zum geschützten Personenkreis dieser Bestimmung auch Arbeitnehmer zählen, die noch nicht im Betrieb tätig sind. (Nickel, NJW 2001, S. 2668 (2669); vgl. dazu Richardi in Richardi, § 75, Rn. 7).

137 Kreutz, in GK-BetrVG, Band II, § 75, Rn. 23 f.; Junker, Grundkurs Arbeitsrecht, Rn. 57; Richardi in Richardi, § 75, Rn. 8; Fitting/Kaiser/Heither/Engels/Schmidt, Betriebsverfassungsgesetz, § 75, Rn. 20.

138 Kreutz in GK-BetrVG, Band II, § 75, Rn. 25; Fitting/Kaiser/Heither/Engels/Schmidt, Betriebsverfassungsgesetz, $\S$ 75, Rn. 20; Besondere Bedeutung erlangt in diesem Zusammenhang die Tatsache, daß $§ 75$ I BetrVG absolute bzw. zwingende Differenzierungsverbote statuiert. (Waas, ZIP 2000, S. 2151 (2154 f.)).

139 Abl. EG 1996, Nr. L 145, S. 4 f.; zur rechtlichen Grundlage der Richtlinie im EGV vgl. Rebhahn in Schwarze, Art. 137 EGV, Rn. 14.

140 In Verbindung mit $\S 2$ Nr. 4 der Rahmenvereinbarung über Elternurlaub vom 14.12.1995 (Anhang zur RL 96/34/EG, Abl. EG 1996, Nr. L 145, S. 6 ff.). 
Außerdem soll der Arbeitnehmer gem. Art. $1^{141}$ im Anschluß an den Elternurlaub das Recht haben,

,$[\ldots]$ an seinen früheren Arbeitsplatz zurückzukehren oder, wenn das nicht möglich ist, entsprechend seinem Arbeitsvertrag oder Arbeitsverhältnis einer gleichwertigen oder ähnlichen Arbeit zugewiesen zu werden.“

Bemerkenswert an der Richtlinie 96/34/EG - und Grund, sie erst an dieser Stelle zu erwähnen - ist, daß sie in ihrem Anwendungsbereich jede Ungleichbehandlung von Personen mit Kind gegenüber Personen ohne Kind verbietet. Differenzierungsmerkmal ist somit nicht allein das Geschlecht, selbst wenn dies vordergründig bezweckt sein mag, ${ }^{142}$ sondern auch der Status: „Person mit Kind“. ${ }^{143}$

Das nationale Recht Deutschlands gewährte zunächst in $\S 18$ des Bundeserziehungsgeldgesetzes (BErzGG) besonderen Kündigungsschutz für den Fall der Inanspruchnahme von Elternzeit. Gemäß $\S 18$ I 1 BErzGG-A durfte

„,[d]er Arbeitgeber [...] das Arbeitsverhältnis ab dem Zeitpunkt, von dem an Elternzeit verlangt worden [war], höchstens jedoch acht Wochen vor Beginn der Elternzeit, und während der Elternzeit nicht kündigen.““144

Es wurde vertreten, die Bestimmung sei in dieser Form mit den Vorgaben der Richtlinie 96/34/EG vereinbar $^{145}$ und erfülle gleichzeitig die dem deutschen Gesetzgeber durch Art. 6 I GG aufgetragene Pflicht zum Schutz und zur Förderung der Familie. ${ }^{146}$ Wie im Falle der Richtlinie 96/34/EG genossen sowohl männliche als auch weibliche Arbeitnehmer den besonderen Kündigungsschutz aus $§ 18$ I 1 BErzGG-A. ${ }^{147}$ Das liegt daran, daß die Norm nicht - zumindest nicht primär - die Geschlechterdiskriminierung unterbinden wollte, sondern vordergründig einer möglichst problemlosen Anfangserziehung des Kindes diente. ${ }^{148}$

Eine dem $\S 18$ I 1 BErzGG-A entsprechende Bestimmung enthält nunmehr $\S 18$ I 1 des Bundeselterngeld- und Elternzeitgesetzes (BEEG), das am 01.01.2007 in Kraft getreten ist. ${ }^{149}$

141 In Verbindung mit $\S 2$ Nr. 5 der Rahmenvereinbarung über Elternurlaub vom 14.12.1995 (Anhang zur RL 96/34/EG, Abl. EG 1996, Nr. L 145, S. 6 ff.).

142 Vergleiche dazu die Präambel der Rahmenvereinbarung über den Elternurlaub.

143 Das BVerfG hat entschieden, daß der Gesetzgeber nicht nur im Bereich des Arbeitsrechts, sondern auch in anderen Bereichen des Privatrechts Regelungen mit besonderer Rücksicht auf Familien mit Kindern zu erwägen hat, so etwa das Verbot einer Kündigung von Mietverträgen über Wohnraum wegen der Aufnahme eines neugeborenen Kindes. (BVerfGE 88, S. 203 (260)).

144 Gemäß Art. 3 II des Gesetzes zur Einführung des Elterngeldes trat der „Zweite Abschnitt des Bundeserziehungsgeldgesetzes [...] am 31. Dezember 2006 außer Kraft. Im übrigen tritt das Bundeserziehungsgeldgesetz am 31. Dezember 2008 außer Kraft.“ (BR-Druck. 698/06)

145 Buchner/Becker, Mutterschutzgesetz und Bundeserziehungsgeldgesetz, Vor $\$ \S 15-21$ BerzGG, Rn. 17.

146 Buchner/Becker, Mutterschutzgesetz und Bundeserziehungsgeldgesetz, Vor $\S \S 15-21$ BerzGG, Rn. 13.

147 Buchner/Becker, Mutterschutzgesetz und Bundeserziehungsgeldgesetz, Vor $\S \S 9$ und 10 MuSchG, Rn. 5.

148 Buchner/Becker, Mutterschutzgesetz und Bundeserziehungsgeldgesetz, Vor $\S \S 9$ und $10 \mathrm{MuSchG}, \mathrm{Rn} .9$.

149 Vergleiche dazu Art. 3 I des Gesetzes zur Einführung des Elterngeldes. (BR-Druck. 698/06). 
Schließlich verbietet das Teilzeit- und Befristungsgesetz (TzBfG) in seinem $\S 4$ I die Diskriminierung eines teilzeitbeschäftigten Arbeitnehmers gegenüber einem vergleichbar vollzeitbeschäftigten Arbeitnehmer, in § 4 II die Diskriminierung eines befristet beschäftigten Arbeitnehmers gegenüber einem vergleichbar unbefristet beschäftigten Arbeitnehmer.

Vor dem Hintergrund der vorgestellten Antidiskriminierungsmaßnahmen auf nationaler wie internationaler Ebene dient der folgende Teil der Arbeit dem Ziel, den Begriff der Diskriminierung zu definieren. 

Den Ausgangspunkt aller Überlegungen bilden die vorgestellten Antidiskriminierungsmaßnahmen, weil sie indizieren, in welchen Bereichen und für welche Merkmale Diskriminierungsschutz notwendig ist. Insofern erfüllen sie die Funktion einer wichtigen Orientierungshilfe für die Beantwortung der aufgeworfenen Fragen.

Darüber hinaus sind sie insofern zwingend zur Grundlage aller weiteren Überlegungen zu machen, als sie - wie im Fall der Antidiskriminierungsrichtlinien - den deutschen Gesetzgeber zu konkretem Tätigwerden verpflichten. ${ }^{1}$ Dies ist auch der Grund dafür, weshalb sich der Verfasser im Rahmen dieser Arbeit nicht an der grundlegenden Diskussion um die Sinnhaftigkeit der Schaffung von Antidiskriminierungsmaßnahmen, insbesondere der Einführung eines allgemeinen Antidiskriminierungsgesetzes, beteiligt. Mit Blick auf die insofern bestehenden Verpflichtungen Deutschlands ist diese Diskussion in gewisser Weise müßig geworden. ${ }^{2}$ Das anerkannten zähneknirschend auch die Gegner eines Antidiskriminierungsgesetzes, die bis zum Inkrafttreten des Allgemeinen Gleichbehandlungsgesetzes am 18.08.2006 nur noch dafür kämpften, daß die Richtlinien nicht ,übererfüllt“ würden, ein Antidiskriminierungsgesetz also keine über den Inhalt der Richtlinien hinausgehenden Regelungen enthalte. ${ }^{3}$

Herausragende Bedeutung für die weiteren Ausführungen erlangen die Antidiskriminierungsrichtlinien 76/207/EWG, 2000/43/EG, 2000/78/EG sowie 2004/113/EG, enthalten sie doch im Vergleich zu den angesprochenen Antidiskriminierungsmaßnahmen die detailiertesten Bestimmungen bezüglich des Diskriminierungsbegriffs. Aus diesem Grund bilden sie im folgenden immer wieder den Ausgangspunkt der Betrachtungen.

\section{A. Diskriminierungsrelevantes Verhalten}

Die Antidiskriminierungsrichtlinien unterscheiden zwischen verschiedenen Formen diskriminierungsrelevanten Verhaltens.

\section{Benachteiligende Ungleichbehandlung - Unmittelbare bzw. direkte Diskriminierung}

Gemäß Art. 2 II lit. a der Richtlinie 2000/43/EG

\footnotetext{
Vergleiche dazu Teil 4, Prüfungspunkt A. II.

So auch Thüsing, NZA 2001, S. 1061 (1061).

„Unsere Mitgliedsunternehmen fordern die Bundesregierung auf, im weiteren Gesetzgebungsverfahren dafür Sorge zu tragen, daß das Gesetz auf die EU-Vorgaben beschränkt wird und die für die Immobilienbranche inakzeptablen Folgewirkungen beseitigt werden. Ich frage: Warum muß in Deutschland immer noch eins draufgesattelt werden? Warum reichen die drei EU-Richtlinien für ein Gesetz nicht aus?" (Präsident der Bundesvereinigung der Landesentwicklungs- und Immobiliengesellschaften von Michaelis in FAZ vom 25.02.2005, S. 49 unter dem Titel: „Was sagen Sie zum geplanten Antidiskriminierungsgesetz?“).
} 
„liegt eine unmittelbare Diskriminierung vor, wenn eine Person aufgrund ihrer Rasse oder ethnischen Herkunft in einer vergleichbaren Situation eine weniger günstige Behandlung als eine andere Person erfährt, erfahren hat oder erfahren würde“. 4

Diese Definition des Begriffs der Diskriminierung deckt sich zum Teil mit seinem allgemeinen Sprachgebrauch. Danach bezeichnet Diskriminierung u. a. die Benachteiligung bzw. Zurücksetzung bestimmter Personen bzw. Personengruppen, die daraus resultieren kann, daß Personen unterschiedlich behandelt werden. ${ }^{5}$

Während das Wort Diskriminierung im allgemeinen Sprachgebrauch einen negativen Charakter erhalten hat, ist dessen ursprüngliche Wortbedeutung neutral. „Discriminare“ bedeutet trennen bzw. absondern. ${ }^{6}$ In diesem neutralen Sinne wird der Begriff in der Fachsprache teilweise noch verwendet. $^{7}$

Bevor mit den weiteren Ausführungen fortgefahren werden kann, muß an dieser Stelle darauf hingewiesen werden, daß der im folgenden in unterschiedlichen Formen verwendete Begriff „Diskriminierung“ jeweils ein bestimmtes diskriminierungsrelevantes Verhalten beschreiben soll der terminus technicus „unmittelbaren Diskriminierung“ etwa eine Ungleichbehandlung. Dadurch soll nicht der Eindruck erweckt werden, jede Ungleichbehandlung stelle eine Diskriminierung dar. Die Entwurfsbegründung des am 18.08.2006 in Kraft getretenen Allgemeinen Gleichbehandlungsgesetzes (AGG) weist in diesem Zusammenhang zu recht darauf hin, ,daß nicht jede unterschiedliche Behandlung, die mit der Zufügung eines Nachteils verbunden ist, diskriminierenden Charakter hat. “8

\section{Offene Diskriminierung}

Unmittelbare bzw. direkte Diskriminierungen können in offener und verdeckter Weise erfolgen. Offen diskriminiert, wer die Ungleichbehandlung durch direkten Verweis auf das unerwünschte Merkmal begründet. ${ }^{9}$

4 Ähnliche Definitionen enthalten die Richtlinie 76/207/EWG in Art. 2 II 1. Spiegelstrich, Richtlinie2000/78/EG in Art. 2 II lit. a sowie Richtlinie 2004/113/EG in Art. 2 lit.a mit dem Unterschied, daß sie an andere Diskriminierungsmerkmale anknüpfen.

5 Duden, Das große Wörterbuch der deutschen Sprache, Band 2 (Bedr - Eink), S. 828; in diesem Sinne auch Nickel, nach dem der Begriff der Diskriminierung nach heutigem allgemeinen Sprachgebrauch eine ungerechtfertigte Ungleichbehandlung bezeichnet. (Nickel, Gleichheit und Differenz in der vielfältigen Republik, S. 69).

Duden, Das große Wörterbuch der deutschen Sprache, Band 2 (Bedr - Eink), S. 828.

Duden, Das große Wörterbuch der deutschen Sprache, Band 2 (Bedr - Eink), S. 828.

Entwurfsbegründung, BT-Druck. 16/1780, S. 30.

Nickel, Gleichheit und Differenz in der vielfältigen Republik, S. 83. 
Beispiel: So sollen Türsteher einer Göttinger Discothek eine Zutrittsverweigerung unter anderem damit begründet haben, daß „,die Geschäftsführung die Anweisung herausgegeben hätte, keine „Schwarzköpfe“ [...] hereinzulassen.“10

Fälle offener Diskriminierung sind mittlerweile - insbesondere wenn es um den Zutritt zu Gaststätten und Discotheken geht - selten geworden. ${ }^{11}$ Inwieweit diese Entwicklung Ergebnis einer gestiegenen Akzeptanz des anderen in der Gesellschaft ist, ist allerdings fraglich. Letztlich dürfte die Tatsache, daß derartige Diskriminierungen rechtlich geahndet wurden, zu einer Dezimierung dieser Erscheinungsform der Diskriminierung geführt haben. ${ }^{12}$

\section{Verdeckte Diskriminierung}

Gegen die Vermutung, in der Gesellschaft sei die Akzeptanz gegenüber dem anderen gestiegen, spricht die Tatsache, daß nach wie vor - wenn auch in subtilerer Art und Weise - diskriminiert wird. Der Diskriminierende unterwirft sich nach außen dem heutzutage gesellschaftlich bestehenden Zwang, ,politisch korrekt“ handeln $\mathrm{zu}$ müssen. ${ }^{13} \mathrm{Er}$ begründet deshalb die Ungleichbehandlung nicht mehr durch direkten Verweis auf das unerwünschte Merkmal, sondern knüpft äußerlich an ein anderes Merkmal an. ${ }^{14}$

Beispiele: Die Türsteher einer Göttinger Discothek verweigerten - ohne auf den wahren, diskriminierenden Grund hinzuweisen - unerwünschten Personen den Zutritt, indem sie darauf hinwiesen, daß an dem betreffenden Tag nur Stammgäste zugelassen seien. ${ }^{15}$

\section{Diskriminierungsdrittwirkung}

Einen Sonderfall der unmittelbaren Diskriminierung stellt die sog. „Diskriminierungsdrittwirkung“ dar. ${ }^{16}$ Mit diesem Begriff verbindet man die Konstellation, daß eine Person, die in einer gewissen

10 Aus einem Brief des Geschäftsführers des Göttinger Integrationsrates an die Geschäftsleitung der Discothek „Savoy“ vom 06.01.2003.

11 Nickel, Gleichheit und Differenz in der vielfältigen Republik, S. 83.

12 Vergleiche dazu OVG Münster, GewArch. 1967, S. 118 f.; OLG Frankfurt, NJW 1985, S. 1720 f.; siehe auch Antwort der Bundesregierung vom 29.07.1982 auf eine Anfrage bzgl. der Diskriminierung von Angehörigen der US-Streitkräfte in der Bundesrepublik Deutschland (BT-Druck. 9/1882).

13 Von Münch propagiert den Schutz der Meinungsfreiheit vor diesem Zwang nach „,political correctness“. (v. Münch, NJW 1999, S. 260 (262)); ebenso Säcker, ZRP 2002, S. 286 (287).

14 Krebber in Calliess/Ruffert, Art. 141 EGV, Rn. 39; Nickel irrt sich, wenn er behauptet, eine verdeckte Diskriminierung liege auch dann vor, wenn der „Diskriminierende“ sein Verhalten auf eine andere Person abwälzt. Wenn ein Gastwirt einen behinderten Gast mit der Begründung ablehnt, er könne den übrigen Gästen den Anblick einer mit Füßen essenden contergangeschädigten Frau nicht zumuten, dann verdeckt er den Grund der Ablehnung des behinderten Gastes gerade nicht. (vgl. Nickel, Gleichheit und Differenz in der vielfältigen Republik, S. 83 u. 84 , Fn. 272).

15 Aus einem Brief des Geschäftsführers des Göttinger Integrationsrates an die Geschäftsleitung der Discothek „Savoy“ vom 06.01.2003.

16 Den Begriff der „Diskriminierungsdrittwirkung“ prägt Nickel. (Nickel, Gleichheit und Differenz in der vielfältigen Republik, S. 191 und 193). 
(z.B. Verwandtschafts-) Beziehung zu der diskriminierten Person steht, ebenfalls Auswirkungen der Diskriminierung erdulden muß. ${ }^{17}$

Beispiel: Einer Frau war der Zutritt zu einer Discothek gewährt worden, ihrem Mann sowie dessen Neffen hingegen nicht. Begründet wurde dies damit, daß Ausländer keinen Zutritt zu der Lokalität hätten. Die Frau verließ daraufhin die Discothek, da sie ohne Mann und Neffen keinen Anreiz sah, in selbiger länger zu verweilen. ${ }^{18}$

In dem Beispielsfall läßt sich eine unmittelbare Diskriminierung auch gegenüber der Frau konstruieren. Die Frau weist dadurch, daß sie mit Ausländern bzw. Personen, die für Ausländer gehalten werden, die Discothek betreten möchte, ein Merkmal auf, aufgrunddessen sie anders behandelt wird als andere Besucher, die die Einrichtung in einer Gruppe betreten möchten.

Untermauern läßt sich die Einordnung der Diskriminierungsdrittwirkung als unmittelbare Diskriminierung gegenüber dem Dritten durch folgende Überlegung: Wenn ein Vermieter keine Kinder in seiner zu vermietenden Wohnung wünscht, dann wird er nicht an Eltern mit Kindern vermieten, selbst wenn ihm die Eltern sympathisch sein sollten und er an sie vermieten würde, wenn sie keine Kinder hätten. In einem solchen Fall stellt sich die Frage einer etwaigen Diskriminierung gegenüber den Eltern, obwohl der Vermieter genaugenommen nicht sie ablehnt, sondern die Kinder. ${ }^{19}$

\section{Einseitig belastende Behandlung}

Die bisherigen Ausführungen haben gezeigt, daß der Diskriminierungsbegriff die benachteiligende Ungleichbehandlung von Personen erfaßt. Fraglich ist, ob auch andere Verhaltensweisen den Tatbestand der Diskriminierung erfüllen können.

\section{Individuelle Vertragsgestaltung}

Beispiel: Arbeitgeber untersagt einer Arbeitnehmerin das - religiös bedingte - Tragen eines Kopftuchs.

17 Dieses Problem ist zum einen in dem Entwurf eines Gesetzes gegen Rassismus und die Diskriminierung ausländischer Bürgerinnen und Bürger (Antirassismusgesetz) der PDS vom 19.05.1995 thematisiert (BT-Druck. 13/1466), zum anderen in dem Entwurf eines Gesetzes zur Durchsetzung des Gleichbehandlungsgebotes des Art. 3 Grundgesetz (Gleichbehandlungsgesetz) der SPD vom 09.03.1998 (BT-Druck. 13/10081).

Beide Entwürfe sprechen zwar von mittelbarer Diskriminierung, meinen aber die Diskriminierungsdrittwirkung. (vgl. dazu die Gesetzesbegründung des PDS-Entwurfs zu $\S 2$ 2, BT-Druck. 13/1466, S. 10 sowie die Gesetzesbegründung des SPD-Entwurfs zu Art. 1, § 1, BT-Druck. 13/10081, S. 11 ).

18 Aus einem Brief des Betroffenen an den Ausländerbeirat der Stadt Göttingen vom 22.11.1999.

19 Erwähnenswert ist in diesem Zusammenhang ein Urteil des BVerfG, in dem das Gericht entschieden hat, daß der Gesetzgeber nicht nur im Bereich des Arbeitsrechts, sondern auch in anderen Bereichen des Privatrechts Regelungen mit besonderer Rücksicht auf Familien mit Kindern zu erwägen hat, so etwa das Verbot einer Kündigung von Mietverträgen über Wohnraum wegen der Aufnahme eines neugeborenen Kindes. (BVerfGE 88, S. $203(260))$. 
In dem Beispielsfall könnte man das Vorliegen einer Diskriminierung mit der Begründung verneinen, der Arbeitgeber „,behandle“ nur eine Person. In der Tat trifft der Arbeitgeber mit dem gegenüber der einzelnen Arbeitnehmerin ausgesprochenen Verbot und der darauf folgenden Kündigung keine Aussage darüber, ob er, vor das Problem gestellt, einem anderen Arbeitnehmer beispielsweise das Tragen der Kippa gestatten würde. Nur in diesem Fall ließe sich aber von einer Ungleichbehandlung aufgrund der Religion sprechen. Denn im Falle der verhaltensbedingten Kündigung kommt eine Verletzung des Gleichbehandlungsgebots nur in Betracht, wenn der Arbeitgeber einem Arbeitnehmer verhaltensbedingt kündigt, während er andere Arbeitnehmer, die sich nicht anderes verhalten, weiterbeschäftigt. ${ }^{20}$

\section{a) Hypothetische Ungleichbehandlung}

Allenfalls von einer hypothetischen Ungleichbehandlung ließe sich in dem Beispielsfall sprechen, wenn man davon ausginge, der Arbeitgeber, vor eine Entscheidung gestellt, würde andere religiöse Symbole tolerieren und demnach nicht mit Verbot und Kündigung reagieren.

Im Rahmen des Art. 141 I EGV ist umstritten, ob die Frage der Diskriminierung durch Vergleich mit einem hypothetischen Arbeitnehmer beantwortet werden kann. ${ }^{21}$ Der EuGH und Teile der Literatur lehnen dies ab. ${ }^{22}$ Man argumentiert, das Diskriminierungsverbot würde andernfalls zu einem Anspruch auf gerechte Bezahlung ausgedehnt. ${ }^{23}$

Für die Zulässigkeit des Vergleichs mit einer hypothetischen Person könnte indes Art. 2 II lit. a der Richtlinie 2000/43/EG sprechen, nach dem eine unmittelbare Diskriminierung vorliegt,

„[...] wenn eine Person [...] eine weniger günstige Behandlung als eine andere Person erfährt, erfahren hat oder erfahren würde“. 24

Fast wortlautgleich bestimmt § 3 I 1 AGG, daß eine unmittelbare Benachteiligung vorliegt,

„[...] wenn eine Person [...] eine weniger günstige Behandlung erfährt, als eine andere Person in einer vergleichbaren Situation erfährt, erfahren hat oder erfahren würde. ${ }^{\text {‘25 }}$

Richardi in Münchener Handbuch zum Arbeitsrecht, Band 1, § 14, Rn. 20.

Zum Meinungsstand vgl. Krebber in Calliess/Ruffert, Art. 141 EGV, Rn. 54.

EuGH, Rs. C-129/79, Slg. 1980 1, S. 1275 (1289, Rn. 15); Rs. C-200/91, Slg. 1994 I 4, S. 4389 (4430 f., Rn. 103$)$; Krebber in Calliess/Ruffert, Art. 141 EGV, Rn. 54.

23 Krebber in Calliess/Ruffert, Art. 141 EGV, Rn. 54.

24 Hervorhebung durch den Verfasser; Ähnliche Definitionen enthalten die Richtlinie 76/276/EWG in Art. 2 II 1. Spiegelstrich, Richtlinie2000/78/EG in Art. 2 II lit. a sowie Richtlinie 2004/113/EG in Art. 2 lit.a.

In diesem Sinne ist wohl auch Rust zu verstehen, wenn er etwas nebulös formuliert, die Antwort auf die Frage nach der Zulässigkeit des Vergleichs mit einer hypothetischen Arbeitskraft dürfte künftig in der Legaldefinition der unmittelbaren Diskriminierung in der Richtlinie 2002/73/EG zu finden sein. (Rust in von der Groeben/Schwarze, Band 3, Art. 141 EGV, Rn. 439); Dazu sei angemerkt, daß die Richtlinie 2002/73/EG die Richtlinie 76/207/EWG novelliert hat.

25 Hervorhebung durch den Verfasser. 
Fraglich ist, wie man die Formulierung: „erfahren würde“ verstehen muß. ${ }^{26}$ Sicherlich wird man nicht so weit gehen können, dem Arbeitgeber diskriminierendes Verhalten einfach zu unterstellen. Eine derartige Interpretation des Tatbestandsmerkmals „erfahren würde“ wäre verfehlt.

Auch die Vertreter, die sich grundsätzlich für den Vergleich mit einem hypothetischen Arbeitnehmer aussprechen, ${ }^{27}$ verlangen ,irgendwelche Indizien“, aus denen sich ergibt, daß die beschäftigten Frauen schlechter bezahlt werden als hypothetische männliche Arbeitnehmer für diese Tätigkeit vergütet würden, ${ }^{28}$ bzw. fordern die Bezugnahme auf das Entgelt, welches dem anderen Geschlecht für die gleiche Arbeit in demselben Unternehmen normalerweise gezahlt oder angeboten wird. ${ }^{29} \mathrm{Ob}$ eine andere Person eine bessere Behandlung erfahren würde, soll somit nicht einfach unterstellt werden können, sondern sich aus konkreten Indizien ergeben müssen.

Bei genauer Betrachtung der Argumente stellt man fest, daß beide Ansichten eine Vergleichsbasis in Gestalt eines tatsächlich existierenden Arbeitnehmers fordern, egal ob dieser gleichzeitig mit dem „diskriminierten“ Arbeitnehmer beschäftigt ist, davor oder dannach. ${ }^{30}$

Im Gegensatz zu Art. 141 I EGV wurde im Rahmen des $§ 612$ III 1 BGB-A das Problem des hypothetischen Arbeitgebers nicht thematisiert. Die Notwendigkeit des Vergleich mit einem realen Arbeitnehmer des anderen Geschlechts wurde, so scheint es zumindest, vorausgesetzt. ${ }^{31}$

In diesem Sinne dürfte auch $§ 3$ I 1 AGG zu interpretieren sein. Laut Entwurfsbegründung muß die unmittelbare Benachteiligung ,,[...] entweder noch andauern bzw. bereits abgeschlossen sein; oder aber es muß eine hinreichend konkrete Gefahr bestehen, daß eine solche Benachteiligung eintritt (,erfährt, erfahren hat oder erfahren würde“). Ein nur abstrakte Gefahr löst noch keine Ansprüche aus. $، 32$

Ein Vergleich mit anderen Arbeitnehmern, aus dem das Vorliegen einer Ungleichbehandlung hätte abgeleitet werden können, war im „Kopftuchfall“ scheinbar nicht möglich. Das Bundesarbeitsgericht hat sich mit der Frage des Vorliegens einer Ungleichbehandlung in diesem Fall jedenfalls nicht beschäftigt. Sein Urteil stützte das Gericht nicht auf Art. 3 III GG, erwähnte den Artikel nicht einmal. ${ }^{33}$ Es erklärte die vom Arbeitgeber ausgesprochene Kündigung vielmehr solche hypothetische Betrachtung in der Praxis aussehen soll“. (Bauer, NJW 2001, S. 2672 (2674)).

27 Steindorff, RdA 1988, S. 129 (132); Langenfeld, Die Gleichbehandlung von Mann und Frau im Europäischen Gemeinschaftsrecht, S. 253 f.

28 Steindorff, RdA 1988, S. 129 (133).

29 Langenfeld, Die Gleichbehandlung von Mann und Frau im Europäischen Gemeinschaftsrecht, S. 254.

$30 \quad$ Krebber in Calliess/Ruffert, Art. 141 EGV, Rn. 55.

31 Putzo in Palandt, § 612, Rn. 12; Hanau in Erman, § 612, Rn. 28; Raab in Soergel, Band 4/1, § 612, Rn. 55; Richardi in Staudinger, $\S 612$, Rn. 61.

32 Entwurfsbegründung, BT-Druck. 16/1780, S. 32.

33 BAG, NZA 2003, S. 483 ff. 
deshalb für unwirksam, weil es die Religionsfreiheit der Arbeitnehmerin durch selbige verletzt sah. $^{34}$

\section{b) Resümee - Diskriminierung durch einseitig belastende Behandlung}

Der „Kopftuchfall“ stellt nach den bisherigen Ausführungen keine Diskriminierung dar, weil er das Tatbestandsmerkmal der Ungleichbehandlung nicht aufweist. Gleichwohl wird er teilweise unter dem Stichwort „Diskriminierung“ diskutiert, wird er doch im Zusammenhang mit der Richtlinie 2000/78/EG erwähnt, ${ }^{35}$ die gem. ihres Art. 1 die Bekämpfung der Diskriminierung in Beschäftigung und Beruf unter anderem aufgrund der Religion bezweckt.

Im „Kopftuchfall“ erfährt die Arbeitnehmerin eine für sie ungünstige Behandlung, weil sie Trägerin eines Merkmals ist, das der Arbeitgeber nicht toleriert. Auch die unmittelbare Diskriminierung im Sinne der Richtlinien ist dadurch gekennzeichnet, daß die Trägerschaft eines Merkmals zu einer Ungleichbehandlung führt. In beiden Fällen stellt sich die Frage, ob die Anknüpfung an das jeweilige Merkmal rechtswidrig ist. Auch der „Kopftuchfall“ wirft damit die Frage nach dem Vorliegen einer Diskriminierung auf. Zumindest dann, wenn man davon ausgeht, daß Diskriminierungsschutz nicht nur bezweckt, das Unrecht zu vermeiden, das dadurch entsteht, daß ein an sich legitimes Verhalten durch gleichheitswidrige Unterscheidung ungerecht wird. ${ }^{36}$ Es wird deshalb vorgeschlagen, auch die einseitig belastende Behandlung einer Person als potenziell diskriminierendes Verhalten einzustufen.

Im Zusammenhang mit der hier vorgeschlagenen Problemlösung ist auf die Rechtsprechung des BGH zur Rechtmäßigkeit der Kündigung von Parteikonten hinzuweisen. Dort hat das Gericht in Anlehnung an die Rechtsprechung des BVerfG festgestellt: „Der Gleichheitssatz des Art. 3 I GG erschöpft sich nicht in dem Verbot einer ungerechtfertigten Ungleichbehandlung verschiedener Personen und Personengruppen, [die im Falle der Kündigung eines Parteikontos dann vorliegen kann, wenn das Kreditinstitut für andere Parteien Konten führt, $\left.{ }^{37}\right]$ sondern bringt als fundamentales Rechtsprinzip ein Willkürverbot zum Ausdruck.“38 „Das Willkürverbot ist verletzt, wenn sich bei verständiger Würdigung der das Grundgesetz beherrschenden Gedanken ein sachgerechter Grund für eine Maßnahme der öffentlichen Gewalt nicht finden läßt. ‘39

\footnotetext{
$B A G$, NZA 2003, S. 483 (486 f.).

So jedenfalls Thüsing, NJW 2003, S. 405 (406).

So aber Thüsing, ZfA 2001, S. 397 (411); Wiedemann/Thüsing, Der Betrieb 2002, S. 463 (466).

In dem vom BGH entschiedenen Fall hatte das Berufungsgericht nicht festgestellt, daß die Beklagte Girokonten für andere Parteien führt. Auch hatte der Kläger dies nicht geltend gemacht. (BGH, NJW 2003, S. 1658 (1659)).

$B G H$, NJW 2003, S. 1658 (1659); BVerfGE 55, S. 72 (89); 78, S. 232 (248).

$B G H$, NJW 2003, S. 1658 (1659); NJW 2004, S. 1031 (1031); BVerfGE 55, S. 72 (89 f.); 78, S. 232 (248); In einem späteren Urteil formuliert es das $B V \operatorname{erfG}$ enger: „Kommt als Maßstab nur das Willkürverbot in Betracht, so kann ein Verstoß gegen Art. 3 Abs. 1 GG erst festgestellt werden, wenn die Unsachlichkeit der Differenzierung evident ist.“" (BVerfGE 99, S. 367 (389))
} 
Die Überlegungen zu dem in Art. 3 I GG verankerten Willkürverbot haben gezeigt, daß potenziell diskriminierendes Verhalten nicht auf die Ungleichbehandlung beschränkt werden muß.

\section{Exkurs: (Sexuelle) Belästigung}

Das bestätigen die Antidiskriminierungsrichtlinien, indem sie Belästigungen unter den Begriff der Diskriminierung subsumieren. Belästigungen werden gem. Art. 2 III 1 der Richtlinie 2000/43/EG definiert als

„[u]nerwünschte Verhaltensweisen, die im Zusammenhang mit der Rasse oder der ethnischen Herkunft einer Person stehen und bezwecken oder bewirken, daß die Würde der betreffenden Person verletzt und ein von Einschüchterungen, Anfeindungen, Erniedrigungen, Entwürdigungen und Beleidigungen gekennzeichnetes Umfeld geschaffen wird [... $]^{\text {“. } 40}$

Unerwünschte Verhaltensweisen in diesem Sinne sollen in Gestalt sprachlicher Äußerungen und Gesten oder durch Verfassen, Zeigen oder Verbreiten von schriftlichen Äußerungen, Bildern oder sonstigem Material vorgenommen werden können. ${ }^{41}$

Im Sinne des Art. 2 III 1 Richtlinie 2000/43/EG ist gem. § 3 III AGG eine Belästigung

,[...] eine Benachteiligung, wenn unerwünschte Verhaltensweisen, die mit einem in $\S 1$ genannten Grund [ $-\mathrm{d}$. h. mit einem dort genannten Diskriminierungsmerkmal - ] in Zusammenhang stehen, bezwecken oder bewirken, daß die Würde der betreffenden Person verletzt und ein von Einschüchterungen, Anfeindungen, Erniedrigungen, Entwürdigungen oder Beleidigungen gekennzeichnetes Umfeld geschaffen wird.“

Auch nach allgemeinem Sprachgebrauch bezeichnet Diskriminierung unter anderem (unzutreffende) Äußerungen oder Behauptungen in der Öffentlichkeit, die geeignet sind, das Ansehen einer Person zu schädigen bzw. dessen Leistungen herabzuwürdigen, sowie die Verächtlichmachung bestimmter Personen durch das Nähren von Vorurteilen. ${ }^{42}$

Gemäß Art. 2 II 4. Spiegelstrich der Richtlinie 76/207/EWG bezeichnet sexuelle Belästigung

,jede Form von unerwünschtem Verhalten sexueller Natur, das sich in unerwünschter verbaler, nicht-verbaler oder physischer Form äußert und das bezweckt oder bewirkt, daß die Würde der betreffenden Person verletzt wird,

Im Zusammenhang mit dem Begriff der öffentlichen Gewalt muß angemerkt werden, daß es sich bei den in Rede stehenden Banken nicht um Privatbanken handelte, vielmehr auf der einen Seite des Vertragsverhältnisses die öffentliche Verwaltung stand, die sich zur Erfüllung öffentlicher Aufgaben in Form des Privatrechts betätigte (sog. Verwaltungsprivatrecht). (Heinrichs in Palandt, § 242, Rn. 12).

40 Ähnliche Definitionen enthalten die Richtlinie 76/207/EWG in Art. 2 II 3. Spiegelstrich, Richtlinie 2000/78/EG in Art. 2 III sowie Richtlinie 2004/113/EG in Art. 2 lit. c.

41 Coen, AuR 2000, S. 11 (11).

42 Duden, Das große Wörterbuch der deutschen Sprache, Band 2, S. 828. 
insbesondere wenn ein von Einschüchterungen, Anfeindungen, Erniedrigungen, Entwürdigungen und Beleidigungen gekennzeichnetes Umfeld geschaffen wird.“43

In Deutschland existierte seit dem 24.06.1994 zunächst das Gesetz zum Schutz der Beschäftigten vor sexueller Belästigung am Arbeitsplatz (BeSchuG). In $§ 2$ II 1 BeSchuG-A war sexuelle Belästigung am Arbeitsplatz definiert als

,jedes vorsätzliche, sexuell bestimmte Verhalten, das die Würde von Beschäftigten am Arbeitsplatz verletzt.“

Dazu gehörten gem. § 2 II 2 BeSchuG-A

„1. sexuelle Handlungen und Verhaltensweisen, die nach den strafgesetzlichen Vorschriften unter Strafe gestellt sind, sowie

2. sonstige sexuelle Handlungen und Aufforderungen zu diesen, sexuell bestimmte körperliche Berührungen, Bemerkungen sexuellen Inhalts sowie Zeigen und sichtbares Anbringen von pornographischen Darstellungen, die von den Betroffenen erkennbar abgelehnt werden. “44

Das Beschäftigtenschutzgesetz ist gem. Art. 4 Satz 2 des Gesetzes zur Umsetzung europäischer Richtlinien zur Verwirklichung des Grundsatzes der Gleichbehandlung ${ }^{45}$ am 18.08.2006 außer Kraft getreten. $^{46}$

Schutz vor sexueller Belästigung in Beschäftigung und Beruf gewährt nunmehr das AGG. Gemäß 3 IV AGG ist eine sexuelle Belästigung

„[...] eine Benachteiligung in bezug auf $\S 2$ Abs. 1 Nr. 1 bis 4 [ - d. h. im Rahmen von Beschäftigung und Beruf - ], wenn ein unerwünschtes, sexuell bestimmtes Verhalten, wozu auch unerwünschte sexuelle Handlungen und Aufforderungen zu diesen, sexuell bestimmte körperliche Berührungen, Bemerkungen sexuellen Inhalts sowie unerwünschtes Zeigen und sichtbares Anbringen von pornographischen Darstellungen gehören, bezweckt oder bewirkt, daß die Würde der betreffenden Person verletzt wird, insbesondere wenn ein von Einschüchterungen, Anfeindungen, Erniedrigungen und Beleidigungen gekennzeichnetes Umfeld geschaffen wird.“

Die Wortlaut des § 3 IV AGG geht ersichtlich auf Art. 2 II 4. Spiegelstrich Richtlinie 76/207/EWG und $\S 2$ II BeSchuG-A zurück.

\section{a) Belästigungsschutz $=$ Diskriminierungsschutz?}

43 Vergleiche auch Art. 2 lit. d Richtlinie 2004/113/EG.

$44 \mathrm{Zu}$ den verschiedenen Formen sexueller Belästigung vgl. auch die Beispiele bei Baer, Würde oder Gleichheit?, S. $23 \mathrm{ff}$.

45 BGB1. 2006 I, S. $1897 \mathrm{ff}$.

46 Beachte in diesem Zusammenhang ist die Übergangsvorschrift des § 33 I AGG. 
Teilweise wird behauptet, Belästigungsschutz habe nichts mit Diskriminierungsschutz zu tun, es sei falsch, selbigen in ein Antidiskriminierungskonzept zu integrieren. ${ }^{47}$ Das zeige sich schon daran, daß dort, wo wie in Deutschland ein Schutz der Beschäftigten vor sexueller Belästigung am Arbeitsplatz besteht, dieser oftmals durch eigenständige Gesetze realisiert wird. ${ }^{48}$

Eine Beleidigung werde nicht dadurch akzeptabler, daß jemand unterschiedslos jeden beleidigt. ${ }^{49}$ Das Unrecht liege hier in der Handlung selbst. ${ }^{50}$ Diskriminierungsschutz bezwecke demgegenüber, das Unrecht zu vermeiden, das dadurch entsteht, daß ein an sich legitimes Verhalten durch gleichheitswidrige Unterscheidung ungerecht wird. ${ }^{51}$

Kritik begegnet auch der Umstand, daß die Richtlinien den Belästigungsschutz auf bestimmte Diskriminierungsmerkmale beschränken. Nicht einleuchtend sei, warum eine Beleidigung beispielsweise aufgrund des Alters anders bewertet werden sollte als eine Beleidigung aufgrund eines anderen individuellen Merkmals. ${ }^{52}$ Hier wie da liege eine Beleidigung vor, die zu unterlassen sei. ${ }^{53}$ Anzumerken bleibt im Zusammenhang mit diesem begründeten Einwand, daß sich die Frage nach dem Kreis schutzwürdiger Diskriminierungsmerkmale nicht nur bei der Belästigung, sondern allgemein im Rahmen der Suche nach einem adäquaten Diskriminierungsschutz stellt. ${ }^{54}$

Bekräftigt wird die vorgebrachte Kritik dadurch, daß auch der sachliche Geltungsbereich der Richtlinien nicht alle Lebensbereiche umfaßt. Wenn nun aber im Falle der (sexuellen) Belästigung das Unrecht in der Handlung selbst liegen soll, ${ }^{55}$ dann kann doch dieselbe Handlung außerhalb beispielsweise des „Zugang[s] zu und [der] Versorgung mit Gütern und Dienstleistungen, die der Öffentlichkeit zur Verfügung stehen, einschließlich von Wohnraum“, ${ }^{56}$ nicht plötzlich erlaubt sein.

Das Beispiel zeigt, daß der sachliche Geltungsbereich der Richtlinien nicht auf den Belästigungsschutz zugeschnitten ist. Für ihn gelten eigene Kriterien.

Zwar ist auch im Falle der (sexuellen) Belästigung eine Güterabwägung vorzunehmen, um zu klären, ob überhaupt belästigt wurde, jedoch stehen sich hier insbesondere die Meinungs-, Presseund Kunstfreiheit des „Belästigenden“ auf der einen und das allgemeine Persönlichkeitsrecht des „Belästigten“ auf der anderen Seite gegenüber. ${ }^{57}$ Es stellen sich anders als bei der „klassischen“ deutschen ADG-Entwurf beziehen (Wiedemann/Thüsing, Der Betrieb 2002, S. 463 (466)).

48 Thüsing, ZfA 2001, S. 397 (411); Wiedemann/Thüsing, Der Betrieb 2002, S. 463 (466).

49 Thüsing, ZfA 2001, S. 397 (411); Thüsing, NZA 2001, S. 1061 (1064); Wiedemann/Thüsing, Der Betrieb 2002, S. 463 (466).

$50 \quad$ Thüsing, ZfA 2001, S. 397 (411); Wiedemann/Thüsing, Der Betrieb 2002, S. 463 (466).

51 Thüsing, ZfA 2001, S. 397 (411); Wiedemann/Thüsing, Der Betrieb 2002, S. 463 (466); a.A. Baer: „Diskriminierung ist nicht die schlichte Differenzierung als unterschiedliche Behandlung, sondern soziale Hierarchisierung" bzw. Dominierung. (Baer, Würde oder Gleichheit?, S. 48 mit Fn. 238).

52 Thüsing, ZfA 2001, S. 397 (411 f.); Wiedemann/Thüsing, Der Betrieb 2002, S. 463 (466).

53 Thüsing, ZfA 2001, S. 397 (412); Wiedemann/Thüsing, Der Betrieb 2002, S. 463 (466).

$54 \quad$ Vergleiche dazu Teil 2, Prüfungspunkt B.

55 So Thüsing, ZfA 2001, S. 397 (411); Wiedemann/Thüsing, Der Betrieb 2002, S. 463 (466).

56 Art. 3 I lit. h RL 2000/43/EG.

57 Sprau in Palandt, § 823, Rn. 95. 
Diskriminierung im rechtsgeschäftlichen und quasi-rechtsgeschäftlichen Bereich nicht die Fragen nach dem „Ob“ und „Wie“ einer Einschränkung der Privatautonomie des „Diskriminierenden“.

Aufgrund der genannten Besonderheiten im Zusammenhang mit dem Belästigungsschutz wird vorgeschlagen - hält man eine Gesetzesänderung im Hinblick auf die europäischen Vorgaben für erforderlich -, § 823 I BGB entsprechend der bisherigen Rechtsprechung um das Rechtsgut des allgemeinen Persönlichkeitsrechts zu ergänzen, eventuell versehen mit einem ,insbesondereVermerk“, der die diskriminierende Belästigung ausdrücklich nennt. ${ }^{58}$ Die Belästigung stünde dann im Deliktsrecht an der systematisch korrekten Stelle. ${ }^{59}$

Trotz der genannten Widersprüchlichkeiten wird die Inkorporierung der Belästigung in ein Diskriminierungsschutzkonzept teilweise für möglich gehalten, obwohl dies über das spezifische Anliegen des Gleichheitssatzes hinausgreife. ${ }^{60}$

Es wurde an anderer Stelle vorgeschlagen, auch die einseitig belastende Behandlung einer Person als potenziell diskriminierendes Verhalten einzustufen. ${ }^{61}$ Vor diesem Hintergrund bestehen keine Einwände dagegen, auch die Belästigung als Diskriminierung zu begreifen, stellt sie doch einen besonderen Fall einseitig belastender Behandlung einer Person dar.

Außerdem sind die „klassische“ Diskriminierung im (quasi-)rechtsgeschäftlichen Verkehr und die Belästigung eng miteinander verknüpft. Erstere wird zum Teil als Verletzung des allgemeinen Persönlichkeitsrechts und damit letztlich als Belästigung qualifiziert. ${ }^{62}$

Das gelte beispielsweise für die Nichtberücksichtigung einer Stellenbewerberin wegen ihres Geschlechts ${ }^{63}$ oder dann, wenn der Gastwirt einen Farbigen nicht bedient. ${ }^{64}$

Zusammenfassend kann gesagt werden, daß die Belästigung als eine diskriminierungsrelevante Verhaltensweise eingestuft und somit grundsätzlich in ein Diskriminierungsschutzkonzept integriert werden kann, obwohl sie Besonderheiten aufweist, die sie deutlich von den bisher dargestellten Formen der Diskriminierung unterscheidet.

Der für den weiteren Gang der Arbeit wesentliche Unterschied besteht, wie bereits erwähnt, darin, daß sich im Rahmen des Belästigungsschutzes - anders als beim Diskriminierungsschutz im rechtsgeschäftlichen und quasi-rechtsgeschäftlichen Bereich - nicht die Frage nach einer etwaigen Einschränkung der Privatautonomie des „Belästigenden“ stellt. Hier geht es nicht um (vor-

58 Wiedemann/Thüsing, Der Betrieb 2002, S. 463 (467).

59 Wiedemann/Thüsing, Der Betrieb 2002, S. 463 (467).

60 Thüsing, ZfA 2001, S. 397 (412).

61 Vergleiche Teil 2, Prüfungspunkt A. II. 1. b).

62 Hager in Staudinger, § 823, Rn. C 239; Sprau in Palandt, § 823, Rn. 110.

63 BAG, NJW 1990, S. 65 (65); Hager in Staudinger, § 823, Rn. C 239; Sprau in Palandt, § 823, Rn. 110.

64 Hager in Staudinger, § 823, Rn. C 239. 
)vertragliche Rechte und Pflichten von (potenziellen) Vertragspartnern. Die Lösung gerade dieses Spannungsverhältnisses ist aber erklärtes Ziel der vorliegenden Arbeit.

Aus diesem Grund wird das Problem der (sexuellen) Belästigung im weiteren Verlauf der Arbeit nicht vertieft. Nichtsdestotrotz sollen im folgenden die Probleme, die im Zusammenhang mit dem Belästigungsschutz und insbesondere den insofern bestehenden europarechtlichen Verpflichtungen auftauchen, in einem Exkurs kurz angerissen werden.

\section{b) Tatbestandsmerkmal ,,bezwecken oder bewirken““}

Während die Richtlinien sowie § 3 III, IV AGG eine Belästigung annehmen, wenn ein bestimmtes Verhalten bezweckt oder bewirkt, daß die Würde einer Person verletzt wird, definierte $\S 2$ II 1 BeSchuG-A die Belästigung als vorsätzliches Verhalten, das die Würde einer Person verletzt.

Nach dem Wortlaut der Richtlinien und des AGG soll eine Belästigung zum einen schon dann vorliegen, wenn die Verletzung der Würde einer Person bezweckt ist. Damit ist der Belästigungsbegriff gegenüber dem BeSchuG-A auf der einen Seite enger, auf der anderen aber auch weiter gefaßt.

Enger deshalb, weil „,bezwecken“ im Sinne der Richtlinien und des AGG sicherlich mehr verlangen wird als das von $\S 2$ II 1 BeSchuG-A verlangte vorsätzliche Verhalten - im Rahmen jener Norm sollte dolus eventualis ausreichen. ${ }^{65}$

Weiter aus dem Grunde, weil die Richtlinien und das AGG nach ihrem insofern gleichlautenden Wortlaut neben dem subjektiven Element „,bezwecken“ kein objektives verlangen, um das Vorliegen einer Belästigung zu bejahen. § 2 II 1 BeSchuG-A setzte demgegenüber voraus, daß neben dem vorsätzlichen Verhalten die Würde des „Belästigten“ tatsächlich verletzt wurde.

Beispiel: Die anzügliche Bemerkung einer Frau gegenüber einem Mann, die bezweckt, den Mann in dessen Würde zu verletzen, würde nach dem Wortlaut der Richtlinien und des AGG eine Belästigung auch dann darstellen, wenn der Mann die Bemerkung als Kompliment auffassen würde. Dagegen hätte eine sexuelle Belästigung in einem solchen Fall nach dem BeSchuG-A nicht vorgelegen, da nach diesem Gesetz eine sexuelle Belästigung nur dann bejaht werden konnte, wenn das jeweilige Verhalten von dem Betroffenen erkennbar abgelehnt worden war. ${ }^{66}$

Das Beispiel zeigt, daß die Bejahung einer Belästigung unter Verzicht des objektiven Tatbestandes der Verletzung der Würde des Betroffenen, wie es die Richtlinien und auch das AGG vorsehen, zu absurden Ergebnissen führen würde. 
Die Richtlinien und das deren Vorgaben übernehmende AGG sind auch insoweit zu kritisieren, als nicht einzusehen ist, warum eine Belästigung nicht schon dann vorliegen können soll, wenn der „Belästigende“ billigend in Kauf genommen hat, daß sein Verhalten für den Betroffenen eine Belästigung darstellen könnte.

Nach dem Wortlaut der Richtlinien und des AGG soll das Vorliegen einer Belästigung zum anderen schon dann bejaht werden können, wenn ein bestimmtes Verhalten die Verletzung der Würde der betreffenden Person bewirkt. Damit gehen die Richtlinien und das AGG wiederum über den Begriff der Belästigung i.S.d. BeSchuG-A hinaus. Gegenüber jenem Gesetz verzichten die Richtlinien und das AGG in dieser Alternative auf das Vorsatzelement.

In dem Beispielsfall bezweckt die Frau mit ihrer Bemerkung nicht, den Mann in dessen Würde zu verletzen, bewirkt dies aber tatsächlich.

Der Verzicht auf das Vorsatzelement ist folgerichtig, wenn man davon ausgeht, daß eine objektiv belästigende Handlung stets zu unterlassen ist, egal auf welchem Motiv sie beruht. Unter dieser Prämisse ist für das Vorliegen einer Belästigung unerheblich, ob der „Belästigende“ vorsätzlich gehandelt hat.

In diesem Sinne wurde kritisiert, die verschuldensabhängige Struktur des BeSchuG-A sei nicht geeignet gewesen, sexueller Diskriminierung wirksam entgegenzutreten. ${ }^{67}$ Würden Benachteiligungsverbote an den Nachweis bestimmter Motive gebunden, böten sich Umgehungsmöglichkeiten geradezu an. ${ }^{68}$

\section{c) Feindliche Umfeldprägung}

Ein weiteres Problem, das sich im Zusammenhang mit dem Belästigungsschutz des Art. 2 III 1 Richtlinie 2000/43/EG stellt, besteht darin, daß die Norm eine feindliche Umfeldprägung verlangt, was dazu führen könne, daß der bisherige Schutz vor Belästigungen durch § 823 I BGB verringert wird. ${ }^{69}$ In der Tat verwundert es, daß über die Verletzung der Würde des Belästigten hinaus verlangt wird, daß ein ,von Einschüchterungen, Anfeindungen, Erniedrigungen, Entwürdigungen und Beleidigungen gekennzeichnetes Umfeld geschaffen wird“, um das Vorliegen einer Belästigung im Sinne der Richtlinien zu bejahen. ${ }^{70}$ Dasselbe Problem stellt sich i. R. d. § 3 III AGG.

67 Baer, Würde oder Gleichheit?, S. 245 ff. (250).

Baer, Würde oder Gleichheit?, S. 248.

Wiedemann/Thüsing, Der Betrieb 2002, S. 463 (467).

70 Der Grund für diese Einschränkung wird darin gesehen, daß sich der Richtliniengesetzgeber bei seiner Rechtsetzung vom U.S.-amerikanischen Arbeitsrecht hat leiten lassen, wo Gerichte durch Interpretation des Titel 
Das deutsche Recht habe eine solche Erheblichkeitsschwelle bislang nicht gekannt. ${ }^{71}$ Außerdem sei sie nicht notwendig, da insofern das einschränkende Kriterium der Verletzung der Würde ausreiche, das in der Rechtsprechung bereits anerkannt sei. ${ }^{72}$

Anzumerken ist, daß der Wortlaut der Richtlinien in bezug auf die Notwendigkeit der feindlichen Umfeldprägung widersprüchlich ist. Während Art. 2 III 1 der Richtlinie 2000/43/EG für das Vorliegen einer Belästigung verlangt, „daß die Würde der betreffenden Person verletzt und ein von Einschüchterungen, Anfeindungen, Erniedrigungen, Entwürdigungen und Beleidigungen gekennzeichnetes Umfeld geschaffen wird“" ${ }^{73}$ reicht es gem. Art. 2 II 4. Spiegelstrich der Richtlinie 76/207/EWG für das Vorliegen einer sexuellen Belästigung aus, „daß die Würde der betreffenden Person verletzt wird, insbesondere wenn ein von Einschüchterungen, Anfeindungen, Erniedrigungen, Entwürdigungen und Beleidigungen gekennzeichnetes Umfeld geschaffen wird.“74 Besonders deutlich tritt dieser Widerspruch in der Richtlinie 2004/113/EG zutage, die in Art. 2 lit. c bzw. d beide Definitionen enthält. Dasselbe gilt für das AGG und dessen $\S 3$ III auf der einen und $\S$ 3 IV auf der anderen Seite. Warum aber bei der nicht-sexuellen Belästigung das Merkmal der feindlichen Umfeldprägung neben dem der Verletzung der Menschenwürde vorliegen muß, bei der sexuellen Belästigung die Verletzung der Menschenwürde dagegen insbesondere bei feindlicher Umfeldprägung vorliegen soll, ist nicht ersichtlich.

Anzumerken bleibt, daß im U.S.-amerikanischen Arbeitsrecht auch der Arbeitnehmer klagebefugt ist, der zwar nicht belästigt wurde, jedoch durch das feindliche Arbeitsumfeld beeinträchtigt ist, weil er zur geschützten Gruppe des tatsächlich belästigten Arbeitnehmers gehört. ${ }^{75}$

\section{d) Zurechnung fremden Verhaltens}

Besteht die Möglichkeit der Zurechnung belästigenden Verhaltens Dritter? Diese Frage stellt sich im Arbeitsrecht im Rahmen der Haftung des Arbeitgebers, wenn man davon ausgeht, daß auch die (sexuelle) Belästigung eines Arbeitnehmers gegenüber einem anderen Arbeitnehmer von den Richtlinien erfaßt ist. ${ }^{76}$

VII des Civil Rights Act die Rechtsfigur des „hostile enviroment harassment“ entwickelt haben, worunter jedes schuldhafte Verhalten des Arbeitgebers subsumiert wird, das eine Arbeitsumgebung schafft oder billigt, in der es leichter zu auf bestimmte Diskriminierungsmerkmale bezogene Belästigungen kommt. (Thüsing, ZfA 2001, S. 397 (412); Wiedemann/Thüsing, Der Betrieb 2002, S. 463 (467)) Es wird gemutmaßt, der europäische Gesetzgeber habe, obwohl nicht an den Titel VII des Civil Rights Act gebunden, das Merkmal der feindlichen Umfeldprägung übernommen, weil er nur Belästigungen von einer bestimmten Erheblichkeit von der Richtlinie erfaßt wissen wollte. (Thüsing, ZfA 2001, S. 397 (412 f.); Wiedemann/Thüsing, Der Betrieb 2002, S. 463 (467)). Thüsing, ZfA 2001, S. 397 (413).

Wiedemann/Thüsing, Der Betrieb 2002, S. 463 (467).

Hervorhebung durch den Verfasser.

Hervorhebung durch den Verfasser.

Thüsing, ZfA 2001, S. 397 (413).

So Bauer, NJW 2001, S. 2672 (2676). 
Im U.S.-amerikanischen Arbeitsrecht muß sich der Arbeitgeber das Verhalten vorgesetzter Arbeitnehmer nach Stellvertretungsregeln zurechnen lassen, für das Verhalten aller übrigen Arbeitnehmer soll er demgegenüber aufgrund Organisationsverschuldens haftbar sein, wenn er nicht geeignete Vorkehrungen zur Verhinderung von Belästigungen getroffen hat. ${ }^{77}$

$\S 2$ I BeSchuG-A war auf Schutzpflichten des Arbeitgebers gegenüber seinen Beschäftigten beschränkt, so daß ein Schadensersatzanspruch gegenüber dem Arbeitgeber nur bei schuldhafter Verletzung dieser Pflichten in Betracht kam. ${ }^{78}$ Gemäß dieser Norm hatten

„Arbeitgeber und Dienstvorgesetzte [...] die Beschäftigten vor sexueller Belästigung am Arbeitsplatz zu schützen. Dieser Schutz umfaßt auch vorbeugende Maßnahmen.“

In Anlehnung an $\S 2 \mathrm{I}$ BeSchuG-A ${ }^{79}$ bestimmt nunmehr $\S 12$ I AGG, daß der Arbeitgeber verpflichtet ist,

,[...] die erforderlichen Maßnahmen zum Schutz vor Benachteiligungen wegen eines in $\S 1$ genannten Grundes [ - d. h. wegen eines dort genannten Diskriminierungsmerkmals - ] zu treffen. Der Schutz umfaßt auch vorbeugende Maßnahmen.“

Als Rechtsfolge eines Verstoßes gegen diese Bestimmung kommt sowohl ein verschuldensabhängiger Anspruch auf Ersatz des materiellen Schadens als auch ein verschuldensunabhängiger Anspruch auf Ersatz des immateriellen Schadens in Betracht (vgl. dazu $§$ 15 I und II AGG). ${ }^{80}$

\section{Benachteiligende Gleichbehandlung - Mittelbare bzw. indirekte Diskriminierung}

Diskriminierungsrelevantes Verhalten kann auch in einer benachteiligenden Gleichbehandlung von Personen bestehen.

\section{1. (Potenzielle) Benachteiligung}

Gemäß Art. 2 II lit. b der Richtlinie 2000/43/EG

„liegt eine mittelbare Diskriminierung vor, wenn dem Anschein nach neutrale Vorschriften, Kriterien oder Verfahren Personen, die einer Rasse oder ethnischen Gruppe angehören, in besonderer Weise benachteiligen können, es sei denn,

\footnotetext{
Thüsing, ZfA 2001, S. 397 (413).

Bauer, NJW 2001, S. 2672 (2677).

Entwurfsbegründung, BT-Druck. 16/1780, S. 37.

Vergleiche dazu Teil 4, Prüfungspunkt C. II. 1.
} 
die betreffenden Vorschriften, Kriterien oder Verfahren sind durch ein rechtmäßiges Ziel sachlich gerechtfertigt, und die Mittel sind zur Erreichung dieses Ziels angemessen und erforderlich. “ ${ }^{* 81}$

Erstmals wurde die mittelbare bzw. indirekte Diskriminierung in der Richtlinie 97/80/EG über die Beweislast bei Diskriminierung aufgrund des Geschlechts ${ }^{82}$ vom 15.12.1997 legaldefiniert. Gemäß Art. 2 II der Richtlinie ist eine mittelbare Diskriminierung gegeben,

„wenn dem Anschein nach neutrale Vorschriften, Kriterien oder Verfahren einen wesentlich höheren Anteil der Angehörigen eines Geschlechts benachteiligen, es sei denn, die betreffenden Vorschriften, Kriterien oder Verfahren sind angemessen und notwendig und sind durch nicht auf das Geschlecht bezogene sachliche Gründe gerechtfertigt. “ ${ }^{483}$

Die Definitionen der mittelbaren Diskriminierung in den Richtlinien, die auf den ersten Blick identisch zu sein scheinen, unterscheiden sich in einem wesentlichen Punkt. Während das Vorliegen einer mittelbaren Diskriminierung im Sinne der Richtlinie 97/80/EG nur durch entsprechende statistische Nachweise geführt werden kann, verzichtet die Richtlinie 2000/43/EG in Anknüpfung an die diesbezügliche Rechtsprechung des EuGH zur Arbeitnehmerfreizügigkeit ${ }^{84}$ auf einen solchen Nachweis, da es nach ihr bereits ausreicht, daß ,dem Anschein nach neutrale Vorschriften [...] in besonderer Weise benachteiligen können“. ${ }^{85}$

Dem Wortlaut des Art. 2 II lit. b Richtlinie 2000/43/EG entsprechend bestimmt $§ 3$ II AGG, daß eine mittelbare Benachteiligung nach diesem Gesetz vorliegt,

„[...] wenn dem Anschein nach neutrale Vorschriften, Kriterien oder Verfahren Personen wegen eines in $\S 1$ genannten Grundes [ - d. h. wegen eines dort genannten Diskriminierungsmerkmals - ] gegenüber anderen Personen in besonderer Weise benachteiligen können, es sei denn, die betreffenden Vorschriften, Kriterien oder Verfahren sind durch ein rechtmäßiges Ziel sachlich gerechtfertigt und die Mittel sind zur Erreichung dieses Ziels angemessen und erforderlich.“

\section{2. Überwiegende Betroffenheit}

81 Ähnliche Definitionen enthalten die Richtlinie 76/207/EWG in Art. 2 II 2. Spiegelstrich, Richtlinie 2000/78/EG in Art. 2 II lit. b sowie Richtlinie 2004/113/EG in Art. 2 lit. b.

82 ABl. EG 1998 Nr. L 14, S. 6 ff.

83 Die Richtlinie hat damit die Rechtsprechung des EuGH übernommen. (Waas, ZIP 2000, S. 2151 (2152, Fn. 16); zur Rspr. des Gerichts vgl. EuGH, Rs. C-96/80, Slg. 1981 1, S. 911 (925 f., Rn. 13 u. 15); NZA 1986, S. 599 (599, 1. Leitsatz)); Auch im deutschen Arbeitsrecht galt das Verbot mittelbarer Diskriminierung schon vor Erlaß der Richtlinien 2000/43/EG und 2000/78/EG. Das BAG hat in seiner Rechtsprechung zum arbeitsrechtlichen Diskriminierungsverbot mehrfach festgestellt, daß dieses auch mittelbare Diskriminierungen erfaßt. (BAG, NJW 1992, S. 1125 (1125); NJW 1993, S. 3091 (3093)); bzgl. § 611 a BGB vgl. BAG, NZA 1993, S. 739 (740)); Das deutsche Verfassungsrecht kennt das Verbot der mittelbaren Diskriminierung ebenfalls. So verbietet beispielsweise Art. 3 III 1 GG auch die mittelbare Diskriminierung. (Heun in Dreier, Band I, Art. 3, Rn. 116).

84 Coen, AuR 2000, S. 11 (11); Hornung-Draus, Arbeitgeber 2000, S. 14 (14) Anzumerken ist, daß beide Autoren die Kommissionsvorschläge thematisieren, nicht die Richtlinien als solche.; Waas, ZIP 2000, S. 2151 (2153).

85 Waas, ZIP 2000, S. 2151 (2152 f.); Hervorhebung durch den Verfasser. 
Mittelbar diskriminierend ist eine Vorschrift, die formell gleich behandelt, weil sie für alle Unterworfenen der Vorschrift gleichermaßen gilt, aber tatsächlich bestimmte Gruppen stärker belastet als andere. ${ }^{86}$

Beispiele: So wurden geringere Stundensätze für Teilzeitbeschäftigte vom EuGH als mittelbare Diskriminierung eingestuft, ${ }^{87}$ da Teilzeitarbeit nach wie vor eine Domäne der Frau sei. ${ }^{88}$ Das gleiche soll für die Forderung langer Berufserfahrung in klassischen Männer- oder Frauenberufen dann gelten, wenn die Tätigkeit ohne weiteres von einem Berufsanfänger ausgeübt werden kann. ${ }^{89}$ Mittelbar benachteiligend soll auch sein, wenn die Einstellung von einer Mindestlänge oder einem Mindestgewicht abhängig gemacht wird, die Frauen kaum, Männer aber in der Regel erreichen, oder wenn Altershöchstgrenzen für die Einstellung festgelegt werden, die von Frauen wegen des zeitweiligen Ausscheidens aus dem Beruf zum Zwecke der Kinderbetreuung häufiger überschritten werden. ${ }^{90}$

Der wesentliche Unterschied zur unmittelbaren Diskriminierung soll darin bestehen, daß die mittelbare Diskriminierung kein vorsätzliches Handeln des Diskriminierenden voraussetzt. ${ }^{91}$ Der Wortlaut der Richtlinien spricht weder für noch gegen das Erfordernis vorsätzlichen Handelns im Rahmen der mittelbaren Diskriminierung.

Die Worte: „dem Anschein nach“ könnte man in der Weise interpretieren, daß der „Diskriminierende“ diesen Anschein gerade wissentlich erzeugen muß. Fraglich ist dann aber, wie die mittelbare Diskriminierung von der verdeckten unmittelbaren Diskriminierung abgegrenzt werden kann. Der Verdeutlichung des Problems soll folgender Beispielsfall dienen: Der Verheiratetenzuschlag privater Arbeitgeber knüpft nicht unmittelbar an die sexuelle Identität des Arbeitnehmers an und wird aus diesem Grund unter dem Stichwort „,mittelbare Diskriminierung“ diskutiert. ${ }^{92}$ Handelt es sich bei diesen Fällen nicht vielmehr um solche verdeckter unmittelbarer Diskriminierung? Wenn Homosexuelle nicht heiraten dürfen, trifft sie eine scheinbar neutrale Regelung nicht nur häufiger als andere, sondern schlechterdings immer. Dies weiß der Arbeitgeber, der Fahrvergünstigungen explizit nur für Ehepaare schafft. ${ }^{93}$

Gegen das Vorliegen einer verdeckten unmittelbaren Diskriminierung in besagtem Fall ließe sich vorbringen, allein das Wissen, daß sich eine Regelung ungleich auswirken wird, könne nicht ausreichen. In diesem Sinne wird vertreten, eine verdeckte Diskriminierung liege immer dann vor,

86 Nickel, Gleichheit und Differenz in der vielfältigen Republik, S. 71.

87 EuGH, Rs. C-96/80, Slg. 1981 1, S. 911 (925 f., Rn. 13 u. 15); Krebber in Calliess/Ruffert, Art. 141 EGV, Rn. 45 mit weiteren Beispielen der Rspr. des EuGH.

88 Richardi/Annuß in Staudinger, $\$ 611$ a, Rn. 45.

89 Beispiel bei Raab in Soergel, $\S 611$ a, Rn. 24.

90 BAG, NZA 1986, S. 739 (740).

91 Nickel, Gleichheit und Differenz in der vielfältigen Republik, S. 72; Pfarr/Bertelsmann, Diskriminierung im Erwerbsleben, S. 128; implizit auch Waas, ZIP 2000, S. 2151 (2155).

92 So Thüsing, NZA 2001, S. 1061 (1062).

93 In diesem Sinne unterscheiden Pfarr/Bertelsmann zwischen mittelbarer Diskriminierung und geschlechtsneutral formulierter Ungleichbehandlung, die im Ergebnis nur die Beschäftigten eines Geschlechts diskriminiert (verdeckte Diskriminierung). (Pfarr/Bertelsmann, Diskriminierung im Erwerbsleben, S. 113). 
wenn die merkmalsneutrale Formulierung sich lediglich als Tarnversuch erweise. ${ }^{94}$ Von einem Tarnversuch kann aber nur dann gesprochen werden, wenn neben dem Wissen auch der Wille zur Ungleichbehandlung besteht. Der Arbeitgeber, der einen Verheiratetenzuschlag gewährt, kann diesen Willen haben. Es ist aber auch denkbar, daß einziges Motiv seines Handelns die Förderung der Familien seiner verheirateten Arbeitnehmer ist. Ist also der Wille das entscheidende Abgrenzungskriterium zwischen verdeckter unmittelbarer und mittelbarer Diskriminierung?

Es wird vertreten, der bloße Benachteiligungswille könne dann nicht zu einer unmittelbaren, sondern lediglich zu einer mittelbaren Diskriminierung führen, wenn es an der ausschließlichen Betroffenheit nur eines Geschlechts fehlt. ${ }^{95}$

Beispiel: Wenn der Arbeitgeber im Falle klassischer Männer- oder Frauenberufe eine lange Berufserfahrung fordert und durch diese Regelung ein Geschlecht ausschließen möchte, dann kann die Regelung durchaus auch Berufsanfänger des von ihm erwünschten Geschlechts treffen.

Von der verdeckten Diskriminierung unterscheide sich die mittelbare Diskriminierung gerade dadurch, daß bei ihr zumindest die Möglichkeit besteht, daß von der benachteiligenden Maßnahme sowohl Männer als auch Frauen erfaßt werden. ${ }^{96}$ Nach dieser Ansicht ist Abgrenzungskriterium zwischen verdeckter unmittelbarer und mittelbarer Diskriminierung nicht der Benachteiligungswille des „Diskriminierenden“. Eine mittelbare Diskriminierung kann demnach auch dann vorliegen, wenn der „Diskriminierende“ ungleich behandeln möchte. In diesem Sinne sind auch die Antidiskriminierungsrichtlinien $\mathrm{zu}$ verstehen, die das Merkmal vorsätzlichen Handelns nicht ausdrücklich zum Abgrenzungskriterium zwischen unmittelbarer und mittelbarer Diskriminierung machen.

\section{Anweisung zur Diskriminierung}

Gemäß Art. 2 IV der Richtlinie 2000/43/EG gilt auch

„[d]ie Anweisung zur Diskriminierung einer Person aus Gründen der Rasse oder der ethnischen Herkunft [...] als Diskriminierung im Sinne [der Richtlinie.]“97

94 Richardi/Annuß in Staudinger, $\$ 611$ a, Rn. 30.

95 Richardi/Annu $\beta$ in Staudinger, $\S 611$ a, Rn. 30; Edenfeld unterscheidet zwischen Benachteiligungsabsicht und dolus eventualis: Die Besonderheit der mittelbaren Diskriminierung bestehe darin, daß sie ,gerade keine subjektive Benachteiligungsabsicht wegen des Geschlechts voraussetzt, sondern bei der allenfalls das Ergebnis einer objektiven Benachteiligung billigend in Kauf genommen wird.“( Edenfeld in Erman, Band I, § 611 a, Rn. 7).

96 Richardi/Annuß in Staudinger, $\$ 611$ a, Rn. 32.

97 Ähnliche Definitionen enthalten die Richtlinie 76/276/EWG in Art. 2 IV, Richtlinie 2000/78/EG in Art. 2 IV sowie Richtlinie 2004/113/EG in Art. 4 IV. 
Mit dieser Regelung dürfte die Absicht verfolgt worden sein, dem Umstand Rechnung zu tragen, daß es gesellschaftliche Bereiche gibt, in denen Aufgaben delegiert werden. Zu denken ist insofern vor allem an die Tätigkeit in großen Unternehmen.

Beispiel: Erinnert sei an den Personal-Manager, dem gekündigt worden war, weil er sich geweigert hatte, Bewerbungen von Türkinnen bei künftigen Einstellungen nicht mehr zu berücksichtigen. ${ }^{98}$

In dem Beispielsfall wurde der Personal-Manager angewiesen, türkische Bewerberinnen in benachteiligender Weise gegenüber Bewerberinnen anderer Nationalität ungleich zu behandeln. Eine Anweisung kann freilich auch auf eine einseitig belastende Behandlung oder eine benachteiligende Gleichbehandlung gerichtet sein.

Das AGG hat in seinem $\S 3 \mathrm{~V}$ die Anweisung zur Diskriminierung nach europarechtlichem Vorbild ausdrücklich geregelt:

„Die Anweisung zur Benachteiligung einer Person aus einem in $\S 1$ genannten Grund [ - d. h. wegen eines dort genannten Diskriminierungsmerkmals - ] gilt als Benachteiligung. Eine solche Anweisung liegt in bezug auf $\S 2$ Abs. 1 Nr. 1 bis 4 [ - d. h. im Rahmen von Beschäftigung und Beruf - ] insbesondere vor, wenn jemand eine Person zu einem Verhalten bestimmt, das einen Beschäftigten oder eine Beschäftigte wegen eines in $\S 1$ genannten Grundes benachteiligt oder benachteiligen kann.“

Für das Vorliegen einer Anweisung im Sinne des § 3 V AGG soll es nicht darauf ankommen, „ob die angewiesene Person die Benachteiligung tatsächlich ausführt. “99 Die Bedeutung des $§ 3$ V AGG wird durch die Existenz der allgemeinen zivilrechtlichen Zurechnungsnormen relativiert. Dies hat auch der Gesetzgeber erkannt: „Im Bereich des allgemeinen Zivilrechts sind die in Absatz 5 geregelten Sachverhalte regelmäßig über die zivilrechtlichen Zurechnungsnormen zu erfassen (§§ $31,278,831$ BGB). “100

\section{Zusammenfassung}

Die bisherigen Ausführungen haben sich mit der Frage beschäftigt, welche Verhaltensweisen diskriminierenden Charakter haben können. Dabei wurden folgende Feststellungen getroffen: Eine Diskriminierung kann vorliegen, wenn eine Person gegenüber einer anderen in benachteiligender Weise ungleich behandelt wird, weil sie ein unerwünschtes Merkmal bzw. eine unerwünschte Eigenschaft aufweist. In Anlehnung an die Antidiskriminierungsrichtlinien wird insofern von

98 Müller-Münch in Frankfurter Rundschau vom 11.12.2003, S. 1 unter dem Titel: „Türkinnen unerwünscht - und das Gericht gab dem Chef Recht“.

99 Entwurfsbegründung, BT-Druck. 16/1780, S. 33.

100 Entwurfsbegründung, BT-Druck. 16/1780, S. 33. 
unmittelbarer Diskriminierung gesprochen. Diese Form der Diskriminierung kann in offener und verdeckter Weise erfolgen. Offen diskriminiert, wer die Ungleichbehandlung durch direkten Verweis auf das unerwünschte Merkmal begründet, verdeckt dagegen, wer nach außen hin an ein anderes Merkmal anknüpft. Einen Sonderfall der unmittelbaren Diskriminierung stellt die sogenannte Diskriminierungsdrittwirkung dar. Gemeint ist damit die Konstellation, daß eine Person, die in einer gewissen (z.B. Verwandtschafts-) Beziehung zu der diskriminierten Person steht, ebenfalls Auswirkungen der Diskriminierung erdulden muß.

Es gibt Fälle, in denen eine Person aufgrund eines bestimmten Merkmals eine belastende Behandlung erfährt, ohne jedoch gleichzeitig gegenüber einer anderen Person ungleich behandelt zu werden. Es wurde diskutiert, ob in einem derartigen Fall die Frage des Vorliegens einer Ungleichbehandlung hypothetisch beantwortet werden kann. Im Ergebnis wurde festgestellt, daß es stets einer tatsächlich existierenden Vergleichsperson bedarf, daß es jedoch unerheblich ist, ob diese ihre begünstigende Behandlung gleichzeitig mit der belastenden des „Diskriminierten“ erfahren hat, davor oder danach. Schließlich wurde vorgeschlagen, auch die einseitig belastende Behandlung aufgrund eines bestimmten Merkmals als potenziell diskriminierendes Verhalten einzustufen, ist doch gerade auch mit Blick auf das in Art. 3 I GG enthaltene Willkürverbot nicht recht einzusehen, warum Diskriminierungsschutz darauf beschränkt sein sollte, das Unrecht zu vermeiden, das dadurch entsteht, daß ein an sich legitimes Verhalten durch gleichheitswidrige Unterscheidung ungerecht wird.

Auch die (sexuelle) Belästigung stellt nach den Antidiskriminierungsrichtlinien eine Diskriminierung dar. Eine Belästigung liegt etwa vor, wenn jemand aufgrund der Tatsache, daß er Träger eines bestimmten Merkmals ist, beleidigt wird. Es wurde einerseits festgestellt, daß auch die (sexuelle) Belästigung als diskriminierungsrelevante Verhaltensweise eingestuft werden kann, daß diese Form der Diskriminierung jedoch andererseits Fragen aufwirft, die nicht Gegenstand der Arbeit sein sollen. Im Rahmen des Belästigungsschutzes stellt sich - anders als beim Diskriminierungsschutz im rechtsgeschäftlichen und quasi-rechtsgeschäftlichen Bereich - nicht die Frage nach einer etwaigen Einschränkung der Privatautonomie des „Belästigenden“. Hier geht es nicht um (vor-)vertragliche Rechte und Pflichten von (potenziellen) Vertragspartnern. Die Lösung gerade dieses Spannungsverhältnisses ist aber erklärtes Ziel der vorliegenden Arbeit. Die Probleme, die im Zusammenhang mit dem Belästigungsschutz und insbesondere den insofern bestehenden europarechtlichen Verpflichtungen auftauchen, wurden deshalb lediglich in einem Exkurs kurz angerissen.

Auch eine formelle Gleichbehandlung kann sich tatsächlich unterschiedlich auswirken, je nachdem, ob man Träger eines bestimmten Merkmals ist oder nicht. Auch die benachteiligende Gleichbehandlung wirft somit die Frage nach dem Vorliegen einer Diskriminierung auf. Auf die Antidiskriminierungsrichtlinien bezug nehmend wird insofern von mittelbarer Diskriminierung 
gesprochen. Das Vorliegen einer mittelbaren Diskriminierung hängt nicht vom Nachweis einer tatsächlichen Benachteiligung ab. Vielmehr kann sie bereits dann vorliegen, wenn die Gleichbehandlung geeignet ist, tatsächlich benachteiligend $\mathrm{zu}$ wirken. Nicht der Vorsatz ist Abgrenzungskriterium zur unmittelbaren Diskriminierung - auch der mittelbar Diskriminierende kann vorsätzlich handeln -, sondern vielmehr das Merkmal der ausschließlichen Betroffenheit. Nur bei der mittelbaren Diskriminierung sind überwiegend die Personen, die Träger des unerwünschten Merkmals sind, von der benachteiligenden Maßnahme betroffen, nicht aber ausschließlich, wie im Fall der unmittelbaren Diskriminierung.

Die Antidiskriminierungsrichtlinien regeln ausdrücklich, daß auch die Anweisung zur Diskriminierung selbst eine Diskriminierung darstellen kann. Dies ist begrüßenswert angesichts der Tatsache, daß in vielen gesellschaftlichen Bereichen „Über-Unterordnungsverhältnisse“ bestehen, im Rahmen derer Aufgaben delegiert werden. Durch diese legitime Aufgabenverteilung soll es dem Hintermann freilich nicht möglich sein, ein sich an ihn richtendes Diskriminierungsverbot zu umgehen.

\section{B. Diskriminierungsmerkmale}

„Solange man sich auf eine Liste unzulässiger Diskriminierungsmerkmale beschränkt, wird es immer auch sachfremde Diskriminierungen geben, die zulässig bleiben." 101

Die bisherigen Ausführungen haben gezeigt, daß sich die Frage nach dem Vorliegen einer Diskriminierung immer dann stellt, wenn eine Person eine sie belastende oder sich für sie belastend auswirkende Behandlung erfährt, weil sie Trägerin eines bestimmten Merkmals ist bzw. eine bestimmte Eigenschaft aufweist, welche sie von anderen Personen abgrenzt. Die Definition des Begriffs der Diskriminierung beinhaltet demnach die Beantwortung der Frage, aufgrund welcher Merkmale überhaupt diskriminiert werden kann.

Ausgangspunkt für die Beantwortung der Frage nach den schutzwürdigen Merkmalen bilden die untersuchten internationalen und nationalen Antidiskriminierungsmaßnahmen. Festgestellt wurde, daß der Diskriminierungsschutz dieser Maßnahmen größtenteils auf bestimmte Diskriminierungsmerkmale, wie insbesondere dem Geschlecht beschränkt ist. So ist gem. § 1 AGG

„Ziel des Gesetzes [...], Benachteiligungen aus Gründen der Rasse oder wegen der ethnischen Herkunft, des Geschlechts, der Religion oder Weltanschauung, einer Behinderung, des Alters oder der sexuellen Identität zu verhindern oder zu beseitigen.“ 
Es konnten aber auch Diskriminierungsverbote nachgewiesen werden, die in bezug auf die zu schützenden Merkmale einen nicht abschließenden Katalog aufstellen. ${ }^{102}$ Zudem wurden Maßnahmen ausgemacht, die ein Diskriminierungsverbot statuieren, ohne sich überhaupt auf irgendwelche Merkmale festzulegen. ${ }^{103}$

Die Antidiskriminierungsmaßnahmen haben zweifellos Indizfunktion in bezug auf die Frage der Schutzwürdigkeit bestimmter Merkmale. So kann beispielsweise angesichts der Fülle von Maßnahmen, die dem Schutz vor Diskriminierung aufgrund des Geschlechts gewidmet sind, nicht ernsthaft an der Schutzwürdigkeit dieses Merkmals gezweifelt werden.

Nicht vergessen werden darf, daß die Mehrzahl der untersuchten Maßnahmen über ihre Indizfunktion hinaus verpflichtenden Charakter haben. ${ }^{104}$ Das gilt beispielsweise für die Antidiskriminierungsrichtlinien, die dem Schutz der Rasse oder ethnischen Herkunft, der Religion, einer Behinderung, des Alters, der sexuellen Identität etc. dienen.

Nicht zuletzt lassen sich aus Beispielen in der Rechtsprechung Rückschlüsse über die Schutzwürdigkeit einzelner Merkmale ableiten.

\section{Unbeeinflußbares Merkmal}

Die Untersuchung von Fällen, bei denen sich die Frage nach dem Vorliegen einer Diskriminierung stellt, führt zu der Vermutung, daß Diskriminierung vorwiegend Personen trifft, die äußerlich erkennbare Merkmale aufweisen, die sie von anderen Personen unterscheiden. Es handelt sich dabei zumeist um Merkmale, die, wie Hautfarbe, Geschlecht und Behinderung, vom einzelnen grundsätzlich nicht beeinflußt werden können. ${ }^{105}$ Bestätigt wird diese Wahrnehmung durch die untersuchten Antidiskriminierungsmaßnahmen, die schwerpunktmäßig gerade auch die drei genannten Unterscheidungsmerkmale zum Gegenstand haben.

Die besondere Schutzwürdigkeit der Träger dieser Merkmale ergibt sich daraus, daß sie keinen Einfluß auf das jeweilige Merkmal haben, vielmehr ,auf Gedeih und Verderb“ an selbiges gebunden sind.

Unter dieser Prämisse sind nicht nur die Merkmale Hautfarbe, Geschlecht und Behinderung schutzwürdig, sondern in gleicher Weise alle Merkmale, die vom einzelnen nicht beeinflußt werden können. Als Beispiel dafür kann die Schwangerschaft genannt werden. Daß Schwangere besonderen Schutz verdienen, ist allgemein anerkannt. Das bestätigen eine Reihe (inter-)nationaler Maßnahmen zum Schutz werdender Mütter.

102 Vergleiche dazu z. B. Art. 26 des Internationalen Paktes über bürgerliche und politische Rechte: ,[...] insbesondere $[\ldots]$ oder des sonstigen Status $[\ldots]^{\prime \prime}$.

103 Vergleiche dazu Art. 3 I GG: „Alle Menschen sind vor dem Gesetz gleich.“.

104 Vergleiche dazu Teil 4, Prüfungspunkt A.

105 Schiek spricht im Falle unveränderbarer, nach außen wahrnehmbarer Merkmale von ,askriptiven Persönlichkeitsmerkmalen“. (Schiek, Differenzierte Gerechtigkeit, S. 26); Nussberger unterscheidet zwischen verfügbaren und unverfügbaren Eigenschaften. (Nussberger, JZ 2002, S. 524 (524)). 
Es ist nicht immer einfach zu entscheiden, ob das Vorhandensein eines bestimmten Merkmals vom einzelnen beeinflußbar ist oder nicht. Das gilt zum Beispiel für das Merkmal der sexuellen Identität. ${ }^{106}$ Selbst in den Fällen des Geschlechts und der Hautfarbe ließe sich argumentieren, diese Merkmale seien durch medizinische Eingriffe veränderbar. Gleichwohl wird man in den Fällen, in denen die Veränderbarkeit des Merkmals lediglich daraus resultiert, daß in die körperliche Unversehrtheit der betreffenden Person eingegriffen wird, von unbeeinflußbaren Merkmalen sprechen können.

Ein vom einzelnen unbeeinflußbares Merkmal liegt selbst dann vor, wenn es der betreffenden Person, wie im Fall der Schwangerschaft oder einer Krankheit, temporär unbeeinflußbar anhaftet. Das gilt im besonderen für lang andauernde Krankheiten. ${ }^{107}$

Selbst die Tatsache, daß eine Person vorsätzlich Trägerin eines von ihr unbeeinflußbaren Merkmals geworden ist, schließt deren Schutzwürdigkeit nicht aus. Als Beispiel kann die gewollte Schwangerschaft angeführt werden.

Die Tatsache, daß besonders häufig Menschen aufgrund unbeeinflußbarer und zumeist von außen wahrnehmbarer Merkmale diskriminiert werden, könnte dazu verleiten, Antidiskriminierungsmaßnahmen mit der Begründung auf diese Merkmale zu beschränken, allein sie verdienten Schutz. ${ }^{108}$ Eine solche Schlußfolgerung würde indes dem Ziel der Arbeit, eine Antwort auf die Frage nach der rechtlichen Ausgestaltung eines umfassenden und nach Möglichkeit abschließenden Diskriminierungsschutzes zu bieten, zuwiderlaufen. Zu prüfen ist demnach, ob es weitere Merkmale gibt, die Diskriminierungsschutz verdienen.

\section{Status als Merkmal}

Menschen können Träger von Merkmalen sein, deren Vorhandensein sie zwar beeinflussen können, die aber in einer Weise mit ihren Trägern verknüpft sind, daß sie in die Nähe der unbeeinflußbaren Merkmale rücken.

106 Die sexuelle Identität als vom einzelnen beeinflußbares Merkmal betrachtend: Adomeit, der in diesem Zusammenhang von unentschiedenen Gemütern spricht (Adomeit, NJW 2002, S. 1622 (1622)); als dem Willen des einzelnen entzogenes Merkmal betrachtend: $v$. Hoyningen-Huene/Linck, die darauf hinweisen, daß die Homosexualität allenfalls ein personenbedingter, nicht aber verhaltensbedingter Kündigungsgründ sein kann. ( $v$. Hoyningen-Huene/Linck, Kündigungsschutzgesetz, § 1, Rn. 258); Geck und Schimmel meinen, es sei heute unstreitig, daß die sexuelle Identität nicht zur Disposition des erwachsenen Menschen steht. (Geck/Schimmel, ArbuR 1995, S. 177 (183)).

107 Bei Krankheiten, die nach derzeitigem medizinischen Stand als unheilbar gelten, ist Art. 3 III 2 GG zu beachten, dessen Schutzbereich auch chronisch Kranke regelmäßig erfaßt, nicht aber vorübergehend Erkrankte mit voller Genesungserwartung. (Jarass in Jarass/Pieroth, Art. 3, Rn. 127) Unheilbare Krankheiten lassen sich demnach auch als Behinderung einstufen.

108 Wegen der besonderen Dringlichkeit des Schutzes in diesem Bereich beschränkt Schiek ihre Betrachtungen auf askriptive Persönlichkeitsmerkmale, ohne jedoch $\mathrm{zu}$ behaupten, nur diese Merkmale verdienten Diskriminierungsschutz. (Schiek, Differenzierte Gerechtigkeit, S. 27). 
Beispiele: Religionszugehörigkeit, ${ }^{109}$ Familienstand, ${ }^{110}$ Staatsangehörigkeit

\section{Status und statusbedingtes Verhalten}

Im Gegensatz zu dem bereits angesprochenen „Kopftuchfall“ knüpft der Arbeitgeber, der die Einstellung des Bewerbers von einer bestimmten Religionszugehörigkeit abhängig macht, nicht an ein Verhalten des Bewerbers - Tragen des Kopftuchs -, sondern an den Status „Religionszugehörigkeit“ an.

Die Unterscheidung zwischen Status und Verhalten einer Person ist im Arbeitsrecht bekannt. So lasse sich aus der Trennung zwischen personen- und verhaltensbedingter Kündigung die Unterscheidung zwischen Status und Wahlhandlung gewinnen. ${ }^{111}$ Knüpft der Arbeitgeber eine Sanktion an das (Nicht-)Vorhandensein eines bestimmten Status, stehe im Zentrum seines Vorwurfs weniger ein einmaliges Handeln wie z. B. der Eheschließung, als vielmehr der dadurch herbeigeführte Zustand. ${ }^{112}$

Doch selbst wenn man auf die einmalige Handlung der Heirat abstellt, stelle selbige weniger eine Wahlentscheidung als vielmehr schicksalhaftes Verhalten dar: ${ }^{113}$, „[... [aber die Liebe wählt man sich ja nicht, man wird davon angesteckt, gerät in ihre Fänge, sie kommt über einen wie eine Krankheit, wie ein Unglück.“114

Für die Unterscheidung zwischen Status und Verhalten spricht die Tatsache, daß der Status als solcher auf die ordnungsgemäße Erfüllung vertraglicher Pflichten in der Regel keinen bzw. weniger (negativen) Einfluß hat als ein bestimmtes Verhalten. Daß eine Arbeitnehmerin Muslimin ist, berührt das Arbeitsverhältnis in keiner Weise. Ein bestimmtes Verhalten beeinflußt dagegen in aller Regel das Vertragsverhältnis. So ist denkbar, daß sich die nach außen manifestierte Religionszugehörigkeit - beispielsweise durch Tragen eines Kopftuchs - (negativ) auf das Betriebsklima auswirkt oder zu Umsatzeinbußen des Arbeitgebers führt. ${ }^{115}$ Das Beispiel zeigt, daß der Arbeitgeber, der seinem Arbeitnehmer ein bestimmtes statusbedingtes Verhalten verbietet, nicht unbedingt den Status als solchen ablehnen muß.

109 Nussberger spricht im Zusammenhang mit dem religiösen Bekenntnis von einer verfügbaren Eigenschaft. (Nussberger, JZ 2002, S. 524 (524)).

110 Gemäß Art. 11 lit. a des Übereinkommens zur Beseitigung jeder Form von Diskriminierung der Frau müssen die Vertragsstaaten u.a. Maßnahmen ergreifen, die das „Verbot der Diskriminierung aufgrund des Familienstands bei Entlassungen“ durchsetzen. (BGB1. 1985 II 1, S. 648 ff.).

111 Geck/Schimmel, ArbuR 1995, S. 177 (183).

112 Geck/Schimmel, ArbuR 1995, S. 177 (183).

113 Weiss, ArbuR Sonderheft 1979, S. 28 (31); zustimmend Geck/Schimmel, ArbuR 1995, S. 177 (183).

114 Amos $O z$ in „Eine Geschichte von Liebe und Finsternis“.

115 Das $B A G$ hat in seinem Urteil festgestellt, daß betriebliche Störungen oder Umsatzeinbußen entscheidende Faktoren für die Beantwortung der Frage sein können, ob das religiös bedingte Tragen eines Kopftuchs am Arbeitsplatz zulässig ist. (BAG, NJW 2003, S. 1685 (1687)). 
Die Ausführungen haben gezeigt, daß der Status als solcher nicht als verhaltensbedingtes Diskriminierungsmerkmal eingestuft werden kann, selbst wenn er durch ein Verhalten, wie beispielsweise dem Beitritt $\mathrm{zu}$ einer Religionsgemeinschaft, begründet wurde. ${ }^{116}$ Es muß unterschieden werden zwischen dem Status einerseits und dem auf dem Status beruhenden Verhalten andererseits. Diese Unterscheidung führt beispielsweise zu der Konsequenz, daß in der Kündigungssituation der Status als solcher ein personenbedingter Kündigungsgrund, das statusbedingte Verhalten dagegen ein verhaltensbedingter Kündigungsgrund sein kann. ${ }^{117}$ Diese Unterscheidung ist wichtig, unterliegt die personenbedingte Kündigung doch strengeren Rechtfertigungsanforderungen als die verhaltensbedingte Kündigung. ${ }^{18}$

\section{Staatsangehörigkeit}

Eine Sonderstellung unter den Statusmerkmalen nimmt die Staatsangehörigkeit ein. Gemäß Art. 3 II betrifft die Richtlinie 2000/43/EG

„,nicht unterschiedliche Behandlungen aus Gründen der Staatsangehörigkeit und berührt nicht [...] eine Behandlung, die sich aus der Rechtsstellung von Staatsangehörigen dritter Staaten oder staatenlosen Personen ergibt.“119

Laut Erwägungsgrund 13 läßt die Richtlinie unter anderem Vorschriften über den Zugang von Drittstaatsangehörigen zu Beschäftigung und Beruf unberührt. ${ }^{120}$

Beispiel: Dürfen Gastwirte Schilder aufstellen, auf denen sie Ausländern untersagen, das Lokal zu betreten? ${ }^{121}$

In scheinbarem Widerspruch $\mathrm{zu}$ den bisherigen Ausführungen hebt Erwägungsgrund 13 der Richtlinie hervor, daß das Diskriminierungsverbot grundsätzlich auch gegenüber Drittstaatsangehörigen gilt. Daraus kann der Schluß gezogen werden, daß die Ausnahmenvorschrift des Art. 3 II grundsätzlich nicht im Privatrechtsverkehr gilt, die Norm vielmehr deshalb eingeführt wurde, um spezielle Vorschriften beispielsweise bzgl. der Einreise und des Aufenthaltsrechts von Drittstaatsangehörigen nicht als Diskriminierung und damit als rechtwidrig qualifizieren zu

116 Hier zeigt sich eine Parallele zu den unbeeinflußbaren Merkmalen, die vorsätzlich herbeigeführt wurden.

Schiek qualifiziert dagegen Merkmale wie Weltanschauung, Religion und politische Orientierung als verhaltensbezogene Diskriminierungsmerkmale. (Schiek, Differenzierte Gerechtigkeit, S. 26)

117 In diesem Sinne Geck/Schimmel, ArbuR 1995, S. 177 (183).

118 Geck/Schimmel, ArbuR 1995, S. 177 (183); Praktische Relevanz erlangt die Unterscheidung im Rahmen kirchlicher Arbeitsverhältnisse bei der Frage, ob eine Kündigung mit der Begründung zulässig ist, der Arbeitnehmer habe eine geschiedene Person geheiratet bzw. sich als geschiedene Person neu vermählt. (Geck/Schimmel, ArbuR 1995, S. 177 (182)).

119 Eine identische Regelung findet sich auch in Art. 3 II der Richtlinie 2000/78/EG.

120 Abl. EG 2000 Nr. L 180, S. 22 (23).

121 Vergleiche dazu OVG Münster, GewArch. 1967, S. 118 f.; OLG Frankfurt, NJW 1985, S. 1720 f.; siehe auch Antwort der Bundesregierung vom 29.07.1982 auf eine Anfrage bzgl. der Diskriminierung von Angehörigen der US-Streitkräfte in der Bundesrepublik Deutschland (BT-Druck. 9/1882). 
müssen. $^{122}$ Art. 3 II, der so $\mathrm{zu}$ lesen sei, daß nur hoheitliche Handlungen, die an die Staatsangehörigkeit anknüpfen, grundsätzlich zulässig sind, diene lediglich der Sicherung nationalstaatlicher Souveränität im Umgang mit Drittstaatsangehörigen. ${ }^{123}$

Fälle wie im Beispiel geschildert vom Anwendungsbereich des Diskriminierungsverbots herauszunehmen, entspricht ersichtlich nicht dem Ziel und dem Zweck der Richtlinie. ${ }^{124}$ Die pauschale Aussage, eine auf die Staatsangehörigkeit beruhende Ungleichbehandlung falle aufgrund ihres Art. 3 II nicht unter das von der Richtlinie 2000/43/EG statuierte Diskriminierungsverbot, ${ }^{125}$ ist, wenn nicht falsch, so doch zumindest ungenau und irreführend.

Im Zusammenhang mit dem Unterscheidungsmerkmal „Staatsangehörigkeit“ ist auch auf die Richtlinie 2003/109/EG betreffend die Rechtsstellung der langfristig aufenthaltsberechtigten Drittstaatsangehörigen $^{126}$ vom 25.11.2003 hinzuweisen. Gemäß Art. 11 I der Richtlinie werden langfristig Aufenthaltsberechtigte unter anderem auf folgenden Gebieten grundsätzlich wie eigene Staatsangehörige behandelt:

„a) Zugang zu einer unselbständigen oder selbständigen Erwerbstätigkeit, wenn diese nicht, auch nicht zeitweise, mit der Ausübung öffentlicher Gewalt verbunden ist, sowie Beschäftigungs- und Arbeitsbedingungen, einschließlich Entlassungsbedingungen und Arbeitsentgelt;

f) Zugang zu Waren und Dienstleistungen sowie zur Lieferung von Waren und Erbringung von Dienstleistungen für die Öffentlichkeit und zu Verfahren für den Erhalt von Wohnraum;

g) Vereinigungsfreiheit sowie Mitgliedschaft und Betätigung in einer Gewerkschaft, einem Arbeitgeberverband oder einer sonstigen Organisation, deren Mitglieder einer bestimmten Berufsgruppe angehören, sowie Inanspruchnahme der von solchen Organisationen angebotenen Leistungen, unbeschadet der nationalen Bestimmungen über die öffentliche Ordnung und die öffentliche Sicherheit“.

\section{Verhalten als Merkmal}

Die unter dem Prüfungspunkt „Status als Merkmal“ gemachten Ausführungen haben verdeutlicht, daß auch ein bestimmtes Verhalten Anknüpfungspunkt für eine „Diskriminierung“ sein kann.

Beispiel: Arbeitgeber kündigt einer Arbeitnehmerin, weil sie in Zukunft während der Arbeitszeit religiös bedingt ein Kopftuch tragen möchte. ${ }^{127}$

Daß die Unterscheidung zwischen Status und Verhalten als Diskriminierungsmerkmal sinnvoll ist, wurde bereits festgestellt. An dieser Stelle soll ein weiterer Beleg dafür erbracht werden.

Abl. EG 2000 Nr. L 180, S. 22 (23).

Nickel, NJW 2001, S. 2668 (2670).

So auch Nickel, NJW 2001, S. 2668 (2670).

25 So Waas, ZIP 2000, S. 2151 (2152, Fn. 13).

126 Abl. EG 2004 Nr. L 16, S. 44 ff.

$127 B A G$, NJW 2003, S. $1685 \mathrm{ff}$. 
Im Zusammenhang mit dem Statusmerkmal der Weltanschauung ist fraglich, ob dieser Begriff allein weltanschaulich-universalistische Gemeinschaften ohne metaphysische Aspiration (,große“ Ideologien, wie der Kommunismus) meint oder auch partikuläre politisch-ideologische Stellungnahmen („kleine“ Ideologien, wie Veganer, AKW-Gegner, Tierversuchsgegner) einbezieht. ${ }^{128}$ Sicherlich ist es nicht gerechtfertigt, Diskriminierungsschutz von der „Ranghöhe der Ideologie“ - Gibt es eine solche Rangordnung überhaupt? - abhängig zu machen. ${ }^{129}$ Ein AKWGegner sollte grundsätzlich ebenso wenig ob seiner „Ideologie“ diskriminiert werden, wie der Kommunist.

Ein ähnliches Problem stellt sich im Zusammenhang mit dem Merkmal „Religion“. Das BAG hat darauf hingewiesen, daß es nicht darauf ankommt, ob das Tragen des Kopftuchs Ausdruck eines zwingenden religiösen Gebots des Koran ist, da Art. 4 I und II GG nicht nur die persönliche Freiheit gewährleiste, nach Maßgabe einer autoritativen oder allgemein anerkannten Lehre einer Religionsgemeinschaft zu leben, sondern auch die individuelle Religionsfreiheit als Recht des einzelnen, sein gesamtes Verhalten an den Lehren seines Glaubens auszurichten und seiner inneren Glaubensüberzeugung gemäß zu handeln. ${ }^{130}$ Insbesondere überlasse das Grundrecht dem einzelnen, welche religiösen Symbole er anerkennt und verwendet. ${ }^{131}$

Durch die Unterscheidung zwischen Status und Verhalten als Diskriminierungsmerkmal ist es nicht erforderlich, eine Einteilung in anerkannte und nicht anerkannte Religionen und Weltanschauungen vorzunehmen, um den „Diskriminierenden“ davor zu schützen, daß sich der „Diskriminierte“ uferlos auf seine Religions- bzw. Weltanschauungsfreiheit beruft. Der „Diskriminierende“ hat grundsätzlich jede Religion bzw. Weltanschauung als Status zu akzeptieren, nicht dagegen jedes statusbedingte Verhalten des „Diskriminierten“.

\section{Unbegrenzte Anzahl potenzieller Diskriminierungsmerkmale}

Die bisherigen Ausführungen führen zu der Erkenntnis und gleichlautenden These, daß es zumindest potenziell unendlich viele Merkmale gibt, aufgrund derer diskriminiert werden kann. Es ist schlechterdings kein Merkmal denkbar, bei dem sich nicht Fälle bilden ließen, die man als Diskriminierung einstufen müßte.

Die Mehrzahl der diskutierten internationalen wie nationalen Antidiskriminierungsmaßnahmen sind demgegenüber auf den Schutz bestimmter Merkmale beschränkt. Diesem Ansatz folgend wurde bis zum Inkrafttreten des AGG immer wieder gefordert, der deutsche Gesetzgeber solle sich bei der

\footnotetext{
28 Zu diesen Begriffen vgl. Säcker, ZRP 2002, S. 286 (289).

So auch Säcker, ZRP 2002, S. 286 (289).

$B A G$, NJW 2003, S. 1685 (1687).

$B A G$, NJW 2003, S. 1685 (1687).
} 
Umsetzung der Antidiskriminierungsrichtlinien an die Vorgaben der Richtlinien halten, selbige in bezug auf die Diskriminierungsmerkmale nicht „übererfüllen““ ${ }^{132}$

Im Gegensatz dazu und im Einklang mit der hier aufgestellten These wird teilweise gerade die Beschränkung des Diskriminierungsschutzes auf bestimmte Merkmale kritisiert. Der europäische Gesetzgeber taste sich in „legislativer Kasuistik“ von einem zum anderen Diskriminierungsverbot vor, ohne die Begrenztheit dieses selektiven Ansatzes zu überwinden. ${ }^{133}$ Denn solange man sich auf eine Liste unzulässiger Diskriminierungsmerkmale beschränke, werde es immer auch sachfremde Diskriminierungen geben, die zulässig bleiben: ${ }^{134}$ „Ein Farbiger, ein Türke, ein Homosexueller, ein Behinderter und eine Familie mit drei Kindern bewerben sich um eine Wohnung. Wen darf der Vermieter ablehnen, ohne gegen das ADG zu verstoßen? Nur die Familie mit den Kindern. “" ${ }^{355}$ Es wird zudem darauf hingewiesen, daß eine Beschränkung des Diskriminierungsschutzes auf bestimmte Merkmale den geltenden Diskriminierungsschutz im deutschen Recht verkürzen würde, erfaßten die Generalklauseln des Zivilrechts doch alle denkbaren Benachteiligungen, und hier vor allem auch die sachfremden: ,,auch wenn keine Diskriminierung nach Geschlecht, Rasse, Religion etc. vorliegt, kann eine ungerechtfertigte Ungleichbehandlung vorliegen, wenn einem Bewerber/einer Bewerberin die zu vermietende Wohnung versagt wird, z. B. weil dem Vermieter die Haarfarbe, Kleidung, der Name des Bewerbers nicht paßt.“136 In diesem Zusammenhang und um auf den soeben erwähnten Beispielsfall zurückzukommen ist darauf hinzuweisen, daß im geltenden Recht Ansätze eines Diskriminierungsschutzes aufgrund des Merkmals „Person mit Kind“" nachweisbar sind. So soll die Verpflichtung zur Kinderlosigkeit im Mietvertrag sittenwidrig und damit nichtig sein. ${ }^{137}$

132 So z.B. Säcker, ZRP 2002, S. 286 (288 f.); von Michaelis in FAZ vom 25.02.2005, S. 49 unter dem Titel: „Was sagen Sie zum geplanten Antidiskriminierungsgesetz?“.

133 Thüsing, ZfA 2001, S. 397 (415); In bezug auf die Diskriminierungsmerkmale wurde die Richtlinie 76/207/EWG durch die Richtlinien 2000/43/EG und 2000/78/EG erweitert, Richtlinie 2000/43/EG ihrerseits durch die Richtlinie 2004/113/EG.

134 Thüsing, ZfA 2001, S. 397 (414); Thielmann hat schon 1973 im Zusammenhang mit der Frage der Sittenwidrigkeit von Verfügungen von Todes wegen festgestellt, daß eine Begrenzung des Diskriminierungsverbots auf die Merkmale des Art. 3 III GG unmöglich sei. (Thielmann, Sittenwidrige Verfügungen von Todes wegen, S. 304); Wiese hat im Zusammenhang mit $§ 611$ a BGB darauf hingewiesen: „Die Fixierung auf das Problem angemessener Sanktionen im Falle geschlechtsspeziefischer Benachteiligung bei der Einstellung, [...], läßt auch unberücksichtigt, daß die Nichteinstellung aus anderen Gründen durchaus diskriminierender sein kann. [...] Daran wird deutlich, daß $\S 611$ a BGB eine durch die EG-Richtlinie veranlaßte Sonderregelung ist, die aus politischen Erwägungen vordringlich erschien, zugleich aber eine Privilegierung einer wegen des Geschlechts nicht erfolgten Einstellung bedeutet, ohne daß möglicherweise ungleich gravierendere Benachteiligungen und damit die Einheit der Rechtsordnung beachtet worden wäre." (Wiese, JuS 1990, S. 357 (359)).

135 Dieses Beispiel bildet Braun im Zusammenhang mit dem ADG-Entwurf des Bundesjustizministeriums vom 10.12.2001. (Braun, JuS 2002, S. 424 (424)).

136 Von Koppenfels bezieht ihre Kritik auf den Gesetzesentwurf des Bundesjustizministeriums vom 10.12.2001 (von Koppenfels, WM 2002, S. 1489 (1495)).

137 Heinrichs in Palandt, $\S 138$, Rn. 92; Das BVerfG hat entschieden, daß der Gesetzgeber nicht nur im Bereich des Arbeitsrechts, sondern auch in anderen Bereichen des Privatrechts Regelungen mit besonderer Rücksicht auf Familien mit Kindern zu erwägen habe, so etwa das Verbot einer Kündigung von Mietverträgen über Wohnraum wegen der Aufnahme eines neugeborenen Kindes. (BVerfGE 88, S. 203 (260)); Das Zentrum für Arbeitsrecht und Arbeitsbeziehungen (ZAAR) beklagt, daß den besonderen Bedürfnissen von Eltern nicht genügend Aufmerksamkeit geschenkt wird: „Ob in Gesetzen, Tarifverträgen oder Betriebsvereinbarungen: Quer durch das Arbeitsrecht sei die 


\section{1. „Entstehung“ neuer Diskriminierungsmerkmale}

Die einzelnen Diskriminierungsmerkmale sind nicht naturgegeben, sondern beruhen auf gesellschaftlicher Wahl. ${ }^{138}$ Daraus folgt, daß Diskriminierungsmerkmale infolge gesellschaftlichen Wandels neu „entstehen“ können. Beispielhaft kann insofern das Merkmal „Raucher“ genannt werden. Die Europäische Kommission hat sich auf Anfrage einer schottischen Abgeordneten des Europäischen Parlaments dahingehend geäußert, EU-Unternehmen dürften in Stellenanzeigen Raucher explizit aus dem Bewerberkreis ausschließen. ${ }^{139}$ Der Grund für die Anfrage war die Stellenanzeige eines irischen Callcenterbetreibers, „der diese mit dem Zusatz „Raucher müssen sich gar nicht erst bewerben“ eingeschränkt hatte. “140 Das Unternehmen begründete den grundsätzlichen Ausschluß der Raucher damit, diese machten mehr Pausen als Nichtraucher, seinen öfter krank und zudem nicht so intelligent wie jene. Letzteres zeige sich daran, daß sie die Warnhinweise auf den Zigarettenpackungen mißachteten. ${ }^{141}$ Die Kritiker eines Diskriminierungsschutzgesetzes haben die Stellungnahme der EU-Kommission genutzt, um in diesem Zusammenhang - zu recht - auf den willkürlichen Charakter der Antidiskriminierungsrichtlinien im Hinblick auf den Kreis der von ihnen geschützten Diskriminierungsmerkmale hinzuweisen. ${ }^{142}$ Kritikwürdig ist die EU-Kommission besonders deshalb, weil sie in keiner Weise zwischen dem Statusmerkmal „Raucher“ und dem Verhaltensmerkmal „Rauchen“ unterscheidet. Warum nun aber die Religion und damit auch und vor allem das Statusmerkmal „Religionszugehörigkeit“ diskriminierungsschutzwürdig sein soll, das Statusmerkmal „Raucher“ dagegen nicht, ist nicht nachvollziehbar. Es fragt sich in der Tat, ob es der Kommission um Diskriminierungsschutz oder vielmehr darum geht, ,ihr „,politisch korrektes“ Weltbild in der EU zu verankern.“ 143

\section{2. „Merkmalsbezogenes Merkmal“6}

Im Arbeitsrecht wird die Frage nach einer bestehenden Schwangerschaft von der Rechtsprechung als unmittelbare Benachteiligung wegen des Geschlechts eingestuft und in der Regel für unzulässig erklärt. $^{144}$

Gemäß Art. 2 VI Richtlinie 76/207/EWG ${ }^{145}$ können die Mitgliedstaaten vorsehen,

Benachteiligung von Eltern oder zumindest eine Gleichgültigkeit gegenüber ihren Bedürfnissen erkennbar.“ (FAZ vom 16.11.2006, S. 13, unter dem Titel: „Väter und Mütter als Verlierer des Arbeitsrechts“).

138 In diesem Sinne auch Thüsing, wenn sagt: „Die Gründe eines besonderen Diskriminierungsschutzes sind nicht naturrechtlich vorgegeben, sondern beruhen auf gesellschaftlicher Wahl.“ (Thüsing, ZfA 2001, S. 397 (414)).

139 FAZ vom 08.08.2006, S. 13, unter dem Titel: „Im grauen Dunstbereich“.

140 FAZ vom 08.08.2006, S. 13, unter dem Titel: „Im grauen Dunstbereich“.

141 FAZ vom 08.08.2006, S. 13, unter dem Titel: „Im grauen Dunstbereich“.

142 FAZ vom 08.08.2006, S. 11, unter dem Titel: „Verbotene Raucher“.

143 FAZ vom 08.08.2006, S. 11, unter dem Titel: „Verbotene Raucher“.

$144 E u G H$, NZA 1991, S. 171 (172, Rn. 12); BAG, NZA 1993, S. 257 (257, Leitsatz). 
„daß eine Ungleichbehandlung wegen eines geschlechtsbezogenen Merkmals keine Diskriminierung darstellt, wenn das betreffende Merkmal [...] eine wesentliche und entscheidende berufliche Anforderung darstellt, [...].“146

Es ist anzunehmen, daß der Richtliniengesetzgeber bei der Formulierung „wegen eines geschlechtsbezogenen Merkmals“ in Art. 2 VI Richtlinie 76/207/EWG und der damit einhergehenden Ausdehnung des Diskriminierungsschutzes an die angesprochene Problematik gedacht hat und im Sinne der Rechtsprechung des EuGH zur Benachteiligung Schwangerer lösen wollte. $^{147}$

In diesem Zusammenhang ist auf den Entwurf eines Gesetzes zur Umsetzung europäischer Antidiskriminierungsrichtlinien vom 16.12.2004 ${ }^{148}$ hinzuweisen, der in $\S 8$ III ADG-E folgende Bestimmung enthält:

„Die Absätze 1 und 2 gelten auch für eine unterschiedliche Behandlung wegen eines Merkmals, das im Zusammenhang mit einem in $\S 1$ genannten Grund steht.“

Weder die vom Bundestag am 17.06.2005 verabschiedete Fassung dieses ADG-Entwurfs (ADG-E$2005)^{149}$ noch das am 18.08.2006 in Kraft getretene AGG enthalten eine dem § 8 III ADG-E-2004 vergleichbare Bestimmung. Dies ist insofern begrüßenswert, als Merkmale im Sinne des $\S 8$ III ADG-E-2004 - Merkmale im Zusammenhang mit dem Diskriminierungsmerkmal „Geschlecht“ sollten Schwanger- und Mutterschaft $\operatorname{sein}^{150}$ - eigene Diskriminierungsmerkmale darstellen. Dehnt man den Katalog der Diskriminierungsmerkmale entsprechend aus, bedarf es einer $\S 8$ III ADG-E2004 vergleichbaren Bestimmung nicht. Diese Konsequenz zieht jedoch weder der ADG-E-2005 noch das AGG. Die Merkmalskataloge der $\S 1$ ADG-E-2005, 1 AGG sind mit dem des $\S 1$ ADGE-2004 identisch. Insofern ist die Streichung des § 8 III ADG-E-2004 zu kritisieren.

Insbesondere enthält $\S 1$ AGG kein Diskriminierungsmerkmal „Schwangerschaft“. Im Sinne der eingangs erwähnten Rechtsprechung und mit Blick auf Art. 2 VI Richtlinie 76/207/EWG bestimmt $\S 3$ I 2 AGG jedoch, daß

145 In ihrer durch die Richtlinie 2002/73/EG geänderten Fassung.

146 Hervorhebung durch den Verfasser; Art. 4 Richtlinie 2000/43/EG enthält eine ähnliche Formulierung: „,...] aufgrund eines mit der Rasse oder ethnischen Herkunft zusammenhängenden Merkmals [...]“; ebenso Art. 4 I Richtlinie 2000/78/EG: ,[...] daß eine Ungleichbehandlung wegen eines Merkmals, das im Zusammenhang mit einem der in Artilel 1 genannten Diskriminierungsgründe steht, [...]“.

147 A.A. wohl Thüsing, der im Zusammenhang mit Art. 4 Richtlinie 2000/43/EG die Ansicht vertritt, die Formulierung ,[...] aufgrund eines mit der Rasse oder ethnischen Herkunft zusammenhängenden Merkmals [...]“ betreffe Fälle mittelbarer Diskriminierung. (Thüsing, ZfA 2001, S. 397 (401)) Einer solchen Sichtweise steht Art. 2 II lit. b der Richtlinie entgegen, der für Fälle mittelbarer Diskriminierung einen eigenen Rechtfertigungsmaßstab statuiert.

148 BT-Druck. 15/4538.

149 BR-Druck. 445/05.

150 Entwurfsbegründung, BT-Druck. 15/4538, S. 32.

60 
,[e]ine unmittelbare Benachteiligung wegen des Geschlechts [...] in bezug auf $\S 2$ Abs. 1 Nr. 1 bis 4 [ - d. h. im Rahmen von Beschäftigung und Beruf - ] auch im Falle einer ungünstigen Behandlung einer Frau wegen Schwangerschaft oder Mutterschaft vor[liegt]. “151

Dem Ergebnis mag man folgen. Die Begründung dieses Ergebnisses ist indes fragwürdig. Zumindest dann, wenn sich um eine Arbeitsstelle ausschließlich Frauen bewerben, kann die Benachteiligung einer Schwangeren schwerlich damit begründet werden, sie sei aufgrund ihres Geschlechts benachteiligt worden. ${ }^{152}$ Im Rahmen des $\S 611$ a BGB-A wurde deshalb vorgeschlagen: „Soweit eine Benachteiligung von Schwangeren beim Zugang zu einer Beschäftigung verhindert werden soll, wäre eine Ergänzung des $\S 611$ a um ein ausdrückliches Verbot der Benachteiligung wegen einer Schwangerschaft [...] der adäquate Weg.“153 Gleiches gilt für das Merkmal der sexuellen Identität, das in Art. 3 III GG nicht genannt ist. ${ }^{154}$ Benachteiligungen von Hetero-, Bi- oder Transsexuellen knüpfen nicht an das Geschlecht an, ${ }^{155}$ wie teilweise behauptet wird, ${ }^{156}$ so daß Art. 3 II, III 1 GG in diesen Fällen nicht einschlägig ist. $§ 1$ AGG unterscheidet insofern auch konsequenterweise zwischen den Diskriminierungsmerkmalen „Geschlecht“ und ,sexuelle Identität“.

\section{Geschützte Merkmale im Rahmen des Allgemeinen Gleichheitssatzes}

Vor dem Hintergrund der bisherigen Ausführungen kann gesagt werden, daß Opfer hierarchisiert werden, wenn man den Diskriminierungsschutz auf einzelne Merkmale beschränkt. ${ }^{157}$ „Wer einzelne Gruppen herausgreift und sie unter einen Gleichheitsschutz stellt, den er anderen nicht gewährt, trifft eine Ungleichbehandlung, für die Gründe nicht immer zu finden sind." ${ }^{\text {‘58 }}$ In den USA gebe es deshalb Stimmen, die einen Kündigungsschutz nicht vom Diskriminierungsschutz her begründen wollen, sondern allgemein einen arbeitsbezogenen, sachlichen Grund verlangen. ${ }^{159}$ Auch in Frankreich bemerke man, daß das Diskriminierungsverbot des Code du Travail um immer mehr Diskriminierungsmerkmale erweitert werde und daß es sinnvoller wäre, der Gesetzgeber entschiede

151 Eine entsprechende Regelung enthält auch § 3 I 2 ADG-E-2005.

152 So treffend $B A G$, NZA 1986, S. 739 (740); zustimmend Söllner in MüKo (2. Auflage), § 611 a, Rn. 8; Der EuGH hat indes anders entschieden. (EuGH, NZA 1991, S. 171 (172, Rn. 17); Das BAG hat daraufhin seine Rechtsprechung in NZA 1986, S. 739 (740) aufgegeben. (BAG, NZA 1993, S. 257 (257, Leitsatz); NZA 1993, S. 933 (934)).

153 Raab in Soergel, § 611 a, Rn. 27.

154 Vergleiche aber Art. 10 II BerlVerf; Art. 12 II BrandenbVerf; Art. 2 III ThürVerf.

155 Auch der $E u G H$ subsumiert „Diskriminierungen“ aufgrund der sexuellen Orientierung nicht unter das in Art. 141 EGV sowie der Richtlinie 75/117/EWG enthaltene Verbot der Diskriminierung aufgrund des Geschlechts. (EuGH, Rs. C-249/96, Slg. 1998 I 2, S. 621 (651 f., Rn. 47 u. 50)).

156 So Baer, ZRP 2002, S. 290 (292, Fn. 22).

157 Baer, ZRP 2002, S. 290 (294).

158 Thüsing, ZfA 2001, S. 397 (414).

159 Thüsing, ZfA 2001, S. 397 (414 f.). 
sich stattdessen dafür, einen allgemeinen arbeitsrechtlichen Gleichbehandlungsgrundsatz einzuführen. ${ }^{160}$

Der Vorteil eines Konzepts, das Diskriminierungsschutz nicht auf bestimmte Merkmale beschränkt, besteht auch darin, daß der „Diskriminierende“ dann das Diskriminierungsverbot nicht so leicht umgehen könnte, indem er seine Entscheidung auf ein Merkmal stützt, daß vom Diskriminierungsverbot nicht umfaßt ist. Schließlich ist niemand ,[...] nur Mann oder nur behindert, sondern männlich, heterosexuell, christlich usw. Niemand ist nur Frau, niemand nur schwul, niemand nur Christ. “"161

Das AGG erkennt diese Gefahr nicht, beschränkt es den Diskriminierungsschutz doch auf bestimmte Merkmale (vgl. § 1 AGG). Auch i. R. d. § 611 a BGB-A stellte sich das Problem der Umgehung des Diskriminierungsverbotes durch Berufung auf ein nichtgeschütztes Merkmal, statuierte die Norm Diskriminierungsschutz doch nur bzgl. des Merkmals „Geschlecht“. In diesem Zusammenhang muß allerdings auf $\S 611$ a I 3 BGB-A hingewiesen werden, der wie folgt lautete:

„Wenn im Streitfall der Arbeitnehmer Tatsachen glaubhaft macht, die eine Benachteiligung wegen des Geschlechts vermuten lassen, trägt der Arbeitgeber die Beweislast dafür, daß nicht auf das Geschlecht bezogene, sachliche Gründe eine unterschiedliche Behandlung rechtfertigen oder das Geschlecht unverzichtbare Voraussetzung für die auszuübende Tätigkeit ist.“

Die Gefahr der Umgehung des Verbots der Diskriminierung aufgrund des Geschlechts wurde hier dadurch begrenzt, daß selbst dann, wenn das Geschlecht für die unterschiedliche Behandlung irrelevant war, ein sachlicher Grund vorliegen mußte, um selbige zu rechtfertigen.

Eine dem $\S 611$ a I 3 BGB-A vergleichbare Regelung enthält das AGG nicht (vgl. insofern $\S 22$ AGG). ${ }^{162}$

Wenn man davon ausgeht, daß unter Sachkriterien solche Kriterien zu verstehen sind, die einen Bezug zu Tätigkeit oder Betrieb aufweisen, ${ }^{163}$ dann dürfte ein sachlicher Grund beispielsweise dann nicht gegeben gewesen sein, wenn ein Arbeitgeber, dem das Geschlecht seiner Arbeitnehmer egal war, grundsätzlich keine Homosexuellen einstellen wollte.

\section{Geschützte Merkmale im Rahmen des § 611 a BGB-A}

160 Thüsing, ZfA 2001, S. 397 (415).

161 Baer, ZRP 2002, S. 290 (294).

162 Die Entwurfsfassung des $\S 22$ AGG enthielt dagegen eine dem $\S 611$ a I 3 BGB-A nachgebildete Regelung. (Entwurfsbegründung, BT-Druck. 16/1780, S. 47).

163 So Herrmann, ZfA 1996, S. 19 (27). 
An diese Feststellung knüpft die verblüffende Frage an, ob § 611 a BGB-A damit nicht auch vor Benachteiligungen aufgrund anderer Merkmale schützte. Dies bejahend wurde vertreten, $§ 611$ a BGB-A, der die Umsetzung der Richtlinie 76/207/EWG und damit Schutz vor Diskriminierung aufgrund des Geschlechts bezweckte, schieße durch die Regelung in Absatz 1 Satz 3 BGB über sein eigentliches Ziel hinaus. ${ }^{164} \S 611$ a I BGB-A verbiete nicht nur, nach dem Geschlecht zu differenzieren, sondern gebiete zudem, die Bewerber bei Vertragsanbahnung nur nach Sachgesichtspunkten zu beurteilen und schließlich auch auszuwählen. ${ }^{165}$ Deshalb sei $\S 611$ a BGBA nicht weniger als ,Zivilrecht gewordener Art. $3 \mathrm{GG}^{\text {“166 }}$ und, speziell die Einstellungssituation betrachtet, „Zivilrecht gewordener Art. 33 II GG“، ${ }^{167}$ Selbst bei homogener Bewerberlage, d. h. dann, wenn sich auf eine Stelle nur Frauen oder nur Männer bewerben, soll der Arbeitgeber verpflichtet gewesen sein, nach Sachgesichtspunkten zu entscheiden. Begründet wurde dies damit, es sei kein vernünftiger Grund erkennbar, warum im Fall heterogener Bewerberlage nach Sachkriterien entschieden werden muß, im Fall homogener Bewerberlage aber nicht. ${ }^{168}$

Für den Anhänger des Konzepts, wonach kein Merkmal denkbar ist, aufgrund dessen nicht diskriminiert werden kann, klingt es verlockend, § 611 a BGB-A als Zivilrecht gewordenen Art. 3 GG zu betrachten. Eine solche Sichtweise ist indes mit dem Wortlaut der Norm nur bedingt vereinbar. § 611 a I 3 BGB-A war eine Beweislastregelung, die voraussetzte, daß „der Arbeitnehmer Tatsachen glaubhaft macht, die eine Benachteiligung wegen des Geschlechts vermuten lassen“. Nur wenn diese Bedingung erfüllt war, sollte der Arbeitgeber beweisen müssen, „daß nicht auf das Geschlecht bezogene, sachliche Gründe eine unterschiedliche Behandlung rechtfertigen“. Fühlte sich der Arbeitnehmer beispielsweise ob seiner sexuellen Identität diskriminiert, konnte er seine Klage nicht auf $\S 611$ a BGB-A stützen. ${ }^{169} \S 611$ a BGB-A schützte vor Diskriminierungen aufgrund anderer Merkmale als dem Geschlecht nur dann, wenn eine Diskriminierung aufgrund des Geschlechts glaubhaft gemacht worden war. ${ }^{170}$

Das $B A G$ interpretierte $\S 611$ a I 3 BGB-A noch enger, indem es behauptete, durch das Merkmal „sachlicher Grund“ sei die mittelbare Diskriminierung angesprochen. ${ }^{171}$ Ein derartiges Verständnis

164 Herrmann, ZfA 1996, S. 19 (28).

165 Herrmann, ZfA 1996, S. 19 (27).

166 Herrmann, ZfA 1996, S. 19 (21).

167 Herrmann, ZfA 1996, S. 19 (29); Art. 33 II GG lautet: „Jeder Deutsche hat nach seiner Eignung, Befähigung und fachlichen Leistung gleichen Zugang zu jedem öffentlichen Amte.“.

168 Herrmann, ZfA 1996, S. 19 (30).

169 Unmittelbaren Schutz sollten andere Merkmale als das Geschlecht auf andere Weise erlangen, etwa durch Art. 3 III GG. (Buchner in Münchener Handbuch zum Arbeitsrecht, Band 1, § 39, Rn. 82 - 86); vgl. auch Müller-Glöge: „Das Verbot des Abs. 1 Satz 1 erlaubt aber keinen Umkehrschluß. Der Arbeitgeber ist nicht frei, aus anderen als geschlechtsbezogenen Gründen jede Differenzierung vorzunehmen. Vielmehr unterliegt er vielfältigen Bindungen. Einschränkungen können sich aus anderen Normen und insbesondere dem arbeitrechtlichen Gleichbehandlungsgrundsatz ergeben.“ (Müller-Glöge in MüKo, § 611 a, Rn. 23).

170 Darauf weist auch Buchner hin: „Die Vorschrift beläßt dem Arbeitgeber keineswegs nur die Möglichkeit einer unterschiedlichen Behandlung, die aus sachlichen Gründen gerechtfertigt ist. Erst die Beweislastregelung des $\S 611$ a I [3] BGB kann praktisch eine entsprechende Beschränkung bewirken." (Buchner in Münchener Handbuch zum Arbeitsrecht, Band 1, § 39, Rn. 72 f.).

$171 \quad B A G$, NZA 1999, S. 371 (372); ebenso Richardi/Annuß in Staudinger, § 611 a, Rn. 57 u. 97. 
des $\S 611$ a $\quad$ I 3 BGB-A führt zu der Konsequenz, daß der Arbeitgeber sein diskriminierungsrelevantes Verhalten durch Angabe jedweden unsachlichen Grundes rechtfertigen konnte, solange dieser Grund nicht zu einer mittelbaren Diskriminierung aufgrund des Geschlechts führte. ${ }^{172}$ Eine solche Interpretation ergibt sich jedoch weder aus dem Wortlaut der Bestimmung noch kann dem Gesetzgeber eine derartige Intention unterstellt werden. ${ }^{173}$

\section{Geschützte Merkmale im Rahmen der mittelbaren Diskriminierung}

Auch das Institut der mittelbaren Diskriminierung führt zu einem Diskriminierungsschutz, wo er von der jeweiligen Antidiskriminierungsmaßnahme nicht primär bezweckt ist. Das Verhalten des mittelbar „Diskriminierenden“ hat einen doppelten Charakter. Er behandelt gleich in bezug auf das von der jeweiligen Maßnahme geschützte Diskriminierungsmerkmal und im selben Moment ungleich bezüglich eines Merkmals, das durch selbige nicht geschützt ist. Der Arbeitgeber, der Teilzeitbeschäftigte geringer vergütet als Vollzeitbeschäftigte, knüpft unmittelbar nicht an das Merkmal „Geschlecht“ an. Bezüglich dieses Merkmals behandelt er gleich. Das Merkmal „teilbzw. vollzeitbeschäftigt“ macht er dagegen zum Anknüpfungspunkt einer unterschiedlichen Behandlung. Bezüglich dieses Merkmals behandelt er ungleich. Der EuGH hat diese Vergütungspraxis als mittelbare Diskriminierung aufgrund des Geschlechts eingestuft, da Teilzeitarbeit nach wie vor eine Domäne der Frau sei.

Problematisch an dieser Rechtsprechung ist, daß sie dem teilzeitbeschäftigten Mann, der in gleicher Weise wie seine teilzeitbeschäftigte Kollegin von einer derartigen Vergütungspraxis betroffen ist, keinen Diskriminierungsschutz bietet. Ein solcher ließe sich nur dadurch realisieren, daß man das Merkmal „teil- bzw. vollzeitbeschäftigt“ als Diskriminierungsmerkmal qualifiziert. § 4 I TzBfG beschreitet diesen Weg.

Anzumerken ist, daß das Institut der mittelbaren Diskriminierung durch eine Ausweitung möglicher Diskriminierungsmerkmale zwar an Bedeutung verlieren, aber nicht überflüssig würde, da sich im Einzelfall die Unsachlichkeit einer diskriminierungsrelevanten Maßnahme gerade daraus ergeben kann, daß sie eine bestimmte Personengruppe überproportional belastet.

\section{Offener Merkmalskatalog}

All die genannten Argumente bekräftigen die Überzeugung, daß Diskriminierungsschutz nicht auf bestimmte Merkmale beschränkt sein darf. Wenn nun aber ,unendlich“ viele 
Diskriminierungsmerkmale denkbar sind, stellt sich die Frage, wie diesem Umstand gesetzestechnisch Rechnung getragen werden kann.

Sinnvoll wäre es, einen offenen Merkmalskatalog zum Bestandteil eines Antidiskriminierungsgesetzes $\mathrm{zu}$ machen, würde ein solcher doch, ohne abschließend zu sein, zeigen, daß es bestimmte Diskriminierungsmerkmale gibt, deren Schutz besonders dringlich ist.

Als Beispiel für einen solchen offenen Merkmalskatalog kann Art. 21 der Charta der Grundrechte angeführt werden: „Diskriminierungen, insbesondere wegen [...] sind verboten““. ${ }^{174}$ Art. 3 III GG enthält eine ähnliche Formulierung zwar nicht, jedoch ist umstritten, ob dessen Merkmalskatalog abschließend ist. ${ }^{175}$ Selbst wenn man den abschließenden Charakter des Art. 3 III GG bejaht, ${ }^{176}$ folgt daraus nicht, daß eine Differenzierung nach dort nicht genannten Merkmalen zulässig ist. Insoweit komme vielmehr Art. 3 Abs. 1 GG wieder zur Anwendung. ${ }^{177}$ Art. 3 III GG sei lediglich eine historisch zu erklärende Konkretisierung des allgemeinen Gleichheitssatzes. ${ }^{178}$

Daß den Focus der Betrachtungen zum Diskriminierungsschutz ganz bestimmte Merkmale bilden, liegt auf der Hand. Das haben bereits die Betrachtungen $\mathrm{zu}$ den (inter-)nationalen Antidiskriminierungsmaßnahmen gezeigt. Es ist indes nicht nachvollziehbar, daß bestimmte Merkmale aus den Betrachtungen ausgeschlossen werden sollen, weil diesbezüglich die Gefahr einer bloß symbolischen Gesetzgebung bestehe. ${ }^{179}$ Ein Gesetz verliert nicht an Schlagkraft, weil der selten bzw. nur theoretisch vorkommende Fall unter selbiges ebenso subsumiert werden kann, wie der regelmäßig vorkommende.

\title{
V. Tatsächlicher und vermeintlicher Merkmalsträger
}

\begin{abstract}
„Mein Freund Itzig war blond und blauäugig, hatte eine gerade Nase, feingeschwungene Lippen und gute Zähne. Ich dagegen, Max Schulz, unehelicher, wenn auch rein arischer Sohn der Minna Schulz, hatte schwarze Haare, Froschaugen, eine Hakennase, wulstige Lippen und schlechte Zähne. Daß wir beide oft verwechselt wurden, werden Sie sich ja leicht vorstellen können. “180
\end{abstract}

Diskriminierungsmerkmale sind, wie sich gezeigt hat, personenspezifische Merkmale. Daraus könnte der Schluß gezogen werden, daß nur derjenige, der tatsächlich Träger eines bestimmten Diskriminierungsmerkmals ist, aufgrund dieses Merkmals diskriminiert werden kann. Der Diskriminierungsschutz wäre dann jedoch in einer sachlich nicht gerechtfertigten Weise verkürzt.

174 Diesen Weg beschreitet beispielsweise auch $\S 1$ des ADG-Entwurfs von Bündnis 90/Die Grünen, wonach niemand ,[...] insbesondere wegen seiner [...]“ diskriminiert werden darf. (BT-Druck. 13/9706, S. 3) Gleiches gilt für $\S 1$ I des ADG-Entwurfs der SPD: „Niemand darf insbesondere wegen [...] benachteiligt werden.“ (BT-Druck. 13/10081, S. 4).

175 Starck in von Mangoldt/Klein/Starck, Band 1, Art. 3 Abs. 3, Rn. 339, Fn. 9 f.

176 So BAG, NJW 1990, S. 141 (143); a.A.: Baer, ZRP 2002, S. 290 (292, Fn. 22).

177 Nussberger, JZ 2002, S. 524 (531).

178 Mikat in Festschrift für Nipperdey, Band I, S. 595; ebenso Starck: Art. 3 III 1 GG konkretisiere und spezialisiere den allgemeinen Gleichheitssatz. (Starck in von Mangoldt/Klein/Starck, Band 1, Art. 3 Abs. 1, Rn. 338).

179 Mit diesem Argument lehnt Schiek die Einbeziehung aller Merkmale des Art. 3 III GG in ihre Betrachtungen ab. (Schiek, Differenzierte Gerechtigkeit, S. 464).

180 Aus dem Roman „Der Nazi \& Der Friseur“"von Edgar Hilsenrath. 
Wird jemand etwa „diskriminiert“, weil er als Anhänger einer bestimmten Religion gilt, so stellt sich die Frage nach dem Vorliegen einer Diskriminierung unabhängig davon, ob er tatsächlich dieser Religion angehört, oder irrtümlicherweise für einen Anhänger derselben gehalten wird. ${ }^{181}$

\section{Zusammenfassung}

Die im Rahmen des Prüfungspunktes „Diskriminierungsmerkmale“ gewonnenen Erkenntnisse lassen sich wie folgt zusammenfassen: Anknüpfungspunkt für eine „Diskriminierung“ sind zumeist solche Merkmale - etwa Hautfarbe, Geschlecht oder Behinderung -, die einer Person unveränderbar anhaften. Der Diskriminierungsschutz darf sich indes nicht auf diese unbeeinflußbaren Merkmale beschränken. Auch der Status einer Person - etwa eine bestimmte Religionszugehörigkeit - und sogar deren Verhalten können Gründe für eine Diskriminierung derselben darstellen.

Da es zumindest potenziell unendlich viele Merkmale gibt, aufgrund derer diskriminiert werden kann, ist ein auf ausgewählte Merkmale beschränkter Diskriminierungsschutz verfehlt. Im Zusammenhang mit dieser These gewinnen die folgenden Überlegungen Bedeutung: Diskriminierungsmerkmale sind nicht naturgegeben, sondern beruhen auf gesellschaftlicher Wahl. Durch gesellschaftlichen Wandel können demnach neue Diskriminierungsmerkmale „entstehen“. Ein nicht auf bestimmte Merkmale beschränkter Diskriminierungsschutz kann auf den indirekten Schutz bestimmter Merkmale als „merkmalsbezogene Merkmale“ - die Schwangerschaft soll ein geschlechtsbezogenes Merkmal sein - verzichten. Die Tatsache, daß $§ 611$ a BGB-A vor Diskriminierungen aufgrund anderer Merkmale als dem Geschlecht dann schützte, wenn eine Diskriminierung aufgrund des Geschlechts glaubhaft gemacht worden war, offenbart die Schwäche eines auf bestimmte Merkmale beschränkten Diskriminierungsschutzes. Sichtbar wird diese Schwäche auch bei dem Verbot der mittelbaren Diskriminierung, das lediglich dem mittelbar Diskriminierten Schutz auch hinsichtlich des Merkmals gewährt, aufgrunddessen ungleich behandelt wird - jede mittelbare Diskriminierung aufgrund eines bestimmten Merkmals setzt eine Ungleichbehandlung aufgrund eines anderen Merkmals voraus.

Dem Umstand, daß es zumindest potenziell unendlich viele Diskriminierungsmerkmale gibt, sollte gesetzestechnisch durch einen offenen Merkmalskatalog Rechnung getragen werden, der - ohne abschließend zu sein - die besondere Schutzwürdigkeit einiger Diskriminierungsmerkmale hervorheben würde.

Neben dem tatsächlichen genießt auch der vermeintliche Merkmalsträger, d. h. derjenige, der für den Träger eines bestimmten Merkmals gehalten wird, obwohl er dies in Wirklichkeit nicht ist, Diskriminierungsschutz. 


\section{Rechtswidrigkeit des diskriminierungsrelevanten Verhaltens}

Aufgrund der im Rahmen der Prüfungspunkte „Diskriminierungsrelevantes Verhalten“ und „Diskriminierungsmerkmale“ getroffenen Feststellungen hat der Diskriminierungsbegriff eine Weite erlangt, in Konsequenz derer jede private Maßnahme, die eine Person belastet, eine Diskriminierung darstellen müßte.

$\mathrm{Da}$ dieses Ergebnis nicht richtig sein kann, da es berechtigte Interessen des „Diskriminierenden“ völlig unberücksichtigt ließe, leuchtet unmittelbar ein. Welches sind nun aber die auf Seiten des „Diskriminierenden“ zu berücksichtigenden Interessen?

\section{Privatautonomie contra Diskriminierungsschutz}

Der Widerstand gegen die Einführung von Diskriminierungsschutzmaßnahmen im Zivilrecht wird vor allem damit begründet, derartige Maßnahmen gefährdeten das das Zivilrecht prägende Institut der Privatautonomie. ${ }^{182}$

Dem wird entgegnet, Antidiskriminierungsmaßnahmen hätten immer auch die grundgesetzlich geschützte Privatautonomie zu beachten: „Neben Art. 2 I GG gilt dann auch Art. 3 GG, und beide stehen unter dem Dach des Menschenwürdeschutzes nach Art. 1 I GG. So kann gezeigt werden, wie Privatautonomie zu fassen ist, um sie vor dem strategisch erfolgreich skandalisierenden und doch zu Unrecht beschworenen Tod zu bewahren. “183

In diesem Sinne hat das BVerfG festgestellt: „Bei privatrechtlichen Regelungen, die der Vertragsfreiheit Grenzen setzen [- „Die Privatautonomie ist notwendigerweise begrenzt und bedarf der rechtlichen Ausgestaltung. “184 - ], geht es um den Ausgleich widerstreitender Interessen, die regelmäßig beide grundrechtlich verankert sind. Die kollidierenden Grundrechtspositionen sind in ihrer Wechselwirkung zu erfassen und so zu begrenzen, daß sie für alle Beteiligten möglichst weitgehend wirksam werden.“185 Unter dieser Prämisse verliere „,selbst der verfassungsrechtliche

182 abzuschließen." (FAZ vom 10.12.2004, S. 43 zitiert den Präsidenten des Verbandes Haus \& Grund Dorn unter dem Titel: „Die Wohnungswirtschaft warnt vor weiteren Belastungen“); „Denn der Entwurf für das Antidiskriminierungsgesetz kann [...] der Anfang vom Ende der Vertragsfreiheit sein.“ (FAZ vom 16.12.2004, S. 1); Kritik auch bei Wagner in FAZ vom 22.12.2004, S. 12 unter dem Titel: „Das Antidiskriminierungsgesetz - ein Angriff auf die Vertragsfreiheit“.

183 Baer, ZRP 2002, S. 290 (291).

184 BVerfGE 89, S. 214 (231).

185 BVerfG, NJW 2003, S. 2815 (2815); BVerfGE 7, S. 198 (205), wonach neue Vorschriften des Privatrechts im Einklang mit dem grundrechtlichen Wertesystem stehen müssen; vgl. auch Enneccerus/Nipperdey, Allgemeiner Teil des Bürgerlichen Rechts, 1. Band, 1. Halbband, S. 103; Hager, JZ 1994, S. 373 (383); Schwabe, Die sogenannte Drittwirkung der Grundrechte, S. 152; Dürig in Maunz/Dürig, Band I, Art. 3 Abs. I, Rn. 511; bzgl. der mittelbaren Drittwirkung des Art. 3 I GG im Fall einer Monopolstellung vgl. auch Gubelt in v. Münch/Kunig, Band 1, Art. 3, Rn. 2; Heun in Dreier, Band I, Art. 3, Rn. 70; Alexy, Theorie der Grundrechte, S. 491; ihm folgend Nickel, Gleichheit und Differenz in der vielfältigen Republik, S. 138. 
Gleichheitssatz im Vertragsrecht seinen Schrecken, der doch stets im Geruche stand, das System des Zivilrechts aus den Angeln zu heben.“"186

Zwischen den Rechten des „Diskriminierten“ auf der einen Seite und den Rechten des „Diskriminierenden“ auf der anderen besteht ein Spannungsverhältnis, das es im Wege der vom BVerfG geforderten Abwägung zu lösen gilt. Das Gericht hat dabei klargestellt, daß es grundsätzlich Aufgabe des Gesetzgebers ist, im Rahmen seiner Gesetzgebung diese Abwägung vorzunehmen: „Mit der Pflicht zur Ausgestaltung der Privatrechtsordnung stellt sich dem Gesetzgeber ein Problem praktischer Konkordanz.“ 187

\section{Rechtswidrigkeitsprüfung als Ausdruck praktischer Konkordanz}

Die Interessen des „Diskriminierenden“ berücksichtigt der (inter-)nationale Gesetzgeber dadurch, daß er bestimmte diskriminierungsrelevante Handlungen nicht als Diskriminierung einstuft. So ist gem. $§ 8 \mathrm{I}$ AGG

„[e]ine unterschiedliche Behandlung wegen eines in $\S 1$ genannten Grundes $[-\mathrm{d}$. h. wegen eines dort genannten Diskriminierungsmerkmals - ... ], zulässig, wenn dieser Grund wegen der Art der auszuübenden Tätigkeit oder der Bedingungen ihrer Ausübung eine wesentliche und entscheidende berufliche Anforderung darstellt, sofern der Zweck rechtmäßig und die Anforderung angemessen ist.“"188

Ähnlich formuliert es die Richtlinie 76/207/EWG, wonach die Mitgliedstaaten gem. Art. 2 VI vorsehen können,

„[...] daß eine Ungleichbehandlung wegen eines geschlechtsbezogenen Merkmals keine Diskriminierung darstellt, wenn das betreffende Merkmal aufgrund der Art einer bestimmten beruflichen Tätigkeit oder der Bedingungen ihrer Ausübung eine wesentliche und entscheidende berufliche Anforderung darstellt, sofern es sich um einen rechtmäßigen Zweck und eine angemessene Anforderung handelt.“'189

Beim Vergleich beider Bestimmungen fällt zunächst auf, daß § 8 I AGG als Rechtfertigungsgrund konzipiert ist ${ }^{190}$ - „Eine unterschiedliche Behandlung [...] zulässig, wenn [...]“ -, während das Erfordernis der wesentlichen und entscheidenden beruflichen Anforderung in Art. 2 VI Richtlinie 76/207/EWG negatives Tatbestandsmerkmal ist - ,[...] eine Ungleichbehandlung [...] keine

186 Hager, JZ 1994, S. 373 (377).

187 BVerfGE 89, S. 214 (232).

188 Ähnliche Regelungen enthalten die $\S \S 9,10$ und 20 AGG. Besonders zu beachten ist insofern der unterschiedliche Rechtfertigungsmaßstab (vgl. dazu ausführlich Teil 4, Prüfungspunkt B. I. 4 und 5.). Vgl. auch $§ 611$ a I 2 BGB-A.

189 Entsprechende Regelungen enthalten Art. 4 Richtlinie 2000/43/EG sowie Art. 4 I Richtlinie 2000/78/EG.

190 Das gilt auch für die $\S \S 9,10$ und 20 AGG sowie $\S 611$ a I 2 BGB-A. 
[unmittelbare] Diskriminierung darstellt, wenn [...].“191 Für die mittelbare Diskriminierung gilt dasselbe. Gemäß Art. 2 II 2. Spiegelstrich Richtlinie 76/207/EWG liegt eine mittelbare Diskriminierung nicht vor, wenn $[\ldots]$...192

Es wird darauf hingewiesen, der deutschen Rechtsordnung sei die grundsätzliche Unterscheidung zwischen Tatbestandsmäßigkeit und Rechtswidrigkeit eigen, „,wobei erstere die Tatsache des bloßen Eingriffs in einen fremden Rechtskreis erfaßt, wohingegen letztere danach zu beurteilen ist, ob auch nach Abwägung der jeweils betroffenen Rechtsgüter der Eingriff noch als unangemessen erscheint oder ausnahmsweise von der Rechtsordnung gebilligt wird. “193 Diese Unterscheidung lasse sich auch im Falle der unmittelbaren wie mittelbaren Diskriminierung „ohne weiteres in den Kategorien Tatbestandsmäßigkeit und Rechtswidrigkeit abbilden“. ${ }^{194}$

Im gleichen Atemzug wird jedoch auch darauf hingewiesen, daß der deutsche Gesetzgeber nur insoweit nicht an die europäischen Vorgaben hinsichtlich der Normstruktur gebunden ist, als ,,auch im Rahmen einer anderen Systematik die materiellen europäischen Vorgaben umgesetzt werden können. “195 Es steht außer Frage, daß der Gesetzgeber des AGG die materiell-rechtlichen Vorgaben der Richtlinie 76/207/EWG trotz der abweichenden Gesetzestechnik umsetzen wollte.

Vor dem Hintergrund der soeben gemachten Ausführungen bestehen keine Zweifel an der grundsätzlichen Vereinbarkeit des in $\S 8$ I AGG statuierten Rechtfertigungsgrundes mit der Rechtsprechung des $E u G H$, auch wenn das Gericht im Zusammenhang mit der Richtlinie 76/207/EWG und auf den ersten Blick unverständlich festgestellt hat, daß ,,[...] jeder Verstoß gegen das Diskriminierungsverbot für sich genommen ausreicht, um die volle Haftung seines Urhebers auszulösen, ohne daß die im nationalen Recht vorgesehenen Rechtfertigungsgründe berücksichtigt werden können. “196 Gleichzeitig hat das Gericht hervorgehoben, ,daß die Richtlinie [76/207/EWG] in Art. 2 II-IV Ausnahmen von dem in ihrem Art. 2 I aufgestellten Grundsatz der Gleichbehandlung vorsieht, keineswegs aber die Haftung des Urhebers einer Diskriminierung davon abhängig macht, daß ein Verschulden nachgewiesen wird oder kein Rechtfertigungsgrund vorliegt. “197 Betrachtet

191 Entsprechende Regelungen enthalten Art. 4 Richtlinie 2000/43/EG, Art. 4 I Richtlinie 2000/78/EG sowie Art. 4 V Richtlinie 2004/113/EG.; Aufgrund der Tatsache, daß die „Rechtfertigung“ zum Tatbestand zählt, ergab sich für den europäischen Gesetzgeber die Notwendigkeit, die Begriffe „Ungleichbehandlung“ und „Diskriminierung“ nebeneinander zu verwenden.

192 Entsprechende Regelungen enthalten Art. 2 II lit. b Richtlinie 2000/43/EG, Art. 2 II lit. b Richtlinie 2000/78/EG sowie Art. 2 lit. b Richtlinie 2004/113/EG. Daß der „sachliche Grund“ Teil des Tatbestandes der mittelbaren Diskriminierung ist, meinen auch Classen, JZ 1996, S. 921 (924); Richardi/Annuß in Staudinger, § 611 a, Rn. 34 sowie Müller-Glöge in MüKo, § 611 a, Rn. 26.

193 Richardi/Annuß in Staudinger, $\$ 611$ a, Rn. 35.

194 Richardi/Annu $\beta$ in Staudinger, $\$ 611$ a, Rn. 35.

195 Richardi/Annu $\beta$ in Staudinger, $\S 611$ a, Rn. 34; Anzumerken bleibt, daß das AGG die Rechtfertigung einer unmittelbaren Diskriminierung als Rechtfertigungsgrund betrachtet. Lediglich bei der mittelbaren Benachteiligung seien Fragen der zulässigen Ungleichbehandlung bereits auf Tatbestandsebene zu entscheiden. (Entwurfsbegründung, BT-Druck. 16/1780, S. 43).

$196 E u G H$, NJW 1991, S. 628 (629, Rn. 26).

197 EuGH, NJW 1991, S. 628 (629, Rn. 22); Das Gericht bezieht sich dabei auf die ursprüngliche Fassung der Richtlinie. (Abl. EG 1976 Nr. L 39, S. 40 ff.). 
man sich die in Art. 2 II-IV statuierten Ausnahmen, stellt man fest, daß der in $\S 8$ I AGG statuierte Rechtfertigungsgrund kein solcher in dem vom EuGH verstandenen Sinne darstellt.

Die folgenden Betrachtungen dienen dem Ziel $\mathrm{zu}$ ergründen, was unter ,wesentliche und entscheidende berufliche Anforderung“ im Sinne des $§ 8$ I AGG sowie des Art. 2 VI Richtlinie 76/207/EWG zu verstehen ist.

\section{III. ,,Wesentlich und entscheidend“" - Wortbedeutung}

Sprachlich hat das Wort wesentlich u.a. die Bedeutung von: „den Kern einer Sache ausmachend und daher besonders wichtig bzw. von entscheidender Bedeutung“. ${ }^{198}$ Das Wort entscheidend läßt sich hingegen mit ,ausschlaggebend“, „von richtungsweisender Bedeutung“ bzw. „maßgeblich“ umschreiben. ${ }^{199}$ Die Definitionen zeigen, daß beide Begriffe von ihrer sprachlichen Bedeutung her ineinanderfließen, eine klare Abgrenzung schwer fällt.

Ein Vergleich der Absätze 1 und 2 des Art. 4 Richtlinie 2000/78/EG zeigt, daß der Richtliniengesetzgeber beide Begriffe bewußt gewählt hat und ihnen eine unterschiedliche Bedeutung zumißt. Während Absatz 1 verlangt, daß das betreffende Merkmal „,eine wesentliche und entscheidende berufliche Anforderung darstellt,“ reicht nach Absatz 2 aus, daß das betreffende Merkmal „eine wesentliche, rechtmäßige und gerechtfertigte berufliche Anforderung [...] darstellt.“ 200

Eine Abgrenzung beider Begriffe läßt sich dergestalt vornehmen, daß man als wesentliches Merkmal all diejenigen begreift, die typischerweise, d. h. etwa traditionsbedingt, Voraussetzung einer bestimmten Tätigkeit sind, während man von einem entscheidenden Merkmal dann spricht, wenn sich die Tätigkeit nur bei Vorliegen dieses Merkmals bestimmungsgemäß durchführen läßt.

Bei dem Versuch, die Bedeutung der Begriffe ,wesentlich und entscheidend“ zu ergründen, ist immer wieder eine Parallele zu dem Unverzichtbarkeitserfordernis in $§ 611$ a I 2 BGB-A gezogen worden. Nach dieser Norm war

„[e]ine unterschiedliche Behandlung wegen des Geschlechts [...] zulässig, soweit [...] ein bestimmtes Geschlecht unverzichtbare Voraussetzung für diese Tätigkeit [war]. ‘201

198 Duden, Das große Wörterbuch der deutschen Sprache, Band 10, S. 4497 f.; Duden, Band 10 (Bedeutungswörterbuch), S. 752.

199 Duden, Das große Wörterbuch der deutschen Sprache, Band 3, S. 1046; Duden, Band 8 (Sinn- und sachverwandte Wörter), S. 205.

200 Teilweise wird dennoch vertreten, bei beiden Absätzen gelte der gleiche Maßstab. (Thüsing, ZfA 2001, S. 397 (408); Zu beachten ist, daß Thüsing Art. 4 II mit $\S 611$ a I 2 BGB-A und der Richtlinie 76/207/EWG vergleicht, deren Maßstab aber dem des Art. 4 I Richtlinie 2000/78/EG entsprechen soll.).

201 Hervorhebung durch den Verfasser. 
Hinsichtlich des Verhältnisses der verschiedenen Begriffe wurde gesagt, der Wortlaut des $§ 611$ a I 2 BGB-A sei insofern zwar „etwas strenger“. ${ }^{202}$ Inhaltlich sollte indes kein Unterschied zu den Richtlinien bestehen, da die Merkmale „wesentlich und entscheidend“ im Sinne von „unverzichtbar“ auszulegen seien. ${ }^{203}$

In bezug auf das „Unverzichtbarkeitserfordernis“ in § 611 a I 2 BGB-A ist darauf hinzuweisen, daß es angelehnt war an das Erfordernis der ,unabdingbaren Voraussetzung“, das die Richtlinie 76/207/EWG in ihrer ursprünglichen Fassung ${ }^{204}$ in Art. 2 II statuierte. ${ }^{205}$ Auch bezüglich des Merkmals der unabdingbaren Voraussetzung wurde vertreten, es entspräche funktional dem Erfordernis der wesentlichen und entscheidenden beruflichen Anforderung. ${ }^{206}$

Der Entwurf eines Gesetzes zur Umsetzung europäischer Antidiskriminierungsrichtlinien vom 16.12.2004 ${ }^{207}$ unterschied noch zwischen ,unverzichtbarer Voraussetzung“ - dies sollte gem. § 8 I Nr. 1 ADG-E-2004 für das Diskriminierungsmerkmal „Geschlecht“ gelten - und ,,wesentlicher und entscheidender beruflichen Anforderung“ - dies sollte gem. § 8 I Nr. 2 ADG-E-2004 für alle übrigen Merkmale des $\S 1$ ADG-E-2004 gelten. Begründet wurde diese Differenzierung damit, daß „,bei der Umsetzung der Richtlinien bereits bestehende Vorschriften nicht verschlechtert werden dürfen.“208 Eine Verschlechterung des Diskriminierungsschutzes wurde dabei angesichts der Existenz des $§ 611$ a I 2 BGB-A für das Merkmal „Geschlecht“ befürchtet.

Der Gesetzgeber erblickte demnach einen graduellen Unterschied zwischen „unverzichtbar“ und „wesentlich und entscheidend“. Daß eine sinnvolle Differenzierung zwischen beiden Merkmalen indes nicht möglich ist, hat schließlich auch er erkannt. Dementsprechend unterscheiden $\S 8$ I ADGE-2005 sowie $\S 8$ I AGG nicht mehr zwischen „unverzichtbar“ einerseits und „wesentlich und entscheidend“ andererseits. In der Entwurfsbegründung zum AGG heißt es dazu: „Eine Absenkung des Schutzstandards hinsichtlich des Merkmals Geschlecht ist damit nicht verbunden.“209

Thüsing, ZfA 2001, S. 397 (400); in diesem Sinne auch Coen, nach dem das Merkmal „,wesentliche und entscheidende berufliche Voraussetzung" eng ausgelegt werden, eine Ungleichbehandlung nur dann zulässig sein soll, wenn sie zur Ausführung der betreffenden Tätigkeit unbedingt notwendig ist. (Coen, AuR 2000, S. 11 (11)).

204 Das heißt vor ihrer Änderung durch die Richtlinie 2002/73/EG.

205 Abl. EG 1976 Nr. L 39, S. 40 ff.

206 Thüsing vergleicht die Richtlinie 76/207/EWG in ihrer ursprünglichen Fassung mit der Richtlinie 2000/43/EG. (Thüsing, NZA 2001, S. 1061 (1061 f.).

207 BT-Druck. 15/4538.

208 Entwurfsbegründung, BT-Druck. 15/4538, S. 32; Die Richtlinien bestimmen, daß deren Umsetzung nicht als Rechtfertigung für eine Absenkung des von den Mitgliedstaaten bereits garantierten Schutzniveaus in bezug auf Diskriminierungen in den von den Richtlinien abgedeckten Bereichen benutzt werden darf. (vgl. dazu Art. 6 II Richtlinie 2000/43/EG; Art. 8 II Richtlinie 2000/78/EG; Art. 8 e II Richtlinie 76/207/EWG; Art. 7 II Richtlinie 2004/113/EG).

209 Entwurfsbegründung, BT-Druck. 16/1780, S. 35. 
Nachdem auf die Wortbedeutung der Begriffe „,wesentlich“ und „entscheidend“ und ihr Verhältnis zueinander eingegangen wurde, soll im folgenden ihre rechtliche Dimension beleuchtet werden, die nicht einfach zu bestimmen sein dürfte. Ein diese Vermutung stützendes Indiz bildet die Tatsache, daß sich im Rahmen des $\S 611$ a I 2 BGB-A auch nach 20 Jahren Rechtsprechungspraxis eine allgemein anerkannte Definition dafür, wann ein bestimmtes Geschlecht unverzichtbar war, noch nicht herausgebildet hatte. ${ }^{210}$

$\mathrm{Da}$ - wie sich gezeigt hat - zwischen ,wesentliche und entscheidende berufliche Anforderung“ in $\S$ 8 I AGG und „unverzichtbare Voraussetzung“ in § 611 a I 2 BGB-A kein inhaltlicher Unterschied besteht, kann zur Beantwortung der aufgeworfenen Frage im folgenden auf die umfangreiche Rechtsprechung und Kommentierung zu $§ 611$ a I 2 BGB-A zurückgegriffen werden. ${ }^{211}$

\section{Diskriminierungsmerkmal als objektive Anforderung}

Zweifelsohne stellt ein bestimmtes Geschlecht eine „wesentliche und entscheidende berufliche Anforderung“ im Sinne des § 8 I AGG sowie des Art. 2 VI Richtlinie 76/207/EWG dar, wenn aufgrund von Naturgesetzen oder nach dem Stand von Wissenschaft und Technik die jeweilige Tätigkeit nur von einem Geschlecht durchgeführt werden kann (im folgenden objektive Anforderung). ${ }^{212}$

Beispiel: Männer sind biologisch bedingt nicht in der Lage, die Tätigkeit einer Amme auszuüben. ${ }^{213}$

Um zu überprüfen, ob eine naturgesetzliche bzw. biologische Notwendigkeit besteht, wird vorgeschlagen, die Testfrage zu stellen, ob der Arbeitgeber den Arbeitsplatz dauerhaft unbesetzt gelassen hätte, wenn einzig und allein Bewerber des unerwünschten Geschlechts zur Verfügung gestanden hätten. ${ }^{214}$ Diese Frage ist in dem Beispielsfall zweifelsohne zu bejahen.

Die Ausführungen zur objektiven Anforderung gelten natürlich nicht nur für das Diskriminierungsmerkmal Geschlecht und das Arbeitsrecht, sondern für alle Diskriminierungsmerkmale und sämtliche denkbaren Rechtsverhältnisse.

210 Thüsing, RdA 2001, S. 319 (319).

211 Derzeit - Anfang 2007 - existiert zum AGG selbst (noch) keine umfangreiche Rechtsprechung und Kommentierung.

212 Die Überlegungen zur „,naturgesetzlichen Unmöglichkeit“ werden auf die naturgesetzliche (objektive) Anforderung übertragen. (vgl. zu ersterer Heinrichs in Palandt, § 275, Rn. 14).

213 Adomeit, für den das Merkmal der Unverzichtbarkeit i. S. d. § 611 a I 2 BGB-A streng genommen nie erfüllt war, machte einzig und allein bei der Amme eine Ausnahme. (Adomeit, DB 1980, S. 2388 (2388)); ihm zustimmend Richardi/Annu $\beta$ in Staudinger, $\S 611$ a, Rn. 52; Auch Preis war der Auffassung, daß streng genommen nur bei dieser Tätigkeit ein bestimmtes Geschlecht unverzichtbar sei. (Preis, Arbeitsrecht, S. 343). Gemäß $\S 611$ a I 2 BGB-A war ,,[e]ine unterschiedliche Behandlung wegen des Geschlechts [...] zulässig, soweit [...] ein bestimmtes Geschlecht unverzichtbare Voraussetzung für diese Tätigkeit [war].“ (Hervorhebung durch den Verfasser).

214 Thüsing, RdA 2001, S. 319 (322). 
Im Zusammenhang mit der Richtlinie 2000/43/EG wird teilweise jedoch das Gegenteil behauptet, daß nämlich das Merkmal der Rasse oder ethnischen Herkunft außerhalb des Arbeitsrechts niemals unverzichtbar sei. Begründet wird dies damit, die Richtlinie biete für eine andere Sichtweise keinen Raum, da zum einen Art. 4 der Richtlinie nur für den Bereich des Arbeitsrechts gelte und sich zum anderen die Ausnahmevorschrift des Art. 2 II lit. b nur auf mittelbare Diskriminierungen beziehe. ${ }^{215}$ Dieses Argument ist stichhaltig. Allein die Vorgabe der Richtlinie vermag in diesem Punkt nicht zu überzeugen. Daß sich außerhalb des Arbeitsrechts kaum bzw. keine Fälle bilden lassen, in denen eine bestimmte Rasse bzw. ethnische Herkunft eine ,wesentliche und entscheidende [...]

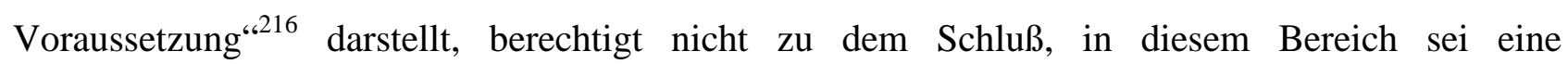
unterschiedliche Behandlung aufgrund jener Merkmale nie gerechtfertigt, d.h. stets verboten. ${ }^{217}$ Teilweise wird in diesem Zusammenhang auch als sinnwidrig angesehen, daß in einem Bereich, in dem ein stärkerer Schutz erforderlich sei - gemeint ist das Arbeitsrecht -, eine Ungleichbehandlung eher zulässig sein soll, als bei der Dienst- und Güterversorgung. ${ }^{218}$

Auch außerhalb des Arbeitsrechts muß eine unterschiedliche Behandlung aufgrund der Rasse bzw. ethnischen Herkunft zulässig sein, soweit diese Merkmale wesentlich und entscheidend für das in Rede stehende Vertragsverhältnis sind. ${ }^{219}$ Unterstützt wird diese Sichtweise durch die Richtlinie 2004/113/EG, deren sachlicher Geltungsbereich nicht den Bereich des Arbeitsrechts umfaßt und dennoch in Art. $4 \mathrm{~V}$ bestimmt, daß eine Ungleichbehandlung aufgrund des Geschlechts unter bestimmten Voraussetzungen gerechtfertigt sein kann.

In dem beschriebenen Sinne objektiv wesentlich und entscheidend ist ein Merkmal, wie sich gezeigt hat, nur in wenigen Fällen. ${ }^{220} \S 8$ I AGG und Art. 2 VI Richtlinie 76/207/EWG sind indes nicht auf diese wenigen Fälle begrenzt.

\section{Diskriminierungsmerkmal als rechtliche Anforderung}

Wesentlich und entscheidend im Sinne des § 8 I AGG sowie des Art. 2 VI Richtlinie 76/207/EWG ist ein bestimmtes Geschlecht vielmehr auch dann, wenn es das Gesetz für die Durchführung einer

215 Nickel, NJW 2001, S. 2668 (2671).

216 So der Wortlaut der Richtlinie; Nickel verwendet dagegen in Anlehnung an $\S 611$ a I 2 BGB-A den Begriff „unverzichtbar“. Es scheint, als differenziere er nicht zwischen „wesentlich und entscheidend“ und „unverzichtbar“. In diesem Sinne auch Thüsing, ZfA 2001, S. 397 (400) sowie Coen, nach dem das Merkmal „wesentliche und entscheidende berufliche Voraussetzung“ eng ausgelegt werden, eine Ungleichbehandlung nur dann zulässig sein soll, wenn sie zur Ausführung der betreffenden Tätigkeit unbedingt notwendig ist. (Coen, AuR 2000, S. $11(11)$ ).

217 So aber Nickel, der den Inhalt der Richtlinie 2000/43/EG wiedergibt, ohne ihn kritisch zu hinterfragen (Nickel, NJW 2001, S. 2668 (2671)).

218 Wiedemann/Thüsing, DB 2002, S. 463 (468); Die Autoren beziehen ihre Kritik auf den ADG-Entwurf des Bundesjustizministeriums vom 10.12.2001.

219 Zu der kritikwürdigen Umsetzung der Richtlinienvorgabe in $\S 20$ AGG vgl. Teil 4, Prüfungspunkt B. I. 4. a).

220 So auch Thüsing, NZA 2001, S. 1061 (1061); ebenso Nickel in bezug auf das Unverzichtbarkeitserfordernis i. S. d. $\S 611$ a I 2 BGB-A (Nickel, Gleichheit und Differenz in der vielfältigen Republik, S. 191 f.). 
Tätigkeit vorschreibt. Die Kommentierung zu $§ 611$ a BGB-A kann insofern 1 : 1 übernommen werden. $^{221}$ Ein Diskriminierungsverbot kann schließlich nicht $\mathrm{zu}$ etwas verpflichten, daß aus anderen Gründen rechtlich verboten ist. Diese Feststellung gilt freilich für alle Rechtsverhältnisse und sämtliche Diskriminierungsmerkmale.

\section{Beschäftigungsverbote}

Das deutsche Arbeitsrecht kennt Vorschriften, die dem Arbeitgeber untersagen, bestimmte Personen in gewissen Situationen zu beschäftigen.

Beispiele: Beschäftigungsverbot gem. $\S 3$ ff. MuSchG bzgl. der (werdenden) Mütter; ${ }^{222}$ Abschlußverbot gem. $§ 5$ I ArbSchG bzgl. Kinder ${ }^{223}$

\section{Fördermaßnahmen}

Maßnahmen, die die Förderung von Trägern bestimmter Merkmale bezwecken, können dazu verpflichten, diese Personen bevorzugt gegenüber Nicht-Merkmalsträgern zu behandeln.

\section{a) Arten von Fördermaßnahmen}

In einer Stellenanzeige der Universität Göttingen vom 29.03.2004, in der ein wissenschaftlicher Mitarbeiter gesucht wird, findet sich folgender Zusatz:

„In diesem Arbeitsbereich sind Frauen an der Universität unterrepräsentiert. Deshalb sind Bewerbungen von Frauen besonders willkommen und werden bei entsprechender Qualifikation im Rahmen der rechtlichen Möglichkeiten mit Vorrang berücksichtigt. Bei gleicher Eignung werden bei der Auswahl Schwerbehinderte bevorzugt.“

\section{aa) Relative Quoten}

Das Bundesgleichstellungsgesetz (BGleiG) ${ }^{224}$ vom 30.11.2001 enthält in seinem $§ 8$ Satz 1 eine einzelfallbezogene relative Quotenregelung. Die Bestimmung lautet wie folgt:

221 Putzo in Palandt, $\S 611$ a, Rn. 12; Thüsing, RdA 2001, S. 319 (322); Richardi/Annuß in Staudinger, $\S 611$ a, Rn. 53.

222 Beschäftigungsverbote haben keinen Einfluß auf die Wirksamkeit des abgeschlossenen Arbeitsvertrages. (Junker, Grundkurs Arbeitsrecht, Rn. 179).

223 Abschlußverbote führen dazu, daß verbotswidrig abgeschlossene Verträge nichtig sind. (Junker, Grundkurs Arbeitsrecht, Rn. 179).

224 BGBl. 2001 I, S. 3234 ff. 
„Sind Frauen in einzelnen Bereichen unterrepräsentiert, hat die Dienststelle sie bei der Vergabe von Ausbildungsplätzen, Einstellung, Anstellung und beruflichem Aufstieg bei Vorliegen von gleicher Eignung, Befähigung und fachlicher Leistung (Qualifikation) bevorzugt zu berücksichtigen, sofern nicht in der Person eines Mitbewerbers liegende Gründe überwiegen.“

$\S 8$ Satz 1 BGleiG enthält eine relative Quotenregelung, weil nach ihr eine Bevorzugung der Frau nur dann in Betracht kommt, wenn beide Geschlechter gleichqualifiziert sind. ${ }^{225}$ Einzelfallbezogen ist sie deshalb, weil die Frau bei gleicher Eignung nicht per se bevorzugt wird. Der Zusatz in der eingangs erwähnten Stellenanzeige stellt in bezug auf das Diskriminierungsmerkmal „Geschlecht“ eine derartige einzelfallbezogene relative Quotenregelung dar.

Beachtet werden muß, daß das BGleiG die Gleichstellung von Frauen und Männern in der Bundesverwaltung und in den Gerichten des Bundes betrifft. Quotenregelungen in bezug auf das Geschlecht existieren bislang nur im öffentlichen Dienst, nicht dagegen im Privatrecht. ${ }^{226}$

\section{bb) Absolute Quoten}

Eine andere Rechtslage ist in bezug auf das Merkmal „Schwerbehinderung“ gegeben. Gemäß § 71 I SGB IX haben private und öffentliche Arbeitgeber

„[...] mit jahresdurchschnittlich monatlich mindestens 20 Arbeitsplätzen im Sinne des $\S 73$ [...] auf wenigstens 5 Prozent der Arbeitsplätze schwerbehinderte Menschen zu beschäftigen. Dabei sind schwerbehinderte Frauen besonders zu berücksichtigen. Abweichend von Satz 1 haben Arbeitgeber mit jahresdurchschnittlich monatlich weniger als 40 Arbeitsplätzen jahresdurchschnittlich je Monat einen schwerbehinderten Menschen, Arbeitgeber mit jahresdurchschnittlich monatlich weniger als 60 Arbeitsplätzen jahresdurchschnittlich je Monat zwei schwerbehinderte Menschen zu beschäftigen. ‘227

§ 71 I SGB IX enthält eine absolute Quotenregelung, die der Arbeitgeber unabhängig davon, ob er tatsächlich die Möglichkeit hat, den Schwerbehinderten in seinem Betrieb zu beschäftigen, zu erfüllen verpflichtet ist. ${ }^{228}$ Auch ist ohne Bedeutung, ob im Betrieb ein Arbeitsplatz für den Schwerbehinderten frei ist oder nicht. ${ }^{229}$ Ist ein solcher nicht vorhanden, muß der Arbeitgeber gegebenenfalls einen neuen Arbeitsplatz schaffen. ${ }^{230}$ Darüber hinaus wird teilweise vertreten, die

225 Definition des Begriffs der relativen Quotenregelung in Abgrenzung zur absoluten Quotenregelung unter http://de.wikipedia.org/wiki/Frauenquote.

226 Hanau in Gedächtnisschrift für Lüderitz, S. 241 (241); Nach Ansicht Starcks wären Frauenquoten im Privatrechtsverkehr mit der Vertragsfreiheit auch nicht vereinbar. (Starck in von Mangoldt/Klein/Starck, Art. 3 Abs. 2, Rn. 310).

227 Säcker empfiehlt, analog zu den $§ \S 71$ ff. SGB IX den Schutz Behinderter etwa im Mietrecht und im Bildungsrecht $\mathrm{zu}$ regeln. Wer beispielsweise 20 Wohnungen oder mehr vermiete, müsse davon mindestens $5 \%$ behindertengerecht ausbauen und anbieten. (Säcker, ZRP 2002, S. 286 (290)).

228 Neumann in Neumann/Pahlen/Majerski-Pahlen, $\S 71$, Rn. 7; Kossens in Kossens/von der Heide/Maaß, $§ 71$, Rn. 6.

229 Neumann in Neumann/Pahlen/Majerski-Pahlen, § 71, Rn. 7; Kossens in Kossens/von der Heide/Maaß, § 71, Rn. 4.

230 Neumann in Neumann/Pahlen/Majerski-Pahlen, $§ 71$, Rn. 7. 
Beschäftigungspflicht des $§ 71$ I SGB IX rechtfertige die Kündigung eines anderen Arbeitnehmers, um auf diese Weise einen Arbeitsplatz für den Schwerbehinderten freizumachen. ${ }^{231}$ Der Arbeitgeber kann sich grundsätzlich nicht mit dem Hinweis auf mangelnde finanzielle Leistungsfähigkeit von seiner Beschäftigungspflicht befreien. ${ }^{232}$ Eine Ausnahme soll insofern bei Unzumutbarkeit oder Unverhältnismäßigkeit der Aufwendungen gelten. ${ }^{233}$ Schließlich ist der Hinweis des Arbeitgebers, in seinem Betrieb könnten nur besonders qualifizierte Arbeitnehmer beschäftigt werden, unbeachtlich. ${ }^{234}$ Der Arbeitgeber hat vielmehr geeignete Arbeitsplätze zu schaffen oder entsprechende Qualifizierungsmaßnahmen anzubieten. ${ }^{235}$

Vor dem Hintergrund der Ausführungen zu § 71 I SGB IX stellt sich die Frage der Rechtmäßigkeit des in der Stellenanzeige enthaltenen Zusatzes: „Bei gleicher Eignung werden bei der Auswahl Schwerbehinderte bevorzugt.“ Der Zusatz stellt im Gegensatz zu jener Norm eine einzelfallunabhängige relative Quotenregelung dar. Die Stellenanzeige widerspricht damit gleichwohl nicht den gesetzlichen Vorgaben des § 71 I SGB IX. Jene Norm verpflichtet die in ihren Geltungsbereich fallenden Arbeitgeber lediglich dazu, in bestimmtem Umfang Schwerbehinderte zu beschäftigen und $\mathrm{zu}$ diesem Zweck geeignete Arbeitsplätze $\mathrm{zu}$ schaffen. Ein Gebot, schwerbehinderte Bewerber auch für solche Tätigkeiten einzustellen, für die sie nicht qualifiziert sind, kann daraus nicht abgeleitet werden. Der Passus in der Stellenanzeige ist demnach nicht zu kritisieren.

Die bisherigen Ausführungen haben gezeigt, daß es Bereiche gibt, in denen der Gesetzgeber den Arbeitgeber verpflichtet, Menschen mit bestimmten Merkmalen unter bestimmten Voraussetzungen bevorzugt zu behandeln. Problematisch an derartigen Fördermaßnahmen ${ }^{236}$ ist die Tatsache, daß sie ihrerseits zu einer „Diskriminierung“ der Personen führen, die nicht Adressaten selbiger Maßnahmen sind. ${ }^{237}$ Darauf hat auch das BVerfG hingewiesen, indem es formuliert hat: ,Einzelne Gruppen fördern heißt bereits, andere ungleich behandeln.“ ${ }^{‘ 238}$ Angesichts der hehren Ziele, die derartige Fördermaßnahmen verfolgen, spricht man in diesem Zusammenhang von ,positiver Diskriminierung.“239

231 Neumann in Neumann/Pahlen/Majerski-Pahlen, $\S 71$, Rn. 7, der auf die a. A. verweist.

232 Kossens in Kossens/von der Heide/Maaß, § 71, Rn. 6.

233 Neumann in Neumann/Pahlen/Majerski-Pahlen, § 71, Rn. 7.

234 Neumann in Neumann/Pahlen/Majerski-Pahlen, § 71, Rn. 7.

235 Kossens in Kossens/von der Heide/Maßß, § 71, Rn. 6; An der Verfassungsmäßigkeit des § 71 I SGB IX sollen trotz der durch ihn verursachten erheblichen Beschränkung der Arbeitgeberrechte keine ernsthaften Zweifel unter den Gesichtspunkten des Verhältnismäßigkeitsprinzips sowie des Übermaßverbotes bestehen. (Kossens in Kossens/von der Heide/Maaß, § 71, Rn. 3).

236 In Anlehnung an das U.S.-amerikanische Recht ist in diesem Zusammenhang teilweise auch von ,,affirmative action“ die Rede. (Nickel, Gleichheit und Differenz in der vielfältigen Republik, S. 164, Fn. 618; Thüsing, ZfA 2001, S. 397 (416)).

237 so auch Nickel, Gleichheit und Differenz in der vielfältigen Republik, S. $163 \mathrm{f}$.

238 BVerfGE 12, S. 354 (367).

239 Wollenschläger, ZAR 1994, S. 10 (11); Nickel verwendet den Begriff der ,umgekehrten Diskriminierung“. (Nickel, Gleichheit und Differenz in der vielfältigen Republik, S. 164). 
Da es der Gesetzgeber ist, der durch Einführung von Quotenregelungen den Arbeitgeber in den genannten Fällen verpflichtet, ungleich zu behandeln, kann der durch die jeweilige Regelung benachteiligte Bewerber bzw. Arbeitnehmer nicht geltend machen, er sei vom Arbeitgeber diskriminiert worden. Die Quotenregelung führt dazu, daß in ihrem Geltungsbereich das geförderte Merkmal rechtlich unverzichtbare Voraussetzung ist.

\section{cc) Nichtdiskriminierende Fördermaßnahmen}

Anders als im Falle der soeben diskutierten Quotenregelungen gibt es Fördermaßnahmen, die Träger eines bestimmten Merkmals fördern, ohne diejenigen zu benachteiligen, die nicht Träger dieses Merkmals sind. Wenn etwa der Unternehmer verpflichtet wird, in seinem Unternehmen eine Rampe für Rollstuhlfahrer zu schaffen, dann hat dies keinerlei diskriminierenden Einfluß auf die nichtbehinderte Belegschaft.

Derartige Fördermaßnahmen spielen unter dem Prüfungspunkt „,rechtliche Unverzichtbarkeit“ keine Rolle, da sie nicht zur Ungleichbehandlung der nicht geförderten Personen verpflichten. Das ist der Grund dafür, weshalb an dieser Stelle nicht näher auf besagte Fördermaßnahmen eingegangen wird. $^{240}$

Im folgenden soll in einem Exkurs auf das Spannungsverhältnis zwischen den Begriffen „Diskriminierungsschutz“ und „positive Diskriminierung“ eingegangen werden. ${ }^{241}$ Im Kern stellt sich die Frage, ob sich das Institut der positiven Diskriminierung überhaupt in ein Antidiskriminierungskonzept eingliedern läßt.

\section{b) Exkurs: Zulässigkeit ,positiver Diskriminierung““}

Für die Beantwortung dieser Frage ist es erforderlich, sich die Ziele zu markieren, die Antidiskriminierungsmaßnahmen erreichen sollen. Konkret geht es um die Frage, welche Gleichheit selbige Maßnahmen anstreben (sollten).

Der „Gleichbehandlungsansatz“ entspricht - vergleichbar mit dem Recht auf „Gleichheit vor dem Gesetz $^{، 242}$ - der Forderung nach formaler Rechtsgleichheit, basiert also auf der Annahme, daß

240 Sie erlangen an anderer Stelle und in anderem Zusammenhang Bedeutung. (vgl. dazu Teil 3, Prüfungspunkte N. und O.).

241 In einem Exkurs deshalb, weil hier der Frage nachgegangen werden soll, ob der Gesetzgeber derartige Fördermaßnahmen treffen darf. Im Rahmen des Prüfungspunktes ,rechtliche Unverzichtbarkeit“ stellt sich demgegenüber wie im Rahmen der gesamten Arbeit die Frage, ob ein bestimmtes Verhalten des Privaten diskriminierend ist.

242 Also dem formellen Anspruch, daß bestehende Gesetze auf alle Rechtsunterworfenen in gleicher Weise angewendet werden müssen. 
allein schon die Verpflichtung zu gleicher Behandlung der Individuen zu gerechten Ergebnissen führen wird. $^{243}$

Nach dem Gleichbehandlungsansatz ist eine mittelbare Diskriminierung nicht möglich, setzt sie doch definitionsgemäß voraus, daß der Diskriminierende formal gleich behandelt. Auch können Fördermaßnahmen zugunsten bestimmter Gruppen nach dem Gleichbehandlungsansatz nicht ergriffen werden.

Der „Ansatz von den gleichen Startchancen“ verlangt demgegenüber, bestehende strukturelle Ungleichheiten zwischen einzelnen Personengruppen durch Fördermaßnahmen und besondere Unterstützung auszugleichen, um so für gleiche Zugangschancen im Wettbewerb um gesellschaftliche Güter zu sorgen. ${ }^{244}$

Diesem Ansatz folgend stellt Art. 5 Richtlinie 2000/43/EG klar, daß der Gleichbehandlungsgrundsatz die Mitgliedstaaten nicht daran hindert,

„,zur Gewährleistung der vollen Gleichstellung in der Praxis spezifische Maßnahmen, mit denen Benachteiligungen aufgrund der Rasse oder ethnischen Herkunft verhindert oder ausgeglichen werden, beizubehalten oder zu beschließen. “245

Die Richtlinie spricht in Art. 5 von Benachteiligungen, nicht von Ungleichbehandlung oder Diskriminierung. Die Verwendung dieses Begriffs deutet darauf hin, daß die Norm in erster Linie nicht die Bekämpfung aktueller Diskriminierung, sondern vielmehr die Überwindung historisch bedingter Unterschiede aufgrund in der Vergangenheit erfolgter Diskriminierung bezweckt. ${ }^{246}$ Dementsprechend seien nach der Rechtsprechung des U.S. Supreme Court Fördermaßnahmen nur dann erlaubt, wenn sie ein geeignetes Mittel darstellten, in der Vergangenheit eingetretene Benachteiligungen durch den Arbeitgeber zu kompensieren, und hierfür angemessen sind. ${ }^{247}$ Zudem dürfe nach U.S.-amerikanischem Recht der Arbeitgeber nur dann bestimmte Arbeitnehmer bevorzugen, wenn Anhaltspunkte dafür bestünden, daß die Gruppe, der der Arbeitnehmer angehört, gerade durch den konkreten Arbeitgeber oder zumindest durch dessen Branche in der Vergangenheit benachteiligt wurde. ${ }^{248}$

Nickel, Gleichheit und Differenz in der vielfältigen Republik, S. 162.

Nickel, Gleichheit und Differenz in der vielfältigen Republik, S. 163.

Vergleiche auch Art. 7 Richtlinie 2000/78/EG; Art. 2 VIII Richtlinie 76/207/EWG; Art. 6 Richtlinie 2004/113/EG sowie Art. 141 IV EGV.

In diesem Sinne auch Thüsing, ZfA 2001, S. 397 (415).

Thüsing, ZfA 2001, S. 397 (416).

Thüsing, ZfA 2001, S. 397 (417). 
Beklagenswert an der Formulierung des Art. 5 Richtlinie 2000/43/EG ist, daß aus ihr nicht hervorgeht, ob und in welchem Umfang die Bestimmung positive Diskriminierung legitimiert. ${ }^{249}$

Das Konzept der „Ergebnisgleichheit“ geht noch einen Schritt weiter, indem es verlangt, daß alle Minderheitengruppen entsprechend ihrem Anteil an der Gesamtbevölkerung auf allen gesellschaftlichen Hierarchieebenen und in allen Bereichen vertreten sind, so daß eine Diskriminierung immer schon dann bejaht werden muß, wenn in irgendeinem Bereich - unabhängig vom jeweiligen Grund - eine ungleiche Verteilung besteht. ${ }^{250}$

Folge dieses Konzepts sind absolute Quoten. Es leuchtet unmittelbar ein, daß sich absolute Quoten im Privatrechtsverkehr nur ausnahmsweise rechtfertigen lassen. In diesem Zusammenhang kann angemerkt werden, daß hinsichtlich der absoluten Quotenregelung des § 71 I SGB IX vertreten wird, an der Verfassungsmäßigkeit dieser Bestimmung bestünden in bezug auf den allgemeinen Gleichheitssatz keine ernsthaften Zweifel. ${ }^{251}$

Im Zusammenhang mit den Fördermaßnahmen, deren Einführung bzw. Beibehaltung die Richtlinien erlauben, wird moniert, die Richtlinien ließen offen, wie sich rechtliche Gleichbehandlung und tatsächliche Ergebnisgleichheit zueinander verhielten. Die Auflösung dieses Spannungsverhältnisses sei aber notwendig, müsse sich doch auch die Bevorzugung grundsätzlich am Gleichheitssatz messen lassen. ${ }^{252}$

In diesem Sinne wird auch im Rahmen des Art. 3 II GG argumentiert, der die Möglichkeit der Frauenförderung vorsieht: ${ }^{253}$ Wenn die Chancengleichheit das Verfassungsziel ist, dürfe selbige nicht hintangestellt werden, um Chancengleichheit zu erreichen. ${ }^{254}$

Ähnlich argumentiert das BVerfG: ,auch das Sozialstaatsprinzip ermächtigt nicht zu beliebiger Sozialgestaltung, die das Gebot der Gleichheit auflösen würde.“255

Den Konflikt zwischen rechtlicher und faktischer Gleichheit hat auch der $E u G H$ gesehen und entschieden, daß europäisches Recht einer nationalen (Quoten-)Regelung entgegensteht, ,nach der ein Bewerber des unterrepräsentierten Geschlechts um eine Stelle im Staatsdienst, der hinreichende Qualifikationen für diese Stelle besitzt, vor einem Bewerber des anderen Geschlechts, der [aufgrund besser Qualifikation] sonst ausgewählt worden wäre, auszuwählen ist, [...].“256 Das Gericht hält

249 [2000/43/EG und 2000/78/EG] wieder kein dogmatisches Konzept der Förderung von diskriminierten Gruppen erkennen läßt [...].“ (Thüsing, ZfA 2001, S. 397 (417)).

250 Nickel, Gleichheit und Differenz in der vielfältigen Republik, S. 163.

251 Kossens in Kossens/von der Heide/Maaß, § 71, Rn. 3.

252 Thüsing, ZfA 2001, S. 397 (415).

253 Art. 3 II GG berechtigt den Gesetzgeber, faktische Nachteile, die typischerweise Frauen treffen, durch begünstigende Regelungen auszugleichen. (BVerfGE 92, S. 91 (109); 85, S. 191 (207)).

254 Rüfner in Bonner Kommentar, Art. 3 Abs. 2 und 3, Rn. 765.

255 BVerfGE 12, S. 354 (367).

256 EuGH, NJW 2000, S. 2653 (2656, Rn. 56). 
dagegen eine Regelung mit europäischem Recht für vereinbar, „nach der ein Bewerber des unterrepräsentierten Geschlechts einem Bewerber des anderen Geschlechts vorgezogen werden kann, wenn die Verdienste der Bewerber als gleichwertig oder fast gleichwertig anzusehen sind, [...], sofern die Bewerbungen Gegenstand einer objektiven Beurteilung sind, bei der die besondere persönliche Lage aller Bewerber berücksichtigt wird. ‘257

Der eingangs erwähnte $\S 8$ Satz 1 BGleiG berücksichtigt durch seine einzelfallbezogene relative Quotenregelung die Vorgaben, die der EuGH an die Zulässigkeit von Frauenquoten im öffentlichen Dienst stellt.

Im Zusammenhang mit der ebenfalls bereits angesprochenen Quotenregelung des $\S 71$ SGB IX wird darauf hingewiesen, Quotenschutz könne zwar ebenso effektiv sein wie Diskriminierungsschutz, sei jenem aber insofern unterlegen, als er dem einzelnen keinen Schutz mehr bieten kann, wenn die Quote bereits erfüllt ist oder es sich um ein Unternehmen handelt, in dem mangels hinreichender Arbeitnehmerzahl das Einstellungsgebot nicht gilt. ${ }^{258}$ Aus diesem Grund wird ein Perspektivwechsel von der Quote hin zum Diskriminierungsschutz gefordert. ${ }^{259}$

Ein solcher Perspektivwechsel erscheint wünschenswert, vergegenwärtigt man sich den sozialen Zündstoff, den Fördermaßnahmen in sich bergen. ${ }^{260}$ Erinnert sei in diesem Zusammenhang an die abwertende Bezeichnung als „Quotenfrau““. ${ }^{261}$ Es wird gemutmaßt, daß Fördermaßnahmen gerade im Kontext von Privatrechtsbeziehungen mit hohen sozialen Kosten verbunden sein würden. ${ }^{262}$

\section{c) Resümee}

Zusammenfassend können folgende Feststellungen getroffen werden: Beschränkt man den Gleichbehandlungsgrundsatz nicht auf den Anspruch auf formale Gleichbehandlung, läßt sich das Institut der positiven Diskriminierung in ein Antidiskriminierungskonzept eingliedern, soweit es restriktiv angewandt wird. Das bedeutet, daß positive Diskriminierung als ultima ratio immer nur dann in Betracht kommt, wenn sich die Benachteiligung der jeweiligen Gruppe nicht schon durch die bloße Gewährung des Anspruchs auf formale Gleichbehandlung verhindern läßt. Die Frage der Zulässigkeit positiver Diskriminierung stellt sich damit für jedes Diskriminierungsmerkmal individuell. $^{263}$

260 Nickel sieht die Gefahr einer Re-Stigmatisierung der geförderten Gruppen. (Nickel, Gleichheit und Differenz in der vielfältigen Republik, S. 201, m.w.N. in Fn. 709; vgl. auch Bsp. S. 201).

261 Nickel, Gleichheit und Differenz in der vielfältigen Republik, S. 202.

262 Nickel, Gleichheit und Differenz in der vielfältigen Republik, S. 202.

263 So auch Nickel, Gleichheit und Differenz in der vielfältigen Republik, S. 164. 
Die Gewährung des Anspruchs auf formale Gleichbehandlung im Wege der Statuierung eines bloßen Diskriminierungsverbotes wird bei einem Großteil der Diskriminierungsmerkmale das gewünschte Ergebnis, Teilhabe statt Ausgrenzung vom gesellschaftlichen Leben, bewirken. ${ }^{264}$

Soweit Quotenregelungen nötig sind, um dieses Ziel erreichen zu können, müssen selbige als relative Quoten ausgestaltet sein. Absolute Quoten lassen sich im Privatrechtsverkehr nur ausnahmsweise rechtfertigen.

Vor der Einführung von Quotenregelungen ist $\mathrm{zu}$ prüfen, ob die angestrebte Teilhabe der benachteiligten Gruppe nicht eventuell dadurch erreicht werden kann, daß Fördermaßnahmen getroffen werden, die nicht zu positiver Diskriminierung führen. Erinnert sei insofern noch einmal an das Beispiel der Rampe für Rollstuhlfahrer.

\section{Diskriminierungsverbot und Sozialstaatsprinzip}

Es gibt im deutschen Arbeitsrecht eine Reihe von Regelungen, die den Arbeitgeber verpflichten oder ihm gestatten, das Alter des Arbeitnehmers zum Anknüpfungspunkt von Entscheidungen zu machen.

Als Beispiel für eine verpflichtende Regelung kann die „Sozialauswahl“ des $§ 1$ III 1 KSchG angeführt werden. Gemäß dieser Norm ist unter mehreren Arbeitnehmern zunächst demjenigen betriebsbedingt zu kündigen, der eine Kündigung unter sozialen Gesichtspunkten am ehesten verkraften kann, ${ }^{265}$ was im Regelfall zur Kündigung des jüngeren Arbeitnehmers führt.

Im Gegensatz dazu gibt es Bereiche, in denen dem Arbeitgeber gestattet ist, das Alter zum Anknüpfungspunkt von Entscheidungen $\mathrm{zu}$ machen. So können im Bereich von Sozialplanabfindungen i.S.d. $\S 112$ BetrVG rentennahe Arbeitnehmer, die durch den baldigen Bezug einer Rente und vorhergehendem Arbeitslosengeld in gewissem Umfang wirtschaftlich abgesichert sind, von Sozialplanleistungen ausgeschlossen werden. ${ }^{266}$

Gemäß $§ 41$ S. 2 SGB VI ist unter bestimmten Voraussetzungen eine Vereinbarung zwischen Arbeitgeber und Arbeitnehmer möglich, „die die Beendigung des Arbeitsverhältnisses [des] Arbeitnehmers ohne Kündigung zu einem Zeitpunkt vorsieht“, in dem der Arbeitnehmer ein Alter erreicht hat, das zur Beziehung von Altersrente berechtigt. Auch ist eine Kündigung bei Erreichen des Rentenalters möglich, wenn der Arbeitnehmer zu diesem Zeitpunkt ein gesetzliches

264 Das gelte beispielsweise für das Merkmal der sexuellen Identität. (Wiedemann/Thüsing, Der Betrieb 2002, S. 463 (468)).

265 Junker, Grundkurs Arbeitsrecht, S. 374.

266 Das $B A G$ hat festgestellt, daß eine derartige Regelung nicht gegen $\S 75$ BetrVG und insbesondere den arbeitsrechtlichen Gleichbehandlungsgrundsatz verstößt. (BAG, NZA 1997, S. 165 (166)); Bauer, NJW 2001 , S. 2672 (2673). 
Altersruhegeld beanspruchen kann und die Kündigung erfolgt, um einen vernünftigen Altersaufbau der Belegschaft zu erreichen und Aufstiegschancen innerhalb des Unternehmens zu eröffnen. ${ }^{267}$

$\S 14$ III Teilzeit- und Befristungsgesetz (TzBfG) gestattet dem Arbeitgeber schließlich in Ausnahme des $\S 14$ I TzBfG mit Arbeitnehmern, die das 58. Lebensjahr vollendet haben, befristete Arbeitsverträge auch dann abzuschließen, wenn für die Befristung kein sachlicher Grund vorliegt. $\mathrm{Zu}$ beachten ist, daß durch das Erste Gesetz für moderne Dienstleistungen am Arbeitsmarkt ${ }^{268}$ vom 23.12.2002 - besser bekannt als „Hartz I“ -, § 14 III TzBfG dergestalt modifiziert worden war, daß bis zum 31.12.2006 an die Stelle des 58. Lebensjahres das 52. Lebensjahr trat.

Schreibt der Gesetzgeber dem Arbeitgeber zwingend vor, das Alter zum Anknüpfungspunkt einer Entscheidung zu machen, so ist die daraufhin durch den Arbeitgeber vorgenommene Ungleichbehandlung aufgrund rechtlicher Unverzichtbarkeit gerechtfertigt.

Das gleiche gilt im Ergebnis auch für die Fälle, bei denen dem Arbeitgeber gestattet ist, nach dem Alter zu unterscheiden, auch wenn hier streng genommen nicht von rechtlicher Unverzichtbarkeit gesprochen werden kann. Entscheidend ist, daß dem Arbeitgeber auch in einem solchen Fall nicht der Vorwurf einer Diskriminierung gemacht werden kann, wenn er von den ihm eingeräumten Befugnissen Gebrauch macht.

Wie bereits bei den Fördermaßnahmen stellt sich auch hier die Frage, inwieweit sich die genannten Bestimmungen in ein Antidiskriminierungskonzept eingliedern lassen. Für ihre Beantwortung erlangt Art. 6 Richtlinie 2000/78/EG eine besondere Bedeutung. Gemäß seines Absatzes 1 Satz 1

„[...] können die Mitgliedstaaten vorsehen, daß Ungleichbehandlungen wegen des Alters keine Diskriminierung darstellen, sofern sie objektiv und angemessen sind und im Rahmen des nationalen Rechts durch ein legitimes Ziel, worunter insbesondere rechtmäßige Ziele aus den Bereichen Beschäftigungspolitik, Arbeitsmarkt und berufliche Bildung zu verstehen sind, gerechtfertigt sind und die Mittel zur Erreichung dieses Ziels angemessen und erforderlich sind."

Es wird vertreten, die Sozialauswahl des § 1 III 1 KSchG sei, wenn nicht durch Art. 6 gerechtfertigt - aus dem Wortlaut der Richtlinie geht nämlich nicht hervor, wer die geschützten Arbeitnehmer sind: „die älteren, die jüngeren oder beide?‘269 -, gleichwohl als Fördermaßnahme i.S.d. Art. 7 zulässig. ${ }^{270}$ 
Auch habe die übliche Praxis, rentennahe Arbeitnehmer von Sozialplanleistungen auszunehmen, mit Diskriminierung nichts $\mathrm{zu}$ tun, sondern entspringe vielmehr der ohne weiteres nachvollziehbaren Erkenntnis, ein Sozialplantopf sei nach sozialen Kriterien zu verteilen. ${ }^{271}$

Im Zusammenhang mit den Altersgrenzen im Sinne des $§ 41$ S. 2 SGB VI besteht die Auffassung, jeder Arbeitgeber habe ein berechtigtes Interesse an einer vernünftigen Altersstruktur seiner Belegschaft. ${ }^{272}$ Auch bestehe ein Allgemeininteresse an einer solchen Regelung, könne es doch nicht sein, daß der Eintritt jüngerer Menschen in den Arbeitsmarkt unnötig erschwert wird, was eintreten würde, wenn einerseits Altersgrenzen nicht mehr zulässig wären und andererseits der allgemeine Kündigungsschutz nach dem KSchG weiterhin „open end“ bliebe. ${ }^{273}$ Gestützt wird diese Ansicht durch Art. 6 I 2 lit. a Richtlinie 2000/78/EG, wonach Ungleichbehandlungen aufgrund des Alters dann keine Diskriminierung darstellen, wenn sie „die berufliche Eingliederung von Jugendlichen bezwecken“.

In bezug auf $\S 14$ III TzBfG wird vertreten, die Norm solle in Zukunft Bestand haben, falle sie doch in den Anwendungsbereich des Art. 6 I Richtlinie 2000/78/EG. ${ }^{274} \S 14$ III TzBfG bezwecke die Erhöhung der Einstellungschancen älterer Arbeitnehmer, die auf dem Arbeitsmarkt überwiegend schlechtere Chancen als jüngere Arbeitssuchende hätten. ${ }^{275}$ Teilweise wird die Norm indes abgelehnt. ${ }^{276}$ Nach $\S 14$ III TzBfG sind beliebig viele Kettenarbeitsverhältnisse zulässig, was, überspitzt formuliert, dazu führen könne, daß der einzelne sein Arbeitsleben als Tagelöhner beenden muß. ${ }^{277}$ Außerdem lasse sich die Regelung nicht mit der Richtlinie 1999/70/EG zu der EGB-UNICE-CEEP-Rahmenvereinbarung über befristete Arbeitsverträge ${ }^{278}$ vom 28.06.1999 vereinbaren, „die bei Kettenarbeitsverhältnissen entweder einen sachlichen Grund oder eine Höchstdauer oder eine Höchstzahl der Verlängerungen vorschreibt. “279 In einem Rechtsstreit vor dem EuGH hat der zuständige Generalanwalt die Ansicht vertreten, zumindest die Herabsetzung der Altersgrenze vom 58. auf das 52. Lebensjahr in $\S 14$ III TzBfG verstoße gegen die Richtlinie 2000/78/EG. ${ }^{280}$

Richtlinie "die Beschäftigungs- und Arbeitsbedingungen, einschließlich der Entlassungsbedingungen und des Arbeitsentgelts" umfaßt.

270 Thüsing, NZA 2001, S. 1061 (1064); im Ergebnis ebenso Bauer, der in diesem Zusammenhang allerdings weder Art. 6 noch Art. 7 der Richtlinie erwähnt. (Bauer, NJW 2001, S. 2672 (2673)); Teilweise wird indes befürchtet, die Antidiskriminierungsrichtlinien könnten bewirken, daß ,bei betriebsbedingten Kündigungen das Kriterium Alter (neben Betriebszugehörigkeit und Unterhaltspflichtigkeit) bei der Sozialauswahl entfallen“ muß. (Sattar zitiert das Vorstandsmitglied der IG-Metall Oberhofer in FAZ vom 16.11.2006, S. 4, unter dem Titel: „„,Auf falschem Bein hurra geschrien" - Drei Monate Gleichbehandlungsgesetz").

272 Ваuеr, NJW 2001, S. 2672 (2674).

273 Bauer, NJW 2001, S. 2672 (2674).

274 Bauer, NJW 2001, S. 2672 (2673).

275 Preis/Gotthardt, DB 2000, S. 2065 (2072); Bauer, NJW 2001, S. 2672 (2673).

276 Däubler, ZIP 2000, S. 1961 (1967); Däubler, ZIP 2001, S. 217 (224).

277 Däubler, ZIP 2000, S. 1961 (1967).

278 Abl. EG 1999, Nr. L 175, S. 43 ff.

279 Däubler, ZIP 2000, S. 1961 (1967).

280 FAZ vom 01.07.2005, S. 11 unter dem Titel: „,Hartz-Gesetz verstößt gegen EU-Recht“““. 
Anzumerken bleibt, daß für die Zulässigkeit einer Regelung, die an das Alter anknüpft, die Tatsache sprechen kann, daß in ihren Anwendungsbereich grundsätzlich jeder Mensch fallen kann - zudem teilweise lediglich temporär ${ }^{281}$-, da sich das Alter eines jeden Menschen ändert. Vor diesem Hintergrund ist etwa eine Regelung zu betrachten, die die Unkündbarkeit vom Erreichen eines bestimmten Alters und einer bestimmten Betriebszugehörigkeit abhängig macht. ${ }^{282}$

Um ein Verständnis dafür zu erlangen, in welchem Verhältnis die genannten Bestimmungen auf der einen Seite und das Verbot der Diskriminierung auf der anderen zueinander stehen, ist die Erkenntnis entscheidend, daß die Antidiskriminierungsrichtlinien eng an der anglo-amerikanischen Tradition des generellen Diskriminierungsverbots angelehnt sind. ${ }^{283}$ In den USA erfülle aufgrund der dort schwach ausgeprägten kollektiven Systeme der sozialen Sicherung und des Fehlens arbeitsrechtlicher Schutzvorschriften für den Arbeitnehmer, wie beispielsweise dem Kündigungsschutz, das generelle Diskriminierungsverbot in hohem Maße die Funktion des sozialen Schutzes desselben. ${ }^{284}$

Im Gegensatz dazu bestünden in Deutschland und den meisten EU-Mitgliedstaaten sehr hoch entwickelte Systeme der kollektiven sozialen Sicherung, was unter anderem dazu geführt habe, daß Personen nach sozialen Kriterien unterschiedlich behandelt werden (z.B. Sozialauswahl bei betriebsbedingten Kündigungen). ${ }^{285}$

Es wird vertreten, beide Institute könnten funktional vergleichbar eingesetzt werden und zu einer vergleichbaren Beschränkung der Flexibilität des Arbeitsverhältnisses führen. ${ }^{286}$ Ungleichbehandlungen, die aufgrund sozialer Gesichtspunkte vorgeschrieben sind bzw. für zulässig erklärt werden, verfolgen letztlich dasselbe, was Diskriminierungsschutz erreichen möchte. Bereits im Rahmen der Ausführungen $\mathrm{zu}$ den Fördermaßnahmen wurde festgestellt, daß Diskriminierungsschutz seinerseits nicht beschränkt ist auf das Gebot der formellen Gleichbehandlung.

Erklärte man mit Blick auf ein etwaig bestehendes Diskriminierungsverbot Regelungen für nichtig, die Ungleichbehandlungen aufgrund sozialer Gesichtspunkte vorschreiben bzw. für zulässig erklären, schaffte man etwas ab, „was gestern noch als soziale Errungenschaft gefeiert wurde““.287 der Regel nur vorrübergehend ist.“ (Nussberger, JZ 2002, S. 524 (526)).

282 Gemäß § 10 Satz 3 Nr. 7 AGG ist eine derartige Regelung zulässig, ,,soweit dadurch nicht der Kündigungsschutz anderer Beschäftigter im Rahmen der Sozialauswahl nach § 1 Abs. 3 des Kündigungsschutzgesetzes grob fehlerhaft gemindert wird“. 
Dem steht, wie das Beispiel des $§ 14$ III TzBfG gezeigt hat, nicht entgegen, daß beide Institute im Lichte des jeweils anderen betrachtet werden müssen. ${ }^{288}$

\section{Verstoß der nationalen Vorschrift gegen europäisches Recht}

Letztlich stellt sich im Rahmen der Prüfung der rechtlichen Unverzichtbarkeit die Frage, welche Konsequenz aus einem Verstoß der nationalen Regelung, die dem einzelnen vorschreibt oder gestattet, ein bestimmtes Merkmal zum Anknüpfungspunkt einer Entscheidung zu machen, gegen höherrangigeres Recht, vor allem gegen die Antidiskriminierungsrichtlinien, resultiert. ${ }^{289}$ Denkbar wäre in einem solchen Fall, dem von der Regelung negativ Betroffenen das Recht einzuräumen, sich unmittelbar auf die einschlägige Bestimmung der betreffenden Antidiskriminierungsrichtlinie zu berufen, seinen geltend gemachten Anspruch mithin unmittelbar auf diese Norm zu stützen. Dem ist jedoch zu entgegnen, daß Richtlinien im Bürger-Bürger- (bzw. horizontalen) Verhältnis nicht unmittelbar anwendbar sind, wenn eine Richtlinienvorschrift in einem nationalen Gerichtsverfahren als Rechtsgrundlage der Entscheidung herangezogen werden müßte. ${ }^{290}$ Das Verbot der unmittelbaren Wirkung von Richtlinien ist nach Ansicht des $E u G H$ demgegenüber dann nicht verletzt, wenn der nationale Richter richtlinienwidriges nationales Recht nicht anwendet. ${ }^{291}$ Der durch die jeweilige nationale Bestimmung Diskriminierte könnte einen Anspruch demnach beispielsweise auf eine Norm des AGG stützen, wenn deren Geltendmachung ein mit europäischem Recht nicht vereinbares Verbotsgesetz entgegenstünde. ${ }^{292}$

\section{Diskriminierungsmerkmal als subjektive Anforderung}

Fälle objektiver wie rechtlicher Anforderung sind dadurch gekennzeichnet, daß sich bei ihnen die Frage, ob ein bestimmtes Merkmal wesentlich und entscheidend ist, völlig selbstverständlich und frei jeglicher Wertung beantworten läßt. Fraglich ist, ob beide Fallgruppen den Begriff der wesentlichen und entscheidenden Anforderung vollständig ausfüllen.

288 Mit Blick auf das AGG wird darauf hingewiesen: „Es kann schon sein, daß nun über einige tarifliche Errungenschaften neu diskutiert wird.“ (Sattar zitiert das Vorstandsmitglied der IG-Metall Oberhofer in FAZ vom 16.11.2006, S. 4, unter dem Titel: „,Auf falschem Bein hurra geschrien“ - Drei Monate Gleichbehandlungsgesetz").

289 Der EuGH hat beispielsweise festgestellt, daß die Richtlinie 76/207/EWG der Anwendung nationaler Bestimmungen entgegensteht, die Frauen allgemein vom Dienst mit der Waffe ausschließen und ihnen nur den Zugang zum Sanitäts- und Militärmusikdienst erlauben. (EuGH, NJW 2000, S. 497 (497 (Leitsatz)) Daraufhin hat Deutschland durch Gesetz vom 19.12.2000 sein Recht durch Änderung des Art. 12 a IV 2 GG den europäischen Vorgaben angepaßt. (BGB1. 2000 I 2, S. 1755) Gemäß der neuen Bestimmung dürfen Frauen ,[...] auf keinen Fall zum Dienst mit der Waffe verpflichtet werden.” Nach altem Wortlaut durften Frauen demgegenüber ,,auf keinen Fall Dienst mit der Waffe leisten.".

290 Streinz, Europarecht, Rn. 399 a.

291 EuGH, Rs. C-443/98, Slg. 2000 I 8/9 (B), S. 7535 (7584 f., Rn. 50-52); Streinz, Europarecht, Rn. 399 a.

292 So auch Raab: „Verstößt ein solches Beschäftigungsverbot gleichzeitig gegen eine Norm des Gemeinschaftsrechts, so ist [es] aufgrund des Vorrangs des Gemeinschaftsrechts auch ohne vorangegangenes förmliches Verfahren nicht anzuwenden.“ (Raab in Soergel, § 611 a, Rn. 36). 
Beispiele: Ist es wesentlich und entscheidend, daß ein Schwarzer die Rolle eines Schwarzen in einem Film spielt, ${ }^{293}$ ausschließlich Frauen Damenmode präsentieren, sich eine Werbeaktion, die den Verkauf von Kaffeemaschinen zum Gegenstand hat, ausschließlich an ein Geschlecht richtet? ${ }^{294}$

Unter Berücksichtigung der bislang gemachten Ausführungen wäre in allen Beispielsfällen das jeweilige Merkmal keine wesentliche und entscheidende Anforderung. In dem Film „Der menschliche Makel“ hat ein weißer Schauspieler (Anthony Hopkins) die Rolle eines Schwarzen gespielt. ${ }^{295} \mathrm{Da}$ ein Mann Damenmode präsentiert, mag unsinnig, unästhetisch, sogar unmoralisch sein. Das führt aber nicht dazu, daß zwingend eine Frau diese Tätigkeit ausüben müßte. ${ }^{296}$ Ein bestimmtes Geschlecht ist hier nicht wesentlich und entscheidend. Noch eindeutiger fällt die Antwort im „Kaffeemaschinenfall“ aus. Selbstverständlich sind beide Geschlechter in der Lage, einen Kaufvertrag über eine Kaffeemaschine abzuschließen. Ein bestimmtes Geschlecht ist nicht wesentliche und entscheidende Voraussetzung für einen derartigen Vertragsschluß.

Die Beispielsfälle zeichnen sich durch die Besonderheit aus, daß der „Diskriminierende“ ein bestimmtes Merkmal als für sich wesentlich und entscheidend erklärt. Das Merkmal ist nicht objektiv oder rechtlich wesentlich und entscheidend, sondern wird für wesentlich und entscheidend erklärt. Insofern besteht eine Parallele zur rechtlichen Anforderung mit dem Unterschied, daß bei dieser der Gesetzgeber das Merkmal für wesentlich und entscheidend erklärt. Hier ist es demgegenüber ein Privatmann in Gestalt des Arbeitgebers bzw. Verkäufers von Kaffeemaschinen.

Der Prozeß der „Wesentlich- und Entscheidendmachung“ ist ein wertender. Natürlich hätte der Verkäufer im Rahmen der Werbeaktion sein Angebot auch ausschließlich an das andere Geschlecht richten können. Fraglich ist, ob unter den Begriff der wesentlichen und entscheidenden Anforderung auch solche wertenden Entscheidungen subsumiert werden können.

\section{1. ,Wesentlich- und Entscheidendmachung“6}

Zum Zweck der Beantwortung dieser Frage sei der Blick noch einmal auf $\S 8$ I AGG gelenkt, der bestimmt, daß das jeweilige Diskriminierungsmerkmal nur dann eine wesentliche und entscheidende berufliche Anforderung bzw. Voraussetzung darstellt,

\section{„[...] sofern der Zweck rechtmäßig und die Anforderung angemessen ist.“ 297}

293 Beispiel aus der U.S.-amerikanischen Gerichtspraxis (Thüsing, NZA 2001, S. 1061 (1061 f.)).

294 Fernsehwerbung des Unternehmens ,Saturn“ im November 2004.

295 Freilich nicht aus dem Grund, weil der Regisseur das amerikanische Antidiskriminierungsrecht in Gestalt des Civil Rights Act fürchtete.

296 So auch Raab in Soergel, § 611 a, Rn. 37.

297 Entsprechende Regelungen enthalten Art. 4 Richtlinie 2000/43/EG, Art. 2 VI Richtlinie 76/207/EWG sowie Art. 4 Richtlinie 2000/78/EG. 
Diese Formulierung verdeutlicht, daß grundsätzlich definierbar ist, was wesentlich und entscheidend für eine bestimmte Tätigkeit ist. ${ }^{298}$ Wäre allein objektiv zu bestimmen, was wesentlich und entscheidend ist, läge bei Bejahung dieser Frage zweifellos immer ein rechtmäßiger Zweck und eine angemessene Anforderung für die Ungleichbehandlung vor. Eines „sofern-Zusatzes“ hätte es in einem solchen Fall nicht bedurft. Dieser macht einzig und allein Sinn, wenn man die Entscheidung dessen, was wesentlich und entscheidend ist, als subjektive Entscheidung begreift. Dann ist es auch zu verstehen, daß in der Literatur gesagt wird, durch den „sofern-Zusatz“ stünden ,,ausreichende Sicherungen zur Verfügung [...], um eine mißbräuchliche Berufung auf ,wesentliche und entscheidende berufliche Anforderungen“" zu verhindern. ‘299

Der soeben getroffenen Feststellung könnte entgegengehalten werden, der „,sofern-Zusatz“ beziehe sich nicht auf die Ungleichbehandlung, sondern auf die Tätigkeit an sich. Daß dem nicht so ist, soll folgendes Beispiel verdeutlichen:

Beispiel: Der Betreiber einer Nachtbar sucht in einer Anzeige Frauen, die in seiner Bar mit einem seiner Angestellten allabendlich vor Publikum die Beiwohnung vollziehen sollen. ${ }^{300}$

Das $B A G$ hat entschieden, daß ein Arbeitsvertrag wegen Verstoßes gegen die guten Sitten $(\S 138$ I BGB) nichtig ist, wenn die nach ihm geschuldete Leistung in der Vorführung des Geschlechtsverkehrs auf einer Bühne besteht. ${ }^{301}$ In dem Beispielsfall ist der Arbeitsvertrag nichtig, weil die Tätigkeit als solche gegen die guten Sitten verstößt, nicht deshalb, weil der Arbeitgeber seine Anzeige nicht geschlechtsneutral formuliert hat. Dies mußte er nicht, weil ein bestimmtes Geschlecht objektiv unverzichtbar für die in Rede stehende Tätigkeit war. Die Anzeige hatte unter dieser Prämisse keinen diskriminierenden Charakter.

Die Kriterien „,rechtmäßiger Zweck“ und ,,angemessene Anforderung“ können sich sinnvollerweise nur auf die Ungleichbehandlung beziehen. Oder sollten in dem Beispielsfall Männer erfolgreich Schadensersatz einklagen können, weil sich die Anzeige nicht auch an sie richtete? Sicherlich nicht. Eine Ungleichbehandlung verliert nicht dadurch ihren unverzichtbaren und dadurch gerechtfertigten Charakter, daß mit ihr verbundene Sekundärziele aus Gründen, die nicht in der Ungleichbehandlung liegen, unrechtmäßig sind. der Arbeit und das Leistungsvermögen des Arbeitnehmers - also objektive, der Definitionsmacht des Arbeitgebers entzogene Maßstäbe -, sondern die Lehre des Arbeitgebers - also von diesem selbst gesetzte Kriterien - die Rechtfertigung einer Ungleichbehandlung beurteilen.“ (Thüsing, ZfA 2001, S. 397 (408)).

299 Nickel, NJW 2001, S. 2668 (2670).

300 Fall nachgebildet nach $B A G$, NJW 1976, S. $1958 \mathrm{ff}$.

$301 \quad B A G$, NJW 1976, S. 1958 (1958, 1. Leitsatz). 
Wenn die Frage der wesentlichen und entscheidenden Anforderung letztlich wertend zu beantworten ist, dann folgt daraus, daß dem „Diskriminierenden“ grundsätzlich die Möglichkeit eingeräumt ist, das in Rede stehende Diskriminierungsmerkmal als für sich wesentlich und entscheidend zu erklären.

Daß diese Möglichkeit objektiv begrenzt sein muß, nicht jede „Wesentlich- und Entscheidendmachung“ zur Bejahung des Erfordernisses der wesentlichen und entscheidenden Anforderung führen kann, ist selbstverständlich. Andernfalls würde die bloße „Wesentlich- und Entscheidendmachung“ zur Rechtfertigung jeden diskriminierungsrelevanten Verhaltens führen, das Erfordernis der wesentlichen und entscheidenden Anforderung damit jeden Sinn verlieren.

\section{Objektive Grenze}

Um die Frage beantworten zu können, wo die objektive Grenze der „Wesentlich- und Entscheidendmachung“ genau verläuft, ist zu klären, was unter den Begriffen ,rechtmäßiger Zweck“ und ,,angemessene Anforderung“ in § 8 I AGG zu verstehen ist. Zu diesem Zweck sei an dieser Stelle noch einmal an die Definition der mittelbaren Diskriminierung erinnert: Gemäß $§ 3$ II AGG liegt eine mittelbare Diskriminierung vor,

„[...] wenn dem Anschein nach neutrale Vorschriften, Kriterien oder Verfahren Personen wegen eines in $\S 1$ genannten Grundes [ - d. h. wegen eines dort genannten Diskriminierungsmerkmals - ] gegenüber anderen Personen in besonderer Weise benachteiligen können, es sei denn, die betreffenden Vorschriften, Kriterien oder Verfahren sind durch ein rechtmäßiges Ziel sachlich gerechtfertigt und die Mittel sind zur Erreichung dieses Ziels angemessen und erforderlich.“ 302

§ 3 II AGG bestimmt, daß ein rechtmäßiges Ziel zu einer sachlichen Rechtfertigung diskriminierungsrelevanten Verhaltens führt, wenn dieses Verhalten zur Erreichung des Ziels angemessen und erforderlich ist.

Wenn man davon ausgeht, daß die Begriffe „rechtmäßiges Ziel“ und „rechtmäßiger Zweck“ identisch sind, kann aus der Definition der mittelbaren Diskriminierung der Schluß gezogen werden, daß auch hinter dem Begriff des rechtmäßigen Zwecks die Notwendigkeit sachlicher Rechtfertigung steht.

Der Begriff der angemessenen Anforderung dürfte seinerseits Hinweis auf die notwendige „ZweckMittel-Relation“ sein, wie sie explizit auch im Rahmen des § 3 II AGG verlangt wird.

302 Ähnliche Definitionen enthalten die Richtlinie 2000/43/EG in Art. 2 II lit. b, Richtlinie 76/207/EWG in Art. 2 II 2. Spiegelstrich, Richtlinie 2000/78/EG in Art. 2 II lit. b sowie Richtlinie 2004/113/EG in Art. 2 lit. b.; vgl. auch Art. 4 V Richtlinie 2004/113/EG der eine ähnliche Formulierung für die Rechtfertigung einer unmittelbaren Diskriminierung enthält. 


\section{Resümee - Unsachliche Behandlung rechtswidrig}

Die Definitionen in den $\S \S 3$ II und 8 I AGG erinnern an den aus dem deutschen Verfassungsrecht bekannten Verhältnismäßigkeitsgrundsatz, ${ }^{303}$ wonach ein Grundrechtseingriff durch die öffentliche Gewalt geeignet, erforderlich und angemessen sein muß. ${ }^{304}$

Es wurde vorgeschlagen, bei der Umsetzung des Art. 2 II lit. b Richtlinie 2000/43/EG - der eine dem § 3 II AGG vergleichbare Regelung enthält - das Unverzichtbarkeitserfordernis des § 611 a I 2 BGB-A entsprechend heranzuziehen, da die Formulierung der Richtlinie ,so allgemein und unbestimmt [sei], daß ihr klare Kriterien für erlaubte Ausnahmen kaum entnommen werden können. “305

Gemäß $§ 611$ a I 2 BGB-A war

„,[e]ine unterschiedliche Behandlung wegen des Geschlechts [...] zulässig, soweit [...] ein bestimmtes Geschlecht unverzichtbare Voraussetzung für diese Tätigkeit [war]. “306

Es ist falsch zu glauben, § 611 a I 2 BGB-A sei durch sein Unverzichtbarkeitserfordernis in dem beschriebenen Sinne klarer formuliert gewesen.

Insofern kann zunächst festgehalten werden, daß das Unverzichtbarkeitsmerkmal des $§ 611$ a I 2 BGB-A nicht nur Fälle objektiver und rechtlicher Unverzichtbarkeit erfassen sollte, wie es der Begriff „unverzichtbar“ auf den ersten Blick nahe legen mag. ${ }^{307}$ Die Begriffswahl wurde aus diesem Grunde als mißglückt bezeichnet. ${ }^{308}$ Letztlich sollte auch im Rahmen des $\S 611$ a I 2 BGB-A wertend $\mathrm{zu}$ entscheiden sein, ob ein bestimmtes Unternehmerinteresse als ausreichend gewichtig anzusehen sei, um das Gleichbehandlungsinteresse des Arbeitnehmers zurückzudrängen. ${ }^{309}$

303

307 Vergleiche dazu die in der Entwurfsbegründung zu § 611 a BGB-A genannten Beispiele (BT-Druck. 8/3317, S. 9); Mitteilung der Regierung der BRD an die Kommission der Europäischen Gemeinschaften, RdA 1988, S. 36 (36); vgl. auch Eich, der meinte, § 611 a BGB-A verlange keine Notwendigkeit (Unverzichtbarkeit) im naturwissenschaftlichen Sinne. (Eich, NJW 1980, S. 2329 (2331)).

308 Thüsing, RdA 2001, S. 319 (322); Richardi/Annuß in Staudinger, § 611 a, Rn. 52; Schlachter in Erfurter Kommentar zum Arbeitsrecht, § 611 a, Rn. 22; Eich hat die Auffassung vertreten, daß der Begriff „notwendig“ angebrachter und im Sinne einer einheitlichen juristischen Terminologie korrekter gewesen wäre. (Eich, NJW 1980, S. 2329 (2331)); Eich folgend Söllner in MüKo (2. Auflage), § 611 a, Rn. 13;

Auch formale Gründe wurden gegen die Bezeichnung ,unverzichtbar“ ins Feld geführt: Sie entspräche nicht der üblichen bürgerlich-rechtlichen Terminologie, da unter „Verzicht“ üblicherweise die Aufgabe einer bestimmten Rechtsposition verstanden werde. (Richardi/Annu $\beta$ in Staudinger, § 611 a, Rn. 52).

309 Richardi/Annuß in Staudinger, § 611 a, Rn. 53; vgl. auch Müller-Glöge: „Gefordert ist eine wertende Entscheidung, die letztendlich von den Gerichten für Arbeitssachen wahrgenommen wird.“ (Müller-Glöge in MüKo, § 611 a, Rn. $42)$. 
Die Frage, wo die objektive Grenze der dem „Diskriminierten“ dadurch eingeräumten Möglichkeit der „Unverzichtbarmachung“ eines bestimmten Geschlechts genau verlaufen sollte, wurde dabei unterschiedlich beantwortet. Einen sachlichen Grund schienen diejenigen genügen zu lassen, die die Meinung vertraten, jede plausible Argumentation müsse zur Unverzichtbarkeit führen. ${ }^{310}$ In diesem Sinne vertraten andere die Ansicht, mit dem Tatbestandsmerkmal der Unverzichtbarkeit sei eine bestimmte Fallgruppe eines sachlichen Grundes angesprochen, deren Besonderheit darin bestehe, daß der sachliche Grund an der Art der ausbedungenen Tätigkeit zu messen sei. ${ }^{311}$ In diese Richtung schien auch das BAG $\mathrm{zu}$ tendieren, wenn es in einem Fall feststellte, „ein sachlicher Differenzierungsgrund [liegt] vor, weil aufgrund der Praxisnotwendigkeiten die Eigenschaften als Frau für den Bekl. und nach seinem unbestrittenen Sachvortrag auch für seine Patienten/innen unverzichtbare Voraussetzung des Arbeitsverhältnisses war.“312 In einem anderen Urteil des Gerichts heißt es, ,ein Verstoß gegen $§ 611$ a BGB[-A] liege nicht vor, wenn die unterschiedliche Behandlung von männlichen und weiblichen Arbeitnehmern durch sachliche Gründe gerechtfertigt werden. “313 Desweiteren wurde behauptet, ein sachlicher Grund allein genüge in der Regel nicht, ${ }^{314}$ was den Umkehrschluß zuläßt, daß nach dieser Ansicht zumindest ausnahmsweise ein solcher ausreichen sollte. ${ }^{315}$

Auf der anderen Seite wurde die Auffassung vertreten, zur Unverzichtbarkeit bedürfe es mehr als bloß eines sachlichen Grundes. ${ }^{316}$ In scheinbarem Widerspruch zu seiner soeben zitierten Rechtsprechung hat dies in einem späteren Urteil auch das $B A G$ festgestellt. ${ }^{317}$

Die angestellten Überlegungen führen zu der Erkenntnis, daß es sinnvoll gewesen wäre, die Richtlinienvorgaben durch Einführung des Begriffs „sachlicher Grund“ umzusetzen. Dies ist teilweise auch geschehen. So ist gem. § 20 I 1 AGG eine Verletzung des zivilrechtlichen Benachteiligungsverbots nicht gegeben,

„wenn für eine unterschiedliche Behandlung wegen der Religion oder der Weltanschauung, einer Behinderung, des Alters, der sexuellen Identität oder des Geschlechts ein sachlicher Grund vorliegt.“ ${ }^{318}$

310 Adomeit, DB 1980, S. 2388 (2388).

311 Eich, NJW 1980, S. 2329 (2331).

312 BAG, NZA 1991, S. 719 (723).

313 BAG, NZA 1993, S. 933 (934).

314 Putzo in Palandt, $\$ 611$ a, Rn. 12.

315 Auch Lorenz scheint das Unverzichtbarkeitserfordernis als sachlichen Grund verstanden zu haben, wenn er sagte, das Differenzierungsgebot sei sonstigen sachlichen Gründen nicht zugänglich. (Lorenz, DB 1980, S. 1745 (1745)); Unabhängig von § 611 a BGB-A scheint auch Baer ,lediglich“ einen sachlichen Grund zu verlangen: „Wer an „Schwarze“ nicht vermieten will, weil sie „schwarz“ sind, wird von der Rechtsordnung nicht geadelt. Sachliche Rechtfertigungen sind hier kaum denkbar: Die „Reinhaltung“ der Wohngegend ist auch rassistisch, wenn Menschen angeblich vor Übergriffen geschützt werden sollen (,Sie passen da nicht hin“). Wer umgekehrt an mißhandelte Frauen vermietet, kann dies sachlich rechtfertigen, da deren Situation objektiv kompensatorische Maßnahmen erforderlich macht.“ (Baer, ZRP 2002, S. 290 (293 mit Fn. 33)).

316 Thüsing, RdA 2001, S. 319 (320); Schlechtriem in Jauernig, $\S 611$ a, 611 b, Rn. 3.

317 BAG, NZA 1999, S. 371 (372).

318 Ausführliche Darstellung des $\S 20$ AGG unter Teil 4, Prüfungspunkt B. I. 4. a). 
Zum einen ist das Erfordernis der wesentlichen und entscheidenden beruflichen Anforderung in $\S 8$ I AGG bedeutungslos neben dem in der Bestimmung außerdem statuierten Erfordernis: ,[...] sofern der Zweck rechtmäßig und die Anforderung angemessen ist.“

Zum anderen haben die Ausführungen zu $§ 611$ a I 2 BGB-A gezeigt, daß gerade der Begriff „unverzichtbar“ nur scheinbar eindeutig weil wertungsfrei war; daß unter ihn Fälle subsumiert wurden, die von seiner eigentlichen Wortbedeutung weit entfernt waren. ${ }^{319}$ Dasselbe gilt natürlich auch für den nunmehr in $\S 8$ I AGG enthaltenen Begriff der wesentlichen und entscheidenden berufliche Anforderung.

Es kann schließlich behauptet werden, daß der Begriff der wesentlichen und entscheidenden Anforderung gegenüber dem des sachlichen Grundes keineswegs die „,enger gefaßte Klausel“3320 darstellt, so daß die Einführung des Begriffs „,sachlicher Grund“ nicht zu einer Absenkung des Schutzniveaus gegenüber dem Erfordernis der wesentlichen und entscheidenden Anforderung führen muß, wie dies teilweise hinsichtlich des Unverzichtbarkeitserfordernisses in $\S 611$ a I 2 BGB-A behauptet wurde. ${ }^{321}$ Denn nicht nur das Erfordernis der wesentlichen und entscheidenden Anforderung ist im Lichte des Diskriminierungsverbotes auszulegen. ${ }^{322}$ Auch die Frage nach dem Vorliegen eines sachlichen Grundes ist mit Blick auf das Diskriminierungsverbot zu beantworten. Das entscheidende Argument ist insofern, daß der auslegungsfähige und -bedürftige Begriff „sachlicher Grund“ im Sinne der Antidiskriminierungsmaßnahmen, die im Zivilrecht Drittwirkung entfalten - das gilt beispielsweise für die Antidiskriminierungsrichtlinien -, auszulegen ist. ${ }^{323}$

Wenn nun aber beide Begriffe geeignet sind, die gleichen Anforderungen an die Rechtfertigung diskriminierungsrelevanten Verhaltens zu stellen, ist der Begriff „,sachlicher Grund“ dem Begriff „wesentliche und entscheidende [...] Anforderung“ vorzuziehen, verdeutlicht er als auslegungsbedürftiger Rechtsbegriff doch, daß die Rechtfertigung diskriminierungsrelevanten Verhaltens letztlich immer eine einzelfallabhängige Abwägungsentscheidung bleibt. ${ }^{324}$

319 Insofern war es falsch, wenn Nickel behauptete, § 611 a BGB-A enthalte keine unbestimmten Rechtsbegriffe, an die eine Einwirkung der Grundrechte anknüpfen könnte. (Nickel, Gleichheit und Differenz in der vielfältigen Republik, S. 121).

320 So Nickel in bezug auf das Unverzichtbarkeitserfordernis in $\S 611$ a I 2 BGB-A (Nickel, NJW 2001, S. 2668 (2671)).

321 Wiedemann/Thüsing, DB 2002, S. 463 (468); BAG: „Eine unverzichtbare Voraussetzung [...] stellt erheblich höhere Anforderungen an das Gewicht des die Ungleichbehandlung rechtfertigenden Umstandes als ein sachlicher Grund, [...].“(BAG, NZA 1999, S. 371 (372)).

322 So das $B A G$ hinsichtlich des Unverzichtbarkeitserfordernisses in $\S 611$ a I 2 BGB-A: “[...] denn das Geschlecht ist nur dann unverzichtbar, wenn ein Angehöriger des jeweils anderen Geschlechts die vertragsgemäße Leistung nicht erbringen könnte und dieses Unvermögen auf Gründen beruht, die ihrerseits der gesetzlichen Wertentscheidung der Gleichberechtigung beider Geschlechter genügen." (BAG, NZA 1999, S. 371 (372); Hervorhebungen durch den Verfasser).

$323 \mathrm{Zu}$ den Auswirkungen der untersuchten Antidiskriminierungsmaßnahmen auf das Privatrechtsverhältnis vgl. Teil 4, Prüfungspunkt A. I.

324 Vergleiche dazu Teil 3, Prüfungspunkt A. 
Im Zusammenhang mit den soeben angestellten Überlegungen ist hinzuweisen auf die unterschiedlichen Begrifflichkeiten im Zusammenhang mit den Anforderungen an die Rechtfertigung diskriminierungsrelevanten Verhaltens in den $\S 8-10$ und 20 AGG. Sie reichen von „,sachlicher Grund“ in $\S 20$ über ,wesentliche und entscheidende berufliche Anforderung [...], sofern der Zweck rechtmäßig und die Anforderung angemessen“ in $\S 8$ I und „gerechtfertigte berufliche Anforderung“ in $\S 9$ I bis zu „objektiv und angemessen und durch ein legitimes Ziel gerechtfertigt“, soweit die „Mittel zur Erreichung dieses Ziels [...] angemessen und erforderlich“ in $\S 10$ Satz 1 und 2. Dieser gesetzestechnische Wirrwarr ist, wie nachgewiesen wurde, nicht erforderlich. Der Begriff des sachlichen Grundes ist aufgrund seiner Flexibilität geeignet, auf die jeweiligen Besonderheiten des Einzelfalls in adäquater Weise zu reagieren.

Mit Blick auf die (nach Inkrafttreten des AGG theoretische) Frage der gesetzestechnischen Umsetzung des Diskriminierungsverbotes ist darauf hinzuweisen, daß der Begriff ,sachlicher Grund“ einen Rechtfertigungsgrund darstellt. Vor dem Hintergrund des hier vertretenen Konzepts, wonach diskriminierungsrelevantes Verhalten grundsätzlich nicht dessen Rechtswidrigkeit indiziert, ${ }^{325}$ müßte - um den Unterschied zwischen Rechtswidrigkeit und Rechtfertigungsgrund deutlich zu machen - die Gesetzesformulierung auf den Begriff „sachlicher Grund“ verzichten und stattdessen den der ,unsachlichen Behandlung“ gebrauchen.

\section{Zusammenfassung}

Ausgangspunkt für die im Rahmen des Prüfungspunktes „Rechtswidrigkeit des diskriminierungsrelevanten Verhaltens“ gemachten Ausführungen war die Feststellung, daß die bis dahin angestellten Überlegungen zum Diskriminierungsbegriff etwaige Interessen des „Diskriminierenden“ völlig unberücksichtigt gelassen hatten. Die vor diesem Hintergrund angestellten Überlegungen führten $\mathrm{zu}$ folgenden Erkenntnissen: Ein dem Schutz des „Diskriminierten“ dienendes zivilrechtliches Diskriminierungsverbot greift in das grundrechtlich geschützte Recht des „Diskriminierenden“ auf privatautonomes Handeln ein und erzeugt damit ein Spannungsverhältnis, das es durch Abwägung der widerstreitenden Interessen der Parteien zu lösen gilt. Der (inter-)nationale Gesetzgeber hat zu diesem Zweck bestimmt, daß eine „Diskriminierung“ unter bestimmten Voraussetzungen gerechtfertigt ist bzw. eine Ungleichbehandlung keine Diskriminierung darstellt.

Die folgenden Überlegungen beschäftigten sich mit der Beantwortung der Frage, wann diskriminierungsrelevantes Verhalten rechtswidrig ist. Zu diesem Zweck wurde untersucht, unter welchen Voraussetzungen diskriminierungsrelevantes Verhalten nach § 8 I AGG gerechtfertigt ist

325 Vergleiche dazu Teil 2, Prüfungspunkt C. III. 
bzw. im Sinne der Antidiskriminierungsrichtlinien keine Diskriminierung darstellt. Dabei wurde zunächst auf den in diesem Zusammenhang in den genannten Rechtsquellen verwendeten Begriff der „wesentlichen und entscheidenden beruflichen Anforderung“ eingegangen und festgestellt, daß „wesentlich und entscheidend“ im Sinne dieser Rechtsquellen ist, was „unverzichtbar“ im Sinne des $\S 611$ a I 2 BGB-A war, und vice versa. Für die Beantwortung der Frage, wann diskriminierungsrelevantes Verhalten nach $\S 8$ I AGG gerechtfertigt ist bzw. im Sinne der Antidiskriminierungsrichtlinien keine Diskriminierung darstellt, konnte so die umfangreiche Rechtsprechung und Kommentierung zu § 611 a I 2 BGB-A fruchtbar gemacht werden. Diesen Vorüberlegungen schlossen sich folgende Erkenntnisse an:

Ein bestimmtes Geschlecht ist „wesentlich und entscheidend“ im Sinne des $§ 8$ I AGG, wenn die in Rede stehende Tätigkeit naturwissenschaftlich bedingt nur von einem Geschlecht ausgeübt werden kann (objektive Anforderung). Dasselbe gilt für alle Diskriminierungsmerkmale und sämtliche denkbaren Rechtsverhältnisse. Die Richtlinie 2000/43/EG, die hinsichtlich des von ihr geschützten Merkmals außerhalb des Arbeitsrechts ein absolutes Diskriminierungsverbot statuiert - das geschützte Merkmal in diesem Bereich also niemals „,wesentlich und entscheidend“ im Sinne des Art. 4 besagter Richtlinie sein kann, diskriminierungsrelevantes Verhalten außerhalb des Arbeitrechts folglich stets rechtswidrig sein soll - ist deshalb zu kritisieren.

Ein bestimmtes Geschlecht ist auch dann wesentlich und entscheidend im Sinne des $\S 8$ I AGG, wenn es für die Durchführung einer Tätigkeit gesetzlich vorgeschrieben ist (rechtliche Anforderung). Beispielhaft können insofern Beschäftigungsverbote sowie Fördermaßnahmen in Form von Quotenregelungen genannt werden. Im Zusammenhang mit letzteren wurde in einem Exkurs der Frage nachgegangen, ob sich eine derartige ,positive Diskriminierung“ - die nicht geförderte Gruppe wird durch eine Quotenregelung benachteiligt - überhaupt in ein Diskriminierungsschutzkonzept eingliedern läßt. Für die Beantwortung dieser Frage war es erforderlich, sich die Ziele von Diskriminierungsschutzmaßnahmen zu vergegenwärtigen. Diese Untersuchung, die auch europarechtliche Vorgaben berücksichtigte, führte $\mathrm{zu}$ den folgenden Erkenntnissen: Positive Diskriminierung kommt als ultima ratio immer nur dann in Betracht, wenn sich das gewünschte Ergebnis - Teilhabe statt Ausgrenzung vom gesellschaftlichen Leben - nicht schon durch die bloße Gewährung des Anspruchs auf formale Gleichbehandlung realisieren läßt. Die Gewährung des Anspruchs auf formale Gleichbehandlung im Wege der Statuierung eines bloßen Diskriminierungsverbotes wird bei einem Großteil der Diskriminierungsmerkmale dieses Ergebnis bewirken. Soweit Quotenregelungen nötig sind, müssen selbige grundsätzlich als relative Quoten ausgestaltet sein. Absolute Quoten lassen sich im Privatrechtsverkehr nur ausnahmsweise rechtfertigen - etwa im Arbeitsrecht für das Merkmal „Schwerbehinderung“. Vor der Einführung von Quotenregelungen ist zu prüfen, ob die angestrebte Teilhabe der benachteiligten Gruppe nicht 
eventuell dadurch erreicht werden kann, daß Fördermaßnahmen getroffen werden, die - wie etwa die Rampe für den Rollstuhlfahrer - nicht zu positiver Diskriminierung führen.

Als Beispiele rechtlicher Unverzichtbarkeit können auch einige sozialstaatlich motivierte Regelungen des deutschen Arbeitsrechts genannt werden, die den Arbeitgeber verpflichten oder ihm gestatten, das Alter des Arbeitnehmers zum Anknüpfungspunkt bestimmter Entscheidungen zu machen. Obwohl dem Grunde nach europarechtlich legitimiert, war auch hier der Frage nachzugehen, wie sich derartige Maßnahmen in ein Diskriminierungsschutzkonzept eingliedern lassen. Folgende Feststellungen wurden insofern getroffen: „Diskriminierung“, die aufgrund sozialer Gesichtspunkte vorgeschrieben ist bzw. für zulässig erklärt wird, verfolgt letztlich dasselbe, was Diskriminierungsschutz erreichen möchte. Beide Institute können also funktional vergleichbar eingesetzt werden. In diesem Zusammenhang wurde noch einmal daran erinnert, daß Diskriminierungsschutz seinerseits nicht beschränkt ist auf das Gebot formeller Gleichbehandlung. Schließlich wurde festgestellt, daß beide Institute stets im Lichte des jeweils anderen betrachtet werden müssen.

Die Ausführungen zur rechtlichen Unverzichtbarkeit schlossen mit Überlegungen zu möglichen Konsequenzen des Verstoßes einer nationalen Regelung gegen höherrangigeres Recht, speziell gegen die Antidiskriminierungsrichtlinien. Es wurde festgestellt, daß eine Richtlinienvorschrift in einem nationalen Gerichtsverfahren nicht als Rechtsgrundlage der Entscheidung fungieren kann, daß dem nationalen Richter aber unbenommen ist, richtlinienwidriges nationales Recht nicht anzuwenden.

Die Tatsache, daß gem. $\S 8$ I AGG das jeweilige Diskriminierungsmerkmal nur dann eine wesentliche und entscheidende berufliche Anforderung bzw. Voraussetzung darstellt, ,[...] sofern der Zweck rechtmäßig und die Anforderung angemessen ist [...]“, führte zu der Erkenntnis, daß nach dieser Bestimmung letztlich wertend $\mathrm{zu}$ entscheiden ist, ob ein bestimmtes Unternehmerinteresse als ausreichend gewichtig anzusehen ist, um das Gleichbehandlungsinteresse des Arbeitnehmers zurückzudrängen; daß das Merkmal der wesentlichen und entscheidenden beruflichen Anforderung i. S. d. § 8 I AGG also nicht beschränkt ist auf die wenigen Fälle objektiver und rechtlicher Anforderung, bei denen die Frage der Rechtfertigung völlig wertungsfrei beantwortet werden kann. Auf der Grundlage dieser Erkenntnis wurden folgende Feststellungen getroffen: Dem Arbeitgeber steht es grundsätzlich frei, etwa ein bestimmtes Geschlecht für die in Rede stehende Tätigkeit für wesentlich und entscheidend zu erklären - subjektive Anforderung. Diese „Wesentlich- und Entscheidendmachung“ ist objektiv begrenzt durch die Begriffe „,rechtmäßiger Zweck“ und ,,angemessene Anforderung“, wobei hinter ersterem die Notwendigkeit sachlicher Rechtfertigung steht, während der zweite ein Hinweis auf die - auch im Rahmen des $\S 3$ II AGG - notwendige „Zweck-Mittel-Relation“ sein dürfte. 
Die zur subjektiven Anforderung angestellten Überlegungen führten schließlich zu der Erkenntnis, daß es sinnvoll gewesen wäre, die Richtlinienvorgaben hinsichtlich der Frage der Rechtswidrigkeit diskriminierungsrelevanten Verhaltens durch Einführung des Begriffs „unsachlich“ umzusetzen, wie in $\S 20$ I 1 AGG durch Einführung des Rechtfertigungsgrundes „sachlicher Grund“ teilweise geschehen. In diesem Zusammenhang wurde nachgewiesen, daß die Verwendung der Formulierung „unsachlich“ nicht zu einer Absenkung des durch § 8 I AGG gewährten und von den Antidiskriminierungsrichtlinien geforderten Schutzniveaus führt. Der Begriff „,unsachlich“ wurde schließlich für vorzugswürdig erklärt, weil er als auslegungsbedürftiger Rechtsbegriff im Gegensatz zu den Begriffen ,wesentlich und entscheidend“ unmißverständlich verdeutlicht, daß die Frage der Rechtswidrigkeit diskriminierungsrelevanten Verhaltens eine einzelfallabhängige Abwägungsentscheidung ist.

\section{Sachlicher Geltungsbereich des Diskriminierungsverbotes}

„Es sind nicht Aufmärsche von Neonazis, welche ein Klima der Angst schaffen, sondern die alltäglichen Diskriminierungen auf der Straße, in der Schule, im Betrieb und vor Discothekentüren. “326

Die Interessen des „Diskriminierenden“ finden neben der Beschränkung des Diskriminierungsbegriffs durch das Merkmal der Rechtswidrigkeit auch dadurch Berücksichtigung, daß der sachlichen Geltungsbereichs des Diskriminierungsverbotes begrenzt ist. Gemäß ihres Art. 3 I gilt die Richtlinie 2000/43/EG im Rahmen der auf die Gemeinschaft übertragenen Zuständigkeiten

„[...] für alle Personen in öffentlichen und privaten Bereichen, einschließlich öffentlicher Stellen, in bezug auf:

a) die Bedingungen - einschließlich Auswahlkriterien und Einstellungsbedingungen - für den Zugang zu unselbständiger und selbständiger Erwerbstätigkeit, unabhängig von Tätigkeitsfeld und beruflicher Position, sowie für den beruflichen Aufstieg;

b) den Zugang $\mathrm{zu}$ allen Formen und allen Ebenen der Berufsberatung, der Berufsausbildung, der beruflichen Weiterbildung und der Umschulung einschließlich der praktischen Berufserfahrung;

c) die Beschäftigungs- und Arbeitsbedingungen, einschließlich Entlassungsbedingungen und Arbeitsentgelt;

d) die Mitgliedschaft und Mitwirkung in einer Arbeitnehmer- oder Arbeitgeberorganisation oder einer Organisation, deren Mitglieder einer bestimmten Berufsgruppe angehören, einschließlich der Inanspruchnahme der Leistungen solcher Organisationen;

e) den Sozialschutz, einschließlich der sozialen Sicherheit und der Gesundheitsdienste;

f) die sozialen Vergünstigungen;

g) die Bildung;

h) den Zugang zu und die Versorgung mit Gütern und Dienstleistungen, die der Öffentlichkeit zur Verfügung stehen, einschließlich von Wohnraum.“

326 Aussage der Abgeordneten Gabriele Andretta (SPD) des niedersächsischen Landtages, wiedergegeben im Göttinger Tageblatt vom 13.01.2001. 
Der Geltungsbereich der Richtlinie 76/207/EWG entspricht dem des Art. 3 I lit. a - d Richtlinie 2000/43/EG. Gleiches gilt für den Geltungsbereich der Richtlinie 2000/78/EG. Der Geltungsbereich der Richtlinie 2004/113/EG läßt sich mit Art. 3 I lit. h Richtlinie 2000/43/EG vergleichen. Gemäß ihres Art. 3 I gilt die Richtlinie 2004/113/EG im Rahmen der auf die Gemeinschaft übertragenen Zuständigkeiten

„[...] für alle Personen, die Güter und Dienstleistungen bereitstellen, die der Öffentlichkeit ohne Ansehen der Person zur Verfügung stehen, und zwar in öffentlichen und privaten Bereichen, einschließlich öffentlicher Stellen, und die außerhalb des Bereichs des Privat- und Familienlebens und der in diesem Kontext stattfindenden Transaktionen angeboten werden.“

In Umsetzung der europarechtlichen Vorgaben enthält $\S 2$ I Nr. 1 bis 8 AGG schließlich eine in Inhalt und Wortlaut weitgehend den Vorgaben des Art. 3 I lit. a bis h Richtlinie 2000/43/EG entsprechende Bestimmung. Konkretisiert wird der sachliche Geltungsbereich des Gesetzes durch die in den $\$ \$ 7$ und 19 statuierten Benachteiligungsverbote. ${ }^{327}$

\section{Rechtsgeschäftliches und quasi-rechtsgeschäftliches Handeln}

Die bisherigen Beispielsfälle haben verdeutlicht, daß sich die Frage nach dem Vorliegen einer Diskriminierung meist im Zusammenhang mit der Begründung, Durchführung und Beendigung eines Vertragsverhältnisses stellt. Dementsprechend statuieren die Antidiskriminierungsrichtlinien Diskriminierungsschutz in diesem Bereich.

Diese Feststellung ist mit der Einsicht verbunden, daß nicht nur der Vertrag als solcher diskriminierenden Charakter haben kann. So stellt etwa die Beendigung des Vertrages in Form der Kündigung keinen Vertrag dar. Das gleiche gilt für den Ausschluß eines Vereinsmitgliedes aufgrund Beschlusses der Mitgliederversammlung. ${ }^{328}$

Die Gemeinsamkeit zwischen Vertrag, Kündigung und Vereinsbeschluß besteht in ihrer Rechtsnatur als Rechtsgeschäft. Der Begriff des Rechtsgeschäfts umfaßt einseitige als auch mehrseitige Rechtsgeschäfte, wobei zu ersteren etwa die Kündigungserklärung und die Auslobung gem. $§ 657$ BGB, zu letzteren Verträge, Gesamtakte und Beschlüsse zählen. ${ }^{329}$ Da sich in all diesen Bereichen die Frage nach dem Vorliegen einer Diskriminierung stellen kann, ${ }^{330}$ umfaßt der

\footnotetext{
327 Vergleiche dazu Teil 4, Prüfungspunkt B. I. 3.

328 Vergleiche zur Beschlußfassung durch die Mitgliederversammlung § 32 I 1 BGB.

329 Brox, Allgemeiner Teil des BGB, Rn. 99 f.

330 „Es sind [...] nicht nur Schuldverträge, sondern auch Gesellschafts- und Vereinssachverhalte betroffen“. (Wiedemann/Thüsing, DB 2002, S. 463 (465)). 
sachliche Geltungsbereich des Diskriminierungsverbotes grundsätzlich das gesamte rechtsgeschäftliche Handeln.

Darüber hinaus umfaßt er auch quasi-rechtsgeschäftliches Handeln, wie die folgenden Ausführungen verdeutlichen: Bei einigen Maßnahmen des „Diskriminierenden“ ist die Frage ihrer Rechtsnatur umstritten. Das gilt etwa für Weisungen des Arbeitgebers, die bereits vom Diskriminierungsverbot des $\S 611$ a I 1 BGB-A ausdrücklich erfaßt waren. Teilweise werden sie als empfangsbedürftige Willenserklärungen eingestuft. ${ }^{331}$ Eine andere Ansicht stellt eine differenzierte Betrachtung an. Weisungen, die nur die Art der vom Arbeitnehmer zu erbringenden Tätigkeit konkretisieren, sollen nicht die Qualität einer Willenserklärung haben. Das gelte etwa für Arbeitskommandos wie dem „Hauruck!“, die nicht auf eine Rechtsgestaltung gerichtet seien, sondern lediglich auf den Vollzug einer privatautonom festgelegten Pflicht. ${ }^{332}$ Eine Weisung, die sich auf die Arbeitszeit oder auf den Ort der Arbeitsleistung bezieht, soll demgegenüber auch nach dieser Ansicht ein Rechtsgeschäft darstellen, weil dadurch die Arbeitsbedingungen festgelegt würden. Gleiches gelte, wenn der Arbeitgeber nicht nur die Art der vom Arbeitnehmer zu erbringenden Tätigkeit konkretisiert, sondern darüber hinaus dem Arbeitnehmer einen anderen Tätigkeitsbereich zuweist. ${ }^{333}$

Eine diskriminierende Weisung kann etwa darin bestehen, daß der Arbeitgeber von einer Arbeitnehmerin islamischen Glaubens verlangt, ohne Kopftuch zu arbeiten. ${ }^{334}$ Nichtig kann eine Kündigung sein, die ausgesprochen wird, weil sich die Arbeitnehmerin weigert, in Zukunft auf das Tragen des Kopftuchs zu verzichten. ${ }^{335}$

Selbst wenn man davon ausgeht, daß die Weisung in dem beschriebenen Fall nur quasirechtsgeschäftliches Handeln darstellt, wird man doch zumindest behaupten können, daß sie ihrem Wesen nach mit dem Rechtsgeschäft „Kündigung“ vergleichbar ist. Beide, die Kündigung wie die Weisung, dienen der Durchsetzung ein und desselben „diskriminierenden“ Interesses des Arbeitgebers.

Unabhängig von der Beantwortung der Frage ihrer Rechtsnatur erscheint es deshalb gerechtfertigt, derartige Weisungen und auch sonstiges quasi-rechtsgeschäftliches Verhalten am Verbot der Diskriminierung zu messen.

\section{Vorfeld des Vertragsschlusses}

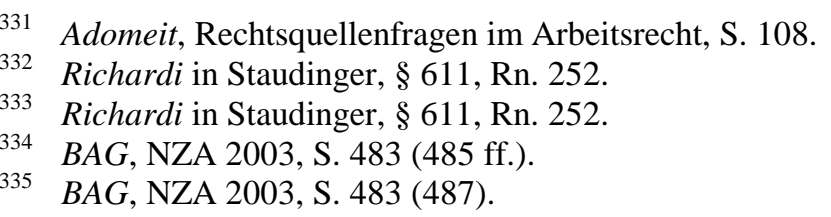


Die Antidiskriminierungsrichtlinien bezwecken Diskriminierungsschutz bereits im Vorfeld des Vertragsschlusses. So erstreckt die Richtlinie 2000/43/EG den sachlichen Geltungsbereich des Diskriminierungsverbots gem. Art. 3 I lit. a auf „die Bedingungen [...] für den Zugang zu unselbständiger und selbständiger Erwerbstätigkeit““ und gem. lit. h auf „,den Zugang zu und die Versorgung mit Gütern und Dienstleistungen“.

Die bisherigen Beispielsfälle haben gezeigt, daß besonders häufig im Vorfeld des Vertragsschlusses diskriminiert wird, in einem Stadium also, in dem ein Vertrag noch nicht geschlossen worden ist. Grund dafür dürfte unter anderem sein, daß sich die potenziellen Vertragsparteien zu diesem Zeitpunkt noch nicht kennen, sich ein Vertrauensverhältnis zwischen ihnen noch nicht aufbauen konnte und eventuell bestehende Vorurteile noch nicht abgebaut werden konnten. In diesem Vertragsstadium zählt der „,erste Eindruck“, der unter Umständen ein völlig falsches Bild von dem Gegenüber vermittelt. $\mathrm{Zu}$ recht wird darauf hingewiesen, daß die Entscheidung, mit dem einen Interessenten den Vertrag zu schließen, mit dem anderen hingegen nicht, ,immer von persönlichen Vorlieben und Abneigungen, Vorurteilen und Animositäten, Illusionen und Schreckhaftigkeiten bestimmt" ist. ${ }^{336}$

\section{Merkmalsneutrale Ausschreibung}

Bereits bei einer Stellenausschreibung, die sich explizit nur an Menschen mit bestimmten Merkmalen richtet, stellt sich die Frage nach dem Vorliegen einer Diskriminierung. Praktische Relevanz hat sie im Zusammenhang mit geschlechtsspezifischen Ausschreibungen erlangt. ${ }^{337}$

Gemäß $§ 11$ AGG darf ein Arbeitsplatz

„[...] nicht unter Verstoß gegen [ das Diskriminierungsverbot des ] § 7 Abs. 1 ausgeschrieben werden.“

Bereits vor Inkrafttreten des AGG enthielt $\S 611$ b BGB-A eine Regelung über die Zulässigkeit geschlechtsspezifischer Ausschreibung. Gemäß dieser Norm durfte der Arbeitgeber

„,einen Arbeitsplatz weder öffentlich noch innerhalb des Betriebs nur für Männer oder nur für Frauen ausschreiben, es sei denn, daß ein Fall des $§ 611$ a Abs. 1 Satz 2 [vorlag].“

$\S 611$ b BGB-A wurde teilweise als eine wertungsmäßig mißratene Regelung angesehen, die nicht länger bestehen, geschweige denn noch weiter ausgedehnt werden sollte. Durch $\S 611 \mathrm{~b}$ BGB-A werde der Ausbietende geradezu verpflichtet, über seine Absichten zu täuschen. Nach klassischem

337 Von einem solchen Fall berichtet Tornau in Frankfurter Rundschau vom 06.02.2004, S. 16 unter dem Titel: „Der diskriminierte Mann“. 
Zivilrecht solle aber gerade enttäuschtes Vertrauen zu Schadensersatz aus vorvertraglichem Schuldverhältnis führen. ${ }^{338}$

Diese Ansicht war und ist auch nach neuem Recht nicht begründet, denn die Pflicht zu neutraler Ausschreibung ist nicht eine bloße Formalie, die gar nicht das Ziel hat, diskriminierten Personen tatsächlich die Chance auf einen Arbeitsplatz zu eröffnen.

Nicht übersehen werden darf in diesem Zusammenhang, daß die Verpflichtung zu geschlechtsneutraler Ausschreibung nur in begrenztem Umfang in der Lage ist, die Einstellungschancen des unerwünschten Geschlechts zu erhöhen. Auf eine Stellenanzeige bewirbt sich für gewöhnlich eine Vielzahl von Interessenten. Die Verpflichtung zu geschlechtsneutraler Ausschreibung führt zwar dazu, daß sich sowohl Männer als auch Frauen bewerben. Sie vermag indes nicht bewirken, daß der Arbeitgeber zwischen mehreren Bewerbern nicht seinen Präferenzen gemäß den Bewerber bzw. die Bewerberin einstellt. ${ }^{339}$

Gleichwohl ist es sinnvoll, den Arbeitgeber zu geschlechtsneutraler Ausschreibung zu verpflichten. Es ist möglich, daß dieser sich trotz bestehender Vorbehalte im Einzelfall für den Bewerber mit dem unerwünschten Geschlecht entscheidet, etwa weil dieser durch seine Bewerbung überzeugt hat oder der einzige Bewerber ist.

Soll das Diskriminierungsverbot auch das Vorfeld des Vertragsschlusses umfassen, ist es außerdem geradezu denknotwendig, ein Verbot geschlechtsspezifischer Ausschreibung zu statuieren, könnte doch andernfalls das Diskriminierungsverbot in diesem Bereich auf einfache Weise umgangen werden.

Eine merkmalsspezifische Ausschreibung ist immer dann zulässig, wenn sie nicht zu einem Verstoß gegen das Benachteiligungsverbot des $\S 7$ I AGG führt. Die Rechtfertigung der merkmalsspezifischen Ausschreibung i. S. d. § 11 AGG unterliegt damit den gleichen Anforderungen, die für die Nichteinstellung des Trägers eines der in $\S 1$ AGG genannten Diskriminierungsmerkmale gelten. ${ }^{340}$ Übertragen auf das im Rahmen dieser Arbeit verfolgte Diskriminierungsschutzkonzept läßt sich formulieren, daß eine merkmalsspezifische Ausschreibung immer dann zulässig ist, wenn auch der Vertragsschluß aufgrund des jeweiligen Merkmals abgelehnt werden könnte.

\section{Merkmalsneutrale Bewerbungsunterlagen}

338 Adomeit, NJW 2002, S. 1622 (1622).

339 Vergleiche dazu die Ausführungen zu dem Problem des „Zusammentreffens der Träger von Diskriminierungsmerkmalen“ unter Teil 3, Prüfungspunkt H.

340 Insofern kann auf die bereits gemachten Ausführungen zur „Rechtswidrigkeit des diskriminierungsrelevanten Verhaltens" verwiesen werden. (vgl. Teil 2, Prüfungspunkt C.). 
In den USA ist der Arbeitgeber nicht berechtigt, in Bewerbungsunterlagen Angaben zu verlangen, aus denen Alter, Religionszugehörigkeit, Geschlecht oder eine etwaige Behinderung hervorgehen. $^{341}$ Wie schon bei der merkmalsneutralen Ausschreibung gilt auch hier, daß merkmalsneutrale Bewerbungsunterlagen nur begrenzt in der Lage sind, die Einstellungschancen von Menschen zu erhöhen, die Träger eines unerwünschten Merkmals sind. In der Mehrzahl der Fälle erlangt der Arbeitgeber spätestens im Bewerbungsgespräch von dem Merkmal Kenntnis, das der Bewerber in seinen Unterlagen nicht angeben müßte. Neutrale Bewerbungsunterlagen können deshalb regelmäßig nicht bewirken, daß der Arbeitgeber zwischen mehreren Bewerbern nicht seinen Präferenzen gemäß den Bewerber mit den erwünschten Merkmalen einstellt. ${ }^{342}$ Denkbar ist allerdings wieder, daß sich der Arbeitgeber trotz bestehender Vorbehalte im Einzelfall für den Bewerber mit dem unerwünschten Merkmal entscheidet: „,...] er hätte, bis auf die Farbe, die ihm anstößig war, schwören mögen, daß er nie etwas Schöneres gesehen. “343

Wesentliche Bedeutung kann das Verbot merkmalsspezifischer Bewerbungsunterlagen bei Merkmalen erlangen, die nach außen nicht unbedingt erkannt werden können. Zu denken ist dabei etwa an die Schwangerschaft, die Zugehörigkeit zu einer Religionsgemeinschaft oder die sexuelle Identität des Bewerbers.

Zusammenfassend läßt sich wiederum formulieren, daß merkmalsspezifische Bewerbungsunterlagen insoweit zulässig sind, als auch die Ablehnung aufgrund des jeweiligen Merkmals gerechtfertigt wäre.

\section{Fragerecht}

Das gleiche gilt für das „Fragerecht des Arbeitgebers“: Die Frage des Arbeitgebers nach dem Vorhanden- bzw. Nichtvorhandensein eines bestimmten Merkmals ist immer dann zulässig, wenn auch die Ablehnung des Vertragsschlusses mit dem Bewerber aufgrund eben dieses Merkmals sachlich begründet wäre. ${ }^{344}$

In diesem Sinne hat das $B A G$ entschieden, daß bei der Einstellung die Frage nach dem Vorliegen einer bestimmten Tatsache zulässig ist, wenn der Arbeitgeber ein berechtigtes, billigenswertes und schutzwürdiges Interesse an der Beantwortung seiner Frage für das Arbeitsverhältnis hat. ${ }^{345}$ Beantwortet der Bewerber die gestellte Frage falsch, kommt eine Anfechtung des geschlossenen

341 Bauer, NJW 2001, S. 2672 (2673).

342 Vergleiche dazu die Ausführungen $\mathrm{zu}$ dem Problem des „Zusammentreffens der Träger von Diskriminierungsmerkmalen“ unter Teil 3, Prüfungspunkt H.

343 Aus der Novelle „Die Verlobung in St. Domingo“ von Heinrich von Kleist.

344 In diesem Sinne auch Thüsing: „Wo eine Rechtfertigung nicht gegeben ist, hat der Arbeitgeber wie bereits jetzt beim Geschlecht auch beim Alter ein „Recht zur Lüge“. (Thüsing, NZA 2001, S. 1061 (1064)).

345 BAG, NJW 1991, S. 2723 (2723). 
Vertrages wegen arglistiger Täuschung gem. § 123 BGB nur dann in Betracht, wenn die Frage zulässigerweise gestellt worden war. ${ }^{346}$

Die Überlegungen zum Fragerecht des Arbeitgebers lassen sich auf sämtliche Schuldverhältnisse übertragen, in denen ein Verbot der Diskriminierung besteht.

Daß der Bewerber im Bewerbungsgespräch die Trägerschaft des nachgefragten Merkmals verneint, führt nicht dazu, daß er sich nach Vertragsschluß nicht auf das Vorhandensein eben dieses Merkmals berufen könnte. Grund dafür ist die Tatsache, daß Arbeitgeber und Bewerber die Vertragsverhandlungen nicht auf gleicher Augenhöhe führen. ${ }^{347}$ Deshalb verwirkt letzterer dadurch, daß er von seinem „Recht zur Lüge“ Gebrauch macht und sich nicht schon bei Vertragsschluß durch Individualvereinbarung Sonderrechte einräumen läßt, nicht seinen unter Umständen bestehenden Anspruch auf Gewährung derartiger Sonderrechte, denn seine ,[...] geringe Verhandlungsstärke macht es ihm praktisch unmöglich, für den Arbeitgeber ungünstige Nebenbedingungen zu vereinbaren. “348

Die folgenden Ausführungen dienen dem Ziel zu ergründen, welche Rechtsgeschäfte vom sachlichen Geltungsbereich des Diskriminierungsverbots umfaßt sein sollen.

\section{III. Öffentlich angebotene Verträge}

Die Beantwortung dieser Frage hängt zum einen von der Art und Weise ihres jeweiligen Zustandekommens ab. Gemäß Art. 3 I lit. h der Richtlinie 2000/43/EG gilt das Diskriminierungsverbot in bezug auf

„den Zugang zu und die Versorgung mit Gütern, die der Öffentlichkeit zur Verfügung stehen, einschließlich von Wohnraum. ${ }^{\text {*349 }}$

Der ADG-Entwurf des Bundesjustizministeriums vom 10.12.2001 hat das Öffentlichkeitserfordernis der Richtlinie 2000/43/EG dadurch umzusetzen gesucht, daß er gem. § 319 a I Nr. 1 lit. a öffentlich angebotene Verträge in den sachlichen Geltungsbereich des Diskriminierungsverbotes einbezogen hat. ${ }^{350}$ Öffentlichkeit soll nach der Entwurfsbegründung

346 BAG, NJW 1991, S. 2723 (2724).

347 So auch LAG Hamm, NJW 2002, S. 1970 (1971).

348 LAG Hamm, NJW 2002, S. 1970 (1971).

349 Hervorhebung durch den Verfasser; Eine ähnliche Formulierung enthält Art. 3 I Richtlinie 2004/113/EG.

350 Http://www.lsvd.de/antidiskriminierung/adgzivil02.html; Ähnlich ist § 319 c I Nr. 1 des ADG-Entwurfs des Bundesfamilienministeriums vom 06.05.2004 formuliert. gesellschaft.de/modules.php?name=News\&file=article\&sid=120; S. 19). 
beispielsweise bei öffentlichen Anpreisungen und Anzeigen in Tageszeitungen gegeben sein. ${ }^{351}$ Entscheidend sei, daß das Angebot den Bereich der Privatsphäre verlassen hat. ${ }^{352}$ Laut Entwurfsbegründung des ADG-Entwurfs des Bundesfamilienminsteriums vom 06.05.2004 erfaßt das Öffentlichkeitserfordernis „den praktisch häufigen Fall, in dem das öffentliche Angebot eine Aufforderung des Schuldners an mögliche Interessenten enthält, ihrerseits einen bindenden Antrag abzugeben (invitatio ad offerendum). Denkbar ist aber auch, daß der Anbietende sich selbst bereits nach $§ 145$ BGB bindet und dieser öffentlich erklärte Antrag nur noch der Annahme bedarf.“353

Mit Blick auf die bisher gemachten Ausführungen kann negativ formuliert werden, daß das Merkmal der Öffentlichkeit dann nicht erfüllt ist, wenn einer ganz bestimmten Person ein Vertragsangebot gemacht wird.

Beispiel: X möchte seine Wohnung vermieten und macht Y ein dementsprechendes Vertragsangebot.

$\mathrm{X}$ ist in dem Beispielsfall eine Ungleichbehandlung nicht vorzuwerfen, da er nur mit der Person in geschäftlichen Kontakt getreten ist, die Vertragspartner werden sollte. Von einer Ungleichbehandlung könnte in einer derartigen Situation nur dann gesprochen werden, wenn es „eine Diskriminierung durch Unterlassen“ gäbe, mithin die Verpflichtung bestünde, Vertragsangebote immer an die Öffentlichkeit zu richten. Art. 3 I lit. h Richtlinie 2000/43/EG verpflichtet jedoch nicht dazu, Güter und Dienstleistungen der Öffentlichkeit zur Verfügung zu stellen, sondern gewährleistet lediglich den gleichen Zugang zu öffentlich angebotenen Gütern und Dienstleistungen. 354

In diesem Sinne ist auch $\S 11$ AGG zu verstehen, der bestimmt, daß dann, wenn der Arbeitgeber einen Arbeitsplatz öffentlich ausschreibt, die Ausschreibung unter Beachtung des Diskriminierungsverbotes des $\S 7$ I AGG und damit grundsätzlich neutral hinsichtlich der in $\S 1$ AGG genannten Diskriminierungsmerkmale formuliert sein muß. Die Norm verpflichtet nicht dazu, jeden Arbeitsplatz öffentlich auszuschreiben.

\section{Entgeltlichkeit - Betrachtungen am Beispiel letztwilliger Verfügungen}

Fraglich ist, ob unentgeltliche Zuwendungen wie Schenkungen, Stipendien und letztwillige Verfügungen in den sachlichen Geltungsbereich des Diskriminierungsverbotes fallen. Die

351 Http://www.lsvd.de/antidiskriminierung/adgzivil02.html; S. 39.

352 Http://www.lsvd.de/antidiskriminierung/adgzivil02.html; S. 39.

353 Http://www.deutsche-gesellschaft.de/modules.php?name=News\&file $=$ article\&sid =1 20; S. 81 .

354 Nickel, NJW 2001, S. 2668 (2669). 
Antidiskriminierungsrichtlinien treffen diesbezüglich keine eindeutige Aussage. Am Beispiel der letztwilligen Verfügung soll die aufgeworfene Frage beantwortet werden.

Von der gem. Art. 14 I 1 GG grundrechtlich gewährleisteten Testierfreiheit ${ }^{355}$ sollen grundsätzlich auch willkürliche Entscheidungen des Erblassers gedeckt sein, etwa über die Bestimmung eines oder mehrerer Erben und deren Anteile oder über die rechtliche oder wirtschaftliche Aufteilung des Vermögens, selbst wenn dessen Motive keine besondere Achtung verdienen. ${ }^{356}$ Das Recht zu willkürlicher Entscheidung bedeutet dabei, daß der Erblasser niemandem zur Rechenschaft über die Gründe seiner Verfügung verpflichtet ist. ${ }^{357}$ Insbesondere soll der Erblasser von Verfassungs wegen nicht zu einer Gleichbehandlung seiner Abkömmlinge gezwungen sein. ${ }^{358}$ Im Gegenteil soll er grundsätzlich auch nahe Angehörige zugunsten familienfremder, nichtverwandter Personen übergehen können, seien doch die nächsten gesetzlichen Erben durch das Pflichtteilsrecht geschützt. $^{359}$

Nichtsdestotrotz ist die Testierfreiheit begrenzt; unter anderem durch das Verbot sittenwidriger Verfügungen von Todes wegen. ${ }^{360}$

Beispiel: „E setzt seinen Sohn zum Erben ein und enterbt seine Tochter, weil er die Frauen nicht leiden kann, weil sie ihm zu dumm ist, weil sie einen Juden, einen Neger geheiratet hat, weil sie einer anderen Kirche beigetreten ist. ${ }^{\text {‘361 }}$

\section{Unbegrenzte Anzahl potenzieller Diskriminierungsmerkmale}

Wenn der Erblasser nicht zur Gleichbehandlung verpflichtet sein und ein Recht zu willkürlicher Entscheidung haben soll, dann dürfte die letztwillige Verfügung des $\mathrm{E}$ in all den genannten Fallkonstellationen nicht dem Verdikt der Sittenwidrigkeit unterfallen. Teilweise wird dies auch vertreten, obwohl die letztwilligen Verfügungen in dem Beispielsfall der Wertentscheidung des Art. 3 III GG zuwiderliefen. ${ }^{362}$

In krassem Gegensatz dazu bejahen andere die Sittenwidrigkeit nicht nur dann, wenn eine Verfügung auf dem Motiv beruht, jemanden wegen eines der in Art. 3 III GG genannten Merkmale

355 BGH, JZ 1999, S. 514 (515); Bryde in von Münch/Kunig, Art. 14, Rn. 47.

356 Edenhofer in Palandt, $\$ 1937$, Rn. 3.

357 Otte in Staudinger, Vorbem zu §§ 2064 - 2086, Rn. 145; von Lübtow, Erbrecht, 1. Halbband, S. 308 f.

358 BVerfG, NJW 1985, S. 1455 (1456); Edenhofer in Palandt, § 1937, Rn. 3; Otte in Staudinger, Vorbem zu $\S 2064-$ 2086, Rn. 145.

359 Von Lübtow, Erbrecht, 1. Halbband, S. 308.

360 Edenhofer in Palandt, § 1937, Rn. 3 f.; Flume, Allgemeiner Teil des Bürgerlichen Rechts, Zweiter Band, § 1, 4 (S. 6); Otte in Staudinger, Vorbem zu §§ 2064 - 2086, Rn. 145; Brox, Erbrecht, Rn. 258.

361 Beispiel bei Brox, Erbrecht, Rn. 255.

362 So Brox, Erbrecht, Rn. 263; Lange-Kuchinke, Lehrbuch des Erbrechts, § 35 IV, S. 779 f.; Dürig in Maunz/Dürig, Band I, Art. 1 Abs. III, Rn. 128; Grunsky, JZ 1972, S. 765 (766); tendenziell ebenso Mayer-Maly, der behauptet, daß derartige letztwillige Verfügungen von Todes wegen ,dennoch gültig sein können“ (Mayer-Maly in MüKo, $\S$ 138, Rn. 16). 
zu diskriminieren, sondern auch dann, wenn sich das Motiv beispielsweise auf körperliche Merkmale, geistige Eigenschaften, besondere Lebensumstände, das Lebensalter oder die Klassenzugehörigkeit bezieht. ${ }^{363}$ Angesichts der Testierfreiheit wird jedoch einschränkend verlangt, daß derartig motivierte erbrechtliche Verfügungen prohibitive Wirkung entfalten, um tatsächlich sittenwidrig zu sein, was dann der Fall sein soll, wenn die von ihr ausgehenden Wirkungen derart intensiv sind, daß sie einen durchschnittlich standhaften Menschen veranlassen würden, das Diskriminierungsmerkmal abzulegen, wäre er dazu imstande. ${ }^{364}$ Sittenwidrig sollen letztwillige Verfügungen nach dieser Ansicht auch dann sein, wenn sie die durch Art. 1 GG geschützte Menschenwürde antasten, mithin erniedrigend wirken. ${ }^{365}$

Schließlich wird vertreten, den Diskriminierungsschutz im Rahmen letztwilliger Verfügungen auf die Merkmale des Art. 3 III GG zu erstrecken. ${ }^{366}$ Andere wollen ihn auf einzelne Merkmale des Art. 3 III GG ${ }^{367}$ bzw. auf besonders anstößige Verstöße gegen Art. 3 GG beschränken. ${ }^{368}$

Die Beschränkung des Diskriminierungsschutzes auf einzelne Merkmale des Art. 3 III GG setzt sich in Widerspruch zu der Tatsache, daß die Merkmale des Art. 3 III GG gleichwertig nebeneinanderstehen. In dieser Beschränkung wird deshalb zu recht eine Entwertung des Art. 3 III GG gesehen: „Beruft man sich nur auf die guten Sitten und gibt ihnen zudem noch selbstherrlich einen willkürlich begrenzten Inhalt, so bedarf es des Art. 3 gar nicht mehr. § 138 Abs. 1 BGB reicht dann aus. Dem Art. 3 GG wird nicht nur eine mittelbare, indirekte Wirkung zugestanden, sondern in Wahrheit gar keine. Was die guten Sitten (oder was der Rechtsanwendende dafür hält) nicht zuwege bringen, vermag dann auch Art. 3 nicht; denn er wird ja in das Korsett der (wirklichen oder angeblichen) guten Sitten gezwängt, und was diese nicht rechtfertigen, darf auch nach Art. 3 nicht rechtens sein. “369

Aber auch die Erstreckung des Diskriminierungsschutzes auf den gesamten Merkmalskatalog des Art. 3 III GG ist zu kritisieren. Dem Verfassungsgesetzgeber sei die Hervorhebung der Merkmale in Art. 3 III GG im Hinblick auf die Erfahrungen der nationalsozialistischen Vergangenheit besonders

363 Thielmann, Sittenwidrige Verfügungen von Todes wegen, S. $304 \mathrm{f}$.

364 Thielmann, Sittenwidrige Verfügungen von Todes wegen, S. 305.

365 Thielmann, Sittenwidrige Verfügungen von Todes wegen, S. 305; BGH, JZ 1999, S. 514 (516); Edenhofer in Palandt, § 1937, Rn. 20.

366 Von Lübtow, Erbrecht, 1. Halbband, S. 309.

367 Mikat in Festschrift für Nipperdey, Band I, S. 597 ff. (Beschränkung auf rassische und bedingt auf religiöse Diskriminierungen; in beiden Fällen jedoch nur bei vom Gesetz gewährter Erberwartung); Otte in Staudinger, Vorbem zu $\S 2064$ ff., Rn. 157.

368 Schlüter, Erbrecht, § 23 I, 2 f, S. 138; Lange-Kuchinke: „Nur dort, wo die Verfügung in einem Ausmaß mißbilligenswert ist, daß die staatliche Ordnung zu ihrer Durchführung nicht die Hand reichen kann, darf Nichtigkeit wegen Sittenwidrigkeit angenommen werden.“ (Lange-Kuchinke, Lehrbuch des Erbrechts, § 35 IV, S. 780) Wann ein solches mißbilligenswertes Ausmaß erreicht sein soll, wird offengelassen.; Stein spricht von „Extremfällen“ und nimmt einen solchen etwa bei einer Diskriminierung ausschließlich wegen der Rasse des gesetzlichen Erben an. Andererseits soll Sittenwidrigkeit nicht vorliegen, soweit der Erblasser eine Differenzierung nach den Merkmalen des Art. 3 III GG vornimmt, ohne objektiv und gezielt den Ausgeschlossenen in seiner Würde herabzusetzen. (Stein in Soergel, § 1937, Rn. 26).

369 Thielmann, Sittenwidrige Verfügungen von Todes wegen, S. 304. 
wichtig erschienen. ${ }^{370}$ Als abschließende Regelung dürfe die Norm indes nicht aufgefaßt werden. ${ }^{371}$ Es gelte vielmehr: „Was dem Abs. 3 des Art. 3 recht ist, muß seinem Abs. 1 billig sein.“372 Beiden Absätzen müsse Drittwirkung im Privatrecht zugestanden werden, wenn man die Drittwirkung der Grundrechte überhaupt anerkenne. ${ }^{373}$ Diese Ansicht deckt sich mit der an anderer Stelle getroffenen Feststellung, Diskriminierungsschutz lasse sich nicht auf einzelne Merkmale beschränken, aufgrund derer besonders häufig diskriminiert wird. „Der Teufel liebt es, sein Gewand zu wechseln, und ebenso wie der gebannt auf den Ungeist des Antisemitismus Starrende die modernen Formen rassischer Diskriminierung im Hinblick auf Gastarbeiter oder farbige Studenten zu leicht aus den Augen verliert, kann auch die Beschränkung auf Rasse und Religion von anderen zumindest gleichwertigen Diskriminierungen ablenken. “374

\section{Diskriminierungsverbot im Rahmen letztwilliger Verfügungen?}

Nachdem geklärt wurde, daß sich Diskriminierungsschutz auch im Rahmen letztwilliger Verfügungen nicht auf einzelne Merkmale beschränken läßt, ist die Frage zu beantworten, ob letztwillige Verfügungen überhaupt am Maßstab eines Diskriminierungsverbotes gemessen werden sollten. Folgende Überlegungen könnten dagegen sprechen:

\section{a) Sozialpflicht des Erblassers}

Gemäß Art. 14 I 1 GG sind Eigentum und Erbrecht gewährleistet. Gemäß Art. 14 II GG verpflichtet Eigentum. Sein Gebrauch soll zugleich dem Wohle der Allgemeinheit dienen. Diese Norm beauftragt den Gesetzgeber, wo geboten, dem Eigentümer die im Interesse Dritter und der Allgemeinheit gebotenen Schranken zu setzen. ${ }^{375}$ Bedürfe etwa ein Dritter der Nutzung des Eigentumsobjekts zu seiner Freiheitssicherung und verantwortlichen Lebensgestaltung, umfasse das grundgesetzliche Gebot einer am Gemeinwohl orientierten Nutzung die Pflicht zur Rücksichtnahme auf den Dritten. ${ }^{376}$ Art. 14 II GG erwähnt das Erbrecht nicht. Daraus ließe sich schlußfolgern, im Erbrecht und somit auch im Rahmen letztwilliger Verfügungen bestünden keine

370 Mikat in Festschrift für Nipperdey, Band I, S. 595 f.

371 Thielmann, Sittenwidrige Verfügungen von Todes wegen, S. $304 \mathrm{f}$.

372 Thielmann, Sittenwidrige Verfügungen von Todes wegen, S. 304 f.; Mikat: „Die Aufzählung in Absatz 3 ist zwar abschließend; daraus folgt aber nicht, daß eine Differenzierung nach dort nicht genannten Merkmalen zulässig sein müßte. Insoweit kommt vielmehr Art. 3 Abs. 1 GG wieder zur Anwendung. Abs. 3 ist eine historisch zu erklärende Konkretisierung des allgemeinen Gleichheitssatzes [...].“ (Mikat in Festschrift für Nipperdey, Band I, S. 595).

373 Thielmann, Sittenwidrige Verfügungen von Todes wegen, S. 305.

374 Thielmann, Sittenwidrige Verfügungen von Todes wegen, S. $303 \mathrm{f}$.

375 Jarass in Jarrass/Pieroth, Art. 14, Rn. 1.

376 Jarass in Jarrass/Pieroth, Art. 14, Rn. 42. 
Rücksichtnahmepflichten des Erblassers gegenüber Dritten. Ganz überwiegend wird indes angenommen, daß eine Sozialpflicht auch im Erbrecht besteht. ${ }^{377}$

\section{b) Rechtmäßiges Alternativverhalten}

Ist nun aber dieser Sozialpflicht nicht auch dann genüge getan, wenn in ,diskriminierender“ Weise vererbt wird? Durch die letztwillige Verfügung wird schließlich jemand begünstigt, wenn auch nicht der „Diskriminierte“. Hinzu kommt, daß dem Erblasser aus der letztwilligen Verfügung keine (materiellen) Vorteile erwachsen. Das gleiche gilt im übrigen für Schenkungen und Stipendien. ${ }^{378}$

Beispiel: Eine in Deutschland lebende islamische Familie bietet per Inserat einer anderen islamischen Familie ihre gebrauchte Küche und ihr Schlafzimmer als Geschenk an. ${ }^{379}$

Sollte man in dem Beispielsfall der Familie mit Sanktionen drohen, obwohl sie aus rein altruistischen Motiven heraus gehandelt hat? Die Familienmitglieder hätten als Eigentümer der Einrichtungsgegenstände selbige zerstören können. Aus diesem Verhalten wären für sie keine negativen Konsequenzen erwachsen. Soll man die Familie dafür „bestrafen“, daß sie, selbst wenn man ihr Motiv als fragwürdig einstufen sollte, durch die Schenkung der Allgemeinheit letztlich einen Dienst erweisen will?

Natürlich läßt sich in gleicher Weise beispielsweise bei Arbeitsverträgen argumentieren. Auch hier handelt der Arbeitgeber bereits dann gemeinnützig, wenn er überhaupt jemanden einstellt. So wird das Diskriminierungsverbot in der Einstellungssituation teilweise mit der Begründung kritisiert, es gäbe hier ein rechtmäßiges Alternativverhalten - gemeint ist die Einstellung des vom Arbeitgeber nicht diskriminierten Bewerbers. ${ }^{380}$ Das Argument, Erblasser und Schenker erfüllten auch bei diskriminierenden Entscheidungen ihre gem. Art. 14 II GG bestehende Sozialpflicht, hilft also nur bedingt weiter.

\section{c) Nachweis des Erblassermotivs}

Gegen die Ansicht, Diskriminierungsschutz auf den Bereich letztwilliger Verfügungen zu erstrecken, spricht indes folgendes Argument. Wenn der Erblasser seine Motivation für die Wahl

377 Bryde in von Münch/Kunig, Art. 14, Rn. 47; Berkemann in Umbach/Clemens, Art. 14, Rn. 721; Jarass in Jarass/Pieroth, Art. 14, Rn. 95; Brox, Erbrecht, Rn. 27; Papier in Maunz/Dürig, Art. 14, Rn. 297.

378 Cremer stellt die Verwandtschaft zwischen Schenkungen und Verfügungen von Todes wegen fest, indem er sagt, $\mathrm{daß}$ bei Schenkungen an eine außereheliche Geliebte oder einen außerehelichen Geliebten im Hinblick auf eine mögliche Sittenwidrigkeit die gleichen Gesichtspunkte zu prüfen seien wie bei einer Zuwendung von Todes wegen. (Cremer in Staudinger, $\S 516$, Rn. 11).

379 Beispiel bei Säcker, ZRP 2002, S. 286 (288).

380 Adomeit, DB 1980, S. 2388 (2388).

106 
der Erben bzw. den Grund dafür, warum bestimmte Personen nicht erben sollen, in seiner letztwilligen Verfügung nicht angibt, entsteht ein Beweisproblem, denn derjenige, „der die Gründe für seine Ungleichbehandlung an sich gleichstehender Personen im Testament in erster Linie und oft sogar als einziger kennt, ist [...] im Zeitpunkt, in dem es auf den Beweis der rechtfertigenden Gründe ankäme, tot.“381 Die durch die Antidiskriminierungsrichtlinien vorgesehene Beweiserleichterung für den „Diskriminierten“382 könnte in diesem Fall unter Umständen ins Leere laufen. Dies allein vermag den Ausschluß der Geltung des Diskriminierungsverbotes im Rahmen letztwilliger Verfügungen natürlich nicht $\mathrm{zu}$ rechtfertigen, würden doch verdeckte Diskriminierungen andernfalls regelrecht belohnt. Außerdem sind beweisrechtliche und materiellrechtliche Ebene grundsätzlich strikt voneinander zu trennen.

\section{d) Pflichtteilsrecht contra Diskriminierungsschutz}

Den Diskriminierungsschutz auf letztwillige Verfügungen zu erstrecken hieße, sich in Widerspruch zum Pflichtteilsrecht zu setzen. Gemäß § 2303 BGB steht bestimmten Personen, den Pflichtteilsberechtigten, eine Mindestbeteiligung am Nachlaß des Erblassers auch dann zu, wenn sie durch Verfügung von Todes wegen von der Erbfolge ausgeschlossen wurden. ${ }^{383}$ Auch dies spricht gegen die Statuierung eines Diskriminierungsverbotes im Rahmen letztwilliger Verfügungen.

\section{e) Recht auf willkürliche Entscheidung oder Diskriminierungsschutz}

Ein weiteres Argument spricht gegen die Gewährung von Diskriminierungsschutz in diesem Bereich. Wenn behauptet wird, die Testierfreiheit erlaube dem Erblasser willkürliches Handeln, ${ }^{384}$ dann müßte er seiner Entscheidung a maiore ad minus auch mißbilligenswerte Gründe zugrunde legen können. In diesem Sinne wird vertreten, § 138 BGB solle nur den Eintritt mißbilligter

381 Otte in Staudinger, Vorbem zu §§ 2064 ff., Rn. 147 a; Das Problem erkennen auch Mikat und Thielmann. (Mikat in Festschrift für Nipperdey, Band I, S. 601; Thielmann, Sittenwidrige Verfügungen von Todes wegen, S. 309 ).

382 Gemäß Art. 8 I Richtlinie 2000/43/EG ergreifen die Mitgliedstaaten im Einklang mit ihrem nationalen Gerichtswesen die erforderlichen Maßnahmen, ,[...] um zu gewährleisten, daß immer dann, wenn Personen, die sich durch die Nichtanwendung des Gleichbehandlungsgrundsatzes für verletzt halten und bei einem Gericht oder einer anderen zuständigen Stelle Tatsachen glaubhaft machen, die das Vorliegen einer unmittelbaren oder mittelbaren Diskriminierung vermuten lassen, es dem Beklagten obliegt zu beweisen, daß keine Verletzung des Gleichbehandlungsgrundsatzes vorgelegen hat." (vgl. auch Art. 10 I RL 2000/78/EG sowie Art. 9 I RL 2004/113/EG); Eine entsprechende Regelung enthält die Richtlinie 76/207/EWG nicht. Jedoch besteht gem. Art. 4 I Richtlinie 97/80/EG über die Beweislast bei Diskriminierung aufgrund des Geschlechts - Abl. EG 1998 Nr. L 14, S. 6 ff. - auch im Anwendungsbereich dieser Richtlinie die in den genannten Richtlinien enthaltene Beweislastverteilung.

383 Daß neben dem Pflichtteilsrecht weitere Schranken der Testierfreiheit in Form der $\S \S 134,138$ BGB in Betracht kommen, sich Pflichtteilsrecht und Diskriminierungsschutz somit nicht zwingend gegenseitig ausschließen, stellt indes der $B G H$ fest. (BGH, JZ 1999, S. 514 (515)) Das Gericht hebt aber gleichzeitig hervor, daß vor dem Hintergrund des Pflichtteilsrechts eine Ungleichbehandlung der Kinder grundsätzlich unbedenklich ist. (BGH, JZ 1999, S. 514 (515 f.)).

384 Otte in Staudinger, Vorbem zu $\S \S 2064$ ff., Rn. 154; BGH: „Selbst wenn der Erblasser eine willkürliche Differenzierung vornimmt, kann dies Ausdruck seiner Testierfreiheit sein." (BGH, JZ 1999, S. 514 (515)). 
Rechtswirkungen verhindern, nicht aber den Erblasser wegen seiner zu mißbilligenden Gesinnung bestrafen. ${ }^{385}$ Wenn jemand nicht verpflichtet sein soll, sein Verhalten zu begründen, ${ }^{386}$ dann muß dies auch dann gelten, wenn die Vermutung diskriminierenden Verhaltens nahe liegt. Andernfalls bestünde eben doch eine Begründungspflicht und damit gerade kein Recht zu willkürlichem Verhalten. Die Erforschung des Erblasserwillens hat deshalb zu unterbleiben, wenn diesem das Recht zu willkürlichem Handeln eingeräumt wird. ${ }^{387}$ Das Recht auf Willkür läßt sich mit einem gleichzeitig bestehenden Diskriminierungsverbot nicht vereinbaren. Das eine steht zu dem anderen in einem „Entweder-Oder-Verhältnis“. Entweder man gewährt dem einzelnen das Recht, willkürliche Entscheidungen zu treffen, und zwar ohne eng begrenzte Ausnahmefälle, ${ }^{388}$ oder man statuiert ein Diskriminierungsverbot. ${ }^{389}$

\section{Resümee}

Ein zwingender Grund für oder gegen die Geltung des Diskriminierungsverbots in den Fällen unentgeltlicher Zuwendungen läßt sich nicht finden. Die Ausführungen haben jedoch gezeigt, daß vieles dafür spricht, im Falle (wirtschaftlich) rein altruistischen Verhaltens kein Diskriminierungsverbot aufzustellen. Daß diese Ansicht vom Ergebnis her billigenswert ist, zeigt folgendes: Es dürfte wenig wahrscheinlich sein, daß in dem letzten Beispielsfall jemand Klage erheben wird, weil sich das Schenkungsangebot in dem Inserat ausschließlich an islamische Familien richtete. Das Inserat wird Andersgläubige nicht empören, weil sie sich durch selbiges nicht ausgeschlossen fühlen werden. Niemand wird bei einem derartigen Inserat ernsthaft davon ausgehen, es bestehe eine Anwartschaft auf die unentgeltliche Zuwendung. ${ }^{390}$ Deshalb wird sich auch das Gefühl, diskriminiert zu sein, nicht einstellen.

Ganz anders liegt der Fall beispielsweise, wenn dem Chinesen, Türken oder Schwarzafrikaner untersagt wird, ein bestimmtes Lokal zu betreten. Die angesprochenen Personen werden sich diskriminiert fühlen, ist es doch Wesen eines Lokalbetriebs, Menschen egal welcher Coleur zu bewirten. Die Zurückgewiesenen erwarten in einem solchen Fall berechtigterweise, bewirtet zu

385 Schlüter, Erbrecht, § 23, I 2 e, S. 135.

386 Im Rahmen letztwilliger Verfügungen bedeutet das Recht zu willkürlicher Entscheidung, daß der Erblasser niemandem Rechenschaft über die Gründe seiner Verfügung ablegen muß. (Otte in Staudinger, Vorbem zu $§ \S 2064$ - 2086, Rn. 145; von Lübtow, Erbrecht, 1. Halbband, S. 308 f.).

387 Nach Mikat ist es dagegen Aufgabe des Tatrichters, die Motive des Erblassers zu ergründen. (Mikat in Festschrift für Nipperdey, Band I, S. 603).

388 Von ,eng begrenzten Ausnahmefällen“ spricht indes der BGH. (BGH, JZ 1999, S. 514 (516)).

389 Das gleiche Problem stellt sich im Arbeitsrecht bei der Frage, ob der Arbeitgeber außerhalb des Kündigungsschutzgesetzes (KSchG) ein Recht zu willkürlicher Kündigung hat. (vgl. dazu Teil 2, Prüfungspunkt D. VI. 3.).

390 Im Falle letztwilliger Verfügungen stellt sich dies etwas anders dar. Dort werden sich nahe Angehörige zurückgesetzt fühlen, wenn sie nicht bedacht werden. Mikat bejaht die Erberwartung gesetzlicher Erben. Nur bei ihnen komme überhaupt eine Diskriminierung in Betracht. (Mikat in Festschrift für Nipperdey, Band I, S. 600); a.A.: Bryde: Das Recht, Erbe zu werden, sei bis zum Erbfall regelmäßig bloße Hoffnung und deshalb von Art. 14 I GG nicht geschützt. (Bryde in von Münch/Kunig, Art. 14, Rn. 45). 
werden. Gleiches gilt etwa für die geschlechtsbezogene Ausschreibung im Falle einer Tätigkeit, die von beiden Geschlechtern ausgeübt werden kann.

Im Falle letztwilliger Verfügungen tritt noch ein verschärfender Umstand hinzu. Während die islamische Familie in dem Beispielsfall an die Öffentlichkeit tritt, indem sie das Inserat aufgibt, ist das Merkmal der Öffentlichkeit im Fall letztwilliger Verfügungen von Todes wegen nicht gegeben. Hier ließe sich allenfalls argumentieren, eine auf bestimmte Personen beschränkte „QuasiÖffentlichkeit“" sei durch deren Erberwartung gegeben.

\section{Gewerbsmäßiges und nichtgewerbsmäßiges Handeln}

Die bisher gemachten Einschränkungen des sachlichen Geltungsbereichs weisen erhebliche Parallelen zum Gewerbebegriff auf - gemeint sind die Erfordernisse des öffentlich angebotenen Vertrages sowie der Entgeltlichkeit. Es liegt deshalb nicht fern $\mathrm{zu}$ fragen, ob das Diskriminierungsverbot ausschließlich bei gewerbsmäßiger Tätigkeit gilt.

Unter Gewerbe wird überwiegend eine berufsmäßige und selbständige von der Absicht dauernder Gewinnerzielung getragene Tätigkeit verstanden. ${ }^{391}$ Kennzeichnend für ein Gewerbe ist seine anbietende Tätigkeit am Markt. ${ }^{392}$ Dieses Merkmal soll nur bei werbender Tätigkeit nach außen erfüllt sein. ${ }^{393}$ Hier zeigt sich die erste Parallele zum sachlichen Geltungsbereich des Diskriminierungsverbotes, der nur öffentlich angebotene Verträge erfaßt.

Die zweite Parallele besteht in der Entgeltlichkeit. Nur eine entgeltliche Tätigkeit am Markt soll ein Gewerbe begründen. ${ }^{394}$

Kennzeichnend für ein Gewerbe ist weiterhin, daß die anbietende Tätigkeit auf unbestimmt viele Geschäfte gerichtet ist, wobei nicht erforderlich ist, daß die Tätigkeit die Haupteinnahmequelle darstellt. ${ }^{395}$ Wer alle zwei Jahre sein Auto weiterverkauft, wer gelegentlich an der Börse spekuliert, wer ererbte Grundstücke parzelliert und zu Auseinandersetzungszwecken verkauft, wer auf einem Flohmarkt hin und wieder alte Familienstücke anbietet oder als Student seine juristischen Lehrbücher an jüngere Kommilitonen weiterverkauft, soll kein Gewerbe betreiben. ${ }^{396}$

Richtlinie 2004/113/EG gilt gem. ihres Art. 3 I

\footnotetext{
391 Schmidt, Handelsrecht, S. 280 f.

392 Schmidt, Handelsrecht, S. 283.

393 Schmidt, Handelsrecht, S. 285.

394 Schmidt, Handelsrecht, S. 288.

Schmidt, Handelsrecht, S. 288.

396 Schmidt, Handelsrecht, S. 288.
} 
„,[...] für alle Personen, die Güter und Dienstleistungen bereitstellen, die der Öffentlichkeit ohne Ansehen der Person zur Verfügung stehen, und zwar in öffentlichen und privaten Bereichen, einschließlich öffentlicher Stellen, und die außerhalb des Bereichs des Privat- und Familienlebens und der in diesem Kontext stattfindenden Transaktionen angeboten werden. ${ }^{\text {‘397 }}$

Die Bestimmung bezieht auf der einen Seite den privaten Bereich in den sachlichen Geltungsbereich des Diskriminierungsverbotes ein. Auf der anderen Seite soll das Diskriminierungsverbot im Privatleben keine Geltung beanspruchen.

Man wird wohl davon ausgehen können, daß derjenige den Bereich des Privatlebens verläßt, der ein Inserat aufgibt, auf das sich zwangsläufig auch Fremde melden werden. Selbst dann, wenn er dies einmalig tut. Richtlinie 2004/113/EG wird deshalb in der Weise interpretiert, daß der von ihr statuierte sachliche Geltungsbereich nicht auf gewerbsmäßiges Handeln beschränkt ist. ${ }^{398}$

Selbst wenn die Richtlinie eine solche Einschränkung machte, stellte sich die Frage, ob es überhaupt gerecht wäre, lediglich den Gewerbetreibenden an das Diskriminierungsverbot zu binden. Man könnte argumentieren, der nicht gewerbsmäßig Handelnde trage ein größeres Risiko in bezug auf seinen Geschäftspartner, weil er in der Regel nicht so finanzkräftig ist wie ein Unternehmen. Der private Vermieter, der eine Wohnung vermietet, sichert unter Umständen durch den Mietzins seinen eigenen Lebensunterhalt. Ein Unternehmen, das gewerbsmäßig Wohnungen vermietet, ist zwar ebenso vom pünktlichen Eingang des Mietzinses abhängig. Gleichwohl wird man behaupten können, daß es einzelne Ausfälle eher verkraften wird als der Private, der lediglich eine Wohnung vermietet.

Trotz des tendenziell höheren Risikos des nicht gewerbsmäßig Handelnden sollte man diesen nicht gegenüber dem Gewerbetreibenden privilegieren. Auch ersterer hat die Möglichkeit, sich vor Vertragsschluß gegen befürchtete Schäden abzusichern. So kann der Vermieter etwa vom Interessenten der Wohnung verlangen, daß dieser den Nachweis seiner Bonität erbringt.

Natürlich bleibt auch dann ein Restrisiko bestehen. Es gibt eben (noch) nicht den gläsernen Vertragspartner, bei dem sich jedes Risiko ausschließen ließe. Da dieses Restrisiko jedoch Wesensmerkmal eines jeden Vertragsverhältnisses ist, läßt sich die alleinige Befreiung des nicht gewerbsmäßig Handelnden vom Verbot der Diskriminierung mit diesem Argument nicht rechtfertigen.

\section{Verträge ohne engen persönlichen Kontakt zum Vertragspartner}

397 Vergleiche auch Art. 3 I lit. h sowie Erwägungsgrund (4) der Richtlinie 2000/43/EG.

398 Das gleiche gilt für die Richtlinie 2000/43/EG. Wiedemann/Thüsing merken jedoch an, daß ,auch eine Ausnahmeklausel für nicht-gewerbliche und nicht-berufliche Geschäfte von Privatpersonen mit dem Europäischen Recht wohl vereinbar wäre.“(Wiedemann/Thüsing, DB 2002, S. 463 (465)). 
Rechtsverhältnisse schaffen ein mehr oder weniger ausgeprägtes Näheverhältnis der Vertragspartner zueinander. Auch dies ist wesentlich für die Bestimmung des sachlichen Bereichs, in dem das Diskriminierungsverbot gelten soll.

\section{Intimsphäre}

Beispiel: X setzt eine Anzeige in die Zeitung, in der er Frauen zwecks Heirat sucht.

Mit dem „Argument“, Männer würden, wenn sie heiraten, Frauen als Ehepartner deutlich bevorzugen, ${ }^{399}$ wurde versucht, das Verbot privater Diskriminierung als tödliche Bedrohung privater Freiheit ${ }^{400} \mathrm{zu}$ brandmarken. Daß ein Diskriminierungsverbot auch den Intimbereich des Menschen umfassen soll, wird sicherlich von niemandem ernsthaft vertreten. ${ }^{401}$ „Daß jeder jeden liebt, das überlassen wir vielleicht besser Jesus. Liebe ist etwas ganz anderes. Sie hat überhaupt keine Ähnlichkeit mit Großzügigkeit und auch nicht mit Mitgefühl. Im Gegenteil.“402 In dem Beispielsfall liegt keine Diskriminierung vor, da er in einen Bereich fällt, der vom Verbot der Diskriminierung nicht umfaßt ist.

\section{Privatsphäre}

Beispiel: Eine ältere Dame möchte per Inserat an eine evangelische Studentin ein Zimmer ihrer Wohnung vermieten. Ist sie gezwungen, ihr Inserat merkmalsneutraler auszugestalten, mit anderen Worten verpflichtet, ihr Zimmer beispielsweise auch katholischen Studenten anzubieten $?^{403}$

\section{Gemäß $§ 573$ a I 1 BGB kann der Vermieter}

„[e]in Mietverhältnis über eine Wohnung in einem vom Vermieter selbst bewohnten Gebäude mit nicht mehr als zwei Wohnungen [...] auch kündigen, ohne daß es eines berechtigten Interesses im Sinne des $§ 573$ bedarf.“

Ratio legis dieser Norm ist es, dem Vermieter aufgrund der Spannungen, die das enge Zusammenleben mit sich bringen kann, eine erleichterte Kündigungsmöglichkeit einzuräumen. ${ }^{404}$ Diese soll unabhängig vom Vorliegen eines sachlichen Grundes für die Zerrüttung bestehen. ${ }^{405}$

Tomuschat in Festschrift für Schlochauer, S. 711

400 Tomuschat in Festschrift für Schlochauer, S. 712.

401 Nickel, Gleichheit und Differenz in der vielfältigen Republik, S. 103.

402 Amos $O z$ in „Eine Geschichte von Liebe und Finsternis“.

403 Beispiel bei Säcker, ZRP 2002, S. 286 (288).

404 Weidenkaff in Palandt, $\S 573$ a, Rn. 1.

405 Teilweise wird der Zweck der erleichterten Kündigungsmöglichkeit gerade darin gesehen, daß in den meisten Fällen die Ursachen der Zerrüttung nicht aufgeklärt werden können. (so Voelskow in MüKo, Band 3, § 564 b, Rn. $8)$. 
Augenscheinlich steigt die Handlungsfreiheit des einzelnen, je stärker eine Regelung seinen Privatbereich betrifft. ${ }^{406}$ Grund dafür ist das durch Art. 2 I GG verfassungsrechtlich anerkannte allgemeine Persönlichkeitsrecht, das dem einzelnen einen Raum gewährt, in dem er unbeobachtet sich selbst überlassen ist oder mit Personen seines besonderen Vertrauens ohne Rücksicht auf gesellschaftliche Verhaltenserwartungen verkehren kann. ${ }^{407}$

In diesem Sinne umfaßt der sachliche Geltungsbereich der Richtlinie 2004/113/EG gem. ihres Art. 3 I nicht den Bereich ,des Privat- und Familienlebens und der in diesem Kontext stattfindenden Transaktionen“. ${ }^{408}$ Dieser Rechtsgedanke ist dem Antidiskriminierungsrecht nicht fremd. So soll der durch Art. 26 des Internationalen Paktes über bürgerliche und politische Rechte gewährte Schutz begrenzt sein auf Diskriminierungen im quasi-öffentlichen Bereich von Arbeitsverhältnissen, Schulen, Verkehrsmitteln, Hotels, Restaurants, Theater, Parks, Stränden etc., da auch die Verfasser dieser Bestimmung grundsätzlich davon ausgegangen seien, daß Diskriminierungen in privaten Beziehungen eine Angelegenheit legitimer persönlicher Entscheidungen sind, geschützt vom Recht auf Privatheit gegen staatliche Eingriffe. ${ }^{409}$ In diesem Sinne verpflichten sich die Vertragsstaaten in Art. 5 lit. f des Internationalen Übereinkommens zur Beseitigung jeder Form von Rassendiskriminierung

„das Recht [jedes einzelnen] auf Zugang zu jedem Ort oder Dienst, der für die Benutzung durch die Öffentlichkeit vorgesehen ist, wie Verkehrsmittel, Hotels, Gaststätten, Cafés, Theater und Parks [, zu gewährleisten.]“

Gemäß § 1 I des ADG-Entwurfs von Bündnis 90/Die Grünen vom 20.01.1998 hat jede Person Anspruch auf Gleichbehandlung im Rechtsverkehr. ${ }^{410}$ In der Entwurfsbegründung heißt es dazu, das Diskriminierungsverbot erfasse nur den Rechtsverkehr, nicht das allgemeine private Verhalten. ${ }^{411}$ Was unter dem Begriff ,allgemeines privates Verhalten“ zu verstehen ist, wird allerdings nicht geklärt. Auch der Gesetzesentwurf der SPD vom 09.03.1998 ist insofern sehr unbestimmt formuliert. $^{412}$

Vor dem Hintergrund der soeben getroffenen Feststellungen kann davon ausgegangen werden, daß $\S 573$ a I 1 BGB auch in Zukunft Bestand haben wird. ${ }^{413}$ Gegen das AGG verstößt die Norm jedenfalls nicht, findet doch gem. § 19 V AGG das zivilrechtliche Benachteiligungsverbot

406 Mager, ZAR 1992, S. 170 (173).

407 BVerfG, NJW 1995, S. 1477 (1477); Säcker, ZRP 2002, S. 286 (288).

408 Vergleiche auch Art. 3 I lit. h sowie Erwägungsgrund (4) der Richtlinie 2000/43/EG.

409 Nowak, der beispielhaft erwähnt, daß niemand gezwungen werden könne, Schwarze, Frauen oder Juden zu einer privaten Party einzuladen (Nowak, CCPR-Kommentar, Art. 26, Rn. 31).

410 BT-Druck. 13/9706, S. 3.

411 Entwurfsbegründung, BT-Druck. 13/9706, S. 11.

412 BT-Druck. 13/10081, S. 11.

413 A.A. insoweit Nickel, der - unter Anführung des Beispiels, daß eine Einliegerwohnung in einem vom Vermieter selbst bewohnten Einfamilienhaus vermietet wird - die Auffassung vertritt, daß ,der Bereich zugelassener Beliebigkeit verlassen wird, sobald der Wohnraum öffentlich angeboten wird.“ (Nickel, NJW 2001, S. 2668 (2669)). 
„keine Anwendung auf zivilrechtliche Schuldverhältnisse, bei denen ein besonderes Näheverhältnis oder Vertrauensverhältnis der Parteien oder ihrer Angehörigen begründet wird. Bei Mietverhältnissen kann dies insbesondere der Fall sein, wenn die Parteien oder ihre Angehörigen Wohnraum auf demselben Grundstück nutzen. [...]“

Noch eindeutiger fällt die Entscheidung im Ausgangsfall aus. Die „eigenen vier Wände“ müssen vom sachlichen Geltungsbereich des Diskriminierungsverbotes unberührt bleiben. ${ }^{414}$

Was für die Kündigung durch $\S 573$ a I 1 BGB explizit geregelt ist, gilt als deren Kehrseite freilich auch für den Vertragsschluß. Ein Diskriminierungsverbot im Vorfeld des Vertragsschlusses macht keinen Sinn, wenn es nicht mit einem ebensolchen Verbot bei der Durchführung und Beendigung des Vertragsverhältnisses einhergeht. Im Falle des § 19 V 1, 2 AGG findet das zivilrechtliche Benachteiligungsverbot des $\S 19$ I AGG in toto und damit auch bei der Begründung des Vertragsverhältnisses keine Anwendung.

Fraglich ist, ob eine Kündigung gem. § 573 a I 1 BGB dem Verdikt der Sittenwidrigkeit unterfallen und deshalb gem. § 138 BGB unwirksam sein kann. Die Anwendbarkeit des $§ 138$ BGB ist nicht von vornherein ausgeschlossen, gilt die Norm doch bei Rechtsgeschäften jeder Art, einschließlich einseitiger wie der Kündigung. ${ }^{415}$ Bei den hier diskutierten Fällen kann die Sittenwidrigkeit jedoch zumindest nicht mit dem Vorliegen einer Diskriminierung begründet werden, da in diesem Bereich ein Diskriminierungsverbot nicht besteht. Ob dies auch für den gegenüber $\S 573$ a I 1 BGB viel weiter gefaßten $\S 19$ I 1, 2 AGG gilt, ist indes ebenso fraglich wie dessen Vereinbarkeit mit den europarechtlichen Vorgaben.

\section{Sozialsphäre - Betrachtungen am Beispiel arbeitsrechtlicher Kündigungen}

Die bisherigen Ausführungen haben gezeigt, daß das Diskriminierungsverbot im Bereich der Intimund Privatsphäre keine Geltung beanspruchen kann. Im Gegensatz dazu gilt es grundsätzlich immer dort, wo ein (potenzielles) Vertragsverhältnis den Bereich der Privatsphäre verläßt und in den Bereich der Sozialsphäre eintaucht. ${ }^{416}$ So verbieten die Antidiskriminierungsrichtlinien Diskriminierung unter anderem im Arbeitsrecht, in einem Bereich also, der der Sozialsphäre zuzuordnen ist.

Fraglich ist, ob das Diskriminierungsverbot die Sozialsphäre vollständig erfaßt, oder ob auch sie Bereiche kennt, in denen der einzelne ein Recht zu willkürlichem Handeln hat.

414 Auch Säcker verneint in dem Ausgangsfall das Vorliegen einer Diskriminierung. (Säcker, ZRP 2002, S. 286 (288)).

415 Heinrichs in Palandt, $\S 138$, Rn. 11.

416 Die Begriffe Intim-, Privat- und Sozialsphäre werden im Zusammenhang mit der im deutschen Verfassungsrecht entwickelten „Sphärentheorie“ verwendet. (vgl. dazu Pieroth/Schlink, Grundrechte, Staatsrecht II, Rn. 376; Dreier in Dreier, Band I, Art. 2 I, Rn. 88). 
Im Arbeitsrecht scheint es derartige Bereiche zu geben. So verlangt die ordentliche Kündigung gem. §§ 620 II, 622 BGB lediglich eine Kündigungsfrist, nicht aber einen Kündigungsgrund.

Nicht übersehen werden darf jedoch, daß in den Fällen, in denen besonderer oder allgemeiner Kündigungsschutz besteht, auch die ordentliche Kündigung eines sachlichen Grundes bedarf. ${ }^{417}$ Der Grundsatz, die ordentliche Kündigung bedürfe keines Grundes, hat sich auf diese Weise praktisch in sein Gegenteil verkehrt.

Allgemeiner Kündigungsschutz besteht gem. § 1 I KSchG nicht während der ersten 6 Monate des Beschäftigungsverhältnisses und gem. $\S 23 \mathrm{I} 2 \mathrm{KSchG}$ nicht in Betrieben mit bis zu fünf Arbeitnehmern bzw. gem. § 23 I 3 KSchG nicht für Arbeitnehmer in Betrieben mit bis zu zehn Arbeitnehmern, deren Arbeitsverhältnis nach dem 31.12.2003 begonnen hat. ${ }^{418}$ Nichtsdestotrotz wird auch für die sog. Probezeit und für Betriebe und Arbeitnehmer, die der sog. Kleinbetriebsklausel unterfallen, die Frage nach einem Kündigungsschutz gestellt. Das BVerfG hat klargestellt, daß Arbeitnehmer außerhalb des Geltungsbereichs des KSchG durch die Generalklauseln des Zivilrechts vor sitten- oder treuwidriger Ausübung des Kündigungsrechts durch den Arbeitgeber geschützt sind. ${ }^{419}$

\section{a) Unbegrenzte Anzahl potenzieller Diskriminierungsmerkmale}

Auch im kündigungsschutzfreien Raum dürfe insbesondere nicht gegen Grundrechte verstoßen werden. ${ }^{420}$ Sitten- bzw. treuwidrig und damit nichtig soll beispielsweise eine Kündigung während der Probezeit sein, die allein aufgrund der Homosexualität des Arbeitnehmers erfolgt. ${ }^{421}$ Gleiches gelte für Fälle, in denen der Arbeitgeber einen Arbeitnehmer allein deshalb entläßt, weil dieser Träger eines der in Art. 3 III GG bezeichneten Merkmale ist ${ }^{422}$ bzw. von seiner durch Art. 5 I 1 GG garantierten Meinungsfreiheit Gebrauch gemacht hat. ${ }^{423}$

417 Zum besonderen und allgemeinen Kündigungsschutz vgl. Junker, Grundkurs Arbeitsrecht, Rn. 338 ff.

418 Nach dem Koalitionsvertrag von SPD und CDU von 2005 sollen Arbeitgeber und Arbeitnehmer eine Ausweitung der gesetzlichen Probezeit von sechs auf vierundzwanzig Monate vereinbaren können. (FAZ vom 14.11.2005, S. 6).

419 BVerfG, NJW 1998, S. 1475 (1476); vgl. bzgl. sittenwidriger Kündigungen in diesem Bereich u. a. v. HoyningenHuene/Linck, Kündigungsschutzgesetz, § 13, Rn. 68; Biebl in APS, § 13 KSchG, Rn. 48

Anzumerken ist in diesem Zusammenhang, daß § 13 II KSchG gegenüber $§ 138$ BGB keine Sperrwirkung entfaltet. Die Bestimmung hat neben $\S 138$ BGB lediglich deklaratorische Funktion. (Preis, Prinzipien des Kündigungsschutzrechts bei Arbeitsverhältnissen, S. 396) § 13 II 1 KSchG lautet: „Verstößt eine Kündigung gegen die guten Sitten, so kann der Arbeitnehmer ihre Nichtigkeit unabhängig von den Vorschriften dieses Gesetzes geltend machen.".

420 Preis, NZA 1997, S. 1256 (1266).

421 Das BAG stützte seine Entscheidung auf § 242 BGB. (BAG, NZA 1994, S. 1080 (1082)) Während Oetker diese Rechtsprechung überzeugend findet, ist nach Preis $\S 138$ BGB bzw. § 612 a BGB i.V.m. Art. 2 I GG sedes materiae. (Oetker, ArbuR 1997, S. 41 (52); Preis, NZA 1997, S. 1256 (1266)) Auch v. Hoyningen-Huene/Linck halten eine derartige Kündigung für sittenwidrig. (v. Hoyningen-Huene/Linck, Kündigungsschutzgesetz, $\S 13$, Rn. 64).

422 BVerfG, NJW 1998, S. 1475 (1476); Preis, NZA 1997, S. 1256 (1266); Oetker, ArbuR 1997, S. 41 (48).

423 Oetker, ArbuR 1997, S. 41 (48). 
Fraglich ist, inwieweit der Arbeitgeber darüber hinaus Kündigungen im kündigungsschutzfreien Raum auf sachliche Gründe stützen muß.

Beispiel: Ein Arbeitgeber beabsichtigt, einem Angestellten während der Probezeit zu kündigen, weil er dem Arbeitgeber - obwohl er seine arbeitsvertraglichen Pflichten in adäquater Weise erfüllt - aufgrund seines äußeren Erscheinungsbildes und persönlichen Auftretens im Betrieb unsympathisch geworden ist, so daß der Arbeitgeber mit ihm nicht mehr zusammenarbeiten will. ${ }^{424}$

Nach einer Ansicht muß ein grundrechtlich geleitetes Verständnis von Treu und Glauben dazu führen, daß eine ordentliche Kündigung nach § 242 BGB nichtig ist, wenn sich der Arbeitgeber für die Beendigung des Arbeitsverhältnisses nicht auf einen sachbezogenen, im Zusammenhang mit dem Arbeitsverhältnis stehenden Grund stützen kann. ${ }^{425}$ Nichtig soll eine ordentliche Kündigung z.B. dann sein, wenn der Arbeitgeber das Arbeitsverhältnis allein deshalb kündigt, weil das äußere Erscheinungsbild und das persönliche Auftreten des Arbeitnehmers dazu führen, daß er dem Arbeitgeber unsympathisch geworden ist. ${ }^{426}$ Den Vertretern dieser Meinung ist zu entgegnen, daß Antipathie sehr wohl ein sachbezogener, im Zusammenhang mit dem Arbeitsverhältnis stehender Grund sein kann.

Nach einer anderen Ansicht benötigt der Arbeitgeber außerhalb des KSchG weder wichtige noch triftige noch sachliche Gründe für eine wirksame Erklärung der ordentlichen Kündigung. ${ }^{427}$ Die willkürliche Kündigung sei außerhalb des KSchG grundsätzlich erlaubt. ${ }^{428}$ Zwar unterfallen auch nach dieser Ansicht die nicht am Maßstab des KSchG überprüfbaren Kündigungen einem allgemeinen Mißbrauchsvorbehalt, doch soll mißbräuchliches Verhalten immer schon dann ausscheiden, wenn ein irgendwie einleuchtender Grund - der nicht notwendig arbeitsvertragsbezogen sein muß - für die Rechtsausübung vorliegt. ${ }^{429}$ Willkürliches Verhalten soll nur dann treuwidrig sein, wenn besondere Umstände hinzutreten. ${ }^{430}$ Als Beispiele werden die ungehörige Kündigung sowie widersprüchliches Verhalten des Arbeitgebers genannt. ${ }^{431}$ Aber auch der Beispielsfall wird aufgrund seines diskriminierenden Charakters als ungerechtfertigte Kündigung eingestuft, ${ }^{432}$ was die Frage provoziert, wann nach dieser Ansicht willkürliches Verhalten überhaupt gerechtfertigt ist.

Im Sinne der hier vertretenen Auffassung, daß sich Diskriminierungsschutz - wo er für notwendig erachtet wird - nicht auf ausgewählte Merkmale beschränken läßt, hat das $B A G$ entschieden, daß 
auch außerhalb des KSchG über $§ 242$ BGB Schutz vor willkürlichen und auf sachfremden Motiven beruhenden Kündigungen bestehen muß. ${ }^{433}$ Der Willkürvorwurf soll dabei bereits dann ausscheiden, wenn ein irgendwie einleuchtender Grund für die Rechtsausübung vorliegt, der beispielsweise darin bestehen könne, daß der Arbeitgeber - tatsachengestützt - das Vertrauen in die Loyalität des Arbeitnehmers verloren hat. ${ }^{434}$

\section{b) Rechtswidrigkeit - Bereichsabhängige Interessenabwägung}

Die Rechtsprechung des BAG zeigt, daß der Arbeitgeber auch außerhalb des Anwendungsbereichs des KSchG kein Recht zu willkürlicher Kündigung haben soll, daß jedoch die Anforderungen an die Sachlichkeit seiner Entscheidung nicht denen entsprechen müssen, die das KSchG verlangt. In diesem Sinne hat das BVerfG festgestellt: „Der durch die Generalklauseln vermittelte Schutz darf nicht dazu führen, daß dem Kleinunternehmer praktisch die im Kündigungsschutzgesetz vorgesehenen Maßstäbe der Sozialwidrigkeit auferlegt werden.““35

Mit dem Ziel, der Lösung des Ausgangsfalls ein Stück näherzukommen, wird im folgenden der Blick auf Sinn und Zweck der Kleinbetriebsklausel gelenkt. Das BVerfG begründet die Existenz der Klausel - und damit letztlich das erleichterte Kündigungsrecht des Kleinunternehmers - u. a. damit, daß gerade in kleinen Teams, die besonders anfällig für Mißstimmungen und Querelen seien, bestimmte Persönlichkeitsmerkmale, die für die Zusammenarbeit, die Außenwirkung und das Betriebsklima erheblich sind, eine entscheidendende Bedeutung hätten. ${ }^{436}$ Störungen des Betriebsklimas könnten zu Leistungsminderungen führen, die bei geringem Geschäftsvolumen spürbar auf das Ergebnis durchschlügen. ${ }^{437} \mathrm{Zu}$ beachten sei auch, daß in kleinen Betrieben der Unternehmer und Arbeitgeber typischerweise selbst als Chef vor Ort mitarbeite, so daß das Vertrauensverhältnis zu jedem seiner Mitarbeiter einen besonderen Stellenwert bekomme. ${ }^{438}$ Aufgrund der (oft) geringen Finanzausstattung sei ein Kleinbetrieb zudem häufig nicht in der Lage, Abfindungen bei Auflösung eines Arbeitsverhältnisses zu zahlen oder weniger leistungsfähiges, weniger benötigtes oder auch nur weniger genehmes Personal mitzutragen. ${ }^{439}$

Aber auch der Arbeitnehmer kann nach Ansicht des Gerichts wichtige Belange in die Waagschale werfen. Seine durch Art. 12 I GG geschützte Berufsfreiheit könne er ausschließlich durch den

433 BAG, NJW 2002, S. 532 (534).

$434 \quad B A G$, NJW 2002, S. 532 (534); Das Gericht überprüfte in dem entschiedenen Fall nicht, ob sich der Arbeitnehmer tatsächlich illoyal verhalten hatte.

435 BVerfG, NJW 1998, S. 1475 (1476).

$436 \quad B$ VerfG, NJW 1998, S. 1475 (1476).

437 BVerfG, NJW 1998, S. 1475 (1476).

$438 \quad B$ VerfG, NJW 1998, S. 1475 (1476).

439 BVerfG, NJW 1998, S. 1475 (1476).

116 
Abschluß und Fortbestand von Arbeitsverträgen realisieren. ${ }^{440}$ Der Arbeitsplatz bilde die wirtschaftliche Existenzgrundlage für den Arbeitnehmer und seine Familie. ${ }^{441}$ Das gilt natürlich nicht nur für den Arbeitnehmer, der schon eine gewisse Zeit in einem Betrieb mit einer bestimmten Größe arbeitet, sondern ebenso für den, dessen Arbeitsverhältnis (noch) nicht vom Kündigungsschutz umfaßt ist. Nicht unberücksichtigt gelassen werden darf zudem, daß der Eintritt in ein neues Arbeitsverhältnis oft von erheblichen finanziellen Belastungen - zu denken ist etwa an einen Umzug - begleitet ist. Durch den Verlust des Arbeitsplatzes wird zudem das soziale Beziehungsgeflecht in Frage gestellt. So kann eine Kündigung beispielsweise zur Beendigung von Nachbarschaftsbeziehungen führen. ${ }^{442}$

Die Argumente des BVerfG machen zum einen deutlich, daß - um auf den Beispielsfall zurückzukommen - selbst bestehende Antipathie gegenüber dem Arbeitnehmer grundsätzlich Anlaß einer rechtmäßigen Kündigung sein kann. Zum anderen zeigen sie aber auch, daß der Vortrag des Arbeitgebers, der Arbeitnehmer sei ihm unsympathisch, nachvollziehbar, mithin sachlich begründet sein muß. In diesem Sinne wird vertreten, Sym- bzw. Antipathie seien nur insoweit zulässige Auswahlkriterien, ,,sofern ihre Berücksichtigung nicht gegen spezielle Diskriminierungsverbote verstößt. So wäre es etwa mit Art. 3 Abs. 3 GG unvereinbar, wenn der Arbeitgeber den Bewerber wegen seiner Hautfarbe ablehnen könnte, selbst wenn er glaubt, wegen seiner Antipathien gegen Menschen fremder Herkunft mit einer solchen Person nicht zusammenarbeiten zu können.“443

\section{c) Resümee}

Die Ausführungen zum Diskriminierungsschutz im Rahmen arbeitsrechtlicher Kündigungen haben zum einen gezeigt, daß selbst in einem Bereich der Sozialsphäre, in dem prima facie willkürliches Handeln gestattet ist, das Diskriminierungsverbot Geltung beansprucht, und zwar unbeschränkt in bezug auf potenzielle Diskriminierungsmerkmale. Zum anderen haben sie verdeutlicht, daß hinsichtlich der im Rahmen der Interessenabwägung und damit der Rechtswidrigkeitsprüfung zu berücksichtigenden Belange der Parteien zwischen verschiedenen Bereichen der Sozialsphäre unterschieden werden muß. Vor dem Hintergrund dieser zweiten Erkenntnis wird die These aufgestellt, daß das Verbot der Diskriminierung außerhalb des Bereichs der Intim- und Privatsphäre uneingeschränkt gilt. Die Beschränkung des sachlichen Geltungsbereichs des Diskriminierungsverbotes auf bestimmte Bereiche der Sozialsphäre ist abzulehnen, da sie ihrerseits

$440 \quad B V e r f G$, NJW 1998, S. 1475 (1476).

$441 \quad B V e r f G$, NJW 1998, S. 1475 (1476); In diesem Sinne wird vertreten, das Arbeitsrecht bezwecke gerade durch seine auf Bestandsschutz abzielenden Vorschriften die Sicherung eben dieser Existenzbasis des Arbeitnehmers und sei zumindest insoweit als spezifische Ausformung des verfassungsrechtlich verankerten Sozialstaatsprinzips zu begreifen. (Weiss, ArbuR, Sonderheft 1979, S. 28 (30)).

442 BVerfG, NJW 1998, S. 1475 (1476).

443 Raab, RdA 1995, S. 36 (38); ebenso Buchner in Münchener Handbuch zum Arbeitsrecht, Band 1, § 39, Rn. 74; Müller-Glöge in MüKo, § 611 a, Rn. 23; a. A.: Wiese, JuS 1990, S. 357 (359). 
diskriminierenden Charakter haben würde, und zwar im Verhältnis der „Diskriminierenden“ zueinander. $^{444}$

\section{Zusammenfassung}

Die Betrachtungen zum Begriff der Diskriminierung schlossen mit der Bestimmung des sachlichen Geltungsbereichs des entsprechenden Diskriminierungsverbotes. Ausgangspunkt aller Überlegungen war dabei die Feststellung, daß schutzwürdige Interessen des „Diskriminierenden“ diesen Bereich begrenzen. Die Frage des konkreten Verlaufs dieser Grenze wurde dabei wie folgt beantwortet:

Diskriminierend kann neben rechtsgeschäftlichem auch quasi-rechtsgeschäftliches Handeln sein, wobei für letzteres beispielhaft die arbeitsrechtliche Weisung genannt werden kann.

Vor dem Hintergrund der Tatsache, daß besonders häufig im Vorfeld des Vertragsschlusses „diskriminiert“ wird und die Antidiskriminierungsrichtlinien in Konsequenz dessen den Diskriminierungsschutz auf diesen Bereich ausweiten, ist - um eine Umgehung des Diskriminierungsverbotes in diesem Bereich $\mathrm{zu}$ verhindern - zwingend erforderlich, merkmalsspezifische Stellenausschreibungen und Bewerbungsunterlagen sowie die Frage nach dem Vorhandensein eines bestimmten Merkmals in der Person des „Diskriminierten“ nur insofern für zulässig zu erklären, als auch der Vertragsschluß aufgrund des jeweiligen Merkmals abgelehnt werden könnte. Der „Diskriminierte“ begibt sich etwaiger Sonderrechte nicht dadurch, daß er von seinem „Recht zur Lüge“ Gebrauch macht.

Das Diskriminierungsverbot kann nur dort Geltung beanspruchen, wo der „Diskriminierende“ eine bestimmte Ware, Dienstleistung etc. etwa durch Anzeigen in Tageszeitungen öffentlich anbietet. Ein Angebot ist dann nicht öffentlich, wenn es sich an eine ganz bestimmte Person richtet. Der „Diskriminierende“ ist nicht verpflichtet, seine Ware, Dienstleistung etc. öffentlich anzubieten. Er ist lediglich verpflichtet, öffentliche Angebote diskriminierungsfrei zu gestalten.

Am Beispiel der letztwilligen Verfügung von Todes wegen wurde der Frage nachgegangen, ob auch unentgeltliche Zuwendungen in den sachlichen Geltungsbereich des Diskriminierungsverbotes fallen. Dabei wurde einmal mehr die Ansicht vertreten, daß ein etwaiges Diskriminierungsverbot auch in diesem Bereich nicht auf bestimmte Merkmale beschränkt sein darf. Im Anschluß daran wurden Für und Wider eines Diskriminierungsschutzes im Rahmen unentgeltlicher Zuwendungen diskutiert. Die Überlegungen ergaben, daß vieles dafür spricht, (wirtschaftlich) rein altruistisches Verhalten nicht am Verbot der Diskriminierung zu messen.

444 Das AGG beschreitet diesen Weg und wird unter anderem deshalb kritisiert. (vgl. dazu Teil 4, Prüfungspunkt B. I. 5.). 
Das Diskriminierungsverbot sollte sowohl gewerbsmäßiges als auch nichtgewerbsmäßiges Handeln umfassen. Die Antidiskriminierungsrichtlinien können in diesem Sinne interpretiert werden.

Vor dem Hintergrund der Überlegung, daß Rechtsverhältnisse ein mehr oder weniger ausgeprägtes Näheverhältnis der Parteien zueinander schaffen, wurde eine Unterteilung in Intim-, Privat- und Sozialsphäre vorgenommen und sodann folgendes festgestellt: Das Diskriminierungsverbot erfaßt selbstverständlich nicht die Intimsphäre des Menschen - also beispielsweise die Wahl seines Lebenspartners.

Für die Privatsphäre, die vor allem die ,eigenen vier Wände“ umfaßt, kann dasselbe konstatiert werden. Begründen läßt sich dies mit dem in Art. 2 I GG verankerten allgemeinen Persönlichkeitsrecht, welches dem einzelnen einen Raum gewährt, in dem er unbeobachtet sich selbst überlassen ist oder mit Personen seines besonderen Vertrauens ohne Rücksicht auf gesellschaftliche Verhaltenserwartungen verkehren kann.

Außerhalb des Bereichs der Intim- und Privatsphäre - mithin im Bereich der Sozialsphäre - sollte das Diskriminierungsverbot dagegen uneingeschränkt gelten. Eine Beschränkung des sachlichen Geltungsbereichs des Diskriminierungsverbotes auf bestimmte Bereiche der Sozialsphäre würde ihrerseits diskriminierenden Charakter haben, und zwar im Verhältnis der „Diskriminierenden“ zueinander. Auch eine Beschränkung des Diskriminierungsschutzes in Teilbereichen der Sozialsphäre - etwa außerhalb des Geltungsbereichs des Kündigungsschutzgesetzes - auf ausgewählte Merkmale ließe sich nicht rechtfertigen. Lediglich im Rahmen der Interessenabwägung und damit der Rechtswidrigkeitsprüfung ist die Unterscheidung einzelner Bereiche der Sozialsphäre von Bedeutung. 

Teil 3: Auswirkungen des Diskriminierungsverbotes auf das rechtsgeschäftliche und quasirechtsgeschäftliche Handeln

„Sehr viel wichtiger [...] ist es, die Folgen von Gesetzen sorgfältig abzuschätzen. Daran hapert es leider [...] immer wieder [...]. Ein Beispiel ist der vorerst gescheiterte Entwurf des Antidiskriminierungsgesetzes. “1

Nachdem die Tatbestandsmerkmale des Diskriminierungsverbotes definiert wurden, soll im folgenden der Frage nachgegangen werden, welche Auswirkungen das Diskriminierungsverbot auf das (quasi-) rechtsgeschäftliche Handeln der Privatrechtssubjekte hat.

\section{A. Vorbemerkungen}

Die bisherigen Ausführungen haben gezeigt, daß nur rechtswidriges diskriminierungsrelevantes Verhalten eine Diskriminierung darstellt und rechtswidrig jedes sachlich nicht begründbare Verhalten ist.

\section{Sachlicher Grund - Wertausfüllungsfähiger und -bedürftiger Rechtsbegriff}

Der Begriff des sachlichen Grundes ist wertausfüllungsfähig und -bedürftig. Das zeigt die Definition dieses Begriffs. Nach dem ADG-Entwurf des Bundesjustizministeriums ist eine Ungleichbehandlung sachlich begründet, wenn sie nicht nur Ausdruck persönlichen Gutdünkens ist, sondern einen Bezug zum Inhalt des fraglichen Rechtsgeschäfts hat und für einen durchschnittlichen Betrachter nachvollziehbar ist. ${ }^{2}$ Diese Formulierung erinnert an die Definition des Willkürverbots des Art. 3 I GG, das verletzt sein soll, wenn sich bei verständiger Würdigung der das Grundgesetz beherrschenden Gedanken ein sachgerechter Grund für eine Maßnahme der öffentlichen Gewalt nicht finden lasse, ${ }^{3}$ wenn selbige also nicht am Gerechtigkeitsgedanken orientiert ist, ${ }^{4}$ sich für sie keine vernünftigen Erwägungen finden lassen, die sich aus der Natur der Sache ergeben oder sonst wie einleuchtend sind. ${ }^{5}$ Wer ist nun aber der durchschnittliche Betrachter, was gerecht, vernünftig und einleuchtend?

Um die weiteren Ausführungen nachvollziehen zu können, ist an dieser Stelle anzumerken, daß die soeben angestellten Überlegungen zu dem Begriff „sachlicher Grund“ natürlich in gleicher Weise

Möllering in FAZ vom 20.07.2005, S. 23 unter dem Titel „Die Gesetze sind besser als ihr Ruf“.

Diskussionsentwurf eines Gesetzes zur Verhinderung von Diskriminierungen im Zivilrecht des Bundesjustizministeriums, S. 47 (http://www.lsvd.de / antidiskriminierung/adgzivil02.html).

$3 \quad B G H$, NJW 2003, S. 1658 (1659); NJW 2004, S. 1031 (1031); BVerfGE 55, S. 72 (89 f.); 78, S. 232 (248); In einem späteren Urteil formuliert es das $B V e r f G$ enger: „Kommt als Maßstab nur das Willkürverbot in Betracht, so kann ein Verstoß gegen Art. 3 Abs. 1 GG erst festgestellt werden, wenn die Unsachlichkeit der Differenzierung evident ist." (BVerfGE 99, S. 367 (389)).

$4 \quad$ BVerfGE 12, S. 354 (367).

5 Heun in Dreier, Band I, Art. 3, Rn. 19. 
für den spiegelbildlichen Begriff der „Unsachlichkeit“ gelten. Letzterer soll - wie an anderer Stelle festgestellt - im Falle der gesetzestechnischen Umsetzung des Diskriminierungsverbotes anstatt des Rechtfertigungsgrundes „sachlicher Grund“ Tatbestandsmerkmal eines Diskriminierungsschutzgesetzes sein. ${ }^{6}$

\section{Rechtswidrigkeitsprüfung einzelfallabhängig}

Durch die Einführung des Begriffs der „Unsachlichkeit“ würde dem Richter die Aufgabe übertragen zu entscheiden, ob diskriminierungsrelevantes Verhalten im Einzelfall rechtswidrig ist oder nicht. Es stellt sich die Frage, ob sich ein Tatbestandsmerkmal „Unsachlichlkeit“ mit der Rechtsprechung des $B V \operatorname{erf} G$ vereinbaren ließe, wonach es grundsätzlich Aufgabe des Gesetzgebers ist, im Rahmen seiner Gesetzgebung die Abwägung der widerstreitenden Interessen der Privatrechtssubjekte vorzunehmen. $^{7}$

Diese Frage muß aufgrund folgender Überlegung bejaht werden: Richtlinie 76/207/EWG bestimmt in Art. 9 II, daß die Mitgliedstaaten

,in regelmäßigen Abständen die unter Artikel 2 Absatz $\left[6^{8}\right]$ fallenden beruflichen Tätigkeiten [prüfen], um unter Berücksichtigung der sozialen Entwicklung festzustellen, ob es gerechtfertigt ist, die betreffenden Ausnahmen aufrechtzuerhalten. Sie übermitteln der Kommission das Ergebnis dieser Prüfung.“9

An der Übermittlungspflicht der Mitgliedstaaten ist kritisiert worden, sie sei auf eine unmögliche Leistung gerichtet. ${ }^{10}$ Einer auch nur annähernd vollständigen und zutreffenden Erfassung der nach $\S$ 611 a I 2 BGB-A zugelassenen Ausnahmen stünde die Abhängigkeit von der jeweils vertraglich geschuldeten Leistung entgegen. ${ }^{11}$ Was an Differenzierungsgründen letzten Endes sachlich gerechtfertigt ist, könne nur anhand der gesamten Umstände des Einzelfalles unter Berücksichtigung der Intentionen des Gleichbehandlungsgesetzes ermittelt werden. ${ }^{12}$ In diesem Sinne ist im Rahmen der Frage nach dem Umfang der mittelbaren Drittwirkung der Grundrechte ${ }^{13}$

Vergleiche dazu die Ausführungen unter Teil 2, Prüfungspunkt C. III.

BVerfGE 89, S. 214 (232).

8 Die Richtlinie verweist tatsächlich auf Absatz 2. Aus der ursprünglichen Fassung der Richtlinie - vgl. dazu Abl. EG 1976 Nr. L 39, S. 40 ff. - kann indes geschlußfolgert werden, daß ein Verweis auf Absatz 6 gemeint ist.

9 Art. 9 II Richtlinie 76/207/EWG wird in Zukunft durch Art. 31 III Richtlinie 2006/54/EG ersetzt.

10 Müller-Glöge kritisiert deshalb den EuGH, der einen Verstoß der Bundesrepublik gegen diese Bestimmung festgestellt habe, ohne deren Erfüllbarkeit anzusprechen. (Müller-Glöge in MüKo, § 611 a, Rn. 40, Fn. 111).

11 Müller-Glöge in MüKo, § 611 a, Rn. 40; in diesem Sinne auch Richardi/Annuß: „Die Grenzen sind notwendig unsicher und lassen sich nicht abstrakt bestimmen, da das jeweilige Resultat entscheidend von dem in vorrechtlicher Wertung ermittelten Gewicht des Unternehmerinteresses einerseits und dem Interesse an einer Gleichbehandlung andererseits abhängt.“(Richardi/Annuß in Staudinger, § 611 a, Rn. 54).

12 Eich, NJW 1980, S. 2329 (2331); Gemeint ist das Gesetz über die Gleichbehandlung von Männern und Frauen am Arbeitsplatz und über die Erhaltung von Ansprüchen bei Betriebsübergang (Arbeitsrechtliches EGAnpassungsgesetz) vom 13.08.1980, aufgrund dessen unter anderem $\S 611$ a BGB-A ins BGB eingefügt wurde. (BGB1. 1980 I 2, S. 1308 f.).

13 Vergleiche dazu Teil 4, Prüfungspunkt A. I. 2. f). 
gesagt worden: „Allerdings wäre es eine Illusion zu folgern, es gäbe formale Kategorien der Intensität der Einwirkung der Grundrechte auf das Privatrecht. Die mittelbare Grundrechtsgeltung tritt vielmehr in zahllosen Abstufungen auf. Man muß eher sagen: in einer beinahe stufenlosen Skala, von (im Ergebnis) weitgehender Annäherung an die volle Grundrechtsbindung - [...] - bis zur unteren Grenze, wo die mittelbare Grundrechtbindung sich praktisch kaum mehr auswirkt -

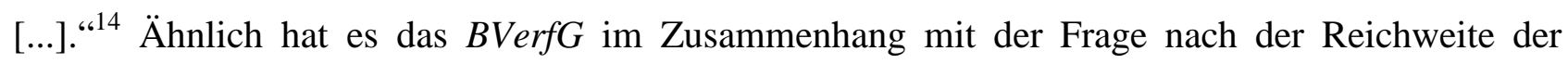
Bindung des Gesetzgebers an Art. 3 I GG formuliert: „Genauere Maßstäbe und Kriterien dafür, unter welchen Voraussetzungen im Einzelfall das Willkürverbot oder das Gebot verhältnismäßiger Gleichbehandlung durch den Gesetzgeber verletzt ist, lassen sich nicht abstrakt und allgemein, sondern nur bezogen auf die jeweils betroffenen unterschiedlichen Sach- und Regelungsbereiche bestimmen. “15

Es ist deshalb zu begrüßen, daß die Richtlinie 2000/43/EG von einer Übermittlungspflicht im Sinne des Art. 9 II Richtlinie 76/207/EWG abgesehen hat. In Erwägungsgrund 18 heißt es lediglich, daß die Bedingungen darüber, wann das Merkmal der wesentlichen und entscheidenden beruflichen Anforderung erfüllt ist, in die Informationen, die die Mitgliedstaaten der Kommission übermitteln vgl. dazu Art. 17 der Richtlinie -, aufgenommen werden sollten. ${ }^{16}$

\section{Kein abschließender Katalog sachlicher Gründe - Rechtsunsicherheit}

Nicht verhehlt werden darf, daß die Unmöglichkeit einer abschließenden Aussage bezüglich der Rechtswidrigkeit diskriminierungsrelevanten Verhaltens $\mathrm{zu}$ Rechtsunsicherheit seitens des „Diskriminierenden“ führen kann. So wird etwa befürchtet, daß ,wohl selbst ein mittelständischer Einzelhändler für Damenunterwäsche künftig schon bei der Stellenanzeige einen Anwalt einschalten [muß], um sicherzugehen, daß er sich bei der Suche nach einer ,jungen dynamischen Verkäuferin mit guten Deutschkenntnissen“ nicht Vorwürfen von drei Seiten aussetzt: den Grauen Panthern, Frauenverbänden und Vertretern ethnischer Minderheiten. “17 Versicherer gehen davon aus, daß sich aus dem am 18.08.2006 in Kraft getretenen AGG Versicherungsbedarf für die „Diskriminierenden“ ergibt: „Im Oktober brachte etwa der Allianz-Konzern ein neues Produkt namens „VH-AGG“ auf den Markt, eine Haftpflichtversicherung von Ansprüchen aus Diskriminierungstatbeständen. Der Versicherungsschutz umfaßt nicht nur die Prozeßkosten,

Dürig in Maunz/Dürig, Band I, Art. 3 I, Rn. 511.

BVerfGE 105, S. 73 (111).

Vergleiche auch Erwägungsgrund 23 der Richtlinie 2000/78/EG.

Groß in FAZ vom 23.02.2005, S. 23 unter dem Titel: „Minderheitenschutz führt in Labyrinth“; Müller-Glöge weist darauf hin, daß die Übertragung der Verantwortung vom Gesetzgeber auf den Arbeitgeber nicht unumstritten ist. (Müller-Glöge in MüKo, $§ 611$ a, Rn. 38, Fn. 110). 
sondern auch Schadensersatzansprüche - eine Art Rundum-sorglos-Diskriminierungspaket für Geschäftsführer, Aufsichtsorgane und leitende Angestellte. “18

Hinzuweisen ist darauf, daß im Rahmen der Frage, wann das Geschlecht unverzichtbare Voraussetzung im Sinne des $\S 611$ a I 2 BGB-A ist, auf die Sicht eines zeitgemäßen, sozialbewußten Arbeitgebers abzustellen sein sollte, der den (Fort-)Bestand und die Leistungsfähigkeit seines Unternehmens anstrebt. ${ }^{19}$ Damit ist klargestellt, daß der Arbeitgeber im Rahmen seiner Auswahlentscheidung neben den eigenen auch den Interessen des „Diskriminierten“ Gewicht beimessen mußte. Dasselbe verlangt nunmehr freilich auch $\S 8$ I AGG vom Arbeitgeber. Natürlich ist es möglich, daß auch der Arbeitgeber, auf den abgestellt wird, eine falsche Interessenabwägung vornimmt bzw. unschlüssig ist, wie die widerstreitenden Interessen im Einzelfall zu gewichten sind. Diese Unsicherheit kann nicht vollends ausgeräumt werden. Insofern ist es richtig, wenn im Zusammenhang mit der Diskussion um die Einführung eines zivilrechtlichen Antidiskriminierungsgesetzes behauptet wurde: „Wahrscheinlich wird erst nach Jahren der Rechtsprechung einigermaßen klar umrissen sein, was verboten und was erlaubt ist. “20 Das ist aber keine spezifische Eigenschaft von Antidiskriminierungsrecht, sondern Wesensmerkmal einer Vielzahl von Rechtsnormen und Grund der meisten Rechtsstreitigkeiten. Im Rahmen des $§ 611$ a BGB-A hatte sich auch nach 20 Jahren Rechtsprechungspraxis eine allgemein anerkannte Definition dafür, wann ein bestimmtes Geschlecht unverzichtbar sein sollte, nicht herausgebildet. ${ }^{21}$

\section{Kriterien für Abwägungsentscheidung bestimmbar}

Trotz der Unmöglichkeit, einen auch nur annähernd vollständigen Katalog sachlicher Gründe zu erstellen, ist es, um eine (möglichst) einheitliche Rechtsprechung mit der daraus resultierenden Rechtssicherheit für die Betroffenen zu erreichen, notwendig, wenigstens Kriterien zu entwickeln, die dem Richter Orientierung bei dessen Entscheidungsfindung bieten. Eine einheitliche Rechtsprechung ist andernfalls nicht unbedingt zu erwarten, gibt doch das Grundgesetz mit den dort verankerten Grundrechten zwar Richtlinien für die im Einzelfall vorzunehmende Abwägung, nicht

18 Gleichbehandlungsgesetz“.

19 Putzo in Palandt, $\S 611$ a, Rn. 12.

20 Groß in FAZ vom 23.02.2005, S. 23 unter dem Titel: „Minderheitenschutz führt in Labyrinth“; vgl. auch Sattar: „Zur Zeit herrscht großes Warten auf die Rechtsprechung. Noch ist völlig unklar, wie scharf das Gleichbehandlungsgesetz ausgelegt wird.“ (Sattar in FAZ vom 16.11.2006, S. 4, unter dem Titel: „,,Auf falschem Bein hurra geschrien" - Drei Monate Gleichbehandlungsgesetz"); Auch der Präsident des BGH, Günter Hirsch, rechne im Zusammenhang mit dem AGG mit einer „Fülle von Auslegungsfragen“, deren Klärung einige Zeit und einige Verfahren in Anspruch nehmen werde. (FAZ vom 27.01.2007, S. 11, unter dem Titel: „Gleichbehandlungsgesetz belastet die Gerichte“).

21 Thüsing, RdA 2001, S. 319 (319). 
aber deren Ergebnis abschließend vor. ${ }^{22}$ Ebenso verhält es sich - wie deren Name bereits andeutet mit den Antidiskriminierungsrichtlinien.

Die folgenden Ausführungen dienen dem Ziel, Kriterien für die erforderliche Abwägungsentscheidung zu entwickeln. Anzumerken ist insofern, daß besagte Kriterien lediglich einen Wegweiser für die Rechtswidrigkeitsprüfung darstellen, die Abwägungsentscheidung aber nicht vorwegnehmen können, da selbige stets unter Berücksichtigung der Besonderheiten des Einzelfalls getroffen werden muß.

\section{B. Unterscheidung zwischen unbeeinflußbarem Merkmal, Status und Verhalten}

Im Rahmen der Interessenabwägung ist von Bedeutung, welche Art Merkmal im konkreten Fall Anknüpfungspunkt für die „Diskriminierung“ war. An anderer Stelle wurde eine Unterteilung in unbeeinflußbare Merkmale, Status sowie Verhalten als Merkmal vorgenommen. Es wurde festgestellt, daß die besondere Schutzwürdigkeit der Träger unbeeinflußbarer Merkmale daraus resultiert, daß sie keinen Einfluß auf das jeweilige Merkmal haben, vielmehr an selbiges gebunden sind. Das ist der wesentliche Unterschied zum Verhalten als Merkmal.

Das Merkmal „Status“ nimmt zwischen diesen beiden Polen eine Zwischenstellung ein. Der Träger eines bestimmten Status kann sich dieses Merkmals grundsätzlich entledigen. Insofern unterscheidet sich das Statusmerkmal vom unbeeinflußbaren Merkmal. Vom Verhaltensmerkmal unterscheidet es sich dadurch, daß der Status als solcher auf die ordnungsgemäße Erfüllung vertraglicher Pflichten in der Regel keinen bzw. weniger (negativen) Einfluß hat als ein bestimmtes Verhalten. Der Grund dafür liegt darin, daß sich im Falle des Status das potenziell vorwerfbare Verhalten in der Statusbegründung erschöpft. Im Rahmen von (potenziellen) Privatrechtsbeziehungen wirkt dieses Verhalten regelmäßig nicht fort, so daß es selbige grundsätzlich nicht negativ beeinflussen kann.

Ohne die jeweiligen Besonderheiten des Einzelfalls zu berücksichtigen, läßt sich an dieser Stelle die generelle Feststellung treffen, daß die Anknüpfung an ein unbeeinflußbares Merkmal den höchsten Rechtfertigungsanforderungen unterliegt, gefolgt vom Status und schließlich dem Verhalten als Merkmal. $^{23}$

\section{C. „,Diskriminierung“6 als bloßer Selbstzweck}

$22 \quad B V e r f G$, NJW 2003, S. 2815 (2816).

23 In diesem Sinne hat das BVerfG unterschiedliche Anforderungen bezüglich der Bindung des Gesetzgebers an Art. 3 I GG formuliert: „Diese ist umso enger, je mehr sich die personenbezogenen Merkmale den in Art. 3 Abs. 3 GG genannten annähern [...]. [...] Bei lediglich verhaltensbezogenen Unterscheidungen hängt das Maß der Bindung davon $\mathrm{ab}$, inwieweit die Betroffenen in der Lage sind, durch ihr Verhalten die Verwirklichung der Merkmale zu beeinflussen, nach denen unterschieden wird.“ (BVerfGE 99, S. 367 (388)). 
Es gibt gesellschaftliche Bereiche, die Trägern bestimmter (Diskriminierungs-)Merkmale gewidmet sind und sich Vertragsangebote dort (vornehmlich) an diese Menschen richten. Zu denken ist u.a. an sog. Nobelrestaurants, Clubs nur für Mitglieder, Frauencafés, Jugendhäuser, ${ }^{24}$ Frauenhotels ${ }^{25}$ oder Unternehmen, die vornehmlich Behinderte einstellen mit dem Ziel, diese in den ersten Arbeitsmarkt zu integrieren. ${ }^{26}$

Gemäß Erwägungsgrund 26 der Richtlinie 2000/78/EG können Fördermaßnahmen

„[...] die Einrichtung und Beibehaltung von Organisationen von Personen mit einer bestimmten Religion oder Weltanschauung, einer bestimmten Behinderung, einem bestimmten Alter oder einer bestimmten sexuellen Ausrichtung zulassen, wenn deren Zweck hauptsächlich darin besteht, die besonderen Bedürfnisse dieser Personen zu fördern. “27

Erwägungsgrund 26 macht deutlich, daß die bezweckte Förderung von Trägern bestimmter Merkmale damit verbundene Benachteiligungen von Personen, die nicht Träger dieser Merkmale sind, im Einzelfall rechtfertigen kann. In diesem Sinne wird vertreten, daß Ausländer immer dann diskriminiert werden, wenn deren Ausgrenzung bloßer Selbstzweck ist. ${ }^{28}$

Nobelrestaurants, Clubs nur für Mitglieder, Frauencafés, Frauenhotels und Jugendclubs lassen sich vor diesem Hintergrund in ein Antidiskriminierungskonzept eingliedern, da sie nicht die Ausgrenzung bestimmter Personengruppen bezwecken. Unter dieser Voraussetzung muß dem jeweiligen Betreiber gestattet sein, seinen Betrieb einem bestimmten Publikum zu widmen. ${ }^{29}$

Auch das Ziel, schwer in den ersten Arbeitsmarkt vermittelbaren Behinderten reguläre Arbeitsplätze zu Tariflöhnen zu schaffen, ist zweifelsfrei ein sachliches Ziel, das in dem geschilderten Fall „durch Spenden, Sponsoren, EU-Gelder und vor allem Lohnkostenzuschüsse - die jede Firma bekommt, wenn sie behinderte Mitarbeiter einstellt“" unterstützt wurde. ${ }^{30}$

Auch bei der Werbeaktion, die den Verkauf von Kaffeemaschinen zum Gegenstand hatte und die sich ausschließlich an Frauen richtete, ${ }^{31}$ war die Ausgrenzung der Männer nicht bezweckt. Es kann vielmehr davon ausgegangen werden, daß das Kaufhaus durch die zeitlich begrenzte Werbeaktion

24 Beispiele bei Timme, ZAR 1997, S. 130 (132).

25 Beispiel bei v. Münch, NJW 1999, S. 260 (260).

26 Von einem derartigen Fall berichtet Roßbach in FAZ vom 28.06.05, S. 8, unter dem Titel: „Jeder macht soviel, wie er kann".

27 Vergleichbare Formulierungen finden sich in Erwägungsgrund 15 der Richtlinie 2002/73/EG sowie Erwägungsgrund 17 der Richtlinie 2000/43/EG.; vgl. auch Erwägungsgrund 16 Richtlinie 2004/113/EG.

28 Timme, ZAR 1997, S. 130 (134).

29 So auch Timme, ZAR 1997, S. 130 (132); Larenz/Wolf: „Jedem Veranstalter muß es frei stehen, für den Besuch seiner Veranstaltung festliche Kleidung oder gar Smoking zu verlangen." (Larenz/Wolf, Allgemeiner Teil des Bürgerlichen Rechts, § 34, Rn. 43).

30 Roßbach in FAZ vom 28.06.05, S. 8, unter dem Titel: „Jeder macht soviel, wie er kann“.

31 Fernsehwerbung des Unternehmens ,Saturn“ im November 2004. 
das Ziel verfolgte, daß seine Einrichtung von den bislang unterrepräsentierten Frauen - es handelte sich um ein Elektronikfachgeschäft - wahrgenommen und besucht wird. ${ }^{32}$ In diesem Fall das Vorliegen einer Diskriminierung zu bejahen, widerspräche jeder Logik, ist doch anzunehmen, daß Männer die besten Kunden dieses Elektronikfachgeschäftes sind. Die Abwägung der widerstreitenden Interessen führt in diesem Fall dazu, daß der unternehmerischen Handlungsfreiheit des Unternehmens Vorrang vor den Interessen der „zurückgesetzten“ Männer einzuräumen ist. Die Werbeaktion verfolgte einen rechtmäßiger Zweck. Es war angemessen, zum Zweck der Gewinnung neuer Kunden, eine derartige Werbeaktion durchzuführen.

Schließlich dürften auch familienfördernde Maßnahmen - zu denken ist etwa an die Unterhaltspflicht für den Nachwuchs als zulässiger Unterscheidungsfaktor bei Kündigungen grundsätzlich mit einem Antidiskriminierungskonzept vereinbar sein. ${ }^{33}$

Im Gegensatz zu den bisher genannten Beispielsfällen bezwecken Restaurantbetreiber mit Schildern, die darauf hinweisen, daß Ausländer generell nicht erwünscht sind, ${ }^{34}$ die Ausgrenzung dieser Personen. Ebenso verhält es sich im umgekehrten Fall, wenn der Betreiber einer Gaststätte nur Ausländer einer bestimmten Nationalität bewirten will. ${ }^{35}$ In beiden Fällen ist die Ausgrenzung der jeweiligen Personengruppe bloßer Selbstzweck und demnach sachlich nicht gerechtfertigt. Auch nicht mit dem Argument, die bezweckte Benachteiligung der einen Personengruppe habe die Förderung einer anderen Personengruppe zur Folge. ${ }^{36}$

\section{Zeitlich (un-)begrenzter Ausschluß}

Ein wesentlicher Unterschied zu der zeitlich begrenzten Werbeaktion des Elektronikfachgeschäfts besteht auch darin, daß besagte Restaurantbesitzer eine bestimmte Personengruppe dauerhaft von der Bewirtung ausschließen. Auch dieser Umstand erlangt für die Beantwortung der Frage der sachlichen Rechtfertigung Bedeutung. Es macht einen Unterschied, ob ein Gastwirt zwecks

32 Ähnliche Gründe lassen sich sicherlich auch in den folgenden Beispielen von Säcker finden: „Sollen besonders günstige Reisepreise, Versicherungsprämien, Fahrschulstundenpreise für junge Leute unter 28 Jahren in Zukunft unerlaubt sein, nachdem der Wegfall des Rabattgesetzes eine differenzierte Preisgestaltung gerade erst möglich gemacht hat? Sollen Seniorenrabatte unerlaubt sein?“ (Säcker, ZRP 2002, S. 286 (288)).

33 Im Zusammenhang mit dem AGG werden insofern aber Bedenken geäußert: „Kaum eine Klausel des AGG ist familienpolitisch neutral, [...]. Jede Maßnahme zur Familienförderung könnte eine mittelbare Ungleichbehandlung zumindest wegen der sexuellen Identität, vielleicht auch wegen des Alters darstellen.“ (zitiert wird die Privatdozentin an der Universität Göttingen Benecke in $F A Z$ vom 16.11.2006, S. 13, unter dem Titel: „Väter und Mütter als Verlierer des Arbeitsrechts").

34 OVG Münster, GewArch. 1967, S. 118 f.; OLG Frankfurt, NJW 1985, S. 1720 f.; siehe auch Antwort der Bundesregierung vom 29.07.1982 auf eine Anfrage bzgl. der Diskriminierung von Angehörigen der US-Streitkräfte in der Bundesrepublik Deutschland (BT-Druck. 9/1882).

35 Timme, ZAR 1997, S. 130 (135).

36 In diesem Sinne argumentiert Adomeit, wenn er in anderem Zusammenhang vom ,rechtmäßigen Alternativverhalten“" spricht. (Adomeit, DB 1980, S. 2388 (2388)). 
Durchführung einer Veranstaltung seine Gaststube an einem bestimmten Tag einem bestimmten Publikum widmet, ${ }^{37}$ oder ob er generell eine bestimmte Kundschaft ausschließt.

\section{E. (Nicht) vertragsbezogene Gründe}

Beispiel: Ist der Einwand zulässig, ein Unternehmen könne Bewerbungen weiblicher Interessenten nicht berücksichtigen, weil die Kontaktpflege mit in- und ausländischen Kunden, die ausschließlich männlichen Geschlechts seien, teilweise die Teilnahme an Unterhaltungen und Vergnügungen beinhalte, die einer Frau nicht zugemutet werden könne, wollte man nicht ihre Würde verletzen $?^{38}$

$\S 8$ I AGG verlangt, daß das Geschlecht eine wesentliche und entscheidende berufliche Anforderung ,wegen der Art der auszuübenden Tätigkeit oder der Bedingungen ihrer Ausübung“ darstellt. $^{39}$ Gründe, die nicht tätigkeitsbezogen sind, sollen eine Differenzierung nach dem Geschlecht nur ausnahmsweise rechtfertigen können, so z.B. wenn der Arbeitgeber selbst oder künftige Kollegen aus verständlichen und zu respektierenden Gründen nicht in der Lage sind, mit Frauen oder Männern zusammenzuarbeiten. ${ }^{40}$ Dem ist grundsätzlich zuzustimmen. Auch ein nichttätigkeitsbezogener Grund wie Antipathie kann das Vertragsverhältnis in einer Weise negativ beeinflussen, die dem „Diskriminierenden“ nicht zugemutet werden kann. Ein Arbeitsverhältnis ist eben nicht nur auf die reine Tätigkeit beschränkt.

In dem Beispielsfall bestand die eigentliche Arbeitsleistung nicht in der Teilnahme an besagten Unterhaltungen und Vergnügungen. Der Arbeitgeber stützte seine Entscheidung mithin auf einen nicht tätigkeitsbezogenen Grund. Ist er dennoch verständlich und respektabel? Sicherlich nicht. Es wäre grotesk, könnte ein Arbeitgeber die Nichteinstellung einer Frau mit dem Argument rechtfertigen, er wolle sie vor einer bei Einstellung zu erwartenden Verletzung der Menschenwürde schützen. Die Menschenwürde würde dann zum Feind ihres Trägers, hier der Arbeitnehmerin, werden. Der Arbeitgeber ist aber gem. § 12 I AGG gerade verpflichtet, seine Arbeitnehmer vor einer Verletzung ihrer Menschenwürde am Arbeitsplatz durch Kollegen oder Dritte, wie etwa

37 Larenz/Wolf nennen als Beispiel den „Weiberfasching“. (Larenz/Wolf, Allgemeiner Teil des Bürgerlichen Rechts, $\S$ 34, Rn. 43).

38 Nachweis eines Gerichtsurteils des ArbG Hamburg bei Thüsing, RdA 2001, S. 319 (320); Vgl. auch folgenden Pressebericht, der vermuten läßt, daß es sich bei dem Beispielsfall nicht um einen Einzelfall handelt: „Auch ist in der Klageschrift von einer „Kultur der Diskriminierung“ bei der Deutschen Bank die Rede. Als Indiz wurden - wie bei der Klage gegen Dresdner Kleinwort sowie bei vergleichbaren Verfahren gegen andere Investmentbanken - die an der Wall Street offenbar unvermeidlichen Besuche von Striptease-Lokalen genannt, bei denen weibliche Mitarbeiter natürlich nicht erwünscht sind.“ (FAZ vom 28.08.2006, S. 9 unter dem Titel: „Deutscher Bank droht Klage wegen Diskriminierung“").

39 Hervorhebung durch den Verfasser; In diesem Sinne verlangte $§ 611$ a I 2 BGB-A, daß, ,ein bestimmtes Geschlecht unverzichtbare Voraussetzung für diese Tätigkeit [war].“ (Hervorhebung durch den Verfasser).

40 So in bezug auf $\S 611$ a BGB-A: Raab in Soergel, § 611 a, Rn. 37; Eich subsumierte diese Fälle unter $\S 611$ a I 3 BGB-A, nahm also sachliche Gründe an, die sich gerade nicht auf das Geschlecht bezogen. (Eich, NJW 1980, S. 2329 (2331)) Diese Sichtweise ist fragwürdig. Wenn, wie in seinem Beispiel, ein Arbeitsteam, aus welchen Gründen auch immer, ein bestimmtes Geschlecht nicht akzeptiert, dann kann man schwerlich behaupten, es liege keine Unterscheidung aufgrund des Merkmals „Geschlecht“ vor. So aber Eich. 
Kunden, zu schützen. ${ }^{41}$ In Verbindung mit $§ 3$ IV AGG gilt dies auch für befürchtete sexuelle Belästigungen. Der Arbeitgeber darf folglich Arbeitnehmer nicht aus dem Grund benachteiligen, weil er derartige Belästigungen befürchtet. $^{42}$ Dasselbe muß mit Blick auf die Antidiskriminierungsrichtlinien auch für den Bewerber um eine Stelle gelten. ${ }^{43}$

Nicht vertragsbezogene Gründe sind vor allem auch solche, die das außervertragliche Verhalten, insbesondere den privaten Lebensbereich betreffen. Im Zusammenhang mit dem Arbeitsrecht ist insofern festgestellt worden: „Die scharfe Trennung zwischen der Pflichtenbindung im Arbeitsverhältnis und dem privaten Lebensbereich zählt $\mathrm{zu}$ den bemerkenswertesten Errungenschaften des modernen Arbeitsrechts. [...] Außerdienstliches Verhalten kann nur ausnahmsweise den Bestand des Arbeitsverhältnisses beeinflussen. “44 Entscheidendes Gewicht komme insofern der Frage zu, inwieweit das außerdienstliche Verhalten Auswirkungen auf die Vertragsbeziehung der Arbeitsvertragsparteien zeitigt. ${ }^{45}$ So könnten beispielsweise Sittlichkeitsdelikte die Beschäftigung von Lehrern, Erziehern und anderen Pädagogen derart belasten, daß es unverantwortlich wäre, sie weiterhin als Erzieher einzusetzen. ${ }^{46}$

\title{
F. Vorurteile und Generalisierungen
}

\begin{abstract}
„In Andorra lebte ein junger Mann, den man für einen Juden hielt. [...] er wußte, er spürte, was alle wortlos dachten; er prüfte sich, ob es wirklich so war, daß er stets an das Geld denke, er prüfte sich, bis er entdeckte, daß es stimmt, es war so, in der Tat, er dachte stets an das Geld. [...] Bis es sich eines Tages zeigt, was er selber nicht hat wissen können, der Verstorbene: daß er ein Findelkind gewesen, dessen Eltern man später entdeckt hat, ein Andorraner wie unsereiner - [...]. Die Andorraner aber, sooft sie in den Spiegel blickten, sahen mit Entsetzen, daß sie selber die Züge des Judas tragen, jeder von ihnen. “47
\end{abstract}

Vorurteile und Generalisierungen bezüglich etwaiger Eigenschaften und Fähigkeiten von Trägern bestimmter Merkmale vermögen diskriminierungsrelevantes Verhalten grundsätzlich nicht $\mathrm{zu}$ rechtfertigen. So ist beispielsweise bei Tätigkeiten, die körperlich anstrengend sind, der grundsätzliche Ausschluß von Frauen mit dem Argument, sie verfügten über eine geringere physische Leistungsfähigkeit, unzulässig. ${ }^{48}$ Im Gegenzug dazu ist nicht einzusehen, warum

41 Entwurfsbegründung, BT-Druck. 16/1780, S. 37; Wie bereits an anderer Stelle erwähnt, ist der Arbeitgeber gem. dieser Norm verpflichtet, ,[...] die erforderlichen Maßnahmen zum Schutz vor Benachteiligungen wegen eines in $\S$ 1 genannten Grundes [ $-\mathrm{d}$. h. wegen eines dort genannten Diskriminierungsmerkmals - ] zu treffen. Der Schutz umfaßt auch vorbeugende Maßnahmen.“

42 Raab in Soergel, $\$ 611$ a, Rn. 37.

43 Für eine entsprechende Erweiterung des BeSchuG-A, das - wie bereits erwähnt - in $§ 2$ I eine dem $\S 12$ I AGG vergleichbare Bestimmung enthielt, war Schlachter. (Schlachter in Erfurter Kommentar zum Arbeitsrecht, 190, § 1 BeSchuG, Rn. 3).

44 Weiss, ArbuR Sonderheft 1979, S. 28 (30).

45 Müller-Glöge in Erfurter Kommentar zum Arbeitsrecht, 230, § 626, Rn. 162.

46 Müller-Glöge in Erfurter Kommentar zum Arbeitsrecht, 230, § 626, Rn. 162.

47 Aus der Novelle „Der andorranische Jude“ von Max Frisch.

48 LAG Köln, NZA-RR 2001, S. 232 (232, Leitsatz); Raab in Soergel, § 611 a, Rn. 35; Eckert in Hk-BGB, § 611 a, Rn. 2. 
Vorgesetzten grundsätzlich das Recht eingeräumt werden sollte, ausschließlich Sekretärinnen statt Sekretäre einzustellen. ${ }^{49}$ In der Entwurfsbegründung zum AGG heißt es schließlich: „Eine beispielsweise auf Xenophobie beruhende pauschale Angst vor „dem Islam“ oder „den Juden“ kann daher eine Ungleichbehandlung nach dem Merkmal der Religion nicht rechtfertigen. “50

Vorurteile und Generalisierungen stellen vor allem dann keinen sachlichen Grund dar, wenn sie nicht vertragsbezogen sind. Der Chefin eines Ladenbetriebes in jedem Fall zu erlauben, Männer zurückzuweisen, weil sie durch bittere Lebenserfahrung männerfeindlich geworden ist, erscheint daher beispielsweise äußerst fragwürdig. ${ }^{51}$ In diesem Sinne wird vertreten, Sym- und Antipathie seien nur insofern zulässige Auswahlkriterien, ,sofern ihre Berücksichtigung nicht gegen spezielle Diskriminierungsverbote verstößt. So wäre es etwa mit Art. 3 Abs. 3 GG unvereinbar, wenn der Arbeitgeber den Bewerber wegen seiner Hautfarbe ablehnen könnte, selbst wenn er glaubt, wegen seiner Antipathien gegen Menschen fremder Herkunft mit einer solchen Person nicht zusammenarbeiten zu können. “52

\section{G. (Vorwerfbare) Störung des Vertragsverhältnisses}

Beispiel: Kann der muslimische Arbeitnehmer von seinem Arbeitgeber verlangen, daß dieser ihm eine dreiminütige Freistellung von der Arbeit gewährt, um täglich sein Morgengebet verrichten zu können? ${ }^{53}$

Zunächst kann festgehalten werden, daß ein allgemeiner Vorrang von Gewissensentscheidungen gegenüber Vertragspflichten nicht besteht. ${ }^{54}$ In diesem Sinne hat das erkennende Gericht in dem Beispielsfall entschieden, daß der Arbeitgeber zwar verpflichtet ist, ,im zumutbaren Umfang durch betriebliche Organisationsmaßnahmen die Religionsausübung durch den [Arbeitnehmer] zu gewährleisten“, daß er aber andererseits ,auch im Hinblick auf den Schutz des Art. 4 II GG nicht verpflichtet [ist], Betriebsablaufstörungen hinzunehmen, damit der [Arbeitnehmer] seine Gebetspausen einhalten kann. Insoweit hat die Vertragstreue Vorrang. “55

Diesem Urteil kann uneingeschränkt gefolgt werden. Das Gericht hat entschieden, daß der Arbeitgeber betriebliche Organisationsmaßnahmen treffen muß, um die Religionsausübung seiner Arbeitnehmer zu gewährleisten. Dies kann nicht bedeuten, daß sich der Betriebsablauf grundsätzlich nach den individuellen Ansprüchen des einzelnen Arbeitnehmers richten müßte.

49 So aber Eich, NJW 1980, S. 2329 (2331); ebenso Wiese: „Warum sollte ein(e) Unternehmer(in) nicht sein (ihr) Vorzimmer stets mit einer Sekretärin besetzen und sich von einem Mann als Chauffeur fahren lassen dürfen?“ (Wiese, JuS 1990, S. 357 (360)); dagegen Söllner in MüKo, § 611 a, Rn. 16; Thüsing, RdA 2001, S. 319 (323).

50

Entwurfsbegründung, BT-Druck. 16/1780, S. 44

51 So aber Adomeit, DB 1980, S. 2388 (2388).

52 Raab, RdA 1995, S. 36 (38); ebenso Buchner in Münchener Handbuch zum Arbeitsrecht, Band 1, § 39, Rn. 74; Müller-Glöge in MüKo, § 611 a, Rn. 23; a. A.: Wiese, JuS 1990, S. 357 (359).

53 LAG Hamm, NJW 2002, S. 1970 ff.

54 Heinrichs in Palandt, $\S 242$, Rn. 8.

55 LAG Hamm, NJW 2002, S. 1970 (1972). 
Zugeständnisse eines jeden Arbeitnehmers bzgl. Ort, Zeit sowie Art und Weise der Erfüllung der geschuldeten Pflichten aus dem Arbeitsvertrag sind gerade, aber nicht nur in großen Betrieben mit automatisiertem Arbeitsablauf notwendig, um überhaupt einen geregelten, für alle Arbeitnehmer akzeptablen Betriebsablauf sicherstellen zu können.

Besondere Bedeutung erlangt in diesem Zusammenhang auch die Tatsache, daß es sich bei den begehrten Gebetspausen um religiös bedingtes Verhalten des Arbeitnehmers handelt. Der Status „Muslim“wird durch das Verbot der Gebetspausen nicht berührt.

Damit der Arbeitgeber sein Direktionsrecht nicht mißbraucht, wird zu recht verlangt, daß er es ausüben muß, um einen ungestörten Betriebsablauf zu gewährleisten.

Im Zusammenhang mit der Verpflichtung des Arbeitgebers, in zumutbarem Umfang durch betriebliche Organisationsmaßnahmen die Religionsausübung durch den Arbeitnehmer zu gewährleisten, ist $\mathrm{zu}$ erwähnen, daß dann, wenn sich ein Arbeitnehmer aufgrund einer Gewissensentscheidung nicht in der Lage sieht, die ihm zugewiesene Arbeit zu verrichten, eine personenbedingte Kündigung gem. $§ 1$ II $1 \mathrm{KSchG}$ nur dann gerechtfertigt sein soll, wenn für den betreffenden Arbeitnehmer eine andere Beschäftigungsmöglichkeit nicht besteht. ${ }^{56}$

Beispiel: „Aber auch der Personalchef im Großbetrieb könnte denken (besser nicht sagen): Abteilungsleiter X ist misogyn, mit weibl. Angestellten kann er nachgewiesenermaßen (Fall 1, Fall 2 ...) nicht zusammenarbeiten, also kommt nur ein männlicher Bewerber in Frage. Oder umgekehrt: er ist ein Casanova felliniesken Ausmaßes, also ist im Interesse sachlicher Betriebsatmosphäre besser ein Mann einzustellen, fast schon Fürsorgepflicht gegenüber den Bewerberinnen, sie dem nicht auszusetzen. “57

Zwar hat das BAG festgestellt, daß nachweisliche Störungen des Betriebsklimas sogar Kündigungen von Personen mit bestimmten Merkmalen rechtfertigen können. ${ }^{58}$ Dies kann aber nicht dazu führen, daß sich der Arbeitgeber seiner Verantwortung, in seinem Betrieb eine für alle Arbeitnehmer akzeptable, belästigungsfreie Atmosphäre zu schaffen, dadurch entledigt, daß er Bewerber mit bestimmten Merkmalen von vornherein nicht einstellt, weil er davon ausgeht, daß es zwischen den bisherigen Arbeitnehmern und eben diesen Bewerbern zu Störungen der Betriebsatmosphäre kommen wird.

In dem vom BAG entschiedenen Fall war es die Arbeitnehmerin, die durch ihr Verhalten die Kündigung verursacht hatte. Demgegenüber wird in den soeben genannten Beispielsfällen die Nichteinstellung mit einem zu erwartenden Verhalten Dritter, nicht des Bewerbers, gerechtfertigt. Die Bewerberinnen sollen die Konsequenzen dafür tragen, daß ihre männlichen Kollegen sie meiden bzw. belästigen würden. Besonders sarkastisch auch vor dem Hintergrund hoher 
Arbeitslosigkeit erscheint es, daß die Nichteinstellung der Bewerberinnen als Fürsorgepflicht deklariert wird.

Im Grundsatz muß gelten, daß nur bei vorwerfbarer Störung des Betriebsklimas und damit des Vertragsverhältnisses diskriminierungsrelevantes Verhalten gerechtfertigt sein kann. ${ }^{59}$ In den Beispielsfällen wäre die Ablehnung von Bewerberinnen unter Angabe der dort genannten Gründe unzulässig. ${ }^{60}$ Das Geschlecht ist ein unbeeinflußbares Diskriminierungsmerkmal. Wie bereits an anderer Stelle festgestellt, resultiert die besondere Schutzwürdigkeit unbeeinflußbarer Diskriminierungsmerkmale gerade daraus, daß ihr Vorhanden- bzw. Nichtvorhandensein durch ihre Träger nicht beeinflußt werden kann. Daraus ergibt sich wiederum die Konsequenz, daß dem Träger eines derartigen Merkmals dessen Trägerschaft nicht vorgeworfen werden kann. Diesem unberechtigten Vorwurf wären aber die Bewerberinnen in dem Beispielsfall ausgesetzt, wenn sie nicht eingestellt würden, weil Abteilungsleiter $\mathrm{X}$ ein Frauenfeind bzw. Casanova felliniesken Ausmaßes ist.

\section{H. Zusammentreffen der Träger von Diskriminierungsmerkmalen}

Die folgenden Überlegungen beschäftigen sich mit der vor allem bei Arbeits- und Mietverträgen typischen Fallkonstellation, daß sich mehrere Personen um ein und denselben Vertrag bemühen, der „Diskriminierende“ also von vornherein die Absicht hat, nur mit einer Person zu kontrahieren.

Beispiel: „Der Vermieter, der mehrere Wohnungen vermietet, hat zwei Interessenten für eine Wohnung, einen Asiaten und einen Italiener. Er entscheidet sich für den Italiener. Der asiatische Interessent klagt und behauptet, er sei wegen seiner Herkunft benachteiligt worden. “61

Es wird behauptet, das Vorliegen einer Diskriminierung müsse zwangsläufig dann bejaht werden, wenn zwei Träger desselben Diskriminierungsmerkmals zusammentreffen und der „Diskriminierende“ sich für einen der beiden entscheidet: ,[Der Arbeitgeber] kann von folgenden Bewerbern auf Schadensersatz und Schmerzensgeld verklagt werden: Von allen Älteren, weil er sich für eine 30 Jahre alte Person entschieden hat; von allen Christen, weil er sich für einen Hindu entschieden hat; von den Ausländern, weil er sich für einen Deutschen entschieden hat; von denen mit weißer Hautfarbe, weil er sich für einen Schwarzen entschieden hat; von den Homosexuellen, weil er sich für einen Heterosexuellen entschieden hat.“62 Die gleiche Behauptung wird aufgestellt,

59 Zur Möglichkeit verhaltensbedingter Kündigung gem. § 1 II 1 KSchG bei Störung des Betriebsfriedens vgl. v. Hoyningen-Huene/Linck, Kündigungsschutzgesetz, § 1, Rn. 330 f.

60 Adomeit hielt indes in beiden Fällen das männliche Geschlecht für unverzichtbar i. S. d. § 611 a I 2 BGB-A. (Adomeit, DB 1980, S. 2388 (2388)).

61 Wagner, in FAZ vom 22.12.2004, S. 12 unter dem Titel: „Das Antidiskriminierungsgesetz - ein Angriff auf die Vertragsfreiheit".

62 Ramsauer, in FAZ vom 20.01.2005 S. 10 unter dem Titel: „Baustein zum Nullwachstum“. 
wenn Träger verschiedener Diskriminierungsmerkmale zusammentreffen. „Ein Farbiger, ein Türke, ein Homosexueller, ein Behinderter und eine Familie mit drei Kindern bewerben sich um eine Wohnung. Wen darf der Vermieter ablehnen, ohne gegen das ADG zu verstoßen? Nur die Familie mit den Kindern. “63

Konsequenz dieser Ansicht ist, daß in jedem Fall, in dem sich mehrere Personen um den Abschluß desselben Vertrages bemühen, eine Diskriminierung vorliegt. Denn schließlich sind alle Menschen Träger von Diskriminierungsmerkmalen. Wenn sich mehrere Bewerber um den Abschluß ein und desselben Vertrages bemühen, treffen infolgedessen stets Träger von (denselben oder verschiedenen) Diskriminierungsmerkmalen aufeinander.

Diese Ansicht ist schon allein deshalb nicht haltbar, weil sie sich in Widerspruch zu der Verpflichtung des Ausbietenden setzt, merkmalsneutral auszuschreiben. ${ }^{64}$ Denn je neutraler die Ausschreibung ist, desto größer ist der Interessentenkreis. Allein die Tatsache, daß der Vermieter in dem Beispielsfall unter mehreren Bewerbern die Wahl trifft, weil er eben nur eine Wohnung zu vermieten hat, kann damit nicht per se zu dem Vorwurf führen, er habe diskriminiert. ${ }^{65}$ Fraglich ist dennoch, ob in derartigen Fällen diskriminiert werden kann.

\section{Grundsatz des freien Auswahlermessens}

Dagegen könnte der Grundsatz des freien Auswahlermessens sprechen. Der Arbeitgeber soll nach deutschem Recht grundsätzlich nicht zu sachbezogenen Entscheidungen zwischen mehreren Bewerbern verpflichtet sein. ${ }^{66}$ Nicht einmal die Begründung: „Die Nase gefällt uns nicht!“ löse arbeitsrechtliche Konsequenzen aus. ${ }^{67}$ Eine Ausnahme soll insoweit allerdings $\S 611$ a BGB-A gebildet haben. ${ }^{68}$ Dasselbe dürfte nunmehr in bezug auf das Diskriminierungsverbot des $§ 7$ I AGG vertreten werden. Fraglich ist, ob es sich bei dieser Norm tatsächlich um eine Ausnahme vom Grundsatz des freien Auswahlermessens handelt.

\section{Einschränkung durch das Diskriminierungsverbot?}

Wenn sich ein Mann und eine Frau für eine Stelle bewerben, für die ein bestimmtes Geschlecht keine wesentliche und entscheidende berufliche Anforderung im Sinne des § 8 I AGG darstellt, dann muß der Arbeitgeber den Mann (oder umgekehrt die Frau) auch mit der Begründung

63 Braun, JuS 2002, S. 424 (424).

64 Vergleiche dazu etwa $§ 11$ AGG.

65 Auf dieses Problem weist auch Adomeit hin. (Adomeit, NJW 2002, S. 1622 (1623)).

66 Waas, ZIP 2000, S. 2151 (2153); vgl. insofern auch eine Stellungnahme der EU-Kommission, die sich auf Anfrage des EU-Parlaments dahingehend geäußert hat, EU-Unternehmen dürften bereits in Stellenanzeigen Raucher explizit aus dem Bewerberkreis ausschließen (FAZ vom 08.08.2006, S. 13, unter dem Titel: „Im grauen Dunstbereich“). 
auswählen können, er wolle einen Mann bzw. eine Frau. Andernfalls würde er sowohl in dem einen wie in dem anderen Fall diskriminieren, da Anknüpfungspunkt für die Entscheidung des Arbeitgebers schließlich niemals das Geschlecht sein darf.

Erlaubte man dem Arbeitgeber in einer solchen Situation nicht ausnahmsweise, den Mann (die Frau) zu wählen, gerade weil er ein Mann (sie eine Frau) ist, dann käme als rechtmäßiges Alternativverhalten nur noch die Nichteinstellung beider Bewerber in Betracht. ${ }^{69}$ Die Wahl des jeweils anderen wäre jedenfalls kein rechtmäßiges Alternativverhalten.

Von einem Arbeitgeber kann nicht verlangt werden, in einer solchen Pattsituation beide Bewerber nicht einzustellen. Andernfalls könnte die groteske Situation entstehen, daß er wiederholte Male ausschreiben muß, um letztlich einen Arbeitnehmer einzustellen.

Im Rahmen der Beantwortung der Frage, ob $\S 7$ I AGG den Grundsatz des freien Auswahlermessens einschränkt, muß aber auch folgendes berücksichtigt werden: Das Geschlecht oder, wie im Ausgangsfall, die Staatsangehörigkeit bzw. ethnische Herkunft zum Anknüpfungspunkt einer Auswahlentscheidung zu machen kann nur dann akzeptiert werden, wenn sich die Bewerber um den Vertrag allein durch das jeweilige Merkmal unterscheiden. Dem Arbeitgeber darf deshalb beispielsweise nicht verwehrt werden, einen Arbeitnehmer einzustellen, bei dem er aufgrund dessen Jugend davon ausgeht, daß dieser leistungsfähiger als ein gleichqualifizierter älterer Kollege ist, selbst wenn objektive Anhaltspunkte hierfür fehlen. ${ }^{70}$

Wenn demgegenüber der Arbeitgeber die Bewerberin nicht einstellt, weil sie eine Frau ist, obwohl sie besser qualifiziert ist als ihr männlicher Konkurrent, dann ist es ein bestimmtes Geschlecht, das durch die Maßnahme ausgegrenzt werden soll. Der Arbeitgeber steht in einer solchen Situation eigentlich nicht vor der „Qual der Wahl“. Man verlangt hier von ihm nichts Unmögliches, wenn man ihn dazu verpflichtet, seine Entscheidung unabhängig vom Geschlecht des Bewerbers zu treffen.

An dieser Stelle kann somit die Feststellung getroffen werden, daß $§ 7$ I AGG den Grundsatz des freien Auswahlermessens nicht unberührt läßt. Das gleiche gilt insbesondere auch für die Antidiskriminierungsrichtlinien. ${ }^{71}$

\section{2. ,Wie“ der Einschränkung}

69 Darauf weist Herrmann hin. (Herrmann, ZfA 1996, S. 19 (29)).

70 A. A. aber Thüsing, NZA 2001, S. 1061 (1063); Thüsing läßt offen, wann sich der Arbeitgeber in einer solchen Situation richtig verhielte.

71 Waas meint in diesem Zusammenhang, ,daß sich [...] weitreichende Wirkungen der Richtlinie [2000/43/EG] nicht ausschließen lassen.“ (Waas, ZIP 2000, S. 2151 (2153)); Bauer vertritt demgegenüber die Ansicht, daß trotz der Richtlinie 2000/78/EG der Grundsatz des freien Auswahlermessens auch künftig aufrechterhalten werden müsse. (Bauer, NJW 2001, S. 2672 (2673)). 
Nachdem die Frage des „Ob“ beantwortet ist, bleibt zu klären, wie weit Antidiskriminierungsrecht den Grundsatz des freien Auswahlermessens einzuschränken vermag.

$\mathrm{Zu}$ diesem Zweck sei an dieser Stelle noch einmal an die Rechtsprechung des EuGH zur Zulässigkeit von Geschlechter-Quotenregelungen erinnert. Das Gericht hat entschieden, daß europäisches Recht einer nationalen Regelung entgegensteht, „nach der ein Bewerber des unterrepräsentierten Geschlechts um eine Stelle im Staatsdienst, der hinreichende Qualifikationen für diese Stelle besitzt, vor einem Bewerber des anderen Geschlechts, der [aufgrund besserer Qualifikation] sonst ausgewählt worden wäre, auszuwählen ist, [...].“72 Das Gericht hält dagegen eine Regelung mit europäischem Recht für vereinbar, „nach der ein Bewerber des unterrepräsentierten Geschlechts einem Bewerber des anderen Geschlechts vorgezogen werden kann, wenn die Verdienste der Bewerber als gleichwertig oder fast gleichwertig anzusehen sind, [...], sofern die Bewerbungen Gegenstand einer objektiven Beurteilung sind, bei der die besondere persönliche Lage aller Bewerber berücksichtigt wird.“73

Die genannten Kriterien könnten auch für die an dieser Stelle diskutierte Fallkonstellation maßgeblich sein, stellt sich doch auch hier im Kern die Frage, wann die unmittelbare Anknüpfung an ein bestimmtes Merkmal zulässig sein soll.

Wenn zwei Bewerber gleichqualifiziert sind, dann muß der Arbeitgeber seine Entscheidung auf das verbleibende Diskriminierungsmerkmal stützen können. Oder sollte man von ihm tatsächlich verlangen, eine Münze zu werfen oder das Los entscheiden zu lassen? Wohl nicht.

Freilich werden sich zwei Bewerber nie wie eineiige Zwillinge gleichen. Schon die Tatsache, daß die Bewerber an unterschiedlichen Universitäten studiert haben, könnte die Ungleichwertigkeit begründen. Deshalb ist es richtig, daß der EuGH annähernde Gleichwertigkeit ausreichen läßt. ${ }^{74}$ Von dem zeitgemäßen, sozialbewußten Arbeitgeber, der den (Fort-)Bestand und die Leistungsfähigkeit seines Unternehmens anstrebt, ${ }^{75}$ wird man verlangen können, daß er die Grenze zwischen (fast) gleichwertig und ungleichwertig zu ziehen vermag. ${ }^{76}$

Fraglich ist in diesem Zusammenhang, ob der Arbeitgeber bei der Prüfung der Gleichwertigkeit der Qualifikationen die besondere persönliche Lage der Bewerber berücksichtigen muß. Der Beantwortung dieser Frage soll das folgende Beispiel dienen: Verzögerungen beim Abschluß der Ausbildung aufgrund der Betreuung von Kindern können bei Männern wie Frauen auftreten. De facto betreffen solche Verzögerungen aber in erster Linie Frauen. ${ }^{77}$ Dem Arbeitgeber sollte aus diesem Grund nicht verwehrt sein, eine solche Verzögerung nicht negativ in die

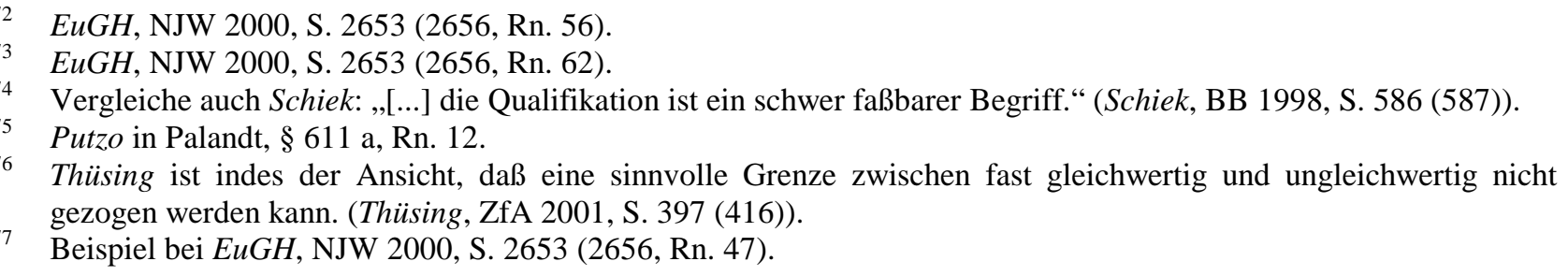


Gleichwertigkeitsprüfung einfließen zu lassen. Es muß ihm mit anderen Worten gestattet sein, in einem Fall, in dem es zu einer derartigen Verzögerung seitens der Bewerberin gekommen ist, bei dem Bewerber hingegen nicht, gleichwohl die Gleichwertigkeit beider Bewerber zu bejahen. Dies läßt sich mit den Zielen, die Antidiskriminierungsmaßnahmen verfolgen, begründen. Selbige wollen nicht nur formelle Gleichheit schaffen, sondern streben, wo erforderlich, auch materielle Gleichheit an. $^{78}$

Der Arbeitgeber ist sogar mittelbar verpflichtet, die besondere persönlichen Lage der Bewerber zu berücksichtigen, kann er sich doch bei deren Nichtbeachtung dem Vorwurf aussetzen, mittelbar zu diskriminieren. Eine mittelbare Diskriminierung soll z. B. dann gegeben sein, wenn Altershöchstgrenzen für die Einstellung festgelegt werden, die von Frauen wegen des zeitweiligen Ausscheidens aus dem Beruf zum Zwecke der Kinderbetreuung häufiger überschritten werden. ${ }^{79}$

\section{II. § 15 II 2 AGG}

Fraglich ist, ob im Arbeitsrecht § 15 II 2 AGG dem hier gewonnenen Ergebnis entgegensteht, wonach der Arbeitgeber in der beschriebenen Pattsituation seine Entscheidung auf das Geschlecht stützen kann. Gemäß $§ 15$ II AGG kann der oder die Beschäftigte

„[w]egen eines Schadens, der nicht Vermögensschaden ist, [...] eine angemessene Entschädigung in Geld verlangen. Die Entschädigung darf bei einer Nichteinstellung drei Monatsgehälter nicht übersteigen, wenn der oder die Beschäftigte auch bei benachteiligungsfreier Auswahl nicht eingestellt worden wäre.“

In der hier diskutierten Fallkonstellation wäre der jeweils benachteiligte Bewerber, d. h. der Mann oder die Frau, auch dann nicht zwangsläufig eingestellt worden, wenn der Arbeitgeber seine Auswahl nicht auf das Geschlecht gestützt, selbige vielmehr der Münze - Kopf oder Zahl? -, d. h. dem Zufall überlassen hätte. § 15 II 2 AGG scheint damit in dieser Fallkonstellation einschlägig zu sein. Fraglich ist, ob die Norm tatsächlich in dieser Weise interpretiert werden kann.

Der $E u G H$ hat entschieden, daß auch der Bewerber einen Schaden erleidet, dessen Bewerbung aufgrund seines Geschlechts schon keine Berücksichtigung gefunden hat, auch wenn er sowieso nicht eingestellt worden wäre. ${ }^{80}$ In dem von dem Gericht entschiedenen Fall, in dem geschlechtsspezifisch ausgeschrieben worden war: „Für unseren Vertrieb suchen wir eine versierte 
Assistentin der Vertriebsleitung.“, hatten sich zwei Männer beworben, von denen der eine bei diskriminierungsfreiem Verhalten eingestellt worden wäre. ${ }^{81}$

Anders als in der hier besprochenen Fallkonstellation, wurde in jenem Fall zum Zeitpunkt der Ausschreibung allein aufgrund des Geschlechts unterschieden. Der sachliche Grund der besseren Qualifikation eines der Bewerber war zu diesem Zeitpunkt noch kein Unterscheidungsmerkmal.

Entsprechend den Vorgaben des EuGH wurde auch $\S 611$ a III BGB-A interpretiert, der in Umsetzung der in jenem Urteil enthaltenen Vorgaben neu ins BGB eingeführt worden war $^{82}$ und eine dem $\S 15$ II AGG entsprechende Regelung enthielt. ${ }^{83}$ Es wurde vertreten, $\S 611$ a III BGB-A betreffe diejenigen Fälle, in denen der Bewerber zwar wegen seines Geschlechts nicht in die Auswahl für den Arbeitsplatz gelangt ist, aber die Stelle sowieso nicht erhalten hätte. ${ }^{84} \S 611$ a III BGB-A setze nur voraus, daß der Bewerber bei der Auswahl geschlechtsbezogen nicht berücksichtigt wurde, nicht aber, daß er nur deswegen nicht eingestellt worden ist. $^{85}$

$\S 15$ II 2 AGG meint demnach nicht die hier diskutierten Fälle, in denen sich der Arbeitgeber in der beschriebenen Entscheidungsnot befindet und seine Entscheidung deshalb zulässigerweise auf das Geschlecht stützen kann. Das Problem, „welche Ansprüche z. B. zwei gleich qualifizierte Klägerinnen haben, denen ein gleich qualifizierter Konkurrent vorgezogen wurde“", und die alle drei „,in der engeren Auswahl waren“, muß demnach nicht gelöst werden, weil insofern kein Problem besteht. ${ }^{86}$ Zumindest eine unmittelbare Diskriminierung liegt in einer derartigen Situation nicht vor.

\section{Mittelbare Diskriminierung}

Fraglich ist, wie zu entscheiden wäre, wenn sich der Vermieter des Ausgangsfalls in derselben Situation immer gleich verhielte. Oder, um ein weniger konstruiertes Beispiel zu nennen, wie wäre zu entscheiden, wenn der Arbeitgeber in wiederholten Einstellungsverfahren stets den Mann bzw. die Frau auswählte? ${ }^{87}$

In einer derartigen Situation ist an das Vorliegen einer mittelbaren Diskriminierung zu denken. Im Unterschied zu den „klassischen“ Fällen mittelbarer Diskriminierung knüpft der „Diskriminierende“ seine Entscheidung in der hier diskutierten Konstellation unmittelbar an das in Rede stehende Diskriminierungsmerkmal. Die bisher unter den Begriff der mittelbaren Diskriminierung subsumierten Fälle zeichnen sich demgegenüber dadurch aus, daß der

$81 \quad E u G H$, DB 1997, S. 983 (983).

82 Richardi in Münchener Handbuch zum Arbeitsrecht, Band 1, § 11, Rn. 37.

$83 \S 611$ a III 1 BGB-A hatte folgenden Wortlaut: „Wäre der Bewerber auch bei benachteiligungsfreier Auswahl nicht eingestellt worden, so hat der Arbeitgeber eine angemessene Entschädigung [...] zu leisten.“.

84 Putzo in Palandt, § 611 a, Rn. 21.

85 Putzo in Palandt, $\$ 611$ a, Rn. 22.

86 A. A.: Schiek, die in dem Beispielsfall eine Diskriminierung bejaht, wenn der Arbeitgeber seiner Entscheidung das Geschlecht zugrunde legt. (Schiek, BB 1998, S. 586 (587)).

$87 \mathrm{Da}$ der Arbeitgeber in Einstellungsverfahren die Wahl zwischen mehreren gleichqualifizierten Bewerbern hat, wird in der Praxis nicht selten vorkommen.; a. A.: Schiek, BB 1998, S. 586 (587). 
Diskriminierende seine Entscheidung nicht auf das in Rede stehende Diskriminierungsmerkmal stützt, sondern auf einen Grund, von dem die Träger des in Rede stehenden Merkmals in stärkerem Maße negativ betroffen sind als Nicht-Träger dieses Merkmals.

Der für sich genommen sachliche Grund, auf den der mittelbar Diskriminierende seine Entscheidung stützt, wird gerade dadurch zu einem unsachlichen. In gleicher Weise läßt sich bei der hier betrachteten Fallkonstellation argumentieren. Die für sich genommen sachlich gerechtfertigte Anknüpfung an das in Rede stehende Diskriminierungsmerkmal wird dadurch unsachlich, daß sie die Träger eben dieses Merkmals in stärkerem Maße negativ betrifft als diejenigen, die nicht Träger dieses Merkmals sind.

Es wäre deshalb gerechtfertigt, dem Vermieter des Ausgangsfalls den Vorwurf der mittelbaren Diskriminierung $\mathrm{zu}$ machen, wenn er in der beschriebenen Pattsituation stets die gleiche Entscheidung träfe. Gleiches würde natürlich auch für den Arbeitgeber gelten, der sich in einer solchen Situation immer für den Mann bzw. die Frau entschiede.

Dem Vorwurf, mittelbar zu diskriminieren, kann der Arbeitgeber (Vermieter) dadurch entgehen, daß er Fördermaßnahmen in Form einer Quotenregelung einführt. ${ }^{88}$

$\mathrm{Ob}$ der Gesetzgeber den privaten Arbeitgeber auch verpflichten darf, Quotenregelungen einzuführen, erscheint vor dem Hintergrund der Möglichkeit, den Arbeitgeber durch das Institut der mittelbaren Diskriminierung zu dem gewünschten Verhalten zu bewegen, als zweitrangiges Problem. ${ }^{89}$

\section{Relativität des Begriffs der Bestqualifizierung}

Die bisherigen Überlegungen könnten zu dem Mißverständnis geführt haben, der Arbeitgeber müsse stets den in objektiver Hinsicht Bestqualifizierten einstellen. Dem ist aber nicht so. ${ }^{90}$ Festgestellt wurde lediglich, daß bei (fast) gleichwertiger Qualifikation die ansonsten unzulässige Berufung auf ein bestimmtes Merkmal zulässig ist, weil dem Arbeitgeber in einer solchen Pattsituation eine sachgerechte Auswahl unmöglich ist.

Natürlich kann der Arbeitgeber bei unterschiedlicher Qualifikation den objektiv „,schlechter“ qualifizierten Bewerber einstellen. Schließlich liegt es im Belieben des Arbeitgebers, welche

88 Von dieser Möglichkeit geht auch Hanau aus: „Die vom EuGH aufgestellten Grundsätze, die zu Quotenregelungen im öffentlichen Dienstrecht ergingen, gelten insofern auch für private Arbeitgeber, als diese sich freiwillig zu einer Quotenregelung entschließen.“(Hanau in Gedächtnisschrift für Lüderitz, S. 241 (267)).

89 Hanau weist auf die Frage hin, läßt sie jedoch unbeantwortet: „Ob der Gesetzgeber private Arbeitgeber dazu verpflichten darf, Quotenregelungen einzuführen, bedarf noch weiterer Überprüfung im Lichte des Amsterdamer Vertrages." (Hanau in Gedächtnisschrift für Lüderitz, S. 241 (267)).

90 In diesem Sinne auch Buchner in Münchener Handbuch zum Arbeitsrecht, Band 1, § 39, Rn. 74; Im Zusammenhang mit $\S 611$ a BGB-A ist beispielhaft erwähnt worden, die Einstellung eines fachlich weniger qualifizierten Sportfreundes des Arbeitgebers unterfalle nicht dem Schutzbereich dieser Norm. (Müller-Glöge in MüKo, § 611 a, Rn. 23). 
Anforderungen er an die Qualifikation der Bewerber stellt. ${ }^{91}$ Er entscheidet, wer der bestqualifizierte Bewerber ist: „Ebenso wie er auf größere Berufserfahrung abstellen kann, steht es ihm frei, Berufsanfänger vorzuziehen, die erst durch die Arbeit in seinem Betrieb ihre Prägung erfahren. Er kann auf höheres, aber auch auf geringeres Lebensalter Wert legen, er darf die Fähigkeit zur kollegialen Zusammenarbeit bevorzugen oder in erster Linie auf Durchsetzungsfähigkeit abstellen. Eine vielseitige berufliche Biographie kann für ihn vorrangiges Auswahlkriterium sein, umgekehrt aber auch Stetigkeit in der Berufspraxis. Ebenso steht es in seinem Belieben, eine spezifische Kombination verschiedener Eigenschaften zu fordern und die einzelnen Qualifizierungsmerkmale unterschiedlich zu gewichten. “92 Auch ist denkbar, daß sich der Minderqualifizierte im Bewerbungsgespräch im Vergleich zu dem Bestqualifizierten als eloquenter, kreativer, spontaner, umgänglicher, höflicher, etc. gezeigt hat, mit der Konsequenz, daß der Arbeitgeber gerade ihm eine besondere Sympathie entgegenbringt. „Auch solche unterhalb der Schwelle des Objektivierbaren liegende Sym- oder Antipathien sind für das vertragliche Erfüllungsinteresse des Arbeitgebers von Bedeutung und kommen als Auswahlkriterien in Betracht." ${ }^{\text {93 }}$ All die genannten Gründe können dazu führen, daß sich der Arbeitgeber in einem solchen Fall berechtigterweise für den objektiv Minderqualifizierten entscheidet.

Da allein der Arbeitgeber die Auswahlkriterien bestimmt, kann er grundsätzlich nicht verpflichtet werden, auf bestimmte sachorientierte Hilfskriterien wie das Lebensalter zurückzugreifen. ${ }^{94}$

Nicht übersehen werden darf, daß die Anforderungen, die der Arbeitgeber an seinen Favoriten stellt, ihrerseits auf einen möglichen Verstoß gegen das Diskriminierungsverbot zu überprüfen sind. So sind Sym- bzw. Antipathie nur insoweit zulässige Auswahlkriterien, „sofern ihre Berücksichtigung nicht gegen spezielle Diskriminierungsverbote verstößt. So wäre es etwa mit Art. 3 Abs. 3 GG unvereinbar, wenn der Arbeitgeber den Bewerber wegen seiner Hautfarbe ablehnen könnte, selbst wenn er glaubt, wegen seiner Antipathien gegen Menschen fremder Herkunft mit einer solchen Person nicht zusammenarbeiten zu können. “95 Auch dürfte dem Arbeitgeber angesichts der Richtlinie 2000/78/EG künftig untersagt sein, nach Belieben das Alter zum Anknüpfungspunkt seiner Auswahlentscheidung zu machen. ${ }^{96}$

\section{Zusammenfassung}

BVerfGE 89, S. 276 (289).

BVerfGE 89, S. 276 (289 f.).

Buchner in Münchener Handbuch zum Arbeitsrecht, Band 1, § 39, Rn. 74; ebenso Raab, RdA 1995, S. 36 (38); Müller-Glöge in MüKo, § 611 a, Rn. 23; Wiese, JuS 1990, S. 357 (360).

94 Herrmann verweist insofern auf Literatur und Rechtsprechung des öffentlichen Rechts. (Herrmann, ZfA 1996, S. $19(29))$.

95 Raab, RdA 1995, S. 36 (38); ebenso Buchner in Münchener Handbuch zum Arbeitsrecht, Band 1, § 39, Rn. 74; Müller-Glöge in MüKo, § 611 a, Rn. 23; a. A.: Wiese, JuS 1990, S. 357 (359).

96 So aber BVerfGE 89, S. 276 (289 f.). 
Die im Rahmen des Prüfungspunktes „Zusammentreffen der Träger von Diskriminierungsmerkmalen“ gewonnenen Erkenntnisse lassen sich wie folgt zusammenfassen: Hat der „Diskriminierende“ die Wahl zwischen mehreren Personen bzw. Merkmalsträgern - um dieselbe Stelle bewerben sich ein Mann und eine Frau -, darf die Entscheidung auf einem an sich unzulässigen Merkmal beruhen - der Arbeitgeber entscheidet sich bewußt für den Mann/die Frau -, wenn eine sachgerechte Differenzierung nicht möglich ist - etwa weil die Bewerber gleichqualifiziert sind.

Nichtsdestotrotz diskriminiert mittelbar, wer in der beschriebenen Pattsituation stets dasselbe Merkmal begünstigt - Arbeitgeber entscheidet sich immer für den Bewerber/die Bewerberin.

$\mathrm{Zu}$ beachten ist schließlich, daß der „Diskriminierende“ - in den Grenzen des Diskriminierungsverbotes - selbst bestimmen kann, ob zwei Personen als „gleichwertig“ anzusehen, zwei Bewerber etwa gleichqualifiziert sind.

$\mathrm{Zu}$ dem Ausgangsfall kann daher gesagt werden: Gegen den Vermieter kann unter der Prämisse, daß es sich bei dem Asiaten und dem Italiener um „gleichwertige“ Interessenten der Wohnung handelte - im Falle eines Mietvertrages dürfte dies in der Regel bei gleichermaßen solventen Interessenten der Fall sein -, nicht der Vorwurf erhoben werden, er habe den Asiaten dadurch diskriminiert, daß er sich bewußt für den Italiener entschieden hat.

Dieses Ergebnis nimmt denjenigen den Wind aus den Segeln, die durch derartige Beispielsfälle Antidiskriminierungsrecht zu diskreditieren suchen. ${ }^{97}$

\section{J. Authentizitätswahrung}

Beispiele: Ist es sachlich begründet, daß ein Schwarzer die Rolle eines Schwarzen in einem Film spielt, ausschließlich Frauen Damenmode präsentieren?

Die Beispielsfälle wurden i. R. d. $\S 611$ a BGB-A teilweise unter dem Stichwort „Authentizitätswahrung“ als Fälle objektiver Unverzichtbarkeit behandelt. ${ }^{98}$ Damenmode könne eben authentisch nur von Frauen vorgeführt, eine Sopranrolle nur von einer Frau gesungen werden,

97 Auch Baer stellt fest, daß bislang ,[...] skandalisierende, aber kaum erhellende Beispiele konstruiert [werden], die Recht gegen Diskriminierung ad absurdum führen sollen, bei genauerer Betrachtung aber Verhaltensweisen beschreiben, die letztlich unproblematisch sind, weil sie sachlich differenzieren, nicht aber diskriminieren." (Baer, ZRP 2002, S. 290 (293)).

98 Thüsing, RdA 2001, S. 319 (322); Richardi/Annuß in Staudinger, § 611 a, Rn. 53; Zu beachten ist, daß Thüsing von tatsächlicher Unverzichtbarkeit spricht, bei der es „objektive Maßstäbe“ gäbe. 
mag es auch Countertenöre geben. ${ }^{99}$ Wenn zwei dasselbe tun, sei es noch lange nicht das gleiche. ${ }^{100}$

Man ist geneigt, dieser Sichtweise uneingeschränkt zu folgen, führt sie doch zu dem gewünschten Ergebnis. Der Weg zu selbigem ist indes zu kritisieren. In den genannten Fällen ist eine objektive Unverzichtbarkeit bzw. eine objektive Anforderung i. S. d. § 8 I AGG nicht gegeben, erfordern doch bei ihnen gerade keine zwingenden objektiven Gründe ein bestimmtes Geschlecht. „Selbstverständlich könnten Dressmen Damen-Bademoden vorführen und umgekehrt, [...].“101 Die Frage der Rechtswidrigkeit des diskriminierungsrelevanten Verhaltens läßt sich in all den genannten Beispielsfällen nur wertend beantworten. ${ }^{102}$ Die objektive Anforderung i. S. d. $\S 8$ I AGG zeichnet sich indes dadurch aus, daß sie ohne jegliche Wertung auszukommen vermag. ${ }^{103}$ Natürlich ist nur die Begründung des ansonsten richtigen Ergebnisses zu kritisieren. In den Fällen, die unter den Oberbegriff der Authentizitätswahrung subsumiert wurden, ist die Entscheidung des „Diskriminierenden“ zweifelsfrei sachlich begründet. Natürlich muß es zulässig sein, die Rolle eines Schwarzen mit einem Schwarzen zu besetzen, ausschließlich Frauen für die Präsentation von Damenmode zu engagieren. ${ }^{104}$

\section{K. Interessen Dritter}

Es gibt Fälle, bei denen der „Diskriminierende“ sein Verhalten damit begründet, Dritte, beispielsweise seine Kunden, würden den „Diskriminierten“ aufgrund der Tatsache, daß dieser Träger eines bestimmten Merkmals ist, ablehnen oder zumindest den Nicht-Träger dieses Merkmals bevorzugen.

\section{Sachlich gerechtfertigte Kundenwünsche}

Beispiele: 1. Läßt sich der Betrieb von „Frauensaunen“ rechtfertigen? 2. Kann Pflegepersonal im Einzelfall nach dem Geschlecht ausgewählt werden? 3. Ist die ausschließliche Einstellung von Stewardessen mit dem Argument zulässig, die Fluggäste würden weibliches Personal bevorzugen? 4. Ist es zulässig, ausschließlich Frauen bei der Besetzung einer Stelle als „Playboy-Bunny“ zu berücksichtigen? ${ }^{105}$

99 Thüsing, RdA 2001, S. 319 (322); In diesem Sinne auch Adomeit: „Parfum verkauft doch besser ein weibl. Verkäufer, Damenoberbekleidung wird vorteilhafter von weibl. Mannequins vorgeführt, [...]!““ (Adomeit, DB 1980, S. 2388 (2388)) sowie Lorenz, der als Beispiel die Mutterrolle im Theater angab. (Lorenz, DB 1980, S. 1745 (1745)); Raab subsumierte den Fall der Sopranstimme unter die biologische Unverzichtbarkeit. (Raab in Soergel, $\S$ 611 a, Rn. 35).

100 Thüsing, RdA 2001, S. 319 (322).

101 Müller-Glöge in MüKo, $\$ 611$ a, Rn. 42.

102 So auch Müller-Glöge in MüKo, § 611 a, Rn. 42.

103 Dies soll zumindest nach der im Rahmen dieser Arbeit vorgeschlagenen Einteilung gelten.

104 Bei derartig eindeutigen Fällen erscheint freilich auch die Ansicht vertretbar, die Frage der wesentlichen und entscheidenden beruflichen Anforderung richte sich hier nach objektiven Maßstäben. (so Thüsing in bezug auf das Unverzichtbarkeitserfordernis des § 611 a BGB-A - Thüsing, RdA 2001, S. 319 (322)).

105 Beispielsfälle aus der amerikanischen Rechtsprechung bei Thüsing, RdA 2001, S. 319 (321). 
Im Fall der Frauensaunen liegt das (gewünschte) Ergebnis wieder auf der Hand. Natürlich müssen solche Einrichtungen zulässig sein, und zwar generell, nicht nur an einzelnen Tagen. Der generelle Ausschluß der Männer bezweckt hier nicht deren generellen Ausschluß vom gesellschaftlichen Leben. Er verfolgt vielmehr das Ziel, Frauen einen Saunabesuch zu ermöglichen, ohne dem Gefühl von Scham ausgesetzt zu sein. Dieser Zweck ist zweifelsohne sachlich begründet. Das gleiche Problem kann sich bei Tätigkeiten stellen, die einen intimen zwischenmenschlichen Kontakt mitsichbringen, wie es bei Pflegepersonal denkbar ist. ${ }^{106}$ Diskriminierungsschutz würde über sein Ziel hinausschießen, verböte man dem Arbeitgeber beispielsweise, bei der Einstellung eines Masseurs für weibliche Hotelgäste nach dem Geschlecht zu unterscheiden. ${ }^{107}$

Um der Mißbrauchsgefahr zu entgehen, die mit der Gewährung dieses Rechtfertigungsgrundes einhergeht, wird man in derartigen Fällen den glaubhaften Nachweis fordern müssen, daß die Kunden bzw. Patienten zur Wahrung ihrer Intimsphäre das andere Geschlecht zurückweisen. ${ }^{108}$ Nicht erforderlich sei indes, daß das Schamgefühl vernünftig und weit verbreitet ist. ${ }^{109}$

Eine Unterscheidung soll auch dann gerechtfertigt sein, wenn ein bestimmtes Geschlecht nicht bloß erwünschte Nebeneigenschaft ist, sondern der Arbeitnehmer gerade hierfür bezahlt wird, das Geschlecht Bestandteil seiner entgoltenen Leistung ist. ${ }^{110}$ Im Fall des „Playboy-Bunnys“ wird man dies zweifellos bejahen können. Dagegen sollen die weiblichen Reize von Stewardessen bloß erwünschte Nebeneigenschaft der Tätigkeit an sich sein, die darin bestehe, beim Ein- und Aussteigen zu helfen, die Sicherheitsausrüstung vorzuführen sowie Mahlzeiten und Getränke zu servieren. ${ }^{111}$

Der Unterschied beider Fälle und zugleich Grund, in dem einen die sachliche Rechtfertigung anzunehmen, in dem anderen dagegen nicht, besteht in folgendem: Es machte gar keinen Sinn, Männer für die Tätigkeit eines „Playboy-Bunnys“ zu engagieren, da männliche Kunden Stripperinnen nicht nur bevorzugen, sondern ausschließlich Vorführungen von Frauen besuchen werden. ${ }^{112}$ Der Arbeitgeber hätte in diesem Fall den Arbeitsplatz dauerhaft unbesetzt gelassen, wenn nur männliche Bewerber zur Verfügung gestanden hätten, während dies in dem anderen Fall

106 So auch Raab in Soergel, § 611 a, Rn. 37; im Ergebnis ebenso Edenfeld, der meint, aus Gründen des Anstands sei in derartigen Fällen eine Ungleichbehandlung gerechtfertigt. (Edenfeld in Erman, § 611 a, Rn. 12; LAG Köln, ArbuR 1996, S. 504 (504)) „Anstand“ dürfte indes ein wenig taugliches Abgrenzungskriterium darstellen. Was ist anständig, was nicht?.

107 So aber nach einem Bericht von Thüsing die US-amerikanische Rechtsprechung (Thüsing, NJW 2003, S. 405 (406)).

108 Thüsing, RdA 2001, S. 319 (323) in Anlehnung an die U.S.-amerikanische Rechtsprechung (ders., S. 322).

109 Thüsing, RdA 2001, S. 319 (323).

110 Thüsing, RdA 2001, S. 319 (321 und 322) in Anlehnung an die U.S.-amerikanische Rechtsprechung, die fragt, ob ein bestimmtes Geschlecht notwendig für die Erfüllung der ,essence of the business“ ist.

111 Thüsing, RdA 2001, S. 319 (321 und 322).

112, ,[...] Animierdamen sind durch normale Männer nicht ersetzbar!“ (Adomeit, DB 1980, S. 2388 (2388)). 142 
nicht denkbar ist. Die Praxis zeigt, daß es auch männliche Reisebegleiter gibt und sich die Reisenden ihre Reiseunternehmen nicht nach dem Geschlecht des Begleitpersonals aussuchen.

Mit Blick auf die im Jahre 2006 in Deutschland geführte Diskussion um die Verbesserung des Nichtraucherschutzes ist anzumerken, daß sachlich begründet auch ein generelles Rauchverbot am Arbeitsplatz sein dürfte, das den Schutz der passiv rauchenden Belegschaft bezweckt. In jedem Fall unzulässig wäre dagegen ein generelles Raucherverbot. In diesem Zusammenhang ist noch einmal auf die kritikwürdige Stellungnahme der EU-Kommission hinzuweisen, in der es heißt, EUUnternehmen dürften in Stellenanzeigen Raucher explizit aus dem Bewerberkreis ausschließen. ${ }^{113}$

\section{Diskriminierende Kundenwünsche}

Beispiele: 1. Ist die Kündigung einer Arbeitnehmerin, die religiös bedingt ein Kopftuch trägt, mit der Begründung zulässig, es könne der Kundschaft ein derartiges Verhalten nicht zugemutet werden? ${ }^{114} 2$. Ist die Ablehnung eines potenziellen Gastes durch den Gastwirt gerechtfertigt, der meint, er könne seinen Gästen den Anblick einer mit Füßen essenden contergangeschädigten Frau nicht zumuten? ${ }^{115}$

In dem „Kopftuchfall“ stützte der Arbeitgeber, ein Kaufhausbetreiber, die Kündigung unter anderem darauf, Verkaufspersonal mit Kopfbedeckung sei einem Großteil der Kunden nicht zuzumuten. $^{116}$ In diesem Sinne entschied die Berufungsinstanz, „[e]s könne keinem Zweifel unterliegen, daß die Bekl. im Hinblick auf den Charakter ihres Kaufhauses, dessen örtliche Lage und die Vorstellungen des ländlich-konservativ geprägten Kundenkreises mit dieser Kleiderordnung [ - vom Verkaufspersonal erwartete man Kleidung ohne auffällige, provozierende, ungewöhnliche fremdartige Akzente - ] berechtigte Interessen verfolge, denen sich die Klägerin als Arbeitnehmerin zu fügen habe. “117

Das $B A G$ hat nicht nur Zweifel an dieser Sichtweise der Berufungsinstanz angemeldet, vielmehr die Religionsfreiheit der Kopftuch tragenden Arbeitnehmerin über die Berufsfreiheit des Unternehmers gestellt und die Kündigung für unwirksam erklärt, die aufgrund der Weigerung der Arbeitnehmerin, ohne Kopftuch zu arbeiten, erfolgt war. ${ }^{118}$ Das Gericht führte in seinem Urteil aus, das darlegungspflichtige Unternehmen hätte keine Tatsachen vorgetragen, aufgrund derer es bei einem

113 FAZ vom 08.08.2006, S. 13, unter dem Titel: „Im grauen Dunstbereich“.

114 BAG, NJW 2003, S. 1685 (1685).

115 Nickel berichtet von einem Artikel in der Frankfurter Rundschau vom 27.09.1996 mit dem Titel: „Wer keine Hände zum Essen hat, ist als Gast unerwünscht“ (Nickel, Gleichheit und Differenz in der vielfältigen Republik, S. 84, Fn. 272).

116 BAG, NJW 2003, S. 1685 (1685).

117 Entscheidungsgründe der Berufungsinstanz abgedruckt in NJW 2003, S. 1685 (1685).

$118 B A G$, NJW 2003, S. 1685 (1685); Das BVerfG hat die daraufhin erfolgte Verfassungsbeschwerde des Arbeitgebers nicht zur Entscheidung angenommen. (BVerfG, NZA 2003, S. 959 (959)). 
weiteren Einsatz der Kopftuch tragenden Arbeitnehmerin als Verkäuferin zu konkreten betrieblichen Störungen oder wirtschaftlichen Einbußen gekommen wäre. ${ }^{119}$

Mit dem Urteil hat das Gericht zum einen deutlich gemacht, daß Vorurteile der Geschäftspartner des Arbeitgebers allein eine Ungleichbehandlung nicht rechtfertigen können. ${ }^{120}$ Auf der anderen Seite hat es klargestellt, daß eine „Diskriminierung“ gerechtfertigt ist, wenn die Ablehnung der Kunden so weit reicht, daß der „Diskriminierende“ finanzielle Einbußen erleidet. Fraglich ist, welchen Grad diese Einbußen erreicht haben müssen, um eine „Diskriminierung“ zu rechtfertigen.

\section{Mindereinnahmen - Zumutbarer Grad der wirtschaftlichen Einbuße}

Es wird vertreten, man könne von einem Arbeitgeber nicht verlangen, „daß er dem Gleichbehandlungsgebot auch dann nachkommt, wenn er dadurch den von ihm unbeeinflußbaren Erwartungen und Einstellungen seiner Geschäftspartner zuwider handeln und den Bestand seines Unternehmens nachhaltig gefährden würde“. ${ }^{121}$ Solange der Bestand des Unternehmens nicht gefährdet sei, seien vom Arbeitgeber sogar erhebliche Umsatzeinbußen hinzunehmen. ${ }^{122}$ Dies sei billig, treffe den Arbeitgeber doch das gleiche Risiko wie seine Konkurrenten, die ebenfalls dem Gleichbehandlungsgebot unterlägen. ${ }^{123}$ Außerdem wäre jede Grenzziehung zwischen gerade noch und nicht mehr hinzunehmenden wirtschaftlichen Einbußen willkürlich; ein klarer Maßstab für diese Grenzziehung sei jedenfalls nicht in Sicht. ${ }^{124}$

Diese Ansicht ist abzulehnen, kann doch ein Unternehmen nicht für gesellschaftliche (Fehl)Entwicklungen verantwortlich gemacht und verpflichtet werden, die Folgen selbiger zu tragen. ${ }^{125}$ Natürlich tragen auch Privatunternehmen eine gesellschaftliche Verantwortung. In erster Linie dienen sie jedoch dem legitimen Zweck, Gewinn zu erzielen. Diesem Umstand würde man nicht in gehörigem Maße Rechnung tragen, wenn man Privatunternehmen gewinnorientierte Entscheidungen verböte. Nimmt der Arbeitgeber nicht auch den bestqualifizierten Bewerber letztlich deshalb, weil er sich gerade durch diesen eine besonderes günstige Entwicklung seines

119 BAG, NJW 2003, S. 1685 (1687).

120 In diesem Sinne auch Richardi/Annuß, die im Rahmen des $§ 611$ a BGB-A die Meinung vertraten, daß ein lediglich tradiertes Rollenverständnis nicht ausreicht, um die Unverzichtbarkeit der geschlechtsspezifischen Differenzierung zu begründen. (Richardi/Annuß in Staudinger, § 611 a, Rn. 54).

121 Richardi/Annuß in Staudinger, $\$ 611$ a, Rn. 54; Thüsing, RdA 2001, S. 319 (324); Richardi/Annuß sind nicht der Ansicht, die Erwartungen der Personen, mit denen der Arbeitgeber in Geschäftsbeziehung tritt, sollten eine unterschiedliche Behandlung stets rechtfertigen, wie von Thüsing behauptet. (vgl. dazu Thüsing, NJW 2003, S. 405 (406)).

122 Thüsing, RdA 2001, S. 319 (323); Diese Ansicht ist an das U.S.-amerikanische Arbeitsrecht angelehnt, das dem Arbeitgeber grundsätzlich zumutet, finanzielle Einbußen hinzunehmen, die einzig und allein auf Vorurteilen der Kunden beruhen. (Thüsing, RdA 2001, S. 319 (321)).

123 Thüsing, RdA 2001, S. 319 (323).

124 Thüsing, RdA 2001, S. 319 (323).

125 Thüsing ist vorzuhalten, daß für ihn das U.S.-amerikanische Recht das Maß aller Dinge zu sein scheint. In anderem Zusammenhang formuliert er: „, [...] und auch die Tatsache, daß auch das U.S.-amerikanische Recht nicht umhinkommt, hier Ausnahmen zu machen, legt es nahe, dem Arbeitgeber die Möglichkeit zur Differenzierung zu geben." (Thüsing, RdA 2001, S. 319 (324)). 
Unternehmens, eine besonders hohe Rendite erhofft? Von niemandem wird bestritten, daß die Qualifikation ein sachlicher Unterscheidungsgrund ist.

Zudem ist das Argument, der Arbeitgeber trage das gleiche Risiko wie seine Konkurrenten, nicht stichhaltig. Richtig ist nur, daß formal alle das gleiche Risiko tragen, tatsächlich sich dieses potenzielle Risiko aber unterschiedlich auswirkt. Der Betreiber einer Szenekneipe, in der sich vornehmlich ausländerfeindlich eingestellte Personen treffen, ist sicherlich stärker von der Pflicht zur Einstellung auch schwarzer Kellner betroffen, als die alternative Studentenkneipe, in der regelmäßig Reggaepartys veranstaltet werden. Das in einer konservativen Gegend gelegene Kaufhaus ist sicherlich von dem Verbot der Kündigung einer kopftuchtragenden Verkäuferin stärker betroffen als das in Berlin-Kreuzberg oder Frankfurt am Main gelegene.

Auch das Argument, jede Grenzziehung zwischen gerade noch und nicht mehr hinzunehmenden wirtschaftlichen Einbußen sei willkürlich, ein klarer Maßstab für diese Grenzziehung jedenfalls nicht in Sicht, kann keine Begründung dafür sein, daß Unternehmen auch erhebliche Umsatzeinbußen hinzunehmen haben. Das BAG hat entschieden, daß wirtschaftliche Einbußen eine „Diskriminierung“ rechtfertigen können. Eine bestimmte Höhe dieser Einbußen, gar erhebliche Umsatzeinbußen, scheint das Gericht damit nicht zu verlangen.

Die hier vertretene Sichtweise führt praktisch nicht dazu, daß auch gänzlich zu vernachlässigende Mindereinnahmen eine „Diskriminierung“ rechtfertigen. Das $B A G$ verlangt nämlich, daß der „Diskriminierende“ konkret darlegt, daß es zu wirtschaftlichen Einbußen gekommen ist bzw. eine reale Gefahr besteht, daß es zu solchen Einbußen kommen wird. ${ }^{126}$ Dieser Nachweis wird bei ganz unerheblichen Einbußen nicht $\mathrm{zu}$ führen sein. ${ }^{127}$ Außerdem wird das Unternehmen selbst wirtschaftliche Verluste erst ab einer bestimmten Höhe wahrnehmen.

\section{Nichtbeachtung des diskriminierenden Kundenwunsches nicht sanktionierbar}

In Übertragung der soeben angestellten Überlegungen kann für den zweiten Beispielsfall formuliert werden, daß der Gastwirt grundsätzlich verpflichtet ist, behinderte Menschen zu bedienen, solange er keine wirtschaftlichen Verluste dadurch erleidet, daß andere Gäste sein Lokal infolgedessen meiden und er durch deren Ausbleiben nachweislich wirtschaftliche Verluste erleidet.

$\mathrm{Zu}$ beachten ist in diesem Zusammenhang folgendes: Wenn der Gastwirt grundsätzlich verpflichtet sein soll, behinderte Menschen zu bedienen, dann kann ihm natürlich andererseits dieses Verhalten nicht zum Vorwurf gemacht werden. Den Gästen, die sich durch die Anwesenheit des Behinderten gestört fühlen, Schadensersatzansprüche gegenüber dem Gastwirt zuzusprechen, wäre fatal. Die

126 BAG, NJW 2003, S. 1685 (1687); Grundrechte sollen nicht durch bloßen Verdacht beiseite gestellt werden können. (BAG, NJW 2003, S. 1685 (1687); Böckenförde, NJW 2001, S. 723 (728)).

127 Wann eine wirtschaftliche Einbuße unerheblich ist, hängt natürlich von der Größe des jeweiligen Unternehmens ab. Was für eine große Bank ,peanuts“ sind, kann für ein mittelständisches Unternehmen bereits eine erhebliche Umsatzeinbuße darstellen. 
Rechtsprechung hat den „Geschädigten“ in derartigen Situationen gleichwohl Schadensersatzansprüche zuerkannt. So wurde entschieden, die Anwesenheit von Behinderten in einem Hotel könne ein Mangel des Reisevertrages sein, da eine Gruppe von Schwerbehinderten bei empfindsamen Menschen eine Beeinträchtigung des Urlaubsgenusses zumindest dann darstellen könne, wenn der Reisende dem Anblick der Behinderten nicht ausweichen kann. ${ }^{128}$ Auch wurde einem Anspruch auf Minderung des Reisepreises mit der Begründung stattgegeben, der ekelerregende Anblick von Schwerstbehinderten, die gefüttert werden müssen, habe dem Kläger den unbeschwerten Genuß der Mahlzeit unmöglich gemacht. ${ }^{129}$

Im Sinne der hier vertretenen Ansicht wird gesagt, aufgrund des Art. 3 III 2 GG $^{130}$ sei das Maß auch zivilrechtlich gebotener gegenseitiger Rücksichtnahme und Toleranz gegenüber behinderten Menschen grundsätzlich neu und anders $\mathrm{zu}$ bestimmen. ${ }^{131}$ „Als schadensersatzfähige Beeinträchtigung des Reisegenusses wird man deshalb die Nähe von Menschen mit Behinderungen angesichts der ausdrücklichen Wertentscheidung des Art. 3 III 2 zukünftig grundsätzlich nicht mehr qualifizieren dürfen und auch die schwierige Abwägung zum beidseitig zumutbaren nachbarschaftlichen Interessenausgleich gem. § 906 BGB hat deutlicher als bisher zu berücksichtigen, daß etwa „unharmonische“ Geräusche der Kommunikation geistig Schwerbehinderter nicht den gewohnten Kategorien des Immissionsschutzrechts („Lästigkeitsfaktor“) zuzuordnen sind.“132

\section{Mehreinnahmen}

Fraglich ist schließlich, wie der umgekehrte Fall zu beurteilen ist, bei dem der Träger eines bestimmten Merkmals dem Unternehmen nachweislich Einnahmen verschafft, die derjenige, der nicht Träger dieses Merkmals ist, nicht zu erreichen vermag.

Beispiel: In einer Bar, in der sich vornehmlich männliche Gäste aufhalten, nimmt weibliche Bedienung in der Regel mehr Geld ein als männliche.

Nachgewiesen wurde bislang, daß finanzielle Verluste diskriminierungsrelevantes Verhalten zu rechtfertigen vermögen. Kann im Gegenzug auch die Aussicht auf Mehreinnahmen derartiges Verhalten rechtfertigen? Diese Frage muß bejaht werden, stehen doch Mehreinnahmen auf der

LG Frankfurt, NJW 1980, S. 1169 (1169 f.).

AG Flensburg, NJW 1993, S. 272 (272).

Art. 3 III 2 GG lautet: „Niemand darf wegen seiner Behinderung benachteiligt werden.“.

31 Osterloh in Sachs, Art. 3, Rn. 307.

132 Osterloh in Sachs, Art. 3, Rn. 307.

146 
einen Seite Mindereinnahmen auf der anderen gegenüber. ${ }^{133}$ Das eine ist Kehrseite des anderen. In dem Beispielsfall könnte man deshalb auch sagen, daß die Kneipe durch den Einsatz von männlichen Servierern finanzielle Verluste erleidet.

Freilich gilt wie bei Stewardessen, daß es nicht Hauptaufgabe von Serviererinnen ist, anziehend auf ihre Gäste zu wirken. Sie sollen in erster Linie Bestellungen aufnehmen und selbige ausführen. Im Unterschied zu jenem Fall wirken sich die weiblichen Reize von Serviererinnen regelmäßig positiv auf die Geschäftsbilanz einer Bar aus. Dies muß Beachtung bei der Beantwortung der Frage nach der Hauptaufgabe von Serviererinnen finden. Unter Berücksichtigung dessen kann behauptet werden, daß die konkrete Arbeit einer Serviererin dem Hauptziel dient, möglichst viel Geld zu erwirtschaften. Das ist ein legitimes Ziel eines jeden Kneipiers, eines jeden Unternehmers.

Wenn man dieses Ziel aber anerkennt, muß man dem Kneipier das Recht einräumen, diejenigen Bewerber auszusuchen, die dieses Ziel am besten erreichen können. ${ }^{134}$ Wenn, was wenig wahrscheinlich ist, sich die Fluggäste ihre Flüge nach dem Geschlecht der Flugbegleiter aussuchen sollten, wenn das Geschlecht selbiger also zu einem finanziellen Faktor werden sollte, dann wäre auch in diesem Bereich die Wahl der Flugbegleiter nach dem Geschlecht als zulässig einzustufen. ${ }^{135}$ „Unterschiedliche Absatzzahlen müssen den überzeugen, der natürliche Unterschiede nicht mehr versteht. “' 136

\section{III. Öffentliche Interessen}

Es sind Fälle denkbar, bei denen der „Diskriminierende“ sein Verhalten mit berechtigten Interessen Dritter begründet, mit denen er nicht in Geschäftskontakt steht. So könne das öffentliche Interesse an der Sicherheit von Gefängnissen, die aufgrund von Vorurteilen der Häftlinge gegenüber weiblichen Bewachern gefährdet ist, die Nichteinstellung von weiblichen Bewachern rechtfertigen. ${ }^{137}$ Wahrscheinlich aus demselben Grund wird gefordert, ein Frauengefängnis solle ausschließlich Wärterinnen beschäftigen können. ${ }^{138}$

\section{Zusammenfassung}

133 Auch Raab hält eine Ungleichbehandlung für zulässig, wenn sich die Nichterfüllung der Erwartungen der Kunden geschäftsschädigend auswirkt. Er nennt in diesem Zusammenhang das Beispiel des überwiegend männlichen Publikums einer Nachtbar, das eher eine weibliche Bedienung erwartet. (Raab in Soergel, § 611 a, Rn. 37).

134 Dagegen aber Thüsing, RdA 2001, S. 319 (324).

135 Dagegen wiederum Thüsing, RdA 2001, S. 319 (324).

136 So treffend Adomeit, DB 1980, S. 2388 (2388).

137 Dieses und weitere Beispiele bei Thüsing, RdA 2001, S. 319 (321); Das öffentliche Interesse als sachlicher Grund für diskriminierungsrelevantes Verhalten wird, wie im Fall des Gefängnisses, seinen Hauptanwendungsbereich dort haben, wo auf der Seite des „Diskriminierenden“ der Staat steht. Insofern erlangt dieser Rechtfertigungsgrund im Rahmen der vorliegenden Arbeit nur eine untergeordnete Bedeutung.

138 Eich, NJW 1980, S. 2329 (2331); Auch der EuGH hält es für zulässig, bestimmte Posten in Gefängnissen nur mit Männern bzw. Frauen zu besetzen. (EuGH, Rs. C-318/86, Slg. 1988 3, S. 3559 (3580, Rn. 17)). 
$\mathrm{Zu}$ der Frage, inwieweit Interessen Dritter diskriminierungsrelevante Entscheidungen des „Diskriminierenden“ rechtfertigen können, kann zusammenfassend wie folgt Stellung genommen werden: Sachlich begründete Wünsche Dritter - etwa Geschäftskunden des „Diskriminierenden“ vermögen diskriminierungsrelevantes Verhalten $\mathrm{zu}$ rechtfertigen. Sachlich begründet ist beispielsweise der Wunsch von Patienten, zur Wahrung ihrer Intimsphäre ausschließlich von weiblichem bzw. männlichem Pflegepersonal betreut zu werden.

Diskriminierende Kundenwünsche vermögen diskriminierungsrelevantes Verhalten zu rechtfertigen, wenn der „Diskriminierende“ andernfalls wirtschaftliche Einbußen von nicht völlig unerheblichem Umfang erleiden würde.

Die Nichtbeachtung eines diskriminierenden Kundenwunsches ist nicht sanktionierbar. So darf etwa die Bedienung eines Behinderten grundsätzlich keine schadensersatzpflichtige Verletzung des Bewirtungsvertrages zwischen dem Gastwirt und dem sich durch die Anwesenheit des Behinderten gestört fühlenden Gastes darstellen.

Unter der Prämisse, daß in einer Marktwirtschaft das Gewinnstreben ein legitimes Ziel des Unternehmers darstellt, muß jenem gestattet sein, diskriminierenden Kundenwünschen nachzukommen, die zu Mehreinnahmen führen. Die sich auf den Umsatz auswirkende ausschließliche Einstellung von Serviererinnen in einer vorwiegend von männlichen Gästen besuchten Bar ist vor diesem Hintergrund rechtmäßig.

Schließlich kann der „Diskriminierende“ sein Verhalten auch mit berechtigten öffentlichen Interessen, d. h. mit Interessen Dritter begründen, mit denen er nicht in Geschäftskontakt steht.

\title{
L. Zusammentreffen von Diskriminierungsmerkmalen in einer Person
}

\begin{abstract}
„Niemand ist nur Mann oder nur behindert, sondern männlich, heterosexuell, christlich usw. Niemand ist nur Frau, niemand nur schwul,
\end{abstract} niemand nur Christ. “139

\section{Unbewußtes Zusammentreffen}

Beispiel: Der Arbeitgeber zahlt dem Arbeitnehmer aufgrund dessen höherer Qualifikation ein höheres Arbeitsentgelt als der Arbeitnehmerin, obwohl beide die gleiche Tätigkeit ausüben. Die Arbeitnehmerin fühlt sich aufgrund ihres Geschlechts diskriminiert.

Die Besonderheit des Beispielsfalls besteht darin, daß die Arbeitnehmerin nicht aufgrund des Merkmals, aufgrund dessen sie sich diskriminiert fühlt, anders gegenüber dem Arbeitnehmer behandelt wurde. Der Arbeitgeber stützte seine Entscheidung gerade nicht auf das Geschlecht der Arbeitnehmerin, sondern auf deren geringere Qualifikation. In diesem Zusammenhang kann 
festgehalten werden, daß jeder Mensch „unendlich“ viele Diskriminierungsmerkmale in sich vereinigt, denn ,[n]iemand ist nur Mann oder nur behindert, sondern männlich, heterosexuell, christlich usw. Niemand ist nur Frau, niemand nur schwul, niemand nur Christ. “140

Fraglich ist, wie sich die Tatsache auswirkt, daß in dem Beispielsfall die Diskriminierungsmerkmale Geschlecht und Qualifikation in einer Person unbewußt zusammentreffen. Unbewußt deshalb, weil der Arbeitgeber seine Entscheidung allein auf die Qualifikation der Arbeitnehmerin, nicht aber auf deren Geschlecht stützte.

Diese Tatsache müßte angesichts der bisher im Rahmen dieser Arbeit angestellten Überlegungen eigentlich dazu führen, daß sich die Frage nach dem Vorliegen einer unmittelbaren Diskriminierung einzig und allein bezüglich des Merkmals „Qualifikation“ stellt. Allenfalls an eine mittelbare Diskriminierung aufgrund des Geschlechts wäre in einem derartigen Fall zu denken.

Fraglich ist indes, ob für absolute Differenzierungsverbote etwas anderes gilt.

\section{Absolute Differenzierungsverbote}

Gleichbehandlung ist zum Teil in der Weise vorgeschrieben, daß jegliche Ungleichbehandlung aufgrund eines bestimmten Merkmals ausgeschlossen ist bzw. eine Ungleichbehandlung aufgrund dieses Merkmals niemals gerechtfertigt werden kann. In solchen Fällen spricht man von absoluten Differenzierungsverboten. ${ }^{141}$

\section{a),,Wegen eines in $§ 1$ genannten Grundes“ $-\S 7$ I AGG}

Fraglich ist in diesem Zusammenhang, wie das Erfordernis zu verstehen ist, daß jegliche Ungleichbehandlung aufgrund eines bestimmten Merkmals ausgeschlossen ist. Zum Zwecke der Beantwortung dieser Frage sei an dieser Stelle der Blick auf $§ 612$ III 1 BGB-A gelenkt, der ein absolutes Differenzierungsverbot statuieren sollte. ${ }^{142}$ Gemäß dieser Norm durfte

„für gleiche oder gleichwertige Arbeit nicht wegen des Geschlechts des Arbeitnehmers eine geringere Vergütung vereinbart werden als bei einem Arbeitnehmer des anderen Geschlechts.“

Dieser Grundsatz der Entgeltgleichheit zwischen Männern und Frauen ergibt sich nunmehr aus § 7 I AGG i. V. m. § 2 I Nr. 2 AGG. ${ }^{143}$ Gemäß $§ 7$ I dürfen Beschäftigte

140 Baer, ZRP 2002, S. 290 (294).

141 Heun in Dreier, Band I, Art. 3, Rn. 122; Schiek, Differenzierte Gerechtigkeit, S. 50.

142 Raab in Soergel, Band 4/1, § 612, Rn. 44.

143 Entwurfsbegründung, BT-Druck. 16/1780, S. 57. 
„,...] nicht wegen eines in $\S 1$ genannten Grundes [ $-\mathrm{d}$. h. wegen eines dort genannten Diskriminierungsmerkmals - ] benachteiligt werden $[\ldots]$. “

§ 7 I AGG geht insofern über § 612 III 1 BGB-A hinaus, als er den Grundsatz der Entgeltgleichheit über das Merkmal Geschlecht hinaus auf alle in $§ 1$ AGG genannten Merkmale erstreckt. ${ }^{144}$

Das Tatbestandsmerkmal, ,wegen eines in $§ 1$ genannten Grundes“ in $§ 7$ I AGG ließe sich im Sinne von ,aufgrund der Existenz“ dieser Gründe interpretieren. So verstanden hätte die Norm unter anderem den Inhalt, daß sich unterschiedliche Entlohnungen von Männern und Frauen durch keinen Grund rechtfertigen ließen.

In diesem Sinne könnte auch und vor allem Art. 141 I EGV verstanden werden, dessen Wortlaut einen „wegen des Geschlechts-Zusatz“ nicht enthält. Gemäß dieser Bestimmung stellt jeder Mitgliedstaat

„[...] die Anwendung des Grundsatzes des gleichen Entgelts für Männer und Frauen bei gleicher oder gleichwertiger Arbeit sicher. “145

\section{b) Kein Recht auf gleichen Lohn für gleiche/gleichwertige Arbeit}

Statuieren § 7 I AGG und Art. 141 I EGV damit ein Recht auf „gleichen Lohn für gleiche bzw. gleichwertige Arbeit" ? Diese Frage ist zu verneinen. ${ }^{146}$ Absolute Differenzierungsverbote führen nicht zum Verbot der Ungleichbehandlung allein deshalb, weil der „Diskriminierte“ Träger eines bestimmten Merkmals ist. Aufgrund eines solchen Verbotes darf eine Ungleichbehandlung lediglich nicht auf das entsprechende Merkmal gestützt werden. ${ }^{147}$ Dabei ist sogar gleichgültig, ob der „Diskriminierte“ überhaupt Träger des Merkmals ist, aufgrunddessen er „diskriminiert“ wird. ${ }^{148}$ Es

144 Entwurfsbegründung, BT-Druck. 16/1780, S. 35.

145 Vergleiche insofern auch Art. 2 I des Übereinkommens der ILO Nr. 100 über die Gleichheit des Entgelts männlicher und weiblicher Arbeitskräfte für gleichwertige Arbeit, der die Vertragsstaaten verpflichtet, ,,[...] die Anwendung des Grundsatzes der Gleichheit des Entgelts männlicher und weiblicher Arbeitskräfte für gleichwertige Arbeit auf alle Arbeitnehmer zu fördern und [...] sicherzustellen.“.

146 Im deutschen Recht stellt dieser Grundsatz keine allgemeingültige Anspruchsgrundlage dar. (BAG, DB $2000, \mathrm{~S}$. 1920 (1920); Bauer, NJW 2001, S. 2672 (2674)); „Vielmehr besteht in Fragen der Vergütung Vertragsfreiheit, die lediglich durch verschiedene rechtliche Bindungen wie Diskriminierungsverbote und tarifliche Mindestentgelte eingeschränkt ist.“ (BAG, DB 2000, S. 1920 (1920 f.)).

147 Waas, ZIP 2000, S. 2151 (2154); Bauer, NJW 2001, S. 2672 (2674); Auch im Rahmen des Art. 141 I EGV könne eine Differenzierung, die nicht aufgrund des Geschlechts erfolgt, im Einzelfall gerechtfertigt sein. (Eichenhofer in Streinz, Art. 141 EGV, Rn. 18); bzgl. § 612 III 1 BGB-A vgl. Weidenkaff, der sagte, die Geschlechtszugehörigkeit müsse Beweggrund für die unterschiedliche Behandlung sein (Weidenkaff in Palandt, § 612, Rn. 13 i.V.m. § 611 a, Rn. 9 f.).

148 Das gilt freilich auch für relative Differenzierungsverbote, die dadurch gekennzeichnet sind, daß das jeweilige Unterscheidungsmerkmal Grund für eine unterschiedliche Behandlung sein kann. (Richardi, Münchner Handbuch zum Arbeitsrecht, Band 1, $\$ 14$, Rn. 27); vgl. dazu vor allem die $\S \S 8-10$ sowie 20 AGG, die eine unterschiedliche Behandlung wegen der in $\S 1$ AGG genannten Gründe unter bestimmten Voraussetzungen zulassen. 
genügt, daß der „Diskriminierende“ seine Entscheidung auf ein vermeintlich in der Person des „Diskriminierten“ vorhandenes Merkmal stützt. ${ }^{149}$

Wenn § 7 I AGG unter anderem untersagt, wegen des Geschlechts unterschiedlich zu entlohnen, dann müßten Lohndifferenzierungen, die nicht wegen des Geschlechts erfolgen, sondern beispielsweise deshalb, weil ein Mann bei gleichwertiger Arbeit aufgrund besseren Zeugnisses eine höhere Vergütung als die Frau erhält oder weil sich die Frau beim Vergütungsgespräch „billiger verkauft“ als ein vergleichbarer männlicher Bewerber, grundsätzlich zulässig sein. ${ }^{150}$ Auch müßte der Arbeitgeber beispielsweise seinen Neffen aus familiären Gründen besser bezahlen dürfen als seine mitarbeitende Ehefrau. ${ }^{151}$

\section{c) „Wegen eines [anderen als] in $§ 1$ AGG genannten Grundes“}

Diese Sichtweise ist mit dem alten, vor dem Inkrafttreten des AGG geltenden Recht vereinbar. Das ergibt sich $\S 611$ a I BGB-A, dessen Wortlaut auch das Verbot der Entgeltdiskriminierung aufgrund des Geschlechts einschloß. ${ }^{152}$ Satz 3 der Norm stellte klar, daß „nicht auf das Geschlecht bezogene, sachliche Gründe eine unterschiedliche Behandlung rechtfertigen“ konnten, während Satz 2 eine unterschiedliche Behandlung wegen des Geschlechts für zulässig erachtete, wenn das Geschlecht „unverzichtbare Voraussetzung“ für die in Rede stehende Tätigkeit war. ${ }^{153}$

Diese Zweiteilung ist auch bei den Antidiskriminierungsrichtlinien $\mathrm{zu}$ beachten. Richtlinie 2000/43/EG statuiert für die Bereiche außerhalb des Arbeitsrechts ein absolutes Diskriminierungsverbot. ${ }^{154}$ Das ergibt ein Umkehrschluß aus Art. 4 der Richtlinie, wonach im

149 Vergleiche insofern $\S 7$ I AGG sowie $\S 1$ I ADG-E aus dem Entwurf eines Gesetzes zum Schutz vor Diskriminierung und zur Stärkung von Minderheitenrechten (Antidiskriminierungs- und Minderheitenrechtsgesetz) der Fraktion Bündnis 90/Die Grünen vom 20.01.1998.

150 So in bezug auf $\S 612$ III BGB-A Bauer, NJW 2001, S. 2672 (2674).

151 So - vor Inkrafttreten des AGG - Otto, Arbeitsrecht, Rn. 167; Daß § 612 III BGB-A an dieser Stelle unerwähnt blieb, wertet der Verfasser in dem Sinne, daß Otto in diesem Beispielsfall - obwohl in einem anderen Zusammenhang gebildet - keinen Verstoß gegen § 612 III BGB-A erblickte.

152 Putzo in Palandt, § 612, Rn. 11; Preis in Erfurter Kommentar zum Arbeitsrecht, 230, § 612 BGB, Rn. 45 Eigenständigen Bedeutung gegenüber $\S 611$ a BGB-A sollte $\S 612$ III BGB-A durch Statuierung einer besonderen Rechtsfolge erlangen. Gemeint ist der unmittelbare Erfüllungsanspruch des diskriminierten Arbeitnehmers auf gleichen Lohn. (Preis in Erfurter Kommentar zum Arbeitsrecht, 230, § 612 BGB, Rn. 68; Putzo in Palandt, § 612, Rn. 11 und 14)

Darüber hinaus dürften sich beide Normen auch dadurch unterschieden haben, daß $\S 612$ III BGB-A ein absolutes, $\S 611$ a BGB-A dagegen ein relatives Differenzierungsverbot statuierte.

153 Junker wies anschaulich auf die wichtige Unterscheidung zwischen sachlichem Grund einerseits und dem Unverzichtbarkeitserfordernis andererseits hin. (Junker, Grundkurs Arbeitsrecht, Rn. 164 a.E.); ebenso Lorenz, der formulierte: „Eine unterschiedliche Behandlung von Arbeitnehmern, die nicht geschlechtsbezogen ist, bleibt auch in Zukunft zulässig, wenn hierfür sachliche Gründe vorliegen. (Lorenz, DB 1980, S. 1745 (1745)); Raab: „Wenn für die Differenzierung nicht an das Geschlecht, sondern ausschließlich an sonstige Umstände angeknüpft wird, so liegt in Wahrheit eine Benachteiligung wegen des Geschlechts gar nicht vor. (Raab in Soergel, § 611 a, Rn. 38); Leisner: ,[...] gestattet ist und bleibt die Unterscheidung aus sachlichen Gründen, auch wenn diese mit den in Art. 3 Abs. 3 GG genannten Differenzierungen zufällig zusammentreffen.“ (Leisner, Grundrechte und Privatrecht, S. 359) Putzo scheint indes den Unterschied zwischen beiden Merkmalen verkannt zu haben, wenn er in einer älteren Auflage des Palandt zur Unverzichtbarkeit schrieb, aus Abs. 1 S. 3 sei zu entnehmen, daß ein sachlicher Differenzierungsgrund genügt. (Putzo in Palandt, 58. Auflage (1999), § 611a, Rn. 12).

154 Vergleiche zu der diesbezüglichen Kritik Teil 2, Prüfungspunkt C. V. 
Bereich des Arbeitsrechts eine Diskriminierung dann nicht gegeben ist, wenn eine bestimmte Rasse oder ethnische Herkunft ,wesentliche und entscheidende berufliche Voraussetzung“ ist. Aus der Tatsache, daß Art. 4 eine Öffnungsklausel nur für das Arbeitsrecht schafft, folgt, daß außerhalb dieses sachlichen Geltungsbereichs eine Ungleichbehandlung aufgrund der Rasse oder ethnischen Herkunft niemals gerechtfertigt werden kann. ${ }^{155}$ Aus dieser Tatsache folgt indes nicht, daß sich diskriminierungsrelevantes Verhalten in diesem Bereich nicht durch einen anderen Grund rechtfertigen ließe.

Nichts anderes gilt im Rahmen der $\S 19$ AGG, der in Umsetzung der Richtlinie 2000/43/EG außerhalb des Arbeitsrechts in bezug auf die Merkmale Rasse und ethnische Herkunft ein absolutes Diskriminierungsverbot statuiert. ${ }^{156}$ Verallgemeinernd läßt sich sagen, daß nach dem AGG „Benachteiligungen“ wegen anderer als der in § 1 AGG genannten Gründe grundsätzlich zulässig sind.

\section{2. ,Diskriminierung“ gestützt auf den (un-)sachlichen Grund?}

Treffen Diskriminierungsmerkmale zusammen, kann, wenn das eine Merkmal die Ungleichbehandlung rechtfertigen würde, das andere dagegen nicht, fraglich sein, ob der „Diskriminierende“ seine Entscheidung auf den sachlichen oder unsachlichen Grund gestützt hat. ${ }^{157}$

Beispiele: 1. Der Vermieter lehnt einen Chinesen ab, weil dieser ihn schon einmal beleidigt hat. 2. Derselbe Vermieter lehnt einen Chinesen mit der Begründung ab, Chinesen würden regelmäßig ihre Vertragspflichten nicht erfüllen. 3. Ein Vater sucht einen Klavierlehrer für seine Tochter. Er lehnt den pädophilen Bewerber ab. ${ }^{158}$ In den ersten beiden Fällen fühlt sich der Bewerber aufgrund der Tatsache, daß er Chinese ist, diskriminiert, im dritten aufgrund seiner sexuellen Identität.

Im ersten Beispielsfall lehnt der Vermieter den Interessenten der Wohnung nicht deshalb ab, weil dieser Chinese ist, sondern allein aufgrund der Tatsache, daß dieser ihn beleidigt hat. Er stützt seine Entscheidung also einzig und allein auf einen sachlichen Grund.

Auf welchen Grund stützt der Vermieter seine Ablehnung im zweiten Beispielsfall, auf die Nichterfüllung der Vertragspflichten oder die Nationalität des Wohnungssuchenden? Da der Vermieter den Interessenten nicht kennt und deshalb auch keine Kenntnis über dessen Vertragstreue hat, stützt er seine Entscheidung auf dessen Nationalität. Er lehnt den Interessenten allein deshalb

155 So auch Nickel, NJW 2001, S. 2668 (2671).

156 Dies ergibt sich aus $§ 20$ AGG, der die Merkmale Rasse und ethnische Herkunft nicht enthält.

157 Angesprochen ist an dieser Stelle nicht das Beweisproblem, das sich im Prozeß aufgrund streitigen Sachverhalts ergeben kann.

158 Beispiel bei Braun, JuS 2002, S. 424 (424).

152 
ab, weil dieser eine Nationalität aufweist, die er mit bestimmten (negativen) Eigenschaften verbindet. ${ }^{159}$ Da die Anknüpfung an die Nationalität in diesem Fall sachlich nicht gerechtfertigt ist, hätte das Vorbringen des Wohnungssuchenden, er sei aufgrund seiner Nationalität diskriminiert worden, vor Gericht Erfolg.

Auch der Vater im dritten Beispielsfall weiß nicht, wie sich der Bewerber verhalten, ob er sich an seiner Tochter vergehen wird oder nicht. Er kennt nur dessen sexuelle Präferenz. Er stützt seine Ablehnung demnach einzig und allein auf die sexuelle Identität des Bewerbers, die ihrerseits auf den angestrebten Vertrag keinen Einfluß hat. Auch ein Pädophiler kann Musikunterricht erteilen, wenn er die nötige fachliche Qualifikation besitzt.

Im Gegensatz zum zweiten Beispielsfall ist dem „Diskriminierenden“ hier die „Probe auf's Exempel“ jedoch nicht zumutbar. Grundrechte dürfen zwar nicht auf möglichen „Verdacht“ hin beiseite gestellt werden. ${ }^{160}$ Dem „Diskriminierenden“ kann aber nicht zugemutet werden, daß er aufgrund bestehenden Diskriminierungsverbotes die Gefahr befürchteter Straftaten trägt, deren Realisierung wahrscheinlich ist. In diesem Sinne ist entschieden worden, allein schon der Verdacht pädophiler Neigungen berechtige zur außerordentlichen Kündigung eines Kindergartenleiters. ${ }^{161}$ Unbegründet ist deshalb die „Befürchtung“, ein Antidiskriminierungsgesetz würde dazu führen, daß man Pädophile nicht mehr aufgrund ihrer Veranlagung als Klavierlehrer für seine Töchter ablehnen darf. $^{162}$

\section{Bewußtes Zusammentreffen - Motivbündel}

Diskriminierungsmerkmale treffen häufig in der Form zusammen, daß der „Diskriminierende“ seine Entscheidung bewußt auf mehrere Merkmale stützt. Der Arbeitgeber beispielsweise wird in den meisten Fällen seine Auswahl eben nicht nur von der Qualifikation der Bewerber abhängig machen. Fraglich ist das Vorliegen einer Diskriminierung in diesen Fällen, wenn die Entscheidung des „Diskriminierenden“ teils auf sachlichen, teils auf unsachlichen Gründen beruht.

Beispiele: 1. Für eine körperlich anstrengende Tätigkeit bewerben sich eine Frau und ein Mann. Die Frau ist schlechter qualifiziert als der Mann. Der Arbeitgeber lehnt die Frau mit der Begründung ab, sie sei schlechter qualifiziert. Außerdem stelle er sowieso lieber einen Mann für die körperlich anstrengende Arbeit ein. 2. Der Arbeitgeber stellt die besserqualifizierte Frau ein, weil sie über bessere Zeugnisse verfügt und er Frauen für Sekretärstätigkeiten lieber einstellt. 3. Die Chefin eines Ladenbetriebs entscheidet sich für die Bewerberin, weil diese besser qualifiziert ist als der Bewerber und sie sowieso lieber Frauen einstellt, weil sie durch bittere Lebenserfahrung männerfeindlich geworden ist.

159 Schiek spricht in diesem Zusammenhang von Askription. (Schiek, Differenzierte Gerechtigkeit, S. 27).

160 BAG, NJW 2003, S. 1685 (1687).

161 ArbG Braunschweig, NZA-RR 1999, S. 192 (192, Leitsatz).

162 So aber Braun, der seine Ausführungen auf den ADG-Entwurf des Bundesjustizministeriums vom 10.12.2001 bezieht (Braun, JuS 2002, S. 424 (424)). 
In all den genannten Beispielsfällen ist die geringere Qualifikation des jeweiligen Bewerbers sachlicher Grund dafür, diesen nicht einzustellen. Das jeweils zusätzlich genannte Motiv ist dagegen unsachlich. Bei Tätigkeiten, die körperlich anstrengend sind, ist der grundsätzliche Ausschluß von Frauen mit dem Argument, sie verfügten über eine geringere physische Leistungsfähigkeit, unzulässig. ${ }^{163}$ Auch ist nicht einzusehen, warum Vorgesetzten grundsätzlich das Recht eingeräumt werden sollte, ausschließlich Sekretärinnen statt Sekretäre einzustellen. ${ }^{164}$ Der Chefin eines Ladenbetriebes in jedem Fall zu erlauben, Männer zurückzuweisen, weil sie durch bittere Lebenserfahrung männerfeindlich geworden ist, erscheint äußerst fragwürdig. ${ }^{165}$

Das BVerfG dürfte in derartigen Fällen das Vorliegen einer Diskriminierung - auch nach Inkrafttreten des AGG - bejahen: „Soll die Beachtung des verfassungsrechtlichen Diskriminierungsverbots auch für den Arbeitgeber bei Einstellungsentscheidungen verbindlich gemacht werden - und darin liegt der Sinn des $\S 611$ a Abs. 1 BGB[-A] -, so muß es diesem verwehrt sein, das Geschlecht eines Bewerbers bei seiner Entscheidung überhaupt zu dessen Lasten zu berücksichtigen. Das ist aber bereits dann der Fall, wenn in dem Motivbündel, das seine Entscheidung beeinflußt hat, das Geschlecht des abgewiesenen Bewerbers als negatives oder das andere Geschlecht als positives Kriterium enthalten ist. “166

Diese Rechtsprechung ist zu kritisieren, verlangt sie doch vom Arbeitgeber, daß er nicht sieht, „was gespielt wird vor seinen Augen, “ die Bewerber also nicht mehr als Mann und Frau wahrnimmt. Das ist aber nicht möglich. Das Geschlecht des Bewerbers dürfte, wenn nicht in jedem, so doch in den meisten Fällen, bei denen sich beide Geschlechter bewerben, Einfluß auf die Auswahlentscheidung haben. Wenn nicht bewußt, so doch zumindest unbewußt. Wenn nicht in entscheidendem Maße, so doch zumindest zu einem geringen Prozentsatz. Nicht selten dürfte in einer solchen Situation ein sexuelles Motiv - also ein irrationales und zugleich unkontrollierbares Gefühl - und damit letztlich das Geschlecht die Auswahlentscheidung mitbeeinflussen:

\footnotetext{
„Finden Männer Frauen immer noch so bezaubernd, wenn Sex keine Rolle mehr spielt? Findet irgend jemand irgendeinen anderen, ganz gleich, welchen Geschlechts, so bezaubernd, wenn Sex zwischen den beiden keine Rolle spielt? Von wem sonst ist man so bezaubert? Von niemandem. “167
}

Wenn es unmöglich ist, Menschen als geschlechtslose Wesen wahrzunehmen, kann vom Arbeitgeber nicht verlangt werden, daß er seine Entscheidung völlig unbeeinflußt vom Geschlecht der Bewerber trifft. Rn. 2.

164 So aber Eich, NJW 1980, S. 2329 (2331); dagegen Söllner in MüKo, § 611 a, Rn. 16.

165 So aber Adomeit, DB 1980, S. 2388 (2388).

166 BVerfGE 89, S. 276 (289).

167 Aus dem Roman „Das sterbende Tier“ von Philip Roth. 


\section{Kausalität des (un-)sachlichen Grundes für die Entscheidung}

Das Vorliegen einer Diskriminierung sollte deshalb immer nur dann bejaht werden, wenn der unsachliche Grund kausal für die Entscheidung des „Diskriminierenden“ " war. ${ }^{168}$ In den Beispielsfällen war das Geschlecht der abgelehnten Bewerber nicht conditio sine qua non für ihre Nichteinstellung. Aufgrund ihrer geringeren Qualifikation wären sie sowieso nicht eingestellt worden. Damit kann die Anknüpfung an ihr Geschlecht hinweggedacht werden, ohne daß ihre Ablehnung entfiele.

Beispiel: Für eine körperlich anstrengende Tätigkeit bewerben sich eine Frau und ein Mann. Die Frau ist besser qualifiziert als der Mann. Der Arbeitgeber entscheidet sich für die Frau, weil sie besser qualifiziert ist als der Mann, obwohl er eigentlich lieber einen Mann für diese Tätigkeit ausgewählt hätte, weil er glaubt, daß Männer für diese Tätigkeit grundsätzlich besser geeignet sind als Frauen, und weil Männer nicht durch Schwangerschaft ausfallen können.

Wenn dem Arbeitgeber verwehrt sein soll, bei seiner Auswahlentscheidung das Geschlecht des Bewerbers zu dessen Lasten zu berücksichtigen, dann müßte in dem Beispielsfall nach der Rechtsprechung des BVerfG dem Arbeitgeber diskriminierendes Verhalten bescheinigt werden, obwohl er die „diskriminierte“ Bewerberin eingestellt hat.

Der Arbeitgeber hat in seine Entscheidung die Tatsache (negativ) einfließen lassen, daß der bestqualifizierte Bewerber eine Frau ist. Gleichwohl hat er die Frau eingestellt, weil ihn letztlich deren bessere Qualifizierung überzeugt hat. Das Merkmal „Qualifikation“ hat in diesem Fall das unerwünschte Merkmal „Geschlecht“ wettgemacht.

Der Beispielsfall verdeutlicht, daß es letztlich immer ein bestimmtes Merkmal ist, daß den Ausschlag für die endgültige Entscheidung gibt. Entweder bildet der unsachliche Grund das Zünglein an der Waage, oder eben nicht.

Sollte man das Vorliegen einer Diskriminierung in dem Beispielsfall dennoch bejahen, stellte sich die Frage nach möglichen Ansprüchen der eingestellten Frau.

\section{Keine Sanktionierung des diskriminierenden Motivs}

Es bedarf keiner Erklärung dafür, daß es unsinnig wäre, der Arbeitnehmerin einen Einstellungsanspruch zu gewähren. Sie wurde schließlich eingestellt. Auch in den Ausgangsfällen

168 In diesem Sinne wird darauf hingewiesen, der EuGH stelle für einen Verstoß gegen die Richtlinie 76/207/EWG darauf ab, „daß der ,wesentliche Grund“ für die Verweigerung der Einstellung ein auf das Geschlecht bezogener ist.“(Zimmer, NJW 1994, S. 1203 (1204)). 
scheidet ein derartiger Anspruch aus, da auch bei ihnen jeweils ein sachlicher Grund dafür vorliegt, den abgelehnten Bewerber nicht einzustellen.

$\mathrm{Zu}$ denken ist in den Ausgangsfällen sowie der Abwandlung an die Gewährung eines Schadensersatzanspruchs. In diesem Zusammenhang sei noch einmal daran erinnert, daß Diskriminierungsschutzmaßnahmen das Ziel verfolgen $\mathrm{zu}$ verhindern, daß Menschen vom gesellschaftlichen Leben aufgrund der Tatsache ausgeschlossen werden, daß sie Träger bestimmter Merkmale sind. In der Abwandlung ist es zu einem derartigen Ausschluß trotz der unsachlichen Vorbehalte des Arbeitgebers gegenüber Frauen nicht gekommen. Gewährte man der Frau in diesem Fall einen Schadensersatzanspruch, sanktionierte man ein bestimmtes, von der Rechtsordnung nicht toleriertes Motiv, schriebe ein zivilistischer Tugendkatalog ${ }^{169}$ dem Bürger mithin eine für richtig befundene Tugend vor. Das kann und darf nicht Aufgabe von Antidiskriminierungsrecht, von Recht im allgemeinen sein. „Von Interesse ist ausschließlich, was getan und bewirkt wird. [...] Entsprechend verbietet die Rechtsordnung auch nicht Gedanken oder Meinungen, sondern Taten, eventuell auch verbales Tun, wenn es tatsächlich verletzende Folgen zeitigt. “170

In den Ausgangsfällen ist es tatsächlich zum Ausschluß des „Diskriminierten“ gekommen, so daß sich die Frage eines möglichen Schadensersatzanspruchs in diesen Fällen neu stellt.

Der in der Abwandlung konstruierte Fall hat deutlich gemacht, daß Motive allein nicht sanktioniert werden dürfen. Genau das würde aber gemacht, gewährte man in den Ausgangsfällen Schadensersatzansprüche einzig und allein aufgrund der Tatsache, daß der Arbeitgeber einen unsachlichen Grund angegeben hat. Seiner Entscheidung letztlich zugrunde gelegt hat er nämlich nicht das Geschlecht der Bewerber, sondern deren Qualifikation.

Wie die Abwandlung gezeigt hat, ist für den Arbeitgeber, der Frauen bzw. Männer grundsätzlich nicht einstellen möchte, ein bestimmtes Geschlecht entweder so wesentlich, daß er seine Entscheidung gerade auf dieses Merkmal stützt, oder es ist ihm ein anderes Merkmal (Qualifikation) derart wichtig, daß er dafür auch das an sich ungeliebte Geschlecht in Kauf nimmt. In den Beispielsfällen war letztlich allein die Qualifikation der Bewerber ausschlaggebend für die Entscheidung des Arbeitgebers, nicht das Geschlecht.

Der Arbeitgeber der Beispielsfälle muß von dem intoleranten Arbeitgeber unterschieden werden, der etwa geschlechtsspezifisch ausschreibt. Jener möchte Bewerber eines bestimmten Geschlechts in keinem Fall einstellen. Dieser hingegen präferiert zwar auch ein bestimmtes Geschlecht, ist aber im Gegensatz zu jenem grundsätzlich bereit, seine persönliche „zweite Wahl“ einzustellen. 
Der Begriff der Toleranz würde mißbraucht, wenn Menschen unter seiner Flagge gezwungen wären, real existierende Unterschiede zu leugnen; wenn ihnen Affektion dort aufgezwungen würde, wo sie sie tatsächlich nicht empfinden.

Ein Schadensersatzanspruch kann somit nicht allein deshalb gewährt werden, weil der Arbeitgeber Vorbehalte einem bestimmten Geschlecht gegenüber hat und diese Vorbehalte auch äußert. Die bloße Nennung eines unsachlichen Grundes begründet keine Diskriminierung, die sanktioniert werden müßte.

Denkbar ist jedoch, daß die Art und Weise der Äußerung seiner Vorbehalte den Arbeitgeber schadensersatzpflichtig werden läßt; dann nämlich, wenn sie belästigenden Charakter hat. ${ }^{171}$ Diskriminierungsschutz bezweckt eben auch den Schutz vor ,,verbale[m] Tun, wenn es tatsächlich verletzende Folgen zeitigt. “" ${ }^{172}$ Nicht die bloße Nennung eines unsachlichen Grundes, sondern deren Art und Weise kann diskriminierenden Charakter haben. Wenn der Arbeitgeber dem abgewiesenen Bewerber eingesteht, er bevorzuge Männer für körperlich anstrengende Tätigkeiten, Frauen für die Sekretärstätigkeit oder generell Frauen aufgrund leidvoller persönlicher Lebenserfahrung, dann wird der abgewiesene Bewerber dadurch nicht belästigt. Auch in dem besonders sensiblen Bereich der Diskriminierung aufgrund der Rasse und Staatsangehörigkeit können geäußerte Vorbehalte nicht per se als Diskriminierung eingestuft werden. Sollte beispielsweise der deutsche Arbeitgeber nicht auf deutsche Arbeitnehmer schwören dürfen?

So sehr der Verfasser gegen Diskriminierung auch innerhalb von Privatrechtsbeziehungen ist, so entschieden ist er gegen eine ,,,political correctness“, die viele von vornherein entmutigt, an einer kontroversen Diskussion mit eigenen Überlegungen teilzunehmen. “173 Korrektheit ist eine Tugend, politische Korrektheit pure Heuchelei, die erschreckende Früchte hervorzubringen vermag. ${ }^{174}$

Deshalb sollte es in den Ausgangsfällen auch nicht zur Umkehr der Beweislast i. S. d. § 22 AGG $^{175}$ kommen, da Arbeitgebern andernfalls tatsächlich geraten werden müßte, „im Auswahlverfahren jede wie auch immer geartete Bezugnahme auf das Geschlecht, die etwa in der Frage nach einer Schwangerschaft liegen kann, zu vermeiden.“176

\section{Zeitlich versetztes Zusammentreffen - § 15 II AGG}


Im Zusammenhang mit dem hier diskutierten Problem des Zusammentreffens von Diskriminierungsmerkmalen in einer Person ist $§ 15$ II AGG zu nennen. Die Bestimmung lautet wie folgt:

„Wegen eines Schadens, der nicht Vermögensschaden ist, kann der oder die Beschäftigte eine angemessene Entschädigung in Geld verlangen. Die Entschädigung darf bei einer Nichteinstellung drei Monatsgehälter nicht übersteigen, wenn der oder die Beschäftigte auch bei benachteiligungsfreier Auswahl nicht eingestellt worden wäre.“

Für das Verständnis des $§ 15$ II 2 AGG erlangt die Rechtsprechung des EuGH Bedeutung, wonach auch der Bewerber einen Schaden erleidet, dessen Bewerbung aufgrund seines Geschlechts schon keine Berücksichtigung gefunden hat, auch wenn er aus einem anderen, sachlichen Grund sowieso nicht eingestellt worden wäre. ${ }^{177}$ In dem konkreten Fall hatten sich zwei Männer beworben, von denen der eine bei diskriminierungsfreiem Verhalten eingestellt worden wäre. ${ }^{178}$ Es war geschlechtsspezifisch ausgeschrieben worden: „Für unseren Vertrieb suchen wir eine versierte Assistentin der Vertriebsleitung. “179 Zum Zeitpunkt der Ausschreibung war demnach allein aufgrund des Geschlechts unterschieden worden. Der sachliche Grund der besseren Qualifikation eines anderen Bewerbers war zu diesem Zeitpunkt noch kein Unterscheidungsmerkmal.

Entsprechend den Vorgaben des EuGH wurde auch $\S 611$ a III 1 BGB-A interpretiert, der in Umsetzung jener Rechtsprechung neu ins BGB eingeführt worden war ${ }^{180}$ und eine dem $§ 15$ II 2 AGG entsprechende Regelung enthielt. ${ }^{181}$ Es wurde vertreten, $\S 611$ a III BGB-A betreffe diejenigen Fälle, bei denen der Bewerber zwar wegen seines Geschlechts nicht in die Auswahl für den Arbeitsplatz gelangt ist, die Stelle aber sowieso nicht erhalten hätte. ${ }^{182}$ Die Norm setze nur voraus, daß der Bewerber bei der Auswahl geschlechtsbezogen nicht berücksichtigt, nicht aber, daß er nur deswegen nicht eingestellt worden ist. ${ }^{183}$

Dementsprechend wurde an anderer Stelle lediglich verlangt, daß der unsachliche Grund kausal für eine Entscheidung im Einstellungsprozeß gewesen sein muß, damit das Vorliegen einer Diskriminierung bejaht werden kann, nicht aber für die Einstellung an sich. Eine Entscheidung in diesem Sinne kann auch die geschlechtsspezifische Ausschreibung sein. Ein unsachlicher Grund führt in diesem Fall dazu, daß Männer bzw. Frauen vom weiteren Einstellungsverfahren ausgeschlossen werden. Ihnen wird damit von vornherein jede Chance genommen, eingestellt zu

177 EuGH, DB 1997, S. 983 (984, Rn. 34).

$178 E u G H$, DB 1997, S. 983 (983).

$179 E u G H$, DB 1997, S. 983 (983).

180 Richardi in Münchener Handbuch zum Arbeitsrecht, Band 1, § 11, Rn. 37.

$181 \S 611$ a III 1 BGB-A lautete wie folgt: „Wäre der Bewerber auch bei benachteiligungsfreier Auswahl nicht eingestellt worden, so hat der Arbeitgeber eine angemessene Entschädigung in Höhe von höchstens drei Monatsverdiensten zu leisten.“.

182 Weidenkaff in Palandt, $\$ 611$ a, Rn. 21.

183 Weidenkaff in Palandt, § 611 a, Rn. 22. 
werden. Die Rechtsprechung des EuGH und der ihre Wertung nunmehr verkörpernde $§ 15$ II 2 AGG verdienen deshalb uneingeschränkte Zustimmung.

\section{Nachschieben von sachlichen Gründen}

Beispiel: Eine Bewerberin, die die formellen Voraussetzungen der Ausschreibung erfüllt, wird mit der Begründung nicht zum Vorstellungsgespräch geladen, die ausgeschriebene Tätigkeit sei für eine Frau nicht geeignet. In der Gerichtsverhandlung behauptet der Arbeitgeber, die zum Vorstellungsgespräch geladenen Bewerber seien der Beschwerdeführerin hinsichtlich ihrer Qualifikation oder Berufserfahrung weit überlegen gewesen, was objektiv zutrifft. $^{184}$

In dem vom BVerfG entschiedenen Fall behauptete der Arbeitgeber, er habe die Ablehnung der Bewerberin mit deren Geschlecht begründet, weil er ihr die Aussichtslosigkeit ihrer Bewerbung möglichst schonend beibringen wollte. ${ }^{185}$

Die Wahrheit dieser Aussage unterstellt, ist das Vorliegen einer Diskriminierung in diesem Fall zu verneinen. Der Arbeitgeber stützte seine Entscheidung darüber, wen er zum Vorstellungsgespräch lädt, unter dieser Prämisse eben nicht auf das Geschlecht, sondern auf Qualifikation und Berufserfahrung der Bewerber.

Handelt es sich bei der Begründung des Arbeitgebers dagegen um eine Schutzbehauptung, hat er die Ablehnung der Bewerberin also einzig und allein auf deren Geschlecht gestützt, dann ist das Vorliegen einer Diskriminierung zweifelsfrei zu bejahen.

\section{Beweisrechtliches Problem}

Der Beispielsfall bereitet demnach materiellrechtlich keine Probleme. Problematisch ist er insofern, als unklar ist, ob die Begründung des Arbeitgebers der Wahrheit entspricht oder ob sie nur dem Ziel diente, dessen diskriminierendes Verhalten $\mathrm{zu}$,rechtfertigen“. Fraglich ist, wie mit dieser Unklarheit prozessual umzugehen ist.

Bedeutung erlangt in diesem Zusammenhang § 22 AGG. Die Bestimmung lautet wie folgt:

„Wenn im Streitfall die eine Partei Indizien beweist, die eine Benachteiligung wegen eines in $\S 1$ genannten Grundes vermuten lassen, trägt die andere Partei die Beweislast dafür, daß kein Verstoß gegen die Bestimmungen zum Schutz vor Benachteiligung vorgelegen hat.“ 
In dem Beispielsfall ist es zweifelsfrei zu einer Beweislastumkehr im Sinne des $§ 22$ AGG gekommen. ${ }^{186}$ Der Arbeitgeber hat zu seiner Verteidigung vorgebracht, die gegebene Begründung für die Ablehnung der Bewerberin sei ungeschickt und unzutreffend gewesen und zum „Beweis“ angeführt, er habe allein Bewerber zum Vorstellungsgespräch geladen und letztlich auch eingestellt, die hinsichtlich ihrer Qualifikation oder Berufserfahrung der abgelehnten Bewerberin weit überlegen gewesen seien. ${ }^{187}$ Genügt der Arbeitgeber seiner Erklärungspflicht durch dieses Vorbringen?

\section{Voraussetzungen zulässigen Nachschiebens sachlicher Gründe}

Das $B V$ erfG verlangte im Rahmen des $\S 611$ a I 3 BGB-A, der eine dem $\S 22$ AGG vergleichbare Beweislastregelung enthielt, ${ }^{188}$ vom Arbeitgeber eine besondere Rechtfertigung für das Nachschieben sachlicher Gründe zu einem späteren Zeitpunkt, da es befürchtete, daß sich dieser anderenfalls in nahezu jedem Fall im Sinne des $\S 611$ a I 3 BGB-A entlasten könne und der gerichtlichen Durchsetzung des Diskriminierungsverbots damit ein praktisch unüberwindliches Hindernis entgegengesetzt sei. ${ }^{189}$ Die Entlastung durch nachträgliche Angabe eines sachlichen Grundes bereite dem Arbeitgeber deshalb so geringe Schwierigkeiten, weil er die Anforderungen an die Qualifikation für eine bestimmte Stelle grundsätzlich nach seinem Belieben festlegen dürfe. ${ }^{190}$ Das Gericht qualifizierte einen nachträglich vorgebrachten Grund für die Bevorzugung eines Bewerbers des anderen Geschlechts deshalb nur dann als „,sachlich“ im Sinne des § 611 a I 3 BGBA, ,wenn besondere Umstände erkennen [ließen], daß der Arbeitgeber diesen Grund nicht nur vorgeschoben [hatte]. “191 Das sollte beispielsweise dann der Fall sein, wenn sich während des Einstellungsverfahrens die Aufgabenstellung und damit die Anforderungen an die Qualifikation des Einzustellenden geändert hatten. Denkbar sei auch - so das Gericht -, daß sich ein Arbeitnehmer bewirbt, der für die ihm zugedachte Aufgabe geradezu prädestiniert ist, mit dessen Bewerbung aber zur Zeit der Ausschreibung vernünftigerweise nicht gerechnet werden konnte. ${ }^{192}$

Es kann davon ausgegangen werden, daß das BVerfG an den nunmehr dem AGG unterworfenen Arbeitgeber die gleichen Anforderungen stellt.

186 So auch das BVerfG (BVerfGE 89, S. 276 (289); Beachte, daß der Entscheidung des Gerichts die alte Rechtslage zugrunde liegt. § 611 a I 3 BGB-A enthielt eine dem § 22 AGG vergleichbare Beweislastregelung. (Vergleiche dazu Teil 4, Prüfungspunkt C. III. 1.).

187 BVerfGE 89, S. 276 (280).

188 Vergleiche dazu Teil 4, Prüfungspunkt C. III. 1.

189 BVerfGE 89, S. 276 (289).

190 BVerfGE 89, S. 276 (289); vgl. auch Schiek: „Teilt der Arbeitgeber die Auswahlkriterien im nachhinein mit, so ist es durchaus möglich, daß sie nunmehr auf den tatsächlich ausgewählten Bewerber „passen“““ (Schiek, BB 1998, S. $586(586))$.

191 BVerfGE 89, S. $276(290)$.

192 BVerfGE 89, S. 276 (290).

160 


\section{Keine Offenlegungspflicht der Entscheidungsgründe}

Beachtet werden muß, daß das BVerfG seine Ausführungen zum „Nachschieben sachlicher Gründe“ auf Fälle bezieht, bei denen der „Diskriminierte“ Tatsachen vorgetragen (und gegebenenfalls bewiesen) hat, die eine Diskriminierung vermuten lassen. In einem solchen Fall soll der bloße Nachweis eines unzweifelhaft vorliegenden sachlichen Grundes - in dem Beispielsfall die Qualifikation und Berufserfahrung - nicht ausreichen, um die glaubhaft gemachte Diskriminierung zu entkräften. Im Kern sagt das Gericht damit - zu recht -, daß ein solcher Nachweis allein noch kein Beweis dafür ist, daß in Wirklichkeit nicht doch der unsachliche Grund - das Geschlecht conditio sine qua non für die Entscheidung gewesen ist. ${ }^{193}$

Beispiel: Der Arbeitgeber entscheidet sich für den im Vergleich zur Mitbewerberin objektiv schlechter qualifizierten Bewerber, weil dieser ihm sympathischer ist. Die abgewiesene Bewerberin klagt vor Gericht, weil sie sich aufgrund ihres Geschlechts diskriminiert fühlt.

Sympathie kann ein Grund sein, jemanden einzustellen; Antipathie, jemanden abzulehnen. Dem Arbeitgeber ist gestattet, den minderqualifizierten Bewerber dem besserqualifizierten vorziehen, weil ersterer ihm sympathischer ist. ${ }^{194}$ Die Bewerberin löst die Beweislastumkehr des $\S 22$ AGG nicht allein durch Verweis auf die geringere Qualifikation des eingestellten Bewerbers aus, ${ }^{195}$ und zwar auch dann nicht, wenn der Arbeitgeber erstmals im Prozeß den sachlichen Grund „Sympathie“ für seine Auswahlentscheidung angibt. Die Rechtsprechung des BVerfG zum Nachschieben von sachlichen Gründen darf nicht in der Weise (fehl-)interpretiert werden, die erstmalige Nennung eines sachlichen Grundes im Prozeß verpflichte den Arbeitgeber stets dazu, dieses Verhalten im Sinne besagter Rechtsprechung besonders zu rechtfertigen. Dem ist nicht so: Bereits „§ 611 a Abs. 1 Satz 3 macht[e] deutlich, daß der Arbeitgeber nicht verpflichtet [war], dem unberücksichtigt gebliebenen Bewerber seine Auswahlgründe mitzuteilen. “ ${ }^{196}$ Durch das Inkrafttreten des AGG ist es insofern nicht zu einer Änderung der Rechtslage gekommen. ${ }^{197}$

Der Arbeitgeber schiebt also keinen sachlichen Grund im Sinne der erwähnten Rechtsprechung des BVerfG nach, wenn er erstmals im Prozeß die Einstellung des Bewerbers damit begründet, dieser sei ihm im Bewerbungsgespräch sympathischer gewesen als die besserqualifizierte Bewerberin. Er

193 Richardi/Anпиß: „Ein Nachschieben objektiv vorliegender, subjektiv jedoch nicht maßgeblicher Rechtfertigungsgründe genügt daher nicht.“ (Richardi/Annuß in Staudinger, § 611 a, Rn. 104); Müller-Glöge: „Ein „Nachschieben“ im Sinne der Entscheidung des BVerfG liegt nur vor, wenn objektiv geeignete Auswahlgründe bei der Entscheidung tatsächlich unberücksichtigt blieben, aber im Streit um die Verletzung von $\S 611$ a [BGB-A] „entdeckt“ und nachträglich vorgebracht werden.“ (Müller-Glöge in MüKo, § 611 a, Rn. 85).

194 Buchner in Münchener Handbuch zum Arbeitsrecht, Band 1, § 39, Rn. 74; Raab, RdA 1995, S. 36 (38); MüllerGlöge in MüKo, § 611 a, Rn. 23; Wiese, JuS 1990, S. 357 (360).

195 Vergleiche dazu Teil 4, Prüfungspunkt C. III. 1. f).

196 Müller-Glöge in MüKo, § 611 a, Rn. 83; in diesem Sinne auch Richardi/Annuß in Staudinger, § 611 a, Rn. 104.

197 Die Einführung einer derartigen Begründungspflicht ist angesichts der Schutzinteressen des „Diskriminierten“ in Betracht zu ziehen. 
muß deshalb nicht beweisen, daß ausschlaggebendes Auswahlkriterium die besondere Zuneigung zu dem eingestellten Bewerber gewesen ist. ${ }^{198}$

\section{Zusammenfassung}

Die im Rahmen des Prüfungspunktes „Zusammentreffen von Diskriminierungsmerkmalen in einer Person“ getroffenen Feststellungen lassen sich wie folgt zusammenfassen: Jeder Mensch ist Träger einer Vielzahl von Diskriminierungsmerkmalen, die im Rahmen einer diskriminierungsrelevanten Maßnahme unbewußt oder bewußt zusammentreffen können.

Ein unbewußtes Zusammentreffen von Diskriminierungsmerkmalen ist etwa gegeben, wenn ein Mann aufgrund besseren Zeugnisses, nicht aufgrund seines Geschlechts, für die gleiche Tätigkeit eine höhere Vergütung erhält als die Frau. In diesem Zusammenhang ist anzumerken, daß das absolute Verbot der Entgeltdiskriminierung aufgrund des Geschlechts nicht zu einem Recht auf gleichen Lohn für gleiche bzw. gleichwertige Arbeit führt. Nicht auf das Geschlecht bezogene, sachliche Gründe vermögen eine unterschiedliche Behandlung stets $\mathrm{zu}$ rechtfertigen. Ob die Entscheidung auf das eine oder andere Diskriminierungsmerkmal gestützt wurde, ihr also ein sachlicher oder unsachlicher Grund zugrunde liegt, ist nicht immer einfach zu beantworten. Das gilt etwa dann, wenn der Vermieter den chinesischen Interessenten mit der Begründung ablehnt, Chinesen würden ihre Vertragspflichten regelmäßig nicht erfüllen.

Ein bewußtes Zusammentreffen von Diskriminierungsmerkmalen liegt vor, wenn der Diskriminierende seiner benachteiligenden Ungleichbehandlung mehrere Diskriminierungsmerkmale zugrunde legt. Allein die Tatsache, daß sich in dem Motivbündel auch ein unsachlicher Grund befindet - der Arbeitgeber etwa entscheidet sich nicht für die Frau, weil sie eine geringere Qualifikation hat als der Mann und weil sie eine Frau ist - führt nicht dazu, daß die diskriminierungsrelevante Entscheidung rechtswidrig ist. Eine Diskriminierung ist in einem solchen Fall nur dann gegeben, wenn der unsachliche Grund kausal für die Entscheidung war. Das diskriminierende Motiv für sich ist nicht sanktionswürdig, solange es nicht in belästigender Weise geäußert wird. So diskriminiert der deutsche Arbeitgeber nicht schon dadurch, daß er auf deutsche Arbeitnehmer schwört.

Diskriminierungsmerkmale treffen zeitlich versetzt zusammen, wenn beispielsweise der Arbeitgeber, der eine Stelle geschlechtsspezifisch ausgeschrieben hat, letztlich zur Grundlage seiner Einstellungsentscheidung die Qualifikation der Bewerber macht. In einem solchen Fall stellt sich die Frage nach dem Vorliegen einer Diskriminierung auch für die durch die geschlechtsspezifische Ausschreibung benachteiligten Männer bzw. Frauen, die schlechter qualifiziert sind als der 
eingestellte Bewerber. Stellenausschreibung und Einstellung stellen nämlich zwei unabhängig voneinander zu betrachtende diskriminierungsrelevante Entscheidungen dar.

Trägt der „Diskriminierte“ Tatsachen vor, die eine Diskriminierung im Sinne des § 22 AGG vermuten lassen, vermag sich der „Diskriminierende“ - sofern diese Tatsachen unstreitig oder bewiesen sind - nicht bereits durch den Nachweis eines objektiv vorliegenden sachlichen Grundes, den er erstmals im Prozeß angibt, zu entlasten. Ein auf diese Weise nachträglich vorgebrachter Grund war nach der Rechtsprechung des BVerfG zu § 611 a I 3 BGB-A, der eine dem $\S 22$ AGG vergleichbare Beweislastregelung enthielt, nur dann als „sachlich“ im Sinne dieser Norm zu qualifizieren, wenn besondere Umstände erkennen ließen, daß der Arbeitgeber diesen Grund nicht nur vorgeschoben hatte. Diese Rechtsprechung muß im Rahmen des $\S 22$ AGG fortbestehen, da sich der Arbeitgeber andernfalls (vermutlich) in nahezu jedem Fall im Sinne des § 22 AGG entlasten könnte, darf er doch grundsätzlich die Anforderungen an die Qualifikation für eine bestimmte Stelle nach seinem Belieben festlegen. Beachtet werden muß, daß die in $\S 22$ AGG geregelte Beweislastumkehr, infolge derer für nachgeschobene sachliche Gründe besondere Begründungsanforderungen bestehen, nicht allein durch die Tatsache eintritt, daß der Arbeitgeber im Prozeß erstmals den (sachlichen) Grund seiner Auswahlentscheidung angibt. Der Arbeitgeber ist nicht verpflichtet, dem unberücksichtigt gebliebenen Bewerber seine Auswahlgründe mitzuteilen. Allein die Tatsache, daß der Arbeitgeber seine Auswahlentscheidung erst im Prozeß beispielsweise damit begründet, ihm sei der Bewerber sympathischer gewesen als die Bewerberin, führt deshalb nicht dazu, daß er diese Behauptung beweisen müßte.

\section{Tendenzschutz}

Im Rahmen der Frage nach den Auswirkungen des Diskriminierungsverbotes auf das (quasi)rechtsgeschäftliche Handeln ist auf das Spannungsverhältnis zwischen Diskriminierungsschutz auf der einen und Tendenzschutz auf der anderen Seite einzugehen. In diesem Zusammenhang ist zunächst der Begriff des Tendenzbetriebes zu klären. Gemäß Art. 118 I BetrVG handelt es sich dabei um einen Betrieb, der unmittelbar und überwiegend

„1. politischen, koalitionspolitischen, konfessionellen, karitativen, erzieherischen, wissenschaftlichen oder künstlerischen Bestimmungen oder

2. Zwecken der Berichterstattung oder Meinungsäußerung, auf die Artikel 5 Abs. 1 Satz 2 des Grundgesetzes Anwendung findet, [dient]“. 
Eine Besonderheit von Tendenzbetrieben soll darin bestehen, daß über den maßgebenden „Tendenzinhalt“ der Tendenzbetrieb entscheidet. ${ }^{199}$ Daraus folge, daß nicht die Natur der Arbeit und das Leistungsvermögen des Arbeitnehmers - also objektive, der Definitionsmacht des Arbeitgebers entzogene Maßstäbe - die Rechtfertigung einer Ungleichbehandlung begründen, sondern die Lehre des Arbeitgebers - also von diesem selbst gesetzte Kriterien. ${ }^{200}$

An anderer Stelle wurde festgestellt, daß auch „Nicht-Tendenzbetriebe“ Diskriminierungsmerkmale für wesentlich und entscheidend erklären können, daß diese „Wesentlich- und Entscheidendmachung“" aber objektiv begrenzt ist.

\section{Geltung des Diskriminierungsverbotes in Tendenzbetrieben}

Fraglich ist, ob dasselbe auch für Tendenzbetriebe gilt, auch deren diskriminierungsrelevanten Entscheidungen also einer objektiven Überprüfung unterliegen.

\section{Art. 137 III, VII WRV i. V. m. Art. 140 GG}

Art. 137 III 1 WRV, der gem. Art. 140 GG Bestandteil des Grundgesetzes ist, beantwortet diese Frage für die Religionsgemeinschaften. Gemäß dieser Bestimmung

„[...] ordnet und verwaltet [jede Religionsgemeinschaft] ihre Angelegenheiten selbständig innerhalb der Schranken des für alle geltenden Gesetzes.“

Das gleiche gilt für die Weltanschauungsgemeinschaften, ordnet Art. 137 VII doch an, daß sie den Religionsgemeinschaften gleichgestellt werden.

Die „Schranken des für alle geltenden Gesetzes“ werden nach der Rechtsprechung des BVerfG überschritten, sobald sich kirchliche Vorgaben in Widerspruch begeben ,zu den Grundprinzipien der Rechtsordnung, wie sie im allgemeinen Willkürverbot (Art. 3 I GG) sowie in dem Begriff der „guten Sitten“ ( $\$ 138$ BGB) und des ordre public [(Art. 6 EGBGB)] ihren Niederschlag gefunden haben.“201 Eine gewisse Homogenität zwischen staatlicher und kirchlicher Ordnung ${ }^{202}$ müsse letztlich auch im Interesse der Glaubensgemeinschaften, auch der christlichen Kirchen liegen. Wollten sie rechtsfreie Räume nutzen, um diskriminieren zu können, verlöre die Bezugnahme auf eine christliche, nämlich tolerante Werteordnung, die auch das Grundgesetz prägen soll, endgültig ihre Berechtigung. ${ }^{203}$ 
$\mathrm{Zu}$ beachten ist auch folgendes: Bedienen sich die Kirchen wie jedermann der Privatautonomie beispielsweise zur Begründung von Arbeitsverhältnissen, so findet auf diese das staatliche Arbeitsrecht Anwendung. ${ }^{204}$ Steht beispielsweise die Frage der Rechtmäßigkeit einer Kündigung im Raum, so ist bei Bejahung der Verletzung einer Loyalitätsobliegenheit in einem zweiten Schritt zu prüfen, ob diese Verletzung eine Kündigung des kirchlichen Arbeitsverhältnisses sachlich rechtfertigt. $^{205} \mathrm{Zu}$ prüfen sind dabei kündigungsschutzrechtliche Vorschriften wie $\S \S 1 \mathrm{KSchG}, 626$ BGB, die als für alle geltendes Gesetz im Sinne des Art. 137 III 1 WRV einer umfassenden arbeitsgerichtlichen Anwendungskompetenz unterliegen. ${ }^{206}$ Nicht vergessen werden darf in diesem Zusammenhang jedoch, daß die Einbeziehung kirchlicher Arbeitsverhältnisse in das staatliche Arbeitsrecht nicht deren Zugehörigkeit zu den ,eigenen Angelegenheiten“ der Kirche aufhebt. ${ }^{207}$ „Art. 137 III 1 WRV gewährleistet mit Rücksicht auf das zwingende Erfordernis des friedlichen Zusammenlebens von Staat und Kirche sowohl das selbständige Ordnen und Verwalten der eigenen Angelegenheiten durch die Kirchen als auch den staatlichen Schutz anderer für das Gemeinwesen bedeutsamer Rechtsgüter. Dieser Wechselwirkung von Kirchenfreiheit und Schrankenzweck ist durch entsprechende Güterabwägung Rechnung zu tragen. Dabei ist dem Selbstverständnis der Kirchen ein besonderes Gewicht beizumessen.“208

Für alle übrigen Tendenzbetriebe kann folgendes gesagt werden: Wenn in besonderem Maße das Handeln der Religions- und Weltanschauungsgemeinschaften vor staatlicher Überprüfung geschützt ist, dann müssen Grenzen, die diesem Handeln dennoch gesetzt sind, erst recht für alle übrigen Tendenzbetriebe gelten.

\section{Art. 4 II Richtlinie 2000/78/EG}

Gemäß Art. 4 II 1 Richtlinie 2000/78/EG können die Mitgliedstaaten

„,...] in Bezug auf berufliche Tätigkeiten innerhalb von Kirchen und anderen öffentlichen oder privaten Organisationen, deren Ethos auf religiösen Grundsätzen oder Weltanschauungen beruht, Bestimmungen [...] beibehalten oder in künftigen Rechtsvorschriften Bestimmungen vorsehen, [...] wonach eine Ungleichbehandlung wegen der Religion oder Weltanschauung einer Person keine Diskriminierung darstellt, wenn die Religion oder die Weltanschauung dieser Person nach der Art dieser Tätigkeiten oder der Umstände ihrer Ausübung eine wesentliche, rechtmäßige und gerechtfertigte berufliche Anforderung angesichts des Ethos der Organisation darstellt.“ 
Bezüglich des Wortlauts der Bestimmung, der von Art. 4 I abweicht, ist festzuhalten, daß gerechtfertigt ist, was rechtmäßig ist und vice versa. ${ }^{209}$ Insofern besteht kein Unterschied zu Art. 4 I, der lediglich von rechtmäßigem Zweck spricht.

Während Absatz 1 verlangt, daß das betreffende Merkmal ,eine wesentliche und entscheidende berufliche Anforderung darstellt,“ reicht nach Absatz 2 aus, daß es „eine wesentliche, rechtmäßige und gerechtfertigte berufliche Anforderung [...] darstellt.“ Unabhängig davon, in welcher Beziehung die Begriffe „,wesentlich“ und „entscheidend“ zueinander stehen, ${ }^{210}$ kann behauptet werden, der Richtliniengesetzgeber habe mit der unterschiedlichen Begriffswahl die Anforderungen an die Rechtswidrigkeit diskriminierungsrelevanten Verhaltens unterschiedlich ausgestalten wollen. Im Geltungsbereich des Absatz 1 soll ein strengerer Maßstab gelten als in dem des Absatz 2.

In jedem Fall setzt auch Art. 4 II 1 Richtlinie 2000/78/EG diskriminierungsrelevantem Handeln des Tendenzbetriebes eine objektive Grenze. ${ }^{211}$ Tendenzbetriebe dürfen nach dieser Vorschrift nur insoweit ungleich behandeln, als eine bestimmte Religion oder Weltanschauung ,[...] wesentliche, rechtmäßige und gerechtfertigte berufliche Anforderung angesichts des Ethos der Organisation darstellt.“212 Gemäß Art. 4 II 2 rechtfertigt eine solche Ungleichbehandlung ,keine Diskriminierung aus einem anderen Grund.“ Sie muß zudem „die verfassungsrechtlichen Bestimmungen und Grundsätze der Mitgliedstaaten sowie die allgemeinen Grundsätze des Gemeinschaftsrechts beachten“.

Die bisherigen Überlegungen haben zu zwei Erkenntnissen geführt: Zum einen sind auch Tendenzbetriebe an das Diskriminierungsverbot gebunden. Zum anderen ist im Rahmen der Überprüfung diskriminierungsrelevanter Entscheidungen von Tendenzbetrieben dem Selbstverständnis dieser Betriebe ein besonderes Gewicht beizumessen. Auf die Frage, wie sich dies auf die Interessenabwägung konkret auswirkt, soll im folgenden eingegangen werden.

\section{Tendenz als Anknüpfungspunkt}

Gemäß Art. 4 II 2 Richtlinie 2000/78/EG rechtfertigt eine Ungleichbehandlung aufgrund der Religion oder Weltanschauung „keine Diskriminierung aus einem anderen Grund.“ Diese Formulierung läßt sich sinnvoll nur in der Weise interpretieren, daß mit „Ungleichbehandlung

209 So Thüsing, ZfA 2001, S. 397 (408).

210 Vergleiche dazu Teil 2, Prüfungspunkt C. IV.

211 Das Verhältnis des Art. 4 II Richtlinie 2000/78/EG zum Tendenzschutz im deutschen Arbeitsrecht beschreibt Thüsing wie folgt: „Dieser Rechtfertigungsgrund dürfte sich 1 : 1 mit den Kriterien des deutschen kirchlichen Arbeitsrechts decken, [...].“ (Thüsing, ZfA 2001, S. 397 (408)).

212 Hervorhebung durch den Verfasser; In Konsequenz der Ausführungen zu den §§ 8 I und 3 II AGG - vgl. dazu Teil 2, Prüfungspunkt C. VII. 2. - stellen die Merkmale „rechtmäßig“ und ,gerechtfertigt“ in Art. 4 II Richtlinie 2000/78/EG die objektive Grenze der „Wesentlichmachung“ durch den Tendenzbetrieb dar. 
aufgrund der Religion oder Weltanschauung“ jede Ungleichbehandlung aufgrund des Status einer bestimmten Religionszugehörigkeit bzw. Zugehörigkeit zu einer bestimmten Weltanschauung gemeint ist. Angesichts des Art. 4 II 2 Richtlinie 2000/78/EG soll im folgenden unterschieden werden zwischen Fällen, bei denen eine bestimmte Tendenzzugehörigkeit Anknüpfungspunkt für die ,diskriminierende“ Entscheidung ist, und solchen, bei denen aufgrund eines anderen Merkmals „diskriminiert“" wird.

\section{Kernbereich der Tätigkeit des Tendenzbetriebes}

Beispiel: Kündigung wegen Austritts aus der Religionsgemeinschaft ${ }^{213}$

Niemand wird bestreiten, daß von einem Pfarrer verlangt werden kann, daß er Mitglied der Glaubensgemeinschaft ist, deren Werte er predigen soll. Der Grund für dieses eindeutige Ergebnis ist in der Tatsache zu suchen, daß die Predigt den Kernbereich kirchlicher Tätigkeit überhaupt betrifft. Allgemein läßt sich formulieren, daß jeder Tendenzbetrieb im Kernbereich seiner tendenzrelevanten Tätigkeit verlangen kann, daß ihn Menschen repräsentieren, die seine Tendenz, in welcher Form auch immer, vertreten.

Im Kernbereich des Tendenzbetriebes werden Handlungen religiöser oder weltanschaulicher Art vorgenommen, die in ihrer konkreten Ausprägung von einem „Nicht-Tendenzbetrieb“ nicht durchgeführt werden, Handlungen also, die gerade das Wesen des Tendenzbetriebes ausmachen. Das gilt etwa für die Predigt in der Kirche sowie für alle Tätigkeiten in einem Presseunternehmen, die den konkreten Inhalt der Zeitung prägen.

\section{Randbereich der Tätigkeit des Tendenzbetriebes}

In dem Beispielsfall war es nicht der Pfarrer, dem gekündigt worden war, sondern ein kaufmännischer Angestellter in einem von einer Ordensgemeinschaft der katholischen Kirche betriebenen Jugendwohnheim. ${ }^{214}$ Man wird behaupten können, daß die Tätigkeit eines kaufmännischen Angestellten in einem Jugendwohnheim nicht den Kernbereich kirchlichen Handelns berührt. Der Angestellte wird im Rahmen seines Arbeitsverhältnisses keine Tätigkeiten ausgeführt haben, die ein konfessionsloser kaufmännischer Angestellter in einem nichtkirchlichen Jugendwohnheim nicht auch durchgeführt hätte.

Der Auftrag der Kirche erfaßt zwar nicht nur den Bereich des Glaubens und des Gottesdienstes, sondern erstreckt sich beispielsweise auch auf das karitative Wirken. ${ }^{215}$ Dennoch dürfte die 
Unterteilung in Kern- und Randbereich kirchlichen Handelns zulässig sein. Fraglich ist, ob Tendenzbetriebe auch im Randbereich „diskriminierende“ Entscheidungen auf die Religion oder Weltanschauung stützen können.

Das $B V \operatorname{erf} G$ hat in dem Beispielsfall implizit die Rechtmäßigkeit der aufgrund des Kirchenaustritts erfolgten Kündigung bejaht: „Der Kirchenaustritt verträgt sich aus der Sicht der Kirche weder mit ihrer Glaubwürdigkeit noch mit der von ih[r] geforderten vertrauensvollen Zusammenarbeit zwischen den Vertragsparteien.“216 Eine personenbedingte Kündigung soll deshalb allein schon dann gerechtfertigt sein können, wenn der Arbeitnehmer eine abweichende Tendenz vertritt. ${ }^{217}$ Es läßt sich behaupten, daß derjenige, der aus der Kirche austritt, nicht mehr die Werte dieser Kirche, mithin eine abweichende Tendenz vertritt. Der Kirchenaustritt kann als äußeres Zeichen innerer Abkehr von der Religionsgemeinschaft gewertet werden. Ist unter diesem Gesichtspunkt die Kündigung des kaufmännischen Angestellten in dem Beispielsfall gerechtfertigt oder bestehen Bedenken gegenüber der Entscheidung des BVerfG?

\section{a) Keine Privilegierung gegenüber dem ,Nicht-Tendenzbetrieb“}

Bedenken resultieren daraus, daß der nichtkirchliche Arbeitgeber - also der „Nicht-Tendenzbetrieb“ - den Kirchenaustritt seines kaufmännischen Angestellten nicht zum Anlaß einer Kündigung desselben nehmen dürfte; selbst dann nicht, wenn auch er religiös motiviert handeln würde. Es ist kein Grund dafür ersichtlich, warum die (katholische) Kirche in einem Bereich, der den Kernbereich ihres religiösen Handelns nicht betrifft, Andersdenkende per se ablehnen können sollte, der „Normalbürger“ bzw. das tendenzlose Unternehmen dagegen nicht. Die Begründung des BVerfG, der Kirchenaustritt vertrage sich aus der Sicht der Kirche weder mit ihrer Glaubwürdigkeit noch mit der von ihr geforderten vertrauensvollen Zusammenarbeit zwischen den Vertragsparteien, ${ }^{218}$ vermag deshalb nicht zu überzeugen.

\section{b) Tendenzträgerschaft}

Der Tendenzbetrieb sollte von dem Arbeitnehmer, der im Randbereich beschäftigt ist, die Trägerschaft eines bestimmten Statusmerkmals nur dann verlangen können, wenn der Arbeitnehmer Tendenzträger ist, also unmittelbar an der geistig-ideellen Zielsetzung des Betriebs - der Tendenz mitwirkt. $^{219}$

\footnotetext{
BVerfG, NJW 1986, S. 367 (369).

Ascheid in Erfurter Kommentar zum Arbeitsrecht, § 1 KSchG, Rn. 269.

$B V e r f G$, NJW 1986, S. 367 (369).

219 Junker, Grundkurs Arbeitsrecht, Rn. 665.

168
} 
Beispiel: Der ADG-Entwurf des Bundesjustizministeriums hält es beispielsweise für sachlich begründet, wenn eine Kirchengemeinde für ihren Kindergarten nur Betreuerinnen einstellt, die ihrer Religionsgemeinschaft angehören, weil sie den Kindergarten als Teil ihrer Verkündung betrachtet. ${ }^{220}$

Ein konfessioneller Kindergarten muß von seinen Betreuerinnen verlangen können, daß sie Angehörige der entsprechenden Religionsgemeinschaft sind, wenn man davon ausgeht, daß sie den Kindern bereits die Werte der Kirche vermitteln sollen.

Die Ausführungen zum Tendenzschutz im Randbereich der Tätigkeit des Tendenzbetriebes können zu folgender Feststellung zusammengefaßt werden: Soweit ein Arbeitnehmer kein Tendenzträger ist - dies ist für den kaufmännischen Angestellten des Ausgangsfalls anzunehmen -, sollte dem Tendenzbetrieb, wie jedem ,normalen“ Unternehmen, grundsätzlich untersagt sein, von diesem einen bestimmten Status, beispielsweise die Mitgliedschaft in einer Religionsgemeinschaft zu verlangen.

\section{Sonstiges Merkmal als Anknüpfungspunkt}

Art. 4 II 2 Richtlinie 2000/78/EG bestimmt, daß eine Ungleichbehandlung aufgrund der Religion oder Weltanschauung im Tendenzbetrieb keine Diskriminierung aus einem anderen Grund rechtfertigt.

\section{Kernbereich der Tätigkeit des Tendenzbetriebes}

Die Bestimmung in Art. 4 II 2 scheint auf den ersten Blick unmißverständlich formuliert und von ihrer Intention nachvollziehbar $\mathrm{zu}$ sein. Warum sollte Tendenzbetrieben gestattet sein, Entscheidungen auf andere Merkmale als ihre Tendenz zu stützen? Privilegierte man sie dann nicht gegenüber ,„normalen“ Unternehmen in einer nicht zu rechtfertigenden Weise?

Beispiel: Ist es der katholischen Kirche aufgrund dieser Norm in Zukunft untersagt, Frauen für das Priesteramt abzulehnen, oder ließe sich dies mit dem Hinweis verneinen, die Kirche unterscheide hier unmittelbar aufgrund der Religion und nur mittelbar aufgrund des Geschlechts?

Das Beispiel zeigt, daß der auf den ersten Blick zweckmäßige Art. 4 II 2 problematische Fragen aufwirft. Sollte der Gesetzgeber tatsächlich beabsichtigt haben, durch diese Norm Kernbestandteile des Selbstverständnisses einer Religionsgemeinschaft zu berühren und neu zu regeln? Sollte es einer Religionsgemeinschaft tatsächlich verwert sein, ausschließlich männliche Priester zu

220 Entwurfsbegründung, S. 49 (http://www.lsvd.de/antidiskriminierung/adgzivil02. html). 
tolerieren? Würde man damit nicht das Selbstbestimmungsrecht der Religionsgemeinschaften untergraben?

Zunächst ist festzuhalten, daß nicht gesagt werden kann, in dem Beispielsfall werde unmittelbar aufgrund der Religion und nur mittelbar aufgrund des Geschlechts unterschieden. Die Formulierung „keine Diskriminierung aus einem anderen Grund“ in Art. 4 II 2 Richtlinie 2000/78/EG läßt sich, wie bereits erwähnt, sinnvoll nur in der Weise interpretieren, daß mit „Ungleichbehandlung aufgrund der Religion oder Weltanschauung“ jede Ungleichbehandlung aufgrund des Status einer bestimmten Religionszugehörigkeit bzw. Zugehörigkeit zu einer bestimmten Weltanschauung gemeint ist. Andernfalls machte die Bestimmung in Art. 4 II 2 keinen Sinn.

Ist nun aber die unmittelbare Anknüpfung an das Geschlecht in dem Beispielsfall zulässig? Um diese Frage beantworten $\mathrm{zu}$ können, ist folgendes $\mathrm{zu}$ beachten: Wesentlich für jede Religionsgemeinschaft ist u.a. das Element des Irrationalen, der Glaube an etwas, das sich nicht nachweisen läßt und was unter Umständen jeder Logik entbehrt. Ist nun aber die Freiheit des Glaubens und das Selbstbestimmungsrecht der Kirchen grundrechtlich geschützt, muß es einen Bereich geben, in dem dieses Unlogische, Irrationale (auch in Gemeinschaft) ohne staatliche Kontrolle ausgelebt werden kann.

Zumindest im Kernbereich kirchlichen Handelns muß es der Kirche gestattet sein, auch aufgrund eines anderen Merkmals, wie beispielsweise dem Geschlecht, unterscheiden zu können, soweit dies nachgewiesenermaßen ihrem Selbstverständnis entspricht. Vor diesem Hintergrund ist der Ausschluß weiblicher Bewerber für das Priesteramt in der katholische Kirche als zulässig zu betrachten. $^{221}$

Gleiches dürfte für homosexuelle Bewerber für das Priesteramt gelten. In diesem Zusammenhang ist entschieden worden, daß eine gesteigerte Loyalitätspflicht von unmittelbaren Tendenzträgern, d. h. von solchen Personen verlangt werden kann, ,die den Verkündungsauftrag der Kirche unmittelbar zu erfüllen haben. ‘222

\section{Randbereich der Tätigkeit des Tendenzbetriebes}

Im Randbereich der Tätigkeit des Tendenzbetriebes muß etwas anderes gelten, will man Art. 4 II 2 Richtlinie 2000/78/EG nicht vollständig entwerten. Aber nicht nur dieser formale Gesichtspunkt zwingt zu einem solchen Schluß. Er ist auch sachlich geboten. Gestattete man beispielsweise kirchlich betriebenen Krankenhäusern, homosexuelle Ärzte oder Ärztinnen abzulehnen, ließe sich

221 Sich auf $\S 611$ a BGB-A beziehend meint Thüsing, in diesem Fall sei das männliche Geschlecht tatsächlich unverzichtbar. (Thüsing, RdA 2001, S. 319 (322)).

222 ArbG Lörrach, ArbuR 1993, S. 151 (153).

170 
nicht mehr rechtfertigen, daß tendenzlose Arbeitgeber an ein diesbezügliches Diskriminierungsverbot gebunden sind. Hat nicht auch der tendenzlose Arbeitgeber unter Umständen Vorbehalte gegenüber homosexuellen Arbeitnehmern oder Arbeitnehmerinnen? Soll er nicht dennoch grundsätzlich verpflichtet sein, (Einstellungs-)Entscheidungen nicht vom Vorhandensein bzw. Nichtvorhandensein dieses Merkmals abhängig zu machen?

Das $B A G$ hat die in einem ,Nicht-Tendenzbetrieb“ aufgrund der Homosexualität des Arbeitnehmers erfolgte Kündigung in der Probezeit als treuwidrig i.S.d. $§ 242$ BGB eingestuft. ${ }^{223}$ Im Gegensatz dazu und im Widerspruch zu dem hier propagierten Gleichlauf von Tendenzbetrieb und tendenzlosem Unternehmen hat das Gericht die im außerdienstlichen Bereich praktizierte homosexuelle Neigung eines im Dienst des Diakonischen Werkes einer evangelischen Landeskirche stehenden, im Bereich der Konfliktberatung eingesetzten Arbeitnehmers als grundsätzlich geeigneten Kündigungsgrund angesehen, sofern der Arbeitnehmer zuvor erfolglos abgemahnt wurde. $^{224}$ Das $B A G$ hat zudem für zulässig erachtet, einer bei einem katholischen Missionsgymnasium beschäftigten katholischen Lehrerin aus personenbedingten Gründen i.S.d. § 1 II KSchG ordentlich zu kündigen, wenn diese einen geschiedenen Mann heiratet. ${ }^{225}$

Diese tendenzbetriebsfreundliche Rechtsprechung des $B A G$ hält den Anforderungen, die Art. 4 II 2 Richtlinie 2000/78/EG stellt, nicht stand. ${ }^{226}$ Zudem ist sie sachlich in zweifacher Hinsicht nicht haltbar. Zum einen bevorzugt sie, wie bereits erwähnt, Tendenzbetriebe gegenüber NichtTendenzbetrieben in ungerechtfertigter Weise. In diesem Zusammenhang sei noch einmal an den bereits mehrfach erwähnten „Kopftuchfall“ erinnert. Dort hat das BAG entschieden, das Tragen eines - islamischen - Kopftuchs rechtfertige regelmäßig noch nicht die ordentliche Kündigung einer Verkäuferin in einem Kaufhaus aus personen- oder verhaltensbedingten Gründen nach $\S 1$ II KSchG. ${ }^{227}$

Die Rechtsprechung des BAG ist auch und vor allem deshalb kritikwürdig, weil sie die Rechte der „Diskriminierten“ nicht in gehöriger Weise berücksichtigt. In diesem Sinne wird vertreten, im Fall

223 BAG, NZA 1994, S. 1080 (1082); Andere halten eine derartige Kündigung für sittenwidrig i. S. d. § 138 I BGB. (v. Hoyningen-Huene/Linck, Kündigungsschutzgesetz, § 13, Rn. 64); Die Berufungsinstanz hatte demgegenüber die Ansicht vertreten, die wegen dieser abartigen Anlage des Geschlechtstriebes - gemeint war die Homosexualität erfolgte Kündigung sei von einem verständlichen und vertretbaren Motiv getragen gewesen. Es widerspräche keineswegs dem Anstandsgefühl aller Billig- und Gerechtdenkenden, persönlichen und geschäftlichen Umgang mit homosexuellen Personen zu meiden und bestehende Kontakte zu ihnen abzubrechen. (wesentliche Gründe des Urteils des LAG München abgedruckt in NZA 1994, S. 1080 (1080)); In einem ähnlich gelagerten Fall hat das ArbG Lörrach festgestellt, die Weigerung der Aufnahme eines Vorpraktikanten in ein Ausbildungsverhältnis allein aufgrund der homosexuellen Veranlagung des Bewerbers verstoße gegen Grundprinzipien der deutschen Rechtsordnung, wie dem allgemeinen Willkürverbot des Art. 3 I GG, den guten Sitten gem. § 138 BGB sowie dem „ordre public“"gem. Art. 6 EGBGB. (ArbG Lörrach, ArbuR 1993, S. 151 (152)).

224 BAG, AP Nr. 15 zu Art. 140 GG, B1. 1169 (Leitsatz); a. A. v. Hoyningen-Huene/Linck, Kündigungsschutzgesetz, § 1, Rn. 258.

225 BAG NJW 1985, S. 1855 (1855, 1. Leitsatz).

226 A. A. im „Homosexuellenfall“ wohl Thüsing: „Durch Art. 4 II der Richtlinie ist den Religionsgemeinschaften jedoch ein weitgehender Freiraum geschaffen worden, der die Vorgaben des kirchlichen Arbeitgebers auch weiterhin als zulässig erscheinen läßt.“ (Thüsing, NZA 2001, S. 1061 (1062)).

227 BAG, NJW 2003, S. 1685 (1685, Leitsatz). 
des Verbots homosexueller Handlungen im außerdienstlichen Bereich klafften kirchliche und staatliche Ordnung in einer Weise auseinander, die die Nichtanwendung der betreffenden Normen wegen Verstoßes gegen den ordre public (Art. 6 EGBGB) rechtfertigten. ${ }^{228}$ Besonders perfide erscheint in diesem Fall das Erfordernis der Abmahnung, geht doch das Gericht scheinbar davon aus, der Homosexuelle könne von heute auf morgen zur „Normalität“ wechseln.

Gegen den ordre public verstoße auch das Verbot der Wiederverheiratung bzw. der Heirat mit einem Geschiedenen. ${ }^{229}$ In diesen Fällen komme erschwerend hinzu, daß sich ein Staat, der sich dem besonderen Schutz von Ehe und Familie verpflichtet fühlt, widersprüchlich verhielte, wenn er solchen Kündigungen Wirksamkeit verleihen würde. ${ }^{230}$

\section{Pflicht zu tendenzdefensivem Verhalten}

Unzulässig soll in den genannten Fällen aber lediglich die Anknüpfung an den Status der Homosexualität und der Wiederheirat sein. ${ }^{231}$

Im Zusammenhang mit dieser Einschränkung ist auf Art. 4 II 3 Richtlinie 2000/78/EG zu verweisen: Gemäß dieser Bestimmung können die Kirchen und anderen öffentlichen oder privaten Organisationen, sofern die Bestimmungen der Richtlinie 2000/78/EG im übrigen eingehalten werden,

„,...] von den für sie arbeitenden Personen verlangen, daß sie sich loyal und aufrichtig im Sinne des Ethos der Organisation verhalten. ${ }^{\text {،232 }}$

In diesem Sinne, aber beschränkt auf Kirchenmitglieder, hat das BVerfG entschieden, daß die Kirche befugt ist, „den ihr angehörenden Arbeitnehmern die Beachtung jedenfalls der tragenden Grundsätze der kirchlichen Glaubens- und Sittenlehre aufzuerlegen und zu verlangen, daß sie nicht gegen die fundamentalen Verpflichtungen verstoßen, die sich aus der Zugehörigkeit zur Kirche ergeben und die jedem Kirchenglied obliegen. Denn für die Kirchen kann ihre Glaubwürdigkeit davon abhängen, daß ihre Mitglieder, die in ein Arbeitsverhältnis zu ihnen treten, die kirchliche Ordnung - auch in ihrer Lebensführung - respektieren. “233

Es wird in diesem Zusammenhang zwischen tendenzaggressivem und tendenzdefensivem Verhalten unterschieden. Bei tendenzaggressivem Verhalten des Arbeitnehmers beispielsweise in Form 
öffentlichkeitswirksamen Auftretens als homosexuell oder als im Widerspruch zum kirchlichen Eherecht lebend sei eine Kündigung nicht per se unzulässig. ${ }^{234}$

In diesem Sinne hat auch das BAG in zwei Fällen entschieden: Zum einen hat das Gericht eine Kündigung für zulässig erklärt, die aufgrund der Tätigkeit des Arbeitnehmers für eine andere Glaubensgemeinschaft ausgesprochen worden war. $^{235}$ Zum anderen hat es festgestellt, daß eine ordentliche Kündigung aufgrund Verstoßes gegen die Loyalitätspflicht gerechtfertigt sein kann, wenn ein in einem katholischen Krankenhaus beschäftigter Arzt öffentlich für den legalen Schwangerschaftsabbruch Stellung bezieht. ${ }^{236}$

\section{Tendenzschutz außerhalb des Arbeitsrechts}

Die Frage nach möglichem Tendenzschutz kann sich auch außerhalb des ArbeitgeberArbeitnehmer-Verhältnisses stellen.

\section{Tendenz als Anknüpfungspunkt}

Beispielhaft können insofern konfessionell gebundene Studentenwohnheime genannt werden, die vom Wohnungssuchenden eine bestimmte Konfession verlangen. Studenten, die der jeweiligen Religionsgemeinschaft nicht angehören, finden in derartigen Studentenwohnheimen keine Aufnahme. Da diese Einrichtungen den Randbereich kirchlichen Handelns darstellen, wird man an die Rechtmäßigkeit der beschriebenen „Diskriminierung“ die Bedingung knüpfen müssen, daß sie nicht auf das bloße Vermieten von Wohnraum, d. h. auf Gewinnerzielung beschränkt sind, wie von „,normalen“ Vermietern primär bezweckt. Denkbar ist insofern, daß diese Studentenwohnheime besonders preisgünstig sind und somit den Charakter eines Quasi-Stipendiums für deren Bewohner haben, ${ }^{237}$ oder daß in ihnen in einer Weise zusammengelebt wird, die die gemeinsame Pflege kirchlicher Handlungen und Traditionen umfaßt, in ihnen also Verkündigungsarbeit geleistet wird.

Für den Fall, daß ein derartiges Studentenwohnheim die genannten Bedingungen nicht erfüllt, sollte ihm wie jedem Vermieter verwehrt sein, den Abschluß von Mietverträgen von der Konfessionszugehörigkeit des Interessenten abhängig zu machen. Es ist nicht einzusehen, warum der „normale“ Vermieter insofern strengeren Verpflichtungen unterliegen sollte.

234 Geck/Schimmel, ArbuR 1995, S. 177 (183); Es ließe sich fragen, ob es mit der Menschenwürde vereinbar ist, von dem Homosexuellen zu verlangen, daß dieser seine sexuelle Identität in der Öffentlichkeit verheimlicht. Es dürfte indes ein tendenzieller Unterschied zwischen dem Verheimlichenmüssen und dem Verbot tendenzaggressiven Verhaltens bestehen.

$235 B A G$, NZA 2001, S. 1136 (1141).

236 BAG, NJW 1984, S. 826 (826, Leitsätze 2 und 4); bestätigt durch BVerfG, NJW 1986, S. 367 (369); Von Hoyningen-Huene/Linck halten diese Rechtsprechung für bedenklich. ( $v$. Hoyningen-Huene/Linck, Kündigungsschutzgesetz, § 1, Rn. 258).

237 An anderer Stelle wurde die Feststellung getroffen, daß im Rahmen unentgeltlicher Zuwendungen das Diskriminierungsverbot keine Geltung beansprucht. (vgl. Teil 2, Prüfungspunkt D. IV.). 
Beispielhaft, obwohl nicht die Vermietung von Wohnraum betreffend, können in diesem Zusammenhang Entscheidungen des BGH angeführt werden, die sich mit der Rechtmäßigkeit der Kündigung von Parteikonten beschäftigen. ${ }^{238} \mathrm{Zu}$ diesen Fällen ist zunächst anzumerken, daß es sich bei den in Rede stehenden Banken nicht um Privatbanken handelte, vielmehr auf der einen Seite des Vertragsverhältnisses die öffentliche Verwaltung stand, die sich zur Erfüllung öffentlicher Aufgaben in Form des Privatrechts betätigte (sog. Verwaltungsprivatrecht). ${ }^{239}$ Der $B G H$ hat in besagten Fällen einen Verstoß gegen das in Art. 3 I GG enthaltene Willkürverbot bejaht. Es sei sachlich nicht gerechtfertigt, die politische Zielrichtung einer Partei zum Anlaß für eine Kontokündigung $\mathrm{zu}$ nehmen, solange das BVerfG die Verfassungswidrigkeit der Partei nicht festgestellt habe. ${ }^{240}$

Die Ansicht des Gerichts läßt sich auf Privatbanken übertragen. Auch ihnen müssen derartig begründete Kündigungen zumindest dann verboten sein, wenn ihnen aus einer derartigen Geschäftsbeziehung keine wirtschaftlichen Nachteile erwachsen. ${ }^{241}$

\section{Sonstiges Merkmal als Anknüpfungspunkt}

Beispiel: Weigerung einer katholischen Kirchengemeinde, ihren Gemeindesaal zwecks Feier einer im Sinne des Lebenspartnerschaftsgesetzes ${ }^{242}$ begründeten Lebensgemeinschaft zur Verfügung zu stellen ${ }^{243}$

In dem Beispielsfall weigerte sich die Kirchengemeinde nach Vertragsschluß, die Räumlichkeiten zu überlassen, nachdem sie erfahren hatte, daß es sich um eine „Hochzeitsfeier“ zwischen zwei Männern handelte. Das zuständige Gericht hat im Sinne der bisher angestellten Überlegungen die Möglichkeit der Anfechtung des Mietvertrages seitens der Kirchengemeinde verneint und selbige zu Schadensersatz verurteilt. ${ }^{244}$

Anzumerken ist in diesem Zusammenhang, daß ein Tendenzbetrieb von seinen Mietern nicht in dem Maße wie von seinen Arbeitnehmern tendenzdefensives Verhalten verlangen kann, da Mietverträge im Gegensatz zu Arbeitsverträgen den Bereich der Privatsphäre berühren, in welchem dem einzelnen grundsätzlich ein größerer Raum für die Entfaltung seiner Persönlichkeit zusteht als im Bereich der Sozialsphäre.

\section{Tendenzschutz im ,Nicht-Tendenzbetrieb“}

238 BGH, NJW 2003, S. 1658 f. (die „NPD“ betreffend); NJW 2004, 1031 f. (die „Republikaner“ betreffend).

239 Heinrichs in Palandt, $\S 242, \mathrm{Rn} .12$.

240 BGH, NJW 2003, S. 1658 (1658, Leitsätze 2 und 3); NJW 2004, S. 1031 (1031, Leitsätze 2 und 3).

241 Darauf weist auch der $B G H$ hin. (BGH, NJW 2004, S. 1031 (1032)).

242 Vom 16.02.2001 (BGBl. I, S. 266 ff.).

243 AG Neuss, NJW 2003, S. 3785 ff.

244 Das Gericht wies aber auch darauf hin, daß sich die Kirchengemeinde grundsätzlich ihre Vertragspartner frei aussuchen dürfe, also keinem Kontrahierungszwang - zumindest nicht in einem Fall, wie dem vorliegenden unterliege. (AG Neuss, NJW 2003, S. 3785 (3787)). 
Es hat sich gezeigt, daß ein Tendenzbetrieb „diskriminierende“ Entscheidungen oft mit seiner Eigenschaft als Betrieb, der eine bestimmte Tendenz vertritt, sachlich begründen kann.

Aus dieser Tatsache ließe sich der Umkehrschluß ziehen, der Nicht-Tendenzbetrieb könne „diskriminierendes“ Verhalten niemals durch Verweis auf zwingende Vorgaben der eigenen Religion oder Weltanschauung rechtfertigen. Dem ist aber nicht so. Die Religions- und Weltanschauungsfreiheit gestattet dem einzelnen unter bestimmten Voraussetzungen ein solches Verhalten.

Beispiele: 1. „Der Vermieter eines für eine Schlachterei geeigneten Ladenlokals lehnt es ab, das Lokal an einen Schlachtermeister zu vermieten, der auf Wunsch religiös motivierter Kunden Tiere ohne Betäubung tötet. Soll der Vermieter nunmehr gezwungen sein, das Lokal an diesen Schlachtermeister zu vermieten, weil das BVerfG das Schächten dem Schutzbereich des Art. 4 GG zuordnet?‘ ${ }^{245}$ 2. Der islamische Metzger lehnt es ab, Kundinnen ohne Kopftuch zu bedienen. ${ }^{246} 3$. „Eine Religionsgemeinschaft hält Homosexualität mit ihren religiösen Überzeugungen für unvereinbar und erwartet von ihren Mitgliedern „Distanz gegenüber Schwulen und Lesben“. Ein Mitglied dieser Religionsgemeinschaft lehnt es deshalb ab, einem homosexuellen Paar eine freie Wohnung zu vermieten, obgleich es den höchsten Mietzins bietet. “247 4 . „Eine von der Waldorfpädagogik überzeugte Familie sucht per Anzeige für ihre auf einer Waldorfschule lernenden Kinder einen Nachhilfelehrer für die Fächer Mathematik und Englisch, der Anhänger der Anthroposophie Rudolf Steiners ist. Soll dies in Zukunft unzulässig sein?‘248

$\mathrm{Zu}$ dem zweiten Beispielsfalls ist angemerkt worden, daß ein Verbot, Kundinnen ohne Kopftuch zu benachteiligen, nur dann den Schutzbereich des Art. 4 I GG betreffen würde, „wenn sich der Metzger auf einen Glaubenssatz berufen könnte, der es ihm verbietet, Fleisch an Frauen zu verkaufen, die kein Kopftuch tragen. [...] Er müßte ernsthaft darlegen können, daß das Betreiben einer Metzgerei nicht nur die Einhaltung bestimmter Regeln bei der Schlachtung der Tiere, sondern auch eine bestimmte Auswahl der Kundschaft erfordert.“249

Nicht vergessen werden darf, daß selbst dann, wenn dieser Nachweis erbracht wird, die Religionsfreiheit begrenzt ist, etwa durch Grundrechte Dritter. Vor diesem Hintergrund ist äußerst fraglich, ob in Deutschland unter Berufung auf die Religionsfreiheit die Möglichkeit besteht, Frauen ohne Kopftuch die Bedienung in einem Geschäft zu verweigern. Das gleiche gilt für die Ablehnung des homosexuellen Paars im dritten Beispielsfall, und zwar auch dann, wenn der „Diskriminierende“ den Nachweis erbringt, daß die Religionsgemeinschaft, deren Mitglied er ist, von ihren Mitgliedern Distanz gegenüber Schwulen und Lesben erwartet.

\footnotetext{
245 Fragt Säcker, ZRP 2002, S. 286 (288).

246 Beispielsfall aus der AGG-Entwurfsbegründung, BT-Druck. 16/1780, S. 45.

47 Säcker, ZRP 2002, S. 286 (289).

248 Fragt Säcker, ZRP 2002, S. 286 (288).

249 AGG-Entwurfsbegründung, BT-Druck. 16/1780, S. 45.
} 
Die Ablehnung des Schlachters dürfte dagegen von der Religions- bzw. Weltanschauungsfreiheit des Vermieters gedeckt sein. Noch eindeutiger fällt die Entscheidung im vierten Beispielsfall aus. Hier hat die Weltanschauungsfreiheit des Bewerbers Einfluß auf das in Rede stehende Arbeitsverhältnis, konkret auf die Art und Weise, wie dieser seinen Unterricht gestaltet. Die Ablehnung eines Bewerbers, der die genannte Voraussetzung nicht erfüllt, muß demnach angesichts der Weltanschauungsfreiheit der Anbietenden gerechtfertigt sein. Um der Gefahr zu entgehen, daß die Berufung auf die eigene Religions- bzw. Weltanschauungsfreiheit bloßer Vorwand für die Ausgrenzung des Andersdenkenden ist, kann auch in diesen Fällen - wie in jedem - von den „Diskriminierenden“ verlangt werden, daß sie ernsthaft darlegen, daß ihnen der eigene Glaube bzw. die eigene Weltanschauung die Ausgrenzung gebieten.

\section{Zusammenfassung}

Die im Rahmen des Prüfungspunktes „Tendenzschutz“ gewonnenen Erkenntnisse lassen sich wie folgt zusammenfassen: Auch sogenannte Tendenzbetriebe - etwa Kirchen und Weltanschauungsgemeinschaften - sind grundsätzlich an das Diskriminierungsverbot gebunden. Die Überprüfung diskriminierungsrelevanter Entscheidungen von Tendenzbetrieben hat jedoch dem Selbstverständnis dieser Betriebe ein besonderes Gewicht beizumessen.

So kann der Tendenzbetrieb im Kernbereich seiner Tätigkeit von seinen Beschäftigten Tendenzzugehörigkeit verlangen - vom Pfarrer etwa, daß er Mitglied der Glaubensgemeinschaft ist, deren Werte er predigen soll. Im Randbereich seiner Tätigkeit ist dem Tendenzbetrieb dies nur dann gestattet, wenn der Arbeitnehmer Tendenzträger ist, also unmittelbar an der geistig-ideellen Zielsetzung des Betriebes - der Tendenz - mitwirkt. Das gilt etwa für Betreuerinnen eines konfessionellen Kindergartens dann, wenn bereits sie den Kindern die Werte der Kirche vermitteln sollen. Ansonsten ist der Tendenzbetrieb wie der „Nicht-Tendenzbetrieb“ zu behandeln. Gestattete man ihm, auch von Nicht-Tendenzträgern Tendenzzugehörigkeit zu verlangen, privilegierte man ihn gegenüber dem „Nicht-Tendenzbetrieb“ in einer nicht zu rechtfertigenden Weise.

Im Kernbereich seiner Tätigkeit ist es dem Tendenzbetrieb gestattet, auch aufgrund eines anderen Merkmals als der Tendenz zu unterscheiden, soweit dies nachgewiesenermaßen seinem Selbstverständnis entspricht. So ist etwa der Ausschluß weiblicher Bewerber für das Priesteramt in der katholischen Kirche als zulässig zu betrachten. Im Randbereich seiner Tätigkeit ist der Tendenzbetrieb wie der „Nicht-Tendenzbetrieb“ zu behandeln; und zwar unabhängig davon, ob der Arbeitnehmer Tendenzträger ist oder nicht.

In jedem Fall kann der Tendenzbetrieb von seinen Arbeitnehmern tendenzdefensives Verhalten verlangen. Tendenzaggressiv verhält sich etwa der in einem katholischen Krankenhaus beschäftigte Arzt, der öffentlich für den legalen Schwangerschaftsabbruch eintritt. 
Die Frage nach möglichem Tendenzschutz kann sich auch außerhalb des ArbeitgeberArbeitnehmer-Verhältnisses stellen - etwa bei konfessionell gebundenen Studentenwohnheimen, die vom Wohnungssuchenden eine bestimmte Religionszugehörigkeit verlangen. Die für das Arbeitsrecht entwickelten Abwägungskriterien gelten auch hier.

Schließlich ist der Tendenzschutz nicht auf den Tendenzbetrieb beschränkt. Auch diskriminierungsrelevante Entscheidungen des Nichttendenzbetriebes lassen sich im Einzelfall mit dessen Religions- bzw. Weltanschauungsfreiheit rechtfertigen. So ist es Eltern etwa gestattet, von dem Nachhilfelehrer zu verlangen, daß er Anhänger der Weltanschauung ist, die er den Kindern vermitteln soll bzw. die Voraussetzung für die von ihnen gewünschte Unterrichtsgestaltung ist.

\section{N. Unmöglichkeit der Vertragsdurchführung}

Der potenzielle Vertragspartner wird die Ablehnung eines Behinderten oft damit begründen, daß ihm die Vertragsdurchführung aufgrund der Behinderung unmöglich ist. Zu denken ist etwa daran, daß der Arbeitgeber den Rollstuhlfahrer mit der Begründung nicht einstellt, ein behindertengerechter Zugang zum Arbeitsplatz sei nicht vorhanden. ${ }^{250}$

\section{Ermöglichungspflicht}

Die sachliche Rechtfertigung der Ablehnung ließe sich in einem solchen Fall nur dann verneinen, wenn der Arbeitgeber verpflichtet wäre, einen behindertengerechten Zugang zum Arbeitsplatz zu schaffen.

\section{Art. 5 Richtlinie 2000/78/EG}

Gemäß Art. 5 Sätze 2 und 3 Richtlinie 2000/78/EG muß

„,...] der Arbeitgeber die geeigneten und im konkreten Fall erforderlichen Maßnahmen [ergreifen], um den Menschen mit Behinderung den Zugang zur Beschäftigung, die Ausübung eines Berufes, den beruflichen Aufstieg und die Teilnahme an Aus- und Weiterbildungsmaßnahmen zu ermöglichen, es sei denn, diese Maßnahmen würden den Arbeitgeber unverhältnismäßig belasten. Diese Belastung ist nicht unverhältnismäßig, wenn sie durch geltende Maßnahmen im Rahmen der Behindertenpolitik des Mitgliedstaates ausreichend kompensiert wird.“

Richtlinie 2000/78/EG fordert in Art. 5 die Gleichstellung behinderter Menschen und geht damit über das bloße Gleichbehandlungsgebot hinaus. ${ }^{251}$ Das ist interessengerecht, vergegenwärtigt man 
sich die besondere Situation behinderter Menschen, die dazu führt, daß sie in den meisten Fällen faktisch benachteiligt sind, sollte sich ihr Gleichbehandlungsanspruch darauf beschränken, formal wie Nicht-Behinderte behandelt zu werden. ${ }^{252}$

\section{2. §81 IV SGB IX}

Bereits das Schwerbehindertengesetz (SchwbG) enthielt in seinem $\S 14$ eine dem Art. 5 Richtlinie 2000/78/EG vergleichbare Regelung. Eine dem mittlerweile aufgehobenen $\S 14 \mathrm{SchwbG}^{253}$ in materieller Hinsicht entsprechende Norm enthält nunmehr § 81 IV 1 SGB IX. Er gewährt den schwerbehinderten Arbeitnehmern gegenüber ihren Arbeitgebern einen Anspruch auf

„1. Beschäftigung, bei der sie ihre Fähigkeiten und Kenntnisse möglichst voll verwerten und weiterentwickeln können,

2. bevorzugte Berücksichtigung bei innerbetrieblichen Maßnahmen der beruflichen Bildung zur Förderung ihres beruflichen Fortkommens,

3. Erleichterungen im zumutbaren Umfang zur Teilnahme an außerbetrieblichen Maßnahmen der beruflichen Bildung,

4. behindertengerechte Einrichtung und Unterhaltung der Arbeitsstätten einschließlich der Betriebsanlagen, Maschinen und Geräte sowie der Gestaltung der Arbeitsplätze, des Arbeitsumfeldes, der Arbeitorganisation und der Arbeitszeit, unter besonderer Berücksichtigung der Unfallgefahr,

5. Ausstattung ihres Arbeitsplatzes mit den erforderlichen technischen Arbeitshilfen unter Berücksichtigung der Behinderung und ihrer Auswirkungen auf die Beschäftigung.“

Es wird vorgeschlagen, analog zu den $\S \S 71 \mathrm{ff}$. SGB IX den Schutz Behinderter etwa im Mietrecht und im Bildungsrecht zu regeln. Wer 20 Wohnungen oder mehr vermiete, müsse davon mindestens $5 \%$ behindertengerecht ausbauen und anbieten. ${ }^{254}$

Verwiesen sei insofern auch auf einen Entwurf für ein Landesgleichstellungsgesetz der hessischen Grünen, nach dem sowohl für private als auch öffentliche Bauten gelten soll, „daß in Gebäuden mit mehr als drei Wohnungen alle Wohnungen erreichbar sein müssen und eine davon voll für Behinderte nutzbar. Fernsehanstalten sollen Untertitel oder Gebärdendolmetscher einblenden. Eltern erhielten für ihre Kinder ein „tatsächliches“ Wahlrecht zwischen Sonder- oder Realschule. Ferner fordern die Grünen den 1. Juli 2005 als Stichtag, von dem an Verkehrsgesellschaften nur noch behindertentaugliche Fahrzeuge anschaffen dürfen. “255

Fraglich ist, wann ein Arbeitsplatz behindertengerecht eingerichtet ist. Im Zusammenhang mit dieser Frage wird darauf hingewiesen, daß die Verpflichtung zur Schaffung behindertengerechter

\footnotetext{
252 So auch Wiedemann/Thüsing, Der Betrieb 2002, S. 463 (468).

253 Steck in Kossens/von der Heide/Maaß, § 81, Rn. 1.

254 Säcker, ZRP 2002, S. 286 (290).

255 Von Schwanenflug in Frankfurter Rundschau vom 09.01.2004, S. 24 unter dem Titel: „Initiative für Behinderte“. 178
} 
Arbeitsplätze wirkungslos sei, wenn der Behinderte keinen Anspruch auf Einstellung hat - die Beschäftigungspflicht des Arbeitgebers aus $\S 71$ SGB IX besteht lediglich im Verhältnis zum Staat $^{256}$-, ,denn die Behinderungen sind zu verschieden, als daß ohne Ansehen des einzelnen behinderten Arbeitnehmers gesagt werden könnte, ein Arbeitsplatz ist behindertengerecht oder nicht. ‘257 $\mathrm{Ob}$ ein Arbeitsplatz behindertengerecht eingerichtet ist oder nicht, hängt demnach vom jeweiligen Bewerber und dessen konkreter Behinderung ab.

\section{3. $\$ 4$ I Nr. 2 a Gaststättengesetz}

Auch außerhalb des Arbeitsrechts bestehen Regelungen im Sinne von Art. 5 Richtlinie 2000/78/EG und $\S 81$ IV SGB IX. Gemäß $§ 4$ I Nr.2 a Gaststättengesetz (GaststättenG) ist die für das Betreiben des Gaststättengewerbes gem. § 2 I 1 GaststättenG erforderliche Erlaubnis zu versagen, wenn

„die zum Betrieb des Gewerbes für Gäste bestimmten Räume von behinderten Menschen nicht barrierefrei genutzt werden können, soweit diese Räume in einem Gebäude liegen, für das nach dem 1. November 2002 eine Baugenehmigung für die erstmalige Errichtung, für einen wesentlichen Umbau oder eine wesentliche Erweiterung erteilt wurde oder das, für den Fall, daß eine Baugenehmigung nicht erforderlich ist, nach dem 1. Mai 2002 fertig gestellt oder wesentlich umgebaut oder erweitert wurde“. ${ }^{258}$

Das Merkmal „barrierefrei“ wird in $\S 4$ des Gesetzes zur Gleichstellung behinderter Menschen (Behindertengleichstellungsgesetz $-B G G)^{259}$ vom 27.04.2002 näher definiert. Gemäß dieser Norm sind barrierefrei

„[...] bauliche und sonstige Anlagen, Verkehrsmittel, technische Gebrauchsgegenstände, Systeme der Informationsverarbeitung, akustische und visuelle Informationsquellen und Kommunikationseinrichtungen sowie andere gestaltete Lebensbereiche, wenn sie für behinderte Menschen in der allgemein üblichen Weise, ohne besondere Erschwernis und grundsätzlich ohne fremde Hilfe zugänglich und nutzbar sind.“

\section{Grenzen der Ermöglichungspflicht}

Die Ansprüche des $\S 81$ IV 1 SGB IX bestehen gem. $§ 81$ IV 3 SGB IX nur soweit, als sie für den Arbeitgeber zumutbar sind. In diesem Sinne macht auch die Richtlinie 2000/78/EG in Art. 5 eine Einschränkung bei unverhältnismäßiger Belastung des Arbeitgebers.

256 Kossens in Kossens/von der Heide/Maaß, § 71, Rn. 3; Neumann in Neumann/Pahlen/Majerski-Pahlen, § 71, Rn. 3.

257 Thüsing, ZfA 2001, S. 397 (405).

258 § 4 I Nr. 2 a GaststättenG wurde durch das Gesetz zur Gleichstellung behinderter Menschen und zur Änderung anderer Gesetze vom 27.04.2002 ins GaststättenG eingefügt. (BGB1. 2002, I 1, S. 1467 ff.).

259 BGBl. 2002, I 1, S. 1468 ff. 
An der Regelung des Art. 5 Richtlinie 2000/78/EG wird bemängelt, sie lasse völlig ungeklärt, wie weit die Pflicht des Arbeitgebers im einzelnen reicht. ${ }^{260}$

Nach dem American with Disabilities Act sollen diesbezüglich neben den Kosten der Maßnahme, der Größe und Finanzkraft des Unternehmens, dem Grad der Behinderung sowie dessen Einfluß auf andere Arbeitnehmer auch etwaige Finanzierungsmöglichkeiten von dritter Seite oder steuerliche Vergünstigungen eine entscheidende Rolle spielen. ${ }^{261}$

Die deutsche Rechtslage gestaltet sich ähnlich: Die Zumutbarkeit richtet sich auch hier nach der finanziellen Leistungsfähigkeit des Arbeitgebers, wobei insofern zu beachten ist, daß der Arbeitgeber gem. § 102 I Nr. 3 SGB IX finanzielle Unterstützung durch die Integrationsämter in Anspruch nehmen kann. ${ }^{262}$

Für den Arbeitgeber stellen die genannten Abwägungskriterien eine nur „schwer wägbare Mischung، ${ }^{6263}$ dar. Eine für ihn günstigere Rechtslage schafft insofern das irische Recht: Nach dem Employment Equality Act ist der Arbeitgeber nur zu solchen Anpassungen verpflichtet, die ihn nichts kosten. $^{264}$

Ein völlig anderes Konzept verfolgt das französische Recht, das den Arbeitgeber „lediglich“ zur formalen Gleichbehandlung verpflichtet. ${ }^{265}$ Auf der anderen Seite gewährt es Quotenschutz, indem der Code $d u$ Travail den Arbeitgeber verpflichtet, in bestimmtem Umfang Behinderte einzustellen. ${ }^{266}$ Der Vorteil dieses Modells ist, daß es auf vage Abgrenzungen im Rahmen der Zumutbarkeit verzichtet. ${ }^{267}$

Gleichwohl sollte vor der Einführung einer Quotenregelung stets geprüft werden, ob sich die gewünschte Förderung eines bestimmten Personenkreises nicht auf andere Weise realisieren läßt, stellt doch die Quotenregelung stets den größeren Eingriff in die ebenfalls schutzwürdigen Interessen des nicht geförderten Personenkreises dar. Fördermaßnahmen, die keine Quotenregelungen darstellen, könnten letztere zudem überflüssig machen. So dürfte beispielsweise die Behauptung zulässig sein, ein Arbeitgeber, der aufgrund gesetzlicher Verpflichtung in einen behindertengerechten Eingang investiert hat, werde künftig in der Einstellungssituation keine bzw. geringere Ressentiments gegenüber Rollstuhlfahrern haben.

260 Thüsing, ZfA 2001, S. 397 (403); Auch die Rechtsprechung des EuGH ist insofern wage. Nach ihr soll einer behinderten Person beispielsweise dann gekündigt werden können, wenn sie zur Erfüllung der wesentlichen Funktionen ihres Arbeitsplatzes nicht kompetent, fähig oder verfügbar ist. (FAZ vom 12.07.06, S. 12, unter dem Titel: „Diskriminierungsverbot schützt Kranke nicht vor Kündigungen“).

261 Thüsing, ZfA 2001, S. 397 (403).

262 Steck in Kossens/von der Heide/Maaß, § 81, Rn. 34, § 102, Rn. 11; Auch EU-Gelder fließen zu diesem Zweck. (Roßbach in FAZ vom 28.06.05, S. 8, unter dem Titel: „Jeder macht soviel, wie er kann“).

263 So Thüsing, ZfA 2001, S. 397 (403).

264 Thüsing, ZfA 2001, S. 397 (404).

265 Thüsing, ZfA 2001, S. 397 (404).

266 Thüsing, ZfA 2001, S. 397 (404).

267 Thüsing, ZfA 2001, S. 397 (404). 


\section{Zusammenfassung}

Die angestellten Überlegungen zur „Unmöglichkeit der Vertragsdurchführung“ lassen sich wie folgt zusammenfassen: Lehnt der potenzielle Vertragspartner den Träger eines bestimmten Merkmals vor allem des Merkmals „Behinderung“ - mit der Begründung ab, ihm sei die Vertragsdurchführung aufgrund des Merkmals unmöglich - beispielsweise deshalb, weil kein behindertengerechter Zugang zum Arbeitsplatz vorhanden ist -, so hängt die Beantwortung der Frage der Rechtmäßig- bzw. Rechtswidrigkeit der Ablehnung davon ab, ob der „Diskriminierende“ verpflichtet war, dem „Diskriminierten“ die Vertragsdurchführung zu ermöglichen - etwa einen behindertengerechten Zugang zu schaffen.

Das geltende Recht kennt derartige Ermöglichungspflichten etwa im Arbeitsrecht. Die Interessen des Verpflichteten werden dadurch gewahrt, daß ihm die Ermöglichungspflicht - vor allem in finanzieller Hinsicht - zumutbar sein muß.

Daß der „Diskriminierende“ in bestimmten Fällen verpflichtet sein soll, (vor allem) Behinderten die Vertragsdurchführung möglich zu machen - etwa durch die Schaffung eines behindertengerechten Zugangs -, ist interessengerecht, vergegenwärtigt man sich die besondere Situation behinderter Menschen, die dazu führt, daß sie in den meisten Fällen faktisch benachteiligt sind, sollte sich ihr Gleichbehandlungsanspruch darauf beschränken, formal wie Nicht-Behinderte behandelt zu werden.

\section{O. Vertragsanpassungspflicht}

In engem Zusammenhang mit der soeben getroffenen Feststellung steht die Frage, ob der „Diskriminierende“ im Einzelfall Vertragsbedingungen einem bestimmten Merkmal anpassen muß.

\section{Diskriminierungsschutz nicht auf formale Gleichbehandlung beschränkt}

In diesem Zusammenhang ist einmal mehr darauf hinzuweisen, daß der bloße Anspruch auf formale Gleichbehandlung nicht in jedem Fall geeignet ist, dem Gleichbehandlungsgrundsatz in adäquater Weise Wirksamkeit zu verleihen. So werde beispielsweise Diskriminierung aufgrund der religiösen Überzeugung in den meisten Fällen in Form einer Gleichbehandlung auftreten, die sich ungleich auswirkt. ${ }^{268}$ Der Muslim, der fünfmal am Tag seine Gebete verrichten und damit, wenn auch nur kurz, seine Arbeit unterbrechen muß, sei durch feste Arbeitszeiten anders betroffen als derjenige, 
dem die Pflicht zum Gebet nicht obliegt. ${ }^{269}$ Ebenso sei der jüdische Arbeitnehmer durch Einteilung zur Arbeit an einem Sonnabend in anderem Maße betroffen als sein nichtjüdischer Kollege. ${ }^{270}$

Vertragsanpassungspflichten sind vor diesem Hintergrund ein wichtiger Baustein für die Ermöglichung adäquater Teilhabe am gesellschaftlichen Leben. ${ }^{271}$ Der ADG-Entwurf des Bundesjustizministeriums $^{272}$ vom 10.12.2001 ist sich dessen bewußt. Gemäß seines $\S 319 \mathrm{~d}$ I 2 ist eine Unterscheidung nur dann durch einen sachlichen Grund gerechtfertigt,

„[...] wenn sie sich auch durch eine zumutbare Anpassung des Vertrages oder seiner Durchführung nicht vermeiden läßt.“

\section{Vertragsanpassungspflichten de lege lata}

Bereits das geltende Recht Deutschlands kennt das Institut der Vertragsanpassungspflicht.

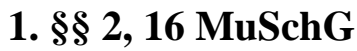

So hat beispielsweise der Arbeitgeber gem. § 2 MuSchG Arbeitsplätze den Bedürfnissen der werdenden oder stillenden Mutter anzupassen. Gemäß $\S 16 \mathrm{MuSchG}$ hat er die Arbeitnehmerin für Untersuchungen freizustellen, die im Rahmen der Schwanger- und Mutterschaft erforderlich sind. Im Zusammenhang mit diesen Bestimmungen des MuSchG ist auf $\S 8$ II AGG hinzuweisen, der bestimmt, daß

„[d]ie Vereinbarung einer geringeren Vergütung für gleiche oder gleichwertige Arbeit wegen eines in $\S 1$ genannten Grundes [ - d. h. wegen eines dort genannten Diskriminierungsmerkmals - ...] nicht dadurch gerechtfertigt [wird], daß wegen eines in $\S 1$ genannten Grundes besondere Schutzvorschriften gelten. “273

\section{2. $\$ 554$ a BGB}

Der Mieter kann vom Vermieter gem. § 554 a I BGB

269 Thüsing, NZA 2001, S. 1061 (1062).

270 Thüsing, NZA 2001, S. 1061 (1062); weitere Beispiele bei Thüsing, ZfA 2001, S. 397 (406).

271 Thüsing weist darauf hin, daß die europäischen Vorgaben allein ein Verbot der Unterscheidung, nicht aber ein Gebot der Unterscheidung statuieren, was bei einzelnen Unterscheidungsmerkmalen aber notwendig sei. Das deutsche Recht kenne demgegenüber Rücksichtnahmepflichten, aus denen sich ein Gebot zur Unterscheidung ergebe. (Thüsing, NZA 2001, S. 1061 (1062)).

272 Http://www.lsvd.de/antidiskriminierung/adgzivil02.html.

273 Schutzvorschriften im Sinne des - durch § 8 II AGG ersetzten (Entwurfsbegründung, BT-Druck. 16/1780, S. 58) -

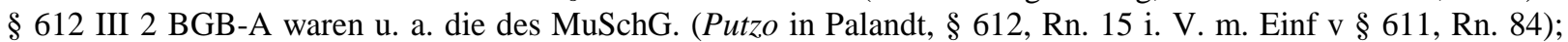
vgl. auch $\S 16$ Satz 3 MuSchG. 
„die Zustimmung zu baulichen Veränderungen oder sonstigen Einrichtungen verlangen, die für eine behindertengerechte Nutzung der Mietsache oder den Zugang zu ihr erforderlich sind, wenn er ein berechtigtes Interesse daran hat. Der Vermieter kann seine Zustimmung verweigern, wenn sein Interesse an der unveränderten Erhaltung der Mietsache oder des Gebäudes das Interesse des Mieters an einer behindertengerechten Nutzung der Mietsache überwiegt. Dabei sind auch die berechtigten Interessen anderer Mieter in dem Gebäude zu berücksichtigen.“

Die Sätze 2 und 3 verdeutlichen, daß auch im Rahmen des $§ 554$ a BGB eine Interessenabwägung durchgeführt werden muß.

\section{3. §§ 242 BGB, 106 GewO}

Auch deutsche Gerichte haben sich immer wieder mit der Frage des Bestehens und der Reichweite von Vertragsanpassungs- bzw. Rücksichtnahmepflichten beschäftigt. So wurde beispielsweise entschieden, es sei grob unbillig, ein bestehendes, zulässiges Hundehaltungsverbot gegenüber einem behinderten Wohnungseigentümer durchzusetzen, selbst wenn das Tier „lediglich“ dem Zweck diene, den durch die körperliche Behinderung bedingten angeschlagenen seelischen Zustand zu stabilisieren. ${ }^{274}$ Die Durchsetzung des Verbots verstoße gegen Treu und Glauben i.S.d. § 242 BGB, dessen Inhalt durch Art. 3 III 2 GG beeinflußt sei, was dazu führe, daß gegenüber behinderten Menschen ein höheres Maß an Rücksichtnahme und Toleranz geboten sei. ${ }^{275}$

Auch wurde entschieden, das Weisungsrecht des Arbeitgebers, das gem. § 106 GewO nach billigem Ermessen auszuüben ist, könne durch das Grundrecht des Arbeitnehmers auf Gewissensfreiheit i.S.d. Art. 4 I GG eingeschränkt sein. ${ }^{276}$ Im Rahmen der Abwägung der Interessenlage beider Vertragsparteien sei vor allem zu berücksichtigen, ob der Arbeitnehmer schon bei Vertragsschluß damit rechnen mußte, daß ihm eine gegen sein Gewissen verstoßende Tätigkeit zugewiesen werden könnte, ob der Arbeitgeber aus betrieblichen Erfordernissen darauf bestehen muß, daß gerade der sich auf den Gewissenskonflikt berufende Arbeitnehmer die in Rede stehende Tätigkeit ausführt, und ob der Arbeitgeber in der Lage ist, dem Arbeitnehmer einen freien Arbeitsplatz anzubieten, an dem der Gewissenskonflikt nicht auftritt. ${ }^{277}$

Der Behinderte hatte sich seinen Hund in dem Beispielsfall während eines bereits bestehenden Vertragsverhältnisses angeschafft. Seine Interessen wären ebenso schutzwürdig gewesen, wenn er bereits vor Eingehung des Vertragsverhältnisses Hundehalter gewesen wäre. In einem derartigen

274 BayObLG, NJW-RR 2002, S. 226 (226 f.).

275 BayObLG, NJW-RR 2002, S. 226 (226 f.); Im Zusammenhang mit dem Mietrecht sei an dieser Stelle auch auf die „Parabolantennen-Problematik“ hingewiesen. (vgl. dazu überblicksmäßig Maaß/Hitpaß, NZM 2003, S. 181 ff.).

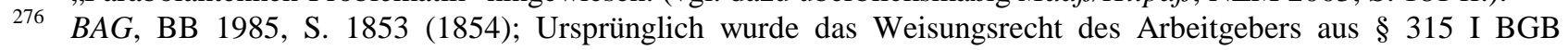
abgeleitet.

$277 B A G$, BB 1985, S. 1853 (1854). 
Fall erscheint es nicht unbillig, den Vertragspartner zu einer zumutbaren Modifikation der grundsätzlich zulässigen Vertragsbedingung „Hundehaltungsverbot“ zu verpflichten.

\section{Zusammenfassung}

Vor dem Hintergrund der Erkenntnis, daß der bloße Anspruch auf formale Gleichbehandlung nicht in jedem Fall geeignet ist, dem Gleichbehandlungsgrundsatz in adäquater Weise Wirksamkeit zu verleihen, wurde festgestellt, daß es zur Ermöglichung adäquater Teilhabe am gesellschaftlichen Leben notwendig sein kann, den „Diskriminierenden“ zu verpflichten, Vertragsbedingungen einem bestimmten Merkmal anzupassen.

Es wurde nachgewiesen, daß dem deutschen Recht das Institut der Vertragsanpassungspflicht nicht unbekannt ist; daß der Arbeitgeber beispielsweise verpflichtet sein soll, in zumutbarem Umfang durch betriebliche Organisationsmaßnahmen die Religionsausübung durch den Arbeitnehmer zu gewährleisten. 
Teil 4: Gesetzestechnische Umsetzung des Diskriminierungsverbotes

Nachdem auf seine Auswirkungen auf das rechts- bzw. quasirechtsgeschäftliche Handeln eingegangen wurde, soll abschließend der Frage nachgegangen werden, wie sich das Diskriminierungsverbot gesetzestechnisch umsetzen läßt.

\section{A. Notwendigkeit legislativer Maßnahmen}

Angesichts der Fülle bereits bestehender Antidiskriminierungsmaßnahmen auf nationaler wie internationaler Ebene stellt sich die Vorfrage, ob in Deutschland legislativer Handlungsbedarf überhaupt besteht. Für die Beantwortung dieser Frage ist zu klären, welche Auswirkungen die angesprochenen (inter-)nationalen Antidiskriminierungsmaßnahmen auf das Privatrechtsverhältnis haben.

Die Tatsache, daß das AGG am 18.08.2006 in Kraft getreten ist, soll dabei an dieser Stelle unberücksichtigt bleiben, im Rahmen dieses Prüfungspunktes mithin die Situation betrachtet werden, die sich vor dessen Inkrafttreten bot. Diese Vorgehensweise dient dem Zweck zu ergründen, ob das AGG Ergebnis eines legislativen Handlungsbedarfs ist, dessen Inkrafttreten insofern also bedeutsam war.

\section{Auswirkungen der Antidiskriminierungsmaßnahmen auf das Privatrechtsverhältnis}

Während teilweise, wie vor Inkrafttreten des AGG im Fall des $§ 611$ a BGB-A, unproblematisch eine unmittelbare Bindung Privater an die jeweilige Maßnahme bzw. Norm konstatiert werden kann, fällt die Beantwortung in anderen Fällen schwer bzw. bedarf selbige näherer Ausführungen.

\section{Innerstaatliche Geltung der internationalen Antidiskriminierungsmaßnahmen}

In diesem Zusammenhang ist zunächst zu prüfen, ob und wie die angesprochenen internationalen Antidiskriminierungsmaßnahmen im innerstaatlichen Recht Deutschlands Geltung erlangt haben.

\section{a) Völkerrechtliche Verträge}

Die internationalen Antidiskriminierungsmaßnahmen sind von ihrer Rechtsnatur her zu einem großen Teil völkerrechtliche Verträge. Damit ein völkerrechtlicher Vertrag Geltung im innerstaatlichen Recht Deutschlands erlangt und somit Bestandteil des objektiven Rechts im 
staatlichen Rechtsraum wird, ${ }^{1}$ müssen folgende Voraussetzungen erfüllt sein: Dem völkerrechtlichen Vertrag muß gem. Art. 59 II GG durch Bundesgesetz zugestimmt worden sein. ${ }^{2}$ Die Notwendigkeit einer weiteren Voraussetzung - gemeint ist das wirksame Inkrafttreten des völkerrechtlichen Vertrages auf völkerrechtlicher Ebene - hängt davon ab, in welchem Verhältnis Zustimmungsgesetz gem. Art. 59 II GG und völkerrechtlicher Vertrag zueinander stehen.

\section{aa) Transformationstheorie}

Nach der sogenannten Transformationstheorie ist das Zustimmungs- bzw. Vertragsgesetz der Transformator, der den völkerrechtlichen Vertrag bzw. die einzelne Vertragsnorm in innerstaatliches Recht umwandelt. ${ }^{3}$ Durch das Zustimmungsgesetz wird gleich lautendes innerstaatliches Recht mit der Folge gesetzt, daß sich Inkrafttreten, Wirksamkeit, Interpretation, Beendigung usw. einerseits nach Völkerrecht - dies gilt für den völkerrechtlichen Vertrag -, andererseits nach innerstaatlichem Recht - dies gilt für die in innerstaatliches Recht umgesetzten Rechtsnormen - richten. ${ }^{4}$ Nach der Transformationstheorie kommt es somit für die innerstaatliche Geltung der transformierten Regelungen eines völkerrechtlichen Vertrages nicht darauf an, ob selbiger auf völkerrechtlicher Ebene in Kraft getreten ist. Allein das Zustimmungs- bzw. Vertragsgesetz führt dazu, daß der Inhalt des völkerrechtlichen Vertrages in der innerstaatlichen Rechtsordnung Geltung erlangt. ${ }^{5}$

\section{bb) Inkorporations-, Adoptions-, Vollzugstheorie}

Nach Inkorporations-, Adoptions- und Vollzugstheorie gilt Völkerrecht innerstaatlich, ohne seinen Charakter als Völkerrecht zu verlieren. ${ }^{6}$ Folge dieser Theorien ist u.a., daß sich das Inkrafttreten des innerstaatlich geltenden Völkerrechts nach Völkerrecht richtet. ${ }^{7}$ Nach besagten Theorien gilt demnach der Inhalt eines völkerrechtlichen Vertrages innerstaatlich erst dann, wenn der völkerrechtliche Vertrag in Kraft getreten ist.

\section{cc) Stellungnahme}

\footnotetext{
Rojahn in v. Münch/Kunig, Band 2, Art. 59, Rn. 34. BVerfGE 1, S. 396 (411); Jarass in Jarass/Pieroth, Art. 59, Rn. 17; Streinz in Sachs, Art. 25, Rn. 29. Schweitzer, Staatsrecht III, Rn. 424; Rojahn in von Münch/Kunig, Band 2, Art. 59, Rn. 33. Schweitzer, Staatsrecht III, Rn. $424 \mathrm{f}$. BVerfGE 1, S. 396 (411); Jarass in Jarass/Pieroth, Art. 59, Rn. 17; Streinz in Sachs, Art. 59, Rn. 60. Rojahn in von Münch/Kunig, Band 2, Art. 59, Rn. 33.

Schweitzer, Staatsrecht III, Rn. 421 und 423, der diese Aussage bzgl. der Adoptionstheorie und der Vollzugslehre trifft.
} 
Der Wortlaut des Art. 59 II 1 GG trifft keine Aussage darüber, welcher Theorie zu folgen ist. ${ }^{8}$ Kritiker der strengen Transformationstheorie weisen darauf hin, daß sie den bestehenden Zusammenhang zwischen Völkerrecht und innerstaatlichem Recht in realitätsfremder Weise auflöse. ${ }^{9}$ Solange der völkerrechtliche Vertrag selbst nicht in Kraft getreten ist, erscheine die Vertragserfüllung durch das innerstaatliche „In-Geltung-Setzen“ der Vertragsnormen durch das Zustimmungsgesetz als ungerechtfertigte Vorleistung. ${ }^{10}$ Dieses Argument gewinnt an Stichhaltigkeit, wenn man sich den Fall konstruiert, daß - aus welchem Grund auch immer - der völkerrechtliche Vertrag niemals in Kraft tritt. Es ist nicht erklärbar, warum ein solcher Vertrag im innerstaatlichen Recht Wirkungen entfalten sollte. Sinnvollerweise wird man deshalb beides, das wirksame Inkrafttreten des völkerrechtlichen Vertrages ${ }^{11}$ wie die wirksame Zustimmung ${ }^{12} \mathrm{zu}$ selbigem verlangen müssen, damit seine Bestimmungen innerstaatlich gelten können. ${ }^{13}$ Daß teilweise lediglich auf das Inkrafttreten des völkerrechtlichen Vertrages abgestellt wird, ${ }^{14}$ dürfte der Tatsache geschuldet sein, daß das Zustimmungsverfahren regelmäßig vor der Ratifikation des völkerrechtlichen Vertrages erfolgt. ${ }^{15}$

Soweit nichts anderes vereinbart ist, tritt ein völkerrechtlicher Vertrag durch seine Ratifikation (= Annahme) in Kraft, ${ }^{16}$ d.h. durch Erklärung des Staates, daß er den Vertrag nunmehr als für sich verbindlich betrachte. ${ }^{17}$ In Deutschland gibt der Bundespräsident diese Erklärung ab, da er gem.

\author{
Rojahn in von Münch/Kunig, Band 2, Art. 59, Rn. 33. \\ Schweitzer, Staatsrecht III, Rn. 432. \\ Rojahn in von Münch/Kunig, Band 2, Art. 59, Rn. 36. \\ 1 BVerfGE 1, S. 396 (411); Rojahn in v. Münch/Kunig, Band 2, Art. 59, Rn. 36; Jarass in Jarass/Pieroth, Art. 59, Rn. \\ 17
}

Die in Rede stehenden völkerrechtlichen Verträge sind in Deutschland in Kraft getreten: die UN-Charta am 18.09.1973 (BGB1. 1974 II 2, S. 1397), das Übereinkommen der ILO Nr. 100 über die Gleichheit des Entgelts männlicher und weiblicher Arbeitskräfte für gleichwertige Arbeit am 08.06.1957 (BGB1. 1957 II 2, S. 1232), das Übereinkommen der ILO Nr. 111 über die Diskriminierung in Beschäftigung und Beruf am 15.06.1962 (BGB1. 1962 II 1, S. 819), das Internationale Übereinkommen zur Beseitigung jeder Form von Rassendiskriminierung am 15. 06. 1969 (BGB1. 1969 II 2, S. 2211), der Internationale Pakt über bürgerliche und politische Rechte am 23.03.1976 (BGB1. 1976 II 2, S. 1068), der Internationale Pakt über wirtschaftliche, soziale und kulturelle Rechte am 03.01.1976 (BGB1. 1976 II 1, S. 428), das Übereinkommen zur Beseitigung jeder Form von Diskriminierung der Frau am 09.08.1985 (BGB1. 1985 II 2, S. 1234), das Rahmenübereinkommen zum Schutz nationaler Minderheiten am 01.02.1998 (BGB1. 1998 II 1, S. 57), die Konvention zum Schutze der Menschenrechte und Grundfreiheiten am 03.09.1953 (BGB1. 1954 II 1, S. 14), die Europäische Sozialcharta am 26.02.1965 (BGB1. 1965 II 2, S. 1122; Die revidierte Fassung der Charta von 1996 ist nicht Gegenstand der vorliegenden Untersuchung, da sie in Deutschland bislang keine Geltung erlangt hat. (Hailbronner in Graf Vitzthum, Völkerrecht, 3. Abschnitt, Rn. 279, Fn. 392)), der EG-Vertrag am 01.01.1958 (BGB1. 1958 II, S. 1; In seiner durch den Vertrag von Nizza geänderten Fassung ist er am 01.02.2003 in Kraft getreten. (BGB1. 2003 II 2, S. 1477)), der EU-Vertrag gem. Art. 52 II EUV am 01.11.1993 (Streinz, Europarecht, Rn. 40; In seiner durch den Vertrag von Nizza geänderten Fassung ist er am 01.02.2003 in Kraft getreten. (BGB1. 2003 II 2, S. 1477)).

12 Das jeweilige Zustimmungsgesetz der in Rede stehenden Verträge ist im Bundesgesetzgebungsblatt an der Stelle veröffentlicht, wo der jeweilige Vertrag abgedruckt ist. Es wird an dieser Stelle auf die insoweit bereits gemachten Angaben verwiesen.

13 So BVerfGE 1, S. 396 (411); Jarass in Jarass/Pieroth, Art. 59, Rn. 17; Verdross/Simma, Universelles Völkerrecht, S. 431 f.; Rojahn in von Münch/Kunig, Band 2, Art. 59, Rn. 36.

14 Vergleiche z.B. Rojahn in von Münch/Kunig, Band 2, Art. 59, Rn. 36.

15 Ipsen, Völkerrecht, § 10, Rn. 17.

16 Ipsen, Völkerrecht, § 10, Rn. 29 i.V.m. Rn. 18.

17 Seidl-Hohenveldern/Stein, Völkerrecht, Rn. 218 f.; Ipsen, Völkerrecht, § 10, Rn. 18. 
Art. 59 I 2 GG „im Namen des Bundes die Verträge mit auswärtigen Staaten“ abschließt. ${ }^{18}$ Anzumerken ist, daß in völkerrechtlichen Verträgen zumeist geregelt ist, daß deren Inkrafttreten erst durch Hinterlegung einer gewissen Anzahl an Ratifikationsurkunden erfolgt. ${ }^{19}$ Bei Staaten, die den völkerrechtlichen Vertrag erst nach dessen Inkrafttreten ratifizieren, tritt der Vertrag in einem durch den Vertrag bestimmten Zeitpunkt nach Hinterlegung der Ratifikationsurkunde des jeweiligen Staates in Kraft. ${ }^{20}$

\section{b) Völkergewohnheitsrecht}

Gemäß Art. 25 S. 1 GG sind die ,allgemeinen Regeln des Völkerrechts [...] Bestandteil des Bundesrechts. “ Regeln in diesem Sinne entstammen hauptsächlich dem Völkergewohnheitsrecht, ${ }^{21}$ so daß selbiges über Art. 25 GG Bestandteil des Bundesrechts wird. ${ }^{22}$ Das gilt auch für solche (seltenen) allgemeinen Regeln des Völkerrechts, die sich direkt an Individuen wenden und diese durch unmittelbare Berechtigung oder Verpflichtung selbst zu Völkerrechtssubjekten machen. ${ }^{23}$

\section{c) EU-Verordnungen}

Gemäß Art. 249 II 2 EGV gilt die Verordnung ,unmittelbar in jedem Mitgliedstaat.“ Das bedeutet, daß sie mit ihrem Inkrafttreten ${ }^{24}$ Geltung in den Mitgliedstaaten erlangt, ohne daß die Legislativorgane des jeweiligen Staates diese Geltung gesondert anordnen müßten. ${ }^{25}$

\section{d) EU-Richtlinien}

Demgegenüber taucht im Schrifttum die Formulierung auf, die Richtlinie gelte erst dann, wenn die nationalen Stellen - speziell die Legislative - tätig geworden sind. ${ }^{26} \mathrm{Zu}$ beachten ist in diesem

18 Vitzthum, Völkerrecht, 1. Abschnitt, Rn. 117.

19 So z.B. im Fall der UN-Charta, vgl. dazu Art. 110 III; Möglich sind auch andere Vereinbarungen bzgl. des Inkrafttretens. So tritt beispielsweise das Klimaschutzprotokoll von Kyoto erst dann in Kraft, wenn Länder ihm beitreten, die 199055 Prozent des weltweiten Kohlendioxydausstoßes verursacht haben. (FAZ vom 01.10.2004, S. 1 unter dem Titel: „Berlin und Brüssel loben Putins Kyoto-Entscheidung“).

20 Vergleiche dazu beispielhaft Art. 110 IV der UN-Charta; Die UN-Charta ist am 24. 10. 1945 für seine ursprünglichen Mitglieder in Kraft getreten (Verdross/Simma, Universelles Völkerrecht, S. 82), in Deutschland am 18.09.1973. (BGB1. 1974 II 2, S. 1397).

21 Streinz in Sachs, Art. 25, Rn. 32.

22 Die Frage der innerstaatlichen Geltung von Völkergewohnheitsrecht stellt sich, da an anderer Stelle darauf hingewiesen wurde, daß zu selbigem allgemeine Menschenrechte zählen, worunter z. B. auch das Recht auf Schutz vor rassistischer Diskriminierung fällt.

23 Streinz in Sachs, Art. 25, Rn. 46.

24 Ist der Tag des Inkrafttretens in der Verordnung nicht festgelegt - dies trifft auf die Verordnung 1612/68 zu -, tritt sie gem. Art. 254 I bzw. II EGV am zwanzigsten Tag nach ihrer Veröffentlichung im Amtsblatt der Europäischen Union in Kraft.

25 Streinz, Europarecht, Rn. 380; Schweitzer/Hummer, Europarecht, Rn. 352.

26 Schweitzer/Hummer, Europarecht, Rn. 364. 
Zusammenhang, daß die Literatur nicht immer zwischen der Frage der Geltung einer Richtlinie und deren Anwendbarkeit im innerstaatlichen Recht unterscheidet. ${ }^{27}$ Da beide Begriffe jedoch ganz unterschiedliche Fragen beantworten, ${ }^{28}$ sind sie strikt voneinander zu trennen. Auch die Richtlinie gilt mit ihrem Inkrafttreten unmittelbar, da auch sie einer Transformation nicht bedarf. ${ }^{29}$

\section{e), ,Soft-Law 6}

Einige der erwähnten Maßnahmen wurden nicht in Form eines völkerrechtlichen Vertrages geschlossen, auch wenn dies der Anschein zuweilen nahe legt. So hat beispielsweise die Allgemeine Erklärung der Menschenrechte als sog. „soft law“30 nicht den Status eines völkerrechtlichen Vertrages. ${ }^{31}$ Obwohl auch soft law erhebliche politische Verpflichtungen in den internationalen Beziehungen und der internen Rechtssetzung, einschließlich der Verfassungsgebung begründen könne, ${ }^{32}$ habe es rechtlich lediglich die Bedeutung einer stets reversiblen Festlegung der politischen Richtung. ${ }^{33}$ Die Allgemeine Erklärung der Menschenrechte hat aus diesem Grund im innerstaatlichen Recht Deutschlands keine rechtliche Geltung erlangt. ${ }^{34}$ Mangels Verbindlichkeit $^{35}$ ist auch die Gemeinschaftscharta der Sozialen Grundrechte der Arbeitnehmer nicht Bestandteil der

27 So benutzt Ruffert den Begriff der unmittelbaren Geltung im Zusammenhang mit der Frage, ob dem einzelnen Rechte verliehen und Pflichten auferlegt werden. (Ruffert in Calliess/Ruffert, Art. 249 EGV, Rn. 41) Damit spricht er eigentlich die Frage der unmittelbaren Anwendbarkeit der Richtlinie an. Auch Schweitzer/Hummer, Bleckmann und Streinz meinen die unmittelbare Anwendbarkeit, wenn sie von unmittelbarer Geltung der Richtlinien sprechen. (Schweitzer/Hummer, Europarecht, Rn. 364; Bleckmann, Europarecht, Rn. 415; Streinz, Europarecht, Rn. 395) Schroeder weist auf die Ungenauigkeiten im Zusammenhang mit der Verwendung der einzelnen Termini hin. (Schroeder in Streinz, Art. 249, Rn. 104).

28 Während es bei der unmittelbaren Geltung darum geht, daß das Gemeinschaftsrecht - hier die Richtlinie - ohne weiteren Transformationsakt Bestandteil des innerstaatlichen Rechts wird, behandelt die unmittelbare Anwendbarkeit die Frage, unter welchen Voraussetzungen das Gemeinschaftsrecht von innerstaatlichen Stellen angewendet werden muß. (Schroeder in Streinz, Art. 249, Rn. 104).

29 Schroeder in Streinz, Art. 249, Rn. 105; Auch hier gilt - wie bei der Verordnung - Art. 254 EGV. Die für die vorliegende Arbeit besonders relevanten Sekundärrechtsakte sind in Kraft getreten: Richtlinie 2000/43/EG gem. Art. 18 am 19.07.2000, Richtlinie 2000/78/EG gem. Art. 20 am 02.12.2000; Richtlinie 76/207EWG in ihrer durch die Richtlinie 2002/73EG geänderten Fassung gem. Art. 3 Richtlinie 2002/73EG am 05.10.2002, Richtlinie 2004/113/EG gem. Art. 18 am 21.12.2004.

30 Pernice in Dreier, Art. 59, Rn. 45; Schweitzer, Staatsrecht III, Rn. 269; Unter den Begriff ,soft law“ fallen u.a. das „Gentlement's Agreement“, politische Absichtserklärungen, aber auch Verhaltenskodizes (,„codes of conduct“), also unverbindliche Empfehlungen an Staaten oder andere Verbandseinheiten. (Ipsen, Völkerrecht, § 9, Rn. 14 ff.; Pernice in Dreier, Art. 59, Rn. 45).

31 Tomuschat, Veröffentlichung der Vereinigung der Deutschen Staatsrechtslehrer, Heft 36, S. 7 (32 f.); Sie sei als solches der Mitentscheidung durch das Parlament im Wege des Art. 59 II 1 GG entzogen. (BVerfGE 68, S. 1 (109); Tomuschat, Veröffentlichung der Vereinigung der Deutschen Staatsrechtslehrer, Heft 36, S. 7 (32); Streinz in Sachs, Art. 59, Rn. 40; für eine teilweise Einbeziehung von soft law durch eine differenzierende Anwendung des Art. 59 II 1 GG dagegen Perince in Dreier, Art. 59, Rn. 46).

32 Pernice in Dreier, Art. 59, Rn. 45.

33 Ipsen, Völkerrecht, § 9, Rn. 15.

$34 \mathrm{Zu}$ der dennoch nicht zu unterschätzenden Bedeutung der Allgemeinen Erklärung der Menschenrechte vgl. Nowak: „Die wegweisende Bedeutung der Allgemeinen Erklärung [...] besteht darin, dem in der Satzung der Vereinten Nationen mehrfach verwendeten Begriff der Menschenrechte inhaltliche Gestalt gegeben und dadurch einen universell anerkannten Mindeststandard geschaffen $\mathrm{zu}$ haben, der auch heute noch als Richtschnur für das Verhalten der Staaten und als inhaltliche Grundlage für eine Reihe von Maßnahmen und Verfahren der Weltorganisation zur internationalen Überwachung der Durchsetzung und Einhaltung der Menschenrechte durch die Regierungen gilt.“ (Nowak, CCPR-Kommentar, Einführung, Rn. 2).

35 Heinze in Kasseler Handbuch zum Arbeitsrecht, Band 2, 12, Rn. 6. 
deutschen Rechtsordnung geworden. Gleiches gilt für die am 07.12.2000 auf dem EU-Gipfel von Nizza feierlich verkündete Charta der Grundrechte der Europäischen Union. ${ }^{36}$ Mangels vertraglich begründeter Geltung ist sie weder für die EU, noch für deren Mitgliedstaaten unmittelbar verbindlich. ${ }^{37}$ Dies könnte sich jedoch in Zukunft ändern, soll doch die Charta der Grundrechte gem. Art. 1 Nr. 8) des Vertrages von Lissabon ${ }^{38}$ vom 13.12.2007 - der gem. seines Art. 6 II frühestens am 01.01.2009 in Kraft tritt - Bestandteil des EU-Vertrages werden.

\section{Unmittelbare Anwendbarkeit der Antidiskriminierungsmaßnahmen}

Nachdem die Frage ihrer innerstaatlichen Geltung beantwortet worden ist, soll nunmehr geklärt werden, ob die internationalen Antidiskriminierungsmaßnahmen im Privatrechtsverhältnis unmittelbar anwendbar sind. Dieselbe Frage stellt sich auch bei einigen nationalen Antidiskriminierungsmaßnahmen; konkret bei den Grundrechten des Grundgesetzes.

\section{a) Völkerrechtliche Verträge}

Die Tatsache, daß ein völkerrechtlicher Vertrag im innerstaatlichen Recht Deutschlands Geltung erlangt hat, sagt nichts darüber aus, ob er in Deutschland auch unmittelbar anwendbar ist. Unmittelbar anwendbar sind nach herrschender Meinung nur solche Vertragsbestimmungen, die sich durch Statuierung von Pflichten auf der einen und Rechten auf der anderen Seite unmittelbar an staatliche Rechtsanwendungsorgane und die Rechtsunterworfenen wenden, mithin derart bestimmt sind, daß zu ihrer Ausführung keine weiteren Akte erforderlich sind. ${ }^{39}$ Man spricht insofern von „,self-executing"-Normen. ${ }^{40}$ Die Auslegung des Inhalts, Zwecks und der Fassung einer Vertragsbestimmung ergibt, ob sie self-executing ist. ${ }^{41}$

Im Zusammenhang mit der Frage, ob Bestimmungen eines völkerrechtlichen Vertrages unmittelbar anwendbar, d.h. ohne weiteren innerstaatlichen Rechtsakt vollzugsfähig bzw. transformabel sind, ${ }^{42}$ muß beantwortet werden, ob die in Rede stehenden Bestimmungen überhaupt den Staat oder - was im Rahmen dieser Arbeit von besonderem Interesse ist - den Privaten verpflichten und auf der

\footnotetext{
Herdegen, Europarecht, Rn. 174 a.

Herdegen, Europarecht, Rn. 174 b; Triebel, JURA 2003, S. 525 (525).

Abl. EG 2007, C 306, S. 1 ff.

Streinz in Sachs, Art. 59, Rn. 67 f.; Schweitzer, Staatsrecht III, Rn. 438, 440.

Streinz in Sachs, Art. 59, Rn. 67; Schweitzer, Staatsrecht III, Rn. 438.

Streinz in Sachs, Art. 59, Rn. 68.

Schweitzer, Staatsrecht III, Rn. 438.
} 
anderen Seite den einzelnen berechtigen. ${ }^{43}$ Unmittelbar anwendbar kann eine Norm schließlich nur dann sein, wenn sie berechtigt und/oder verpflichtet. ${ }^{44}$

Art. 1 III der UN-Charta beschreibt ein in Zukunft $\mathrm{zu}$ erreichendes Ideal, die Achtung der Menschenrechte und Grundfreiheiten ohne Diskriminierung. Die Norm verpflichtet jedoch weder die Staaten noch Private und statuiert auf der anderen Seite auch keinerlei Rechte einzelner Personen. Sie ist folglich ,non-self-executing“. Ebenso verhält es sich mit Art. $2,{ }^{45} 3$ II, ${ }^{46}$ und 137 lit. $b$ und $i^{47} \mathrm{EGV}$.

\section{aa) Unmittelbare Verpflichtung der Vertragsstaaten}

Im Gegensatz dazu verpflichten die meisten der im Rahmen dieser Arbeit bereits angesprochenen Bestimmungen der völkerrechtlichen Verträge die Vertragsstaaten. ${ }^{48}$ Der Wortlaut einiger dieser Bestimmungen bringt dies unzweideutig zum Ausdruck. ${ }^{49}$

Umstritten ist die Bindung der Mitgliedstaaten an die Gemeinschaftsgrundrechte ${ }^{50}$ sowie an Art. 13 EGV. ${ }^{51}$

\section{bb) Unmittelbare Berechtigung Privater}

Der Verpflichtung des Staates steht vereinzelt die Berechtigung des einzelnen gegenüber. Das gilt z.B. für die Art. $12,{ }^{52} 39,{ }^{53}$ und $141 \mathrm{EGV}^{54}$

Uneinheitlich wird die Frage der Statuierung subjektiver Rechte dort beantwortet, wo schon die Verpflichtung des Staates umstritten ist, wie im Fall des Art. 13 EGV. ${ }^{55}$ Gleiches muß für die

43 Teilweise wird die Frage nach dem Bestehen subjektiver Rechte streng von der der Anwendbarkeit unterschieden. (Streinz in Sachs, Art. 59, Rn. 69) Diese strikte Trennung wird im folgenden nicht vorgenommen. (so auch Schweitzer, Staatsrecht III, Rn. 439 b).

44 Schweitzer, Staatsrecht III, Rn. 438, 439 b.

45 Streinz in Streinz, Art. 2 EGV, Rn. 9.

46 Kempen in Streinz, Art. 3 EGV, Rn. 49.

47 In den dort genannten Bereichen wird der Rat gem. Art. 137 II lit. b lediglich ermächtigt, per Richtlinie Mindestvorschriften zur Unterstützung der Mitgliedstaaten zu erlassen.

48 Das gilt für alle Bestimmungen, auf die an dieser Stelle nicht gesondert eingegangen wird.

49 Art. 2 des Übereinkommens zur Beseitigung jeder Form von Diskriminierung der Frau lautet beispielsweise: „Die Vertragsstaaten [...] verpflichten sich zu diesem Zweck, [...]“.

50 Die Bindung verneinend Stumpf in Schwarze, Art. 6 EUV, Rn. 16; die Bindung bejahend, soweit der konkrete Fall dem Anwendungsbereich des Gemeinschaftsrechts unterliegt Streinz, Europarecht, Rn. 367; Kingreen in Calliess/Ruffert, Art. 6 EUV, Rn. 56; Cirkel, NJW 1998, S. 3332 (3333).

51 Dagegen Streinz in Streinz, Art. 13 EGV, Rn. 17; Epiney in Calliess/Ruffert, Art. 13 EGV, Rn. 1 f.; a.A. unter der Prämisse, daß sich die Mitgliedstaaten im Rahmen der durch den EG-Vertrag auf die Gemeinschaft übertragenen Zuständigkeiten bewegen Holoubek in Schwarze, Art. 13 EGV, Rn. 9 f.; Szczekalla, EuZW 1998, S. 215 (216); Cirkel, NJW 1998, S. 3332 (3333).

52 Holoubek in Schwarze, Art. 12 EGV, Rn. 14; Epiney in Calliess/Ruffert, Art. 13, Rn. 1.

53 Franzen in Streinz, Art. 39 EGV, Rn. 4; Streinz, Europarecht, Rn. 705 f.; Eine Ausnahme bildet lediglich Art. 39 III lit. d (Schneider/Wunderlich in Schwarze, Art. 39 EGV, Rn. 2).

54 Eichenhofer in Streinz, Art. 141 EGV, Rn. 7.

55 Dagegen Streinz in Streinz, Art. 13 EGV, Rn. 17; Epiney in Calliess/Ruffert, Art. 13 EGV, Rn. 1 f.; a.A. unter der Prämisse, daß sich die Mitgliedstaaten im Rahmen der durch den EG-Vertrag auf die Gemeinschaft übertragenen 
Gemeinschaftsgrundrechte im Sinne des Art. 6 II EUV gelten, die gegenüber den Gemeinschaftsorganen zweifellos subjektive Rechte des einzelnen statuieren. ${ }^{56}$

Die Verpflichtung des Staates führt nicht zwangsläufig zu einem korrespondierenden Recht des einzelnen. So gewährt Art. 5 lit. f des Internationalen Übereinkommens zur Beseitigung jeder Form von Rassendiskriminierung kein Individualrecht des einzelnen auf ungehinderten Zugang zu den betreffenden Einrichtungen - gemeint ist der Zugang zu Verkehrsmitteln, Hotels, Gaststätten, Cafés etc. -, sondern verpflichtet die Vertragsstaaten lediglich, den einzelnen vor Diskriminierung in diesen Bereichen zu schützen. ${ }^{57}$ Art. 5 lit. f zeigt, daß einer völkerrechtlichen Bestimmung nicht allein deshalb self-executing-Charakter zukommt, weil sie den Staat verpflichtet. ${ }^{58}$ Wesentliches Kriterium der unmittelbaren Anwendbarkeit einer solchen Bestimmung im innerstaatlichen Recht ist vielmehr, daß sie den einzelnen unmittelbar berechtigt und/oder verpflichtet. ${ }^{59}$ Grund dafür, daß Art. 5 lit. f kein Individualrecht des einzelnen begründet, ist, daß ein Recht des einzelnen auf freien Zugang zu besagten Einrichtungen die Verpflichtung Privater - Cafés werden in der Regel privat betrieben - aus Art. 5 lit. f voraussetzte. Gleiches gilt für die meisten der bereits angesprochenen Bestimmungen aus völkerrechtlichen Verträgen, da auch sie die Mitgliedstaaten verpflichten, in Rechtsbeziehungen Privater regelnd einzugreifen.

\section{cc) Unmittelbare Verpflichtung Privater}

Nach der Rechtsprechung des BVerfG konstatieren völkerrechtliche Verträge unmittelbare Pflichten einzelner Bürger jedoch nur dann, ,wenn und soweit dies der Vertragstext unzweideutig zum Ausdruck bringt “. ${ }^{60}$ Art. 5 lit. f ist unzweideutig nicht in diesem Sinne formuliert. Die Norm schafft keine unmittelbare Verpflichtung Privater, ${ }^{61}$ im Gegensatz beispielsweise zu Art. 141 EGV, ${ }^{62}$ obwohl auch dessen Wortlaut gegen eine solche Annahme spricht ${ }^{63}$ und die unmittelbare Drittwirkung dieser Norm deshalb nicht unumstritten ist. ${ }^{64}$

Zuständigkeiten bewegen Holoubek in Schwarze, Art. 13 EGV, Rn. 8 ff.; Szczekalla, EuZW 1998, S. 215 (216); Cirkel, NJW 1998, S. 3332 (3333).

56 Stieglitz, Allgemeine Lehren im Grundrechtsverständnis nach der EMRK und der Grundrechtsjudikatur des EuGH, S. 169.

57 Kühner, NJW 86, S. 1397 (1398).

58 Schweitzer, Staatsrecht III, Rn. 439.

59 Schweitzer, Staatsrecht III, Rn. 439 b.

60 BVerfGE 43, S. 203 (209).

61 Kühner, NJW 86, S. 1397 (1399); Stock, ZAR 99, S. 118 (119).

$62 E u G H$, Rs. C-43/75, Slg. 1976 1, S. 455 (476, Rn. 40); Streinz, Europarecht, Rn. 916; Eichenhofer in Streinz, Art. 141 EGV, Rn. 7.

63 Darauf weisen u. a. auch Streinz/Leible hin. (Streinz/Leible, EuZW 2000, S. 459 (462)).

64 Der unmittelbaren Drittwirkung stehen skeptisch gegenüber: Roth in Festschrift für Everling, Band II, S. 1231 (1240); Birk meint, eine solche Schlußfolgerung sei contra legem. (Birk in Münchener Handbuch zum Arbeitsrecht, $\S 19$, Rn. 312). 
Umstritten ist ebenfalls, inwieweit Art. 12 und 39 EGV Privatpersonen verpflichten. Es wird vertreten, Art. 39 binde Privatpersonen dann, wenn sie eine faktische Machtstellung innehaben, ${ }^{65}$ die sich daraus ergeben könne, daß der Staat seine Normsetzungsbefugnis zugunsten privat- oder öffentlich-rechtlicher Institutionen zurücknimmt ${ }^{66}$ bzw. ihnen sonst hoheitliche Befugnisse verleiht $^{67}$ oder private Verbände aufgrund ihrer gesellschaftlichen Stellung gegenüber dem einzelnen staatsgleich agieren können. ${ }^{68}$ Gebunden an Art. 39 sollen Private demnach dann sein, wenn sie abstrakt-generelle private Regelungen treffen, denen der einzelne nicht ausweichen kann. ${ }^{69}$ Auch im Rahmen des Art. 12 EGV wird teilweise in diesem Sinne argumentiert. ${ }^{70}$ In einer umstrittenen Entscheidung hat der $E u G H$ mittlerweile auch individuelle Abreden unter Privatpersonen am Maßstab des Art. 39 II gemessen. ${ }^{71}$ Auch im Rahmen des Art. 12 gibt es Stimmen, die eine generelle Bindung Privater bejahen. ${ }^{72}$

Wie im Falle der Art. 12 und 39 EGV ist umstritten, ob die Gemeinschaftsgrundrechte Private binden, mithin eine unmittelbare Drittwirkung entfalten. Teilweise wird dies in der Weise verneint, daß auf diese Frage überhaupt nicht eingegangen wird bzw. Private in den Adressatenkreis des Art. 6 II EUV nicht aufgenommen sind. ${ }^{73}$ Eine andere Ansicht hält hingegen eine unmittelbare Drittwirkung in den Fällen für möglich, in denen sich das Rechtsverhältnis zwischen den fraglichen privaten Parteien nach Gemeinschaftsrecht richtet. ${ }^{74}$ Es wird auch vertreten, die unmittelbare Drittwirkung sei - ohne sie generell abzulehnen - verzichtbar, weil sich das Problem privater Beeinträchtigungen der Grundrechte ähnlich wie im Bereich der Grundfreiheiten über die hoheitliche Schutzpflicht gegenüber Übergriffen Privater lösen ließe. ${ }^{75}$

Neben der Frage nach einer etwaigen Bindung Privater ist zudem problematisch, wer Träger der Gemeinschaftsgrundrechte ist. Die Gemeinschaftsgrundrechte berechtigen zunächst

65 Franzen in Streinz, Art. 39 EGV, Rn. 94 f.

66 Wie z.B. im Tarifvertragswesen und der betrieblichen Normsetzung.

67 Wie z.B. im Fall der berufständischen Kammern für Rechtsanwälte, Ärzte, Apotheker, Handwerker etc.

68 Das gelte bspw. für Arbeitgeberverbände, Gewerkschaften, politische Parteien sowie Sport(dach)verbände. (Franzen in Streinz, Art. 39 EGV, Rn. 95).

69 Remmert, JURA 2003, S. 13 (15 f.).

70 Vergleiche dazu im einzelnen Bode, Die Diskriminierungsverbote im Vertrag über die EWG, 1968, S. 299; Epiney in Calliess/Ruffert, Art. 12 EGV, Rn. 23; Streinz in Streinz, Art. 12 EGV, Rn. 39; Holoubek in Schwarze, Art. 12 EGV, Rn. 26.

71 EuGH, Rs. C-281/98, Slg. 2000 I 6, S. 4139 (4173, Rn. 36); Diese Rspr. wird u. a. abgelehnt von Franzen in Streinz, Art. 39, Rn. 97; Calliess hat das Urteil „Angonese“ in einer Europarechtsvorlesung an der Universität Göttingen im Wintersemester 2003/2004 als Einzelfallentscheidung und Fehlurteil bezeichnet.; Im Gegensatz dazu ist Brechmann für eine uneingeschränkte Drittwirkung. (Brechmann in Calliess/Ruffert, Art. 39 EGV, Rn. 51).

72 Von Bogdandy in Grabitz/Hilf, Band I, Art. 6 EGV, Rn. 31; Bleckmann in Bleckmann, Europarecht, Rn. 1769, der allerdings auf Art 6 eingeht (= Art. 12 n.F.)); In diesem Zusammenhang wird jedoch auch daran erinnert, daß im Privatrechtsverhältnis immer auch die Grundrechte des Diskriminierenden zu berücksichtigen seien. (Bleckmann in Bleckmann, Europarecht, Rn. 1770); Streinz/Leible sprechen in diesem Zusammenhang von der herrschenden Meinung. Umstritten sei lediglich der Umfang der unmittelbaren Drittwirkung. (Streinz/Leible, EuZW 2000, S. 459 (462, Fn. 44)).

73 Stumpf in Schwarze, Art. 6 EUV, Rn. 16; Streinz, Europarecht, Rn. 367 f.

74 Borchardt in Lenz, Art. 220 EGV, Rn. 35; Wetter, Die Grundrechtscharta des Europäischen Gerichtshofs, S. 102.

75 Kingreen in Calliess/Ruffert, Art. 6 EUV, Rn. 63; Pernice/Mayer in Grabitz/Hilf, Band I, Art. 6 EUV, Rn. 33; Remmert, JURA 2003, S. 13 (18)); Stieglitz, Allgemeine Lehren im Grundrechtsverständnis nach der EMRK und der Grundrechtsjudikatur des EuGH, S. 174; Szczekalla, Die sogenannten grundrechtlichen Schutzpflichten im deutschen und europäischen Recht, S. 708; Gersdorf, AöR 119, S. 400 (420 f.). 
Staatsangehörige aus den Mitgliedstaaten, d.h. Unionsbürger i.S.d. Art. 17 EGV. ${ }^{76}$ Darüber hinaus sollen auch Drittstaatsangehörige, die sich im Hoheitsgebiet eines Mitgliedstaates aufhalten, Grundrechtsträger sein. ${ }^{77}$ Dem wird entgegengehalten, daß Grundrechte, die in der überwiegenden Zahl der Mitgliedstaaten den eigenen Bürgern vorbehalten sind, ${ }^{78}$ auch auf Gemeinschaftsebene jedenfalls dann nicht jedermann berechtigen, wenn die $\mathrm{EMRK}^{79}$ keinen oder nur eingeschränkten grundrechtlichen Schutz gewährt, als Rechtserkenntnisquelle daher allein die Verfassungsüberlieferungen der Mitgliedstaaten zur Verfügung stehen. ${ }^{80}$ Die Brisanz der Frage nach der Grundrechtsträgerschaft zeigt sich in Deutschland wiederum am Beispiel der Türken, die Statistiken zufolge besonders von Diskriminierung betroffen sind.

\section{dd) Zusammenfassung}

Zusammenfassend kann festgehalten werden, daß sich die Frage der innerstaatlichen Anwendbarkeit von Bestimmungen aus völkerrechtlichen Verträgen nicht einheitlich beantworten läßt. Es gibt zunächst solche Verträge, die als bloße Absichtserklärungen keinerlei Verpflichtungen begründen. In der Mehrzahl der diskutierten Verträge sind die Vertragsstaaten indes Verpflichtungen eingegangen. Diesen Verpflichtungen stehen teilweise unmittelbare Individualrechte gegenüber. Vereinzelt bestehen besagte Rechte auch gegenüber Privatpersonen, was voraussetzt, daß sie ihrerseits aus der in Rede stehenden Bestimmung unmittelbar verpflichtet sind.

\section{b) Völkergewohnheitsrecht}

Es gibt allgemeine Regeln des Völkerrechts, die sich direkt an Individuen wenden und selbige durch unmittelbare Berechtigung oder Verpflichtung zu Völkerrechtssubjekten machen. ${ }^{81} \mathrm{Zu}$ den Individualrechten zählen vor allem die gewohnheitsrechtlich anerkannten allgemeinen Menschenrechte, wie z.B. das Recht auf Schutz vor rassistischer Diskriminierung. Nicht verkannt werden darf jedoch, daß dem Recht des einzelnen (hier des Diskriminierten) nicht zwangsläufig die

76 Kingreen in Calliess/Ruffert, Art. 6 EUV, Rn. 51.

77 Pechstein in Streinz, Art. 6 EUV, Rn. 9; so wohl auch Stumpf, nach dem alle Privatpersonen Grundrechtsträger sind (Stumpf in Schwarze, Art. 6 EUV, Rn. 16).

78 Im deutschen Verfassungsrecht spricht man von sog. „Deutschen-Grundrechten“ im Gegensatz zu den sog. „Jedermann-Grundrechten“, die jede Person unabhängig von ihrer Staatsangehörigkeit berechtigen. (Jarass in Jarass/Pieroth, Art. 19, Rn. 9).

79 Die EMRK ist gem. Art. 6 II EUV eine der beiden Rechtserkenntnisquellen, aus denen die Gemeinschaftsgrundrechte abgeleitet werden.

${ }^{80}$ Kingreen in Calliess/Ruffert, Art. 6 EUV, Rn. 52; Gesagtes gelte etwa bei den klassischen Bürgerrechten wie dem Wahlrecht, der Freizügigkeit, aber auch dem Grundrecht auf Berufsfreiheit. (Kingreen in Calliess/Ruffert, Art. 6 EUV, Rn. 52).

81 Streinz in Sachs, Art. 25, Rn. 46. 
Pflicht des anderen (hier des Diskriminierenden) gegenübersteht. ${ }^{82}$ Es gibt nur wenige allgemeine Regeln des Völkerrechts, die dem einzelnen unmittelbar Pflichten auferlegen. ${ }^{83}$ Das Gebot, nicht zu diskriminieren, zählt nicht dazu. ${ }^{84}$ Selbst wenn dies der Fall wäre, ergäben sich aus der völkerrechtlichen Verpflichtung allein keine Rechtsfolgen. Die Übernahme einer völkerrechtlichen Regel in das innerstaatliche Recht läßt nämlich den Grundsatz vom Vorbehalt des Gesetzes unberührt. $^{85}$

\section{c) EU-Verordnungen}

Verordnungen können Individuen unmittelbar berechtigen und verpflichten. ${ }^{86}$ Dies macht Art. 249 II 2 EGV deutlich, indem er formuliert, daß die Verordnung unmittelbar in jedem Mitgliedstaat und nicht für jeden Mitgliedstaat gilt. ${ }^{87}$ Daraus folgt jedoch nicht, daß die Verordnung in jedem Fall unmittelbar anwendbar wäre. Dies ist sie nur, soweit sie self-executing ist, ${ }^{88}$ d.h. eine klare und unbedingte Verpflichtung begründet, die keiner weiteren Maßnahme der Gemeinschaftsorgane oder der Mitgliedstaaten bedarf und deshalb von staatlichen Behörden und Gerichten angewendet werden kann. ${ }^{89}$ Der self-executing-Charakter ist für jede Bestimmung einer Verordnung gesondert zu prüfen. ${ }^{90}$

Art. 7 I und IV, sowie Art. 8 der Verordnung 1612/68 sind in dem beschriebenen Sinne selfexecuting und demnach im innerstaatlichen Recht Deutschlands unmittelbar anwendbar. Anzumerken bleibt, daß aufgrund des self-executing-Charakters des Art. 7 IV der an anderer Stelle angesprochene Streit über die generelle Bindung Privater an Art. 39 II EGV an Bedeutung verliert. $^{91}$

\section{d) EU-Richtlinien}

82 Vergleiche dazu Streinz in Sachs, Art. 25, der bei den Individualrechten in Rn. 68 ff. die Menschenrechte aufführt, bei den Pflichten in Rn. 74 jedoch nicht.

83 Streinz in Sachs, Art. 25, Rn. 74 mit Beispielen (Verbot der Piraterie, Angriffe auf ausländische Staatsoberhäupter etc.)..

84 Eine solche Pflicht wird in der Literatur jedenfalls nicht erwähnt. (vgl. u.a. Rojahn in v. Münch/Kunig, Band 2, Art. 25, Rn. 30; Streinz in Sachs, Art. 25, Rn. 74).

85 Rojahn in v. Münch/Kunig, Band 2, Art. 25, Rn. 16; Streinz in Sachs, Art. 25, Rn. 46; „Vorbehalt des Gesetzes“ bedeutet, daß die vollziehende Gewalt ohne gesetzliche Grundlage nicht tätig werden darf. (Sachs in Sachs, Art. 20, Rn. 113).

86 Streinz, Europarecht, Rn. 380.

87 Bleckmann, Europarecht, Rn. 409.

88 Schweitzer/Hummer, Europarecht, Rn. 354.

89 EuGH, Rs. C-9/70, Slg. 1970, S. 825 (838, Rn. 5); Schroeder in Streinz, Art. 249, Rn. 60.

90 Schroeder in Streinz, Art. 249, Rn. 60.

91 Stieglitz, Allgemeine Lehren im Grundrechtsverständnis nach der EMRK und der Grundrechtsjudikatur des EuGH, S. 173. 
Richtlinien entfalten Rechtswirkungen in der innerstaatlichen Rechtsordnung regelmäßig erst nach ihrer pflichtgemäßen Umsetzung durch die Mitgliedstaaten. ${ }^{92}$ Es ist jedoch anerkannt, daß auch sie unter bestimmten Voraussetzungen unmittelbar anwendbar ${ }^{93}$ sind $^{94} \mathrm{Zu}$ diesen Voraussetzungen zählt, daß die Richtlinie self-executing-Charakter hat und die Umsetzungsfrist verstrichen ist, ohne daß die Richtlinie vollständig und richtig umgesetzt wurde. ${ }^{95}$

Die Umsetzungsfrist der bereits angesprochenen Richtlinien ist größtenteils verstrichen. ${ }^{96}$ Das gilt insbesondere auch für die Richtlinien 2000/43/EG und 2000/78/EG. ${ }^{97}$

Teilweise wird den zentralen Vorschriften beider Richtlinien self-executing-Charakter attestiert. ${ }^{98}$ Folge sei die unmittelbare Anwendung der Richtlinien im Staat-Bürger- (bzw. vertikalen) Verhältnis. $^{99}$

Während die Möglichkeit der unmittelbaren Anwendbarkeit von Richtlinien im vertikalen Verhältnis allgemein anerkannt ist, ${ }^{100}$ ist umstritten, ob sie auch im Bürger-Bürger- (bzw. horizontalen) Verhältnis in Betracht kommt. ${ }^{101}$ Der $E u G H$ hat dies bislang verneint und so beispielsweise die horizontale Wirkung der Richtlinie 76/207/EWG abgelehnt. ${ }^{102}$

Für die Rechtsprechung des $E u G H$ spricht die Tatsache, daß nur die Mitgliedstaaten Adressaten von Richtlinien sein können. ${ }^{103}$ Die unmittelbare Drittwirkung würde somit zu einer Belastung von Personen führen, an die sich die Richtlinie nicht richtet. ${ }^{104}$

Ohne die Frage nach der vollständigen oder richtigen Umsetzung der Antidiskriminierungsrichtlinien bereits an dieser Stelle zu beantworten, kann angemerkt werden,

92 Streinz, Europarecht, Rn. 395.

93 Teilweise wird in diesem Zusammenhang auch von unmittelbarer Wirkung bzw. Direktwirkung gesprochen. (Streinz, Europarecht, Rn. 395).

94 EuGH, Rs. C-152/84, Slg. 1986 1, S. 723 (748, Rn. 46); Das BVerfG hat die Rechtsprechung des EuGH bestätigt. Nach Auffassung des Gerichts ist es ,,methodisch nicht unvertretbar, wenn der Gerichtshof dann im Hinblick auf das Ziel, dem Marktbürger möglichst weitreichenden Rechtsschutz zu verschaffen, und der unstreitigen Verpflichtung der Mitgliedstaaten, Richtlinien zu befolgen, Nachdruck zu verleihen, zu dem Schluß gelangt, der Marktbürger könne sich in bestimmten begrenzten Fällen gegenüber dem Mitgliedstaat in einem Rechtsstreit auf die Richtlinie „berufen“ und der Mitgliedstaat könne ihm die Nichterfüllung der Richtlinie nicht entgegenhalten.“ (BVerfGE 75, S. 223 (240 f.).

95 EuGH, Rs. C-152/84, Slg. 1986 1, S. 723 (748, Rn. 46); Streinz, Europarecht, Rn. 400; Schmidt in von der Groeben/Schwarze, Band 4, Art. 249 EGV, Rn. 42.

96 Eine Ausnahme bildet insofern die Richtlinie 2004/113/EG. Gemäß ihres Art. 17 ist sie bis spätestens 21.12.2007 umzusetzen.

97 Richtlinie 2000/43/EG war gem. Art. 16 bis spätestens 19.07.2003, Richtlinie 2000/78EG gem. Art. 18 bis spätestens 02.12.2003 umzusetzen.

98 Thüsing, NJW 2003, S. 3441 (3442).

99 Thüsing, NJW 2003, S. 3441 (3442).

100 Streinz, Europarecht, Rn. 397; Hier würde ein andere Entscheidung zu dem grotesken Ergebnis führen, daß der Staat dem Bürger seine rechtswidrige Untätigkeit entgegenhalten könnte.

101 Streinz, Europarecht, Rn. 397.

102 EuGH, Rs. C-152/84, Slg. 1986 1, S. 723 (749, Rn. 48); Pernice/Mayer behaupten, der EuGH habe die unmittelbare Drittwirkung der den Art. 141 EGV ausgestaltenden Richtlinie 75/117/EWG angenommen, ohne dies jedoch zu belegen. (Pernice/Mayer in Grabitz/Hilf, Band I, nach Art. 6 EUV, Rn. 187).

103 Schmidt in von der Groeben/Schwarze, Band 4, Art. 249 EGV, Rn. 37 und 42; Gem. Art. 249 III EGV ist die Richtlinie ,[...] für jeden Mitgliedstaat, an den sie gerichtet wird, [...] verbindlich [...]“. Auch die Richtlinien selbst bezeichnen stets die Mitliedsstaaten als Adressaten selbiger. (siehe z.B. Art. 19 der Richtlinie 2000/43/EG oder Art. 21 der Richtlinie 2000/78/EG).

104 Streinz, Europarecht, Rn. 397.

196 
daß das am 18.08.2006 in Kraft getretene AGG der Umsetzung der Richtlinien 2000/43/EG, 2000/78/EG, 2002/73/EG sowie 2004/113/EG dienen soll.

\section{e) ,Soft-Law"}

Die Allgemeine Erklärung der Menschenrechte ist allein schon aufgrund der Tatsache, daß sie als soft law im innerstaatlichen Recht Deutschlands keine Geltung erlangt hat, nicht unmittelbar anwendbar. Gleiches gilt für die Gemeinschaftscharta der Sozialen Grundrechte der Arbeitnehmer und die Charta der Grundrechte der Europäischen Union.

\section{f) Grundrechte des Grundgesetzes}

Gemäß Art. 1 III GG binden die Grundrechte ,,[...] Gesetzgebung, vollziehende Gewalt und Rechtsprechung als unmittelbar geltendes Recht.“

\section{aa) Unmittelbare Drittwirkung}

Trotz dieses Wortlauts, der eine scheinbar deutliche Aussage gegen die Bindung Privater an die Grundrechte, mithin gegen deren unmittelbare Drittwirkung trifft, ${ }^{105}$ wurde eine solche teilweise angenommen. ${ }^{106}$

Die Vertreter der unmittelbaren Drittwirkung bzw. absoluten Wirkung der Grundrechte ${ }^{107}$ stützen ihre Ansicht auf eine Aussage des Bundesverfassungsgerichtes, wonach die Grundrechte über ihre Funktion als Abwehrrechte des Bürgers gegenüber dem Staat ${ }^{108}$ eine objektive Wertordnung aufgerichtet haben, die für alle Bereiche des Rechts, auch für das bürgerliche Recht, gilt. ${ }^{109}$ Das Bundesverfassungsgericht will den Einfluß dieser Wertordnung auf das Privatrecht jedoch in der Weise verstanden und erschöpft wissen, als neue Vorschriften des Privatrechts im Einklang mit dem grundrechtlichen Wertsystem stehen und bereits existente Vorschriften in seinem Geist ausgelegt werden müssen. ${ }^{110}$ Eine Bindung unabhängig von bereits bestehenden Gesetzen oder solchen, die

105 Pieroth/Schlink, Grundrechte, Staatsrecht II, Rn. 173; Von unmittelbarer Drittwirkung spricht man im Zusammenhang mit der Frage, ob Private in gleicher Weise wie die Staatsgewalt an die Grundrechte gebunden sind. (Dreier in Dreier, Band I, Vorb., Rn. 98).

106 BAGE 1, S. 185 (193); Enneccerus/Nipperdey, Allgemeiner Teil des Bürgerlichen Rechts, 1. Band, 1. Halbband, S. 93; Nipperdey, RdA 1950, S. 121 (125); Hager, JZ 1994, S. 373 (383); Leisner, Grundrechte und Privatrecht, 1960, S. 378; Klein, Der Staat 10 (1971), S. 145 (149)).

107 Nipperdey hält den Begriff Drittwirkung für ungenau. (Enneccerus/Nipperdey, Allgemeiner Teil des Bürgerlichen Rechts, 1. Band, 1. Halbband, S. 93 f.).

108 BVerfGE 7, S. 198 (204).

109 BVerfGE 7, S. 198 (205); Enneccerus/Nipperdey, Allgemeiner Teil des Bürgerlichen Rechts, 1. Band, 1. Halbband, S. 96.

110 BVerfGE 7, S. 198 (205). 
vom einfachen Gesetzgeber in Ausführung der Grundsatznorm erlassen werden, lehnt das Gericht mithin ab. ${ }^{111}$

Die Vertreter der unmittelbaren Drittwirkung erkennen, daß sich - anders als im Staat-BürgerVerhältnis - im Bürger-Bürger-Verhältnis zwei Grundrechtsträger gegenüberstehen. Die Anwendung der Verfassungsnormen im Privatrecht soll die Freiheitssphäre des einzelnen nur dort und insoweit einschränken, als es im Interesse des gesunden sozialen Lebens dringend erforderlich ist, ${ }^{112}$ keinesfalls also uneingeschränkt, wie im Staat-Bürger-Verhältnis. ${ }^{113}$

So dürfe die unmittelbare Drittwirkung des Art. 3 GG nicht zu einer ungebührlichen Einschränkung der durch Art. 2 I GG verbürgten privatrechtlichen Vertragsfreiheit führen. ${ }^{114}$ Letztlich bedürfe die Kollision beider Grundrechte einer Abwägung im Einzelfall, wobei grundsätzlich die Freiheit Vorrang habe, im Falle wirtschaftlicher Überlegenheit - zu denken sei insofern an das Arbeitsrecht - jedoch Art. 3 GG die Freiheitsinteressen überwiegen könne. ${ }^{115}$ Außerdem soll derjenige, der beim Angebot wichtiger Güter oder Dienstleistungen ein Monopol hat, Adressat des Art. 3 I GG sein, ${ }^{116}$ der allgemeine Gleichheitssatz in diesem Bereich mithin unmittelbare Drittwirkung entfalten.

\section{bb) Zurechnung privat bewirkter Beeinträchtigung zum Staat}

Eine andere Ansicht leitet den Grundrechtsschutz im Privatrechtsverkehr nicht über eine - wie auch immer geartete - Bindung Privater an die Grundrechte her. Nach dieser Ansicht wird auch im Privatrechtsverkehr keine ,private Gewalt“ entfaltet, der gegenüber die Grundrechtsgeltung erst noch zu erweisen ist. ${ }^{117}$ So liege beispielsweise bei privatrechtlichen Ansprüchen nichts anderes als staatliche Gewalt in Form von Ge- oder Verboten vor. ${ }^{118}$ Der Vertragspartner fürchte in einem solchen Fall nicht den „Befehl“ seines Kontrahenten, sondern den staatlichen Rechtsbefehl, der durch einen privatrechtlichen Vertrag ausgelöst werde. ${ }^{119}$

Konsequenz dieser Meinung, wonach Grundrechtsbeeinträchtigungen im Privatrechtsverkehr oft von der staatlichen Rechtsmacht herrühren, ist, daß diese Rechtsmacht und die von ihr ausgehenden Beeinträchtigungen gemäß Art. 1 III GG an den Grundrechten zu messen sind. ${ }^{120}$ Zum gleichen Ergebnis gelangt man, wenn man die Wirkung der Grundrechte im Privatrecht mit der staatlichen

111 So aber Enneccerus/Nipperdey, Allgemeiner Teil des Bürgerlichen Rechts, 1. Band, 1. Halbband, S. 93.

112 Enneccerus/Nipperdey, Allgemeiner Teil des Bürgerlichen Rechts, 1. Band, 1. Halbband, S. 95.

113 Nipperdey, Staatsrechtliche Abhandlungen verschiedener Autoren, Sammelband 6, Heft 2, 1961, S. 18; Heun weist deshalb zu recht darauf hin, daß selbst die Vertreter der unmittelbaren Drittwirkung selbige nicht im Sinne einer analog zur Staatsgewalt vorzustellenden vollständigen Grundrechtsbindung der Privatrechtssubjekte vertreten. (Heun in Dreier, Band I, Vorb., Rn. 98, Fn. 404).

114 Enneccerus/Nipperdey, Allgemeiner Teil des Bürgerlichen Rechts, 1. Band, 1. Halbband, S. 103.

115 Enneccerus/Nipperdey, Allgemeiner Teil des Bürgerlichen Rechts, 1. Band, 1. Halbband, S. 103.

116 Jarass in Jarass/Pieroth, Art. 3, Rn. 13.

117 Schwabe, Die sogenannte Drittwirkung der Grundrechte, S. 45.

118 Schwabe, NJW 1973, S. 229 (230).

119 Schwabe, NJW 1973, S. 229 (230).

120 Schwabe, Die sogenannte Drittwirkung der Grundrechte, S. 26. 198 
Schutzpflicht begründet, die eine der Grundrechtsfunktionen darstellen soll. ${ }^{121}$ Die Grundrechtswirkung auf die Privatrechtsverhältnisse ist auch unter dieser Prämisse Konsequenz der Bindung des Staates an die Grundrechte als unmittelbar geltendes Recht gem. Art. 1 III. ${ }^{122}$ Eine Sonderstellung soll Art. 3 GG einnehmen. Diese Norm entfalte prinzipiell keine Wirkung im Zivilrecht, da eine staatliche Teilhabe an privater Ungleichbehandlung fehle. ${ }^{123}$ Doch selbst wenn man diese These ablehnte und den Staat als an der Ungleichbehandlung Beteiligten ansähe, folgte daraus nicht die uneingeschränkte Wirkung des Art. 3 GG im Privatrechtsverkehr. ${ }^{124}$ Der höherwertige Grundsatz der Privatautonomie neutralisiere vielmehr grundsätzlich den Gleichheitssatz. ${ }^{125}$ Die radikale Gleichbehandlung im Privatrecht würde zum einen das System des Zivilrechts aus den Angeln heben und sei zum anderen praktisch nicht durchführbar. ${ }^{126}$

Letztlich kommt es aber auch nach dieser Ansicht auf das Ausmaß der Ungleichbehandlung an, so daß im Einzelfall beispielsweise eine Vertragsbestimmung aufgrund Verstoßes gegen Art. 3 III GG nichtig sein könne. ${ }^{127}$ Im Ergebnis bedarf es somit auch nach dieser Theorie einer Abwägung im Einzelfall für die Beantwortung der Frage, inwiefern Art. 3 GG im Privatrecht wirkt.

\section{cc) Mittelbare Drittwirkung}

Eine dritte Ansicht geht grundsätzlich von der Staatsgerichtetheit der Grundrechte aus. ${ }^{128}$ In der Privatrechtsordnung wirkten dagegen subjektiv private Rechte. ${ }^{129}$ Dieser Dualismus der Konstruktion dürfe aber nicht zu einem Dualismus der Rechtsmoral des Gesamtrechts führen. ${ }^{130}$ Der staatlichen Gewalt obliege nämlich gem. Art. 1 I GG über ihre Achtungspflicht hinaus eine Schutzpflicht gegenüber der Menschenwürde. ${ }^{131}$ Wenn die Verfassung in den einzelnen Grundrechten die wirksamste positivrechtliche Realisierung dieses Wertschutzes sieht, dann folge daraus, daß die in den Grundrechten dem Staat gegenüber abgesicherten Werte von der staatlichen Gewalt auch dann gewahrt werden müssen, wenn sie von privaten einzelnen oder gesellschaftlichen Kollektiven angegriffen werden. ${ }^{132}$ Der Weg, mit dessen Hilfe bei Fehlen spezieller zivilrechtlicher Schutznormen das objektive Privatrecht seinen Schutzauftrag erfüllt, verlaufe dabei über die

121 Murswiek in Sachs, Art. 1, Rn. $104 \mathrm{f}$.

122 Murswiek in Sachs, Art. 1, Rn. 105.

123 Schwabe, Die sogenannte Drittwirkung der Grundrechte, S. 151, Beispiel S. 149.

124 Schwabe, Die sogenannte Drittwirkung der Grundrechte, S. 150.

125 Schwabe, Die sogenannte Drittwirkung der Grundrechte, S. 150.

126 Schwabe, Die sogenannte Drittwirkung der Grundrechte, S. 151.

127 So sei die Vertragsbedingung, ein Grundstück nicht an Juden oder Farbige zu verkaufen, nichtig. Andererseits sei das Ausmaß der Diskriminierung gering, wenn der Grundstücksverkauf aufgrund der politischen Anschauungen eines Mitglieds der Regierungspartei nicht erfolgte. (Schwabe, Die sogenannte Drittwirkung der Grundrechte, S. 152).

128 Dürig in Maunz/Dürig, Band I, Art. 1 Abs. III, Rn. 131.

129 Dürig in Maunz/Dürig, Band I, Art. 1 Abs. III, Rn. 131.

130 Dürig in Maunz/Dürig, Band I, Art. 1 Abs. III, Rn. 131.

131 Dürig in Maunz/Dürig, Band I, Art. 1 Abs. III, Rn. 131.

132 Dürig in Maunz/Dürig, Band I, Art. 1 Abs. III, Rn. 131. 
wertausfüllungsfähigen und wertausfüllungsbedürftigen Begriffe und Generalklauseln des Privatrechts. ${ }^{133}$

Diese Lehre von der mittelbaren Drittwirkung ${ }^{134}$ der Grundrechte steht im Einklang mit der eingangs erwähnten Rechtsprechung des Bundesverfassungsgerichtes, nach der jede bürgerlichrechtliche Vorschrift im Geiste der durch das Grundgesetz aufgestellten objektiven Wertordnung ausgelegt werden muß, ${ }^{135}$ wobei gerade zwingendes Recht aufgrund seiner nahen Verwandtschaft mit dem öffentlichen Recht dem Einfluß des Verfassungsrechts ausgesetzt sein müsse und sich der Rechtsprechung zur Realisierung dieses Einflusses vor allem die Generalklauseln anböten, da sie, wie beispielsweise $\S 826$ BGB, zur Beurteilung menschlichen Verhaltens auf außer-zivilrechtliche bzw. sogar außerrechtliche Maßstäbe, wie die ,guten Sitten“ verwiesen. ${ }^{136}$ Durch Generalklauseln schafft der Gesetzgeber bewußt ${ }^{137}$ unvollständige Regelungen und überträgt dadurch dem Richter die Aufgabe der Rechtsfortbildung. ${ }^{138}$ Der Richter nimmt so im Wege der Auslegung die Funktion eines „Ersatzgesetzgebers“ wahr. ${ }^{139}$

Auch die Lehre von der mittelbaren Drittwirkung führt letztlich zu einer Abwägung wiederstreitender Interessen im Einzelfall, trete doch die mittelbare Grundrechtsgeltung in zahllosen Abstufungen bzw. einer beinahe stufenlosen Skala auf, ,,von (im Ergebnis) weitgehender Annäherung an die volle Grundrechtsbindung - [bei Art. 3 I GG] im Fall, daß ein privates Subjekt existenzwichtige Leistungen vergibt, eine Monopolstellung innehat oder sonst extreme Marktmacht besitzt - bis zur unteren Grenze, wo die mittelbare Grundrechtsbindung sich praktisch kaum mehr auswirkt - unter ,gleich Schwachen“, die sich, abgesehen von Beeinträchtigungen ihrer Persönlichkeitsrechte durch Ehrverletzungen, kaum grundrechtsberührend „wehtun“ können.“ “140

\section{dd) Stellungnahme}

136 BVEFE 7, S. 198 (205).

BVerfGE 7, S. 198 (206); Zwanziger in Kittner/Däubler/Zwanziger, Art. 3 GG, Rn. 22; Hirsch, RdA 1998, S. 194 (198); Gleichwohl sei jede Norm auslegungsfähig, was im Einzelfall zur Rechtfertigung von Entscheidungen gegen den Wortlaut des Gesetzes führen könne. (Alexy, Theorie der Grundrechte, S. 481) Beleg dafür ist etwa die Tatsache, daß die Rechtsprechung in den Kreis der in § 823 I BGB genannten Rechte auch das ,allgemeine Persönlichkeitsrecht" einbezieht, das der Gesetzgeber des Bürgerlichen Gesetzbuchs noch ablehnte. Das BVerfG hat keinen Anlaß gesehen, dieser Rechtsprechung von Verfassungs wegen entgegenzutreten. (BVerfGE 34, S. 269 (280 f.)).

137 Es geht hier nicht um die Fälle einer planwidrigen Regelungslücke.

138 Sack in Staudinger, $\S 138$, Rn. 24; Der Grund für diese partielle Kompetenzverschiebung ist darin zu sehen, daß kein Gesetzgeber jeden denkbaren Fall vorhersehen und spezialgesetzlich regeln kann. Durch Schaffung von Generalklauseln will er erreichen, mit Hilfe bereits bestehender Gesetze auch auf ungeregelte Probleme reagieren zu können. Außerdem sollen Generalklauseln durch ihre flexiblere Anwendbarkeit ermöglichen, daß sich das Recht gesellschaftlichen Veränderungen anpassen kann.

Rüthers in FAZ vom 02.02.2005, S. 7 unter dem Titel: „Diener oder Herren“.

140 Dürig in Maunz/Dürig, Band I, Art. 3 Abs. I, Rn. 511; zur mittelbaren Drittwirkung des Art. 3 I GG im Fall einer Monopolstellung vgl. auch Gubelt in v.Münch/Kunig, Band 1, Art. 3, Rn. 2; Heun in Dreier, Band I, Art. 3, Rn. 70. 
Der Streit um die „richtige Drittwirkungslehre“ wird dadurch relativiert, daß der Gesetzgeber seinen Schutzauftrag teilweise durch Schaffung einfachgesetzlicher Normen realisiert hat. Es besteht nämlich die Möglichkeit der Beeinflussung des Privatrechts durch Art. 3 GG dadurch, daß der Gesetzgeber von seiner Kompetenz Gebrauch macht, das Gleichheitsgebot im Privatrechtsverkehr durchzusetzen. ${ }^{141}$ So verpflichtet beispielsweise $\S 22$ Personenbeförderungsgesetz (PBefG) den Unternehmer, grundsätzlich jeden Antrag auf Abschluß eines bürgerlich-rechtlichen Beförderungsvertrages (Werkvertrag im Sinne der $\S \S 631$ ff. BGB) anzunehmen. ${ }^{142}$ Gemäß $\S 10$ I 1 des Energiewirtschaftsgesetzes (EnWG) haben Energieversorgungsunternehmen „für Gemeindegebiete, in denen sie die allgemeine Versorgung von Letztverbrauchern durchführen, Allgemeine Bedingungen und Allgemeine Tarife für die Versorgung in Niederspannung oder Niederdruck öffentlich bekanntzugeben und zu diesen Bedingungen und Tarifen jedermann an ihr Versorgungsnetz anzuschließen und zu versorgen. “143 Neben den soeben genannten Vorschriften ist auch der arbeitsrechtliche Gleichbehandlungsgrundsatz zu nennen, auf den an anderer Stelle bereits ausführlich eingegangen worden ist.

Da der Gesetzgeber vor der Schaffung des AGG lediglich in Teilbereichen einfachgesetzliche Regelungen zur Durchsetzung des Gleichbehandlungsgebots bzw. Diskriminierungsverbots geschaffen hatte, bleibt die Frage bedeutsam, welcher der vorgestellten Lehren zur Wirkung der Grundrechte im Zivilrecht der Vorzug einzuräumen ist. ${ }^{144}$ Folgendes Beispiel soll der Problemlösung dienen: Es wird vertreten, der arbeitsrechtliche Gleichbehandlungsgrundsatz sei ein Rechtsprinzip des Privatrechts ${ }^{145}$ und als solches strikt vom verfassungsrechtlichen Gleichheitssatz des Art. 3 I GG zu unterscheiden. ${ }^{146}$ Auch im Arbeitsrecht gelte Art. 3 I GG demnach nur mittelbar. ${ }^{147} \mathrm{Ob}$ dem gefolgt werden muß, oder bessere Gründe für die unmittelbare Drittwirkung ins Feld geführt werden können, ist letztlich egal. Denn selbst wenn man die mittelbare Drittwirkung vertritt, ändert dies nichts an der de facto unmittelbaren Drittwirkung des Art. 3 I GG im Arbeitsrecht bzw. in dem Bereich, in dem der Arbeitgeber an den arbeitsrechtlichen Gleichbehandlungsgrundsatz gebunden ist. ${ }^{148}$ Im Ergebnis soll der Arbeitgeber in bestimmten Bereichen an den (verfassungs- bzw. zivilrechtlichen) Gleichbehandlungsgrundsatz gebunden sein.

Ob der einzelne seinen Anspruch direkt aus Art. 3 GG (unmittelbare Drittwirkung) oder aber aus

141 Heun in Dreier, Band I, Art. 3, Rn. 71; Starck in von Mangoldt/Klein/Starck, Band 1, Art. 3 Abs. 1, Rn. 268.

142 Fromm/Fey/Sellmann/Zuck, Personenbeförderungsrecht, § 22, Rn. 1.

143 Soweit nicht einschlägig, sollen die $\S \S 138,826$ BGB und vor allem das Mißbrauchs- und Diskriminierungsverbot des Gesetzes gegen Wettbewerbsbeschränkungen (vgl. 20 I GWB) gewährleisten, die durch $\S 10$ EnWG angestrebten Ziele und statuierten Rechtsfolgen durchzusetzen. Schutz biete zudem $\S 823$ II BGB, da $\S 10$ EnWG Schutzgesetz im Sinne dieser Norm sei. (Danner in Danner/Theobald, I B, § 10 EnWG, Rn. 2 bzw. 35).

144 Beachte, daß im Rahmen des Prüfungspunktes „Notwendigkeit legislativer Maßnahmen“ die Situation betrachtet werden soll, die sich vor dem Inkrafttreten des AGG am 18.08.2006 insofern bot.

145 BAGE 71, S. 29 (35).

146 Osterloh in Sachs, Art. 3, Rn. 191.

147 Osterloh in Sachs, Art. 3, Rn. 75; wohl auch Starck (Starck in von Mangoldt/Klein/Starck, Band 1, Art. 3 Abs. 1, Rn. 272 a.E.).

148 Gubelt in v.Münch/Kunig, Band 1, Art. 3, Rn. 54. 
dem arbeitsrechtlichen Gleichbehandlungsgrundsatz (mittelbare Drittwirkung) herleitet, ist unerheblich, soweit beide Ansprüche den gleichen Inhalt haben.

In diesem Sinne wird behauptet, eine Entscheidung für die Lehre der unmittelbaren oder mittelbaren Drittwirkung der Grundrechte bzw. die Lehre von der Zurechnung privat bewirkter Beeinträchtigung zum Staat sei entbehrlich, da mit ihnen in allen Fällen dasselbe Ergebnis erzielt werden könne. ${ }^{149}$ Diese zunächst verblüffende Behauptung ist nachvollziehbar, wenn man sich noch einmal vergegenwärtigt, daß keine der genannten Lehren, nicht einmal die der unmittelbaren Drittwirkung, eine staatsgleiche Grundrechtsbindung der Privatrechtssubjekte vertritt. Die Vertreter der verschiedenen Ansichten erkennen, daß im Bürger-Bürger-Verhältnis auf beiden Seiten Grundrechtsträger stehen ${ }^{150}$ und demnach letztlich abzuwägen sei, inwieweit den widerstreitenden Grundrechten Wirkung im Privatrechtsverhältnis zukommt. ${ }^{151}$ Unter dieser Prämisse verliert ,,selbst der verfassungsrechtliche Gleichheitssatz im Vertragsrecht seinen Schrecken, der doch stets im Geruche stand, das System des Zivilrechts aus den Angeln zu heben. “152

\section{Mittelbare Drittwirkung der Antidiskriminierungsmaßnahmen}

Vor dem Hintergrund der gewonnenen Erkenntnis, daß nicht alle der untersuchten Antidiskriminierungsmaßnahmen im Privatrechtsverhältnis unmittelbar anwendbar sind, stellt sich die Frage, ob die nicht unmittelbar anwendbaren Maßnahmen wenigstens eine mittelbare Drittwirkung entfalten.

\section{a) Völkerrechtliche Verträge}

Es ist anerkannt, daß völkerrechtliche Verträge das innerstaatliche Recht mittelbar beeinflussen können, auch wenn ihre unmittelbare Anwendbarkeit verneint wird. ${ }^{153}$ Begründen läßt sich dies mit

149 Alexy, Theorie der Grundrechte, S. 483; ihm folgend Nickel, Gleichheit und Differenz in der vielfältigen Republik, S. 137; Starck ist zwar der Meinung, daß man sich nicht mit dem Hinweis auf gleiche Ergebnisse dem Theorienstreit zwischen unmittelbarer und mittelbarer Drittwirkung entziehen sollte, weist jedoch auch darauf hin, $\mathrm{daß}$ sich beide Theorien nicht so fern stehen, wie es vielleicht zunächst scheine. (Starck in von Mangoldt/Klein/Starck, Band 1, Art. 1 Abs. 3, Rn. 265 f.); Auch Schwabe vertritt die Ansicht, die unmittelbare Drittwirkung erzwinge keine härteren Lösungen. (Schwabe, NJW 1973, S. 229 (230)) Heun formuliert im Zusammenhang mit den verschiedenen Drittwirkungslehren, daß ,über deren Lösung nicht selten Einigkeit herrscht". (Heun in Dreier, Band I, Vorb., Rn. 99).

150 Alexy weist darauf hin, daß die Privatautonomie selbst, nicht nur ihre Begrenzung, Gegenstand grundrechtlicher Garantien und damit der Drittwirkung ist. (Alexy, Theorie der Grundrechte, S. 491).

151 Enneccerus/Nipperdey, Allgemeiner Teil des Bürgerlichen Rechts, 1. Band, 1. Halbband, S. 103; Hager, JZ 1994, S. 373 (383); Schwabe, Die sogenannte Drittwirkung der Grundrechte, S. 152; Dürig in Maunz/Dürig, Band I, Art. 3 Abs. I, Rn. 511; bzgl. der mittelbaren Drittwirkung des Art. 3 I GG im Fall einer Monopolstellung vgl. auch Gubelt in v.Münch/Kunig, Band 1, Art. 3, Rn. 2; Heun in Dreier, Band I, Art. 3, Rn. 70; Alexy, Theorie der Grundrechte, S. 491; ihm folgend Nickel, Gleichheit und Differenz in der vielfältigen Republik, S. 138. 
dem in der deutschen Verfassung verankerten Grundsatz der Völkerrechtsfreundlichkeit, ${ }^{154}$ der u.a. besagt, daß innerstaatliches Recht völkerrechtskonform auszulegen ist. ${ }^{155}$

Verwiesen wird in diesem Zusammenhang wiederum vor allem auf die unbestimmten Rechtsbegriffe, wie z.B. die ,wertausfüllungsfähigen und -bedürftigen Generalklauseln des Privatrechts“156 oder die polizeirechtlichen Begriffe der öffentlichen Sicherheit und Ordnung. ${ }^{157}$ Der Richter nimmt so im Wege völkerrechtskonformer Auslegung die Funktion eines „Ersatzgesetzgebers“"158 wahr und setzt in dieser Eigenschaft völkerrechtliche Verpflichtungen anstelle des Gesetzgebers um.

Die Auslegung setzt voraus, daß eine völkerrechtliche Verpflichtung des Gesetzgebers besteht. Der überwiegende Teil der genannten völkerrechtlichen Verträge erfüllt, wie bereits nachgewiesen, diese Voraussetzung.

Desweiteren ist erforderlich, daß sich die Verpflichtung des Gesetzgebers - soll es zur Auslegung von Bestimmungen des Zivilrechts kommen - auf das Privatrecht erstrecken muß. Diese Voraussetzung ist beispielsweise im Fall des Art. 39 EGV erfüllt, umfaßt die Verpflichtung der Mitgliedstaaten aus selbiger Norm doch den Schutz vor sämtlichen freiheitsbeschränkenden Verhaltensweisen durch Private. ${ }^{159}$ Auch bei Art. 5 lit. f des Internationalen Übereinkommens zur Beseitigung jeder Form von Rassendiskriminierung steht die Möglichkeit der mittelbaren Drittwirkung außer Frage, fordert die Norm doch den Zugang zu Einrichtungen - wie z.B. Cafés -, die überwiegend privat betrieben werden.

Überall dort, wo eine Verpflichtung des Gesetzgebers nicht besteht - zu denken ist etwa an Art. 1 III der UN-Charta -, sind die Ziele des betreffenden Vertrages bzw. der betreffenden Bestimmung auf anderem Wege zu erreichen. ${ }^{160}$ So sei z. B. das Internationale Übereinkommen zur Beseitigung jeder Form von Rassendiskriminierung Antwort auf die allgemeine Verpflichtung aus Art. 1 III der UN-Charta. ${ }^{161}$

Es gibt Bestimmungen in völkerrechtlichen Verträgen, die die Vertragsstaaten nur unter bestimmten Voraussetzungen verpflichten. In einem solchen Fall kommt eine Drittwirkung soweit in Betracht, als die Verpflichtung besteht. So sei in dem Umfang, in dem die Mitgliedstaaten an die

154 BVerfGE 6, S. 309 (362).

155 BVerfGE 75, S. 1 (19); Pernice in Dreier, Band II, Art. 25, Rn. 40.

156 Dürig in Maunz/Dürig, Band I, Art. 3 III, Rn. 68.

157 Partsch, GYIL 20, S. 119 (127); Kühner, NJW 86, S. 1397 (1400).

158 Rüthers in FAZ vom 02.02.2005, S. 7 unter dem Titel: „Diener oder Herren“.

159 Streinz/Leible, EuZW 2000, S. 459 (466); Remmert, JURA 2003, S. 13 (17); Die Frage der mittelbaren Drittwirkung des Art. 39 EGV stellt sich freilich nur insoweit, als dessen unmittelbare Drittwirkung abgelehnt wird. (vgl. dazu die bereits an anderer Stelle gemachten Ausführungen).

160 Bei völkerrechtlichen Bestimmungen, bei denen umstritten ist, ob sie die Vertragsstaaten verpflichten - zu denken ist z.B. an Art. $13 \mathrm{EGV} \mathrm{-,} \mathrm{hängt} \mathrm{die} \mathrm{Beantwortung} \mathrm{der} \mathrm{Frage} \mathrm{der} \mathrm{mittelbaren} \mathrm{Drittwirkung} \mathrm{freilich} \mathrm{von} \mathrm{der}$ Beantwortung dieser Vorfrage ab.

161 Haedrich, JA 2003, S. 899 (899). 
Gemeinschaftsgrundrechte gebunden sind, eine mittelbare Drittwirkung selbiger in Form gemeinschaftsgrundrechtskonformer Auslegung nationalen Rechts möglich. ${ }^{162}$

\section{b) EU-Richtlinien}

Obwohl Richtlinien keine unmittelbare Drittwirkung entfalten, können sie doch - trotz Nichtumsetzung - Einfluß auf Rechtsverhältnisse zwischen Privaten haben. Die Mitgliedstaaten sind zur richtlinienkonformen Auslegung allen nationalen Rechts verpflichtet, daß sich im Regelungsbereich einer Richtlinie befindet. ${ }^{163}$ Der $E u G H$ hat in diesem Zusammenhang festgestellt, das Verbot der unmittelbaren Wirkung von Richtlinien sei nicht verletzt, wenn der nationale Richter richtlinienwidriges nationales Recht nicht anwendet. ${ }^{164}$

Die Verpflichtung, nationales Recht richtlinienkonform auszulegen, gilt vom Ablauf der Umsetzungsfrist an ${ }^{165}$ und setzt sich auch dann fort, wenn die Richtlinie bereits ordnungsgemäß in nationales Recht umgesetzt worden ist. ${ }^{166}$

\section{c) ,Soft-Law"}

Da soft law Staaten nicht verpflichtet, kann es keine mittelbare Drittwirkung im innerstaatlichen Recht eines Staates entfalten. ${ }^{167}$

\section{d) Grundrechte des Grundgesetzes}

162 Szczekalla, Die sogenannten grundrechtlichen Schutzpflichten im deutschen und europäischen Recht, S. 708 f.; Hirsch, RdA 1998, S. 194 (197 u. 198).

163 EuGH, Rs. C-106/89, Slg. 1990 I 3, S. 4135 (4159, Rn. 8); verb. Rs. C-240-98 und C-244/98, Slg. 2000 I 6, S. 4941 (4975, Rn. 30); Streinz, Europarecht, Rn. 404.

164 EuGH, Rs. C-443/98, Slg. 2000 8/9 (B), S. 7535 (7584 f., Rn. 50-52); Streinz, Europarecht, Rn. 399 a

Teilweise wird die Rechtsprechung des EuGH zur richtlinienkonformen Auslegung als widersprüchlich bezeichnet, führe sie doch im Ergebnis zu einer unmittelbaren Drittwirkung, die das Gericht gerade ablehnt. (Biervert in Schwarze, Art. 249 EGV, Rn. 30) Bei genauer Betrachtung der Rechtsinstitute zeigt sich jedoch, daß sich beide keineswegs in der von den Kritikern beschriebenen Weise bedingen, unmittelbare Drittwirkung und richtlinienkonforme Auslegung vielmehr strikt voneinander zu trennen sind. (so auch Ruffert in Calliess/Ruffert, Art. 249 EGV, Rn. 111) Bereits an anderer Stelle wurde festgestellt, daß der Richter in bestimmten Bereichen - so z.B. im Rahmen der Auslegung der Generalklauseln des Zivilrechts - die Funktion eines „Ersatzgesetzgebers“ wahrnimmt. Daraus ergibt sich die Konsequenz, daß richtlinienkonforme Auslegung nicht die unmittelbare Drittwirkung der Richtlinie „durch die Hintertür“ ist, sondern deren tatsächliche Umsetzung. Eine umgesetzte Richtlinie kann aber zweifellos in Privatrechtrechtsverhältnisse eingreifen.

$165 E u G H$, verb. Rs. C-240/98 und C-244/98, Slg. 2000 I 6, S. 4941 (4975, Rn. 31).

Fraglich ist, ob schon vor Ablauf der Umsetzungsfrist die Verpflichtung zur richtlinienkonformen Auslegung besteht. (vgl. dazu Streinz, Europarecht, Rn. 407) Dieses Problem kann hier vernachlässigt werden, da die jeweilige Umsetzungsfrist der im Rahmen dieser Arbeit diskutierten Richtlinien größtenteils abgelaufen ist. Eine Ausnahme bildet insofern die Richtlinie 2004/113/EG. Gemäß ihres Art. 17 ist sie bis spätestens 21.12.2007 umzusetzen.

166 EuGH, Rs. C-421/92, Slg. 1994 I 2, S. 1657 (1673, Rn. 9 f.); Streinz, Europarecht, Rn. 397; Thüsing, NJW 2003, S. 3441 (3442).

167 Gesagtes gilt für die Allgemeinen Erklärung der Menschenrechte, die Charta der Grundrechte der Europäischen Union sowie für die Gemeinschaftscharta der Sozialen Grundrechte der Arbeitnehmer. 
Es wurde an anderer Stelle bereits ausführlich erläutert, daß die Grundrechte des Grundgesetzes Einfluß auch auf Privatrechtsbeziehungen haben. Wie dieser Einfluß erfolgt, ist umstritten. Es hat sich jedoch gezeigt, daß die verschiedenen Drittwirkungslehren zu den gleichen Ergebnissen führen können, so daß die Entscheidung für eine der Lehren nicht erforderlich ist. Deshalb ist an dieser Stelle die Behauptung zulässig, die Grundrechte entfalten eine mittelbare Drittwirkung im Zivilrecht.

\section{Resümee - Legislativer Handlungsbedarf}

Die Ausführungen $\mathrm{zu}$ den Auswirkungen der untersuchten (inter-)nationalen Antidiskriminierungsmaßnahmen auf das Privatrechtsverhältnis haben ergeben, daß einige dieser Maßnahmen Private unmittelbar berechtigen und verpflichten. Das gilt selbstverständlich für zivilrechtliche Normen wie - vor Inkrafttreten des AGG - § 611 a BGB-A, ${ }^{168}$ aber auch für die untersuchten Bestimmungen der Verordnung 1612/68 sowie nach nicht unumstrittener Rechtsprechung des EuGH für Art. 141 EGV. Diskutiert wird die unmittelbare Drittwirkung der Art. 12 und 39 EGV, der Gemeinschaftsgrundrechte sowie der Grundrechte des Grundgesetzes.

Das Gros der untersuchten Antidiskriminierungsmaßnahmen entfaltet eine mittelbare Drittwirkung im Privatrechtsverhältnis. Das gilt für alle völkerrechtlichen Verträge, die den Gesetzgeber verpflichten, Schutz vor Diskriminierung auch im Privatrechtsverkehr zu gewährleisten. Dasselbe gilt für die Antidiskriminierungsrichtlinien. Auch die Grundrechte des Grundgesetzes sind mittelbar drittwirkend, sofern man nicht deren unmittelbare Drittwirkung vertritt.

Die Feststellung, daß die meisten der untersuchten Antidiskriminierungsmaßnahmen zumindest mittelbar drittwirkend sind, wirft die Frage auf, ob angesichts dessen vor Inkrafttreten des AGG überhaupt noch legislativer Handlungsbedarf des deutschen Gesetzgebers bestand. Diese Frage stellt sich freilich nur dann, wenn die mittelbare Drittwirkung zu einem Diskriminierungsschutz führt, der von der jeweiligen Maßnahme verlangt wird. Diese Voraussetzung wird teilweise als erfüllt angesehen.

So wird beispielsweise vertreten, der wesentliche Inhalt der Antidiskriminierungsrichtlinien 2000/43/EG und 2000/78/EG könne für das allgemeine Zivilrecht sowie für das Arbeitsrecht durch richtlinienkonforme Auslegung der $\S \S 138,826$ BGB umgesetzt werden. ${ }^{169}$ Ähnliche Argumente

Beachte, daß im Rahmen des Prüfungspunktes „Notwendigkeit legislativer Maßnahmen“ die Situation betrachtet werden soll, die sich vor dem Inkrafttreten des AGG am 18.08.2006 insofern bot.

169 Heinrichs in Palandt, Anh nach $\S 319$, Rn. 2. 
sind auch gegen die Notwendigkeit einer legislativen Umsetzung von Verpflichtungen aus völkerrechtlichen Verträgen ins Feld geführt worden. ${ }^{170}$

Demgegenüber wurde vor Inkrafttreten des AGG vertreten, daß Diskriminierungsschutz in gewissem Umfang und bei moderner Auffassung des (Quasi-)Vertragsrechts de lege lata zwar bestehe, die nach wie vor vorhandenen Schutzlücken indes durch gesetzgeberische Maßnahmen geschlossen werden müßten. ${ }^{171}$

Zumindest für die Antidiskriminierungsrichtlinien, die im Kreis der untersuchten Antidiskriminierungsmaßnahmen eine hervorragende Bedeutung haben, ist festzustellen, daß für ihre Umsetzung legislatives Tätigwerden zwingend erforderlich war. Der EuGH hat entschieden, daß ,....] eine etwa bestehende nationale Rechtsprechung, die innerstaatliche Rechtsvorschriften in einem Sinn auslegt, der als den Anforderungen einer Richtlinie entsprechend angesehen wird, nicht die Klarheit und Bestimmtheit aufweisen kann, die notwendig sind, um dem Erfordernis der Rechtssicherheit zu genügen. “"

Auch bezüglich Art und Weise der Umsetzung von Verpflichtungen aus völkerrechtlichen Verträgen wird teilweise von einem eingeschränkten Ermessen der Vertragsparteien gesprochen. ${ }^{173}$ Es wird vertreten, die Staaten hätten ,durch geeignete gesetzliche Vorschriften und Sanktionen die Einhaltung des Diskriminierungsverbotes durch alle Rechtsunterworfenen zu gewährleisten“ “. ${ }^{174}$

Vor allem aufgrund der soeben erwähnten Rechtsprechung des EuGH zur Umsetzung von Richtlinien ist die eingangs gestellte Frage nach der Notwendigkeit legislativer Maßnahmen zu bejahen. Die weiteren Ausführungen dienen dem Ziel zu ergründen, ob der deutsche Gesetzgeber dem durch Schaffung des AGG in adäquater Weise Rechnung getragen hat. Im Anschluß daran soll ein eigener Vorschlag für die gesetzestechnische Umsetzung des Diskriminierungsverbotes unterbreitet werden.

\section{B. Das Allgemeine Gleichbehandlungsgesetz und alternative Gesetzesentwürfe}

170 Vergleiche insofern bzgl. des Internationalen Übereinkommens zur Beseitigung jeder Form von Rassendiskriminierung Kühner, NJW 1986, S. 1397 (1398).

171 Schiek, Differenzierte Gerechtigkeit, S. 359 ff. und insb. Zusammenfassung S. 409.

172 EuGH, NJW 2001, S. 2244 (2245, Rn. 21).

173 Kühner, dessen Ausführungen sich auf das Internationalen Übereinkommen zur Beseitigung jeder Form von Rassendiskriminierung beziehen (Kühner, NJW 1986, S. 1397 (1398)).

174 Delbrück, dessen Aussage auf die Rassendiskriminierung beschränkt ist (Delbrück, Die Rassenfrage als Problem des Völkerrechts und nationaler Rechtsordnungen, S. 113). Bereits an anderer Stelle wurde festgestellt, daß aus Art. 3 I u. III GG keine Verpflichtung des Gesetzgebers zur Schaffung eines Diskriminierungsschutzgesetzes folgt; daß der Gesetzgeber jedoch aus Art. 3 II GG verpflichtet ist, seine Rechtsunterworfenen - in welcher Form auch immer - vor Diskriminierung aufgrund des Geschlechts durch Private zu schützen. (vgl. Teil 1, Prüfungspunkte A., B. u. F.). 
„Schon in den vergangenen Jahren des parteiübergreifenden Gezänks um die Umsetzung der europäischen Antidiskriminierungsrichtlinien beschlich den unbeteiligten Bürger das Gefühl, einem gesetzgeberischen Laienspiel beizuwohnen. Mal war das eine Ministerium zuständig, mal das andere, es versanken fertige Entwürfe wieder in der Schublade, weil die Legislaturperiode zu Ende war. Dann kam im dritten Anlauf der überstürzte Kompromiß der großen Koalition - der sich als schlecht gemachter Kuhandel entpuppte -, und alles wurde noch skurriler.“175

Als erster Versuch, ein Diskriminierungsschutzgesetz zu schaffen, das in weiten Teilen des Zivilrechtsverkehrs Geltung beanspruchen sollte, kann der auf die Diskriminierungsmerkmale Hautfarbe, Nationalität, ethnische Herkunft, Kultur sowie Religionszugehörigkeit beschränkte Entwurf eines Gesetzes gegen Rassismus und Diskriminierung ausländischer Bürgerinnen und Bürger (Antirassismusgesetz) ${ }^{176}$ der Gruppe der PDS vom 17.05.1995 gewertet werden. Am 19.01.1998 legte die Fraktion von Bündnis 90/Die Grünen den Entwurf eines Gesetzes zum Schutz vor Diskriminierung und zur Stärkung von Minderheitenrechten (Antidiskriminierungs- und Minderheitenrechtsgesetz) ${ }^{177}$ vor. Am 09.03.1998 folgte der Entwurf eines Gesetzes zur Durchsetzung des Gleichbehandlungsgebotes des Artikels 3 Grundgesetz (Gleichbehandlungsgesetz) ${ }^{178}$ der SPD-Fraktion.

Mit dem Diskussionsentwurf eines Gesetzes zur Verhinderung von Diskriminierungen im Zivilrecht $^{179}$ des Bundesjustizministeriums vom 10.12.2001 sollte erstmals eine Antidiskriminierungsrichtlinie - Richtlinie 2000/43/EG - umgesetzt werden. Über dieses primäre Ziel hinausgehend bezog der Gesetzesentwurf neben der Rasse und ethnischen Herkunft auch die Merkmale Geschlecht, Religion, Weltanschauung, Behinderung, Alter sowie sexuelle Identität in den Schutzbereich des von ihm statuierten Diskriminierungsverbotes ein. Der Entwurf eines Gesetzes zum Schutz vor Diskriminierungen (Antidiskriminierungsgesetz $-A D G)^{180}$ des Familienministeriums vom 06.05.2004 bezweckte seinerseits, die Richtlinien 2000/43/EG, 2000/78/EG sowie die durch die Richtlinie 2002/73/EG geänderte Richtlinie 76/207/EWG in innerstaatliches Recht umzusetzen.

Nachdem all die genannten Entwürfe für ein allgemeines Antidiskriminierungsgesetz zur Diskussion gestellt und verworfen worden waren, drohte die Europäische Kommission im Juli 2004 unter anderem Deutschland mit der Einleitung von Vertragsverletzungsverfahren wegen Nichtumsetzung von EU-Richtlinien. ${ }^{181}$ Daraufhin legten die Fraktionen von SPD und Bündnis 90/Die Grünen, deren Parteien zu diesem Zeitpunkt die Regierung stellten, am 16.12.2004 den Entwurf eines Gesetzes zur Umsetzung europäischer Antidiskriminierungsrichtlinien ${ }^{182}$ vor, der mit

175 FAZ vom 01.08.06, S. 11, unter dem Titel: „Groteske Pannenserie“.

176 BT-Druck. 13/1466.

177 BT-Druck. 13/9706.

178 BT-Druck. 13/10081.

179 Http://www.lsvd.de/antidiskriminierung/adgzivil02.html.

180 Http://www.deutsche-gesellschaft.de/modules.php?name=News\&file=article\&sid = 120 .

181 Frankfurter Rundschau vom 20.07.2004, S. 5.

182 BT-Druck. 15/4538. 
rot-grüner Mehrheit am 17.06.2005 vom Bundestag verabschiedet wurde. ${ }^{183}$ Neben den bereits erwähnten Richtlinien sollte durch diesen Gesetzesentwurf auch die Richtlinie 2004/113/EG umgesetzt werden. Der CDU/CSU-dominierte Bundesrat legte am 08.07.2005 Einspruch gegen das Gesetzeswerk ein und beschloß, den Vermittlungsausschuß mit dem Ziel einzuberufen, den Gesetzesentwurf im Sinne einer Beschränkung auf das europarechtlich zwingend gebotene zu überarbeiten. ${ }^{184}$ Aufgrund der vorgezogenen Bundestagswahl im Herbst 2005, die zu neuen Machtverhältnissen im Parlament führte - die Regierungsverantwortung übernahm eine (große) Koalition aus CDU/CSU und SPD -, scheiterte die rot-grüne Gesetzesinitiative aus dem Jahr 2004 letztlich.

Mit dem Ziel, die genannten Antidiskriminierungsrichtlinien in innerstaatliches Recht umzusetzen, legte die nunmehr schwarz/rote Bundesregierung am 08.06.2006 den Entwurf eines Gesetzes zur Umsetzung europäischer Richtlinien zur Verwirklichung des Grundsatzes der Gleichbehandlung ${ }^{185}$ vor. In einer Stellungnahme vom 16.06.2006 forderte der Bundesrat, diverse Änderungen an dem geplanten Gesetz vorzunehmen. ${ }^{186}$ Der Rechtsausschuß des Bundestages überarbeitete daraufhin den Gesetzesentwurf und empfahl dem Bundestag am 28.06.2006, den Gesetzesentwurf in der geänderten Fassung anzunehmen. ${ }^{187}$ Am 29.06.2006 nahm der Bundestag den überarbeiteten Gesetzesentwurf an. ${ }^{188}$ Der Bundesrat verzichtete am 07.07.2006 angesichts der vorgenommenen Änderungen auf eine Einberufung des Vermittlungsausschusses. ${ }^{189}$ Am 14.08.2006 wurde das Gesetz ausgefertigt, am 17.08.2006 im Bundesgesetzblatt verkündet. ${ }^{190}$ Gemäß seines Art. 4 tritt das Gesetz ,am Tag nach der Verkündung in Kraft.“ Das Gesetz zur Umsetzung europäischer Richtlinien zur Verwirklichung des Grundsatzes der Gleichbehandlung ist demnach am 18.08.2006 in Kraft getreten.

Dadurch ist der deutsche Gesetzgeber seinem legislativen Handlungsauftrag, wenn auch verzögert, formal nachgekommen. Fraglich ist, ob es ihm mit dem Gesetz auch gelungen ist - auch mit Blick auf den im Rahmen der Arbeit entwickelten Diskriminierungsbegriff -, eine materiell-rechtlich gelungene Problemlösung zu erarbeiten. Mit dem Ziel, eine Antwort auf diese Frage zu finden, soll das Gesetzeswerk im folgenden eingehend untersucht werden. Als Gegenentwurf sollen im Anschluß daran die bereits erwähnten Gesetzesentwürfe der Fraktionen Bündnis 90/Die Grünen und SPD aus dem Jahr 1998 näher vorgestellt werden.

\footnotetext{
183 BR-Druck. 445/05.

184 BR-Druck. 445/05 (Beschluß).

185 BT-Druck. 16/1780.

186 BT-Druck. 16/1852.

187 BT-Druck. 16/2022.

188 BR-Druck. 466/06.

189 BR-Druck 466/06 (Beschluß).

190 BGBl. 2006 I, S. 1897 ff.
} 


\section{Allgemeines Gleichbehandlungsgesetz (AGG) von 2006}

Das am 18.08.2006 in Kraft getretene Gesetz zur Umsetzung europäischer Richtlinien zur Verwirklichung des Grundsatzes der Gleichbehandlung enthält in seinem Artikel 1 ein Allgemeines Gleichbehandlungsgesetz (AGG). Das AGG unterscheidet in seinen Abschnitten 2 und 3 zwischen dem „Schutz der Beschäftigten vor Benachteiligung“ und dem „Schutz vor Benachteiligung im Zivilrechtsverkehr“. Die zentralen Vorschriften beider Abschnitte bilden die in den $\S \S 7$ und 19 $\mathrm{AGG}^{191}$ statuierten Benachteiligungsverbote. Den besonderen Bestimmungen der Abschnitte 2 und 3 ist in Abschnitt 1 ein „Allgemeiner Teil“" vorangestellt.

\section{Diskriminierungsrelevantes Verhalten - § 3 AGG}

$\S 3$ definiert, was nach dem Gesetz unter dem Begriff der Benachteiligung zu verstehen ist. ${ }^{192}$ Die Absätze 1 bis 5 unterscheiden dabei zwischen unmittelbarer Benachteiligung, mittelbarer Benachteiligung, Belästigung, sexueller Belästigung und der Anweisung zur Benachteiligung. Auf die einzelnen Begriffe muß nicht näher eingegangen werden, da das AGG insofern die Definitionen der Richtlinien im wesentlichen übernommen hat.

$\mathrm{Zu}$ bemängeln ist, daß das Gesetz wie die Antidiskriminierungsrichtlinien nicht bestimmt, daß eine Diskriminierung auch dann vorliegen kann, wenn ein Vergleich zu einer begünstigten Person nicht möglich ist, weil eine solche Person nicht existiert. ${ }^{193}$

\section{Diskriminierungsmerkmale - 1 AGG}

Gemäß seines $§ 1$ ist Ziel des AGG,

„[...] Benachteiligungen aus Gründen der Rasse oder wegen der ethnischen Herkunft, des Geschlechts, der Religion oder Weltanschauung, einer Behinderung, des Alters oder der sexuellen Identität zu verhindern oder zu beseitigen.“194

Die Bestimmung steht mit ihrer Beschränkung des Diskriminierungsschutzes auf ausgewählte Merkmale in Widerspruch zu einer elementaren Aussage der vorliegenden Arbeit, daß nämlich

191 Paragraphen ohne Gesetzesangabe sind im folgenden solche des AGG.

192 Das Gesetz spricht von „Benachteiligung“ und nicht von „Diskriminierung“ um deutlich zu machen, daß nicht jede unterschiedliche Behandlung, die mit der Zufügung eines Nachteils verbunden ist, diskriminierenden Charakter hat. (Entwurfsbegründung, BT-Druck. 16/1780, S. 30).

193 Vergleiche dazu Teil 2, Prüfungspunkt A. II. 1.

194 In bezug auf das Diskriminierungsmerkmal „Geschlecht“ ist auf $\S 3$ I 2 hinzuweisen, der bestimmt, daß eine unmittelbare Benachteiligung wegen des Geschlechts im sachlichen Geltungsbereich des § 2 I Nr. 1 - 4 ,,auch im Fall einer ungünstigeren Behandlung einer Frau wegen Schwangerschaft oder Mutterschaft" vorliegt. 
Diskriminierungsschutz nicht beschränkt sein darf auf bestimmte Merkmale, der Katalog potenzieller Diskriminierungsmerkmale vielmehr „unendlich“ lang ist.

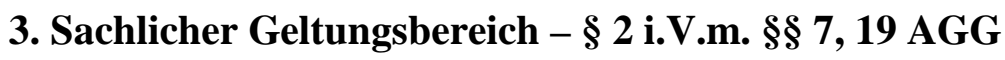

$\S 2$ I Nr. 1 bis 8 bestimmt den sachlichen Geltungsbereich des Gesetzes, der in Inhalt und Wortlaut weitgehend den Vorgaben des Art. 3 I lit. a bis h Richtlinie 2000/43/EG entspricht. Konkretisiert wird die Bestimmung durch die in den $\S \S 7$ und 19 statuierten Benachteiligungsverbote.

\section{a) Benachteiligungsverbot des $§ 19$ AGG}

\author{
Gemäß § 19 I ist
}

„[e]ine Benachteiligung aus Gründen der Rasse oder wegen der ethnischen Herkunft, wegen des Geschlechts, der Religion, einer Behinderung, des Alters oder der sexuellen Identität bei der Begründung, Durchführung und Beendigung zivilrechtlicher Schuldverhältnisse, die

1. typischerweise ohne Ansehen der Person zu vergleichbaren Bedingungen in einer Vielzahl von Fällen zustande kommen (Massengeschäfte) oder bei denen das Ansehen der Person nach der Art des Schuldverhältnisses eine nachrangige Bedeutung hat und die zu vergleichbaren Bedingungen in einer Vielzahl von Fällen zustande kommen oder

2. eine privatrechtliche Versicherung zum Gegenstand haben,

[...] unzulässig.“

Auffällig ist zunächst, daß das in $§ 1$ enthaltene Diskriminierungsmerkmal „Weltanschauung“ vom Schutzbereich der Norm nicht erfaßt ist. ${ }^{195}$ Der Rechtsausschuß des Bundestages begründet dies damit, es bestehe andernfalls - obwohl das Merkmal „Weltanschauung“ die allgemeine politische Gesinnung gerade nicht erfasse - die Gefahr, „daß z. B. Anhänger rechtsradikalen Gedankenguts aufgrund der Vorschrift versuchen, sich Zugang zu Geschäften zu verschaffen, die ihnen aus anerkennenswerten Gründen verweigert wurden.“196 Diese Begründung vermag in mehrfacher Hinsicht nicht zu überzeugen. Zum einen stellt sich die Frage, warum die politische Gesinnung nicht vom Begriff der Weltanschauung umfaßt sein sollte. ${ }^{197}$ Außerdem läßt sich die Privilegierung der Religion - dieses Merkmal ist von $§ 19$ I erfaßt - gegenüber der Weltanschauung jedenfalls nicht mit der gegebenen Begründung rechtfertigen. Schließlich besteht aufgrund der vorzunehmenden Interessenabwägung stets die Möglichkeit, Anhängern rechtsradikalen

195 Der ursprüngliche Gesetzesentwurf enthielt dagegen in $\S 19$ I auch jenes Merkmal: ,, Eine Benachteiligung wegen eines in $\S 1$ genannten Grundes [...] ist unzulässig.“ (BT-Druck. 16/1780, S. 10; Hervorhebung durch den Verfasser).

196 Begründung der Änderungsempfehlung des Rechtsausschusses des Bundestages, BT-Druck. 16/2022, S. 13.

197 Nach Ansicht von Säcker ist bspw. der Kommunismus eine Weltanschauung. (Säcker, ZRP 2002, S. 286 (289)). 210 
Gedankengutes bei Vorliegen sachlicher Gründe den Zugang zu einem Geschäft zu verweigern. Der Ausschluß des Merkmals „Weltanschauung“ aus dem Schutzbereich des § 19 I verdient aus den genannten Gründen keine Zustimmung.

Voraussetzung des $\$ 19$ I Nr. 1 erste Alternative ist das Vorliegen eines sogenannten Massengeschäftes. Durch dieses Erfordernis sollen „einmalige Sachverhalte“ vom Geltungsbereich des Diskriminierungsverbotes ausgeschlossen werden: „So ist etwa der Absatz von Gebrauchtwagen für den gewerblichen Kfz-Händler ein Geschäft, das er in einer Vielzahl von Fällen abwickelt. Anders ist es bei einer Privatperson, die ihren gebrauchten Pkw verkaufen will.“198 Dies hat zur Folge, daß nach dem Gesetzesentwurf vom allgemeinen zivilrechtlichen Benachteiligungsverbot nur diejenigen Leistungen erfaßt sind, „die von Unternehmen erbracht werden, also von natürlichen oder juristischen Personen, die in Ausübung ihrer gewerblichen oder beruflichen Selbständigkeit handeln“. ${ }^{199}$ Begründet wird dies damit, „,[d]er mit dem Benachteiligungsverbot zwangsläufig verbundene Eingriff in die Vertragsfreiheit [lasse] sich bei Unternehmen eher rechtfertigen, weil sie sich mit ihrem Leistungsangebot in die öffentliche Sphäre begeben und es damit grundsätzlich an die Allgemeinheit richten. ‘200

Der Beschränkung des Diskriminierungsverbotes auf den Bereich gewerbsmäßigen Handelns wurde an anderer Stelle eine Absage erteilt. ${ }^{201}$ Die Entwurfsbegründung vermag an dieser Sichtweise nichts zu ändern. Auch die Privatperson, die ihren gebrauchten Pkw verkaufen will, schafft Öffentlichkeit etwa dadurch, daß sie eine Annonce aufgibt.

Wesensmerkmal eines Massengeschäfts im Sinne des § 19 I Nr. 1 erste Alternative ist weiterhin, daß das Geschäft „typischerweise ohne Ansehen der Person“, d.h. ohne nach den in § 19 I genannten Merkmalen zu unterscheiden, zustande kommt. Beispielhaft nennt der Gesetzesentwurf insofern den Einzelhandel, die Gastronomie und das Transportwesen. ${ }^{202}$

Konsequenterweise müßte das Vorliegen dieser Voraussetzung und damit das Vorliegen eines Massengeschäfts verneint werden, wenn sich eine der genannten Branchen in Gänze dazu entschlösse, mit Trägern eines der in § 19 I genannten Merkmale keine Verträge mehr zu schließen. Diese Überlegung zeigt, daß die Beantwortung der Frage des Vorliegens einer Diskriminierung nicht davon abhängig gemacht werden darf, ob das in Rede stehende Rechtsgeschäft typischerweise ohne Ansehen der Person vorgenommen wird.

Der gleichen Kritik ist auch das Merkmal „,zu vergleichbaren Bedingungen“ in $\S 19$ I Nr. 1 erste Alternative ausgesetzt. 
Von der zweiten Alternative des $§ 19$ I Nr. 1 sollen Rechtsgeschäfte erfaßt sein, „bei denen „das Ansehen der Person“"zwar eine Rolle spielt; diese Voraussetzung jedoch eine nachrangige Bedeutung hat.“ ${ }^{203}$ Beispielhaft wird der Vermieter genannt, der eine Vielzahl von Wohnungen anbietet. ${ }^{204}$ Im Zusammenhang mit diesem Beispiel ist jedoch auf $\S 19 \mathrm{~V} 3$ hinzuweisen, der in dem ursprünglichen Gesetzesentwurf noch nicht enthalten war. Nach dieser Bestimmung ist

„[d]ie Vermietung von Wohnraum zum nicht nur vorübergehenden Gebrauch [...] in der Regel kein Geschäft im Sinne des Absatzes 1 Nr. 1, wenn der Vermieter insgesamt nicht mehr als 50 Wohnungen vermietet.“

Der Rechtsausschuß des Bundestages begründet die Notwendigkeit dieser Bestimmung unter anderem damit, es müsse davon ausgegangen werden, „daß jedenfalls dann, wenn der Vermieter insgesamt nicht mehr als 50 Wohnungen vermietet, das Ansehen der Person des konkreten Mieters für ihn nicht ohne Bedeutung oder nicht nur von nachrangiger Bedeutung ist. Der Nachweis, daß es im Einzelfall anders ist, bleibt ebenso möglich wie der Nachweis, daß auch größere Vermieter dem Ansehen der Person des konkreten Mieters mehr als nur nachrangige Bedeutung beimessen. “205 Die Bestimmung des $\S 19$ V 3 scheint eher das Ergebnis einer erfolgreichen Lobbyarbeit denn das einer sachgerechten Abwägung widerstreitender Vermieter-Mieter-Interessen $\mathrm{zu}$ sein. Der Rechtsausschuß vermag jedenfalls nicht zu begründen, worin er die Rechtfertigung der von ihm für notwendig erachteten (Vermutungs-)Regelung in $\S 19$ V 3 sieht. Auch ist nicht nachvollziehbar, warum der Rechtsausschuß im Einzelfall den Gegenbeweis in die eine wie die andere Richtung zuläßt. Er setzt sich damit in Widerspruch zu dem ursprünglichen Gesetzesentwurf, in dessen Begründung zu $\S 19$ I Nr. 1 es heißt: „Differenziert der Unternehmer im Einzelfall bei der Auswahl des Vertragspartners oder bei der Erbringung der Leistung dennoch von vornherein nach den in $\S 1$ genannten Merkmalen, ändert sich nichts an der Anwendbarkeit der Vorschrift. Die Einordnung als Massengeschäft erfolgt nämlich nach einer allgemeinen, typisierenden Betrachtungsweise. So sind etwa Freizeiteinrichtungen (Badeanstalten, Fitneßclubs etc.) typischerweise für Angehörige jeden Geschlechts und jeden Alters zugänglich.“206

Die spezielle Regelung in $\S 19$ I Nr. 2 wird damit begründet, im Bereich der Privatversicherung bestehe auch bei individueller Risikoprüfung ein Bedürfnis, sozial nicht $\mathrm{zu}$ rechtfertigende Unterscheidungen zu unterbinden, deckten Versicherungen doch häufig elementare Lebensrisiken $\mathrm{ab}$, weshalb der verweigerte Vertragschluß in diesem Bereich schwerwiegende Auswirkungen für den Benachteiligten haben könne. ${ }^{207}$

203 Entwurfsbegründung, BT-Druck. 16/1780, S. 42.

204 Entwurfsbegründung, BT-Druck. 16/1780, S. 42.

205 Begründung der Änderungsempfehlung des Rechtsausschusses des Bundestages, BT-Druck. 16/2022, S. 13.

206 Entwurfsbegründung, BT-Druck. 16/1780, S. 41.

207 Entwurfsbegründung, BT-Druck. 16/1780, S. 42. 
Gemäß $§ 19$ II ist

„[e]ine Benachteiligung aus Gründen der Rasse oder wegen der ethnischen Herkunft [...] darüber hinaus auch bei der Begründung, Durchführung und Beendigung sonstiger zivilrechtlicher Schuldverhältnisse im Sinne des $§ 2$ Abs. 1 Nr. 5 bis 8 unzulässig.

Schuldverhältnisse im Sinne des § 2 I Nr. 5 bis 8 betreffen

„5. den Sozialschutz, einschließlich der sozialen Sicherheit und der Gesundheitsdienste;

6. die sozialen Vergünstigungen;

7. die Bildung;

8. den Zugang zu und die Versorgung mit Gütern und Dienstleistungen, die der Öffentlichkeit zur Verfügung stehen, einschließlich von Wohnraum. ‘208

$\mathrm{Zu}$ den Nummern 5 bis 7 des $\S 2$ I heißt es in der Gesetzesbegründung, daß die meisten dieser Sachverhalte öffentlich-rechtlichen Regelungen unterliegen werden, ,denn beim Sozialschutz sowie den sozialen Vergünstigungen und auch bei der Bildung wird es sich überwiegend um staatliche Leistungen handeln. ${ }^{\text {209 }}$

Herausragende Bedeutung erlangt dagegen $§ 2$ I Nr. 8, dessen Geltungsbereich erheblich über den des $§ 19$ I Nr. 1 hinausgeht. Er erfaßt auch Geschäfte Privater, sofern der Vertragsschluß öffentlich angeboten wird. Beispielhaft wird insofern der Verkauf des gebrauchten privaten Pkw über eine Zeitungsannonce genannt. ${ }^{210}$

Gemäß $§ 19$ II gilt das Diskriminierungsverbot im sachlichen Geltungsbereich des $§ 2$ I Nr. 8 nur im Falle einer Benachteiligung aus Gründen der Rasse oder wegen der ethnischen Herkunft. Den sachlichen Geltungsbereich des Diskriminierungsverbotes und damit das Diskriminierungsverbot an sich auf bestimmte Merkmale zu beschränken, steht in eklatantem Widerspruch zu der hier vertretenen Ansicht. Die Bestimmung des § 19 II ist insofern abzulehnen.

Gemäß $§ 19$ III ist bei der Vermietung von Wohnraum

„eine unterschiedliche Behandlung im Hinblick auf die Schaffung und Erhaltung sozial stabiler Bewohnerstrukturen und ausgewogener Siedlungsstrukturen sowie ausgeglichener wirtschaftlicher, sozialer und kultureller Verhältnisse zulässig.“ 
Die Verortung dieser Bestimmung in $\S 19$ ist $\mathrm{zu}$ kritisieren, spricht sie doch die Frage der Rechtfertigung diskriminierungsrelevanten Verhaltens an, nicht die des sachlichen Geltungsbereichs des Diskriminierungsverbotes. Inhaltlich ist darauf hinzuweisen, daß vertreten wird, die ,„,Reinhaltung“ einer Wohngegend ist auch dann rassistisch, wenn Menschen angeblich vor Übergriffen geschützt werden sollen (,Sie passen da nicht hin.“).“211 Das Beispiel zeigt, daß die Bestimmung des $\S 19$ III äußerst restriktiv anzuwenden ist.

Gemäß $§ 19$ IV findet das zivilrechtliche Benachteiligungsverbot

„keine Anwendung auf familien- und erbrechtliche Schuldverhältnisse.“

In der Gesetzesbegründung heißt es dazu, die im Familien- und Erbrecht geregelten Schuldverhältnisse würden sich, ,grundlegend von den Verträgen des sonstigen Privatrechts unterscheiden“. ${ }^{212}$ Begründen läßt sich dies damit, daß familien- und erbrechtliche Rechtsgeschäfte die Intim- bzw. Privatsphäre eines Menschen betreffen, Bereiche also, in dem das Diskriminierungsverbot keine Geltung beanspruchen darf. ${ }^{213}$

Gemäß $§ 19$ V findet das zivilrechtliche Benachteiligungsverbot

„keine Anwendung auf zivilrechtliche Schuldverhältnisse, bei denen ein besonderes Näheverhältnis oder Vertrauensverhältnis der Parteien oder ihrer Angehörigen begründet wird. Bei Mietverhältnissen kann dies insbesondere der Fall sein, wenn die Parteien oder ihre Angehörigen Wohnraum auf demselben Grundstück nutzen. Die Vermietung von Wohnraum zum nicht nur vorübergehenden Gebrauch ist in der Regel kein Geschäft im Sinne des Absatzes 1 Nr. 1, wenn der Vermieter insgesamt nicht mehr als 50 Wohnungen vermietet.“

Klare Kriterien dafür, wann ein besonderes Nähe- oder Vertrauensverhältnis vorliegen soll, liefert der Gesetzesentwurf nicht. In der Entwurfsbegründung heißt es nebulös, ein besonderes Nähe- oder Vertrauensverhältnis erfordere eine Beziehung, „die über das hinausgeht, was ohnehin jedem Schuldverhältnis an persönlichem Kontakt zugrunde liegt. ‘214

Die Vorschrift ist zu begrüßen, soweit sie die Privatsphäre des Menschen vom Verbot der Diskriminierung ausschließt. Daß sie nur diesen Bereich erfassen möchte, ist indes zu bezweifeln. So kann laut Entwurfsbegründung ein besonderes Nähe- und Vertrauensverhältnis „,beispielsweise darauf beruhen, daß es sich um ein für die durch das Benachteiligungsverbot verpflichtete Person besonders bedeutsames Geschäft handelt, oder daß der Vertrag besonders engen oder lang

Baer, ZRP 2002, S. 290 (293, Fn. 33).

Entwurfsbegründung, BT-Druck. 16/1780, S. 42.

Erinnert sei an dieser Stelle auch an die Ausführungen zur „Entgeltlichkeit“ unter Teil 2, Prüfungspunkt D. IV.

214 Entwurfsbegründung, BT-Druck. 16/1780, S. 42. 
andauernden Kontakt der Vertragspartner mitsichbringen würde. ${ }^{\text {“215 }}$ Es kann behauptet werden, daß auch und gerade im Bereich der Sozialsphäre besonders bedeutsame Geschäfte getätigt werden; daß auch in diesem Bereich Verträge geschlossen werden, die einen besonders engen oder lang andauernden Kontakt der Vertragspartner mitsichbringen.

$\mathrm{Zu}$ \& $19 \mathrm{~V} \mathrm{3}$, der bereits an anderer Stelle eingehend untersucht und kritisiert wurde, ist anzumerken, daß er unter anderem verdeutlichen soll, „daß die Verneinung eines persönlichen Nähe- oder Vertrauensverhältnisses nicht bereits zur Annahme eines Massengeschäfts [im Sinne des $§ 19$ I Nr. 1] führt.“ ${ }^{216}$ Das Tatbestandsmerkmal ,zum nicht nur vorübergehenden Gebrauch“ soll seinerseits klarstellen, daß die Bestimmung ,nicht Vermietungen im Sinne des § 549 Abs. 2 Nr. 1 BGB, also beispielsweise die Vermietung von Hotelzimmern oder Ferienwohnungen“ " betrifft. ${ }^{217}$

\section{b) Benachteiligungsverbot des $§ 7$ AGG}

Gemäß § 7 I dürfen Beschäftigte

,[...] nicht wegen eines in $\S 1$ genannten Grundes benachteiligt werden; dies gilt auch, wenn die Person, die die Benachteiligung begeht, das Vorliegen eines in $\S 1$ genannten Grundes bei der Benachteiligung nur annimmt.“

$\S 2$ I Nr. 1 bis 3 bestimmt den sachlichen Geltungsbereich, in dem Benachteiligungen im Sinne des $\S 7$ I unzulässig sind. Inhalt und Wortlaut des $\S 2$ I Nr. 1 bis 3 entsprechen weitgehend den Vorgaben des Art. 3 I lit. a bis c Richtlinie 2000/43/EG. ${ }^{218}$ Der Begriff des Beschäftigten wird in $\S$ 6 I, III definiert; die Person die gem. § 7 I ,die Benachteiligung begeht““ in $§ 6$ II. Obwohl von $§ 6$ II

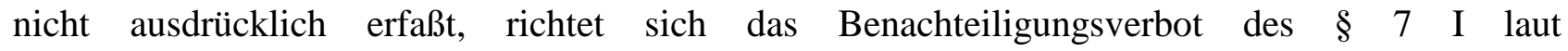
Entwurfsbegründung , auch gegen Arbeitskollegen und Dritte, wie z. B. Kunden des Arbeitgebers.“219 Gemeint kann damit nur die Diskriminierung in Form der (sexuellen) Belästigung sein, gegen die der Arbeitgeber gem. $\S 12$ geeignete Maßnahmen zu treffen hat. Die Entwurfsbegründung nennt in diesem Zusammenhang beispielhaft den Auslieferungsfahrer, der von den Kunden seines Arbeitgebers wegen seiner ethnischen Herkunft schikaniert wird. ${ }^{220}$

$\S 7$ I weist ausdrücklich darauf hin, daß Opfer einer Diskriminierung auch sein kann, wer für den Träger eines bestimmten Merkmals gehalten wird, dies in Wirklichkeit aber gar nicht ist. Anzumerken ist in diesem Zusammenhang, daß das in $\S 19$ statuierte Benachteiligungsverbot eine derartige Bestimmung nicht enthält, wodurch der Eindruck erweckt wird, diese Norm biete insofern

\footnotetext{
215 Entwurfsbegründung, BT-Druck. 16/1780, S. 42 f.

216 Begründung der Änderungsempfehlung des Rechtsausschusses des Bundestages, BT-Druck. 16/2022, S. 13.

217 Begründung der Änderungsempfehlung des Rechtsausschusses des Bundestages, BT-Druck. 16/2022, S. 13.

218 Entsprechende Vorgaben enthalten die Richtlinien 76/207/EWG und 2000/78/EG.

219 Entwurfsbegründung, BT-Druck. 16/1780, S. 34.

220 Entwurfsbegründung, BT-Druck. 16/1780, S. 37.
} 
geringeren Diskriminierungsschutz als jene. Sachlich begründen ließe sich eine derartige Inkongruenz - sollte sie vom Gesetzgeber beabsichtigt sein, was unwahrscheinlich erscheint freilich nicht.

Besondere Erwähnung im Zusammenhang mit dem in $\S 7$ statuierten Benachteiligungsverbot verdient $\S 2 \mathrm{IV}$, der bestimmt, daß

„[f]ür Kündigungen [...] ausschließlich die Bestimmungen zum allgemeinen und besonderen Kündigungsschutz [gelten].“

Ursprünglich sollten gem. § 2 IV AGG-Entwurf

„,[f]ür Kündigungen [...] vorrangig die Bestimmungen des Kündigungsschutzgesetzes [gelten].، ‘221

Vor dem Hintergrund der Tatsache, daß $\S 2$ IV AGG-Entwurf von „Bestimmungen des Kündigungsschutzgesetzes“ spricht, stellt sich die Frage, ob § 2 IV, in dem von „Bestimmungen zum allgemeinen und besonderen Kündigungsschutz" die Rede ist, auf arbeitsrechtliche Kündigungen beschränkt ist. Der Bundesrat, von dem die Initiative zur Änderung des § 2 IV ausging, verlangte lediglich, daß für Kündigungen ausschließlich das Kündigungsschutzgesetz gelten sollte. ${ }^{222}$ Das Wort „Kündigungsschutzgesetz“ selbst sollte hingegen nicht geändert werden. Erst die entsprechende Änderungsempfehlung des Rechtsausschusses des Bundestages enthielt die Formulierung: „Bestimmungen zum allgemeinen und besonderen Kündigungsschutz““ ${ }^{223}$ In seiner Begründung heißt es dazu einerseits, ,,[d]as Verhältnis beider Gesetze [- gemeint sind das AGG und das KSchG - ] zueinander soll dahin präzisiert werden, daß für Kündigungen ausschließlich die Bestimmungen zum allgemeinen und besonderen Kündigungsschutz Anwendung finden.“224 $§ 2$ IV scheint im Lichte dieser Formulierung auf das Arbeitsrecht beschränkt zu sein. Auf der anderen Seite wird in der Begründung darauf hingewiesen, die wesentlichen Bestimmungen des allgemeinen Kündigungsschutzes fänden sich unter anderem im Bürgerlichen Gesetzbuch. ${ }^{225}$ Da das BGB nun aber Kündigungsschutz unter anderem auch zugunsten von Mietern statuiert, ist es nicht fernliegend, § 2 IV als nicht auf arbeitsrechtliche Kündigungen beschränkt anzusehen.

221 BT-Druck. 16/1780; Hervorhebungen durch den Verfasser; Die Änderung des $\S 2$ IV erfolgte aufgrund einer Forderung des Bundesrates - BT-Druck. 16/1852, S. 2 (Nr. 3. e)) - und einer entsprechenden Änderungsempfehlung des Rechtsausschusses des Bundestages - BT-Druck. 16/2022, S. 6 (unter a) I. 1.).

222 BT-Druck. 16/1852, S. 2 (Nr. 3. e)).

223 BT-Druck. 16/2022, S. 6 (unter a) I. 1.).

224 Begründung der Änderungsempfehlung des Rechtsausschusses des Bundestages, BT-Druck. 16/2022, S. 12.

225 Begründung der Änderungsempfehlung des Rechtsausschusses des Bundestages, BT-Druck. 16/2022, S. 12. 216 
Der Geltungsausschluß des AGG im Bereich von Kündigungen wird als sachgerecht angesehen, seien doch die Regelungen des allgemeinen und besonderen Kündigungsschutzes „speziell auf Kündigungen zugeschnitten“. ${ }^{226}$ Angesichts der Tatsache jedoch, daß der sachliche Geltungsbereich des AGG gem. § 2 I Nr. 2 unter anderem auch „Entlassungsbedingungen“, gem. § 19 I, II unter anderem auch die „Beendigung (sonstiger) zivilrechtlicher Schuldverhältnisse“ umfaßt, muß die Regelung des $\S 2$ IV verwundern. Es stellt sich die Frage nach der Intention des Gesetzgebers. Offensichtlich möchte er verhindern, daß durch das AGG ein Kündigungsschutz statuiert wird, der über den bisherigen allgemeinen und besonderen Kündigungsschutz hinausgeht. Wenn dem so wäre, stellte sich natürlich die Frage, inwieweit der Gesetzgeber Unterschiede zwischen dem Diskriminierungsschutz des AGG und dem, den der bisherige allgemeine und besondere Kündigungsschutz bietet, sieht. Unabhängig von der Beantwortung dieser Frage sei an dieser Stelle noch einmal an eine wesentliche These der vorliegenden Arbeit erinnert, daß nämlich das Diskriminierungsverbot in der gesamten Sozialsphäre gelten sollte. Schon unter dieser Prämisse muß die Regelung des $\S 2$ IV als verfehlt angesehen werden. Sie sei zudem nicht mit der Rechtsprechung des EuGH, nach der auch Kündigungen im Lichte der Antidiskriminierungsrichtlinien bewertet werden müßten, vereinbar. ${ }^{227}$

\section{Rechtfertigung des diskriminierungsrelevanten Verhaltens}

Auch im Bereich der Rechtfertigung diskriminierungsrelevanten Verhaltens unterscheidet das Gesetz zwischen der Benachteiligung im Zivilrechtsverkehr und der Benachteiligung der Beschäftigten.

\section{a) Zulässige unterschiedliche Behandlung gem. § 20 AGG}

Gemäß $§ 20$ I 1 ist eine Verletzung des zivilrechtlichen Benachteiligungsverbots nicht gegeben,

„,wenn für eine unterschiedliche Behandlung wegen der Religion oder der Weltanschauung, einer Behinderung, des Alters, der sexuellen Identität oder des Geschlechts ein sachlicher Grund vorliegt.“

Aus der Tatsache, daß $§ 20$ I 1 die Merkmale Rasse und ethnische Herkunft im Gegensatz zu allen übrigen Merkmalen des $\S 1$ nicht erwähnt, folgt, daß eine Verletzung des zivilrechtlichen Benachteiligungsverbots immer dann gegeben sein soll, wenn die unterschiedliche Behandlung auf

226 Begründung der Änderungsempfehlung des Rechtsausschusses des Bundestages, BT-Druck. 16/2022, S. 12.

227 FAZ vom 12.07.06, S. 12, unter dem Titel: „Diskriminierungsverbot schützt Kranke nicht vor Kündigungen“; „Es kann sein, daß dieser Paragraph dem EU-Recht widerspricht.“ (Sattar zitiert das Vorstandsmitglied der IG-Metall Oberhofer in FAZ vom 16.11.2006, S. 4, unter dem Titel: „,,Auf falschem Bein hurra geschrien“ - Drei Monate Gleichbehandlungsgesetz"). 
die Merkmale Rasse und ethnische Herkunft gestützt wird. Der Gesetzesentwurf folgt insofern einer kritikwürdigen Vorgabe der Richtlinie 2000/43/EG. ${ }^{228}$

Begrüßenswert ist die Bestimmung des $§ 20$ insofern, als sie den Begriff des sachlichen Grundes einführt. Der Gesetzgeber ist sich dabei bewußt, daß es für dessen Feststellung einer wertenden Entscheidung im Einzelfall nach den Grundsätzen von Treu und Glauben bedarf und wegen der Reichweite des allgemeinen zivilrechtlichen Benachteiligungsverbotes eine abschließende nähere Konkretisierung unmöglich ist. ${ }^{229}$

$\S 20$ I 2 beschränkt sich deshalb auf die Nennung von Regelbeispielen, die laut Entwurfsbegründung ,die wichtigsten Fallgruppen umreißen und zugleich eine Richtschnur für die Auslegung des Grundtatbestandes geben können. “230 Ein sachlicher Grund soll danach insbesondere vorliegen, wenn die unterschiedliche Behandlung

„1. der Vermeidung von Gefahren, der Verhütung von Schäden und anderen Zwecken vergleichbarer Art dient,

2. dem Bedürfnis nach Schutz der Intimsphäre oder der persönlichen Sicherheit Rechnung trägt,

3. besondere Vorteile gewährt und ein Interesse an der Durchsetzung der Gleichbehandlung fehlt,

4. an die Religion eines Menschen anknüpft und im Hinblick auf die Ausübung der Religionsfreiheit oder auf das Selbstbestimmungsrecht der Religionsgemeinschaften, der ihnen zugeordneten Einrichtungen ohne Rücksicht auf ihre Rechtsform sowie der Vereinigungen, die sich die gemeinschaftliche Pflege einer Religion zur Aufgabe machen, unter Beachtung des jeweiligen Selbstverständnisses gerechtfertigt ist.“

Der Zweck der Nummer 1 soll vor allem in der Notwendigkeit bestehen, bei Massengeschäften die Beachtung der Verkehrssicherungspflichten durchzusetzen. So könne es beispielsweise in Freizeitparks erforderlich sein, den Zugang zu Fahrgeschäften für Menschen mit einer körperlichen Behinderung zu beschränken oder aber auf einer Begleitperson zu bestehen. Als weiteres Beispiel werden Einrichtungen genannt, die Opfern sexueller Gewalt gewidmet sind und die nur Angehörigen eines Geschlechts offenstehen. ${ }^{231}$

Die Nummer 2 erfaßt in erster Linie Unterscheidungen nach dem Geschlecht. Dem Bedürfnis nach Schutz der Intimsphäre dienten etwa getrennte Öffnungszeiten in Schwimmbädern und Saunen, der persönlichen Sicherheit unter anderem Frauenparkplätze. ${ }^{232}$ Nicht jedes subjektive Sicherheitsbedürfnis soll jedoch ausreichen, um eine Unterscheidung zu rechtfertigen: „Eine beispielsweise auf Xenophobie beruhende pauschale Angst vor „dem Islam“ oder „den Juden“ kann daher eine Ungleichbehandlung nach dem Merkmal der Religion nicht rechtfertigen. “233 
Vorteile im Sinne der Nummer 3 sollen vor allem Preisnachlässe oder andere Sonderkonditionen bei der Anbahnung, Durchführung oder Beendigung von Massengeschäften sein. In diesen Fällen bestehe kein Anlaß, den Grundsatz der Gleichbehandlung durchzusetzen: „Die gewährten Vergünstigungen reagieren nämlich entweder darauf, daß bestimmte Gruppen typischerweise weniger leistungsfähig sind: Rabatte für Schüler und Studenten etwa sind damit zu begründen, daß sie meist nicht über ein Erwerbseinkommen verfügen. Oder aber die Vergünstigungen bezwecken die gezielte Ansprache von Kundenkreisen, die der Anbieter anlocken möchte. ${ }^{\text {‘234 }}$ Es wird darauf hingewiesen, daß ein Verbot dieser Praktiken den objektiv benachteiligten Personenkreisen nicht helfen würde, ,denn der Anbieter würde nicht mit der Erstreckung der Vorteile auf alle Kunden reagieren, sondern mit dem Verzicht auf jegliche Vergünstigung. ‘235

Der Wortlaut der Nummer 4 und ein Vergleich mit der insofern eingeschränkter formulierten Bestimmung des $\S 9$ zeigen, daß „Benachteiligender“ im Sinne dieses Regelbeispiels nicht nur die Religionsgemeinschaft und die ihr zugeordneten Einrichtungen sein können, sondern auch jede natürliche Person. Beispielhaft wird der islamische Metzger genannt, der Kundinnen ohne Kopftuch die Bedienung verweigert. ${ }^{236}$ In bezug auf die Religionsgemeinschaften wird die Bedeutung des Art. 140 GG in Verbindung mit Art. 137 III WRV hervorgehoben. ${ }^{237}$

Für private Versicherungsverträge nach $\S 19$ I Nr. 2 enthält $§ 20$ II eine besondere Bestimmung:

„Eine unterschiedliche Behandlung wegen des Geschlechts ist im Falle des $\S 19$ Abs. 1 Nr. 2 bei den Prämien oder Leistungen nur zulässig, wenn dessen Berücksichtigung bei einer auf relevanten und genauen versicherungsmathematischen und statistischen Daten beruhenden Risikobewertung ein bestimmender Faktor ist. Kosten im Zusammenhang mit Schwangerschaft und Mutterschaft dürfen auf keinen Fall zu unterschiedlichen Prämien oder Leistungen führen. Eine unterschiedliche Behandlung wegen der Religion oder Weltanschauung, einer Behinderung, des Alters oder der sexuellen Identität ist im Falle des $\S 19$ Abs. 1 Nr. 2 nur zulässig, wenn diese auf anerkannten Prinzipien risikoadäquater Kalkulation beruht, insbesondere auf einer versicherungsmathematisch ermittelten Risikobewertung unter Heranziehung statistischer Erhebungen.“

In der Entwurfsbegründung heißt es $\mathrm{zu} \quad \S \quad 20$ II, die Einbeziehung sämtlicher Privatversicherungsverträge in den Anwendungsbereich des allgemeinen privatrechtlichen Benachteiligungsverbots solle vor Willkür schützen, nicht aber die auch im Interesse der Versicherten erforderliche Differenzierung nach dem ex ante beurteilten individuellen Risiko

234 Entwurfsbegründung, BT-Druck. 16/1780, S. 44; Bezüglich des zweiten Grundes sei an dieser Stelle noch einmal an den „Kaffeemaschinenfall“ erinnert.

235 Entwurfsbegründung, BT-Druck. 16/1780, S. 44.

236 Entwurfsbegründung, BT-Druck. 16/1780, S. 45; Auf dieses Beispiel wurde bereits unter Teil 3, Prüfungspunkt M. VI. näher eingegangen.

237 Entwurfsbegründung, BT-Druck. 16/1780, S. 44. 
unmöglich machen, gehöre diese Differenzierung doch zu den Grundprinzipien der privatrechtlichen Versicherung. ${ }^{238}$

Auch § 20 II ist zunächst insofern zu kritisieren, als er den Versicherern implizit einschränkungslos verbietet, Rasse und ethnische Herkunft als Risikofaktoren zu verwenden.

Durch $§ 20$ II 1 werden die Vorgaben des Art. 5 II 1 Richtlinie 2004/113/EG in innerstaatliches Recht umgesetzt. Die Tatsache, daß sich besagte Vorgaben des europäischen Gesetzgebers nur auf das Merkmal „Geschlecht“ beziehen, dürfte der Grund dafür sein, daß der deutsche Gesetzgeber für die Merkmale Religion, Weltanschauung, Behinderung, Alter sowie sexuelle Identität in $\S 20$ II 3 eine Sonderregelung geschaffen hat, die gegenüber $\S 20$ II 1 ,weicher“ formuliert ist. Vergleicht man den Wortlaut beider Bestimmungen, erscheint die vorgenommene Unterscheidung äußerst gekünstelt. So setzt Satz 1 voraus, daß das Geschlecht ein ,[bestimmender Faktor] bei einer auf relevanten und genauen versicherungsmathematischen und statistischen Daten beruhenden Risikobewertung“ ist, während Satz 3 verlangt, daß eine unterschiedliche Behandlung der dort genannten Merkmale ,,auf anerkannten Prinzipien risikoadäquater Kalkulation beruht, insbesondere auf einer versicherungsmathematisch ermittelten Risikobewertung unter Heranziehung statistischer Erhebungen.“ In diesem Zusammenhang ist darauf hinzuweisen, daß der am 17.06.2005 vom Bundestag verabschiedete ${ }^{239}$ aber letztlich nicht in Kraft getretene Gesetzesentwurf in $\S 20$ Satz 2 Nr. 5 ADG-Entwurf eine dem $\S 20$ II 1 vergleichbare Regelung mit dem Unterschied enthielt, daß jene Bestimmung sämtliche Merkmale umfaßte, die nunmehr auf die Sätze 1 und 3 des $\S 20$ II verteilt sind.

$\S 20$ II 2, der die Bestimmung des Art. 5 III Richtlinie 2004/113/EG wortwörtlich übernimmt, stellt laut Entwurfsbegründung eine sozialpolitisch motivierte Ausnahme von der grundsätzlich zulässigen Differenzierung im Rahmen der Risikobewertung dar. ${ }^{240}$

\section{b) Zulässige unterschiedliche Behandlung gem. §§ 8 - 10 AGG}

Gemäß § 8 I ist eine unterschiedliche Behandlung wegen eines in $§ 1$ genannten Grundes zulässig,

„[...] wenn dieser Grund wegen der Art der auszuübenden Tätigkeit oder der Bedingungen ihrer Ausübung eine wesentliche und entscheidende berufliche Anforderung darstellt, sofern der Zweck rechtmäßig und die Anforderung angemessen ist."

238 Entwurfsbegründung, BT-Druck. 16/1780, S. 45; In diesem Sinne wird beispielsweise vertreten, der Privatgesetzgeber sei „verfassungsrechtlich nicht verpflichtet, für die private Krankenversicherung eine UnisexTarifierung vorzuschreiben.“ Vielmehr sei er „angesichts der herausragenden Bedeutung der dauernden Erfüllbarkeit der Verpflichtungen des Versicherers berechtigt, eine Unisex-Tarifierung - wie er es getan hat - zu verbieten.“(Wandt, VersR 2004, S. 1341 (1346)). 
Eine zulässige unterschiedliche Behandlung im Sinne des $\S 8$ I soll laut Entwurfsbegründung beispielsweise vorliegen, ,wenn bei Organisationen der in Deutschland anerkannten nationalen Minderheiten und der anerkannten Regional- oder Minderheitensprachen Personen bevorzugt eingestellt werden, die der jeweiligen Gruppe angehören. ‘241

Bereits an den Antidiskriminierungsrichtlinien, die Vorlage für die Formulierung des $\S 8$ I waren, wurde das Kriterium der ,wesentlichen und entscheidenden beruflichen Voraussetzung“ kritisiert. Diese Kritik gilt in gleichem Maße für die vorliegende Bestimmung.

Positiv hervorzuheben ist jedoch, daß das Gesetz nicht zwischen „unverzichtbar“ für das Merkmal Geschlecht und „,wesentlich und entscheidend“ für die übrigen Merkmale des $§ 1$ unterscheidet, wie noch der Entwurf eines Gesetzes zur Umsetzung europäischer Antidiskriminierungsrichtlinien vom 16.12.2004 in $\S 8$ I Nr. 1 und 2 ADG-E. ${ }^{242} \mathrm{Zu}$ recht weist die Entwurfsbegründung in diesem Zusammenhang darauf hin, daß damit der - bis zum Inkrafttreten des AGG durch § 611 a I 2 BGBA bestehende - Schutzstandard hinsichtlich des Merkmals Geschlecht nicht abgesenkt wird. ${ }^{243} \S 8$ I verstößt folglich nicht gegen Art. 8 e II Richtlinie 76/207/EWG, wonach

„,[d]ie Umsetzung dieser Richtlinie [...] keinesfalls als Rechtfertigung für eine Absenkung des von den Mitgliedstaaten bereits garantierten Schutzniveaus in Bezug auf Diskriminierungen in den von der Richtlinie abgedeckten Bereichen benutzt werden [darf]. ${ }^{244}$

Diese Gefahr sah noch der Verfasser des Gesetzesentwurfs vom 16.12.2004, der in seiner Entwurfsbegründung darauf hinwies, daß ,bei der Umsetzung der Richtlinien bereits bestehende Vorschriften nicht verschlechtert werden dürfen.“245

Der Gesetzgeber hat durch Einführung des $§ 9$ von der ihm durch Art. 4 II Richtlinie 2000/78/EG eingeräumten Möglichkeit Gebrauch gemacht, eine Sonderregelung in bezug auf die Zulässigkeit unterschiedlicher Behandlung wegen der Religion oder Weltanschauung durch Tendenzbetriebe zu schaffen. Auffällig ist, daß das Merkmal ,wesentliche, rechtmäßige und gerechtfertigte berufliche Anforderung“ des Art. 4 II 1 der Richtlinie zu ,gerechtfertigte berufliche Anforderung“ in § 9 I verkürzt wurde. An anderer Stelle wurde herausgearbeitet, daß die Begriffe ,wesentlich und entscheidend“, „unverzichtbar“ bzw. „sachlicher Grund“ geeignet sind, gleichwertigen Diskriminierungsschutz zu gewähren. Unter dieser Prämisse führt die in $\S 9$ I gewählte Formulierung nicht zu einer Verminderung des Diskriminierungsschutzes gegenüber dem in Art. 4 II 1 Richtlinie 2000/78/EG statuierten. Der Gesetzgeber müßte dies, will er sich nicht in

241 Entwurfsbegründung, BT-Druck. 16/1780, S. 35.

242 BT-Druck. 15/4538.

243 Entwurfsbegründung, BT-Druck. 16/1780, S. 35.

244 Entsprechende Vorschriften enthalten Art. 6 II Richtlinie 2000/43/EG; Art. 8 II Richtlinie 2000/78/EG und Art. 7 II Richtlinie 2004/113/EG.

245 Entwurfsbegründung, BT-Druck. 15/4538, S. 32. 
Widerspruch zum Wortlaut seines Gesetzes setzen, allerdings anders sehen. Wenn er zwischen „wesentliche und entscheidende berufliche Anforderung“ in $\S 8$ I und ,gerechtfertigte berufliche Anforderung“ in § 9 I unterscheidet, muß er denknotwendig diesen Begriffen eine unterschiedliche Bedeutung beimessen. Wenn dem nun aber so ist, dann müßte es die Intention des Gesetzgebers gewesen sein, das Schutzniveau in $\S 9$ I gegenüber dem in Art. 4 II 1 Richtlinie 2000/78/EG statuierten zu senken. Es verwundert deshalb, daß es in der Entwurfsbegründung gleichwohl heißt, $\S 9$ I entspräche den Vorgaben besagter Richtlinie. ${ }^{246}$ An dieser Stelle zeigt sich einmal mehr, daß die Verwendung unterschiedlicher Begriffe hinsichtlich der Rechtfertigung diskriminierungsrelevanten Verhaltens zu Widersprüchen führt, die durch einen einheitlichen Terminus vermieden werden können.

Der Gesetzgeber hat schließlich durch $\S 10$ von der ihm durch Art. 6 Richtlinie 2000/78/EG verliehenen Kompetenz Gebrauch gemacht, eine Sonderregelung bezüglich der Zulässigkeit unterschiedlicher Behandlung wegen des Alters zu schaffen. § 10 gibt dabei größtenteils wortwörtlich Art. 6 Richtlinie 2000/78/EG wieder.

\title{
c) Positive Maßnahmen - 5 AGG
}

\author{
Gemäß $§ 5$ ist
}

„[u]ngeachtet der in den $\S \S 8$ bis 10 sowie in $\S 20$ benannten Gründe [...] eine unterschiedliche Behandlung auch zulässig, wenn durch geeignete und angemessene Maßnahmen bestehende Nachteile wegen eines in $\S 1$ genannten Grundes verhindert oder ausgeglichen werden sollen.“

Positiv hervorzuheben ist zunächst, daß $\S 5$ laut Entwurfsbegründung ,gezielte Maßnahmen zur Förderung bisher benachteiligter Gruppen nicht nur durch den Gesetzgeber [...], sondern auch durch Arbeitgeber, Tarifvertrags- und Betriebspartner sowie seitens der Parteien eines privatrechtlichen Vertrags“" legitimiert. ${ }^{247}$

$\mathrm{Zu}$ erwähnen ist außerdem, daß die Vorschrift „Maßnahmen zur Behebung bestehender Nachteile ebenso zu[lassen soll] wie präventive Maßnahmen zur Vermeidung künftiger Nachteile.“248 $\mathrm{Zu}$ Umfang und Grenzen möglicher Maßnahmen im Sinne des $\S 5$ heißt es in der Entwurfsbegründung, selbige „müssen nach objektivem Maßstab geeignet und angemessen sein und bedürfen im konkreten Fall der Abwägung mit Rechtspositionen der von ihnen negativ Betroffenen. Das schließt nach der Rechtsprechung des Europäischen Gerichtshofes einen absoluten Vorrang der 
zu fördernden Gruppe aus. ${ }^{649}$ Aus der Entwurfsbegründung geht hervor, daß der Gesetzgeber unter Maßnahmen im Sinne des $\S 5$ auch solche versteht, die zu einer sog. positiven Diskriminierung führen, spricht er doch von den „,negativ Betroffenen“. In diesem Zusammenhang deutet die Entwurfsbegründung an, daß allenfalls relative einzelfallbezogene Quoten durch $\S 5$ gerechtfertigt sein sollen. ${ }^{250}$

\section{Resümee}

Das AGG ist dadurch gekennzeichnet, daß es im wesentlichen die Vorgaben der Antidiskriminierungsrichtlinien 1: 1 umsetzt. Das gilt zumindest für den untersuchten Teil des Gesetzes. Nur vereinzelt geht es über die Vorgaben der Richtlinien hinaus bzw. bleibt hinter selbigen zurück.

Da schon die Antidiskriminierungsrichtlinien kein umfassendes Diskriminierungsschutzkonzept verfolgen, bleibt auch das Gesetz insoweit lückenhaft. So beschränkt es den Diskriminierungsschutz auf bestimmte Diskriminierungsmerkmale. Auch der sachliche Geltungsbereich des Diskriminierungsverbots ist in nicht gerechtfertigter Weise eingeschränkt. Beispielhaft kann insofern § 2 IV genannt werden, der bestimmt, daß das AGG für Kündigungen nicht gilt, oder § 19 $\mathrm{V}$ 3, der Vermieter, die insgesamt nicht mehr als 50 Wohnungen vermieten, in einer nicht zu rechtfertigen Weise privilegiert. Schließlich hat der Gesetzgeber nicht erkannt, daß eine Diskriminierung auch ohne das Vorhandensein einer Vergleichsperson vorliegen kann. Dieses Problem relativiert sich freilich dadurch, daß das AGG in $\S 2$ IV Kündigungen aus seinem Geltungsbereich und damit einen Bereich ausschließt, in dem sich das Problem des Nichtvorhandenseins einer Vergleichsperson besonders häufig stellen kann.

An der Bestimmung des $\S 19$ V 3 zeigt sich exemplarisch, daß das AGG selbst diskriminierenden Charakter hat, und zwar im Verhältnis der „Diskriminierenden“ zueinander. Warum der Vermieter, der 30, 40 oder 50 Wohnungen vermietet, gegenüber dem, der 60, 70, 80 und mehr Wohnungen anbietet, privilegiert sein soll, ist nicht ersichtlich. Das gleiche gilt hinsichtlich der Privilegierung des Vermieters von Wohnungen gegenüber dem Hotelier.

Zudem ist die Gesetzestechnik teilweise zu kritisieren. Das gilt etwa für die Formulierung des § 19 I Nr. 1, die dazu führt, daß das Vorliegen einer Diskriminierung beispielsweise zu verneinen wäre, wenn - aus welchen Gründen auch immer - in Deutschland typischerweise Juden keinen Zutritt zu deutschen Geschäften hätten - ein Ergebnis, daß vom Gesetzgeber sicherlich nicht gewollt sein dürfte. Zwar ist es unwahrscheinlich, daß in Deutschland jemals wieder nationalsozialistische Zustände herrschen werden. Die praktische Bedeutung dieses provokanten Beispiels tendiert gegen

249 Entwurfsbegründung, BT-Druck. 16/1780, S. 34.

250 Zum Begriff der relativen einzelfallbezogenen Quote vgl. Teil 2, Prüfungspunkt C. VI. 2. a) aa). 
null. Es ist jedoch geeignet zu verdeutlichen, daß die Bestimmung des $§ 19$ I Nr. 1 von seinem theoretischen Ansatz her nicht stimmig ist.

Auch sind die unterschiedlichen Begrifflichkeiten im Zusammenhang mit den Anforderungen an die Rechtfertigung diskriminierungsrelevanten Verhaltens in den $\S \S 8-10$ und 20 kritikwürdig. Sie reichen von „sachlicher Grund“ in § 20 über ,wesentliche und entscheidende berufliche Anforderung [...], sofern der Zweck rechtmäßig und die Anforderung angemessen“ in $\S 8$ I und „gerechtfertigte berufliche Anforderung“ in $§ 9 \mathrm{I}$ bis zu „objektiv und angemessen und durch ein legitimes Ziel gerechtfertigt““, soweit die „Mittel zur Erreichung dieses Ziels [...] angemessen und erforderlich“ in $\S 10$ Satz 1 und 2. Dieser gesetzestechnische Wirrwarr ist, wie nachgewiesen wurde, nicht erforderlich. Der Begriff des sachlichen Grundes ist aufgrund seiner Flexibilität geeignet, auf die jeweiligen Besonderheiten des Einzelfalls in adäquater Weise zu reagieren.

Neben inhaltliche Fehler gesellen sich handwerkliche. Ein Beispiel dafür bildet $\S 33$ I, wonach

„,[b]ei Benachteiligungen nach den $\S \S 611$ a, 611 b und 612 Abs. 3 des Bürgerlichen Gesetzbuchs oder sexuellen Belästigungen nach dem Beschäftigtenschutzgesetz [...] das vor dem 18. August maßgebliche Recht anzuwenden [ist].“

Das „maßgebliche [alte] Recht“ im Sinne dieser Bestimmung bilden die genannten Bestimmungen des BGB sowie das Beschäftigtenschutzgesetz. Nach dem Wortlaut des $§ 33$ I soll das alte Recht über den 18.08.2006 hinaus anwendbar sein. Angesichts der Tatsache, daß mit Inkrafttreten des Gesetzes zur Umsetzung europäischer Richtlinien zur Verwirklichung des Grundsatzes der Gleichbehandlung am 18.08.2006 die $\S \S 611$ a, 611 b und 612 Abs. 3 BGB aufgehoben wurden ${ }^{251}$ und das BeSchuG außer Kraft getreten ist, ${ }^{252}$ erscheint eine derartige Interpretation des $\S 33$ I jedoch fraglich. In der Entwurfsbegründung zu $\S 33$ I heißt es dazu, daß das alte Recht nur auf Benachteiligungen anzuwenden ist, die zeitlich vor dem Inkrafttreten des AGG liegen. ${ }^{253}$ Wie diese - freilich einzig sinnvolle - Interpretation des $\S 33$ I mit dem Wortlaut der Norm vereinbar ist, bleibt ein Geheimnis des Gesetzgebers. In diesem Zusammenhang ist zu erwähnen, daß die Bundesregierung noch vor dem Inkrafttreten des AGG angekündigt hatte, „,kleinere redaktionelle Ungenauigkeiten“ des Gesetzes - zu denen zweifellos auch § 33 I AGG zählt - zu beheben. ${ }^{254}$

Ein Verdienst des Gesetzes ist, daß es in $§ 10$ Satz 3 Nr. 1-8 sowie $§ 20$ I 2 Nr. 1-4 Regelbeispiele nennt und damit eine Richtschnur dafür gibt, wann in den jeweiligen Bereichen eine

251 Art. 3 XIV des Gesetzes zur Umsetzung europäischer Richtlinien zur Verwirklichung des Grundsatzes der Gleichbehandlung.

252 Art. 4 Satz 2 des Gesetzes zur Umsetzung europäischer Richtlinien zur Verwirklichung des Grundsatzes der Gleichbehandlung.

253 Entwurfsbegründung, BT-Druck. 16/1780, S. 53.

254 FAZ vom 03.08.06, S. 1, unter dem Titel: ,„,Redaktionelle Ungenauigkeiten“““. 
unterschiedliche Behandlung zulässig sein soll. Zu erwähnen ist in diesem Zusammenhang auch § 20 II.

Zusammenfassend kann die Feststellung getroffen werden, daß das AGG, das weitgehend auf die „bloße“ Umsetzung der Antidiskriminierungsrichtlinien beschränkt ist, kein umfassendes Diskriminierungsschutzkonzept verfolgt und aus diesem Grunde und aufgrund der aufgezeigten Mängel nicht als gelungene Gesetzgebung angesehen werden kann. Die Frage nach einem adäquaten Diskriminierungsschutzgesetz bleibt damit trotz des AGG bestehen. ${ }^{255}$

\section{Gesetzesentwürfe von 1998}

Vorlage für ein Diskriminierungsschutzgesetz könnte der Entwurf eines Gesetzes zur Durchsetzung des Gleichbehandlungsgebotes des Artikel 3 Grundgesetz (Gleichbehandlungsgesetz) der SPDFraktion vom 09.03.1998 sein, dessen Name schon vermuten läßt, daß er ein umfassendes Diskriminierungsschutzkonzept verfolgt.

Der Gesetzesentwurf statuiert in Artikel 1, § 1 I ein Benachteiligungsverbot:

„Jede Person hat Anspruch auf Gleichbehandlung im Rechtsverkehr. Niemand darf insbesondere wegen seines Geschlechts, seiner Abstammung, seiner Rasse, seiner Sprache, seiner Heimat und Herkunft, seines Glaubens, seiner religiösen oder politischen Anschauungen, seiner Behinderung oder seiner sexuellen Identität benachteiligt werden.“

Erklärtes Ziel dieser Bestimmung ist es, „das Benachteiligungsverbot des Art. 3 GG über den öffentlichen Bereich auch auf den privaten Rechtsverkehr aus[zudehnen].“256

Eine dem Art. 1, § 1 I des SPD-Entwurfs vergleichbare Regelung enthält Art. 2, § 1 I des Entwurfs eines Gesetzes zum Schutz vor Diskriminierung und zur Stärkung von Minderheitenrechten (Antidiskriminierungs- und Minderheitenrechtsgesetz) der Fraktion Bündnis 90/Die Grünen vom 20.01.1998. ${ }^{257}$ Gemäß dieser Bestimmung hat jede Person

„[...] Anspruch auf Gleichbehandlung im Rechtsverkehr. Niemand darf insbesondere wegen seiner tatsächlichen oder vermeintlichen

a) ethnischen Abstammung, Herkunft oder Zugehörigkeit, Hautfarbe, Nationalität, religiösen Anschauungen oder

b) sexuellen Identität oder

255 In diesem Zusammenhang sei auf den von Henssler und Preis erarbeiteten Entwurf eines Arbeitsvertragsgesetzes (ArbVG) hingewiesen - vgl. www.arbvg.de -, der auch Regeln zum Diskriminierungsschutz enthält, die ob ihrer Übersichtlichkeit auf positive Resonanz gestoßen sind: „Schlanke sechs Paragraphen reichen den Wissenschaftlern aus, um 33 AGG-Normen zusammenzufassen.“ (Budras in FAZ vom 27.01.2007, S. 11, unter dem Titel: „Aufräumen im Arbeitsrecht"“).

256 Entwurfsbegründung, BT-Druck. 13/10081, S. 11.

257 BT-Druck. 13/9706. 
c) Behinderung

diskriminiert werden.“

\section{Diskriminierungsrelevantes Verhalten}

Die Diskriminierungshandlung des SPD-Entwurfs kann laut Entwurfsbegründung in einer unmittelbaren und mittelbaren Benachteiligung bestehen. Mittelbar sei sie dann, wenn sie sich gegen Angehörige der Träger von unerwünschten Merkmalen richtet. ${ }^{258}$ Der Gesetzesentwurf erfaßt damit die sogenannte Diskriminierungsdrittwirkung, nicht aber das, was im Rahmen dieser Arbeit unter den Begriff der mittelbaren Diskriminierung subsumiert wurde. ${ }^{259}$ Der Diskriminierungsschutz des Gesetzesentwurfs ist insofern lückenhaft. ${ }^{260}$

Der Gesetzesentwurf von Bündnis 90/Die Grünen definiert den Begriff der Diskriminierung in Art. 2, § 1 II als ,,jede nicht gerechtfertigte Ungleichbehandlung.“ Im Gegensatz zum SPD-Entwurf umfaßt diese Bestimmung auch die mittelbare Diskriminierung in dem hier verstandenen Sinne. ${ }^{261}$ Aber auch die Diskriminierungsdrittwirkung findet in dem Gesetzesentwurf Berücksichtigung. Gemäß Art. 2, § 1 III ist eine Ungleichbehandlung nicht gerechtfertigt,

„[...] wenn sie ausschließlich oder überwiegend auf Umständen beruht, die in mittelbarem oder unmittelbarem Zusammenhang mit den in Absatz 1 genannten Zugehörigkeitsmerkmalen stehen.“

Durch diese Bestimmung sollen auch Fälle von Diskriminierung erfaßt sein, „die Familienangehörigen von Personen aus den benachteiligten Gruppen widerfahren können, beispielsweise dem deutschen Ehepartner eines Migranten oder den Eltern eines behinderten Kindes. “262

\section{Diskriminierungsmerkmale}

Der SPD-Gesetzesentwurf ist insofern begrüßenswert, als er das im Rahmen der Arbeit vorgeschlagene Modell des offenen Merkmalskataloges realisiert hat. ${ }^{263}$

Gleiches gilt für den Entwurf von Bündnis 90/Die Grünen, wenngleich darauf hingewiesen werden muß, daß die Entwurfsbegründung den Eindruck vermittelt, als sei der Diskriminierungsschutz trotz

258 Entwurfsbegründung, BT-Druck. 13/10081, S. 11.

259 Vergleiche dazu Teil 2, Prüfungspunkte A. I. 3. sowie A. III.

260 So auch Nickel, Gleichheit und Differenz in der vielfältigen Republik, S. 195.

261 Vergleiche dazu Entwurfsbegründung, BT-Druck. 13/9706, S. 11.

262 Entwurfsbegründung, BT-Druck. 13/9706, S. 11.

263 Vergleiche dazu Teil 2, Prüfungspunkt B. IV. 6. 
des „,insbesondere-Zusatzes“ auf die explizit genannten Merkmale beschränkt: „Andere Unterscheidungsmerkmale nach Artikel 3 Abs. 3 GG wie z. B. das Geschlecht waren vorliegend nicht zu berücksichtigen, da insoweit bereits andere gesetzgeberische Initiativen ergriffen worden sind bzw. der hier weiterhin bestehende gesetzgeberische Handlungsbedarf nicht unter dem Vorzeichen der Minderheitenrechte geregelt werden kann. ‘264

Positiv hervorzuheben ist, daß der Gesetzesentwurf ausdrücklich klarstellt, daß es nicht darauf ankommt, daß der Diskriminierte tatsächlich Träger des Merkmals ist, aufgrund dessen er diskriminiert wird.

\section{Sachlicher Geltungsbereich}

Der sachliche Geltungsbereich des in Artikel $1, \S 1$ des SPD-Entwurfs statuierten Benachteiligungsverbotes wird durch den Begriff des Rechtsverkehrs umschrieben. Laut Entwurfsbegründung umfaßt er ,,...] den Abschluß, die Ausgestaltung, die Abwicklung und die Beendigung von Rechtsgeschäften. Auch solche Rechtshandlungen sind mitumfaßt, die der Anbahnung und Vorbereitung von Verträgen dienen.“265 Es wird vertreten, das Benachteiligungsverbot des $\S 1$ I ergreife damit ,sämtliche relevanten Tatbestände der Diskriminierung in privaten Rechtsbeziehungen. “266

Eingeschränkt wird der sachliche Geltungsbereich dadurch, daß vom Begriff des Rechtsverkehrs „,nur rechtlich relevante Handlungen, nicht das private allgemeine Verhalten“ umfaßt sein soll. Um letzteres rechtlich zu binden, reichten ,die dafür vorgesehenen Regelungen, insbesondere des Strafrechts (Beleidigung, üble Nachrede, Verleumdung) aus. “267

Begrüßenswert ist, daß der Gesetzesentwurf in den sachlichen Geltungsbereich allgemein rechtsgeschäftliches Handeln einbezieht. Auch der Hinweis darauf, daß das Benachteiligungsverbot auch das Vorfeld des Vertragsschlusses erfaßt, ist positiv hervorzuheben. Zu unbestimmt ist indes die Beschränkung des sachlichen Geltungsbereichs auf „,rechtlich relevante Handlungen“ bzw. der Ausschluß des ,,privaten allgemeinen Verhaltens“. Der Hinweis auf die strafrechtlichen Tatbestände der Beleidigung, üblen Nachrede sowie Verleumdung lassen den Schluß zu, daß das private allgemeine Verhalten dasjenige umfassen soll, das im Rahmen der Arbeit unter dem Begriff der (sexuellen) Belästigung diskutiert wurde. Wenn der Gesetzgeber tatsächlich in diesem Sinne verstanden werden wollte, fragt sich, welche Position er etwa in bezug auf unentgeltliche Verträge oder Rechtsgeschäfte im Bereich der Intim- und Privatsphäre bezieht.

264 Entwurfsbegründung, BT-Druck. 13/9706, S. 11.

265 Entwurfsbegründung, BT-Druck. 13/10081, S. 11.

266 Nickel, Gleichheit und Differenz in der vielfältigen Republik, S. 193.

267 Entwurfsbegründung, BT-Druck. 13/10081, S. 11. 
Auch der Gesetzesentwurf von Bündnis 90/Die Grünen läßt in bezug auf die Reichweite des sachlichen Geltungsbereichs Fragen offen. Laut Entwurfsbegründung erfaßt das Diskriminierungsverbot ,nur den Rechtsverkehr, nicht das allgemeine private Verhalten. Für nichtrechtsgeschäftliches Handeln reichen die hierfür vorgesehenen Regelungen, insbesondere die strafrechtlichen Vorschriften gegen Beleidigung ( $\S 185$ ff. StGB) und Volksverhetzung ( $\$ 130$ StGB) aus.“268 Auch hier fragt sich wiederum, welche Position der Gesetzesentwurf in bezug auf unentgeltliche Verträge oder Rechtsgeschäfte im Bereich der Intim- und Privatsphäre bezieht, wobei insofern Art. 2, § 2 I gewisse Rückschlüsse zuläßt.

Art. 2, § 2 I konkretisiert den sachlichen Geltungsbereich des von dem Gesetzesentwurf statuierten Diskriminierungsverbotes, indem er Regelbeispiele für das Vorliegen einer Diskriminierung nennt. Danach verhält sich insbesondere diskriminierend unter Verstoß gegen Art. 2, § 1, wer

„1. die Gestaltung, den Abschluß, die Aufrechterhaltung oder die Fortsetzung eines Rechtsgeschäfts auf Grund eines der in $\S 1$ Abs. 1 genannten Merkmale verweigert, dessen der andere Teil zur Gestaltung seiner Lebensverhältnisse bedarf; hierzu zählen insbesondere Mietverträge, Verträge über Dienst-, Arbeits- und Ausbildungsverhältnisse oder Versicherungsverträge;

2. den Abschluß, die Aufrechterhaltung oder die Fortsetzung eines Rechtsgeschäfts auf Grund eines der in $\S 1$ Abs. 1 genannten Merkmale verweigert, das öffentlich oder einem unbestimmten Personenkreis angeboten wird, insbesondere im Bereich der Gastronomie, bei Dienstleistungen oder Veranstaltungen;

3. bei einem öffentlichen Angebot oder der Aufforderung zur Abgabe eines Angebotes zum Abschluß eines Vertrages, insbesondere in Inseraten, den Vertragsabschluß vom Vorliegen oder Nichtvorliegen eines der in $\S 1$ Abs. 1 genannten Merkmale abhängig macht;

4. innerhalb eines Arbeitsverhältnisses Vergünstigungen, Beförderungen oder freiwillige Leistungen, Schulungen, Maßnahmen der Weiterbildung oder disziplinarische Maßnahmen vom Vorliegen oder Nichtvorliegen eines der in $\S 1$ Abs. 1 genannten Merkmale abhängig macht;

5. den Zugang zu einem Gebiet oder Raum, der einer unbestimmten Anzahl von Personen offengehalten wird, vom Vorliegen oder Nichtvorliegen eines der in $§ 1$ Abs. 1 genannten Merkmale abhängig macht;

6. die Aufnahme in, die Zugehörigkeit zu oder den Ausschluß aus einem Verein, einer Gesellschaft oder einer Partei vom Vorliegen oder Nichtvorliegen eines der in $§ 1$ Abs. 1 genannten Merkmale abhängig macht.“

Die Nummern 1 bis 6 des Art. 2, § 2 I stehen in bezug auf den durch sie definierten und eingeschränkten sachlichen Geltungsbereich des Diskriminierungsverbotes im Einklang mit den insofern im Rahmen der Arbeit angestellten Überlegungen.

\section{Rechtfertigung des diskriminierungsrelevanten Verhaltens}

Gemäß Art. 1, § 1 II des SPD-Entwurfs ist eine Benachteiligung nicht gegeben, 
„wenn eine Berücksichtigung der in Absatz 1 genannten persönlichen Merkmale der Sache nach geboten ist.“

An dieser Bestimmung wurde - vor Inkrafttreten des AGG - mit Blick auf das Unverzichtbarkeitserfordernis des $\S 611$ a I 2 BGB-A kritisiert, sie bleibe hinter dem mittlerweile anerkannten Schutzniveau von Antidiskriminierungsrecht zurück. ${ }^{269}$ Dem ist entgegenzuhalten, daß nach der Entwurfsbegründung eine Unterscheidung nur dann zulässig sein soll, ,,wenn die Gründe dafür unabweisbar sind." Insbesondere bei Abschluß und Ausgestaltung von Arbeitsverträgen habe das zur Folge, „daß etwa die Besonderheiten eines Arbeitsplatzes nicht ohne weiteres als gegeben hingenommen werden können, sondern auch die Zumutbarkeit in finanzieller und technischer Hinsicht mit überprüft werden kann.،270

Art. 1, § 1 II steht im Einklang mit der hier vertretenen Ansicht, wonach ein sachlicher Grund diskriminierungsrelevantes Verhalten rechtfertigt. ${ }^{271}$

Gemäß Art. 2, § 1 IV des Entwurfs von Bündnis 90/Die Grünen ist eine nicht gerechtfertigte Ungleichbehandlung nicht gegeben,

„,wenn eine Berücksichtigung der Merkmale nach Absatz 1 der Sache nach unverzichtbar geboten ist oder die Berücksichtigung der Merkmale nach Absatz 1 zur Wahrung der berechtigten Interessen von Diskriminierten erforderlich ist.“

Die Interessenklausel des Absatz IV, 2. Alternative soll laut Entwurfsbegründung verhindern, „daß das Diskriminierungsverbot gegen die benachteiligten Gruppen selbst gewendet wird“. Möglich soll beispielsweise weiterhin sein, ,im Rahmen von Lesbenveranstaltungen männliches Publikum auszuschließen. “272

Es wird zu recht darauf hingewiesen, daß es nicht die benachteiligten Gruppen selbst sein dürfen, die definieren, was berechtigte Interessen im Sinne des Absatz IV, 2. Alternative sind, könnte doch andernfalls ,,beispielsweise ein türkischstämmiger Arbeitgeber einen deutschen Bewerber auf einen Arbeitsplatz ohne weiteres mit der Begründung abweisen, daß es die Interessen der türkischstämmigen Bürger gebieten, nur türkischstämmige Bewerber zu berücksichtigen.“273

\section{Resümee}

269 Nickel, Gleichheit und Differenz in der vielfältigen Republik, S. 193.

270 Entwurfsbegründung, BT-Druck. 13/10081, S. 11.

271 Vergleiche dazu Teil 2, Prüfungspunkt C. VII. 3.

272 Entwurfsbegründung, BT-Druck. 13/9706, S. 11.

273 Nickel, Gleichheit und Differenz in der vielfältigen Republik, S. 197. 
Trotz der aufgezeigten Kritikpunkte enthalten die Gesetzesentwürfe von SPD und Bündnis 90/Die Grünen „,eine Reihe vielversprechender Ansätze für ein umfassendes und wirksames Antidiskriminierungsgesetz““ ${ }^{274}$ Vor allem die Gesetzestechnik, mithin die auf das wesentliche beschränkte Formulierung des Diskriminierungsverbotes, muß denjenigen überzeugen, der das Diskriminierungsverbot ins BGB integrieren möchte. ${ }^{275}$ Die Gesetzesentwürfe von SPD und Bündnis 90/Die Grünen bilden deshalb im folgenden die Grundlage für die gesetzestechnische Umsetzung des Diskriminierungsverbotes im Zivilrecht.

\section{Vorschlag für die gesetzestechnische Umsetzung des Diskriminierungsverbotes}

Mit Blick auf die im Rahmen dieser Arbeit gewonnenen Erkenntnisse wird vorgeschlagen, das Verbot der Diskriminierung im Zivilrecht gesetzestechnisch wie folgt umzusetzen:

\section{Tatbestand - $\$ 115$ I, II BGB-E}

\section{$\S 115$ BGB-E}

(1) Unzulässig ist eine Willenserklärung, die den Erklärungsempfänger insbesondere aufgrund seiner tatsächlichen oder vermeintlichen Rasse oder ethnischen Herkunft, seines Geschlechts, seiner Religion oder Weltanschauung, seiner Behinderung, seines Alters oder seiner sexuellen Identität unsachlich behandelt (diskriminiert). Unzulässig ist zudem, eine Willenserklärung, der ein öffentliches Angebot vorausgeht, in diskriminierender Weise nicht abzugeben. Auf das öffentliche Angebot ist Satz 1 entsprechend anzuwenden. Das gleiche gilt für sonstige Maßnahmen, die ihrem Wesen nach einer Willenserklärung im Sinne des Satz 1 vergleichbar sind.

(2) Absatz 1 Satz 1 findet insbesondere keine Anwendung auf Willenserklärungen, die den Intimbereich oder den Privatbereich des Erklärenden betreffen. Satz 1 gilt entsprechend im Fall des Absatz 1 Satz 2.

Die Gesetzesentwürfe von SPD und Bündnis 90/Die Grünen sind von dem Begriff „Rechtsverkehr“ geprägt. Es wurde nachgewiesen, daß die Entwürfe darunter rechtsgeschäftliches Handeln verstehen. Anstelle der Begriffe „Rechtsverkehr“ bzw. „Rechtsgeschäft““ verwendet der vorliegende Entwurf den allgemeineren Begriff „,Willenserklärung“. ${ }^{276}$ Der Grund dafür besteht in folgendem: Eine Vielzahl von Diskriminierungsfällen sind dadurch geprägt, daß der „Diskriminierende“ die Annahme des vom „Diskriminierten“ angetragenen Vertragsschlusses verweigert oder seinerseits gegenüber dem „Diskriminierten“ keinen Antrag auf Vertragsschluß abgibt. Angebot und Annahme

274 Nickel, Gleichheit und Differenz in der vielfältigen Republik, S. 199.

275 In diesem Sinne Baer: „Zwar wäre es systematisch konsequenter gewesen, Diskriminierungsschutz in den allgemeinen Regeln für das gesamte Zivilrecht zu verankern, [...].“(Baer, ZRP 2002, S. 290 (292)).

276 Die Willenserklärung ist notwendiger Bestandteil eines jeden Rechtsgeschäftes. (Brox, Allgemeiner Teil des BGB, Rn. 96). 
sollen nun aber keine einseitigen Rechtsgeschäfte, sondern als einseitige, empfangsbedürftige Willenserklärungen lediglich Teile des zweiseitigen Rechtsgeschäfts „,Vertrag“ sein. ${ }^{277}$

Eine Willenserklärung ist eine private Willensäußerung, die auf die Erzielung einer Rechtsfolge gerichtet ist. ${ }^{278}$ Bei den Willenserklärungen tritt der Rechtserfolg ein, weil er gewollt ist. ${ }^{279}$ Insofern unterscheidet sich die Willenserklärung von den sogenannten Rechtshandlungen, an die die Rechtsordnung Rechtsfolgen unabhängig davon knüpft, ob sie vom Handelnden gewollt sind oder nicht. ${ }^{280} \mathrm{Zu}$ den Rechtshandlungen zählen unter anderem unerlaubte Handlungen im Sinne der $\S \S$ $823 \mathrm{ff} . ~ B G B .^{281}$ (Sexuelle) Belästigungen sind als Verletzung des allgemeinen Persönlichkeitsrechts des Belästigten unerlaubte Handlungen in diesem Sinne. Dementsprechend wird vorgeschlagen, „§ 823 Abs. 1 BGB entsprechend der bisherigen Rechtsprechung um das Rechtsgut des allgemeinen Persönlichkeitsrechts zu ergänzen, vielleicht noch versehen mit einem „Insbesondere-Vermerk“, der diskriminierende Belästigungen ausdrücklich nennt. Dann würde die Belästigung hiervon zweifelsohne erfaßt werden, und der deliktische Tatbestand wäre in die richtige Systematik des Gesetzes eingebaut.“282 Dieser Ansicht folgend erfaßt die unter $§ 115$ BGB-E zu subsumierende Diskriminierungshandlung nicht die (sexuelle) Belästigung. ${ }^{283}$

Durch die Verwendung des Begriffs der Willenserklärung wird der Standort des Diskriminierungsverbotes im Allgemeinen Teil des BGB bei den Regelungen über die „Willenserklärungen“ in Abschnitt 3, Titel 2 bestimmt. ${ }^{284}$ Der Vorschlag, das Diskriminierungsverbot als $\S 115$ BGB zu normieren, ist allein der Tatsache geschuldet, daß ein $\S$ 115 BGB zur Zeit nicht existiert. Anstatt eine Bestimmung im Sinne des $§ 611$ a BGB-A, d. h. eine Bestimmung mit Buchstabenzusatz zu schaffen, wird eine vorhandene freie Stelle im BGB besetzt. Das Diskriminierungsverbot als $\S 115$ BGB zu normieren und damit an den Anfang der Bestimmungen über die Willenserklärungen zu setzen, führt nicht zu einem Systembruch in bezug auf die Reihenfolge, in der die Bestimmungen in Abschnitt 3, Titel 2 genannt werden, da eine zwingende Systematik insofern nicht besteht.

$\S 115$ I 1 BGB-E enthält einen offenen Merkmalskatalog, wobei er die Diskriminierungsmerkmale der Richtlinien 76/207/EWG, 2000/43/EG, 2000/78/EG sowie 2004/113/EG ausdrücklich nennt.

\footnotetext{
277 Heinrichs in Palandt, $\S 145$, Rn. 1; $\S 148$, Rn. 1.

278 Brox, Allgemeiner Teil des BGB, Rn. 82.

279 Brox, Allgemeiner Teil des BGB, Rn. 94.

280 Heinrichs in Palandt, Überbl v $§ 104$, Rn. 4.

281 Heinrichs in Palandt, Überbl v $\$ 104$, Rn. 4 f.

282 Wiedemann/Thüsing, DB 2002, S. 463 (467).

283 Vergleiche hinsichtlich der von $\S 115$ BGB-E erfaßten Diskriminierungshandlung Teil 2, Prüfungspunkt A.

284 Für die Verankerung des Diskriminierungsverbotes in den allgemeinen Regeln für das gesamte Zivilrecht Baer, ZRP 2002, S. 290 (292); vgl. auch von Koppenfels, WM 2002, S. 1489 (1496); Wiedemann/Thüsing halten dabei eine Regelung ,in der Nachbarschaft der $\S \S 134,138$ BGB“ für möglich. (Wiedemann/Thüsing, DB 2002, 463 (465)).
} 
Auch hebt die Bestimmung hervor, daß unerheblich ist, ob der „Diskriminierte“ tatsächlich Träger des Diskriminierungsmerkmals ist, aufgrund dessen er „diskriminiert“ wird.

$\S 115$ I 1 BGB bestimmt, daß eine Willenserklärung nur dann diskriminierend und damit rechtswidrig ist, wenn sie den Erklärungsempfänger aufgrund der Tatsache, daß er (vermeintlicher) Träger eines bestimmten Merkmals ist, unsachlich behandelt, sie also sachlich nicht gerechtfertigt ist. ${ }^{285}$ Für die Beantwortung der Frage, ob im Einzelfall ein sachlicher Grund vorliegt oder nicht, erlangen die untersuchten Antidiskriminierungsmaßnahmen Bedeutung, die im Zivilrecht eine mittelbare Drittwirkung entfalten. Der auslegungsfähige und -bedürftige Begriff „unsachlich“ ist im Sinne dieser Maßnahmen auszulegen.

$\S 115$ II BGB-E beschränkt - ohne abschließend zu sein, wie das Wort „,insbesondere“ verdeutlicht - den sachlichen Geltungsbereich des Diskriminierungsverbotes auf die sogenannte Sozialsphäre. ${ }^{286}$ Eine weitere Einschränkung ist etwa für unentgeltliche Zuwendungen wie Schenkungen erforderlich. In den $\S \S 516$ ff. BGB ist zu diesem Zweck eine Bestimmung mit dem Inhalt aufzunehmen, daß $§ 115$ BGB-E auf Schenkungen keine Anwendung findet.

Auch die Nichtabgabe einer Willenserklärung kann diskriminierenden Charakter haben. Einschränkend bestimmt $\S 115$ I 2 BGB-E insofern, daß dies nur bei Willenserklärungen der Fall sein kann, denen ein öffentliches Angebot vorausgeht.

Der Begriff des öffentlichen Angebots meint in erster Linie die invitatio ad offerendum. In diesem Sinne wird im Zusammenhang mit § 319 I Nr. 1 lit. a ADG-E des Bundesjustizministeriums, der das Merkmal des öffentlichen Angebots enthält, vertreten, damit seien typischerweise „Einzelgeschäfte und Warenhäuser, Gaststätten und Hotels, Zeitungsannoncen und Internetangebote usw.“ betroffen. ${ }^{287}$ Der Begriff „öffentliches Angebot“ ist streng von dem „Antrag“ im Sinne der $\S \S$ 145 ff. BGB zu trennen, obwohl oder gerade weil auch insofern oft von dem „Angebot“ die Rede ist. $^{288}$ Der im Sinne des $\S 145$ BGB Antragende schlägt einem anderen in verbindlicher und annahmefähiger Weise den Abschluß eines Vertrages vor. ${ }^{289}$ Dem öffentlich Anbietenden in dem hier verstanden Sinne fehlt ein derartiger Rechtsbindungswille. ${ }^{290}$

Der Begriff des öffentlichen Angebots ist nicht auf die invitatio ad offerendum beschränkt. Er erfaßt vielmehr auch die Fälle, bei denen der „Diskriminierende“ Öffentlichkeit etwa dadurch schafft, daß er eine Regel aufstellt und befolgt, die abstrakt an allgemeine Merkmale anknüpft und generell für

285

Kriterien für die Beantwortung der Frage, wann eine Maßnahme im Einzelfall unsachlich und damit diskriminierend ist, wurden in Teil 3 der Arbeit herausgearbeitet.

286 Vergleiche dazu Teil 2, Prüfungspunkt D. VI.

287 Wiedemann/Thüsing, DB 2002, 463 (465).

288 Bork in Staudinger, $\S 145$, Rn. 1.

289 Bork in Staudinger, § 145, Rn. 1.

Bork in Staudinger, $\S 145$, Rn. 3. 
eine Mehrzahl von Fällen gelten soll. Ein Beispiel dafür ist die Zahlung einer Weihnachtsgratifikation durch den Arbeitgeber.

Gemäß $§ 115$ I 3 BGB-E ist auf das öffentliche Angebot das Diskriminierungsverbot des Absatz 1 Satz 1 entsprechend anzuwenden. Dieser Regelung bedarf es für den Fall, daß das öffentliche Angebot selbst keine Willenserklärung darstellt. Das gilt für die invitatio ad offerendum, die mangels erkennbar fehlendem Geschäftswillen kein Angebot im Rechtssinne und damit auch keine Willenserklärung ist. ${ }^{291}$ Aber auch die unterschiedliche Entlohnung von Arbeitnehmern beruht nach Ansicht des $B A G$ nicht in jedem Fall auf einer Regelung, sondern kann sich vielmehr auch aus der vom Arbeitgeber tatsächlich geübten Lohnpolitik ergeben. ${ }^{292}$ Das Gericht hat in diesem Zusammenhang entschieden: „Die bereits angeführte Richtlinie 75/117/EWG verbietet nämlich jede tatsächliche Diskriminierung beim Arbeitsentgelt ohne Rücksicht darauf, ob die diskriminierende Wirkung auf einer Rechtsnorm, einer Vereinbarung oder dem tatsächlichen Verhalten des Arbeitgebers beruht. “293 In diesem Sinne hat der EuGH festgestellt, das Vorbringen, eine Vergünstigung werde nicht aufgrund einer vertraglichen Verpflichtung gewährt, führe nicht zur Unanwendbarkeit des Art. $141 \mathrm{EGV}$, komme es doch für dessen Anwendbarkeit nicht auf die Rechtsnatur der Vergünstigung an. ${ }^{294}$

Gemäß $§ 115$ I 4 BGB-E ist $\S 115$ I 1 BGB-E über das öffentliche Angebot hinaus auch auf sonstige Maßnahmen ${ }^{295}$ entsprechend anzuwenden, „,die ihrem Wesen nach einer Willenserklärung im Sinne des Absatz 1 Satz 1 vergleichbar sind.“ Diese Bestimmung trägt wie schon $§ 115$ I 3 BGB-E dem Umstand Rechnung, daß auch quasi-rechtsgeschäftliches Handeln diskriminierenden Charakter haben kann. Beispielhaft kann die diskriminierende Weisung des Arbeitgebers genannt werden.

Das Merkmal der Vergleichbarkeit soll verhindern, daß unter den Begriff der Maßnahme auch die (sexuelle) Belästigung subsumiert wird, die, wie bereits erwähnt, an anderer Stelle geregelt werden soll. ${ }^{296}$ Diese Trennung ist vor allem deshalb erforderlich, weil das Verbot der (sexuellen) Belästigung nicht, wie in $§ 115$ II BGB-E vorgesehen, auf den Bereich der Sozialsphäre beschränkt ist, sondern absolut gilt. Das ist der Unterschied zur ,diskriminierenden“ Weisung, die - sofern sie keinen belästigenden Charakter hat - im Rahmen der Privat- und Intimsphäre zulässig sein muß, da in diesem Bereich u. a. auch die „diskriminierende“ Kündigung zulässig ist.

291 Brox, Allgemeiner Teil des BGB, Rn. 167; Heinrichs in Palandt, § 145, Rn. 2.

$292 B A G$, NJW 1993, S. 3091 (3093 f.).

293 BAG, NJW 1993, S. 3091 (3094).

294 EuGH, Rs. C-12/81, Slg. 1982 1, S. 359 (370, Rn. 10).

295 Der Begriff „Maßnahme“ wurde bereits in $\S 611$ a I 1 BGB-A verwendet.

296 Vielleicht war Heinrichs in diesem Sinne zu verstehen, wenn er verlangte, die Maßnahme im Sinne des § 611 a I 1 BGB-A - ein Begriff, der bürgerlichrechtlich kaum noch eingegrenzt werden könne - müsse jedenfalls irgendeinen konkreten Bezug zum Arbeitsverhältnis haben. (Heinrichs in Palandt, § 611 a, Rn. 8). 


\section{Rechtsfolgen}

Gemäß Art. 15 Richtlinie 2000/43/EG legen die Mitgliedstaaten die Sanktionen fest,

„[...] die bei einem Verstoß gegen die einzelstaatlichen Vorschriften zur Anwendung dieser Richtlinie zu verhängen sind, und treffen alle geeigneten Maßnahmen, um deren Durchsetzung zu gewährleisten. Die Sanktionen, die auch Schadensersatzleistungen an die Opfer umfassen können, müssen wirksam, verhältnismäßig und abschreckend sein.“297

\section{Schadensersatzanspruch}

Wie von der Bestimmung explizit genannt, kommt als wirksame, verhältnismäßige und abschreckende Sanktion im Sinne der Richtlinie ein Schadensersatzanspruch des Diskriminierten in Betracht.

\section{a) § 115 BGB-E als Schutzgesetz im Sinne des $\$ 823$ II BGB}

Es wird insofern vorgeschlagen, dem Diskriminierungsverbot in $\S 115$ BGB-E den Status eines Schutzgesetzes im Sinne des $§ 823$ II BGB zu verleihen. ${ }^{298}$ Gemäß $§ 823$ II BGB trifft denjenigen eine Schadensersatzpflicht,

„[...] welcher gegen ein den Schutz eines anderen bezweckenden Gesetzes verstößt. Ist nach dem Inhalt des Gesetzes ein Verstoß gegen dieses auch ohne Verschulden möglich, so tritt die Ersatzpflicht nur im Falle des Verschuldens ein.“

\section{b) Materielle Belastung als Folge des Gesetzesverstoßes}

Eine Schadensersatzpflicht besteht dabei in den hier diskutierten Fällen erst dann, wenn der Diskriminierte durch den Verstoß gegen $§ 115$ BGB-E materiell - nicht nur formell - belastet ist. Dieses einschränkende Erfordernis wird vor dem Hintergrund folgender Tatsache verständlich: Vor Inkrafttreten des AGG führte der Verstoß gegen die Pflicht zu geschlechtsneutraler Ausschreibung gem. § 611 b BGB-A zu einem Entschädigungsanspruch aus $§ 611$ a II BGB-A. Nun war es in der Vergangenheit vorgekommen, daß sich „Arbeitsuchende“ allein deshalb auf Stellen beworben hatten, um bei erfolgreicher Ablehnung - selbige war bezweckt - den Anspruch aus $§ 611$ a II

297 Identische Bestimmungen enthalten Art. 8 d Richtlinie 76/207/EWG, Art. 17 Richtlinie 2000/78/EG sowie Art. 14 Richtlinie 2004/113/EG.

298 Vorbild ist insoweit $\S 4$ ADG-E von Bündnis 90/Die Grünen. (BT-Druck. 13/9706); Auch das in Art. 1 , 1 des SPD-Entwurfs statuierte Diskriminierungsverbot ist laut Entwurfsbegründung ,systematisch als Schutzgesetz i. S. des $§ 823$ Abs. 2 konzipiert.“ (Entwurfsbegründung, BT-Druck. 13/10081, S. 11). 
BGB-A geltend machen zu können. ${ }^{299}$ Dieses Verhalten wurde als rechtsmißbräuchlich eingestuft. Das $B A G$ verlangte deshalb in der Folgezeit für den Anspruch aus $\S 611$ a II BGB-A eine ernsthafte Bewerbung. ${ }^{300}$ Es kann davon ausgegangen werden, daß diese Rechtsprechung im Rahmen des AGG fortbestehen wird.

Klargestellt ist damit auch, daß ein diskriminierendes öffentliches Angebot im Sinne des $\S 115$ I BGB-E - also beispielsweise eine geschlechtsspezifische Stellenausschreibung - für sich allein keinen Schadensersatzanspruch zu begründen vermag.

\section{c) Vermögens- und Nichtvermögensschaden}

Inhalt und Umfang des Schadensersatzanspruchs aus § 823 II BGB bemessen sich nach den $\S 249$ ff. BGB. Vermögensschäden hat der Schädiger nach Maßgabe der §§ 249-252 BGB bei Verschulden - das ergibt sich aus $\S 823$ II 2 BGB - voll zu ersetzen. ${ }^{301}$ In diesem Sinne statuieren $\S$ 15 I sowie § 21 II 1, 2 AGG verschuldensabhängige Ansprüche auf Ersatz des entstandenen Vermögensschadens, wobei in beiden Fällen zunächst vermutet wird, daß der Diskriminierende den Verstoß gegen das Diskriminierungsverbot zu vertreten hat. Wie bei $§ 280$ I 2 BGB, ${ }^{302}$ nach dessen Vorbild beide Bestimmungen formuliert worden $\operatorname{sind}^{303}$ führt dies dazu, daß es dem Diskriminierenden obliegt zu beweisen, daß er die Diskriminierung nicht zu vertreten hat.

Neben Vermögensschäden kann der Benachteiligte nach § 15 II 1 sowie $\S 21$ II 3 AGG „,[w]egen eines Schadens, der nicht Vermögensschaden ist, [...] eine angemessene Entschädigung in Geld verlangen.“ In der Entwurfsbegründung zu § 15 AGG heißt es dazu: „Der Anspruch auf Entschädigung erfüllt die Forderungen der Richtlinien sowie der Rechtsprechung des Europäischen Gerichtshofes nach einer wirksamen und verschuldensunabhängig ausgestalteten Sanktion bei Verletzung des Benachteiligungsverbotes durch den Arbeitgeber. Der aus § 611 a BGB[-A] bekannte Grundgedanke wird hier auf alle Tatbestände einer Benachteiligung übertragen.، ${ }^{304}$ Eine Ausnahme macht der Gesetzgeber lediglich bei kollektiv-rechtlichen Vereinbarungen, ${ }^{305}$ die gem. $\S$

299 Von einem derartigen Fall berichtet Adomeit, NJW 1997, S. 2295 (2295). Dasselbe Problem stellt sich im Rahmen des AGG: ,[...] sogenannten AGG-Hoppern, die auf ihrer Bewerbungstour durch die deutsche Wirtschaftswelt nur auf Schadensersatz aus sind.“ (Sattar in FAZ vom 16.11.2006, S. 4, unter dem Titel: „,AAuf falschem Bein hurra geschrien“ - Drei Monate Gleichbehandlungsgesetz").

300 BAG, NZA 1999, S. 371 (373).

301 Heinrichs in Palandt, Vorbem v $§ 249$, Rn. 7.

302 Ernst in MüKo, § 280, Rn. 31.

303 Entwurfsbegründung, BT-Druck. 16/1780, S. 38 u. 46.

304 Entwurfsbegründung, BT-Druck. 16/1780, S. 38.

$305 \mathrm{Da} ß$ auch dieser Bereich Zündstoff birgt, zeigt beispielhaft die Anfang November 2006 bekannt gewordene Klage von zwölf Lufthansa-Piloten, ,,die den Tarifvertrag des Konzerns mit der Pilotenvereinigung Cockpit nicht mit dem Gleichbehandlungsgesetz für vereinbar halten. Nach der Tarifvereinbarung darf eine Lufthansa-Maschine nur steuern, wer nicht älter als 60 Jahre ist. Die zwölf Piloten sehen in der vermeintlichen sozialen Errungenschaft eine Altersdiskriminierung.“ (Sattar in FAZ vom 16.11.2006, S. 4, unter dem Titel: „,Auf falschem Bein hurra geschrien“- Drei Monate Gleichbehandlungsgesetz"). 
15 III AGG nur dann zu einer Entschädigungspflicht des Arbeitgebers führen sollen, „wenn er vorsätzlich oder grob fahrlässig handelt.“ Der Gesetzgeber begründet diese Ausnahme damit, ,,[d]ie vermutete „höhere Richtigkeitsgewähr“ rechtfertig[e] es, die Rechtsfolgen benachteiligender kollektiver Regelungen anders auszugestalten als bei Maßnahmen, für die der Arbeitgeber allein verantwortlich ist. “306

\section{d) Verschulden}

Die in den $\S \S 15$ I, 21 II 1, 2 AGG statuierten verschuldensabhängigen Schadensersatzansprüche stehen im Widerspruch zu dem verschuldensunabhängigen Anspruch aus § 611 a II BGB-A, der seinerseits ,,als Anspruchsgrundlage für den Ersatz der materiellen Schäden gleichermaßen wie für den Ausgleich der immateriellen Schäden in Betracht [kam].“307

Die Bestimmung des $\S 611$ a II BGB-A ist jedoch zu recht mit dem Argument angegriffen worden, „eine verschuldensunabhängige Kompensationsvorschrift [lasse] sich nicht in die geltende Privatrechtsordnung einfügen, da in dieser eine Schadensverlagerung vom Geschädigten auf den Schädiger grundsätzlich nur bei Verletzung der im Verkehr erforderlichen Sorgfalt [stattfinde].،308 Vor diesem Hintergrund ist es begrüßenswert, daß der Gesetzgeber des AGG dem Diskriminierten nur dann einen Anspruch auf Ersatz seines materiellen Schadens gewährt, wenn der Diskriminierende sein Verhalten zu vertreten hat.

Die auf den Ersatz immaterieller Schäden gerichteten Entschädigungsansprüche aus $§ § 15$ II 1, 21 II 3 AGG sind im Gegensatz zu den auf den Ersatz materieller Schäden gerichteten Ansprüchen aus $\S \S 15$ I, 21 II 1, 2 AGG verschuldensunabhängig und damit im Einklang mit § 611 a II BGB-A das Vorbild für $\S 15$ II 1 und $\S 21$ II 3 AGG - ausgestaltet. Es verwundert, daß der Entschädigungsanspruch, der dem Zivilrecht eigentlich fremd ist, da dieses in erster Linie Kompensation bezweckt, schärfer formuliert ist, als der systemkonforme Anspruch auf Ersatz des materiellen Schadens.

Diejenigen, die $\S 611$ a II BGB-A bereits seines Charakters als verschuldensunabhängige Kompensationsvorschrift wegen kritisierten, behaupteten zu recht, die Bestimmung verstoße gegen die Verfassung, soweit ,dem Arbeitgeber eine über die bloße Kompensation hinausgehende [verschuldensunabhängige] „Entschädigungspflicht“ auferlegt wird“. ${ }^{309}$ Das im Grundgesetz verankerte Bild eines frei verantwortlich handelnden Menschen gestatte nur dann eine Bestrafung,

306 Entwurfsbegründung, BT-Druck. 16/1780, S. 38.

307 Richardi/Annuß in Staudinger, $\$ 611$ a, Rn. 84.

308 Richardi/Annu $\beta$ in Staudinger, $\S 611$ a, Rn. 81; im Ergebnis ebenso Raab in Soergel, § 611 a, Rn. 49; Ehmann/Emmert, SAE 1997, S. 253 (259 f.).

309 Richardi/Annu $\beta$ in Staudinger, $\$ 611$ a, Rn. 82. 
wenn der jeweilige Akteur unter Berücksichtigung seiner individuellen Anlagen im konkreten Fall anders hätte handeln können. ${ }^{310}$

Die Notwendigkeit des Verschuldenserfordernisses zeigt sich in besonderem Maße in den Fällen, in denen dem „Diskriminierenden“ einzig und allein „,vorzuwerfen“ ist, eine diskriminierende nationale Bestimmung befolgt zu haben. Es läßt sich in keiner Weise begründen, daß sich derjenige schadensersatzpflichtig machen soll, der den Vorgaben eines nationalen - wenn auch diskriminierenden - Gesetzes gemäß handelt.

Im Falle des $\S 611$ a II BGB-A schlug man deshalb vor, die Bestimmung verfassungskonform dergestalt $\mathrm{zu}$ reduzieren, daß der dort geregelte Anspruch auf Ersatz immaterieller Schäden Verschulden voraussetze. ${ }^{311}$

Dies wäre indes der Rechtsprechung des EuGH zur Richtlinie 76/207/EWG zuwider gelaufen, aufgrund derer $\S 611$ a II BGB-A seine Änderung von einem verschuldensabhängigen zu einem verschuldensunabhängigen Schadensersatzanspruch erfahren hatte. ${ }^{312}$ In diesem Zusammenhang ist jedoch folgendes zu beachten:

Das Bundesverfassungsgericht ging in seiner Rechtsprechung zunächst davon aus, daß sich sekundäres Gemeinschaftsrecht - Verordnungen und Richtlinien - vor den Grundrechten des Grundgesetzes bewähren müsse, solange das Gemeinschaftsrecht selbst keinen Grundrechtskatalog habe, der dem Grundrechtskatalog des Grundgesetzes adäquat sei. ${ }^{313}$ Später relativierte das Gericht seine Rechtsprechung, da es, wie die herrschende Meinung in der Literatur schon zuvor, ${ }^{314}$ nunmehr davon ausging, „,im Hoheitsbereich der Europäischen Gemeinschaften [sei] ein Maß an Grundrechtsschutz erwachsen, das nach Konzeption, Inhalt und Wirkungsweise dem Grundrechtsstandart des Grundgesetzes im wesentlichen gleichzuachten“ sei. ${ }^{315}$ Es wollte abgeleitetes Gemeinschaftsrecht nicht mehr am Maßstab der Grundrechte des Grundgesetzes überprüfen, „,[s]olange die Europäischen Gemeinschaften, insbesondere die Rechtsprechung des Gerichtshofs der Gemeinschaften einen wirksamen Schutz der Grundrechte gegenüber der Hoheitsgewalt der Gemeinschaften generell gewährleist[et]en, der dem vom Grundgesetz unabdingbar gebotenen Grundrechtsschutz im wesentlichen gleich zu achten [sei], zumal den

310 Richardi/Annuß in Staudinger, $\S 611$ a, Rn. 82.

311 Richardi/Annu $\beta$ in Staudinger, $\S 611$ a, Rn. 82.

312 Seit seiner Änderung durch das Gesetz zur Änderung des Bürgerlichen Gesetzbuchs und des Arbeitsgerichtsgesetzes vom 29.06.1998 - (BGB1. 1998 I 2, S. 1694 f.) - war $\S 611$ a II BGB-A verschuldensunabhängig ausgestaltet. Zur Entwicklung des $§ 611$ a II BGB-A von einer verschuldensabhängigen zu einer verschuldensunabhängigen Schadensersatzregelung siehe Richardi/Annu $\beta$ in Staudinger, $\S 611$ a, Rn. 72 ff.; Der deutsche Gesetzgeber hatte durch die Abschaffung des Verschuldenserfordernisses eine Vorgabe des $E u G H$ umgesetzt. (vgl. dazu EuGH, NJW 1991, S. 628 (629); NJW 1997, S. 1839 (1840)).

313 BVerfGE 37, S. 271 (285).

314 Schweitzer, Staatsrecht III, Rn. 76.

315 BVerfGE 73, S. 339 (378). 
Wesensgehalt der Grundrechte generell [verbürge]“. ${ }^{316}$ Das Bundesverfassungsgericht hat mit dieser Rechtsprechung seine Forderung nach völliger Adäquanz des Grundrechtsschutzes aufgegeben $^{317}$ und sich auf eine ,generelle Gewährleistung des unabdingbaren Grundrechtsstandards ${ }^{\text {318 }}$ zurückgezogen. ${ }^{319}$

Vor dem Hintergrund der Rechtsprechung des BVerfG wäre es dem deutschen Gesetzgeber trotz der Vorgaben des EuGH unbenommen geblieben, auf die Schaffung einer verschuldensunabhängigen Entschädigungspflicht in $§ 611$ a II BGB-A und $\S 15$ II 1, 21 II 3 AGG zu verzichten, verletzte bzw. verletzt sie doch den durch das Grundgesetz gewährleisteten unabdingbaren Kern grundrechtlichen Schutzes zugunsten des „Diskriminierenden“.

Anzumerken ist, daß die Frage nach der Notwendigkeit des Verschuldenserfordernisses dadurch relativiert wird, „daß bei unmittelbaren Diskriminierungen ein auch subjektives Verschulden nur in den seltensten Fällen [...] verneint werden kann.“320 Praktische Bedeutung erlangt das Verschuldenserfordernis dagegen im Rahmen der mittelbaren Diskriminierung, ,da hier zahlreiche Fälle denkbar sind, in denen der Arbeitgeber auf Grund mangelnder Tatsachentransparenz selbst bei Anwendung aller ihm zumutbaren Sorgfalt einen Verstoß gegen das Diskriminierungsverbot nicht vermeiden kann. “321

\section{e) $\$ 253$ II BGB-E}

Die Ablehnung verschuldensunabhängiger Entschädigungsansprüche im Sinne des $§ 611$ a II BGBA sowie der $\S \S 15$ II 1 und 21 II 3 AGG führt nicht dazu, daß der Ersatz immaterieller Schäden generell ausgeschlossen ist. Der Schadensbegriff des BGB umfaßt, wie § 253 BGB verdeutlicht, auch den Nichtvermögensschaden. Dabei hat der Verletzte auch bei immateriellen Schäden, soweit Naturalrestitution möglich ist, den Herstellungsanspruch aus $\S 249$ BGB. ${ }^{322}$ Der Anspruch auf

316 BVerfGE 73, S. 339 (387).

317 Schweitzer, Staatsrecht III, Rn. 81.

318 BVerfGE 89, S. 155 (175).

319 Auch die Zustimmungsgesetze zu völkerrechtlichen Verträgen sollen der Jurisdiktion des Bundesverfassungsgerichtes unterliegen. (Streinz in Sachs, Art. 59, Rn. 71) Selbst eine Verfassungsbeschwerde lasse sich auf eine Grundrechtsverletzung durch das Zustimmungs- bzw. Vertragsgesetz stützen. (Pernice in Dreier, Band II, Art. 59, Rn. 53) Das Vertragsgesetz und damit letztlich die Wirkung des völkerrechtlichen Vertrages im innerstaatlichen Recht könne das Gericht dadurch erhalten, daß es das Vertragsgesetz verfassungskonform auslegt. (Streinz in Sachs, Art. 59, Rn. 73; Pernice in Dreier, Band II, Art. 59, Rn. 54) Auch bestehe bezüglich des Völkervertragsrechts ein „Souveränitätsvorbehalt“, der den Gesetzgeber verpflichte, selbiges nicht zu beachten, sofern nur auf diese Weise ein Verstoß gegen tragende Grundsätze der Verfassung abzuwenden sei. (Müller in FAZ vom 13.04.2005, S. 5 unter dem Titel: „Identität des deutschen Verfassungsstaates“).

Richardi/Annuß in Staudinger, § 611 a, Rn. 83; ebenso Ehmann/Emmert, SAE 1997, S. 253 (259); Pfarr/Bertelsmann vertreten sogar die Ansicht, daß „,bei Fällen unmittelbarer Diskriminierung Verschulden in Form der Fahrlässigkeit immer gegeben“ ist. (Pfarr/Bertelsmann, Diskriminierung im Erwerbsleben, S. 77); vgl. auch Entwurfsbegründung zum AGG, BT-Druck. 16/1780, S. 46.

321 Richardi/Annuß in Staudinger, $\S 611$ a, Rn. 83; ähnlich Pfarr/Bertelsmann, Diskriminierung im Erwerbsleben, S. 128.

322 Heinrichs in Palandt, Vorbem v $\S 249$, Rn. 7. 
Naturalherstellung, der unter anderem im Widerruf einer ehrverletzenden Äußerung bestehen kann, hat aber nur geringe praktische Bedeutung, da Naturalbeseitigung meist nicht möglich ist. ${ }^{323}$ Doch selbst dann, wenn im Falle einer Diskriminierung Naturalbeseitigung der immateriellen Schäden möglich sein sollte, dürfte allein dadurch die Forderung der Richtlinien nach wirksamen, verhältnismäßigen und abschreckenden Sanktionen nicht erfüllt sein.

Gemäß § 253 BGB kann in bestimmten Fällen auch für Nichtvermögensschäden eine Entschädigung in Geld gefordert werden. Das gilt gem. § 253 II BGB unter anderem dann, wenn wegen einer Verletzung des Körpers, der Gesundheit, der Freiheit oder der sexuellen Selbstbestimmung Schadensersatz zu leisten ist. Angesichts der Tatsache, daß Diskriminierung vielfach keine oder nur sehr geringe Vermögensschäden verursacht, ${ }^{324}$ die Richtlinien aber Sanktionen fordern, die wirksam, verhältnismäßig und abschreckend sind, ${ }^{325}$ wird vorgeschlagen, $\S$ 253 II BGB um den Begriff der Diskriminierung zu erweitern. ${ }^{326}$

\section{$\S 253$ II BGB-E}

Ist wegen einer Verletzung des Körpers, der Gesundheit, der Freiheit oder der sexuellen Selbstbestimmung oder wegen Verstoßes gegen das Diskriminierungsverbot Schadensersatz zu leisten, kann auch wegen des Schadens, der nicht Vermögensschaden ist, eine billige Entschädigung in Geld gefordert werden.

Daß dieser Anspruch schuldhaftes Handeln des Diskriminierenden voraussetzt, ergibt sich dabei aus $\S 823$ II 2 BGB.

\section{f) $\S \$ 280,826 \mathrm{BGB}$}

Da Ansprüche aus den $\S \S 823$ ff. BGB grundsätzlich selbständig nebeneinander und neben anderen Ansprüchen stehen, ${ }^{327}$ können neben dem Schadensersatzanspruch aus $§ 823$ II BGB in Verbindung

323 Heinrichs in Palandt, $\S 253$, Rn. 3.

324 Nickel spricht insofern von dem „Problem der „schadlosen“ Diskriminierungen“. (Nickel, Gleichheit und Differenz in der vielfältigen Republik, S. 197); Die Entwurfsbegründung zum AGG nennt in diesem Zusammenhang nur ein Beispiel, das zudem äußerst konstruiert erscheint: „Weigert sich etwa ein Taxiunternehmer, einen Fahrgast wegen seiner ethnischen Herkunft zu befördern, und entgeht dem Benachteiligten hierdurch ein Geschäft, weil er einen entsprechenden Termin nicht einzuhalten vermag, so ist dieser Vermögensschaden nach $\S 21$ Abs. 2 Satz 1 zu ersetzen.“ (Entwurfsbegründung, BT-Druck. 16/1780, S. 46).

325 Das $B A G$ hat in diesem Zusammenhang festgestellt, daß etwa der Ersatz der Bewerbungskosten in Höhe von 0,15 DM (Kosten für einen Briefumschlag) nicht zu einer abschreckenden Sanktion in diesem Sinne führt. (BAG, NJW 1990, S. 65 (67)).

326 In diesem Sinne auch Wiedemann/Thüsing, die $\S 253$ BGB ,um die Verletzung des allgemeinen Persönlichkeitsrechts“ erweitern wollen (Wiedemann/Thüsing, DB 2002, 463 (469)); Schon der Gesetzesentwurf von Bündnis 90/Die Grünen sah einen derartigen Anspruch vor. Zu diesem Zweck war die Einführung eines $\S 847$ a in das BGB vorgesehen.

327 Sprau in Palandt, Einf v $§ 823$, Rn. 4. 
mit $\S 115$ BGB-E bei Vorliegen der speziellen Voraussetzungen auch solche aus den $\S \S 280$ und 826 BGB hergeleitet werden. ${ }^{328}$

\section{Beseitigungs- und Unterlassungsanspruch $-\S 1004$ BGB analog}

In analoger Anwendung des $\S 1004$ I BGB kommt als mögliche Rechtsfolge ein verschuldensunabhängiger Beseitigungs- und Unterlassungsanspruch des Diskriminierten in Betracht. § 1004 I BGB schützt in entsprechender Anwendung alle deliktisch geschützten Rechtsgüter, also auch die von $§ 823$ II BGB i. V. m. dem jeweiligen Schutzgesetz umfaßten. ${ }^{329}$ Das Allgemeine Gleichbehandlungsgesetz statuiert in $\S 21$ I explizit einen Beseitigungs- und Unterlassungsanspruch. Darüber hinaus verweist es in $\S 15 \mathrm{~V}$ implizit auf $\S 1004$ BGB. ${ }^{330}$ Der Unterlassungsanspruch aus § 21 I AGG kann laut Entwurfsbegründung beispielsweise darauf gerichtet sein, künftig die Verweigerung des Zugangs zu einer Einkaufspassage zu unterlassen. ${ }^{331}$

Bezüglich des Beseitigungsanspruchs ist zu beachten, daß der Inhalt dessen, was als Beseitigung verlangt werden kann, vom Schadensersatz in Form der Naturalrestitution abgegrenzt werden muß. „Denn Schadensersatz kann der Verletzte nach $\S \S 823$ ff. nur bei Verschulden des Täters fordern. Die Beseitigung muß also wesentlich enger sein, damit man über den Beseitigungsanspruch nicht zu einer Schadensersatzpflicht ohne Verschulden kommt. “332 Auch die Entwurfsbegründung zu § 21 I AGG stellt klar, daß es sich bei dem Beseitigungsanspruch um einen verschuldensunabhängigen Anspruch handelt: „Satz 1 gibt bereits beim objektiven Verstoß gegen das Benachteiligungsverbot einen Beseitigungsanspruch.“333

\section{Anspruch auf Vertragsschluß}

Der Unterlassungsanspruch aus § 21 I AGG kann laut Entwurfsbegründung - wie bereits erwähnt darauf gerichtet sein, die Verweigerung des Zugangs zu einer Einkaufspassage zu unterlassen. ${ }^{334}$ Dieses Beispiel wirft die Frage auf, ob der Eigentümer der Einkaufspassage als Verkäufer der in selbiger zum Verkauf angebotenen Waren auch verpflichtet ist, Kaufverträge mit der Person abzuschließen, der er den Zugang zu seinen Verkaufräumen gewähren muß. Kommt, allgemeiner

328 Heinrichs in Palandt, Anh nach $\S 319$, Rn. 23; Larenz/Wolf, Allgemeiner Teil des Bürgerlichen Rechts, $\S 34$, Rn. 42.

329 Bassenge in Palandt, § 1004, Rn. 4.

330 Entwurfsbegründung, BT-Druck. 16/1780, S. 38.

331 Entwurfsbegründung, BT-Druck. 16/1780, S. 46.

332 Medicus, Bürgerliches Recht, Rn. 629.

333 Entwurfsbegründung, BT-Druck. 16/1780, S. 46.

334 Entwurfsbegründung, BT-Druck. 16/1780, S. 46. 
gefragt, als mögliche Rechtsfolge eines Verstoßes gegen das Diskriminierungsverbot ein Anspruch auf Vertragsschluß in Betracht?

Dem geltenden Recht sind Fälle gesetzlichen Kontrahierungszwangs nicht fremd, so daß die Diskussion um eine solche Rechtsfolge im Antidiskriminierungsrecht nicht von vornherein abwegig ist. § 22 Personenbeförderungsgesetz (PBefG) verpflichtet beispielsweise den Unternehmer, grundsätzlich jeden Antrag auf Abschluß eines bürgerlich-rechtlichen Beförderungsvertrages

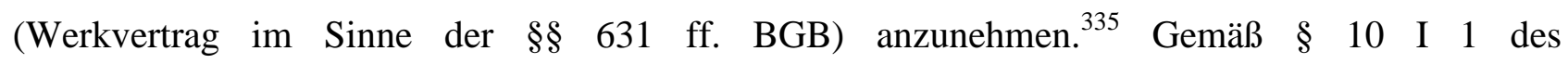
Energiewirtschaftsgesetzes (EnWG) haben Energieversorgungsunternehmen ,für Gemeindegebiete, in denen sie die allgemeine Versorgung von Letztverbrauchern durchführen, Allgemeine Bedingungen und Allgemeine Tarife für die Versorgung in Niederspannung oder Niederdruck öffentlich bekanntzugeben und $\mathrm{zu}$ diesen Bedingungen und Tarifen jedermann an ihr Versorgungsnetz anzuschließen und zu versorgen." Bei fehlender expliziter gesetzlicher Anordnung wurde ein Kontrahierungszwang aus $\S 826$ BGB abgeleitet. ${ }^{336}$ Auch in Diskriminierungsfällen wurde die Frage aufgeworfen, ob $§ 826$ BGB zu einem Anspruch des Diskriminierten auf Abschluß des angestrebten Vertrages führen kann. ${ }^{337}$

Die Antidiskriminierungsrichtlinien schreiben einen Kontrahierungszwang als Rechtsfolge nicht explizit vor. Die Richtlinie 2000/43/EG macht nur insoweit Vorgaben, als gem. Art. 15 Satz 2 ,„[...] Sanktionen, die auch Schadensersatzleistungen an die Opfer umfassen können, [...] wirksam, verhältnismäßig und abschreckend sein [müssen]. ${ }^{338}$

\section{a) $\$ 611$ a II 2. Halbsatz BGB-A}

Es wurde festgestellt, daß nach den Antidiskriminierungsrichtlinien bereits das Vorfeld des Vertragsschlusses zum sachlichen Geltungsbereich des Diskriminierungsverbots zählt. In diesem Sinne durfte auch gem. $§ 611$ a I 1 BGB-A der Arbeitgeber einen Arbeitnehmer

„,...] insbesondere bei der Begründung des Arbeitsverhältnisses [...] nicht wegen seines Geschlechts benachteiligen.“

Unter Berücksichtigung der Verpflichtung aus $§ 611$ a I 1 BGB-A hätte diskriminierungsfreies Verhalten in einem Fall, in dem sich um eine Stelle nur ein Interessent bewirbt, der zwar hinreichend qualifiziert ist, aber das vom Arbeitgeber nicht erwünschte Geschlecht aufweist, bedeutet, diesen einzustellen. Zu diesem Schluß kommt man jedenfalls dann, wenn man das Verbot

\footnotetext{
335 Fromm/Fey/Sellmann/Zuck, Personenbeförderungsrecht, § 22, Rn. 1.

336 Vergleiche dazu Timme, ZAR 1997, S. 130 (131).

337 Vergleiche dazu Timme, ZAR 1997, S. 130 (132 ff.).

338 Vergleiche auch Art. 8 d S. 2 Richtlinie 76/207/EG; Art. 17 S. 2 Richtlinie 2000/78/EG sowie Art. 14 S. 2 Richtlinie 2004/113/EG.
} 
der Nicht-Einstellung als Gebot der Einstellung begreift. ${ }^{339}$ So gesehen vermittelt $§ 611$ a I 1 BGBA den Eindruck, die Norm habe einen Kontrahierungszwang statuiert. ${ }^{340}$

$\S 611$ a II 2. HS BGB-A belehrt jedoch eines besseren. Gemäß dieser Norm bestand bei einem Verstoß gegen das Benachteiligungsverbot in der Einstellungssituation kein Anspruch auf Begründung des Arbeitsverhältnisses.

Zwischen Tatbestand und Rechtsfolge des $§ 611$ a BGB-A bestand insofern ein Widerspruch als Fälle denkbar sind, bei denen das einzig nicht-diskriminierende Verhalten des Arbeitgebers darin bestanden hätte, den unerwünschten Bewerber einzustellen. Einen Einstellungsanspruch schloß das Gesetz jedoch explizit aus. Der im Einzellfall bestehenden Einstellungspflicht des Arbeitgebers stand also kein Anspruch des Bewerbers auf Einstellung gegenüber.

\section{b) $§ 15$ VI AGG und $§ 15$ VI, 21 II ADG-E-2005}

Angesichts der Tatsache, daß der deutsche Gesetzgeber bei der Umsetzung der Richtlinie 76/207/EWG durch Einführung des $§ 611$ a BGB-A einen Kontrahierungszwang als Rechtsfolge explizit ausgeschlossen hatte, war zu vermuten, daß er diesen Weg auch bei der Umsetzung der Richtlinien aus den Jahren 2000 und 2004 beschreiten würde.

Es verwundert deshalb nicht, daß $\S 15$ VI AGG in Anlehnung an $\S 611$ a II u. V BGB-A ${ }^{341}$ bestimmt, daß

„[e]in Verstoß des Arbeitgebers gegen das Benachteiligungsverbot des $§ 7$ Abs. 1 [...] keinen Anspruch auf Begründung eines Beschäftigungsverhältnisses, Berufsausbildungsverhältnisses oder einen beruflichen Aufstieg [begründet], es sei denn ein solcher ergibt sich aus einem anderen Rechtsgrund. ‘342

§ 21 AGG enthält eine vergleichbare Bestimmung nicht. Dies ist bemerkenswert angesichts der Tatsache, daß § 21 I u. II AGG Beseitigungs- und Schadensersatzansprüche statuiert und gem. § 21 III AGG Ansprüche aus unerlaubter Handlung unberührt bleiben. Nun läßt sich der Anspruch des Diskriminierten auf Vertragsschluß einerseits aus $\S 249$ I BGB herleiten. Besteht die Diskriminierungshandlung in der Ablehnung des Vertragsschlusses, führt die Verpflichtung des Schädigers zur Naturalrestitution zu einem Kontrahierungszwang, ${ }^{343}$ soweit dies möglich ist (§ 251

339 So Adomeit, DB 1980, S. 2388 (2388); Bydlinski, AcP 180, S. 1 (13); Larenz, SchuldR I, § 4 I. a).

340 Adomeit, DB 1980, S. 2388 (2388).

341 Entwurfsbegründung, BT-Druck. 16/1780, S. 38.

342 Als ,anderen Rechtsgrund“ im Sinne der Bestimmung nennt die Entwurfsbegründung beispielhaft den tariflichen Bewährungsaufstieg. (Entwurfsbegründung, BT-Druck. 16/1780, S. 38).

343 BGHZ 21, S. 1 (8), 44 , S. 279 (283); Darauf weisen auch Wiedemann/Thüsing hin. (Wiedemann/Thüsing, DB 2002, 463 (469)) Daß die Verpflichtung zur Naturalrestitution zu einem Kontrahierungszwang führen kann, übersieht Nickel, wenn er behauptet, die Gesetzesentwürfe von SPD und Bündnis 90/Die Grünen enthielten keinen Kontrahierungszwang, sondern nur ,die üblichen zivilrechtlichen Rechtsfolgen (Unterlassung, Schadensersatz, Schmerzensgeld).“ (Nickel, Gleichheit und Differenz in der vielfältigen Republik, S. 197). 
I BGB). Auf der anderen Seite wird vertreten, der Anspruch auf Vertragsschluß könne auch als quasinegatorischer Folgebeseitigungsanspruch, der kein Verschulden voraussetzt, ${ }^{344}$ geltend gemacht werden. ${ }^{345}$

Läßt sich aus § 21 I bzw. II AGG somit ein Anspruch auf Vertragsschluß herleiten? Nach den bisherigen Ausführungen müßte diese Frage bejaht werden. Eine dem $\S 15$ VI AGG vergleichbare Regelung enthält $§ 21$ AGG nicht. Außerdem macht ein Anspruch auf Zugang zu einem Kaufhaus nur Sinn, wenn er mit einem solchen auf Vertragsschluß verbunden ist.

Betrachtet man sich jedoch die historische Entwicklung des Allgemeinen Gleichbehandlungsgesetzes, regen sich Bedenken gegen diese Schlußfolgerung. Das AGG basiert auf dem Antidiskriminierungsgesetz-Entwurf vom 16.12.2004, ${ }^{346}$ der mit rot-grüner Mehrheit am 17.06.2005 vom Bundestag verabschiedet worden war. ${ }^{347} \S 21$ II ADG-E sah explizit einen Kontrahierungszwang vor:

„Im Fall einer Vertragsverweigerung kann der Benachteiligte den Abschluß eines Vertrages nur verlangen, wenn dieser ohne Verstoß gegen das Benachteiligungsverbot erfolgt wäre. Die Leistung muß hinreichend bestimmt sein; die Gegenleistung ist im Zweifel nach $§ 315$ Abs. 3 und $\S 316$ des Bürgerlichen Gesetzbuchs zu ermitteln.“

Der Bundesrat, der am 08.07.2005 Einspruch gegen den Gesetzesentwurf eingelegt und beschlossen hatte, den Vermittlungsausschuß mit dem Ziel einzuberufen, das Gesetzeswerk im Sinne einer Beschränkung auf das europarechtlich zwingend gebotene zu überarbeiten, verlangte unter anderem die Streichung des in $\S 21$ II ADG-E verankerten Anspruchs auf Abschluß eines Vertrages als mögliche Folge eines Verstoßes gegen das zivilrechtliche Benachteiligungsverbot. ${ }^{348}$

Es kann vermutet werden, daß es der Gesetzgeber des AGG aus diesem Grund unterlassen hat, eine dem $§ 21$ II ADG-E vergleichbare Bestimmung in $§ 21$ AGG aufzunehmen, woraus geschlußfolgert werden kann, daß es Intention des Gesetzgebers sein dürfte, auch bei einem Verstoß gegen das zivilrechtliche Benachteiligungsverbot des § 19 AGG keinen Anspruch auf Vertragsschluß zu gewähren. Ein Indiz für einen derartigen Willen des Gesetzgebers liefert die Entwurfsbegründung, wonach der Anspruch aus $\S 21$ I AGG ,tatsächliches Handeln“ betreffen könne. ${ }^{349}$ Ob damit der Ausschluß rechtsgeschäftlichen Handels zum Ausdruck gebracht werden sollte, ist indes fraglich.

Zusammenfassend kann festgehalten werden, daß sich aus § 21 I AGG, § 21 II AGG sowie aus $\S$ 21 III AGG i. V. m. §§ 823 ff. BGB ein Anspruch auf Vertragsschluß ableiten läßt, die historische Entwicklung des $§ 21$ AGG indes die Vermutung nahe legt, daß diese Rechtsfolge vom Gesetzgeber wohl nicht gewollt ist.

344 Heinrichs in Palandt, Einf v $§ 145$, Rn. 9.

345 Heinrichs in Palandt, Anh nach § 319, Rn. 20; ebenso Bydlinski, AcP 180, S. 1 (13); Larenz, SchuldR I, § 4 I. a).

346 BT-Druck. 15/4538.

347 BR-Druck. 445/05.

348 BR-Druck. 445/05 (Beschluß) unter Punkt 9.

349 Entwurfsbegründung, BT-Druck. 16/1780, S. 46. 


\section{c) $§ 1004$ BGB analog}

Gegner eines zivilrechtlichen Antidiskriminierungsgesetzes sehen durch dessen Einführung den im Zivilrecht verankerten Grundsatz der Privatautonomie gefährdet: „Denn der Entwurf für das Antidiskriminierungsgesetz kann [...] der Anfang vom Ende der Vertragsfreiheit sein.“350 ${ }^{\text {In }}$ besonderer Kritik steht dabei die Beschränkung der Freiheit des Privaten, allein mit den Personen Verträge abzuschließen, die ihm genehm sind: „In Deutschland stirbt wieder ein Stück Freiheit. Vermieter sollen gezwungen werden, Verträge gegen ihren Willen abzuschließen.““351

Auf der anderen Seite wird vertreten, Antidiskriminierungsmaßnahmen könnten ,in zumutbaren Fällen“ einen Kontrahierungszwang statuieren. ${ }^{352}$

Die Befürworter des Kontrahierungszwangs als mögliche Rechtsfolge im Antidiskriminierungsrecht begründen ihre Ansicht damit, daß derjenige, der durch öffentliche Angebote bekunde, es sei irrelevant, wer Vertragspartei werde, wenn nur die sachlichen Bedingungen erfüllt werden, die das Angebot enthält, an genau dieses Angebot gebunden werden kann. ${ }^{353}$ Diese Begründung vermag nicht zu überzeugen. Natürlich ist denkbar, daß der Anbietende undifferenzierte Angebote in Anzeigen macht, weil ihm bestimmte Eigenschaften und Merkmale des potenziellen Vertragspartners egal sind. Denkbar ist aber auch, daß er die öffentlichen Angebote einzig und allein deshalb merkmalsneutral ausgestaltet, weil er dazu verpflichtet ist. ${ }^{354}$ Aus der Befolgung eines solchen Gebots kann demnach nicht der Schluß gezogen werden, für den „Diskriminierenden“ sei irrelevant, wer Vertragspartei wird.

Auch den Gegnern des Kontrahierungszwangs mangelt es an einer überzeugenden Begründung ihrer Ansicht. Daß gerade der geplante Eingriff in die Abschlußfreiheit kritisiert wird ist der Tatsache geschuldet, daß es sich beim Abschluß eines Vertrages grundsätzlich um einen rechtlich freien Akt handelt $\mathrm{t}^{355}$ - ob es überhaupt zum Vertragsschluß kommt, hängt vom Willen der

350 FAZ vom 16.12.2004, S. 1; Kritik auch bei Wagner in FAZ vom 22.12.2004, S. 12 unter dem Titel: „Das Antidiskriminierungsgesetz - ein Angriff auf die Vertragsfreiheit“.

351 FAZ vom 10.12.2004, S. 43 zitiert den Präsidenten des Verbandes Haus \& Grund Dorn unter dem Titel: „Die Wohnungswirtschaft warnt vor weiteren Belastungen“".

352 Baer, ZRP 2002, S. 290 (293); Ein solcher bestehe sogar dann, wenn der Abschluß eines Arbeitsvertrages richtlinienwidrig abgelehnt worden ist. (Heinrichs, dessen Feststellung nicht mit einer Kritik an dieser Rechtsfolge einhergeht. Zu beachten ist jedoch, daß er seine Aussage nur auf die Richtlinie 2000/43/EG bezieht. (Heinrichs in Palandt, Anh nach $\S 319$, Rn. 20)).

353 Baer, ZRP 2002, S. 290 (293).

354 Auch Baer anerkennt die Pflicht zu merkmalsneutraler Ausschreibung bzw. merkmalsneutralem Angebot, wenn sie sagt, ein öffentliches Warenangebot exklusiv an Menschen bestimmter sexueller Identität sei eindeutig problematisch. (Baer, ZRP 2002, S. 290 (294)).

355 Adomeit, DB 1980, S. 2388 (2388). 
Vertragsparteien ab. Daraus wird der Schluß gezogen, daß man das schlecht tun dürfe, was man nicht tun müsse. ${ }^{356}$

Es läßt sich nicht bestreiten, daß beispielsweise die Einstellung insofern ein rechtlich freier Akt ist, als der Arbeitgeber entscheiden kann, ob er überhaupt jemanden einstellt. Diese Freiheit bzgl. des „Ob“ führt aber nicht zwangsläufig zu einer Freiheit des „Wie“. Wer einen Verkehr eröffnet ist verpflichtet, Dritte vor Schäden zu schützen, die dieser Verkehr mitsichbringen kann. Auch hier ist derjenige frei in der Entscheidung, ob er einen Verkehr eröffnet. Das führt aber nicht dazu, daß er dies in einer für Dritte schädigenden Form tun darf. Das Argument: „Was man nicht tun muß, das darf man auch schlecht machen."vermag nicht zu überzeugen.

Es wird behauptet, der Vertrag als Instrument gelungener zweiseitiger Selbstbestimmung trage seine Legitimität und Dignität in sich; die Willenseinigung der Privatrechtssubjekte biete typischerweise eine größere Chance auf eine materielle Richtigkeitsgewähr für die Vertragsbeteiligten als jede staatliche Zensur des Vertragsschlusses und seiner Motive. ${ }^{357}$ Diese Ansicht läßt völlig unberücksichtigt, daß sich die potenziellen Vertragspartner oft nicht auf gleicher Augenhöhe gegenüberstehen. Das gilt z. B. dann, wenn der Abschluß eines Arbeitsvertrages in Rede steht: „Bei Arbeitsvertragsschluß stehen sich Arbeitgeber und Arbeitnehmer nicht als gleichwertige Vertragspartner gegenüber. Die Vertrags- und Verhandlungsschwäche des Arbeitnehmers ergibt sich schon daraus, daß er auf den Arbeitsplatz angewiesen ist und der Arbeitsmarkt in der Regel wenig nutzbare Ausweichmöglichkeiten bietet. “358 Der Diskriminierende vermag seine Verhandlungstärke in der Weise auszunutzen, daß er den Vertrag mit der von ihm abgelehnten Person nicht schließt. Dementsprechend sind die meisten Fälle, bei denen sich die Frage nach dem Vorliegen einer Diskriminierung stellt, dadurch gekennzeichnet, daß es zu einem Vertragsschluß gar nicht erst kommt.

Beispiel: Von gelungener zweiseitiger Selbstbestimmung und übereinstimmendem Willen der Vertragspartner ${ }^{359}$ kann nicht die Rede sein, wenn einem Schwarzafrikaner der Eintritt in eine Discothek mit der Begründung verwehrt wird, der Zutritt sei für „Schwarzköpfe“ nicht gestattet. ${ }^{360}$

In einem derartigen Fall spricht kein vernünftiger, am Gerechtigkeitsgedanken orientierter Grund dagegen, dem Diskriminierten einen Anspruch auf Vertragsschluß zu gewähren. Ein Anspruch - um

356 Adomeit, DB 1980, S. 2388 (2388).

357 Säcker, ZRP 2002, S. 286 (287).

358 LAG Hamm, NJW 2002, S. 1970 (1971).

359 „Im Vertragsrecht ergibt sich der sachgerechte Interessenausgleich [grundsätzlich] aus dem übereinstimmenden Willen der Vertragspartner." (BVerfGE 89, S. 214 (232)).

360 Das Aktenmaterial der Göttinger Antidiskriminierungs-Beratungsstelle schildert einen Fall, der sich am 28.12.2002 zugetragen hat. Ein Türsteher der Göttinger Discothek „Savoy“ soll gesagt haben, es bestehe die Anweisung, „Schwarzköpfen“ den Zutritt zu verwehren. 
auf das Beispiel der Entwurfsbegründung zum AGG zurückzukommen - auf Gewährung des Zutritts zu einem Kaufhaus ${ }^{361}$ macht doch nur Sinn, wenn der Diskriminierende grundsätzlich auch verpflichtet ist, (Kauf-)Verträge mit dem Diskriminierten abzuschließen.

Diejenigen, die diese Rechtsfolge kategorisch ausschließen, seien daran erinnert, daß sich die Frage des Anspruchs auf Vertragsschluß erst auf Rechtsfolgenseite stellt, in einem Stadium also, in dem das Vorliegen einer Diskriminierung bereits bejaht worden ist. Da diese Voraussetzung des Anspruchs auf Vertragsschluß gerade in den Fällen, in denen der Kontrahierungszwang besonders vehement abgelehnt wird - gemeint sind Arbeits- und Mietrecht -, vielfach gar nicht vorliegen wird, relativiert sich die Gefahr, die im Kontrahierungszwang gesehen wird, von vornherein. Bei Arbeits- und Mietverträgen besteht die Besonderheit, daß sich um den angebotenen Arbeitsplatz oder die angebotene Wohnung in der Regel mehrere Interessenten bemühen. Diese Tatsache führt dazu, daß der Arbeitgeber bzw. Vermieter die Ablehnung eines Bewerbers bzw. Interessenten eher begründen kann, als beispielsweise der Discothekenbetreiber. ${ }^{362}$

Andere sehen demgegenüber gerade in dieser Tatsache den Grund für die Ablehnung des Kontrahierungszwangs bei derartigen Verträgen. Daß diese „Begründung“ auf tönernen Füßen steht, beweist schon die insofern widersprüchliche Argumentation: ,[...] denn der Vermieter kann nicht allen Interessenten die Wohnung zukommen lassen, sondern muß notwendig eine Auswahl treffen. Welchen Interessenten er dabei aussucht, muß ihm überlassen bleiben. Er darf jeden Interessenten ablehnen. Ablehnungsgrund darf jedoch nicht einer der unter das Diskriminierungsverbot fallenden Gründe sein. Eine Abschlußpflicht ist daraus jedoch nicht abzuleiten.“ ${ }^{363}$ Die Vertreter dieser Ansicht, die bei einem „unbegrenzten oder breiten öffentlichen Angebot“ - beispielhaft sei der Zugang zur Discothek genannt - eine Abschlußpflicht bejahen, ${ }^{364}$ müssen sich fragen lassen, warum sie gerade in einem Bereich, in dem der „Diskriminierte“ besonders schutzwürdig ist - gemeint ist vor allem der Bereich des Arbeitsrechts - bezüglich der Sanktionen geringeren Schutz gewähren. Hier besteht ein Wertungswiderspruch ${ }^{365}$ auch wenn berücksichtigt wird, daß auch der „Diskriminierende“ gerade dann, wenn Dauerschuldverhältnisse in Rede stehen, seinerseits besonders schutzwürdige Interessen in die Waagschale werfen kann.

Abzulehnen ist schließlich auch die Ansicht, die den Kontrahierungszwang ablehnt, wenn dem abgewiesenen Interessenten zumutbare Ausweichmöglichkeiten zur Verfügung stehen. Der Abgewiesene soll in einem solchen Fall die Ausweichmöglichkeiten nutzen, „statt mit dem Unternehmer, der ihn nicht bedienen will, zu prozessieren. Die Rechtsordnung [habe] keinen

361 Entwurfsbegründung, BT-Druck. 16/1780, S. 46.

362 Im Falle des Discothekenbesuchs bemühen sich - zumindest solange die Kapazitätsgrenze der Einrichtung nicht erreicht ist - nicht mehrere Interessenten um den Abschluß ein und desselben Vertrages.; vgl. zu diesem wesentlichen Unterschied Teil 3, Prüfungspunkt H.

363 Larenz/Wolf, Allgemeiner Teil des Bürgerlichen Rechts, § 34, Rn. 41.

364 Larenz/Wolf, Allgemeiner Teil des Bürgerlichen Rechts, § 34, Rn. 41.

365 Auf diesen Wertungswiderspruch weisen in anderem Zusammenhang auch Wiedemann/Thüsing hin. (Wiedemann/Thüsing, DB 2002, S. 463 (468)). 
Grund, querulatorisches Verhalten zu fördern oder gar dazu anzuregen. ${ }^{\text {*366 }}$ Zum einen läßt diese Ansicht offen, wann eine Ausweichmöglichkeit zumutbar ist. Zum anderen - und das ist der entscheidende Grund - ist demjenigen, der etwa seinen Abend in einer bestimmten Discothek verbringen will, querulatorisches Verhalten nicht vorzuwerfen, selbst wenn er die Wahl zwischen verschiedenen Einrichtungen hat.

Anzumerken ist letztlich, daß der Anspruch auf Vertragsschluß nicht in Widerspruch zu dem Institut der „invitatio ad offerendum“ steht. Obwohl umgangsprachlich oft von dem „Stellenangebot“ die Rede ist, beinhaltet eine Stellenausschreibung regelmäßig noch kein Angebot im Rechtssinne, sondern nur eine Aufforderung an das Publikum, Bewerbungen zu unterbreiten. ${ }^{367}$ Sinn und Zweck der invitatio ad offerendum ist es, den Anbietenden vor einer unkontrollierbaren Fülle von Vertragspartnern zu schützen, denen gegenüber er nicht erfüllen kann. Dieser Zweck wird durch die Statuierung eines Kontrahierungszwangs nicht unterwandert.

Wie bereits erwähnt, läßt sich der Anspruch des Diskriminierten auf Vertragsschluß einerseits aus $\S$ 249 I BGB herleiten. Besteht die Diskriminierungshandlung in der Ablehnung des Vertragsschlusses, führt die Verpflichtung des Schädigers zur Naturalrestitution zu einem Kontrahierungszwang, ${ }^{368}$ soweit dies möglich ist ( $\$ 251$ I BGB).

Auf der anderen Seite wird vertreten, der Anspruch auf Vertragsschluß könne auch als quasinegatorischer Folgebeseitigungsanspruch, der kein Verschulden voraussetzt, ${ }^{369}$ geltend gemacht werden. ${ }^{370}$

Dieser Ansicht kann gefolgt werden, handelt es sich doch bei dem Anspruch auf Vertragsschluß auch um präventiven Rechtsgüterschutz. ${ }^{371}$ Denn solange der Vertrag nicht abgeschlossen ist, besteht für den Diskriminierten die Gefahr des Schadenseintritts bzw. der Schadensvertiefung. Damit unterscheidet sich der Anspruch auf Vertragsschluß in seiner Zielrichtung wesentlich von dem ,gewöhnlichen“ Schadensersatzanspruch. Es ist daher gerechtfertigt, daß er in analoger Anwendung des $§ 1004$ BGB als verschuldensunabhängiger Beseitigungsanspruch geltend gemacht werden kann.

\section{Nichtigkeit der diskriminierenden Willenserklärung - § 115 III BGB-E}

366 Bydlinski, AcP 180, S. 1 (14 f.).

367 Junker, Grundkurs Arbeitsrecht, Rn. 166.

368 BGHZ 21, S. 1 (8), 44 , S. 279 (283); Darauf weisen auch Wiedemann/Thüsing hin. (Wiedemann/Thüsing, DB 2002, 463 (469)) Daß die Verpflichtung zur Naturalrestitution zu einem Kontrahierungszwang führen kann, übersieht Nickel, wenn er behauptet, die Gesetzesentwürfe von SPD und Bündnis 90/Die Grünen enthielten keinen Kontrahierungszwang, sondern nur ,die üblichen zivilrechtlichen Rechtsfolgen (Unterlassung, Schadensersatz, Schmerzensgeld).“ (Nickel, Gleichheit und Differenz in der vielfältigen Republik, S. 197).

369 Heinrichs in Palandt, Einf v $§ 145$, Rn. 9.

370 Heinrichs in Palandt, Anh nach § 319, Rn. 20; ebenso Bydlinski, AcP 180, S. 1 (13); Larenz, SchuldR I, § 4 I. a).

371 So auch Bydlinski, AcP 180, S. 1 (13). 
Schließlich kommt als Rechtsfolge die Nichtigkeit der diskriminierenden Willenserklärung in Betracht. ${ }^{372}$ Denkbar ist dies im Falle diskriminierender Kündigungen. ${ }^{373}$ Bereits nach geltendem Recht sind derartige Kündigungen als sittenwidrig betrachtet und damit gem. § 138 I BGB für nichtig erklärt worden.

$\mathrm{Zu}$ beachten ist jedoch, daß der Diskriminierte unter Umständen an der Wirksamkeit der Willenserklärung interessiert ist. $\mathrm{Zu}$ denken ist etwa an das diskriminierende Vertragsangebot. Der Diskriminierte hat in einem solchen Fall regelmäßig ein Interesse an der Wirksamkeit des Vertragsangebotes. Er möchte lediglich, daß dies diskriminierungsfrei gestaltet wird. ${ }^{374}$ In diesem Sinne wird im Zusammenhang mit der Richtlinie 2000/43/EG vertreten, daß nach deren Schutzzweck nicht der Vertrag im Ganzen unwirksam ist. Nichtig seien nur die benachteiligenden Bedingungen. 375

Daß die diskriminierende Willenserklärung wirksam ist, weil der Diskriminierte ein Interesse an der Wirksamkeit hat, bedeutet also nicht, daß dieser damit genötigt ist, auch die diskriminierenden Bestandteile der Willenserklärung - etwa diskriminierende Bedingungen eines Vertragsangebotes gegen sich gelten zu lassen. In diesem Sinne kann sich gem. § 21 IV AGG der Benachteiligende

„,[a]uf eine Vereinbarung, die von dem Benachteiligungsverbot abweicht, [...] nicht berufen.“

In der Entwurfsbegründung heißt es dazu, die Bestimmung meine einzelne diskriminierende Vertragsabreden. § 139 BGB sei nicht anwendbar. Das Schuldverhältnis bleibe vielmehr im übrigen erhalten, „denn mit der Rückabwicklung des Vertrages wäre dem Benachteiligten oftmals nicht geholfen.“376 Mit der in $§ 21$ IV AGG gewählten Formulierung möchte der Gesetzgeber klarstellen, daß ein diskriminierender und damit nichtiger Teil eines Schuldverhältnisses nicht zur Unwirksamkeit des ganzen Schuldverhältnisses führt. Implizit wird damit § 139 BGB für unanwendbar erklärt. ${ }^{377}$

Auch Interessen Dritter können im Einzelfall gegen die Nichtigkeit der diskriminierenden Willenserklärung sprechen. Das gilt beispielsweise für die Auslobung gem. § 657 BGB. Der

372 Vorbild ist insofern $\S 319$ e II ADG-E des Bundesjustizministeriums, der aber von der Nichtigkeit einseitiger Rechtsgeschäfte spricht.

373 Vergleiche dazu Entwurfsbegründung zu § 21 IV AGG, BT-Druck. 16/1780, S. 47.

374 Darauf weist auch die Entwurfsbegründung des ADG-Entwurfs des Bundesjustizministeriums hin. (http://www.lsvd.de/antidiskriminierung/adgzivil 02.html, S. 51).

375 Heinrichs in Palandt, Anh nach $\S 319$, Rn. 21; ebenso Pfarr/Bertelsmann, Diskriminierung im Erwerbsleben, S. 76 f. und 127; Richardi/Annuß in Staudinger, § 611 a, Rn. 64.

376 Entwurfsbegründung, BT-Druck. 16/1780, S. 47.

377 Eine vergleichbare Formulierung enthält u. a. § 475 I BGB. Auch hier dient sie dem Ziel, § 139 BGB für unanwendbar zu erklären. (Putzo in Palandt, § 475, Rn. 3 a. E. u. 5). 
Diskriminierende könnte hier trotz Nichtigkeit der Auslobung mit deren Befolgung rechnen. ${ }^{378}$ Deshalb wird $\mathrm{zu}$ recht darauf hingewiesen, daß sämtliche mit Dritten begründeten Rechtsbeziehungen in ihrer Rechtswirksamkeit unberührt bleiben. ${ }^{379}$

Vor dem Hintergrund der soeben angestellten Überlegungen wird vorgeschlagen, die Rechtsfolge „Nichtigkeit der Willenserklärung“ wie folgt in § 115 III BGB-E umzusetzen:

Eine Willenserklärung im Sinne des Absatz 1 Satz 1 ist nichtig, sofern nicht Interessen des Erklärungsempfängers oder eines Dritten dem entgegenstehen. Hat der Erklärungsempfänger ein Interesse an der Wirksamkeit der Willenserklärung, kann sich der Erklärende insoweit nicht auf den Inhalt der Willenserklärung berufen, als dieser den Erklärungsempfänger diskriminiert.

$\mathrm{Zu}$ beantworten bleibt die Frage, was an die Stelle des diskriminierenden und damit nichtigen Teils der Willenserklärung tritt. Im Zusammenhang mit der Richtlinie 2000/43/EG wird insofern vertreten, die benachteiligenden und damit nichtigen Vertragsbedingungen würden durch diejenigen ersetzt, zu denen der Anbieter mit anderen Kunden abschließe. ${ }^{380}$ In diesem Sinne gewährte $§ 612$ III BGB-A dem Diskriminierten einen unmittelbaren Erfüllungsanspruch auf gleichen Lohn, ${ }^{381}$ welcher sich nunmehr aus § 7 I AGG i. V. m. § 2 I Nr. 2 AGG ableitet.

Dieser Lösungsansatz berücksichtigt nicht, daß eine Diskriminierung - zumindest nach der hier vertretenen Ansicht - auch dann vorliegen kann, wenn eine Vergleichsperson nicht vorhanden ist. Er vermag deshalb die aufgeworfene Frage nicht befriedigend zu beantworten.

Wenn dem Diskriminierenden gem. § 115 I 2 BGB-E untersagt ist, eine Willenserklärung, der ein öffentliches Angebot - etwa eine invitatio ad offerendum - vorausgeht, in diskriminierender Weise nicht abzugeben, dann muß diesem erst recht verboten sein, das Verlangen des Diskriminierten auf diskriminierungsfreie Ausgestaltung der ihn diskriminierenden Willenserklärung abzulehnen. Die wenn auch diskriminierende - Willenserklärung schafft schließlich eine noch viel engere Bindung der Parteien zueinander als es das öffentliche Angebot vermag.

Es ist deshalb systematisch konsequent, dem Diskriminierten in diesem Fall in analoger Anwendung des $\S 1004$ BGB einen unmittelbaren Erfüllungsanspruch auf diskriminierungsfreie Ausgestaltung der ihn diskriminierenden Willenserklärung zu gewähren.

Der konkrete Inhalt dieses Anspruchs ist einzelfallabhängig. Der Anspruch kann beispielsweise darauf gerichtet sein, dem diskriminierten Arbeitgeber gleichen - d. h. höheren - Lohn zu zahlen oder ihm ein bestimmtes Verhalten - etwa das Tragen des Kopftuchs - zu gestatten.

378 Darauf weist auch die Entwurfsbegründung des ADG-Entwurfs des Bundesjustizministeriums. hin. (http://www.lsvd.de/antidiskriminierung/adgzivil 02.html, S. 51 f.).

379 Richardi/Annuß in Staudinger, § 611 a, Rn. 64; ebenso Raab in Soergel, § 611 a, Rn. 41.

380 Heinrichs in Palandt, Anh nach $\S 319$, Rn. 21; ebenso Pfarr/Bertelsmann, Diskriminierung im Erwerbsleben, S. 76 f. und 127; Richardi/Annu $\beta$ in Staudinger, § 611 a, Rn. 64.

$381 B A G$, NJW 1993, S. 3091 (3091, 4. Leitsatz und 3095). 


\section{Beweislast}

Gemäß Art. 4 I Richtlinie 97/80/EG über die Beweislast bei Diskriminierung aufgrund des Geschlechts $^{382}$ vom 15.12.1997 ergreifen die Mitgliedstaaten im Einklang mit dem System ihrer nationalen Gerichtsbarkeit die erforderlichen Maßnahmen,

„,nach denen dann, wenn Personen, die sich durch die Verletzung des Gleichbehandlungsgrundsatzes für beschwert halten und bei einem Gericht bzw. einer anderen zuständigen Stelle Tatsachen glaubhaft machen, die das Vorliegen einer unmittelbaren oder mittelbaren Diskriminierung vermuten lassen, es dem Beklagten obliegt zu beweisen, daß keine Verletzung des Gleichbehandlungsgrundsatzes vorgelegen hat. “383

In den Erwägungsgründen 17 und 18 der Richtlinie heißt es dazu, „,[d]er klagenden Partei stünde unter Umständen kein wirksames Mittel zur Verfügung, um die Einhaltung des Gleichbehandlungsgrundsatzes vor den nationalen Gerichten durchzusetzen, wenn der Beweis des Anscheins einer Diskriminierung nicht dazu führte, dem Beklagten die Beweislast dafür aufzuerlegen, daß sein Verhalten in Wirklichkeit nicht diskriminierend ist. Der Gerichtshof der Europäischen Gemeinschaften [habe] daher entschieden, daß eine Änderung der Regeln für die Beweislastverteilung geboten [sei], wenn der Anschein einer Diskriminierung besteht, und daß in solchen Fällen zur wirksamen Anwendung des Gleichbehandlungsgrundsatzes eine Verlagerung der Beweislast auf die beklagte Partei erforderlich [sei].“ 384

\section{1. $§ 22$ AGG}

Gemäß $§ 22$ AGG trägt dann,

„[w]enn im Streitfall die eine Partei Indizien beweist, die eine Benachteiligung wegen eines in $\S 1$ genannten Grundes vermuten lassen, [...] die andere Partei die Beweislast dafür, daß kein Verstoß gegen die Bestimmungen zum Schutz vor Benachteiligung vorgelegen hat.“

\section{a) ,Vermuten lassen“ im Sinne des $§ 22$ AGG}

382 Abl. EG 1998 Nr. L 14, S. 6 ff.; Richtlinie 97/80/EG findet gem. ihres Art. 3 I lit. a unter anderem Anwendung auf die von der Richtlinie 76/207/EWG erfaßten Situationen.

$\mathrm{Zu}$ beachten ist, daß die Richtlinie 97/80/EG gem. Art. 34 I der Richtlinie 2006/54/EG mit Wirkung vom 15.08.2009 aufgehoben und durch jene Richtlinie ersetzt wird.

383 Entsprechende Bestimmungen enthalten Art. 8 I Richtlinie 2000/43/EG, Art. 10 I Richtlinie 2000/78/EG sowie Art. 9 I Richtlinie 2004/113/EG.

384 Abl. EG 1998 Nr. L 14, S. 6 (7); EuGH, Rs. C-127/92, Slg. 1993 I 5, S. 5535 (5572, Rn. 14; 5573, Rn. 18). 
Mit dem Ziel zu ergründen, was unter ,vermuten lassen“ im Sinne des § 22 AGG zu verstehen ist, soll an dieser Stelle der Blick auf $\S 611$ a I 3 BGB-A, dem $§ 22$ AGG nachgebildet sei, ${ }^{385}$ gelenkt werden.

Bereits vor Erlaß der Richtlinie 97/80/EG hatte der deutsche Gesetzgeber in $\S 611$ a I 3 BGB-A eine dem Art. 4 I besagter Richtlinie entsprechende Regelung ${ }^{386}$ geschaffen. $§ 611$ a I 3 BGB-A bestimmte:

„Wenn im Streitfall der Arbeitnehmer Tatsachen glaubhaft macht, die eine Benachteiligung wegen des Geschlechts vermuten lassen, trägt der Arbeitgeber die Beweislast dafür, daß nicht auf das Geschlecht bezogene, sachliche Gründe eine unterschiedliche Behandlung rechtfertigen oder das Geschlecht unverzichtbare Voraussetzung für die auszuübende Tätigkeit ist.“

Das $B A G$ hat im Zusammenhang mit dieser Vorschrift festgestellt, die Glaubhaftmachung durch den Arbeitnehmer lasse die Beweisverteilung zunächst unberührt, sie senke nur das Beweismaß. Dabei sei Glaubhaftmachung nicht als Glaubhaftmachung im Sinne des $§ 294$ ZPO zu verstehen. ${ }^{387}$ Auch handele es sich nicht um eine Vermutungsregelung im Sinne des $\S 292$ ZPO. ${ }^{388} \S 611$ a I 3 BGB-A sei vielmehr so $\mathrm{zu}$ verstehen, „daß der klagende Arbeitnehmer eine Beweislast des Arbeitgebers dadurch herbeiführen kann, daß er Hilfstatsachen darlegt und ordnungsgemäß unter Beweis stellt, die eine Benachteiligung wegen des Geschlechts vermuten lassen. Hierzu genüg[e] die Überzeugung des Gerichts von der überwiegenden Wahrscheinlichkeit für die Kausalität zwischen Geschlechtszugehörigkeit und Nachteil.“389

Es kann davon ausgegangen werden, daß auch § 22 AGG im Sinne dieser Rechtsprechung zu interpretieren ist. Die folgenden Ausführungen gelten deshalb grundsätzlich auch für diese Bestimmung.

\section{b) Überwiegende Wahrscheinlichkeit}

Das erkennende Gericht soll vom Vorliegen der Kausalität zwischen Geschlechtszugehörigkeit und Nachteil bereits dann ausgehen können, wenn diese überwiegend wahrscheinlich ist. Dieses Beweismaß ist dann erreicht, wenn das Vorliegen einer Tatsache wahrscheinlicher ist als deren Nichtvorliegen, in Prozentzahlen ausgedrückt also bei $51 \%{ }^{390}$ Die vom BAG angesprochene Senkung des Beweismaßes besteht darin, daß der Arbeitnehmer ausnahmsweise keinen Vollbeweis erbringen muß. Nach diesem Grundsatz ist ein Beweis erst dann erbracht, wenn das Gericht von der

385 Entwurfsbegründung, BT-Druck. 16/1780, S. 47.

386 Annu $\beta$ in Staudinger (2005), § 611 a, Rn. 117.

$387 B A G$, NJW 2004, S. 2112 (2114).

$388 B A G$, NJW 2004, S. 2112 (2114).

$389 B A G$, NJW 2004, S. 2112 (2114).

390 Reichold in Thomas/Putzo, $\S 286$, Rn. 2 a. 
Wahrheit einer Behauptung überzeugt ist. „Hierfür genügt, da eine absolute Gewissheit nicht zu erreichen und jede Möglichkeit des Gegenteils nicht auszuschließen ist, ein für das praktische Leben brauchbarer Grad von Gewissheit [...], ein für einen vernünftigen, die Lebensverhältnisse klar überschauenden Menschen so hoher Grad von Wahrscheinlichkeit, daß er den Zweifeln Schweigen gebietet, ohne sie völlig auszuschließen [...]. ${ }^{391}$ Nicht ausreichend ist die bloße - wenn auch überwiegende - Wahrscheinlichkeit einer Tatsache. ${ }^{392}$

\section{c) Hilfstatsachen im Sinne des $§ 22$ AGG}

Die Überzeugung des Gerichts vom Vorliegen der Kausalität zwischen Geschlechtszugehörigkeit und Nachteil soll aus vom Arbeitnehmer darzulegenden und ordnungsgemäß unter Beweis zu stellenden Hilfstatsachen resultieren können. Als Hilfstatsachen sollen dabei beispielsweise eine geschlechtsspezifische Stellenausschreibung, diskriminierende Äußerungen des Arbeitgebers oder seiner Vertreter, die Unterrepräsentation eines Geschlechts im Unternehmen oder etwaige vorangegangene Benachteiligungen eines Geschlechts in Betracht kommen. ${ }^{393}$ Laut Entwurfsbegründung zum AGG können unter anderem auch Ergebnisse von Statistiken oder sogenannten Testing-Verfahren Hilfstatsachen in diesem Sinne sein. Bei letzteren handele es sich um Verfahren, bei denen eine Vergleichsperson eingesetzt wird, „um zu überprüfen, ob ein Verhalten gegenüber einer Person, bei der eines der in $\S 1$ [AGG] genannten Merkmale vorliegt, gleichermaßen auch gegenüber der Vergleichsperson, bei der dies nicht der Fall ist, erfolgt، “394

\section{d) Vollbeweis der Hilfstatsachen}

Von Hilfstatsachen bzw. Indizien ${ }^{395}$ auf eine entscheidungserhebliche Haupttatsache zu schließen, ist Wesen des sog. Indizienbeweises. Fraglich ist, ob - wie bei diesem ${ }^{396}$ - auch im Rahmen des $\S$ 22 AGG die Hilfstatsachen voll zu beweisen sind. Der Wortlaut scheint diese Frage eindeutig zu bejahen,,$-[\ldots]$ Indizien beweist $[\ldots]$..

Es ist auch nicht ersichtlich, warum die bereits erwähnte Senkung des Beweismaßes auch für die streitige Hilfstatsache selbst gelten sollte. Der Arbeitnehmer wird nicht überfordert, wenn man von

391 Reichold in Thomas/Putzo, § 286, Rn. 2.

392 Oberheim, § 7, Rn. 44.

393 So in bezug auf $\S 611$ a I 3 BGB-A: Annuß in Staudinger (2005), § 611 a, Rn. 114; In einem Verfahren vor einem US-amerikanischen Gericht hat die zuständige Richterin festgestellt: „Vorkommnisse von wahrgenommenem Sexismus können zugelassen werden, um eine diskriminierende Absicht des Beklagten nachzuweisen." (FAZ vom 28.08.2006, S. 9 unter dem Titel: „Deutscher Bank droht Klage wegen Diskriminierung“).

394 Entwurfsbegründung, BT-Druck. 16/1780, S. 47.

395 Das heißt ,tatbestandsfremde Umstände, die den Schluß auf das Vorliegen oder Nichtvorliegen des Tatbestandsmerkmals selbst rechtfertigen“" (Begründung der Änderungsempfehlung des Rechtsausschusses des Bundestages, BT-Druck. 16/2022, S. 13).

396 Oberheim, § 27 a, Rn. 14. 
ihm den Vollbeweis etwa dafür verlangt, daß die Stelle, auf die er sich beworben hat, geschlechtsspezifisch ausgeschrieben wurde.

Der Gesetzgeber des AGG scheint dies anders zu sehen: „Bleiben Vermutungstatsachen streitig, [...] genügt [es], wenn das Gericht ihr Vorliegen für überwiegend wahrscheinlich hält. “ ${ }^{397}$ Dies verblüfft vor allem vor dem Hintergrund der Tatsache, daß nach dem - dem $\S 611$ a I 3 BGB-A entsprechenden - Wortlaut der Entwurfsfassung des § 22 AGG die Indizien tatsächlich „nur“ glaubhaft zu machen waren:

„Wenn im Streitfall die eine Partei Tatsachen glaubhaft macht, die eine Benachteiligung wegen eines in $\S 1$ genannten Grundes vermuten lassen, trägt die andere Partei die Beweislast dafür, daß andere als in $\S 1$ genannte, sachliche Gründe die unterschiedliche Behandlung rechtfertigen oder die unterschiedliche Behandlung wegen eines in $\S 1$ genannten Grundes nach Maßgabe dieses Gesetzes zulässig ist. “398

Die sprachliche Neufassung des $§ 22$ AGG ist angesichts der hier vertretenen Ansicht, daß die Hilfstatsachen voll zu beweisen sind, begrüßenswert.

Der Gesetzgeber indes scheint durch die Änderung des Wortlauts der Bestimmung nicht seine Ansicht aufgeben zu wollen, für die streitige Hilfstatsache selbst genüge es, ,wenn das Gericht ihr Vorliegen für überwiegend wahrscheinlich hält““ ${ }^{399}$ soll doch die sprachliche Neufassung lediglich das Mißverständnis ausräumen, der Begriff der Glaubhaftmachung in § 22 AGG-Entwurf meine die Glaubhaftmachung im Sinne des § 294 ZPO, nicht aber zu einer Rechtsänderung führen. ${ }^{400}$

Wie auch immer sich der Gesetzgeber verstanden wissen will, es ist nicht begründbar, warum die bereits erwähnte Senkung des Beweismaßes auch für die streitige Hilfstatsache selbst gelten sollte. Insofern besteht keine derartige Situation, die es dem „Diskriminierten“ von vornherein unmöglich macht, seine Rechte durchzusetzen. ${ }^{401}$

Bestätigt wird diese Ansicht durch die Rechtsprechung des BAG zu § 611 a I 3 BGB-A. Wie bereits erwähnt, waren nach dem Wortlaut dieser Bestimmung die Indizien, die eine Benachteiligung wegen des Geschlechts vermuten lassen sollten, ,[...] glaubhaft [...]“ zu machen. Trotz dieses (scheinbar eindeutigen) Wortlauts entschied das Gericht, § 611 a I 3 BGB-A sei so zu verstehen, „daß der klagende Arbeitnehmer eine Beweislast des Arbeitgebers dadurch herbeiführen kann, daß er Hilfstatsachen darlegt und ordnungsgemäß unter Beweis stellt, die eine Benachteiligung wegen

397 Entwurfsbegründung, BT-Druck. 16/1780, S. 47; Unumstritten ist dagegen, daß der Arbeitnehmer das Vorliegen einer benachteiligenden Maßnahme voll beweisen muß. (Entwurfsbegründung, BT-Druck. 16/1780, S. 47 sowie in bezug auf $\S 611$ a I 3 BGB-A: Annuß in Staudinger (2005), § 611 a, Rn. 111; Die Regelung in $\S 611$ a I 3 BGB-A sollte sich nämlich nur ,auf den Benachteiligungsgrund, also auf die Tatsache der Benachteiligung aus geschlechtsspezifischen Gründen [beziehen].“(BAG, NJW 2004, S. 2112 (2114)). BT-Druck. 16/1780.

399 Entwurfsbegründung, BT-Druck. 16/1780, S. 47; Hinsichtlich der Tatsache des Vorliegens einer gegenüber einer anderen Person ungünstigen Behandlung soll dagegen, wie bereits erwähnt, auch nach Ansicht des Gesetzgebers dem Kläger der Vollbeweis obliegen. (Entwurfsbegründung, BT-Druck. 16/1780, S. 47).

400 Begründung der Änderungsempfehlung des Rechtsausschusses des Bundestages, BT-Druck. 16/2022, S. 13.

401 EuGH, Rs. C-127/92, Slg. 1993 I 5, S. 5535 (5572, Rn. 14; 5573, Rn. 18). 
des Geschlechts vermuten lassen. “402 Der Arbeitnehmer hatte nach dieser Rechtsprechung die Hilfstatsachen voll zu beweisen. Der Wortlaut des $\S 611$ a I 3 - „Wenn [...] der Arbeitnehmer Tatsachen glaubhaft macht, [...].“ - war demnach irreführend. Es hätte vielmehr heißen müssen: „Wenn [...] der Arbeitnehmer Tatsachen beweist, die eine Benachteiligung wegen des Geschlechts vermuten lassen, [...].“

\section{e) Entkräftung der glaubhaften Diskriminierung - Beweis des Gegenteils}

Die Regelung des $§ 22$ AGG weist aber auch entscheidende Unterschiede zum Indizienbeweis auf. Das gilt zum einen hinsichtlich der aus den Hilfstatsachen zu ziehenden Schlußfolgerung. Beim Indizienbeweis steht die entscheidungserhebliche Haupttatsache erst dann fest, „wenn andere Schlüsse aus den Indiztatsachen ernstlich nicht in Betracht kommen.“403 Bei $§ 22$ AGG soll bereits ausreichen, daß das erkennende Gericht eine Benachteiligung wegen des Geschlechts für überwiegend wahrscheinlich hält. Ein weiterer Unterschied zum Indizienbeweis besteht darin, daß anders als bei jenem ${ }^{404}$ - der Arbeitgeber eine überwiegend wahrscheinliche Diskriminierung nicht bereits durch Führen des Gegenbeweises entkräften kann. ${ }^{405}$ Er muß vielmehr „den vollen Beweis führen, daß die Benachteiligung aus rechtlich zulässigen Gründen erfolgte. “406

\section{f) Nicht bzw. schwer beweisbare Tatsachen - Beweislastentscheidung}

Beispiel: Der Arbeitgeber entscheidet sich für den im Vergleich zur Mitbewerberin objektiv schlechter qualifizierten Bewerber, weil dieser ihm sympathischer ist. Die abgewiesene Bewerberin klagt vor Gericht, weil sie sich aufgrund ihres Geschlechts diskriminiert fühlt.

Wie bereits an anderer Stelle im Zusammenhang mit diesem Beispielsfall festgestellt, kann Sympathie ein Grund sein, jemanden einzustellen; Antipathie, jemanden abzulehnen. Dem Arbeitgeber ist gestattet, den minderqualifizierten Bewerber dem besserqualifizierten vorziehen, weil ersterer ihm sympathischer ist. ${ }^{407}$

Unbeantwortet blieb bislang die Frage, ob der Arbeitgeber in dem Beispielsfall seiner Beweispflicht i. S. d. § 22 AGG durch die bloße Erklärung genügt, der Bewerber sei ihm sympathischer gewesen. Man ist geneigt, diese Frage zu bejahen. Sympathie und Antipathie sind Gefühle, die sich nicht

402

403

404

405

406

407 254

$B A G$, NJW 2004, S. 2112 (2114); Hervorhebung durch den Verfasser.

Reichold in Thomas/Putzo, Vorbem $\S 284$, Rn. 11.

Oberheim, § 27 a, Rn. 19.

So in bezug auf $\S 611$ a I 3 BGB-A: Annuß in Staudinger (2005), § 611 a, Rn. 113.

$B A G$, NJW 2004, S. 2112 (2115); in diesem Sinne auch Entwurfsbegründung, BT-Druck. 16/1780, S. 47.

Buchner in Münchener Handbuch zum Arbeitsrecht, Band 1, § 39, Rn. 74; Raab, RdA 1995, S. 36 (38); Müller-

Glöge in MüKo, § 611 a, Rn. 23; Wiese, JuS 1990, S. 357 (360). 
immer rational erklären lassen. So haben etwa Versuchspersonen in einer Studie „gutaussehende“ Bewerber als sympathischer bewertet. ${ }^{408}$

Ein Arbeitgeber, der nicht die Absicht hat, aufgrund des Geschlechts zu diskriminieren, könnte in einer wie im Beispielsfall beschriebenen Situation deshalb unter Umständen überfordert sein, verlangte man von ihm den Vollbeweis dafür, daß er sich für den Bewerber deshalb entschieden hat, weil dieser ihm sympathischer gewesen ist. „Ein Rechtfertigungszwang könnte nämlich beispielsweise dazu führen, daß eine Einstellungsentscheidung selbst dann als objektiv rechtswidrig angesehen würde, wenn der Arbeitgeber zwar sachbezogene Gründe für die Ablehnung des Bewerbers hatte, diese aber mangels Objektivierbarkeit einem Beweis nicht oder nur schwer zugänglich sind.“409

Dem Arbeitgeber, der diskriminieren möchte, könnte man indes die Möglichkeit sanktionsloser verdeckter Diskriminierung schaffen, gestattete man ihm, sein Verhalten durch die bloße Erklärung bestehender Sympathie bzw. Antipathie zu rechtfertigen.

Das Dilemma besteht demnach darin, daß die eine wie die andere Lösung im Einzelfall zu ungerechten Ergebnissen führen würde. Wie ist nun aber der Beispielsfall zu lösen? Der Schlüssel zur Beantwortung dieser Frage liegt in der differenzierten Beweislastverteilung des $\S 22$ AGG. Demnach muß die abgewiesene Bewerberin zunächst Tatsachen vortragen (und gegebenenfalls beweisen), die vermuten lassen, daß sie aufgrund ihres Geschlechts diskriminiert worden ist. Gelingt ihr dies, obliegt es dem Arbeitgeber (durch Indizien) voll zu beweisen, daß er sich für den Bewerber allein deshalb entschieden hat, weil dieser ihm sympathischer gewesen ist.

Ersteres blieb im Rahmen der bisherigen Betrachtungen völlig unberücksichtigt. Läßt allein der Verweis auf die geringere Qualifikation des eingestellten Bewerbers das Vorliegen einer Diskriminierung überhaupt vermuten? Diese Frage ist zu verneinen. Die Einstellung ist vor allem, aber nicht nur, von der Qualifikation des Bewerbers abhängig. Allein die Tatsache, daß der Arbeitgeber den Bewerber der besserqualifizierten Bewerberin vorgezogen hat, läßt eine Diskriminierung aufgrund des Geschlechts nicht überwiegend wahrscheinlich erscheinen.

In dem Beispielsfall ist es zu einer Verlagerung der Beweislast auf den Arbeitgeber demnach gar nicht gekommen, so daß dieser auch nicht beweisen muß, daß ausschlaggebendes Auswahlkriterium die besondere Zuneigung zu dem eingestellten Bewerber gewesen ist.

$\mathrm{Zu}$ einer Verlagerung der Beweislast wäre es in dem Beispielsfall demgegenüber etwa dann gekommen, wenn der Arbeitgeber die Stelle geschlechtsspezifisch ausgeschrieben hätte. In diesem Fall müßte der Arbeitgeber voll beweisen, daß er sich für den Bewerber letztlich deshalb entschieden hat, weil dieser ihm sympathischer gewesen ist. Natürlich wird er diesen Beweis oft nicht führen können, so daß tatsächlich die Gefahr besteht, daß es in Einzelfällen zur 
Sanktionierung materiell-rechtlich zulässiger Auswahlentscheidungen kommen wird. ${ }^{410}$ Dieses Ergebnis ist jedoch vor dem Hintergrund der Tatsache, daß der „Diskriminierte“ zunächst Tatsachen, die eine Diskriminierung vermuten lassen, vortragen (und gegebenenfalls beweisen) muß, bevor sich die Beweislast umkehrt, billigenswert. Zwischen den Parteien besteht insofern „Waffengleichheit“, als dem „Diskriminierten“ - wie der Ausgangsfall gezeigt hat - auch oft nicht gelingen wird, die Beweislastumkehr i. S. d. § 22 AGG herbeizuführen.

Das Problem, daß eine zu beweisende Tatsache von vornherein nicht bzw. nur selten bewiesen werden kann, stellt sich auch außerhalb des Diskriminierungsschutzrechts. Ein Beispiel dafür bildet die Kausalität zwischen Behandlungsfehler und Schaden im Arzthaftungsrecht. Wohl wissend, daß sich die Kausalität (oft) nicht beweisen läßt, versucht der Geschädigte hier den Beweis dafür zu erbringen, daß es sich bei dem Behandlungsfehler um einen groben handelt. Wenn ihm dies gelingt, obliegt es nämlich nicht mehr ihm, den (nicht selten unmöglichen) Beweis zu erbringen, daß der Behandlungsfehler kausal für den entstandenen Schaden war. Vielmehr muß in diesem Fall der Arzt beweisen, daß keine Kausalität zwischen Behandlungsfehler und Schaden vorliegt - was natürlich auch ihm (häufig) nicht möglich ist. Im Arzthaftungsprozeß ergeht demnach häufig eine Beweislastentscheidung, deren Tenor davon abhängt, wer die Beweislast für das Vorliegen bzw. Nichtvorliegen der Kausalität zwischen Behandlungsfehler und Schaden trägt. Auch hier wurde durch die Unterscheidung zwischen leichtem und grobem Behandlungsfehler versucht, eine gerechte Risikoverteilung zwischen den Parteien zu schaffen.

\section{2. $\$ 115$ IV BGB-E}

Vor dem Hintergrund der zu $\S 22$ AGG und $\S 611$ a I 3 BGB-A angestellten Überlegungen wird vorgeschlagen, die Beweislastverteilung wie folgt in § 115 IV BGB-E zu regeln:

Beweist der Erklärungsempfänger Tatsachen, die darauf schließen lassen, daß die Willenserklärung überwiegend wahrscheinlich eine solche im Sinne des Absatz 1 Satz 1 ist, obliegt dem Erklärenden der Beweis des Gegenteils. Dem Erklärenden obliegt in diesem Fall auch der Beweis dafür, daß er die Diskriminierung nicht zu vertreten hat. Satz 2 gilt im Falle der mittelbaren Diskriminierung jedoch nur dann, wenn der Erklärungsempfänger zuvor Tatsachen beweist, die darauf schließen lassen, daß der Erklärende die mittelbare Diskriminierung überwiegend wahrscheinlich zu vertreten hat. Satz 1 gilt entsprechend im Fall des Absatz 1 Satz 2.

\section{a) Diskriminierung - $\$ 115$ IV 1 BGB-E}


Der Wortlaut des § 115 IV 1 BGB-E läßt keinen Zweifel daran, daß der „Diskriminierte“ - dem „Günstigkeitsprinzip“411 folgend - zunächst das Vorliegen einer ihn materiell belastenden Willenserklärung voll zu beweisen hat.

Der „Diskriminierte“ hat ferner die Tatsachen voll zu beweisen, aus denen der diskriminierende Charakter der Willenserklärung geschlossen werden soll. ${ }^{412}$ Der Wortlaut der Bestimmung bringt dies unmißverständlich zum Ausdruck - „Beweist der Erklärungsempfänger Tatsachen, [...].“

Lediglich für die aus den Hilfstatsachen zu ziehende Schlußfolgerung hinsichtlich der Frage nach dem diskriminierenden Charakter der Willenserklärung gilt das gesenkte Beweismaß. § 115 IV nennt dabei explizit den insofern geltenden Wahrscheinlichkeitsgrad - ,überwiegend wahrscheinlich“.

Liegt danach eine Diskriminierung überwiegend wahrscheinlich vor, obliegt dem Erklärenden gem. $\S 115$ IV BGB-E der Beweis des Gegenteils. ${ }^{413}$ Dieser ist seiner Natur nach Hauptbeweis, ${ }^{414}$ also erst dann geführt, wenn der nicht-diskriminierende Charakter der Willenserklärung voll bewiesen ist. Die Beweisführung folgt dabei den allgemeinen Regeln. ${ }^{415}$

$\S 115$ IV 4 BGB-E bestimmt schließlich, daß die in $\S 115$ IV 1 BGB-E geregelte Beweislastverteilung entsprechend auch für den Fall „diskriminierender“ Nichtabgabe einer Willenserklärung im Sinne des § 115 I 2 BGB-E gilt. Insofern gelten keine Besonderheiten.

\section{b) Verschulden - § 115 IV 2, 3 BGB-E}

Wie bereits an anderer Stelle ausgeführt, kann der Diskriminierte Schadensersatzansprüche geltend machen, wenn der Diskriminierende sein Verhalten zu vertreten hat. ${ }^{416}$ In diesem Zusammenhang regelt $\S 115$ IV 2 BGB-E, daß dann, wenn die Voraussetzungen des $\S 115$ IV 1 BGB-E vorliegen und dem Diskriminierenden der Beweis des Gegenteils im Sinne dieser Bestimmung nicht möglich oder das Vorliegen einer Diskriminierung zwischen den Parteien gar unstreitig ist, es dem Diskriminierenden obliegt zu beweisen, „daß er die Diskriminierung nicht zu vertreten hat.“

411 Danach trägt eine Partei grundsätzlich die Beweislast für die tatsächlichen Voraussetzungen der Rechtsnormen, deren Rechtswirkungen ihr zugute kommen können. (Oberheim, § 7, Rn. 54).

412 Die Bestimmung verwendet statt des in $\$ 22$ AGG verwendeten Begriffs „Indizien“ den allgemeineren Begriff „Tatsachen“, wie er bereits in $\S 611$ a I 3 BGB-A gebraucht wurde. Inhaltlich bestehen insofern keine Unterschiede. Als Indizien bezeichnet man tatbestandsfremde Tatsachen. (Reichold in Thomas/Putzo, Vorbem $\S$ 284, Rn. 11).

413 Dabei handelt es sich um einen in $\S 292$ ZPO enthaltenen terminus technicus. Wenn dieser nun in $\S 115$ IV BGB-E Verwendung findet, bedeutet das nicht, daß es sich bei dieser Bestimmung um eine gesetzliche Vermutung im Sinne des § 292 ZPO handelt.

414 Reichold in Thomas/Putzo, Vorbem $§ 292$, Rn. 3.

415 Reichold in Thomas/Putzo, Vorbem $\$ 292$, Rn. 3.

416 Vergleiche dazu Teil 4, Prüfungspunkt C. II. 1. c). 
Entsprechende Regelungen enthalten die $\S 15$ I 2, 21 II 2 AGG hinsichtlich des Ersatzes materieller Schäden. Ohne die Frage des „Warum?“ dieser Vorschriften zu beantworten, heißt es in der Entwurfsbegründung zu den $\S 15$ I 2, 21 II 2 AGG lapidar, die Bestimmungen orientierten sich an $\S 280$ I 2 BGB. ${ }^{417}$

Hinsichtlich der ,unmittelbaren Diskriminierung“ läßt sich die Beweislastverteilung der $\S 15$ I 2, 21 II 2 AGG, 115 IV 2 BGB-E damit begründen, daß bei ihr ,ein auch subjektives Verschulden nur in den seltensten Fällen [...] verneint werden kann. ${ }^{4118}$ Der Tatbestand indiziert also im Falle der unmittelbaren Diskriminierung das Verschulden. Das gleiche gilt für die Diskriminierung in Form der ,einseitig belastenden Behandlung“.

Der Tatbestand der „mittelbaren Diskriminierung“ strahlt diese Indizwirkung nicht aus, „da hier zahlreiche Fälle denkbar sind, in denen der [Diskriminierende] auf Grund mangelnder Tatsachentransparenz selbst bei Anwendung aller ihm zumutbaren Sorgfalt einen Verstoß gegen das Diskriminierungsverbot nicht vermeiden kann. “419 Eine Beweislastverteilung nach dem Vorbild der $\S \S 15$ I 2, 21 II 2 AGG erscheint hier deshalb nicht angebracht. Auf der anderen Seite ist zu beachten, daß dem Diskriminierten unter Umständen kein wirksames Mittel zur Verfügung stünde, um die Einhaltung des Gleichbehandlungsgrundsatzes vor Gericht durchzusetzen, wenn es diesem obläge, voll zu beweisen, daß der Diskriminierende die mittelbare Diskriminierung zu vertreten hat. $\S 115$ IV 3 BGB-E bestimmt deshalb, daß dann, wenn der Erklärungsempfänger (Diskriminierte) Tatsachen beweist, die darauf schließen lassen, daß der Erklärende (Diskriminierende) die mittelbare Diskriminierung überwiegend wahrscheinlich zu vertreten hat, dem Erklärenden der Beweis des Gegenteils obliegt. ${ }^{420}$

Die Sätze 2 und 3 des $\S 115$ IV BGB-E gelten - ohne daß es insofern eines ausdrücklichen Verweises in $\S 115$ IV 4 BGB-E bedurft hätte - entsprechend auch für den Fall „diskriminierender“ Nichtabgabe einer Willenserklärung im Sinne des § 115 I 2 BGB-E. Das ergibt sich mittelbar aus dem Verweis in $§ 115$ IV 4 BGB-E auf $\S 115$ IV 1 BGB-E.

\section{c) Praktische Bedeutung des $§ 115$ IV BGB-E}

417 Entwurfsbegründung, BT-Druck. 16/1780, S. 38 u. 46.

418 Richardi/Anпи $\beta$ in Staudinger, § 611 a, Rn. 83; ebenso Ehmann/Emmert, SAE 1997, S. 253 (259); Pfarr/Bertelsmann vertreten sogar die Ansicht, daß „,bei Fällen unmittelbarer Diskriminierung Verschulden in Form der Fahrlässigkeit immer gegeben“ ist. (Pfarr/Bertelsmann, Diskriminierung im Erwerbsleben, S. 77); vgl. auch Entwurfsbegründung zum AGG, BT-Druck. 16/1780, S. 46.

419 Richardi/Annu $\beta$ in Staudinger, $\S 611$ a, Rn. 83; ähnlich Pfarr/Bertelsmann, Diskriminierung im Erwerbsleben, S. 128; vgl. auch Entwurfsbegründung zum AGG, BT-Druck. 16/1780, S. 46.

420 § 115 IV BGB-E ist insofern lex specialis gegenüber $§ 280$ I 2 BGB. 
Die Ausführungen zur Sympathie als sachlicher Grund haben angedeutet, daß der praktischen Durchsetzung von Diskriminierungsverboten große Hindernisse entgegenstehen. ${ }^{421}$ Man muß kein Pessimist sein, um zu behaupten, in vielen Fällen werde der Diskriminierende Wege finden, sein diskriminierendes Verhalten als nicht-diskriminierendes zu tarnen.

Beispiel: „Bewerbung für Außendiensttätigkeit (Katalogübergabe an Privathaushalte): Der Deutsche erhält einen Termin, um sich persönlich vorzustellen. Zehn Minuten später ruft der Türke an und erhält eine Absage, da alles bereits vergeben sei. Im direkten Anschluß daran ruft der Deutsche nochmals unter anderem Namen an und bekommt erneut einen Vorstellungstermin genannt. “422

Das Beispiel läßt vermuten, daß eine Vielzahl von Diskriminierungsfällen unerkannt bleiben und aus diesem Grund nicht $\mathrm{zu}$ einem Prozeß führen werden. Auch deshalb relativiert sich die praktische Bedeutung des 115 IV BGB-E.

\section{Zusammenfassung}

Ausgangspunkt des eigenen Vorschlags für die gesetzestechnische Umsetzung des Diskriminierungsverbotes bildeten Überlegungen zum „Wo“ und „Wie“ der Wiedergabe des im Rahmen der Arbeit entwickelten Diskriminierungsbegriffs in einem gesetzlichen Tatbestand. Folgender Lösungsvorschlag wurde unterbreitet:

\section{$\S 115$ BGB-E}

(1) Unzulässig ist eine Willenserklärung, die den Erklärungsempfänger insbesondere aufgrund seiner tatsächlichen oder vermeintlichen Rasse oder ethnischen Herkunft, seines Geschlechts, seiner Religion oder Weltanschauung, seiner Behinderung, seines Alters oder seiner sexuellen Identität unsachlich behandelt (diskriminiert). Unzulässig ist zudem, eine Willenserklärung, der ein öffentliches Angebot vorausgeht, in diskriminierender Weise nicht abzugeben. Auf das öffentliche Angebot ist Satz 1 entsprechend anzuwenden. Das gleiche gilt für sonstige Maßnahmen, die ihrem Wesen nach einer Willenserklärung im Sinne des Satz 1 vergleichbar sind.

(2) Absatz 1 Satz 1 findet insbesondere keine Anwendung auf Willenserklärungen, die den Intimbereich oder den Privatbereich des Erklärenden betreffen. Satz 1 gilt entsprechend im Fall des Absatz 1 Satz 2.

Zu § 115 I, II BGB-E wurden folgende Erläuterungen gemacht: Die Norm verwendet den Begriff „Willenserklärung“, da die diskriminierungsrelevante Handlung überwiegend in der Ab- bzw.

421 Darauf weist auch Wiese hin: „Da [den Arbeitgeber, der Vorbehalte gegenüber einem Geschlecht hat] niemand hindern kann, seine Auswahlentscheidung nach Sympathie oder Antipathie zu treffen, würde er sich sogar darauf berufen dürfen, solange er eine Ablehnung nicht mit dem Geschlecht, sondern nur der Person eines (einer) Bewerbers (Bewerberin) begründet. Die Beweislastregel des § 611 a I 3 BGB[-A] hilft dann wenig." (Wiese, JuS 1990, S. 357 (360)).

422 Beispiel aus einer Studie bei Nickel, Gleichheit und Differenz in der vielfältigen Republik, S. 79. 
Nichtabgabe einer Willenserklärung besteht. Die (sexuelle) Belästigung ist von dem Begriff „Willenserklärung“ nicht erfaßt. Durch seine Verwendung wird der Standort des Diskriminierungsverbotes im Allgemeinen Teil des BGB bei den Regelungen über die „Willenserklärungen“ bestimmt.

$\S 115$ I 1 BGB-E enthält einen offenen, d. h. einen nicht abgeschlossenen Merkmalskatalog. Die Bestimmung stellt zudem explizit klar, daß Diskriminierungsschutz auch derjenige erfährt, der nicht Träger des Merkmals ist, aufgrund dessen er „diskriminiert“ wird.

Das Tatbestandsmerkmal „unsachlich“ in $\S 115$ I 1 BGB-E verdeutlicht, daß nur rechtswidriges diskriminierungsrelevantes Verhalten eine Diskriminierung darstellt. Der auslegungsfähige und bedürftige Begriff „unsachlich“ ist im Sinne der in Teil 1 der Arbeit untersuchten Antidiskriminierungsmaßnahmen, die im Zivilrecht Drittwirkung entfalten, auszulegen.

$\S 115$ II BGB-E beschränkt den sachlichen Geltungsbereich des Diskriminierungsverbotes auf die Sozialsphäre. Eine entsprechende Bestimmung ist in den $\S \S 516$ ff. BGB mit dem Inhalt aufzunehmen, daß $§ 115$ BGB-E auf Schenkungen keine Anwendung findet.

Der Begriff „öffentliches Angebot“ in § 115 I 2 BGB-E ist streng von dem Begriff „Antrag“ im Sinne der $\S \S 145$ ff. BGB zu trennen. Er erfaßt neben der invitatio ad offerendum auch die Fälle, bei denen der „Diskriminierende“ Öffentlichkeit etwa dadurch schafft, daß er eine Regel aufstellt und befolgt, die abstrakt an allgemeine Merkmale anknüpft und generell für eine Mehrzahl von Fällen gelten soll. Beispielhaft kann insofern die Zahlung einer Weihnachtsgratifikation durch den Arbeitgeber genannt werden.

§ 115 I 3 BGB-E trägt dem Umstand Rechnung, daß das öffentliche Angebot nicht in jedem Fall eine Willenserklärung darstellt. Das gilt etwa für die invitatio ad offerendum.

Der Bestimmung des $§ 115$ I 4 BGB-E bedarf es, weil auch quasi-rechtsgeschäftliches Handeln etwa bestimmte Weisungen des Arbeitgebers - diskriminierenden Charakter haben können. Das Merkmal der Vergleichbarkeit dient dem Zweck zu verhindern, daß unter den Begriff der Maßnahme auch die (sexuelle) Belästigung subsumiert wird. Belästigungsschutz ist durch $§ 823$ I BGB zu realisieren.

An die Ausführungen zum Diskriminierungstatbestand schlossen sich Überlegungen zu möglichen Rechtsfolgen eines Verstoßes gegen das Diskriminierungsverbot an. Grundlage dieser Überlegungen bildeten dabei immer wieder die $\S \S 611$ a BGB-A, 15, 21 AGG. Im einzelnen wurden folgende Feststellungen getroffen:

$\S 115$ BGB-E ist ein Schutzgesetz im Sinne des $\S 823$ II BGB. Ein Verstoß gegen das Diskriminierungsverbot des $§ 115$ BGB-E führt zu einem Schadensersatzanspruch aus $§ 823$ II BGB i. V. m. § 115 BGB-E. Inhalt und Umfang dieses Anspruchs bemessen sich nach den $\S \S 249$ 
ff. BGB. Vermögensschäden hat der Diskriminierende nach Maßgabe der §§ 249-252 BGB voll zu ersetzen.

Vor dem Hintergrund der Erkenntnis, daß Diskriminierung vielfach keine oder nur sehr geringe Vermögensschäden verursacht, die Richtlinien aber Sanktionen fordern, die wirksam, verhältnismäßig und abschreckend sind, wurde vorgeschlagen, § 253 II BGB um den Begriff der Diskriminierung zu erweitern.

\section{$\S 253$ II BGB-E}

Ist wegen einer Verletzung des Körpers, der Gesundheit, der Freiheit oder der sexuellen Selbstbestimmung oder wegen Verstoßes gegen das Diskriminierungsverbot Schadensersatz zu leisten, kann auch wegen des Schadens, der nicht Vermögensschaden ist, eine billige Entschädigung in Geld gefordert werden.

Beide Ansprüche - sowohl der auf Ersatz des Vermögens- als auch der auf Ersatz des Nichtvermögensschadens - setzen schuldhaftes Handeln des Diskriminierenden voraus. Das ergibt sich aus $\S 823$ II 2 BGB. Eine verschuldensunabhängige Kompensationsvorschrift - wie sie $\S 611$ a II BGB-A statuierte - ließe sich nicht in die geltende Privatrechtsordnung einfügen, da in dieser eine Schadensverlagerung vom Geschädigten auf den Schädiger grundsätzlich nur bei Verletzung der im Verkehr erforderlichen Sorgfalt stattfindet. Eine über die bloße Kompensation hinausgehende verschuldensunabhängige Entschädigungspflicht - wie sie die § 611 a II BGB-A vorsah und nunmehr die $\S \S 15$ II 1, 21 II 3 AGG statuieren - verstieße sogar gegen die Verfassung. Das im Grundgesetz verankerte Bild eines frei verantwortlich handelnden Menschen gestattet nur dann eine Bestrafung, wenn der jeweilige Akteur unter Berücksichtigung seiner individuellen Anlagen im konkreten Fall anders hätte handeln können. Entsprechende Vorgaben des EuGH - § 611 a II BGB-A war, $\S 15$ II 1, 21 II 3 AGG sind Ergebnis dieser Vorgaben - verletzen den durch das Grundgesetz gewährleisteten unabdingbaren Kern grundrechtlichen Schutzes zugunsten des „Diskriminierenden“ und sind deshalb nicht zu beachten. Die Bedeutung der Frage nach der Notwendigkeit des Verschuldenserfordernisses relativiert sich dadurch, daß zumindest bei der unmittelbaren Diskriminierung schuldhaftes Handeln zumeist vorliegen wird.

Bei Vorliegen der speziellen Voraussetzungen kommen neben dem Schadensersatzanspruch aus $\S$ 823 II BGB in Verbindung mit $\S 115$ BGB-E auch solche aus den $\S \S 280$ und 826 BGB in Betracht. $\S 1004$ I BGB analog gewährt dem Diskriminierten einen verschuldensunabhängigen Beseitigungsund Unterlassungsanspruch. Der Beseitigungsanspruch ist streng vom Schadensersatzanspruch in Form der Naturalrestitution abzugrenzen, damit man durch ihn nicht doch zu einer verschuldensunabhängigen Schadensersatzpflicht gelangt. Der Unterlassungsanspruch kann beispielsweise darauf gerichtet sein, dem Diskriminierten in Zukunft nicht mehr den Zugang zu einer Einkaufspassage zu verweigern. 
Dieses Beispiel provozierte die Frage, ob der Eigentümer der Einkaufspassage als Verkäufer der in selbiger zum Verkauf angebotenen Waren auch verpflichtet ist, Kaufverträge mit der Person abzuschließen, der er den Zugang zu seinen Verkaufräumen gewähren muß. Die daraufhin angestellten Überlegungen zum Anspruch auf Vertragsschlu $\beta$ als mögliche Rechtsfolge eines Verstoßes gegen das Diskriminierungsverbot führten zu folgenden Feststellungen: Das geltende Recht kennt Fälle gesetzlichen Kontrahierungszwangs - vgl. etwa $\S 22$ PBefG. In Diskriminierungsfällen wird diskutiert, ob $§ 826$ BGB zu einem Anspruch des Diskriminierten auf Abschluß des angestrebten Vertrages führen kann. Die Antidiskriminierungsrichtlinien schreiben den Kontrahierungszwang als mögliche Rechtsfolge nicht explizit vor. $§ 611$ a II 2. HS BGB-A schloß ihn explizit aus. Dasselbe gilt nunmehr für $\S 15$ VI AGG. Eine dem $\S 15$ VI AGG vergleichbare Bestimmung enthält § 21 AGG zwar nicht, jedoch legt die historische Entwicklung dieser Norm nahe, daß auch in ihrem Anwendungsbereich der Kontrahierungszwang als Rechtsfolge vom Gesetzgeber nicht gewollt ist.

Der im Einzelfall nach dem AGG bestehenden Pflicht des „Diskriminierenden“ auf Vertragsschluß steht also kein ebensolcher Anspruch des „Diskriminierten“ gegenüber. Kein vernünftiger, am Gerechtigkeitsgedanken orientierter Grund vermag diese Inkongruenz zwischen Tatbestand und Rechtsfolge zu erklären. Der Diskriminierungsschutz wird zudem in nicht zu rechtfertigender Weise verkürzt, schließt man den Kontrahierungszwang als mögliche Rechtsfolge eines Verstoßes gegen des Diskriminierungsverbot aus. Ein Anspruch beispielsweise auf Gewährung des Zutritts zu einem Kaufhaus macht doch nur Sinn, wenn der Diskriminierende grundsätzlich auch verpflichtet ist, (Kauf-)Verträge mit dem Diskriminierten abzuschließen.

Der Anspruch auf Vertragsschluß ist nicht beschränkt auf bestimmte Verträge. Er ist auch nicht dadurch ausgeschlossen, daß dem abgewiesenen Interessenten zumutbare Ausweichmöglichkeiten zur Verfügung stehen.

Der Anspruch auf Vertragsschluß kann - da er auch präventiven Rechtsgüterschutz bezweckt - in analoger Anwendung des $\S 1004$ BGB als verschuldensunabhängiger Beseitigungsanspruch geltend gemacht werden.

Ein Verstoß gegen das Diskriminierungsverbot kann schließlich die Nichtigkeit der diskriminierenden Willenserklärung bewirken. Das gilt beispielsweise für die diskriminierende Kündigung.

Diese Rechtsfolge tritt nicht ein, wenn der Diskriminierte - wie beispielsweise im Falle des diskriminierenden Vertragsangebotes - ein Interesse an der Wirksamkeit der Willenserklärung hat. Diskriminierende Bestandteile der wirksamen Willenserklärung muß der Diskriminierte gleichwohl nicht gegen sich gelten lassen.

Auch Interessen Dritter können bewirken, daß eine diskriminierende Willenserklärung wirksam bleibt. $\mathrm{Zu}$ denken ist etwa an die diskriminierende Auslobung gem. $§ 657$ BGB. Grundsätzlich 
bleiben sämtliche mit Dritten begründeten Rechtsbeziehungen in ihrer Rechtswirksamkeit unberührt.

Die Rechtsfolge „Nichtigkeit der Willenserklärung“ ist gesetzestechnisch in § 115 III BGB-E wie folgt umgesetzt:

Eine Willenserklärung im Sinne des Absatz 1 Satz 1 ist nichtig, sofern nicht Interessen des Erklärungsempfängers oder eines Dritten dem entgegenstehen. Hat der Erklärungsempfänger ein Interesse an der Wirksamkeit der Willenserklärung, kann sich der Erklärende insoweit nicht auf den Inhalt der Willenserklärung berufen, als dieser den Erklärungsempfänger diskriminiert.

Im Rahmen der Überlegungen zur gesetzestechnischen Umsetzung des Diskriminierungsverbotes wurde schließlich die prozessuale Frage der Beweislast diskutiert. Den Ausgangspunkt der Betrachtungen bildeten einmal mehr die Antidiskriminierungsrichtlinien, das AGG - konkret $\S 22$ AGG - sowie § 611 a BGB-A. Die insofern gewonnenen Erkenntnisse - insbesondere die Feststellung, daß es einer differenzierten Beweislastverteilung bedarf, um die Einhaltung des Diskriminierungsverbotes gerichtlich durchsetzen zu können - waren Grundlage für folgende Beweislastregelung:

\section{$\S 115$ IV BGB-E}

Beweist der Erklärungsempfänger Tatsachen, die darauf schließen lassen, daß die Willenserklärung überwiegend wahrscheinlich eine solche im Sinne des Absatz 1 Satz 1 ist, obliegt dem Erklärenden der Beweis des Gegenteils. Dem Erklärenden obliegt in diesem Fall auch der Beweis dafür, daß er die Diskriminierung nicht zu vertreten hat. Satz 2 gilt im Falle der mittelbaren Diskriminierung jedoch nur dann, wenn der Erklärungsempfänger zuvor Tatsachen beweist, die darauf schließen lassen, daß der Erklärende die mittelbare Diskriminierung überwiegend wahrscheinlich zu vertreten hat. Satz 1 gilt entsprechend im Fall des Absatz 1 Satz 2.

Der „Diskriminierte“ hat - dem „Günstigkeitsprinzip“ folgend - zunächst das Vorliegen einer ihn materiell belastenden Willenserklärung voll zu beweisen. Dasselbe gilt für die „Tatsachen“ im Sinne des $\S 115$ IV 1 BGB-E, zu denen unter anderem die geschlechtsspezifische Stellenausschreibung gezählt werden kann. Für die aus den „Tatsachen“ zu ziehende Schlußfolgerung hinsichtlich der Frage nach dem diskriminierenden Charakter der Willenserklärung - mithin der Kausalität zwischen Diskriminierungsmerkmal und Belastung - gilt indes ein gesenktes Beweismaß - „überwiegend wahrscheinlich“. Es ist - im Unterschied zu den Anforderungen, die der Vollbeweis verlangt - bereits dann erreicht, wenn das Vorliegen einer Tatsache wahrscheinlicher ist als deren Nichtvorliegen, in Prozentzahlen ausgedrückt also bei $51 \%$. Die Beweiserleichterung ist geboten, da der klagenden Partei unter Umständen kein wirksames Mittel zur Verfügung stünde, um die Einhaltung des Gleichbehandlungsgrundsatzes gerichtlich 
durchzusetzen, wenn der Beweis des Anscheins einer Diskriminierung nicht dazu führte, dem Beklagten die Beweislast dafür aufzuerlegen, daß sein Verhalten in Wirklichkeit nicht diskriminierend ist.

Der „Beweis des Gegenteils“ im Sinne des $§ 115$ IV 1 BGB-E ist seiner Natur nach Hauptbeweis, im Gegensatz zum „Gegenbeweis“ also erst dann geführt, wenn der nicht-diskriminierende Charakter der Willenserklärung voll bewiesen ist. Die Beweisführung folgt dabei den allgemeinen Regeln.

Im Falle der „unmittelbaren Diskriminierung“ indiziert der Tatbestand das Verschulden des Diskriminierenden. Das gleiche gilt für die Diskriminierung in Form der „einseitig belastenden Behandlung“. In diesen Fällen obliegt es deshalb dem Diskriminierenden gem. § 115 IV 2 BGB-E zu beweisen, „daß er die Diskriminierung nicht zu vertreten hat“, wenn ihm der Beweis des Gegenteils nicht möglich oder das Vorliegen einer Diskriminierung zwischen den Parteien gar unstreitig ist.

Der Tatbestand der ,mittelbaren Diskriminierung“ strahlt diese Indizwirkung nicht aus, da hier zahlreiche Fälle denkbar sind, in denen der Diskriminierende auf Grund mangelnder Tatsachentransparenz selbst bei Anwendung aller ihm zumutbaren Sorgfalt einen Verstoß gegen das Diskriminierungsverbot nicht vermeiden kann. $§ 115$ IV 2 gilt deshalb nicht im Falle der mittelbaren Diskriminierung. Da dem Diskriminierten jedoch unter Umständen kein wirksames Mittel zur Verfügung stünde, um die Einhaltung des Gleichbehandlungsgrundsatzes vor Gericht durchzusetzen, wenn es diesem obläge, voll zu beweisen, daß der Diskriminierende die mittelbare Diskriminierung $\mathrm{zu}$ vertreten hat, bestimmt $\S 115$ IV 3 BGB-E, daß dann, wenn der Erklärungsempfänger (Diskriminierte) Tatsachen beweist, die darauf schließen lassen, daß der Erklärende (Diskriminierende) die mittelbare Diskriminierung überwiegend wahrscheinlich zu vertreten hat, dem Erklärenden der Beweis des Gegenteils obliegt.

Die Gründe der diskriminierungsrelevanten Entscheidung lassen sich bisweilen nicht bzw. nur schwer beweisen. Das gilt etwa für das Auswahlkriterium „Sympathie“. Nicht in jedem „Diskriminierungsverfahren“ wird deshalb die materiell-rechtlich richtige Lösung ergehen können.

Die Beweislastregelung des $\S 115$ IV BGB-E führt dazu, daß das Risiko nicht bzw. nur schwer beweisbarer Tatsachen gerecht verteilt wird.

Es ist zu vermuten, daß eine Vielzahl von Diskriminierungen unerkannt bleiben werden, weil der Diskriminierende geschickt genug sein wird, sein diskriminierendes Verhalten als nichtdiskriminierendes zu tarnen. Oft wird der Diskriminierte deshalb einen Prozeß gar nicht erst anstrengen bzw. die Beweislastumkehr nicht herbeiführen können. Die praktische Bedeutung des $\S$ 115 IV BGB-E darf angesichts dessen nicht überbewertet werden. 


\section{Gesamtzusammenfassung}

Den Ausgangspunkt der Arbeit bildete eine Untersuchung bereits bestehender Antidiskriminierungsmaßnahmen auf internationaler wie nationaler Ebene. Sie diente dem Ziel herauszuarbeiten, in welchen Bereichen und für welche Merkmale besagte Maßnahmen Schutz vor Diskriminierung bieten.

Die im Rahmen dieser Untersuchung gewonnenen Erkenntnisse bildeten die Grundlage für die Bestimmung des Diskriminierungsbegriffs im zweiten Teil der Arbeit. Konkret herausgearbeitet wurde dort, welche Verhaltensweisen diskriminierenden Charakter haben können, aufgrund welcher Merkmale oder Eigenschaften eines Menschen überhaupt diskriminiert werden kann, wann diskriminierungsrelevantes Verhalten rechtswidrig ist und schließlich, in welchen Bereichen zwischenmenschlichen Lebens das Diskriminierungsverbot Geltung beanspruchen kann. Im einzelnen wurden folgende Feststellungen getroffen:

Eine Diskriminierung kann vorliegen, wenn eine Person gegenüber einer anderen in benachteiligender Weise ungleich behandelt wird, weil sie ein unerwünschtes Merkmal bzw. eine unerwünschte Eigenschaft aufweist (unmittelbare Diskriminierung). Offen „diskriminiert“ dabei, wer die Ungleichbehandlung durch direkten Verweis auf das unerwünschte Merkmal begründet, verdeckt dagegen, wer nach außen hin an ein anderes Merkmal anknüpft. Die sogenannte Diskriminierungsdrittwirkung - ein Sonderfall der unmittelbaren Diskriminierung - meint die Konstellation, daß eine Person, die in einer gewissen (z.B. Verwandtschafts-) Beziehung zu der diskriminierten Person steht, ebenfalls Auswirkungen der Diskriminierung erdulden muß.

Auch die einseitig belastende Behandlung aufgrund eines bestimmten Merkmals stellt potenziell diskriminierendes Verhalten dar. Gemeint sind damit die Fälle, in denen eine Person aufgrund eines bestimmten Merkmals eine belastende Behandlung erfährt, ohne jedoch gleichzeitig gegenüber einer anderen Person ungleich behandelt zu werden.

Ebenso kann die (sexuelle) Belästigung als diskriminierungsrelevante Verhaltensweise eingestuft werden. Da diese Form der Diskriminierung jedoch Fragen aufwirft, die nicht Gegenstand der Arbeit sein sollen, wurden die Probleme, die im Zusammenhang mit dem Belästigungsschutz und insbesondere den insofern bestehenden europarechtlichen Verpflichtungen auftauchen, lediglich in einem Exkurs kurz angerissen.

Auch die formale Gleichbehandlung, die sich - je nachdem, ob man Träger eines bestimmten Merkmals ist oder nicht - unterschiedlich auswirkt, wirft die Frage nach dem Vorliegen einer 
Diskriminierung auf (mittelbare Diskriminierung). Das Vorliegen einer mittelbaren Diskriminierung hängt nicht vom Nachweis einer tatsächlichen Benachteiligung ab. Vielmehr kann sie bereits dann vorliegen, wenn die Gleichbehandlung geeignet ist, tatsächlich benachteiligend zu wirken. Nur bei der mittelbaren Diskriminierung sind überwiegend die Personen, die Träger des unerwünschten Merkmals sind, von der benachteiligenden Maßnahme betroffen, nicht aber ausschließlich, wie im Fall der unmittelbaren Diskriminierung.

Schließlich stellt auch die Anweisung zur Diskriminierung diskriminierungsrelevantes Verhalten dar.

Anknüpfungspunkt für eine „Diskriminierung“ sind zumeist solche Merkmale - etwa Hautfarbe, Geschlecht oder Behinderung -, die einer Person unveränderbar anhaften. Der Diskriminierungsschutz darf sich indes nicht auf diese unbeeinflußbaren Merkmale beschränken. Auch der Status einer Person - etwa eine bestimmte Religionszugehörigkeit - und sogar deren Verhalten können Gründe für eine Diskriminierung derselben darstellen.

Da es zumindest potenziell unendlich viele Merkmale gibt, aufgrund derer diskriminiert werden kann, ist ein auf ausgewählte Merkmale beschränkter Diskriminierungsschutz verfehlt. Gesetzestechnisch könnte diesem Umstand durch Einführung eines offenen Merkmalskataloges Rechnung getragen werden, der - ohne abschließend zu sein - die besondere Schutzwürdigkeit einiger Diskriminierungsmerkmale hervorheben würde.

Neben dem tatsächlichen genießt auch der vermeintliche Merkmalsträger, d. h. derjenige, der für den Träger eines bestimmten Merkmals gehalten wird, obwohl er dies in Wirklichkeit nicht ist, Diskriminierungsschutz.

Für die zu beantwortende Frage nach der Rechtswidrigkeit des diskriminierungsrelevanten Verhaltens bildeten das Allgemeine Gleichbehandlungsgesetz (AGG), die Antidiskriminierungsrichtlinien sowie $§ 611$ a BGB-A einmal mehr eine wichtige Erkenntnisquelle. Die Untersuchung führte zu folgenden Erkenntnissen: Ein bestimmtes Geschlecht ist „wesentlich und entscheidend“ im Sinne des $\S 8$ I AGG, wenn die in Rede stehende Tätigkeit naturwissenschaftlich bedingt nur von einem Geschlecht ausgeübt werden kann (objektive Anforderung). Dasselbe gilt für alle Diskriminierungsmerkmale und sämtliche denkbaren Rechtsverhältnisse.

Ein bestimmtes Geschlecht ist auch dann wesentlich und entscheidend im Sinne des $\S 8$ I AGG, wenn es für die Durchführung einer Tätigkeit gesetzlich vorgeschrieben ist (rechtliche Anforderung). Dasselbe gilt wiederum für alle Diskriminierungsmerkmale und sämtliche denkbaren Rechtsverhältnisse. Unter die rechtliche Anforderung können auch die Fälle subsumiert werden, bei 
denen dem „Diskriminierenden“ per Gesetz gestattet ist, ein bestimmtes Merkmal zum Anknüpfungspunkt einer diskriminierungsrelevanten Entscheidung zu machen.

Die Tatsache, daß gem. $§ 8$ I AGG - in Übereinstimmung mit den Antidiskriminierungsrichtlinien das jeweilige Diskriminierungsmerkmal nur dann eine wesentliche und entscheidende berufliche Anforderung bzw. Voraussetzung darstellt, ,[...] sofern der Zweck rechtmäßig und die Anforderung angemessen ist [...]“, führte zu der Erkenntnis, daß letztlich wertend zu entscheiden ist, ob ein bestimmtes Unternehmerinteresse als ausreichend gewichtig anzusehen ist, um das Gleichbehandlungsinteresse des Arbeitnehmers zurückzudrängen. Dem Arbeitgeber steht es damit grundsätzlich frei, etwa ein bestimmtes Geschlecht für die in Rede stehende Tätigkeit für wesentlich und entscheidend $\mathrm{zu}$ erklären - subjektive Anforderung. Diese „Wesentlich- und Entscheidendmachung“ ist jedoch objektiv begrenzt durch die Begriffe „rechtmäßiger Zweck“ und „,angemessene Anforderung“.

Die zur subjektiven Anforderung angestellten Überlegungen führten schließlich zu der Erkenntnis, daß es sinnvoll gewesen wäre, die Richtlinienvorgaben hinsichtlich der Frage der Rechtswidrigkeit diskriminierungsrelevanten Verhaltens durch Einführung des Begriffs „unsachlich“ umzusetzen, wie in $\S 20$ I 1 AGG durch Einführung des Rechtfertigungsgrundes „sachlicher Grund“ teilweise geschehen. In diesem Zusammenhang wurde nachgewiesen, daß die Verwendung der Formulierung „unsachlich“ nicht zu einer Absenkung des durch § 8 I AGG gewährten und von den Antidiskriminierungsrichtlinien geforderten Schutzniveaus führt. Der Begriff ,unsachlich“ wurde schließlich für vorzugswürdig erklärt, weil er als auslegungsbedürftiger Rechtsbegriff im Gegensatz zu den Begriffen „,wesentlich und entscheidend“ unmißverständlich verdeutlicht, daß die Frage der Rechtswidrigkeit diskriminierungsrelevanten Verhaltens eine einzelfallabhängige Abwägungsentscheidung ist.

Diskriminierend kann neben rechtsgeschäftlichem auch quasi-rechtsgeschäftliches Handeln sein, wobei für letzteres beispielhaft die arbeitsrechtliche Weisung genannt werden kann.

Vor dem Hintergrund der Tatsache, daß besonders häufig im Vorfeld des Vertragsschlusses „diskriminiert“ wird und die Antidiskriminierungsrichtlinien in Konsequenz dessen den Diskriminierungsschutz auf diesen Bereich ausweiten, ist - um eine Umgehung des Diskriminierungsverbotes in diesem Bereich $\mathrm{zu}$ verhindern - zwingend erforderlich, merkmalsspezifische Stellenausschreibungen und Bewerbungsunterlagen sowie die Frage nach dem Vorhandensein eines bestimmten Merkmals in der Person des „Diskriminierten“ nur insofern für zulässig zu erklären, als auch der Vertragsschluß aufgrund des jeweiligen Merkmals abgelehnt werden könnte. Der „Diskriminierte“ begibt sich etwaiger Sonderrechte nicht dadurch, daß er von seinem „Recht zur Lüge“ Gebrauch macht. 
Das Diskriminierungsverbot kann nur dort Geltung beanspruchen, wo der „Diskriminierende“ eine bestimmte Ware, Dienstleistung etc. etwa durch Anzeigen in Tageszeitungen öffentlich anbietet. Ein Angebot ist dann nicht öffentlich, wenn es sich an eine ganz bestimmte Person richtet. Der „Diskriminierende“ ist nicht verpflichtet, seine Ware, Dienstleistung etc. öffentlich anzubieten. Er ist lediglich verpflichtet, öffentliche Angebote diskriminierungsfrei zu gestalten.

Am Beispiel der letztwilligen Verfügung von Todes wegen wurde der Frage nachgegangen, ob auch unentgeltliche Zuwendungen in den sachlichen Geltungsbereich des Diskriminierungsverbotes fallen. Die Überlegungen ergaben, daß vieles dafür spricht, (wirtschaftlich) rein altruistisches Verhalten nicht am Verbot der Diskriminierung zu messen, so daß die aufgeworfene Frage verneint wurde.

Das Diskriminierungsverbot sollte sowohl gewerbsmäßiges als auch nichtgewerbsmäßiges Handeln umfassen. Die Antidiskriminierungsrichtlinien können in diesem Sinne interpretiert werden.

Das Diskriminierungsverbot erfaßt - selbstverständlich - nicht die Intimsphäre des Menschen also beispielsweise die Wahl seines Lebenspartners. Für die Privatsphäre, die vor allem die „eigenen vier Wände“ umfaßt, gilt dasselbe. Außerhalb des Bereichs der Intim- und Privatsphäre mithin im Bereich der Sozialsphäre - sollte das Diskriminierungsverbot dagegen uneingeschränkt gelten. Eine Beschränkung des sachlichen Geltungsbereichs des Diskriminierungsverbotes auf bestimmte Bereiche der Sozialsphäre würde ihrerseits diskriminierenden Charakter haben, und zwar im Verhältnis der „Diskriminierenden“ zueinander. Auch eine Beschränkung des Diskriminierungsschutzes in Teilbereichen der Sozialsphäre - etwa außerhalb des Geltungsbereichs des Kündigungsschutzgesetzes - auf ausgewählte Merkmale ließe sich nicht rechtfertigen. Lediglich im Rahmen der Interessenabwägung und damit der Rechtswidrigkeitsprüfung ist die Unterscheidung einzelner Bereiche der Sozialsphäre von Bedeutung.

Ausgangspunkt und Grund für die im dritten Teil der Arbeit angestellten Überlegungen zu den „Auswirkungen des Diskriminierungsverbotes auf das rechtsgeschäftliche und quasirechtsgeschäftliche Handeln“ war die Feststellung, daß die im Rahmen der Frage nach dem Vorliegen einer Diskriminierung erforderliche Rechtswidrigkeitsprüfung eine einzelfallabhängige Abwägungsentscheidung darstellt; daß es aus diesem Grund unmöglich ist, einen abschließenden Katalog sachlicher Gründe zu erstellen; daß aber Kriterien entwickelt werden können, die einen Wegweiser für die Rechtswidrigkeitsprüfung darstellen, die Abwägungsentscheidung aber nicht vorwegnehmen können, da selbige - wie gesagt - stets unter Berücksichtigung der Besonderheiten des Einzelfalls getroffen werden muß. Die Suche nach besagten Kriterien führte zu folgenden Erkenntnissen: 
Im Rahmen der Interessenabwägung ist von Bedeutung, welche Art Merkmal im konkreten Fall Anknüpfungspunkt für die „Diskriminierung“ war. Die Anknüpfung an ein unbeeinflußbares Merkmal unterliegt grundsätzlich den höchsten Rechtfertigungsanforderungen, gefolgt vom Status und schließlich dem Verhalten als Merkmal.

Die bezweckte Förderung von Trägern bestimmter Merkmale kann die damit einhergehende Benachteiligung von Personen, die nicht Träger dieser Merkmale sind, im Einzelfall rechtfertigen. Andererseits wird immer dann diskriminiert, wenn die Ausgrenzung der benachteiligten Personengruppe bloßer Selbstzweck ist. In diesem Zusammenhang wurde erkannt, daß im Rahmen der Interessenabwägung von Bedeutung sein kann, ob eine Maßnahme zu einem dauerhaften oder nur vorübergehenden Ausschluß der durch die Maßnahme belasteten Person(en) führt.

Einer belastenden Entscheidung des (potenziellen) Vertragspartners müssen grundsätzlich vertragsbezogene Gründe - etwa die Qualifikation des Bewerbers - zugrunde liegen. Im Einzelfall können aber auch nicht bzw. nur mittelbar vertragsbezogene Gründe - etwa Antipathie gegenüber einem Bewerber - und sogar außervertragliche Gründe - etwa strafrechtlich relevantes Verhalten des Arbeitnehmers im außerdienstlichen Bereich - das (potenzielle) Vertragsverhältnis in einer Weise negativ beeinflussen, die dem „Diskriminierenden“ nicht zugemutet werden kann.

Vorurteile und Generalisierungen bezüglich etwaiger Eigenschaften und Fähigkeiten von Trägern bestimmter Merkmale vermögen diskriminierungsrelevantes Verhalten grundsätzlich nicht zu rechtfertigen; insbesondere dann nicht, wenn es sich um nicht-vertragsbezogene Vorurteile und Generalisierungen handelt.

Eine diskriminierungsrelevante Maßnahme, die aufgrund einer Störung des Vertragsverhältnisses getroffen wird, ist grundsätzlich nur dann rechtmäßig, wenn dem „Diskriminierten“ die Störung des Vertragsverhältnisses vorwerfbar ist.

Wenn der „Diskriminierende“ die Wahl zwischen mehreren Personen bzw. Merkmalsträgern hat um dieselbe Stelle bewerben sich ein Mann und eine Frau -, darf die Entscheidung auf einem an sich unzulässigen Merkmal beruhen - der Arbeitgeber entscheidet sich bewußt für den Mann/die Frau -, wenn eine sachgerechte Differenzierung nicht möglich ist - etwa weil die Bewerber gleichqualifiziert sind. Nichtsdestotrotz diskriminiert mittelbar, wer in der beschriebenen Pattsituation stets dasselbe Merkmal begünstigt - Arbeitgeber entscheidet sich immer für den Bewerber/die Bewerberin. Zu beachten ist schließlich, daß der „Diskriminierende“ - in den 
Grenzen des Diskriminierungsverbotes - selbst bestimmen kann, ob zwei Personen als „gleichwertig“ anzusehen, zwei Bewerber etwa gleichqualifiziert sind.

Diskriminierungsrelevantes Verhalten kann zum Zwecke der Authentizitätswahrung sachlich begründet sein - Arbeitgeber stellt ausschließlich Frauen zur Präsentation von Damenmode ein.

Sachlich begründete Wünsche Dritter - etwa Geschäftskunden des „Diskriminierenden“ vermögen diskriminierungsrelevantes Verhalten zu rechtfertigen. Diskriminierende Kundenwünsche vermögen diskriminierungsrelevantes Verhalten $\mathrm{zu}$ rechtfertigen, wenn der „Diskriminierende“ andernfalls wirtschaftliche Einbußen von nicht völlig unerheblichem Umfang erleiden würde bzw. auf Mehreinnahmen verzichten müßte. Die Nichtbeachtung eines diskriminierenden Kundenwunsches ist nicht sanktionierbar. Schließlich kann der „Diskriminierende“ sein Verhalten auch mit berechtigten öffentlichen Interessen, d. h. mit Interessen Dritter begründen, mit denen er nicht in Geschäftskontakt steht.

Jeder Mensch ist Träger einer Vielzahl von Diskriminierungsmerkmalen, die im Rahmen einer diskriminierungsrelevanten Maßnahme unbewußt oder bewußt zusammentreffen können. Ein unbewußtes Zusammentreffen von sachlichem und unsachlichem Diskriminierungsmerkmal ist etwa gegeben, wenn ein Mann aufgrund besseren Zeugnisses - nicht aufgrund seines Geschlechts - für die gleiche Tätigkeit eine höhere Vergütung erhält als die Frau. Ein bewußtes Zusammentreffen von Diskriminierungsmerkmalen liegt vor, wenn der Diskriminierende seiner benachteiligenden Ungleichbehandlung mehrere Diskriminierungsmerkmale zugrunde legt. Allein die Tatsache, daß sich in dem Motivbündel auch ein unsachlicher Grund befindet, führt nicht dazu, daß die diskriminierungsrelevante Entscheidung rechtswidrig ist. Eine Diskriminierung ist in einem solchen Fall nur dann gegeben, wenn der unsachliche Grund kausal für die Entscheidung war. Das diskriminierende Motiv für sich ist nicht sanktionswürdig, solange es nicht in belästigender Weise geäußert wird.

Diskriminierungsmerkmale treffen zeitlich versetzt zusammen, wenn beispielsweise der Arbeitgeber, der eine Stelle geschlechtsspezifisch ausgeschrieben hat, letztlich zur Grundlage seiner Einstellungsentscheidung die Qualifikation der Bewerber macht. In einem solchen Fall stellt sich die Frage nach dem Vorliegen einer Diskriminierung auch für die durch die geschlechtsspezifische Ausschreibung benachteiligten Männer bzw. Frauen, die schlechter qualifiziert sind als der eingestellte Bewerber. Stellenausschreibung und Einstellung stellen nämlich zwei unabhängig voneinander zu betrachtende diskriminierungsrelevante Entscheidungen dar.

Trägt der „Diskriminierte“ Tatsachen vor, die eine Diskriminierung im Sinne des $§ 22$ AGG vermuten lassen, vermag sich der „Diskriminierende“ - sofern diese Tatsachen unstreitig oder 
bewiesen sind - nicht bereits durch den Nachweis eines objektiv vorliegenden sachlichen Grundes, den er erstmals im Prozeß angibt, zu entlasten. Ein auf diese Weise nachträglich vorgebrachter Grund war nach der Rechtsprechung des BVerfG zu § 611 a I 3 BGB-A, der eine dem $§ 22$ AGG vergleichbare Beweislastregelung enthielt, nur dann als „sachlich“ im Sinne dieser Norm zu qualifizieren, wenn besondere Umstände erkennen ließen, daß der Arbeitgeber diesen Grund nicht nur vorgeschoben hatte. Diese Rechtsprechung muß im Rahmen des $§ 22$ AGG fortbestehen, da sich der Arbeitgeber andernfalls (vermutlich) in nahezu jedem Fall im Sinne des $§ 22$ AGG entlasten könnte, darf er doch grundsätzlich die Anforderungen an die Qualifikation für eine bestimmte Stelle nach seinem Belieben festlegen. Beachtet werden muß, daß die in $\S 22$ AGG geregelte Beweislastumkehr, infolge derer für nachgeschobene sachliche Gründe besondere Begründungsanforderungen bestehen, nicht allein durch die Tatsache eintritt, daß der Arbeitgeber im Prozeß erstmals den (sachlichen) Grund seiner Auswahlentscheidung angibt. Der Arbeitgeber ist nicht verpflichtet, dem unberücksichtigt gebliebenen Bewerber seine Auswahlgründe mitzuteilen. Allein die Tatsache, daß der Arbeitgeber seine Auswahlentscheidung erst im Prozeß beispielsweise damit begründet, ihm sei der Bewerber sympathischer gewesen als die Bewerberin, führt deshalb nicht dazu, daß er diese Behauptung beweisen müßte.

Auch sogenannte Tendenzbetriebe - etwa Kirchen und Weltanschauungsgemeinschaften - sind grundsätzlich an das Diskriminierungsverbot gebunden. Die Überprüfung diskriminierungsrelevanter Entscheidungen von Tendenzbetrieben hat jedoch dem Selbstverständnis dieser Betriebe ein besonderes Gewicht beizumessen. So kann der Tendenzbetrieb im Kernbereich seiner Tätigkeit von seinen Beschäftigten Tendenzzugehörigkeit verlangen - vom Pfarrer etwa, daß er Mitglied der Glaubensgemeinschaft ist, deren Werte er predigen soll. Im Randbereich seiner Tätigkeit ist dem Tendenzbetrieb dies nur dann gestattet, wenn der Arbeitnehmer Tendenzträger ist, also unmittelbar an der geistig-ideellen Zielsetzung des Betriebes - der Tendenz - mitwirkt. Das gilt etwa für Betreuerinnen eines konfessionellen Kindergartens dann, wenn bereits sie den Kindern die Werte der Kirche vermitteln sollen. Ansonsten ist der Tendenzbetrieb wie der „Nicht-Tendenzbetrieb“ zu behandeln.

Im Kernbereich seiner Tätigkeit ist es dem Tendenzbetrieb gestattet, auch aufgrund eines anderen Merkmals als der Tendenz zu unterscheiden, soweit dies nachgewiesenermaßen seinem Selbstverständnis entspricht. Im Randbereich seiner Tätigkeit ist der Tendenzbetrieb wie der „Nicht-Tendenzbetrieb“ zu behandeln; und zwar unabhängig davon, ob der Arbeitnehmer Tendenzträger ist oder nicht.

In jedem Fall kann der Tendenzbetrieb von seinen Arbeitnehmern tendenzdefensives Verhalten verlangen. Tendenzaggressiv verhält sich etwa der in einem katholischen Krankenhaus beschäftigte Arzt, der öffentlich für den legalen Schwangerschaftsabbruch eintritt. 
Die Frage nach möglichem Tendenzschutz kann sich auch außerhalb des ArbeitgeberArbeitnehmer-Verhältnisses stellen. Die für das Arbeitsrecht entwickelten Abwägungskriterien gelten auch hier.

Auch diskriminierungsrelevante Entscheidungen des Nichttendenzbetriebes lassen sich im Einzelfall mit dessen Religions- bzw. Weltanschauungsfreiheit rechtfertigen.

Begründet der potenzielle Vertragspartner die Ablehnung eines Behinderten damit, daß ihm die Vertragsdurchführung aufgrund der Behinderung unmöglich ist - beispielsweise deshalb, weil kein behindertengerechter Zugang zum Arbeitsplatz vorhanden ist -, so hängt die Beantwortung der Frage der Rechtmäßig- bzw. Rechtswidrigkeit der Ablehnung davon ab, ob der „Diskriminierende“ verpflichtet war, dem Behinderten die Vertragsdurchführung zu ermöglichen. Das geltende Recht kennt derartige Ermöglichungspflichten etwa im Arbeitsrecht. Die Interessen des Verpflichteten werden dadurch gewahrt, daß ihm die Ermöglichungspflicht - vor allem in finanzieller Hinsicht zumutbar sein muß. Daß der „Diskriminierende“ in bestimmten Fällen verpflichtet sein soll, dem Behinderten die Vertragsdurchführung - etwa durch die Schaffung eines behindertengerechten Zugangs - möglich zu machen, ist interessengerecht, vergegenwärtigt man sich die besondere Situation behinderter Menschen, die dazu führt, daß sie in den meisten Fällen faktisch benachteiligt sind, sollte sich ihr Gleichbehandlungsanspruch darauf beschränken, formal wie Nicht-Behinderte behandelt zu werden.

Aus demselben Grund kann es notwendig sein, den „Diskriminierenden“ zu verpflichten, Vertragsbedingungen einem bestimmten Merkmal anzupassen. Das Institut der Vertragsanpassungspflicht ist dem deutschen Recht nicht unbekannt.

Ausgangspunkt der Überlegungen zur ,gesetzestechnischen Umsetzung des Diskriminierungsverbotes“ bildete die Feststellung, daß die Antidiskriminierungsrichtlinien zwingend auf legislativem Wege umgesetzt werden müssen; der deutsche Gesetzgeber seinem legislativen Handlungsauftrag durch das - am 18.08.2006 in Kraft getretene - Allgemeine Gleichbehandlungsgesetz ( $A G G$ ) formell nachgekommen ist.

Mit Blick vor allem auch auf den im Rahmen der Arbeit entwickelten Diskriminierungsbegriff stellte sich sodann die Frage, ob der Gesetzgeber eine inhaltlich gelungene Problemlösung entwickelt hatte. Das Gesetzeswerk wurde zu diesem Zweck eingehend untersucht und wie folgt beurteilt: Das AGG ist dadurch gekennzeichnet, daß es im wesentlichen die Vorgaben der Antidiskriminierungsrichtlinien $1: 1$ umsetzt. Da schon die Antidiskriminierungsrichtlinien kein umfassendes Diskriminierungsschutzkonzept verfolgen, bleibt auch das Gesetz insoweit lückenhaft. 
Auch inhaltlich bietet das AGG Anlaß zu erheblicher Kritik. Schließlich ist es auch handwerklich teilweise mißlungen. Das AGG kann nicht als Ergebnis einer gelungenen Gesetzgebung bezeichnet werden.

Da das AGG inhaltlich abgelehnt wurde, blieb die Frage nach der adäquaten Umsetzung des Diskriminierungsverbotes bestehen. Mit dem Ziel, der Beantwortung dieser Frage ein Stück näher zu kommen, wurden nun zwei Gesetzesentwürfe aus dem Jahr 1998 näher vorgestellt. Diese Entwürfe zeichneten sich - in krassem Gegensatz zum AGG - durch eine generalklauselartige Formulierung des Diskriminierungsverbotes aus. Damit verdeutlichten sie, daß es möglich sein mußte, das Diskriminierungsverbot - systematisch konsequent - ins BGB zu integrieren.

Die Gesetzesentwürfe-1998 bildeten so - unter Berücksichtigung sämtlicher im Rahmen der Arbeit gewonnenen Erkenntnisse - die Grundlage folgenden „Vorschlags für die gesetzestechnische Umsetzung des Diskriminierungsverbotes“ im Zivilrecht:

\section{$\S 115$ BGB-E}

(1) Unzulässig ist eine Willenserklärung, die den Erklärungsempfänger insbesondere aufgrund seiner tatsächlichen oder vermeintlichen Rasse oder ethnischen Herkunft, seines Geschlechts, seiner Religion oder Weltanschauung, seiner Behinderung, seines Alters oder seiner sexuellen Identität unsachlich behandelt (diskriminiert). Unzulässig ist zudem, eine Willenserklärung, der ein öffentliches Angebot vorausgeht, in diskriminierender Weise nicht abzugeben. Auf das öffentliche Angebot ist Satz 1 entsprechend anzuwenden. Das gleiche gilt für sonstige Maßnahmen, die ihrem Wesen nach einer Willenserklärung im Sinne des Satz 1 vergleichbar sind.

(2) Absatz 1 Satz 1 findet insbesondere keine Anwendung auf Willenserklärungen, die den Intimbereich oder den Privatbereich des Erklärenden betreffen. Satz 1 gilt entsprechend im Fall des Absatz 1 Satz 2.

(3) Eine Willenserklärung im Sinne des Absatz 1 Satz 1 ist nichtig, sofern nicht Interessen des Erklärungsempfängers oder eines Dritten dem entgegenstehen. Hat der Erklärungsempfänger ein Interesse an der Wirksamkeit der Willenserklärung, kann sich der Erklärende insoweit nicht auf den Inhalt der Willenserklärung berufen, als dieser den Erklärungsempfänger diskriminiert.

(4) Beweist der Erklärungsempfänger Tatsachen, die darauf schließen lassen, daß die Willenserklärung überwiegend wahrscheinlich eine solche im Sinne des Absatz 1 Satz 1 ist, obliegt dem Erklärenden der Beweis des Gegenteils. Dem Erklärenden obliegt in diesem Fall auch der Beweis dafür, daß er die Diskriminierung nicht zu vertreten hat. Satz 2 gilt im Falle der mittelbaren Diskriminierung jedoch nur dann, wenn der Erklärungsempfänger zuvor Tatsachen beweist, die darauf schließen lassen, daß der Erklärende die mittelbare Diskriminierung überwiegend wahrscheinlich zu vertreten hat. Satz 1 gilt entsprechend im Fall des Absatz 1 Satz 2.

$\S 115$ BGB-E steht in einer besonderen Beziehung zu folgenden Bestimmungen des BGB: Daß $\S$ 115 BGB-E auf unentgeltliche Zuwendungen keine Anwendung findet, wäre - im Falle seiner Einführung ins BGB - in den $\S \S 516$ ff. BGB ausdrücklich zu regeln.

$\S 115$ BGB-E gewährt keinen Belästigungsschutz. Dieser ist durch § 823 I BGB zu realisieren. 
$\S 115$ BGB-E ist ein Schutzgesetz im Sinne des $\S 823$ II BGB. Ein Verstoß gegen das Diskriminierungsverbot des $\S 115$ BGB-E führt zu einem Schadensersatzanspruch aus $\S 823$ II BGB i. V. m. § 115 BGB-E. Inhalt und Umfang dieses Anspruchs bemessen sich nach den $\S \S 249$ ff. BGB. Vermögensschäden hat der Diskriminierende nach Maßgabe der $\S \S 249-252$ BGB voll zu ersetzen.

Vor dem Hintergrund der Erkenntnis, daß Diskriminierung vielfach keine oder nur sehr geringe Vermögensschäden verursacht, die Richtlinien aber Sanktionen fordern, die wirksam, verhältnismäßig und abschreckend sind, wurde vorgeschlagen, § 253 II BGB um den Begriff der Diskriminierung zu erweitern.

\section{$\S 253$ II BGB-E}

Ist wegen einer Verletzung des Körpers, der Gesundheit, der Freiheit oder der sexuellen Selbstbestimmung oder wegen Verstoßes gegen das Diskriminierungsverbot Schadensersatz zu leisten, kann auch wegen des Schadens, der nicht Vermögensschaden ist, eine billige Entschädigung in Geld gefordert werden.

Beide Ansprüche - sowohl der auf Ersatz des Vermögens- als auch der auf Ersatz des Nichtvermögensschadens - setzen schuldhaftes Handeln des Diskriminierenden voraus. Das ergibt sich aus $\S 823$ II 2 BGB.

Bei Vorliegen der speziellen Voraussetzungen kommen neben dem Schadensersatzanspruch aus $\S$ 823 II BGB in Verbindung mit $\S 115$ BGB-E auch solche aus den $\S \S 280$ und 826 BGB in Betracht. $\S 1004$ I BGB analog gewährt dem Diskriminierten einen verschuldensunabhängigen Beseitigungsund Unterlassungsanspruch. Bei Vorliegen der entsprechenden Voraussetzungen kann aus dieser Bestimmung u. a. auch ein Anspruch auf Vertragsschluß hergeleitet werden.

Die Ausführungen endeten mit folgenden Gedanken zur praktischen Bedeutung des $§ 115$ BGB-E: Es ist zu vermuten, daß eine Vielzahl von Diskriminierungen unerkannt bleiben werden, weil der Diskriminierende geschickt genug sein wird, sein diskriminierendes Verhalten als nichtdiskriminierendes zu tarnen. Oft wird der Diskriminierte deshalb einen Prozeß gar nicht erst anstrengen bzw. die Beweislastumkehr nicht herbeiführen können. 
Abschließend bleibt folgendes anzumerken: Die Arbeit hat sich nicht mit allen Fragen beschäftigt, die sich im Zusammenhang mit der Umsetzung der Antidiskriminierungsrichtlinien stellen, ${ }^{1}$ sondern lediglich mit den angesichts der gesetzten Aufgabenstellung bedeutsamen. Sie erhebt insofern nicht den Anspruch, umfassend zu sein.

Die Frage der Statuierung eines adäquaten Diskriminierungsschutzes im Zivilrecht hat sich durch das Inkrafttreten des AGG am 18.08.2006 nicht erledigt. So wird derzeit - 2007 - beispielsweise über den Entwurf eines Arbeitsvertragsgesetzes (ArbVG) ${ }^{2}$ diskutiert, der unter anderem Regeln zum Diskriminierungsschutz enthält, insofern aber ein gegenüber dem AGG eigenständiges Konzept verfolgt. $^{3}$

1 Vergleiche dazu etwa folgende Bestimmungen der Richtlinie 2000/43/EG: Art. 7 II (,Verbandsklagerecht“), Art. 11 (,Sozialer Dialog“), Art. 12 („Dialog mit Nichtregierungsorganisationen“), Art. 13 („Mit der Förderung der Gleichbehandlung befaßte Stellen").

2 Vergleiche dazu www.arbvg.de.

3 „Schlanke sechs Paragraphen reichen den Wissenschaftlern aus, um 33 AGG-Normen zusammenzufassen.“ (Budras in FAZ vom 27.01.2007, S. 11, unter dem Titel: „Aufräumen im Arbeitsrecht“). 



\section{Literaturverzeichnis:}

Adomeit, Klaus:

Adomeit, Klaus:

Adomeit, Klaus:

Adomeit, Klaus:

Alexy, Robert:

Ascheid, Reiner;

Preis, Ulrich;

Schmidt, Ingrid:

Baer, Susanne:

Baer, Susanne:

Bauer, Jobst-Hubertus:

Bezzenberger, Tilman:

Bittner, Claudia:

Bleckmann, Albert:

Böckenförde,

Ernst-Wolfgang:

Braun, Johann:

Brox, Hans:

Brox, Hans:

Brox, Hans;

Walker, Wolf-Dietrich:
Rechtsquellenfragen im Arbeitsrecht, 1. Auflage, München 1969

Gleichstellung von Mann und Frau bei Einstellung von Arbeitnehmern, in: DB 1980, S. 2388 f.

Arbeitsrecht auf Abwegen, in: NJW 1997, S. 2295 f.

Diskriminierung - Inflation eines Begriffs, in: NJW 2002, S. $1622 \mathrm{f}$.

Theorie der Grundrechte, 1. Auflage, Baden-Baden 1985

Kündigungsrecht, Großkommentar zum gesamten Recht der Beendigung von Arbeitsverhältnissen, 2. Auflage, München 2004

Würde oder Gleichheit? - Zur angemessenen grundrechtlichen Konzeption von Recht gegen Diskriminierung am Beispiel sexueller Belästigung am Arbeitsplatz in der Bundesrepublik Deutschland und den USA, 1. Auflage, Baden-Baden 1995

„Ende der Privatautonomie“ oder grundrechtlich fundierte Rechtsetzung?, in: ZRP 2002, S. 290 ff.

Europäische Antidiskriminierungsrichtlinien und ihr Einfluß auf das deutsche Arbeitsrecht, in: NJW 2001, S. 2672 ff.

Ethnische Diskriminierung, Gleichheit und Sittenordnung im Bürgerlichen Recht, in: AcP 196 (1996), S. 395 ff.

„Islamisches Kopftuch“ als Grund zur ordentlichen Kündigung einer Verkäuferin?, in: JURA 2004, S. 39 ff.

Europarecht, 6. Auflage, Köln, Berlin, Bonn, München 1997

„Kopftuchstreit“ auf dem richtigen Weg?, in: NJW 2001, S. 723 ff.

Forum: Übrigens - Deutschland wird wieder totalitär, in: JuS 2002, S. $424 \mathrm{f}$.

Allgemeiner Teil des BGB, 28. Auflage, Köln, Berlin, München 2004

Erbrecht, 19. Auflage, Köln, Berlin, Bonn, München 2001

Allgemeines Schuldrecht, 29. Auflage, München 2003 
Buchner, Herbert;

Becker, Ulrich:

Bydlinski, Franz:

Caspar, Johannes:

Calliess, Christian;

Ruffert, Matthias:

Cirkel, Johannes:

Classen, Claus Dieter:

Coen, Martin:

Danner, Wolfgang;

Theobald, Christian:

Däubler, Wolfgang:

Däubler, Wolfgang:

Delbrück, Jost:

Dieterich, Thomas;

Müller-Glöge, Rudi;

Preis, Ulrich;

Schaub, Günter:

Dolzer, Rudolf;

Vogel, Klaus;

Graßhof, Karin:

Dreier, Horst:
Mutterschutzgesetz und Bundeserziehungsgeldgesetz (Kommentar), 7. Auflage, München 2003

$\mathrm{Zu}$ den dogmatischen Grundfragen des Kontrahierungszwanges, in: AcP 180, S. 1 ff.

Das Diskriminierungsverbot behinderter Personen nach Art. 3 Abs. 3 S. 2 GG und seine Bedeutung in der aktuellen Rechtsprechung, in: EuGRZ 2000, S. 135 ff.

EUV/EGV (Kommentar), 2. Auflage, Neuwied, Kriftel 2002

Gleichheitsrechte im Gemeinschaftsrecht, in: NJW 1998, S. 3332 f.

Wie viele Wege führen zur Gleichberechtigung von Männern und Frauen? - Gemeinsamkeiten und Unterschiede von deutschem und europäischem Recht, in: JZ 1996, S. 921 ff.

Neue EU-Antidiskriminierungs-Richtlinien, in: AuR 2000, S. 11 f.

Energierecht (Kommentar), Band 1, 47. Ergänzungslieferung (Stand Mai 2004), München 2004

Das geplante Teilzeit- und Befristungsgesetz, in: ZIP 2000, S. 1961 ff.

Das neue Teilzeit- und Befristungsgesetz, in: ZIP 2001, S. 217 ff.

Die Rassenfrage als Problem des Völkerrechts und nationaler Rechtsordnungen, 1. Auflage, Frankfurt am Main 1971

Erfurter Kommentar zum Arbeitsrecht, 5. Auflage, München 2005

Bonner Kommentar zum Grundgesetz, 119. Aktualisierung (September 2005), Heidelberg 2005

Grundgesetz (Kommentar), Band I (Präambel, Artikel 1-19), 2. Auflage, Tübingen 2004; Band II (Artikel 20-82), 1. Auflage, Tübingen 1998 
Ehmann, Horst;

Emmert, Angela:

Eich, Rolf-Achim:

Enneccerus, Ludwig;

Nipperdey, Hans Carl:

Fabricius, Fritz;

Kraft, Alfons;

Wiese, Günther;

Kreutz, Peter;

Oetker, Hartmut,

Raab, Thomas;

Weber, Christoph:

Fahr, Ulrich;

Kaulbach, Detlef:

Fitting, Karl;

Kaiser, Heinrich;

Heither, Friedrich;

Engels, Gerd;

Schmidt, Ingrid:

Flume, Werner:

Friauf, Karl Heinrich;

Höfling, Wolfram:

Fromm, Günter;

Fey, Michael;

Sellmann, Klaus-Albrecht;

Zuck, Holger:

Frowein, Jochen Abraham:
Grundlage, Zweck und Mißbrauch der Sanktionierung des Diskriminierungsverbots - kritische Bemerkungen zum Diskriminierungsurteil des EuGH vom 22.04.1997 - Rs. C-180/95, in: SAE 1997, S. $253 \mathrm{ff}$.

Das Gesetz über die Gleichbehandlung von Männern und Frauen am Arbeitsplatz, in: NJW 1980, S. 2329 ff.

Allgemeiner Teil des Bürgerlichen Rechts (Lehrbuch), 1. Band, 1. Halbband, 15. Auflage, Tübingen 1959

GK-BetrVG, Gemeinschaftskommentar zum Betriebsverfassungsgesetz, Band II, 7. Auflage, Neuwied, Kriftel 2002

Versicherungsaufsichtsgesetz / Gesetz über die Errichtung eines Bundesaufsichtsamtes für Versicherungswesen (Kommentar), 2. Auflage, München 1997

Betriebsverfassungsgesetz (Handkommentar), 21. Auflage, München 2002

Allgemeiner Teil des Bürgerlichen Rechts, Zweiter Band (Das Rechtsgeschäft), 4. Auflage, Berlin, Heidelberg, New York, London, Paris, Tokyo, Hong Kong, Barcelona, Budapest 1992

Berliner Kommentar zum Grundgesetz, Band 1, 10. Ergänzungslieferung, Berlin 2004

Personenbeförderungsrecht (Kommentar), 3. Auflage, München 2001

Die Überwindung von Diskriminierung als Staatsauftrag in Art. 3 Abs. 3 GG, in: Festschrift für Hans F. Zacher, S. 157 ff., Heidelberg 1998 
Frowein, Jochen Abraham; Peukert, Wolfgang:

Gebauer, Jochen:

Geck, Barbara;

Schimmel, Roland:

Gersdorf, Hubertus:

Grabitz, Eberhard; Hilf, Meinhard:

Graf Vitzthum, Wolfgang:

Gramlich, Ludwig:

Hadeler, Indra:

Haedrich, Martina:

Hager, Johannes:

Hagmann, Joachim:

Hähnel, Manfred:

Hanau, Peter:

Herdegen, Matthias:

Hirsch, Günter:
Europäische Menschenrechtskonvention (EMRK-Kommentar), 2. Auflage, Kehl am Rhein, Straßburg, Arlington 1996

Die Grundfreiheiten des EG-Vertrages als Gemeinschaftsgrundrechte, Berlin 2004

Grenzen der Kündigung kirchlicher Arbeitsverhältnisse Glaubwürdigkeit der Verkündung kontra Menschenwürde des Arbeitnehmers?, in: ArbuR 1995, S. 177 ff.

Funktionen der Gemeinschaftsgrundrechte im Lichte des Solange II-Beschlusses des Bundesverfassungsgerichts, in: AöR 119, Tübingen 1994, S. 400 ff.

Das Recht der Europäischen Union (Kommentar), Band I (EUV/EGV), 23. Ergänzungslieferung (Januar 2004), München 2004

Völkerrecht, 2. Auflage, Berlin, New York 2001

Grundfreiheiten contra Grundrechte im Gemeinschaftsrecht?, in: DÖV 1996, S. $801 \mathrm{ff}$.

Die Revision der Gleichbehandlungsrichtlinie 76/207/EWG Umsetzungsbedarf für das deutsche Arbeitsrecht, in: NZA 2003, S. $77 \mathrm{ff}$.

Rassismusbekämpfung nach Völkerrecht und innerstaatliche Wirkungen, in: JA 2003, S. 899 ff.

Grundrechte im Privatrecht, in: JZ 1994, S. 373 ff.

Das Verbot der Benachteiligung Behinderter gemäß Art. 3 Abs. 3 S. 2 GG und seine Auswirkungen auf das Zivilrecht, 1. Auflage, Aachen 1999

Situation der ausländischen Arbeitnehmer und ihrer Familienangehörigen in der Bundesrepublik Deutschland, in: ZAR 2002, S. $356 \mathrm{ff}$.

Frauenförderung bei Ausschreibung und Besetzung von Arbeitsplätzen im deutschen und europäischen Recht, in: Gedächtnisschrift für Alexander Lüderitz, S. 241 ff., München 2000

Europarecht, 3. Auflage, München 2001

Die Grundrechte in der Europäischen Union, in: RdA 1998, S. 194 ff. 
Herrmann, Elke:

Hoffmann, Lutz:

Hornung-Draus, Renate:

Immenga, Ulrich;

Mestmäcker, Ernst-Joachim:

Ipsen, Knut:

Jarass, Hans D.;

Pieroth, Bodo:

Jauernig, Othmar:

Jochum, Georg:

Junker, Abbo:

Kittner, Michael;

Däubler, Wolfgang;

Zwanziger, Bertram:

Klein, Hans Hugo:

Kossens, Michel;

von der Heide, Dirk;

Maaß, Michael:

Kühner, Rolf:

Lange, Heinrich;

Kuchinke, Kurt:

Langenfeld, Christine:

Larenz, Karl:
Die Abschlußfreiheit - ein gefährdetes Prinzip, Zugleich der Versuch einer dogmatischen Erfassung der vorvertraglichen Regelungen des $\S 611$ a BGB, in: ZfA 1996, S. 19 ff.

Ausländerbeiräte in der Krise, in: ZAR 2002, S. 63 ff.

Diskriminierungsverbot - Ein EU-Vorschlag birgt Zündstoff, in: Arbeitgeber 2000, S. $14 \mathrm{f}$.

GWB - Gesetz gegen Wettbewerbsbeschränkungen (Kommentar), 3. Auflage, München 2001

Völkerrecht, 5. Auflage, München 2004

Grundgesetz (Kommentar), 7. Auflage, München 2004

Bürgerliches Gesetzbuch (Kommentar), 10. Auflage, München 2003

Der neue Art. 13 EGV oder ,political correctness“ auf europäisch?, in: ZPR 1999 S. 279 ff.

Grundkurs Arbeitsrecht, 4. Auflage, München 2004

Kündigungsschutzrecht, 5. Auflage, Frankfurt/Main 2001

Öffentliche und private Freiheit - Zur Auslegung des Grundrechts der Meinungsfreiheit, in: Der Staat 10 (1971), S. 145 ff.

Praxiskommentar zum Behindertenrecht (SGB IX) - Rehabilitation und Teilhabe behinderter Menschen, 1. Auflage, München 2002

Das Recht auf Zugang zu Gaststätten und das Verbot der Rassendiskriminierung, in: NJW 1986, S. 1397 ff.

Lehrbuch des Erbrechts, 4. Auflage, München 1995

Die Gleichbehandlung von Mann und Frau im Europäischen Gemeinschaftsrecht, Baden-Baden 1990

Lehrbuch des Schuldrechts, Erster Band, Allgemeiner Teil, 13. Auflage, München 1982 
Larenz, Karl;

Wolf, Manfred:

Leinemann, Wolfgang:

Leisner, Walter:

Lenz, Carl Otto:

Lorenz, Martin:

Mager, Ute:

Maaß, Rainer;

Hitpaß, Peter:

Maunz, Theodor;

Dürig, Günter:

Medicus, Dieter:

Mikat, Paul:

Neumann, Dirk;

Pahlen, Ronald;

Majerski-Pahlen, Monika:

Nickel, Rainer:

Nickel, Rainer:

Nipperdey, Hans Carl:

Nipperdey, Hans, Carl:
Allgemeiner Teil des Bürgerlichen Rechts, 9. Auflage, München 2004

Kasseler Handbuch zum Arbeitsrecht, Band 2, 2. Auflage, Neuwied, Kriftel 2000

Grundrechte und Privatrecht, München 1960

EG-Vertrag (Kommentar), 2. Auflage, Köln, Basel, Genf, München, Wien 1999

Gesetz über die Gleichbehandlung von Männern und Frauen am Arbeitsplatz und über die Erhaltung von Ansprüchen bei Betriebsübergang, in: DB 1980, S. 1745 ff.

Möglichkeiten und Grenzen rechtlicher Maßnahmen gegen die Diskriminierung von Ausländern, in: ZAR 1992, S. 170 (173)

Entwicklung der Parabolantennen-Rechtsprechung seit 2000, in: NZM 2003, S. $181 \mathrm{ff}$.

Grundgesetz (Kommentar), Band I (Art. 1-5), 42. Ergänzungslieferung (Februar 2003), München 2003; Band II (Art. 6-16 a), 43. Ergänzungslieferung (Februar 2004), München 2004

Bürgerliches Recht, 20. Auflage, Köln, Berlin, München 2004

Gleichheitsgrundsatz und Testierfreiheit, in: Festschrift für Hans Carl Nipperdey, Band I, München, Berlin 1965, S. 581 ff.

Sozialgesetzbuch IX - Rehabilitation und Teilhabe behinderter Menschen (Kommentar), 10. Auflage, München 2003

Gleichheit und Differenz in der vielfältigen Republik (Plädoyer für ein erweitertes Antidiskriminierungsrecht), 1. Auflage, BadenBaden 1999

Handlungsaufträge zur Bekämpfung von ethnischen Diskriminierungen in der neuen Gleichbehandlungsrichtlinie 2000/43/EG, in: NJW 2001, S. 2668 ff.

Gleicher Lohn der Frau für gleiche Leistung, in: RdA 1950, S. 121 ff.

Grundrechte und Privatrecht, in: Staatsrechtliche Abhandlungen verschiedener Autoren, Sammelband 6, Heft 2, Krefeld 1961 
Nowak, Manfred:

Nussberger, Angelika:

Oberheim, Rainer:

Oetker, Hartmut:

Otto, Hansjörg:

Otto, Hansjörg:

Palandt, Otto:

Partsch, Karl Josef:

Pfarr, Heide M.;

Bertelsmann, Klaus:

Pieroth, Bodo;

Schlink, Bernhard:

Preis, Ulrich:

Preis, Ulrich:

Preis, Ulrich:

Preis, Ulrich;

Gotthardt, Michael:

Raab, Thomas:
UNO-Pakt über bürgerliche und politische Rechte und Fakultativprotokoll (CCPR-Kommentar), 1. Auflage, Kehl am Rhein, Straßburg, Arlington 1989

Altersgrenzen als Problem des Verfassungsrechts, in: JZ 2002, S. $524 \mathrm{ff}$.

Zivilprozeßrecht für Referendare, 6. Auflage, Düsseldorf 2004

Gibt es einen Kündigungsschutz außerhalb des Kündigungsschutzgesetzes?, in: ArbuR 1997, S. 41 (52)

Personale Freiheit und soziale Bindung, 1. Auflage, München 1978

Arbeitsrecht, 3. Auflage, Berlin 2003

Bürgerliches Gesetzbuch (Kommentar), 58. Auflage, München 1999; 64. Auflage, München 2005

Die Strafbarkeit der Rassendiskriminierung nach dem Internationalen Abkommen und die Verwirklichung der Verpflichtungen in nationalen Strafrechtsordnungen, in: German Yearbook of International Law (GYIL), Volume 20 (1977), Berlin 1978, S. 119 ff.

Diskriminierung im Erwerbsleben - Ungleichbehandlungen von Frauen und Männern in der Bundesrepublik Deutschland, 1. Auflage, Baden-Baden 1989

Grundrechte, Staatsrecht II, 19. Auflage, Heidelberg 2003

Prinzipien des Kündigungsrechts bei Arbeitsverhältnissen, 1. Auflage, München 1987

Der Kündigungsschutz außerhalb des Kündigungsschutzgesetzes, in: NZA 1997, S. 1256 ff.

Arbeitsrecht (Praxis-Lehrbuch zum Individualarbeitsrecht), 1. Auflage, Köln 1999

Neuregelung der Teilzeitarbeit und befristeten Arbeitsverhältnisse Zum Gesetzesentwurf der Bundesregierung, in: DB 2000, S. 2065 ff.

Das Fragerecht des Arbeitgebers nach schwebenden Strafverfahren und die Unschuldsvermutung des Bewerbers, in: RdA 1995, S. 36 ff. 
Rebmann, Kurt;

Säcker, Franz Jürgen:

Rebmann, Kurt;

Säcker, Franz Jürgen;

Rixecker, Roland:

Remmert, Barbara:

Richardi, Reinhard:

Richardi, Reinhard;

Wlotzke, Otfried:

Roellecke, Gerd:

Roth, Wulf-Henning:

Sachs, Michael:

Säcker, Franz-Jürgen:

Schiek, Dagmar:

Schiek, Dagmar:

Schlüter, Wilfried:

Schmidt, Karsten:

Schmoeckel, Mathias:

Schulze, Reiner;

Dörner, Heinrich;

Ebert, Ina; Eckert, Jörn;

Hoeren, Thomas;

Kemper, Rainer;

Saenger, Ingo;

Schulte-Nölke, Hans;

Staudinger, Ansgar:
Münchener Kommentar zum Bürgerlichen Gesetzbuch, Band 1 ( $\S$ 1-240, AGB-Gesetz), 3. Auflage, München 1993; Band 3, 1. Halbband (§§ 433-651 k), 2. Auflage, München 1988

Münchener Kommentar zum Bürgerlichen Gesetzbuch, Band 4 ( $\S$ 611-704, EFZG, TzBfG, KSchG), 4. Auflage, München 2005

Grundfreiheiten und Privatrechtsordnung, in: JURA 2003, S. 13 ff.

Betriebsverfassungsgesetz mit Wahlordnung (Kommentar), 9. Auflage, München 2004

Münchener Handbuch zum Arbeitsrecht, Band 1 (Individualarbeitsrecht I), 2. Auflage, München 2000

Antidiskriminierung auf europäisch, in: NJW 1996, S. 3261 f.

Drittwirkung der Grundfreiheiten, in: Festschrift für Ulrich Everling, Band II, Baden-Baden 1995

Grundgesetz (Kommentar), 3. Auflage, München 2003

„Vernunft statt Freiheit!“ - Die Tugendrepublik der neuen Jakobiner, in: ZRP 2002, S. 286 ff.

Draehmpaehl und die Folgen - Gesetzentwurf zum Schadensersatz bei Einstellungs- und Beförderungsdiskriminierung (Geschlechtsdiskriminierung), in: BB 1998, S. 586 f.

Differenzierte Gerechtigkeit (Diskriminierungsschutz und Vertragsrecht), 1. Auflage, Baden-Baden 2000

Erbrecht, 12. Auflage, München 1986

Handelsrecht, 5. Auflage, Köln, Berlin, Bonn, München 1999

Anmerkung zu BGH, JZ 1999, S. 514 ff., in: JZ 1999, S. 517 ff.

Bürgerliches Gesetzbuch (Handkommentar), 3. Auflage, BadenBaden 2003 
Schwabe, Jürgen:

Schwabe, Jürgen:

Schwarze, Jürgen:

Schweitzer, Michael:

Schweitzer, Michael;

Hummer, Waldemar:

Seidl-Hohenveldern, Ignaz;

Stein, Torsten:

Sen, Faruk;

Sauer, Martina;

Halm, Dirk:

Soergel, Hs. Th.;

Siebert, W:

Steindorff, Ernst:

Stieglitz, Edgar:

Stock, Remmert A.:

Streinz, Rudolf:

Streinz, Rudolf:

Streinz, Rudolf;

Leible, Stefan:

Szczekalla, Peter:

Szczekalla, Peter:
Die sogenannte Drittwirkung der Grundrechte - Zur Einwirkung der Grundrechte auf den Privatrechtsverkehr, München 1971

„Drittwirkung“ und kein Ende, in: NJW 1973, S. 229 f.

EU-Kommentar, 1. Auflage, Baden-Baden 2000

Staatsrecht III - Staatsrecht, Völkerrecht, Europarecht, 8. Auflage, Heidelberg 2004

Europarecht, 5. Auflage, Neuwied, Kriftel, Berlin 1996

Völkerrecht, 10. Auflage, Köln, Berlin, Bonn, München 2000

Integration oder Abschottung? Zur Situation türkischer Zuwanderer in Deutschland, in: ZAR 2001, S. 214 ff.

Bürgerliches Gesetzbuch (Kommentar), Band 2 (§§ 241-432), 12. Auflage, Stuttgart, Berlin, Köln 1990, Band 4/1 (§§ 516-651), 12. Auflage, Stuttgart, Berlin, Köln 1998; Band 21 (§§ 1922-2063), 13. Auflage, Stuttgart 2001

Gleichbehandlung von Mann und Frau nach dem EG-Recht, in: RdA 1988, S. 129 ff.

Allgemeine Lehren im Grundrechtsverständnis nach der EMRK und der Grundrechtsjudikatur des EuGH - Zur Nutzbarmachung konventionsrechtlicher Grundrechtsdogmatik im Bereich der Gemeinschaftsgrundrechte, 1. Auflage, Baden-Baden 2002

Zugang zu Gaststätten und Rassendiskriminierung, in: ZAR 1999, S. $118 \mathrm{ff}$.

Europarecht, 6. Auflage, Heidelberg 2003

EUV/EGV (Kommentar), 1. Auflage, München 2003

Die unmittelbare Drittwirkung der Grundfreiheiten, in: EuZW 2000, S. $459 \mathrm{ff}$.

Anmerkung zu EuGH, EuZW 1998, S. 212 ff., in: EuZW 1998, S. $215 \mathrm{ff}$.

Die sogenannten grundrechtlichen Schutzpflichten im deutschen und europäischen Recht, 1. Auflage, Berlin 2002 
Thielmann, Georg:

Thomas, Heinz;

Putzo, Hans:

Thüsing, Gregor:

Thüsing, Gregor:

Thüsing, Gregor:

Thüsing, Gregor:

Thüsing, Gregor:

Timme, Michael:

Tomuschat, Christian:

Tomuschat, Christian:

Triebel, Matthias:

Umbach, Dieter C.;

Clemens, Thomas:

Verdross, Alfred;

Simma, Bruno:

Von der Groeben, Hans;

Schwarze, Jürgen:
Sittenwidrige Verfügungen von Todes wegen, 1. Auflage, Berlin 1973

Zivilprozeßordnung, 27. Auflage, München 2005

Zulässige Ungleichbehandlung weiblicher und männlicher Arbeitnehmer - Zur Unverzichtbarkeit i. S. des § 611a Abs. 1 Satz 2 BGB, in: RdA 2001, S. 319 ff.

Der Fortschritt des Diskriminierungsschutzes im Europäischen Arbeitsrecht - Anmerkungen zu den Richtlinien 2000/43/EG und 2000/78/EG, in: ZfA 2001, S. 397 ff.

Handlungsbedarf im Diskriminierungsrecht - Die Umsetzungserfordernisse auf Grund der Richtlinien 2000/78/EG und 2000/43/EG, in: NZA 2001, S. $1061 \mathrm{ff}$.

Vom Kopftuch als Angriff auf die Vertragsfreiheit, in: NJW 2003, S. $405 \mathrm{ff}$.

Richtlinienkonforme Auslegung und unmittelbare Geltung von EGRichtlinien im Anti-Diskriminierungsrecht, in: NJW 2003, S. 3441 ff.

Rechtliche Behandlung von Zutrittsverweigerungen gegenüber Ausländern im Gaststättengewerbe, in: ZAR 1997, S. 130 ff.

Der Verfassungsstaat im Geflecht der internationalen Beziehungen, in: Veröffentlichungen der Vereinigung der Deutschen Staatsrechtslehrer, Heft 36, Berlin, New York 1978, S. 7 ff.

Equality and Non-Discrimination under the International Covenant on Civil and Political Rights, in: Festschrift für Hans-Jürgen Schlochauer, Berlin, New York 1981 S. 691 ff.

Die rechtliche Bedeutung der Grundrechtecharta, in: JURA 2003, S. $525 \mathrm{ff}$.

Grundgesetz (Mitarbeiterkommentar und Handbuch), Band I (Art. 1-37 GG), 1. Auflage, Heidelberg 2002

Universelles Völkerrecht, 3. Auflage, Berlin 1984

EUV/EGV (Kommentar), Band 3 (Art. 98-188 EGV), 6. Auflage, Baden-Baden 2003; Band 4 (Art. 189-314 EGV), 6. Auflage, Baden-Baden 2004 
Von der Groeben, Hans;

Thiesing, Jochen;

Ehlermann, Claus-Dieter:

Von Hoyningen-Huene,

Gerrick; Linck, Rüdiger:

Von Koppenfels, Katharina:

Von Lübtow, Ulrich:

Von Mangoldt, Hermann;

Klein, Friedrich;

Starck, Christian:

Von Münch, Ingo:

Von Münch, Ingo;

Kunig, Philip:

Von Staudinger, Julius:

Waas, Bernd:

Wandt, Manfred:

Weiss, Manfred:

Westermann, Harm Peter:
EUV/EGV (Kommentar), Band 3 (Art. 102 a-136 a EGV), 5. Auflage, Baden-Baden 1999

Kündigungsschutzgesetz (Kommentar), 13. Auflage, München 2002

Das Ende der Vertragsfreiheit? - Erkenntnisse aus dem vorläufig gescheiterten zivilrechtlichen Anti-Diskriminierungsgesetz für die Umsetzung der Richtlinien 2000/43/EG und 2000/78/EG -, in: WM 2002, S. 1489 ff.

Erbrecht, 1. Halbband, 1. Auflage, Berlin 1971

Grundgesetz (Kommentar), Band 1 (Präambel, Artikel 1 bis 19), 4. Auflage, München 1999

Antidiskriminierungsgesetz - notwendig oder überflüssig?, in: NJW 1999, S. $260 \mathrm{ff}$.

Grundgesetz (Kommentar), Band 1 (Präambel bis Art. 19), 5. Auflage, München 2000; Band 2 (Art. 20 bis 69), 4./5. Auflage, München 2001

Bürgerliches Gesetzbuch (Kommentar), Erstes Buch, allgemeiner Teil ( $\S ~ 134-163), 13$. Auflage, Berlin 1996; Zweites Buch, Recht der Schuldverhältnisse ( $\S 433-534), 13$. Auflage, Berlin 1995, ( $\S$ 611-615), 13. Auflage, Berlin 1999 und 14. Auflage, Berlin 2005, ( $\S 823-825), 13$. Auflage, Berlin 1999; Fünftes Buch, Erbrecht ( $\S$ 2064-2196), 12. Auflage, Berlin 1989, 14. Auflage, Berlin 2003

Die neue EG-Richtlinie zum Verbot der Diskriminierung aus rassischen oder ethnischen Gründen im Arbeitsverhältnis, in: ZIP 2000, S. $2151 \mathrm{ff}$.

Geschlechtsabhängige Tarifierung in der privaten Krankenversicherung - Gebietet die Verfassung Unisex-Tarife?, in: VersR 2004, S. $1341 \mathrm{ff}$.

Die Kirchen und ihre Einrichtungen als Tendenzbetriebe, in: ArbuR, Sonderheft 1979, S. 28 ff.

Erman, Bürgerliches Gesetzbuch (Kommentar), Band I (§§ 1- 811 BGB; UKlaG), 11. Auflage, Köln, Münster 2004 
Wetter, Irmgard:

Wiedemann, Herbert; Thüsing, Gregor:

Wiese, Günther:

Wissenschaftlicher Rat der Dudenredaktion (Drosdowski, Günther; Köster, Rudolf; Müller, Wolfgang; Scholze-Stubenrecht, Werner):

Wissenschaftlicher Rat Der Dudenredaktion:

Wollenschläger, Michael

Zimmer, Maximilian:
Die Grundrechtscharta des Europäischen Gerichtshofes - Die Konkretisierung der gemeinschaftlichen Grundrechte durch die Rechtsprechung des EuGH zu den allgemeinen Rechtsgrundsätzen, 1. Auflage, Frankfurt am Main, Berlin, Bern, New York, Paris, Wien 1998

Fragen zum Entwurf eines zivilrechtlichen AntiDiskriminierungsgesetzes, in: DB 2002, S. 463 ff.

Verbot der Benachteiligung wegen des Geschlechts bei der Begründung eines Arbeitsverhältnisses - BAG, Betr 1989, 2279, in: JuS 1990, S. 357 ff.

Der Duden in 10 Bänden, Band 8 (Sinn- und sachverwandte Wörter), 1. Auflage, Mannheim, Wien, Zürich 1972; Band 10 (Bedeutungswörterbuch), 2. Auflage, Mannheim, Wien, Zürich 1985

Duden, Das große Wörterbuch der deutschen Sprache, Band 2 (Bedr - Eink), Band 3 (Einl - Geld), Band 10 (Vide - Zz), 3. Auflage, Mannheim, Leipzig, Wien, Zürich 1999

Rechtsfragen eines Konzeptes gegen Ausländerdiskriminierung, in: ZAR 1994, S. 10 ff.

Diskriminierung wegen des Geschlechts bei der Einstellung von Arbeitnehmern und Art. 3 II GG, in: NJW 1994, S. 1203 ff. 
Lebenslauf des Verfassers:

Geburtstag / -ort:

Staatsangehörigkeit:

\section{Schulausbildung:}

1982-90

1990-95

Dienstzeit:

\section{Studium:}

WS 1996/97

WS 1999/2000 u. SS 2000

03/2001-02/2002

$10 / 2002-04 / 2003$

WS 2003/2004

$12 / 2005$

10/2007 - 03/2008
29.04.1976 in Wernigerode als Sohn von Dr. med. Michael Zeckei und Dr. med. Monika Zeckei

deutsch

Polytechnische Oberschule August-Hermann-Francke Wernigerode

Carl-Friedrich-Gauß-Gymnasium Schwedt/Oder Abschluß: Allgemeine Hochschulreife

01.07.1995 - 30.04.1996 (zuletzt in der Topographiebatterie 400 der Uckermark-Kaserne Prenzlau)

Beginn des Studiums der Rechtswissenschaften an der GeorgAugust-Universität Göttingen

Studium der Rechtswissenschaften an der Universidad de Granada (Spanien) im Rahmen des ErasmusAustauschprogramms

Teilnahme an einem juristischen Repetitorium zur Vorbereitung auf das erste Staatsexamen

Abschluß des Studiums durch Ablegen der ersten juristischen Staatsprüfung

Beginn der Promotion

Beginn des Referendariats im Landgerichtsbezirk Göttingen

Abschluß des Referendariats durch Ablegen der zweiten juristischen Staatsprüfung 
